\title{
A spatiotemporal model for snow crab (Chionoecetes opilio) stock size in the southern Gulf of St. Lawrence
}

\author{
Cadigan, Noel G.; Wade, Elmer; Nielsen, Anders
}

Published in:

Canadian Journal of Fisheries and Aquatic Sciences

Link to article, DOI:

$10.1139 /$ cjfas-2016-0260

Publication date:

2017

Document Version

Peer reviewed version

Link back to DTU Orbit

Citation (APA):

Cadigan, N. G., Wade, E., \& Nielsen, A. (2017). A spatiotemporal model for snow crab (Chionoecetes opilio) stock size in the southern Gulf of St. Lawrence. Canadian Journal of Fisheries and Aquatic Sciences, 74(11), 1808-1820. https://doi.org/10.1139/cjfas-2016-0260

\section{General rights}

Copyright and moral rights for the publications made accessible in the public portal are retained by the authors and/or other copyright owners and it is a condition of accessing publications that users recognise and abide by the legal requirements associated with these rights.

- Users may download and print one copy of any publication from the public portal for the purpose of private study or research.

- You may not further distribute the material or use it for any profit-making activity or commercial gain

- You may freely distribute the URL identifying the publication in the public portal 


\section{Canadian Journal of Fisheries and Aquatic Sciences}

Canadian Science Publishing

Journal canadien des sciences halieutiques et aquatiques

\section{A spatial-temporal model for snow crab (Chionoecetes opilio) stock size in the Southern Gulf of St. Lawrence}

\begin{tabular}{|r|l|}
\hline Journal: & Canadian Journal of Fisheries and Aquatic Sciences \\
\hline Manuscript ID & cjfas-2016-0260.R2 \\
\hline Manuscript Type: & Article \\
\hline Date Submitted by the Author: & $28-$ Oct-2016 \\
\hline Complete List of Authors: & $\begin{array}{l}\text { Cadigan, Noel; Memorial University of Newfoundland, Marine Institute } \\
\text { Wade, Elmer; Fisheries and Oceans Canada Gulf Region } \\
\text { Nielsen, Anders; Technical University of Denmark (DTU Aqua), Marine } \\
\text { living resources }\end{array}$ \\
\hline Keyword: & $\begin{array}{l}\text { Catch per unit of effort, depletion model, density-dependence catchability, } \\
\text { STOCK ASSESSMENT < General, Gaussian markov random field }\end{array}$ \\
\hline &
\end{tabular}


1 A spatial-temporal model for snow crab (Chionoecetes opilio) stock size in the Southern Gulf of 2 St. Lawrence.

Noel G. Cadigan ${ }^{1}$, Elmer Wade ${ }^{2}$, Anders Nielsen ${ }^{3}$

${ }^{1}$ Centre for Fisheries Ecosystems Research

Marine Institute of Memorial University of Newfoundland

P.O. Box 4920

St. John's, NL

Canada A1C 5R3

${ }^{2}$ Aquatic Resources Division

Oceans and Science Branch

Department of Fisheries and Oceans

343 Université Avenue

Moncton, NB

Canada E1C 5K4

${ }^{3}$ National Institute of Aquatic Resources

Technical University of Denmark

Charlottenlund Slot Jægersborg Allé 1

2920 Charlottenlund

Corresponding author: Noel Cadigan, tel:1 709778 0603; fax: 1709778 0669; email: noel.cadigan@mi.mun.ca 


\section{Abstract}

We develop a high resolution spatio-temporal model of stock size and harvest rates for snow crab (Chionoecetes opilio) in the Southern Gulf of St. Lawrence, which supports an economically important fishery off the east coast of Canada. It is a spatial and weekly model during 1997-2014 that utilizes within-season depletion based on catch per unit of effort (CPUE; $\mathrm{kg}$ per pot), and also biomass values from a survey designed specifically for this stock. The model is formulated in a state-space framework. The main contribution of the model is to provide a better understanding of fishery-dependent factors that affect CPUE. There is strong evidence of density-dependence in the relationship with CPUE and stock biomass, in addition to a general increase in CPUE catchability over time that may be related to changes in gear soak time, and spatial variation in catchability. We also find that a natural mortality rate of 0.4 provides a better fit to survey results. Model results suggest that there is no evidence of effort saturation in the fishery.

41 Keywords: Catch per unit of effort; depletion model; Gaussian markov random field; density42 dependence catchability; stock assessment.

44 Introduction

45 Snow crab (Chionoecetes opilio) have been fished commercially in the southern Gulf of St.

47 of this fishery is based on logbook data, dockside monitoring of the catch, and at-sea sampling

48 by observers. Snow crab normally molt every year until maturity (Conan and Comeau 1986) 
49 prior to the fishery (e.g. Hébert et al. 2002). After molting, crabs have a soft shell for 8 to 10

50 months. Males live approximately 5 years (Sainte-Marie et al. 1995) to 7.7 years (Fonseca et al.

$512008)$ after their terminal molt. The fishery targets hard-shelled adult male animals with a

52 minimum landing size of $95 \mathrm{~mm}$ carapace width $(\mathrm{CW})$. The management of this fishery is based

53 on total allowable catch (TAC), effort controls (number of licenses, trap limits, and seasons), and

54 a limit on the percentage of the low commercial value soft-shelled crabs in the catch.

55 This stock is assessed using size and/or stage-based survey indices of biomass and abundance

56 from a post-season trawl survey designed specifically for this stock (Moriyasu et al. 2016;

57 Hébert et al. 2016b). A grid-based sampling design has been used in all survey years, although

58 the survey coverage and grid cell size has changed over time. Total survey biomass for various

59 life stages of crab is estimated using kriging with external drift using depth as a secondary

60 variable (Hébert et al. 2016b). These stages include residual biomass of commercial sized males

61 remaining after the fishery, and recruitment biomass (soft-shelled adult males $>=95 \mathrm{~mm} \mathrm{CW}$ )

62 that will be available to the fishery in the following fishing season. The exploitable biomass of

63 commercial males is inferred from the residual and recruited biomass from the previous year's

64 survey. A harvest decision rule including precautionary approach reference points based on

65 commercial biomass is used to provide total allowable catch (TAC) advice (Chaput et al. 2014).

66 A simple model has been used to project biomass in the next several years based on survey

67 estimates of pre-recruit stages (Surette and Wade, 2006). The only other model published for this

68 stock was an age-, sex- and stage-structured model by Siddeek et al. (2009), and the purpose of

69 this model was for developing limit and target harvest control rules, but not directly for stock

70 assessment. 
71 Commercial catch rates are considered in sGSL snow crab stock assessments but the relationship

72 between commercial-sized adult male biomass estimated from the snow crab survey and CPUE

73 calculated from logbooks is weak; $\mathrm{r}^{2}=0.22$ (Hébert et al. 2016a) and varies across different

74 locations. This results in differences in perception of stock abundance between the fishing industry observations and those from the survey. Fishing industry perceptions not only involve the overall level of CPUE but also the rate of decline in CPUE as the fishing season progresses. They consider that if CPUE does not decline much then this is an indication of a larger stock size than when CPUE declines substantially. This is also related to the sedentary nature of snow crabs (Biron et al. 2008) during the sGSL fishery.

It is possible to estimate absolute biomass based on the within-season rate of decline of a biomass index in response to the fishery removals. The Leslie method (Leslie 1952) has been used for many crustacean species (see review by Smith and Addison 2003) and others (e.g. Bishir and Lancia 1996). Its popularity is partly related to its simplicity. If $C_{t}$ and $E_{t}$ are the catch and effort of a fishery at time $t$, and CPUE is $X_{t}=C_{t} / E_{t}$ and is proportional to stock biomass $B_{t}$, season, as a function of either catch or effort, to estimate initial stock size. These are two

91 examples of depletion models.

92 In practise there are many problems with assumptions for these simple depletion estimators.

93 Miller and Mohn (1993) examined issues with the Leslie method for species like crabs and 
94 lobsters. An important assumption is that catchability is the same for all individuals throughout

95 the fishing season. Of particular relevance to sGSL commercial snow crab is the potential for 1)

96 a change (decrease or increase) in capture probability depending on how many crabs are in a

97 trap, which will result in density-dependent CPUE catchability, and 2) increased activity in

98 warmer waters could lead to higher catchability in nearshore areas during the spring and

99 summer. The physical habitat and temperature may affect crab walking rates, and current

100 direction and speed may affect the bait "plume" and trap detection. CPUE catchability may also

101 change for many other reasons (also see Miller 1990, Bacheler et al. 2013). Varying

102 characteristics of fishing effort such as soak time, bait type and amount, and trap design and

103 spacing can affect catchability. Trap saturation may happen with longer soak times for many

104 reasons including space limitation of the gear, inter-species or intra-species interactions, or the

105 loss and degradation of bait (Shertzer et al. 2015). However, Charles et al. (2014) found soak

106 time had little effect in a study of sGSL snow crab. Temporal, spatial, and density-dependent

107 variations in CPUE catchability have been examined extensively in the fisheries literature.

108 Density-dependence relationships between CPUE and stock size (i.e. catchability) are commonly

109 reported (e.g. Harley 2001, Ward et al. 2013) but are not ubiquitous (e.g. Zhou et al. 2007).

110 Many factors may produce a power-law relationship between CPUE and stock size (e.g. Cooke

111 and Beddington 1984). If such factors change in a systematic way, bias can be introduced to

112 depletion estimators.

113 Another important assumption is that fishing effort is uniformly distributed over the area

114 occupied by the stock. This is not the case for sGSL snow crab. Fishing fleets may adjust their

115 fishing locations when catch rates decline and this can mask the decline in stock size due to

116 fishing (e.g. Hilborn and Walters 1987). Depletion methods also require landings and effort to be 
117 accurately reported. Fisheries monitoring for sGSL snow crab is rigorous so reported catch

118 should be reliable. However, effort is not evaluated in dockside monitoring and may be

119 inaccurate. The Leslie method assumes a closed population, with no emigration, natural

120 mortality, or recruitment. The last two issues are relevant for snow crab because of the length of

121 the fishing season. Concerns about within-season recruitment and fleet movements led scientists

122 to conclude that the Leslie method was inappropriate for stock assessment of sGSL snow crab

123 (Loch et al. 1995). Corrections for these problems, when they exist, require additional

124 information (Miller and Mohn 1993).

Various extensions of depletion models have been proposed to address some of the above

127 problems. Gedamke et al. (2004) used information from vessel monitoring systems to

128 disentangle the effects of nonrandom fishing patterns for scallops so that a depletion model could

129 be applied. They used spatial analyses to define regions where assumptions of the depletion

130 method were met. Walter et al. (2007) extended this stratification approach and conducted a

131 geostatistical analysis of their depletion model results. Robert et al. (2010) formulated the

132 DeLury model in a Bayesian framework and included natural mortality and within-season

133 recruitment for an octopus fishery, with simulation testing of various model formulations and

134 data configurations. They also modelled a range of years in a hierarchical framework in which

135 initial abundance and catchability were related across years. Roa-Ureta (2012) proposed

136 generalized depletion models for open populations and a nonlinear relationship between catch

137 and effort. His model allowed for waves of within-season immigration to the fishing grounds. He

138 applied his model to a squid fishery. Roa-Ureta (2015) extended the generalized depletion model

139 to be multi-annual and to include multiple fleets with different catchabilities for a Spanish 
140 mackerel fishery. Zhou et al. $(2011,2015)$ presented a generalized Leslie-type model to estimate

141 natural mortality rates as well as stock size and harvest rates in a Bayesian framework. Their

142 multi-annual biomass model included within-season somatic growth and a parametric year-

143 varying catchability function.

144 There is often little evidence of depletion in total fishery CPUE for sGSL snow crab. In the 2014 145 fishery, total CPUE (total catch divided by total effort) increased overall and only decreased for 146 three short periods (Figure 3). Average soak times rapidly increased from about 50 hours at the 147 start of the fishery to about 120 hours by week 25 . This may partly explain the stability of CPUE 148 during this period. However, another factor is a change in the distribution of the fishery (Figure 149 4). There is a pronounced change in the fleet distribution starting in August (week 29). This is a 150 consistent feature throughout the time-series. Also, in some weeks there may be a large 151 concentration of effort in just a few grids cells and pots may compete for crab in these cases. If 152 multiple pots are placed within their effective fishing area then this will eventually produce a 153 saturation effect in which catch is no longer proportional to effort. Not surprisingly, in 154 preliminary analyses we found poor correspondence between stock size and harvest rates from a 155 sGSL spatial depletion model and survey results. Hence, in this paper we also explore changes in 156 model catchability assumptions, both temporal and spatial, to account for changes in the fishery 157 to improve this correspondence.

158 We propose a novel CPUE and survey integrated spatial biomass depletion model that provides 159 spatio-temporal estimates of snow crab stock size and also density-dependent and within-season 160 spatio-temporal changes in CPUE catchability. We provide more precision on how to interpret 161 CPUE catchability for snow crab, or more generally for stocks in which it is reasonable to 162 assume that the gear catches all fish in the area the gear fishes. We use high resolution spatio- 
163 temporal (i.e. weeks and grids) catch and effort data, and also spatial survey data, for model

164 estimation. Extracting more information about stock size using data at the highest resolution

165 possible can produce more reliable stock assessments and more relevant information for fisheries

166 managers, but an important cost of doing this is the need for a highly parameterized model that is

167 potentially computationally complex. Another contribution of this paper is a high resolution

168 spatial biomass depletion model that is restricted in certain ways to make the model

169 computationally efficient. We also include a novel effort saturation effect that is spatial because

170 of differences in the fraction of crab "grounds" within grid cells. Our model is implemented in a

171 state-space modelling framework which has become a favored approach in modeling time

172 varying population dynamics (e.g. Nielsen and Berg, 2014; Cadigan, 2016).

\section{Materials and Methods}

175 Model notation and parameters are described in the text and also Table 1. The data we used are

176 weekly fisherman logbook observations of catch and effort for 314 spatial grid cells in sGSL

177 during 1997-2014. Also, we use the post fishery snow crab survey trawl catches of hard-shelled

178 adult males (i.e. exploitable crab). These data are displayed in Supplementary figures, for catch

179 weight (Figures SC.1-20), effort (Figures SE.1-20), CPUE (Figures SX.1-20), and the snow crab

180 survey results for exploitable biomass (Figures SSEB.1-19) and total biomass (Figures SSTB.1-

181 19) although the latter information is not used in our model but is used in the DFO stock

182 assessment.

183 The biomass $(B)$ depletion model for grid cell $i$ and week $t$ in year $y$ is 


$$
B_{y, t, i}=B_{y, t-1, i} \exp \left(-Z_{y, t-1, i}\right)
$$

184 where the weekly total mortality rate is $Z_{y, t, i}=M / 52+F_{y, t, i}, F_{y, t, i}$ is the fishing mortality rate, 185 and $M$ is an assumed constant annual value for the natural mortality rate which we prorate 186 equally to weeks. We examine $\mathrm{M}=0.2,0.3$, and 0.4 which are values that are consistent with the 187 survival times in Sainte-Marie et al. (1995) and Fonseca et al. (2008). The depletion model starts 188 at an initial biomass $B_{y, o, i}$ in year $y$ and we project this to the start of the fishery using Equation 189 (1) with no $F$. Our model does not include movement of crab between grid cells which provides 190 substantial computational efficiencies. Snow crab in the sGSL are relatively sedentary during the 191 fishery (Biron et al. 2008) but they may move somewhat between grid cells during the winter 192 months before the fishery so $B_{y, o, i}$ is only a nominal initial biomass for a grid cell and the actual 193 biomass may be different. However, we assume that $\sum_{i} B_{y, o, i}$ is the total stock biomass at the 194 beginning of the year because errors due to movements will cancel out in the total biomass. The 195 catch equation is

$$
C_{y, t, i}=B_{y, t, i}\left\{1-\exp \left(-Z_{y, t, i}\right)\right\} \frac{F_{y, t, i}}{Z_{y, t, i}}
$$

197 A basic assumption underlying our spatial depletion model is that $C P U E=C / E=q B$ where $E$ is 198 the effort which we define as the number of pots fished. This implies that the harvest rate is $H \equiv$ $199 C / B=q E$ and $q$ represent the harvest rate of a single pot. Some boundary grid cells are small so 200 an area-adjustment is useful. Let $a_{f t i}$ be the area fished by a pot which we assume varies spatially 201 (i) and temporally $(t)$ because of a variety of factors related to the fishery (e.g. soak time);

202 however, we assume the area fished by a pot is constant within a grid cell and a week. Let $A_{i}$ be 
203 the area of the grid cell and $p_{i}$ be the fraction of area that are crab grounds. If crab are

204 homogeneously distributed within the crab grounds and if a pot catches all crab within the area it 205 fishes then the expected catch from a single randomly placed pot in cell $i$ with total biomass $B_{y, t, i}$ 206 is $q_{y, t, i} B_{y, t, i} / A_{i}$ where $q_{y, t, i}=a_{f y, t, i} / p_{i}$ is the potential area fished; that is, $a_{f y, t, i} \leq q_{y, t, i}$. The total catch 207 from $E_{y, t, i}$ pots is $C_{y, t, i}=q_{y, t, i} E_{y, t, i} D_{y, t, i}$ where $D_{y, t, i}=B_{y, t, i} / A_{i}$ is the biomass density in grid cell $i$. 208 Here we assumed that $E_{y, t, i}<<q_{y, t, i}$ so that pots fish independently. Hence the expected CPUE is $209 X_{y, t, i}=q_{y, t, i} D_{y, t, i}$. The CPUE biomass catchability is proportional to grid cell size and varies across 210 years, weeks and grids.

211 We have incomplete information on the factors that may cause catchability to change over time 212 but we expect that these factors will be auto-correlated. Hence, we assume that

$$
q_{y, t, i}=q_{y, i} \exp \left(\delta_{y, t, i}\right)
$$

213 where $\delta_{y, t, i}$ are weekly $q$ process errors that we assume for simplicity are $\operatorname{AR}(1)$ auto-correlated 214 normal random variables with mean zero, standard deviation $\sigma_{\delta}$, and auto-correlation parameter $215 \varphi_{\delta}$. These errors are assumed to be correlated across weeks within grid cells and years but 216 otherwise are independent. Including process error is typical of contemporary stock assessment 217 models (Maunder and Piner, 2014). The $q_{y, i}$ annual spatial effects are further separated into 218 independent year and space effects

$$
q_{y, i}=q_{y} q_{i}
$$

219 The $q_{y}$ year-effects account for long-term changes in fishing practices and are modelled as a 220 random walk, 


$$
\log \left(q_{y+1} / q_{y}\right) \sim N\left(0, \sigma_{q y}^{2}\right)
$$

221 The $q_{i}$ spatial effects are described below. Hence, we model catchability at high resolution using 222 correlated spatial effects, a random walk year effect, and auto-correlated weekly process errors 223 within grid cells.

224 The idealized maximum effective effort that can be fished without pot competition is $E_{\max , y, t, i}=$ $225 A_{i} / q_{y, t, i}$. This is the total number of distinct pot fishing areas in grid $i$ with total area $A_{i}$. The 226 model-predicted effort corresponding to $C_{y, t, i}$ is $E_{y, t, i}=C_{y, t, i} / q_{y, t, i} D_{y, t, i}$ and

$$
E_{y, t, i}=\frac{C_{y, t, i} A_{y, t, i}}{q_{y, t, i} B_{y, t, i}}=H_{y, t, i} E_{\max , y, t, i}
$$

227 We will use Equation (5) to model observed effort. Note that unlike much of the fisheries

228 literature we do not assume effort is a covariate that is known without error and we are therefore

229 better able to account for the reliability of this data. However, if there is pot saturation then pots

230 may compete for crabs and the effective (i.e. independent) effort is less than the model

231 prediction.

232 If fishermen randomly chose fishing locations regardless of gear that may already be present then 233 this behavior can be approximated as a Poisson process. We assume that each grid cell is 234 comprised of $E_{\max , y, t, i}$ distinct fishing locations and that all pots within one location exploit the 235 same as a single pot. Let $E_{o, y, t, i}$ be the nominal total effort and let $Y$ be the number of pots in a 236 fishing location which has an approximate Poisson distribution with mean $237 \lambda_{y, t, i}=E_{o, y, t, i} / E_{\max , y, t, i}$. The probability that a location has at least one pot is $\operatorname{Prob}(Y>0)=$ $2381-\exp \left(-\lambda_{y, t, i}\right)$. The number of fishing locations with at least one pot, which is the effective 239 effort $E_{y, t, i}$, is 


$$
E_{y, t, i}=E_{\max , y, t, i}\left\{1-\exp \left(-E_{o, y, t, i} / E_{\max , y, t, i}\right)\right\}
$$

240 However, crab fishermen are aware of gear already present at a location and they will usually

241 avoid setting new gear close to existing gear. In this case Equation (6a) will under-estimate

242 effective effort. We propose an alternative smooth "hockey-stick" formulation (e.g. Mesnil and

243 Rochet, 2010) to account for this behavior,

$$
E_{y, t, i}=1 / 2 E_{o, y, t, i}+1 / 2 E_{\max , y, t, i}\left\{\sqrt{1+\gamma^{2} / 4}-\sqrt{\left(E_{\max , y, t, i}^{-1} E_{o, y, t, i}-1\right)^{2}+\gamma^{2} / 4}\right\} .
$$

244 Note that we modified the model formulation of Mesnil and Rochet (2010) so that $\gamma$ is a fraction 245 of $E_{\max , y, t, i}$

246 Equations (6a) and (6b) are illustrated in Figure 5 for $E_{\max , y, t, i}=2000$. We set $\gamma=0.3$ in 247 Equation (6b). Effective effort based on Equation (6b) and nominal effort are almost the same 248 until effort is close to $E_{\max }$ but effective effort based on Equation (6a) is usually much less than 249 nominal effort unless effort is low. Both Equations (6a) and (6b) imply that $E_{y, t, i} \leq E_{o, y, t, i}$. If $250 E_{o, y, t, i} \ll E_{\max , y, t, i}$ then $E_{y, t, i} \approx E_{o, y, t, i}$ but as $E_{o, y, t, i} \rightarrow \infty$ then $E_{y, t, i} \rightarrow E_{\max , y, t, i}$. We can use 251 the inverse of these equations as functions of $E_{o, y, t, i}$ to get the model predicted nominal fishing 252 effort. For Equation (6a) this is

$$
E_{o, y, t, i}=-E_{\max , y, t, i} \log \left(1-H_{y, t, i}\right)
$$

253 Let $U_{y, t, i}=2 H_{y, t, i}-1-\sqrt{1+\gamma^{2} / 4}$. The inverse of Equation (6b) is

$$
E_{o, y, t, i}=E_{\max , y, t, i}\left\{1+\frac{U_{y, t, i}^{2}-\gamma^{2} / 4}{2 U_{y, t, i}}\right\}
$$


254 If there is negligible effort saturation then

$$
E_{o, y, t, i}=H_{y, t, i} E_{\max , y, t, i}
$$

256 We also include a density dependence effect in $q$. Our basic generating equation is $\mathrm{CPUE}=q D^{\beta}$ 257 and $\beta=1$ means no density dependence. However, $\beta$ changes the interpretation of $q$ so we use a 258 slightly modified version, $C P U E=q D D_{s}^{\beta-1}$ where $D_{s}=D / \bar{D}$ is the density relative to the 259 average over space and time. We computed an average value of $D$ for all years, weeks, and grid 260 cells in which fishing occurred, using a specific model formulation (M1; see Results) but kept 261 this same value (i.e. $867 \mathrm{~kg} / \mathrm{km}^{2}$ or $49 \mathrm{Kt}$ for the sGSL total area) in other model formulations.

262 The re-scaling of density only affects estimation of $q$ and not $\beta$. When $D=\bar{D}$ then $C P U E=q D$, 263 so $q$ represents catchability at average stock density. Our density-dependence model only 264 requires modifying Equation (3),

$$
q_{y, t, i}=D_{s, y, t, i}^{\beta-1} q_{y, i} \exp \left(\delta_{y, t, i}\right)
$$

265 There is otherwise no change in the way annual and spatial effects $\left(q_{y, i}\right)$ or weekly effects $\left(\delta_{y, t, i}\right)$ 266 are modelled. However, this density dependence may be confounded with effort saturation.

267 There are two groups of spatial effects in our model, the yearly initial biomass densities $D_{y, o, i}=$ $268 B_{y, o, i} / A_{i}$ and the catchabilities $q_{i}$. We have insufficient data to estimate these effects freely, nor 269 would this be a good idea because we expect spatial correlation in these effects. Hence, we also 270 model these spatial effects as latent random variables on the snow crab survey grid. Let $G$ be the 271 total number of grid cells. The covariance structure we use for these random variables is the 272 same as that described in Kristensen et al. (2013) and is based on a precision matrix (i.e. inverse 
273 covariance matrix) that implies that a grid cell is, conditional on the cell's neighbors,

274 independent of all other cells. The precision matrix $\Psi_{G \times G}$ for the logs of $D_{y, o . i}$ or $q_{i}$ has $i, j$ 'th

275 element

$$
\Psi_{i, j}= \begin{cases}-\tau, & \text { if cell } i \text { neighbors cell } j \\ \tau\left(m_{i}+\vartheta\right), & \text { if } i=j \\ 0, & \text { otherwise, }\end{cases}
$$

276 where $m_{i}$ is the number of neighbors of grid cell $i$ which will typically be four but will be less

277 for boundary cells. The $\tau$ and $\vartheta$ parameters are estimated separately for each spatial effect. We

278 denote these as $\tau_{D}$ and $\vartheta_{D}$, etc. The precision matrix approach is typical of those used in

279 Gaussian Markov random fields (GMRF) and implies that the correlation decreases with distance

280 traveled through water and not simply the straight-line distance between two grid cells. This is

281 particularly relevant for the sGSL because of irregular coast lines (i.e. Chaleur Bay) and island

282 obstacles (i.e. the Magdalen Islands; see Figure 1). Let $\underline{D}_{y, o}$ be a vector of spatial density effects

283 for year $y$. We also expect that the spatial distribution of biomass will be similar from year to

284 year. We accommodate this behavior by modelling the density in the first year, $\log \left(\underline{D}_{1, o}\right)$, as a

285 GMRF with parameters $\tau_{D 1}$ and $\vartheta_{D 1}$, and the relative difference in densities in successive years,

$286 \log \left(\underline{D}_{y+1, o} / \underline{D}_{y, o}\right)$, as independent GMRFs with parameters $\tau_{D 2}$ and $\vartheta_{D 2}$. These latter parameters

287 are assumed to be the same for all years. Hence, our model involves a spatially correlated

288 random walk for biomass density from year to year.

289 The data available to estimate model parameters $(\theta$, see next Section) are spatial catch and effort

290 measurements each week during 1997-2014, and the snow crab survey catches for the same

291 years (see Supplementary figures). Our model is for male commercial biomass so we only use

292 survey catch information for hard-shelled legal size males that were exploited in the same year of 
293 the survey (i.e. residual exploitable biomass). We assume a small and fixed value for log-catch 294 measurement error (ME) standard deviation (SD; i.e. $\sigma_{C}=0.05$ ) because this data is obtained 295 from dockside monitoring with reportedly high accuracy. This is approximately the catch 296 measurement error CV. The state-space model log-likelihood (i.e. observation) equation for 297 catch is

$$
l\left(\theta \mid\left\{C_{o b s}\right\}\right)=\sum_{y} \sum_{t} \sum_{i} \log \left[\sigma_{C}^{-1} \varphi_{N}\left\{\frac{\log \left(C_{o b s, y, t, i}\right)-\log \left(C_{y, t, i}\right)}{\sigma_{C}}\right\}\right]
$$

311 effort is

$$
\log \left(E_{o b s, y, t, i}\right)=\log \left(H_{y, t, i}\right)+\log \left(A_{y, t, i}\right)-\log \left(q_{y, i}\right)+\varepsilon_{y, t, i}-\delta_{y, t, i}
$$

The $\varepsilon$ effort ME's and the $\delta$ catchability process errors are confounded. We can only estimate their total effect. We still refer to these errors as $\delta$ process errors although they are a mixture of changes in catchability and ME in effort. Let $t_{f, y, i}$ denote the first week of the fishery in year $y$ and grid cell $i$. We re-define $\delta_{y, t, i}=\log \left(E_{o b s, y, t, i}\right)-\log \left(E_{o, y, t, i}\right)$ and the likelihood equation for 


$$
l\left(\theta \mid\left\{E_{o b s}\right\}\right)=\sum_{y} \sum_{i} \sum_{t>t_{f, y, i}} \log \left[\sigma_{\delta}^{-1} \varphi_{N}\left\{\frac{\delta_{y, t, i}-\varphi_{\delta} \delta_{y, t-1, i}}{\sigma_{\delta}}\right\}\right] .
$$

312 For the first week of the fishery, when $t=t_{f, y, i}$ then $\delta_{y, t-1, i}=0$.

313 The snow crab survey biomass values, $S_{o b s, y, i}$, are assumed to be absolute estimates of the

314 biomass in the trawl swept area. We re-scaled these biomass values to be equivalent to a tow

315 with a standard swept area of $2700 \mathrm{~m}^{2}$ which is typical in the survey design. The model predicted

316 survey biomass values are $S_{y, i}=2700 B_{y, 34, i} / A_{i}$ where the grid cell area is in $\mathrm{m}^{2}$. We assumed

317 this survey occurred in week 34 which is about the end of August. This is considered further in

318 the Discussion. In preliminary analysis we found that the $\operatorname{ME~} \operatorname{SD}\left(\sigma_{S}\right)$ did not depend much on

319 predicted values. Also, there are a large number of zero's in the survey time-series so log-

320 transformation was not an option and did not seem necessary. Hence, the survey likelihood

321 equation is

$$
l\left(\theta \mid\left\{S_{o b s}\right\}\right)=\sum_{y} \sum_{i} \log \left[\sigma_{S}^{-1} \varphi_{N}\left\{\frac{S_{o b s, y, i}-S_{y, i}}{\sigma_{S}}\right\}\right] .
$$

323 The $F$ 's in Equation (2) are also treated as unstructured random effects with a common mean and

324 variance. Let $\mu_{F}=E(F)$ and $\sigma_{F}^{2}=\operatorname{Var}\{\log (F)\}$. The likelihood equation for $F^{\prime}$ 's is

$$
l(\{F\} \mid \theta)=\sum_{y} \sum_{t} \sum_{i} \log \left[\sigma_{F}^{-1} \varphi_{N}\left\{\frac{\log \left(F_{y, t, i}\right)-\log \left(\mu_{F}\right)}{\sigma_{F}}\right\}\right] .
$$

325 We will show that the estimate of $\sigma_{F}$ is fairly large and so the $F$ 's are estimated almost freely.

\section{Estimation}


327 The model is high dimensional, based on 314 spatial grid cells and 936 time steps for the 18 328 years and 52 weeks per year, resulting in slightly over 290000 space-time cells. However, there 329 are only a small number of parameters to estimate. Most of the model effects are random and are 330 not freely estimated. Fixed effect parameters, denoted collectively as the parameter vector $\theta$, are 331 estimated via maximum likelihood (MLE) based on the total marginal likelihood, $L(\theta)$, in which 332 random effects are "integrated out". Let $\Gamma$ denote a vector of all random effects. There are 13 or $33314 \theta$ 's, depending on the model formulation, but there are almost $27000 \Gamma$ 's. Note that there are 334 far fewer $\Gamma$ 's than the total number of space-time model cells because $\delta$ 's and $F$ 's are only 335 estimated for cells that had reported catches, and in many weeks there is no fishing in a grid cell 336 (see Supplementary figures).

337 Let $S$ denote the set of all survey, catch, and effort data used in the model. The marginal $338 \quad$ likelihood is

$$
L(\theta)=\iiint_{\Gamma} f_{\theta}(S \mid \Gamma) g_{\theta}(\Gamma) \partial \Gamma
$$

339 where $f_{\theta}(S \mid \Gamma)$ is the pdf of the data and $g_{\theta}(\Gamma)$ is the pdf for the $\Gamma$ random effects. The 340 template model builder (TMB; Kristensen, 2015) package within R (R Core Team, 2016) was 341 used to implement the model. The MLE's of $\theta$ maximize $L(\theta)$. The user has to provide $\mathrm{C}++$ 342 computer code to calculate $f_{\theta}(S \mid \Gamma)$ and $g_{\theta}(\Gamma)$ but the integration in Equation (14) and 343 calculation of $\theta$ to maximize this equation is then provided by TMB. The high dimensional 344 integral is numerically evaluated in TMB using the Laplace approximation. The random effects $345 \Gamma$ can be predicted by maximizing the joint likelihood, $f_{\theta}(S \mid \Gamma) g_{\theta}(\Gamma)$. Additional information 346 on these procedures is provided by Skaug and Fournier (2006). TMB uses automatic 347 differentiation to evaluate the gradient function of Equation (14) and in the Laplace 
348 approximation. The gradient function is produced automatically from $f_{\theta}(S \mid \Gamma)$ and $g_{\theta}(\Gamma)$. This

349 greatly improves parameter estimation using a derivative-based optimizer. We use the $n \operatorname{lminb}$

350 function within R (R Core Team, 2016) to find the MLE for $\theta$.

351 The reliability of model estimates was assessed through detailed examination of model estimates

352 and residuals, and also retrospective analyses in which the model was fit to subsets of data with

353 recent years left out. Some covariance parameters are shared across years in the model so it is

354 possible that there may be retrospective patterns in model estimates. This was done for

355 retrospective years 2007 to 2014 . Sensitivity analyses to model assumptions about M $(0.2,0.3$, or

356 0.4), saturation (none or hockey-stick type), and density-dependence (none or estimated) were

357 also performed, leading to a total of $3 \times 2 \times 2=12$ model formulations that were fitted.

\section{Results}

360 Including a saturation effect did not make much difference to estimates and model fits because

361 the saturation level was usually estimated to be much greater than typical levels of effort. For

362 example, two models described in Table 2 (i.e. M1 and M2), that differed only in whether a

363 "hockey-stick" saturation effect was included or not, produced nearly identical parameter

364 estimates and the model without effort saturation (i.e. M2) produced a better fit. This was a

365 consistent feature in other model formulations we compared for different values of $\mathrm{M}$ and the

366 density dependence parameter $\beta$. There is also no evidence of saturation in plots of catch versus

367 effort (unreported results) for various regions in sGSL. 
368 The statistical evidence for density dependence was very strong (e.g. compare M2 and M3, and 369 M4 and M5) and the estimate of $\beta \ll 1$ indicating high hyper-stability in CPUE. This effect 370 resulted in a much improved fit to the snow crab survey residual biomass values, as evidenced by 371 the lower value of $\sigma_{S}$ for M3 and M4. Density dependence is evident in plots of CPUE versus 372 mean biomass for each grid cell and year (Figure 6) even from a model (i.e. M2 in Table 2) with 373 no density dependence. The slope of log CPUE versus log biomass should be close to one if 374 there is no density dependence but this is clearly not the case. Note that to simplify this figure we 375 only plotted average CPUE and biomass for all weeks within each grid cell and year. Results 376 based on weekly values were very similar. Including density dependence also resulted in substantially different estimates of spatial effects. For example, the total variation, $\operatorname{trace}\left(\Psi^{-1}\right)$, of the spatial effects for CPUE catchability (i.e. $q_{i}$ ) was 303.0 for M2 but only 51.8 for M3, indicating more between grid variation in $q$ for M2. Conversely, the total variation of the D2 spatial random walk for between year changes in biomass density was 152.1 for M3 but only 69.9 for M2 indicating larger between year changes in the spatial distribution of biomass for M3 compared to M2. The total variation of the D1 spatial distribution of biomass in the first year (i.e. 1997) was 16221 for M2 and 17671 for M3. The D1 total variance is much larger than the D2 total variance which indicates relatively small changes in the spatial distribution of biomass from 385 year to year for both models. The $\vartheta$ parameters control the decorrelation range in the spatial 386 covariance matrix. When these parameters are small then the decorrelation range is large (see Kristensen et al. 2013); that is, the spatial correlation is high. The relatively large CV's for $\vartheta$ 's 389 precision than other parameters. 
Another model formulation issue was the choice of $M$. We investigated three choices $(\mathrm{M}=0.2$, 0.3 , and 0.4 ). All values resulted in fairly similar fits (see Table 2 for $M=0.3$ or 0,4 ). Models in which $\beta=1$ indicated $M=0.4$ was the best choice. When $\beta$ was estimated and no effort saturation was assumed then the best fit was obtained with $M=0.2$. The fit was 2.6 AIC units lower than the fit for $M=0.3$. However, models with $\mathrm{M}=0.4$ produced estimates of beginning of year commercial biomass that agreed more closely with the stock assessment kriging biomass estimates $(\mathrm{DFO}, 2016)$ scaled by $\exp (-18 M / 52)$ to account for natural mortality between the time of the post-season survey and the end of the year (Figure 7). This scaling is described in more detail later. We fit the model only to survey catches of residual biomass so the improved fit to DFO kriging estimates of residual plus recruited biomass provides additional and partially independent support for $M=0.4$. Hence, our preferred model formulation (i.e. M4 in Table 2) is $M=0.4$, with density dependence in CPUE, but no effort saturation effect. More specific results from this model are presented in Table 3. Detailed spatial models results are provided in Supplementary Figures S5-S22.

On a tow-by-tow basis the M4 fit to the snow crab survey catches was highly variable (Figure 8; Figures S1, S5-S22). The mean predicted survey catch was 1.0799 and $\hat{\sigma}_{S}=1.153$ (Table 2) indicating that the survey CV was $108 \%$. The snow crab survey catches have substantial spatial variability (see Figures SSEB.1 - SSEB.19) and many zero catches that the model did fit well. Of particular concern is the tendency of the model to under-estimate high values (Figure 8) which suggests potential model mis-specification. The model is constructed to fit catches almost exactly. Effort observations were fit less closely but without apparent bias (Figures S2-S4). There was very little retrospective variation in model results (unreported results). 
412 All models indicated significant changes in catchability over year. The standard deviation $\left(\sigma_{q y}\right)$

413 of the random walk for the $q_{y}$ year-effects was around 0.1 (Table 2). There was also significant

414 spatial variability in q's (Figure 9). We computed the effort-weighted average of $q_{y, i}$ in Equation

415 (3) for all grid cells each year, which is proportional to $q_{y}$, and divided this by the average grid

416 area to estimate the catchability of a pot which is also the exploitation rate of a single pot (Figure

417 10). We compared this with average soak times and the correlation was high $(0.75)$ suggesting

418 that changes in soak times may be a major factor related to change in trap catchability. The

419 effort-weighted average of $q_{y, i}$ is also an estimate of the average potential pot fishing area and

420 the model results indicate that this has ranged from 46000 to $79000 \mathrm{~m}^{2}$ and has increased over

421 time (unreported results). Recall that these values are upper bounds because they are based on

422 the assumption that an entire grid is snow crab "grounds". We computed the average $E_{\max }$ over

423 years for each grid cell, $\bar{E}_{\max , i}=A_{i} / q_{i} \bar{q}_{y}$, and $\bar{q}_{y}$ is the average of $q_{1997}, \ldots, q_{2014}$ Figure 11).

424 Regions where the area fished by a pot is higher, as indicated in Figure 9, have lower values for

$425 \mathrm{E}_{\max }$. Typical levels of effort (i.e. hundreds of pots per grid cell per week) are far less than these

426 saturation levels.

427 The M4 model harvest rates and those from the stock assessment were usually similar (Figure

428 12). When computing harvest rates from stock assessment estimates of commercial male

429 biomass, we also projected post-season biomass from year $y$ - 1 to beginning of year biomass in

430 year $y$ using $\exp (-18 M / 52)$ to account for natural mortality between the time of the survey and

431 the beginning of the following year. For $M=0.4$ this results in a $13 \%$ reduction in biomass and a

$43215 \%$ increase in harvest rates compared to stock assessment values (see Figure 9 in DFO, 2016).

433 Although similar in magnitude to values from the stock assessment, the M4 harvest rates were

434 more variable. If the goal of snow crab fisheries management is a constant harvest rate then the 
435 M4 model indicates that past quotas have resulted in landings and harvest rates that were more 436 variable than required, especially the large decreases in 2010 and 2011 (Figure 2). Landings were 437 reduced from around $25 \mathrm{Kt}$ in $2008-2009$ to $10 \mathrm{Kt}$ in 2010-2011 which resulted in a sharp drop in 438 M4 exploitation rates, well below potential targets. The M4 model suggests that quotas around $43915 \mathrm{Kt}$ in 2010 and 2011 would have resulted in more constant harvest rates.

\section{Discussion}

442 We developed a spatial depletion model for sGSL snow crab based on high resolution (weekly 443 and spatial) catch and effort data, and spatial survey data, to provide more precise estimates of 444 snow crab biomass and population dynamics. Additional information on population size is 445 obtained from the high resolution temporal information by using a within-season biomass 446 depletion model based on the change in fishery CPUE. Such information is often not utilized in 447 stock assessments. However, there are many problems when interpreting CPUE as an index of 448 stock size and we had to incorporate spatio-temporal changes in CPUE catchability. Nonetheless 449 our model did provide somewhat different trends in a few years compared to the snow crab 450 survey that the stock assessment is based on. In particular, our model suggested that some

451 historic reductions in fishery quotas may not have been completely warranted if the objective of 452 snow crab fisheries management was a constant harvest rate near some target level. Also, our 453 model results provide additional information about how CPUE catchability may change and 454 problems with interpreting CPUE as an index of stock size. Some aspects of the model we 455 proposed are specific to sGSL snow crab, or to cases where the fishing gear catches most or all 456 of the fish available to the gear. However, we suggest the basic approach can be used for other 
457 stocks where: 1) fish movement within the time-frame of the fishery is expected to be low, 2)

458 high resolution spatio-temporal catch and effort data are available, and 3) high resolution spatial 459 data from a research survey are available.

460 The original motivation for this research was to validate a spatial CPUE depletion model for 461 application with Newfoundland and Labrador (NL) snow crab. The depletion model was 462 originally formulated with a spatially and temporally constant $q$ and without density-dependence 463 in $q$ or saturation effects. It was felt that sGSL snow crab was a good test case for that model 464 because there were independent estimates of exploitable biomass from the snow crab survey to 465 compare with depletion model estimates. Research surveys in NL have low catchability for snow 466 crab and did not provide a useful comparison. However, this original model based only on 467 seasonal CPUE did not produce plausible values for sGSL snow crab because of the general lack 468 of a CPUE depletion signal for that fishery. This is very different from NL snow crab that often 469 does show a decline in CPUE throughout the fishing season (e.g. see Figures 47, 61, and 78 in 470 Mullowney et al 2016). We then extended the sGSL model to integrate the snow crab survey 471 catches which provided a basis to estimate various changes (i.e. seasonal, spatial, annual, 472 density-dependent) in CPUE catchability.

473 Our results indicate that there have been substantial changes in sGSL snow crab CPUE 474 catchability and these changes seem to be linked with changes in soak time, among other 475 reasons. There is strong evidence of density-dependence in the relationship with CPUE and stock 476 biomass, in addition to a general increase in CPUE catchability over time, very consistent with 477 changes in fishery soak times across years, and spatial variation in catchability. However, we did 478 not find evidence of CPUE saturation. Hence, our model has contributed additional insights into 479 problems with inferring sGSL snow crab resource status based on CPUE. There are many factors 
480 influencing CPUE in addition to stock size. This has been a source of uncertainty in the

481 assessment of this stock (DFO 2016). In addition, the model also indicated potential snow crab

482 survey year effects in 2009 for exploitable biomass and 2011 for total biomass, a problem that

483 has been identified in snow crab assessments (e.g. DFO 2012).

484 We used our model and additional information from the snow crab survey on soft-shelled male 485 biomass that will recruit to the fishery in the following year to infer that $M=0.4$ was a more 486 plausible value. This implies 5\% survival at the 7.7 year male longevity estimate of Fonseca et 487 al. (2008) which is reasonably consistent. At $M=0.3$ the survival at 7.7 years is $10 \%$. We 488 showed that with $M=0.4$ our model provided a good fit to both M-projected beginning of year 489 commercial biomass and post-season residual biomass from the DFO kriging analyses of the 490 snow crab survey. It has often been noted in sGSL snow crab assessments (e.g. DFO, 2016) that 491 the estimated commercial biomass from the survey tends to be higher than the sum of the 492 residual biomass and the landings of the following year, and the difference is attributed to a 493 number of factors. Our results indicate that additional natural mortality at $M=0.4$ between the 494 end of the post-season survey and the start of the fishery in the following year can explain this 495 difference, but $\mathrm{M}=0.3$ does not completely explain the discrepancy.

496 The standard deviation of the process error $\left(\sigma_{\delta}\right)$ was estimated to be large for all model 497 formulations we investigated. This reflects measurement error in effort and also within-season 498 changes in catchability. Reducing this source of error will require additional information from 499 the snow crab fishing fleet, such as accurate measurements of effort and soak time, pot spacing, 500 and other characteristics that may affect catch rates. Such information could be used as 501 covariates to model and explain some of the variation in catchability, and this seems like a useful 502 area for future research. Nonetheless, the model uncertainty in estimates of stock size and harvest 
503 rates is low, with CV's in the 2-3\% range, which is consistent with the low retrospective

504 variation we found in model M4. Hence, reducing the process error variation may not lead to 505 more precise estimates of stock size or harvest rates.

506 The low CV's on stock size and harvest rates may seem unrealistic given the highly flexible 507 model with a CPUE $q$ that may vary across years, spatial locations, weeks, and stock density. 508 However, the model used a large amount of data, nearly 21000 catch and effort measurements 509 and 4200 survey catch values, and the model was constrained to fit the catches very closely, 510 which will result in low CV's. There is some evidence of model mis-specification in the lack of

511 fit to the larger catches from the snow crab survey which leads to an overall average positive 512 residual in most years. This suggests that the CV's may be too small because they do not reflect 513 uncertainty due to model mis-specification. There are many zero catches in the snow crab survey 514 that the model also does not fit well. This could be due to site effects in the fixed-station survey 515 sampling design or other operational issues. There have been changes in snow crab survey 516 vessels during 1997 to 2015, including a change in 2013 from the vessel which had been used 517 during 2003 to 2012. Comparative fishing experiments were not conducted to assess if there 518 were changes in catchability between vessels; however, Benoît and Cadigan (2016) found some 519 evidence of a change in vessel catchability by comparing snow crab survey catches from those 520 from the DFO multi-species survey. Hence, changes in survey catch rates over time may not 521 accurately reflect changes in stock size if survey vessels have different catchabilities and this is 522 another source of unaccounted variability.

523 We assumed that all snow crab survey sets occurred in a single week. In reality the survey takes 524 around 80 days to complete. It would be fairly simple to use the week a grid cell was sampled 525 because the model predicts weekly and spatial biomass. 
526 To simplify the model we treated stock dynamics separately each year and no connection is

527 made between biomass from year to year. Roa-Ureta (2012) took a similar approach. However,

528 population dynamics are of course linked across years, and the biomass remaining at the end of a

529 year is part of the biomass at the start of the next year in addition to recruitment. Linking across

530 years creates additional challenges related to movement between grid cells which may be

531 ignored within a single fishing season because of the low mobility of sGSL snow crab (Biron et

532 al. 2008), but movement will become more of an issue if crab dynamics are modelled over longer

533 time-scales. Linking across years will also create other computational problems related to

534 reduced sparseness of the covariance matrix of the random effects. This may significantly

535 increase model run times, which are about 6-7 minutes for model formulations we investigated.

536 Best stock assessment practise should involve an assessment model that integrates all of the

537 relevant productivity data for sGSL snow crab, including fishery CPUE, both the snow crab and

538 DFO multi-species trawl survey catch rates, and their stage (maturity/length) composition

539 information. Shell-stage (new/intermediate/old) compositions for mature males can provide

540 important information on mortality rates. An assessment model should include a growth

541 component between immature and newly mature soft-shell crabs and legal-sized hard-shell

542 males. This will provide some capability to provide short-term projections for tactical harvest

543 advice. A spatial assessment model can better integrate data collected at varying spatial scales

544 and provide spatial management advice which is an issue for sGSL snow crab (e.g. DFO, 2016).

545 The results of this paper provide a good basis for further development of such an integrated

546 spatial stock assessment model. 


\section{References}

549 Note that most DFO documents cited in this paper are freely available online, at http://www.dfo550 mpo.gc.ca/csas-sccs/index.htm.

552 Bacheler, N.M., Bartolino, V. and Reichert, M.J., 2013. Influence of soak time and fish 553 accumulation on catches of reef fishes in a multispecies trap survey. Fishery Bulletin, 111: 218554232.

Benoit, H.P., and Cadigan, N. 2016. Trends in the biomass, distribution, size composition and model-based estimates of commercial abundance of snow crab (Chionoecetes opilio) based on the multi-species bottom trawl survey of the southern Gulf of St. Lawrence, 1980-2014. DFO

559 Can. Sci. Advis. Sec. Res. Doc. 2015/084. v + 25 p.

Biron, M., Ferron, C. and Moriyasu, M. 2008. Movement of adult male snow crab, Chionoecetes opilio, in the southern Gulf of St. Lawrence and eastern Nova Scotia, Canada. Fisheries

563 Research, 91: 260-270.

Bishir, J. and Lancia, R.A., 1996. On catch-effort methods of estimating animal abundance.

Cadigan, N.G. 2016. A state-space stock assessment model for northern cod, including underreported catches and variable natural mortality rates. Canadian Journal of Fisheries and Aquatic Sciences 73: 296-308.

572 Chaput, G., and DFO/Industry Joint Working Group. 2014. Evaluation of compliance to the 573 Precautionary Approach of harvest decision rules for the snow crab (Chionoecetes opilio) stock 574 of the southern Gulf of St. Lawrence. DFO Can. Sci. Advis. Sec. Res. Doc. 2014/083. v + 57 p.

578 standardization. Canadian Journal of Fisheries and Aquatic Sciences 71: 1817-1829. 
580 Conan, G.Y., and Comeau, M. 1986. Functional maturity of male snow crab, (Chionoecetes

581 opilio). Canadian Journal of Fisheries and Aquatic Sciences, 43: 1710-1719.

583 Cooke, J.G. and Beddington, J.R., 1984. The relationship between catch rates and abundance in 584 fisheries. Mathematical Medicine and Biology, 1: 391-405.

DeLury, D.B., 1947. On the estimation of biological populations. Biometrics, 3: 145-167.

DFO. 2012. Assessment of snow crab in the southern Gulf of St. Lawrence (Areas 12, 19, 12E and 12F) and advice for the 2012 fishery. DFO Can. Sci. Advis. Sec. Sci. Advis. Rep.

$590 \quad 2012 / 003$. (Areas 12, 19, 12E and 12F) and advice for the 2016 fishery. DFO Can. Sci. Advis. Sec. Sci.

594 Advis. Rep. 2016/010.

Fonseca, D.B., Sainte-Marie, B., and Hazel, F. 2008. Longevity and change in shell condition of

598 Transactions of the American Fisheries Society, 137: 1029-1043.

600 Gedamke, T., DuPaul, W.D. and Hoenig, J.M., 2004. A spatially explicit open-ocean DeLury

601 analysis to estimate gear efficiency in the dredge fishery for sea scallop Placopecten

602 magellanicus. North American Journal of Fisheries Management, 24: 335-351.

603

604

605

606 
607 Harley, S.J., Myers, R.A. and Dunn, A., 2001. Is catch-per-unit-effort proportional to 608 abundance? Canadian Journal of Fisheries and Aquatic Sciences, 58: 1760-1772.

609

610 Hébert, M., Benhalima, K., Miron, G., and Moriyasu, M. 2002. Molting and growth of male 611 snow crab, Chionoecetes opilio, (O. Fabricius, 1788) (Crustacea: Majidae) in the southern Gulf 612 of St. Lawrence. Crustaceana 75: 671-702.

613

614 Hébert, M., Wade, E., DeGrâce, P., and Moriyasu, M. 2016a. Review of the 2014 snow crab 615 (Chionoecetes opilio) fishery in the southern Gulf of St. Lawrence (Areas 12, 19, 12E and 12F). 616 DFO Can. Sci. Advis. Sec. Res. Doc. 2015/081. v + 43 p.

617

Hébert, M., Wade, E., DeGrâce, P., and Moriyasu, M. 2016b. The 2014 assessment of the snow crab (Chionoecetes opilio) stock in the southern Gulf of St. Lawrence (Areas 12, 19, 12E and 12F). DFO Can. Sci. Advis. Sec. Res. Doc. 2015/083. v + 43 p.

Hilborn, R. and Walters, C.J., 1987. A general model for simulation of stock and fleet dynamics in spatially heterogeneous fisheries. Canadian Journal of Fisheries and Aquatic Sciences, 44:

624 1366-1369.

625

626

Kristensen, K., Thygesen, U.H., Andersen, K.H. and Beyer, J.E., 2013. Estimating spatio627 temporal dynamics of size-structured populations. Canadian Journal of Fisheries and Aquatic 628 Sciences: 326-336.

630 Kristensen, K., Nielsen, A., Berg, C.W., Skaug, H. and Bell, B., 2015. Template model builder 631 TMB .Journal of Statistical Software, 70: 1-21.

632

633 Leslie, P.H., 1952. The estimation of population parameters from data obtained by means of the 634 capture-recapture method. II. The estimation of total numbers. Biometrika, 39: 363-388. 
636

637

638

639

640

641

642

643

644

645

646

647

648

649

650

651

652

653

654

655

656

657

658

659

660

661

662

663

664

665

Loch, J.S., Moriyasu, M. and Jones, J.B., 1995. An improved link between industry, management and science: review of case history of the Southwestern Gulf of St. Lawrence snow crab fishery. Aquatic Living Resources, 8: 253-265.

Maunder, M. N., and Piner, K. R. 2014. Contemporary fisheries stock assessment: many issues still remain. ICES Journal of Marine Science, doi:10.1093/icesjms/fsu015.

Mesnil, B. and Rochet, M.J., 2010. A continuous hockey stick stock-recruit model for estimating MSY reference points. ICES Journal of Marine Science: Journal du Conseil, 67: 1780-1784.

Mullowney, D. Dawe, E., Coffey, W., Quilty, S., Colbourne, E., and Maddock Parsons, D. 2016. An Assessment of Newfoundland and Labrador Snow Crab (Chionoecetes opilio) in 2014. DFO Can. Sci. Advis. Sec. Res. Doc. 2016/026. v+179 p.

Nielsen, A., Berg, C. W. 2014. Estimation of time-varying selectivity in stock assessments using state-space models. Fisheries Research, 158, 96-101..

Miller, R.J., 1990. Effectiveness of crab and lobster traps. Canadian Journal of Fisheries and Aquatic Sciences, 47: 1228-1251.

Miller, R.J. and Mohn, R.K., 1993. Critique of the Leslie method for estimating sizes of crab and lobster populations. North American Journal of Fisheries Management, 13: 676-685.

Moriyasu, M., Wade, E., Landry, J.F., DeGrâce, P., Surette, T., and Hébert, M. 2016. Summary of 2014 snow crab trawl survey activities in the southern Gulf of St. Lawrence. DFO Can. Sci. Advis. Sec. Res. Doc. 2015/082. v 39 p.

R Core Team (2016). R: A language and environment for statistical computing. R Foundation for Statistical Computing, Vienna, Austria. URL https://www.R-project.org/. 
666 Roa-Ureta, R.H., 2012. Modelling in-season pulses of recruitment and hyperstability-

667 hyperdepletion in the Loligo gahi fishery around the Falkland Islands with generalized depletion 668 models. ICES Journal of Marine Science: Journal du Conseil, 69:1403-1415. in Saudi waters of the Arabian Gulf with generalized depletion models under data-limited 672 conditions. Fisheries Research, 171: 68-77. the De Lury depletion model: strengths and limitations of the method, and application to the Moroccan octopus fishery. ICES Journal of Marine Science: Journal du Conseil, 67: 1272-1290. stages of male snow crab, Chionoecetes opilio (Brachyura: Majidae). Canadian Journal of Fisheries and Aquatic Sciences, 52: 903-924.

681

682

683

684

685

686

687

688

689

690

691

692

693

694

695

696
Siddeek, M.S.M., Wade, E. and Moriyasu, M., 2009. Proposed harvest control rule for managing the snow crab stock in the southwestern Gulf of St. Lawrence, Canada. Fisheries Research: 268279.

Skaug H.J., and Fournier D.A. 2006. Automatic approximation of the marginal likelihood in non-Gaussian hierarchical models. Computational Statistics \& Data Analysis, 51: 699-709.

Smith, M.T. and Addison, J.T., 2003. Methods for stock assessment of crustacean fisheries. Fisheries Research, 65: 231-256.

Surette, T.J. and Wade, E., 2006. Bayesian serial linear regression models for forecasting the short-term abundance of commercial snow crab (Chionoecetes opilio). Canadian Technical Report of Fisheries and Aquatic Sciences, 2672: vi + 33 p. 
698 Ward, H.G., Askey, P.J. and Post, J.R., 2013. A mechanistic understanding of hyperstability in 699 catch per unit effort and density-dependent catchability in a multistock recreational fishery.

700 Canadian Journal of Fisheries and Aquatic Sciences, 70: 1542-1550.

701

702 Walter, J.F., Hoenig, J.M. and Gedamke, T., 2007. Correcting for effective area fished in fishery703 dependent depletion estimates of abundance and capture efficiency. ICES Journal of Marine 704 Science: Journal du Conseil, 64: 1760-1771.

705

706

Zhou, S., Dichmont, C., Burridge, C.Y., Venables, W.N., Toscas, P.J. and Vance, D., 2007. Is

707 catchability density-dependent for schooling prawns?. Fisheries Research, 85: 23-36.

708

709

Zhou, S., Punt, A.E., Deng, R. and Bishop, J., 2011. Estimating multifleet catchability

710 coefficients and natural mortality from fishery catch and effort data: comparison of Bayesian state-space and observation error models. Canadian Journal of Fisheries and Aquatic Sciences, 712 68: 1171-1181.

713

714 Zhou, S., Buckworth, R.C., Ellis, N., Deng, R.A. and Pascoe, S., 2015. Getting all information 715 out of logbooks: estimating banana prawn fishable biomass, catchability, and fishing power 716 increase, with a focus on natural mortality. ICES Journal of Marine Science: Journal du Conseil, $717 \quad 72: 54-61$. 


\section{Figure Captions}

720 Figure 1. Locations of snow crab (Chionoecetes opilio) fishing grounds and management areas in 721 the southern Gulf of St. Lawrence. PEI indicates the province of Prince Edward Island. 20 and 722200 meter depth contours are shown with heavy grey lines.

723 Figure 2. Landings (tonnes) by fishery management areas in the southern Gulf of St. Lawrence 724 snow crab fishery, 1969 to 2015.

725 Figure 3. CPUE and average soak time versus week for the 2014 snow crab fishery.

726 Figure 4. Weekly catch per unit of effort (CPUE) of snow crab in the southern Gulf of St.

727 Lawrence in 2014. Colors of spatial grids correspond to CPUE levels, as indicated in the legend 728 on the right-hand side. Weeks are indicated in the top-left corner of each panel. Darkest red grids 729 indicate CPUE $>110 \mathrm{Kg} /$ pot which is the 95 th percentile.

730 Figure 5. Illustration of two models for effort saturation. Effective effort is the number of 731 independent fishing locations, with a maximum of Emax $=2000$. PP (Poisson Process) indicates 732 randomly chosen fishing locations and HS (hockey-stick) indicates locations chosen to avoid 733 existing gear. Dashed grey lines are references for Emax and Emax/2, and the 1:1 line. Vertical 734 lines indicate the nominal effort corresponding to Emax/2 effective effort.

735 Figure 6. Log CPUE (total catch divided by total effort) versus log mean biomass for all weeks 736 in each grid cell. Panels correspond to years. Biomass values were obtained from model M1 (see 737 Table 2). The dashed reference lines indicate average log CPUE. The grey lines are linear 738 regressions of $\log$ CPUE versus log biomass. Red lines are linear regressions with the slope fixed 739 at one (i.e. no density dependence). 
740 Figure 7. DFO estimates of biomass from the snow crab survey (SCS; green and blue lines) and

741 M4 model estimates (red and black lines). Colors correspond to sources and shaded regions

742 indicate $95 \%$ confidence intervals. CPUE is shown using dashed lines. Horizontal lines indicate

743 the series average. SCS biomass values were obtained using kriging estimation (see Hébert et al.

$7442016 b$

745 Figure 8. Snow crab survey (SCS) M4 residuals versus predicted values. The red curve is

746 smoother of residuals versus biomass.

747 Figure 9. Spatial effects in snow crab catchability. Colors of spatial grids correspond catchability

748 effects, as indicated in the legend on the right hand side and directly on the figure. Darkest red

749 grids indicate effects $>95$ th percentile.

750 Figure 10. Pot catchability (solid lines) and soak time (dashed lines) versus year. Catchability is

751 effort-weighted across grid cells and standardized by grid area.

752 Figure 11. Annual average maximum effective effort (pots x 1000). Colors of spatial grids

753 correspond to effort, as indicated in the legend on the right hand side and directly on the figure.

754 Darkest red grids indicate effort $>5000$ pots.

755 Figure 12. Harvest rate estimates from the M4 model and values based on projected stock

756 assessment estimates of snow crab survey (SCS) commercial biomass. Colors correspond to

757 sources and shaded regions indicate $95 \%$ confidence intervals. Horizontal lines indicate the

758 series average. 
Table 1. Acronyms, model notation and parameters.

\begin{tabular}{|c|c|}
\hline & Acronyms and notation \\
\hline sGSL & Southern Gulf of St. Lawrence \\
\hline $\mathrm{CW}$ & Carapace width \\
\hline $\mathrm{TAC}$ & Total allowable catch \\
\hline $\mathrm{ME}$ & Measurement error \\
\hline SD & Standard deviation \\
\hline $\mathrm{CV}$ & Coefficient of Variation \\
\hline CPUE, $\mathrm{X}$ & Catch per unit of effort \\
\hline $\mathrm{C}_{\mathrm{obs}}$ & Reported fishery Catch \\
\hline $\mathrm{E}_{\mathrm{obs}}$ & Reported fishery effort \\
\hline$q$ & CPUE Catchability/potential area fished \\
\hline$E_{\max }$ & Maximum effective effort \\
\hline $\mathrm{E}_{\mathrm{o}}$ & Nominal fishing effort without adjustment for saturation \\
\hline $\mathrm{S}_{\mathrm{obs}}$ & Snow crab survey trawl catch (biomass) \\
\hline$A$ & Grid cell area \\
\hline$p$ & Fraction of $\mathrm{A}$ that is crab grounds \\
\hline$a_{f}$ & Area fished by a pot \\
\hline$y$ & year \\
\hline$i$ & Grid cell, $i=1, \ldots, G$ \\
\hline$G$ & Total number of grid cells. \\
\hline$t$ & week \\
\hline$G M R F$ & Gaussian Markov random field \\
\hline$\varphi_{N}$ & Standard normal probability density function \\
\hline$\theta$ & Fixed effect model parameters \\
\hline$\Gamma$ & Model random effects \\
\hline & model parameters $(P)$ and random effects $(R E)$ \\
\hline$F$ & Fishing mortality rate $(\mathrm{RE})$ \\
\hline$\mu_{F}$ & mean $F(\mathrm{P})$ \\
\hline$\sigma_{F}$ & $\mathrm{CV} F(\mathrm{P})$ \\
\hline$q_{y}$ & Catchability year effects (RE) \\
\hline$\sigma_{q y}$ & $\mathrm{CV}$ for $q_{y}(\mathrm{P})$ \\
\hline$\delta$ & Weekly process errors in catchability and ME in effort (RE) \\
\hline$\sigma_{\delta}$ & $\mathrm{SD}$ for $\delta(\mathrm{P})$ \\
\hline$\varphi_{\delta}$ & Auto-correlation for $\delta(\mathrm{P})$ \\
\hline$q_{i}$ & Catchability spatial effects (RE) \\
\hline$\tau_{q}, \vartheta_{q}$ & Precision matrix parameters for $q_{i}(\mathrm{P})$ \\
\hline$D_{y, o, i}$ & Initial biomass density $(\mathrm{RE})$ \\
\hline$\tau_{D 1}, \vartheta_{D 1}$ & $\Psi$ parameters for $D_{1, o, i}$ in first year $(\mathrm{P})$ \\
\hline$\tau_{D 2}, \vartheta_{D 2}$ & $\Psi$ parameters for log-deviations in $D_{y, o, i}$ in other years $(\mathrm{P})$ \\
\hline$\beta$ & Density-dependence catchability parameter $(\mathrm{P})$ \\
\hline$\sigma_{C}$ & Catch measurement error CV $(\mathrm{P})$ \\
\hline$\sigma_{S}$ & Standard deviation of snow crab survey catch $(\mathrm{P})$ \\
\hline
\end{tabular}


model derived quantities

$Z \quad$ Total mortality rate, $Z=F+M$

$\Psi \quad$ Spatial precision matrix

$q_{y, t, i} \quad$ CPUE catchability in year $y$, week $t$ and grid cell $i$

$B_{y, o, i} \quad$ Initial annual biomass, $B_{y, o, i}=D_{y, o, i} A_{i}$

$C_{y, t, i} \quad$ Catch

$H_{y, t, i} \quad$ Harvest rate, $H_{y, t, i}=C_{y, t, i} / B_{y, t, i}$

$E_{y, t, i} \quad$ Model predicted effort

$D_{y, o, i} \quad$ Initial biomass density,

M Natural mortality rate

fixed model inputs

$\gamma \quad$ Fixed parameter controlling the transition of effective effort to $\mathrm{E}_{\max }$

$m_{i} \quad$ The number of neighbors of grid cell $i$ 
Table 2. Estimates (Est) of model parameters (see Table 1) and some population quantities, with percent coefficients of variation (CVx100), for five model formulations. nll denotes the negative loglikelihood and $\triangle \mathrm{AIC}$ is the difference in Akaike information criterion from the minimum of the models.

\begin{tabular}{|c|c|c|c|c|c|c|c|c|c|c|}
\hline \multirow{4}{*}{$\begin{array}{r}\Delta \mathrm{AIC}: \\
\text { nll: } \\
\text { Quantity }\end{array}$} & \multicolumn{2}{|c|}{ M1 } & \multicolumn{2}{|c|}{ M2 } & \multicolumn{2}{|c|}{ M3 } & \multicolumn{2}{|c|}{ M4 } & \multicolumn{2}{|c|}{ M5 } \\
\hline & \multicolumn{2}{|c|}{3442.1} & \multicolumn{2}{|c|}{3429.4} & \multicolumn{2}{|c|}{0} & \multicolumn{2}{|c|}{2.7} & \multicolumn{2}{|c|}{3409.7} \\
\hline & \multicolumn{2}{|c|}{56899} & \multicolumn{2}{|c|}{56893} & \multicolumn{2}{|c|}{55177} & \multicolumn{2}{|c|}{55178} & \multicolumn{2}{|c|}{56883} \\
\hline & Est & $\mathrm{CV} \%$ & Est & $\mathrm{CV} \%$ & Est & $\mathrm{CV} \%$ & Est & $\mathrm{CV} \%$ & Est & $\mathrm{CV} \%$ \\
\hline$q_{1997}\left(x 10^{-4}\right)$ & 6.609 & 18.7 & 6.568 & 18.8 & 6.474 & 13.8 & 6.439 & 13.8 & 6.454 & 18.7 \\
\hline$\beta$ & - & - & - & - & 0.387 & 2.0 & 0.388 & 1.9 & - & - \\
\hline$\mu_{F}$ & 0.053 & 1.3 & 0.053 & 1.3 & 0.078 & 2.1 & 0.076 & 2.0 & 0.052 & 1.3 \\
\hline$\sigma_{F}$ & 1.149 & 0.5 & 1.149 & 0.5 & 1.130 & 0.7 & 1.130 & 0.7 & 1.149 & 0.5 \\
\hline$\sigma_{q y}$ & 0.090 & 23.0 & 0.090 & 23.0 & 0.106 & 18.3 & 0.106 & 18.3 & 0.089 & 23.1 \\
\hline$\sigma_{\delta}$ & 0.474 & 0.6 & 0.474 & 0.6 & 0.463 & 0.6 & 0.464 & 0.6 & 0.474 & 0.6 \\
\hline$\sigma_{S}$ & 1.409 & 1.4 & 1.409 & 1.4 & 1.145 & 4.4 & 1.153 & 3.6 & 1.409 & 1.5 \\
\hline$\tau_{D 1}$ & 0.614 & 10.2 & 0.610 & 10.2 & 0.511 & 11.0 & 0.514 & 11.0 & 0.611 & 10.2 \\
\hline$\vartheta_{D 1}$ & 0.000 & 141.8 & 0.000 & 141.8 & 0.000 & 141.9 & 0.000 & 141.9 & 0.000 & 141.8 \\
\hline$\tau_{D 2}$ & 3.936 & 4.8 & 3.932 & 4.8 & 1.440 & 5.4 & 1.467 & 5.1 & 4.016 & 5.0 \\
\hline$\vartheta_{D 2}$ & 0.042 & 24.5 & 0.042 & 24.5 & 0.090 & 22.1 & 0.087 & 21.9 & 0.041 & 24.8 \\
\hline$\tau_{q}$ & 0.543 & 11.4 & 0.535 & 11.4 & 3.913 & 13.3 & 3.911 & 13.3 & 0.538 & 11.4 \\
\hline$\vartheta_{q}$ & 0.227 & 52.9 & 0.229 & 52.8 & 0.110 & 66.6 & 0.109 & 66.7 & 0.229 & 52.8 \\
\hline$\varphi_{q}$ & 0.359 & 2.8 & 0.360 & 2.8 & 0.289 & 3.2 & 0.288 & 3.1 & 0.359 & 2.8 \\
\hline$B_{2014}(\mathrm{Kt})$ & 66.815 & 1.9 & 66.802 & 1.9 & 56.498 & 2.3 & 59.792 & 2.3 & 70.799 & 1.9 \\
\hline$B_{2014} / \bar{B}$ & 1.118 & 1.8 & 1.118 & 1.8 & 1.116 & 2.1 & 1.119 & 2.1 & 1.119 & 1.8 \\
\hline$H_{2014}(\%)$ & 34.803 & 1.9 & 34.810 & 1.9 & 41.155 & 2.3 & 38.887 & 2.3 & 32.844 & 1.9 \\
\hline$H_{2014} / \bar{H}$ & 1.047 & 1.8 & 1.047 & 1.8 & 1.049 & 2.1 & 1.047 & 2.1 & 1.046 & 1.8 \\
\hline$a_{f, 2014}\left(x 10^{-4}\right)$ & 7.315 & 3.4 & 7.269 & 3.4 & 7.566 & 2.3 & 7.521 & 2.3 & 7.146 & 3.4 \\
\hline$a_{f, 2014} / \bar{a}_{f}$ & 1.052 & 2.8 & 1.052 & 2.8 & 1.128 & 2.0 & 1.128 & 2.0 & 1.053 & 2.8 \\
\hline
\end{tabular}

M1: No density dependence $(\boldsymbol{\beta}=\mathbf{1})$, effort saturation, $\mathrm{M}=0.3$.

M2: No density dependence $(\boldsymbol{\beta}=\mathbf{1})$, no effort saturation, $\mathrm{M}=0.3$.

M3: Density dependence ( $\boldsymbol{\beta}$ estimated), no effort saturation, $\mathrm{M}=0.3$.

M4: Density dependence, no effort saturation, $\mathrm{M}=0.4$.

M5: No density dependence, no effort saturation, $\mathrm{M}=0.4$. 
Table 3. Southern Gulf of St. Lawrence snow crab M4 model estimates (Est) and coefficients of variation ( $\mathrm{CV}, \%)$ of 1 ) beginning of year (i.e. initial) biomass, 2) post-season biomass, 3 ) harvest rates, and 4) area fished. See Table 2 footnotes for M4 formualtion.

\begin{tabular}{ccccccccr}
\hline & $\begin{array}{c}\text { Initial Biomass } \\
(\mathrm{Kt})\end{array}$ & \multicolumn{2}{c}{$\begin{array}{c}\text { Post-Season } \\
\text { Biomass }(\mathrm{Kt})\end{array}$} & \multicolumn{2}{c}{$\begin{array}{c}\text { Harvest Rate } \\
(\%)\end{array}$} & \multicolumn{2}{c}{$\begin{array}{c}\text { Area Fished } \\
\left(\mathrm{x} 10^{4} \mathrm{~m}^{2}\right)\end{array}$} \\
\hline Year & Est & CV\% & \multicolumn{1}{c}{ Est } & CV\% & Est & CV\% & Est & CV\% \\
\hline 1997 & 57.3 & 2.9 & 28.3 & 4.5 & 29.9 & 2.9 & 6.3 & 2.3 \\
1998 & 53.1 & 2.5 & 28.3 & 3.7 & 25.7 & 2.5 & 5.8 & 2.4 \\
1999 & 57.4 & 2.7 & 30.2 & 3.9 & 26.6 & 2.7 & 6.0 & 2.5 \\
2000 & 37.7 & 2.6 & 12.3 & 6.1 & 48.2 & 2.6 & 5.2 & 2.2 \\
2001 & 40.7 & 2.5 & 14.7 & 5.3 & 44.8 & 2.5 & 5.9 & 2.3 \\
2002 & 46.7 & 1.9 & 12.4 & 5.5 & 55.5 & 1.9 & 5.5 & 2.2 \\
2003 & 57.7 & 2.6 & 25.6 & 4.4 & 35.1 & 2.6 & 6.1 & 2.4 \\
2004 & 66.8 & 1.9 & 22.5 & 4.4 & 47.0 & 1.9 & 6.6 & 2.1 \\
2005 & 75.1 & 1.7 & 26.0 & 3.7 & 46.1 & 1.7 & 7.4 & 2.0 \\
2006 & 57.8 & 1.9 & 20.5 & 4.0 & 46.2 & 1.9 & 7.5 & 2.1 \\
2007 & 61.3 & 2.1 & 25.1 & 4.0 & 39.6 & 2.1 & 7.3 & 2.3 \\
2008 & 53.9 & 2.5 & 21.5 & 4.7 & 40.1 & 2.5 & 6.7 & 2.3 \\
2009 & 47.4 & 1.9 & 16.4 & 4.1 & 46.2 & 1.9 & 6.5 & 2.1 \\
2010 & 30.7 & 3.0 & 15.4 & 4.7 & 29.9 & 3.0 & 7.0 & 2.7 \\
2011 & 54.4 & 3.2 & 32.4 & 4.2 & 19.1 & 3.2 & 6.7 & 2.9 \\
2012 & 59.6 & 2.2 & 26.9 & 3.8 & 35.5 & 2.2 & 8.3 & 2.3 \\
2013 & 65.2 & 2.0 & 27.5 & 3.7 & 38.1 & 2.0 & 9.0 & 2.3 \\
2014 & 59.8 & 2.3 & 24.5 & 4.3 & 39.0 & 2.3 & 7.5 & 2.3 \\
\hline
\end{tabular}




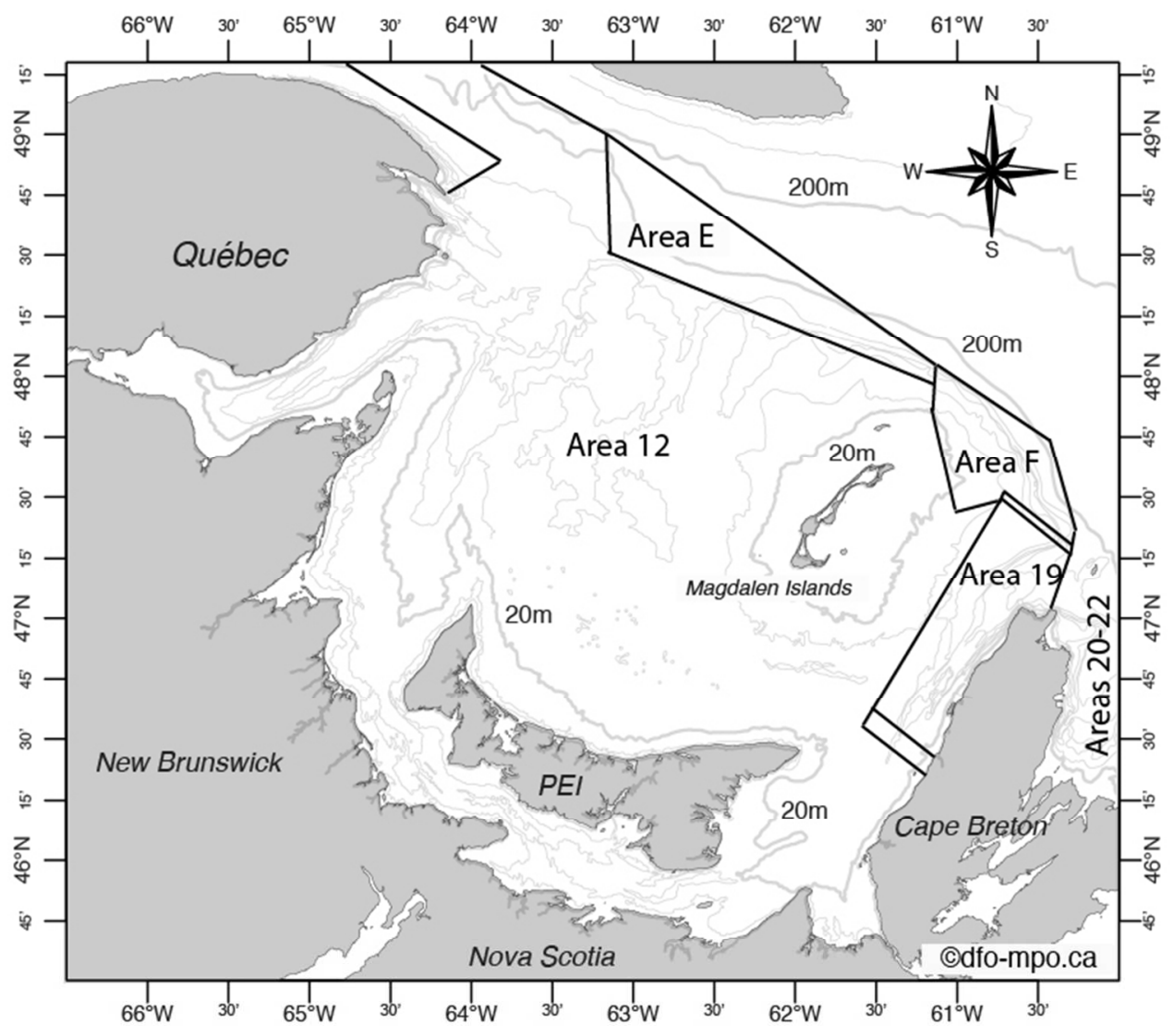

$140 \times 125 \mathrm{~mm}(150 \times 150 \mathrm{DPI})$ 


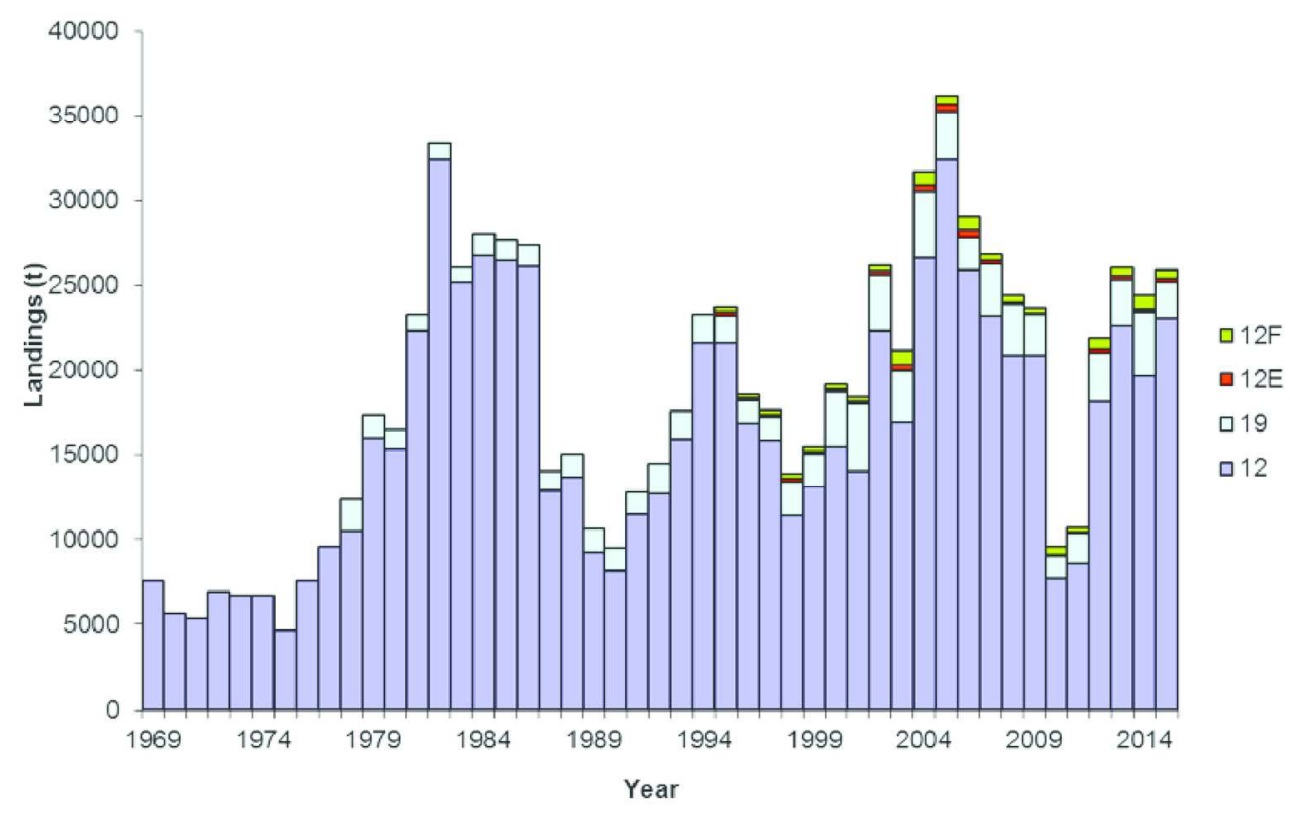

Landings (tonnes) by fishery management areas in the southern Gulf of St. Lawrence snow crab fishery, 1969 to 2015.

$275 \times 179 \mathrm{~mm}(150 \times 150 \mathrm{DPI})$ 


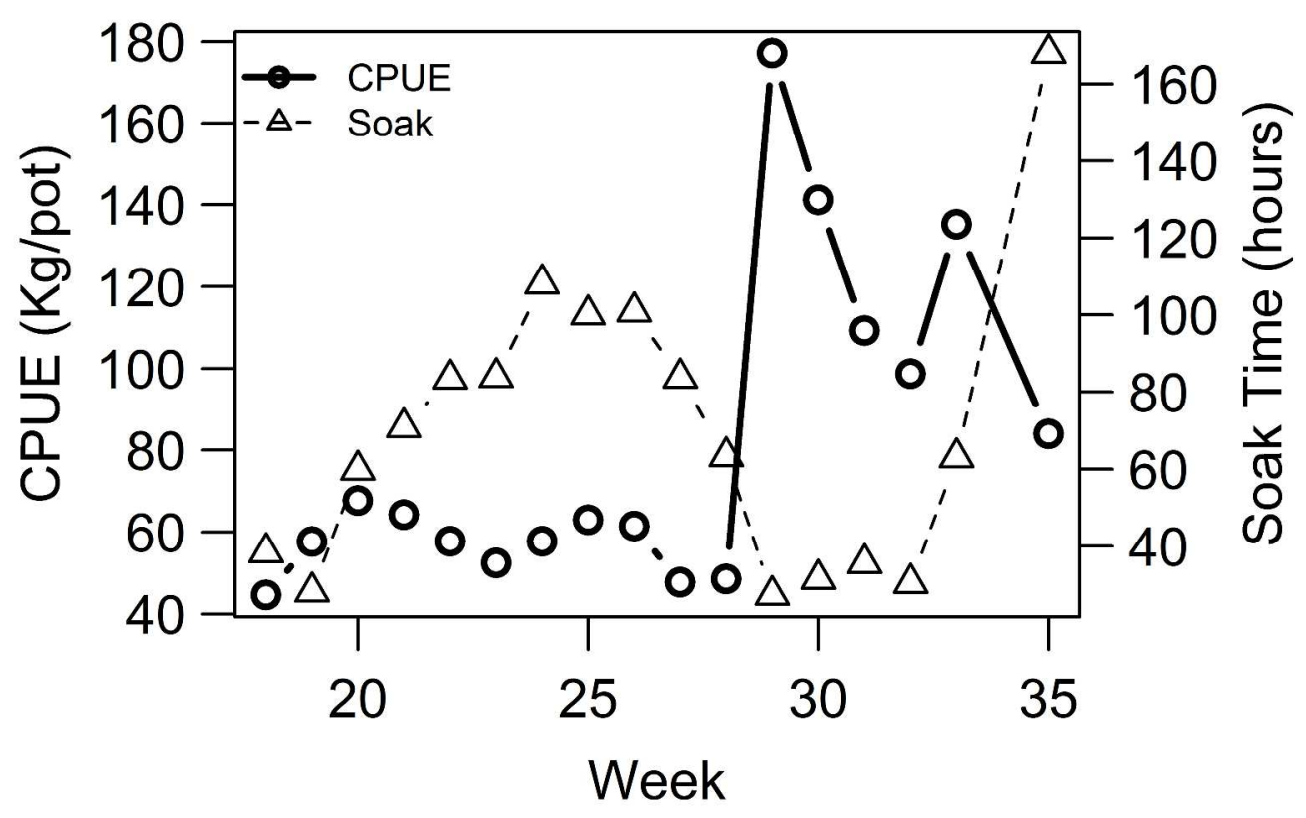

CPUE and average soak time versus week for the 2014 snow crab fishery. 


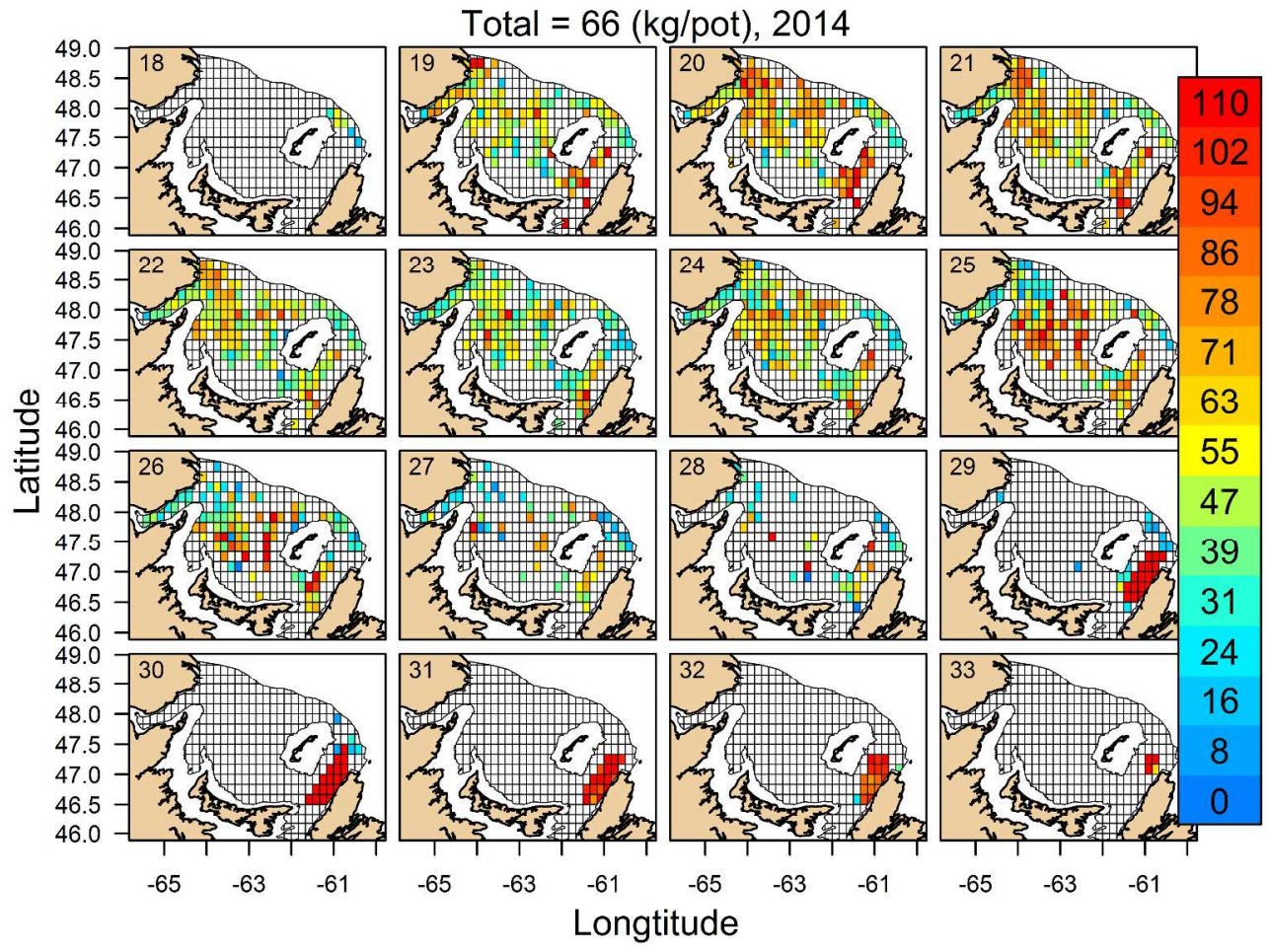

Weekly catch per unit of effort (CPUE) of snow crab in the southern Gulf of St. Lawrence in 2014. Colors of spatial grids correspond to CPUE levels, as indicated in the legend on the right-hand side. Weeks are indicated in the top-left corner of each panel. Darkest red grids indicate CPUE $>110 \mathrm{Kg} /$ pot which is the 95th percentile. 


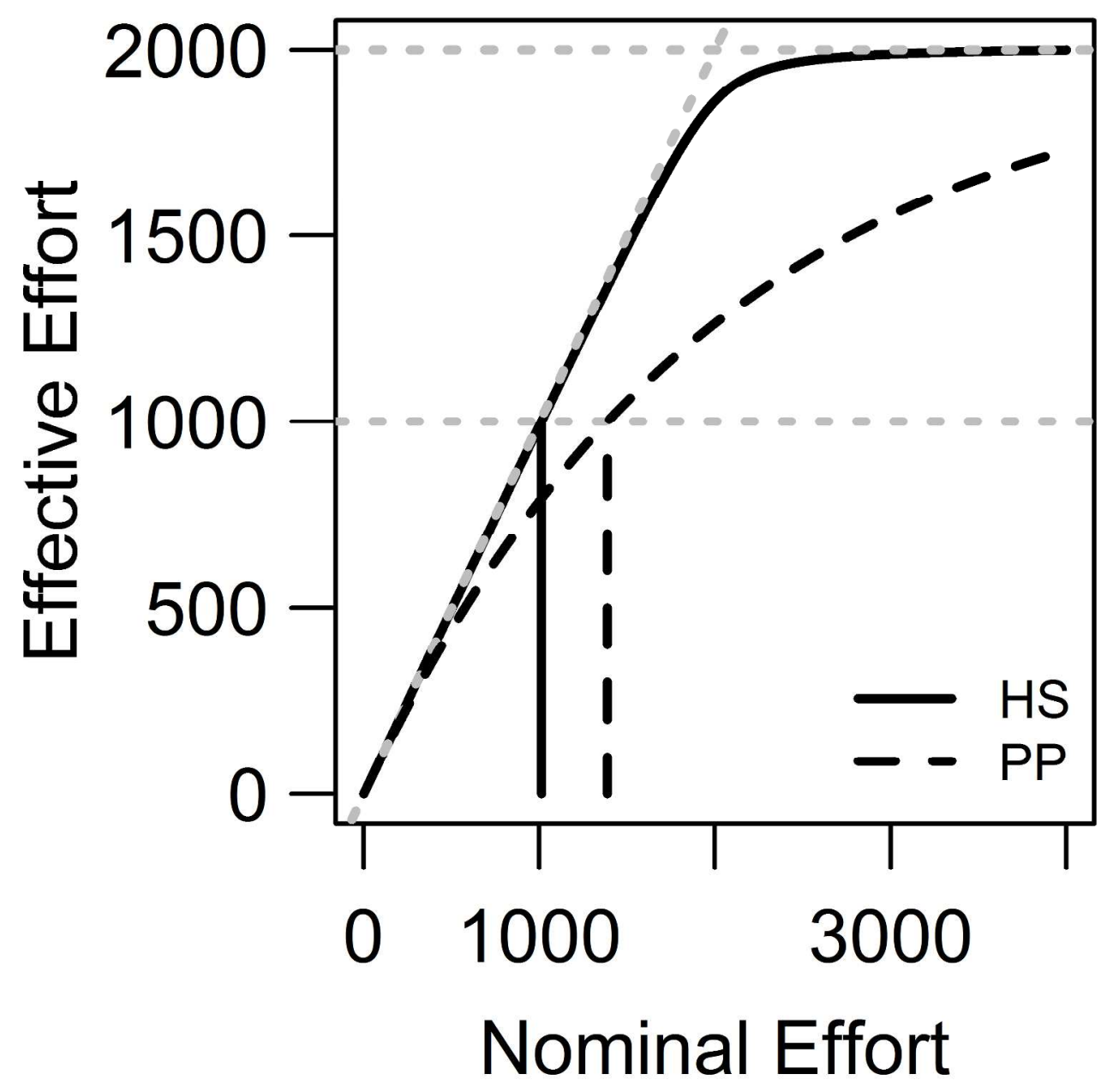

Illustration of two models for effort saturation. Effective effort is the number of independent fishing locations, with a maximum of $E_{\max }=2000$. PP (Poisson Process) indicates randomly chosen fishing locations and HS (hockey-stick) indicates locations chosen to avoid existing gear. Dashed grey lines are references for $E_{\max }$ and $E_{\max } / 2$, and the $1: 1$ line. Vertical lines indicate the nominal effort corresponding to $E_{\max } / 2$ effective effort. 

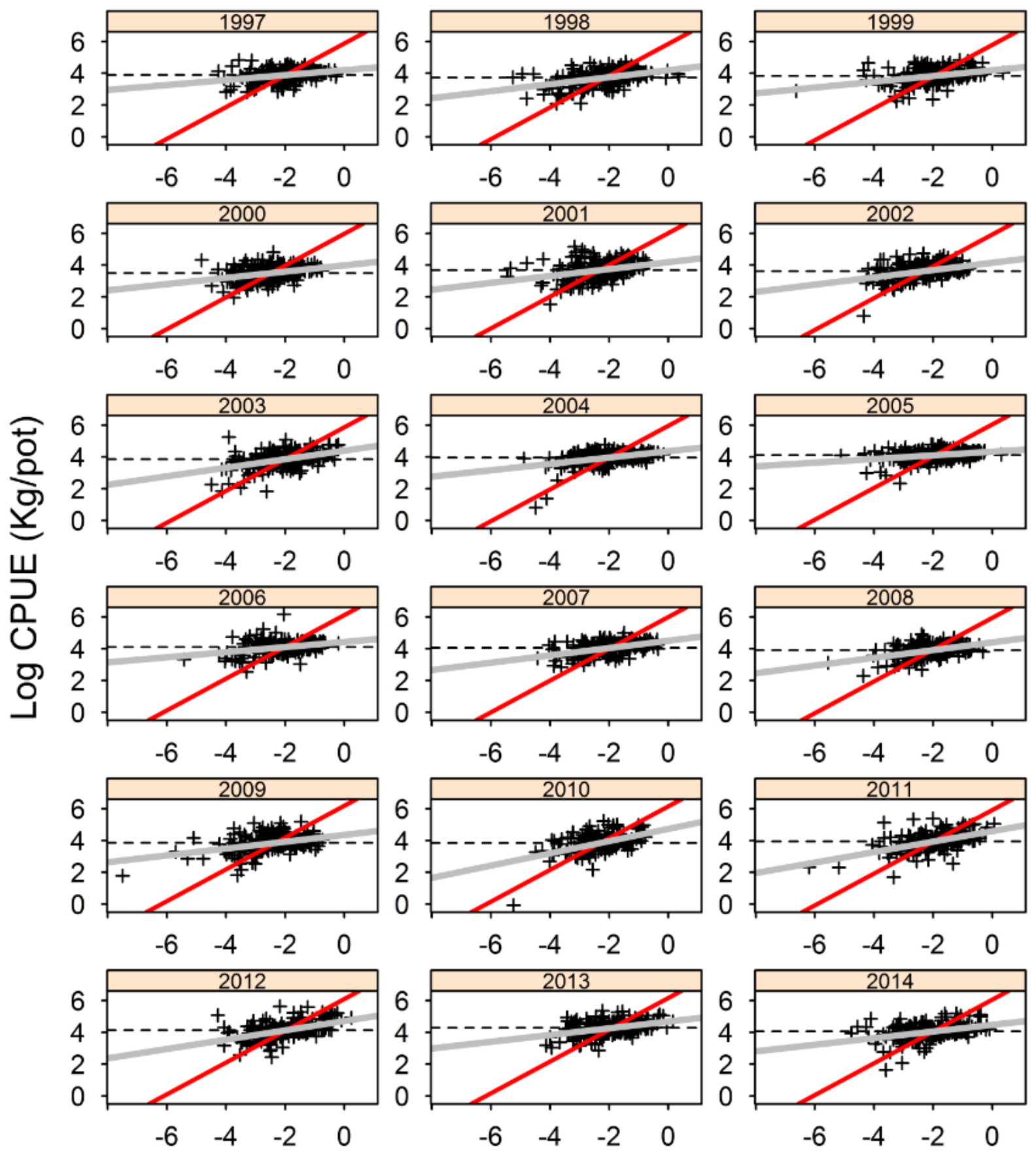

Log Mean Grid Biomass (Kt) 


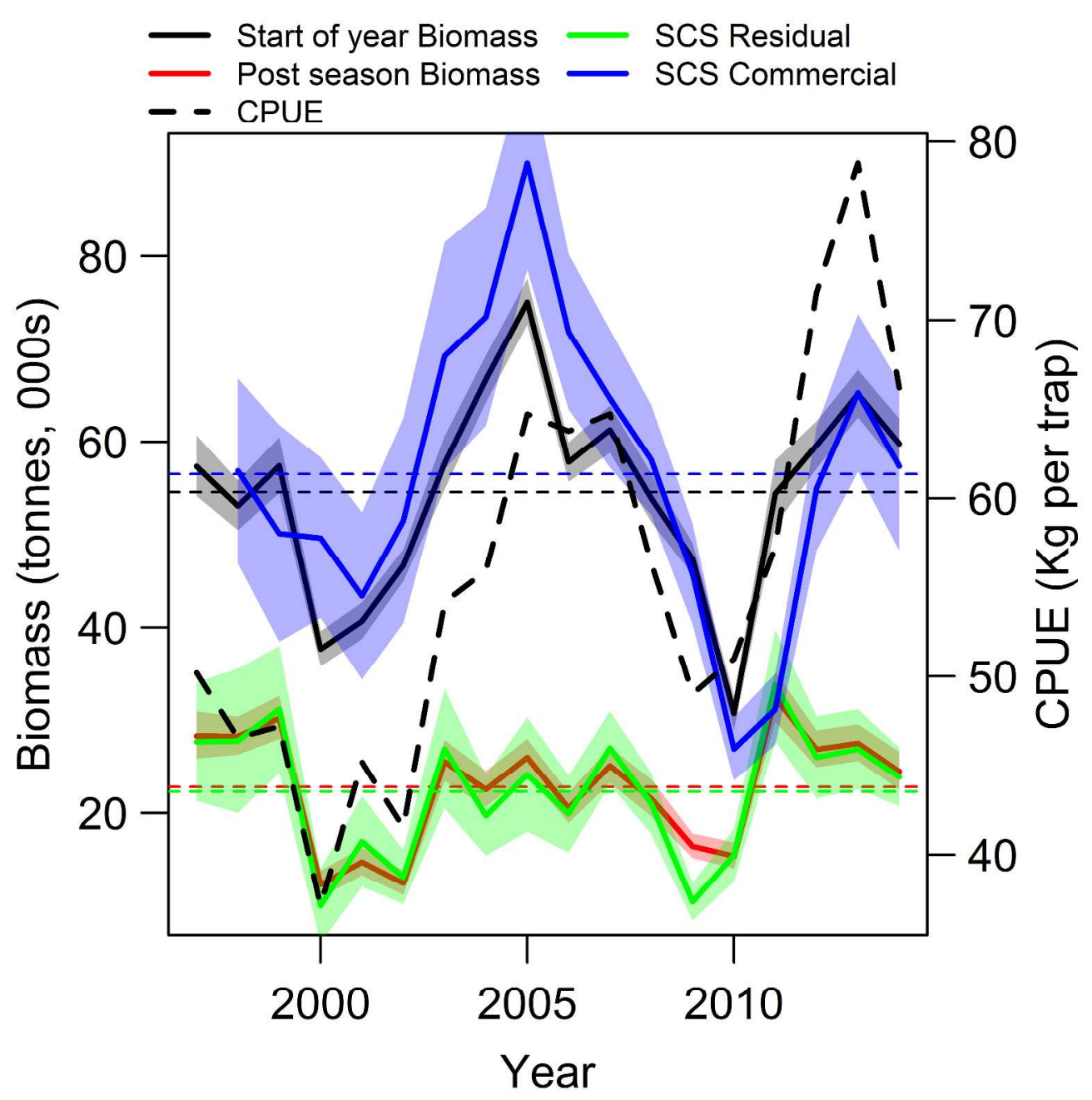

DFO estimates of biomass from the snow crab survey (SCS; green and blue lines) and M4 model estimates (red and black lines). Colors correspond to sources and shaded regions indicate $95 \%$ confidence intervals. CPUE is shown using dashed lines. Horizontal lines indicate the series average. SCS biomass values were obtained using kriging estimation (see Hébert et al. 2016b). 


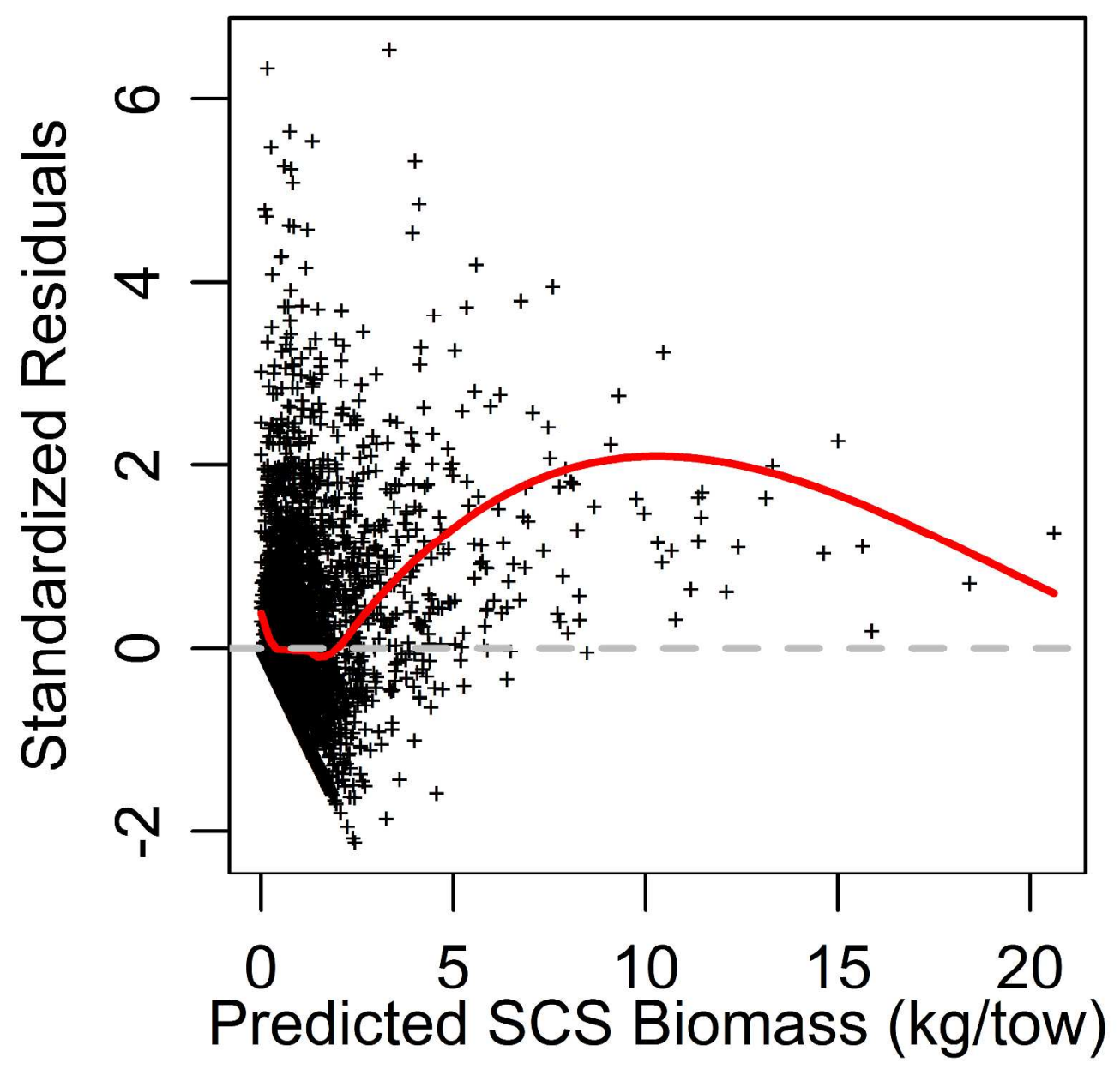

Snow crab survey (SCS) M4 residuals versus predicted values. The red curve is smoother of residuals versus biomass. 


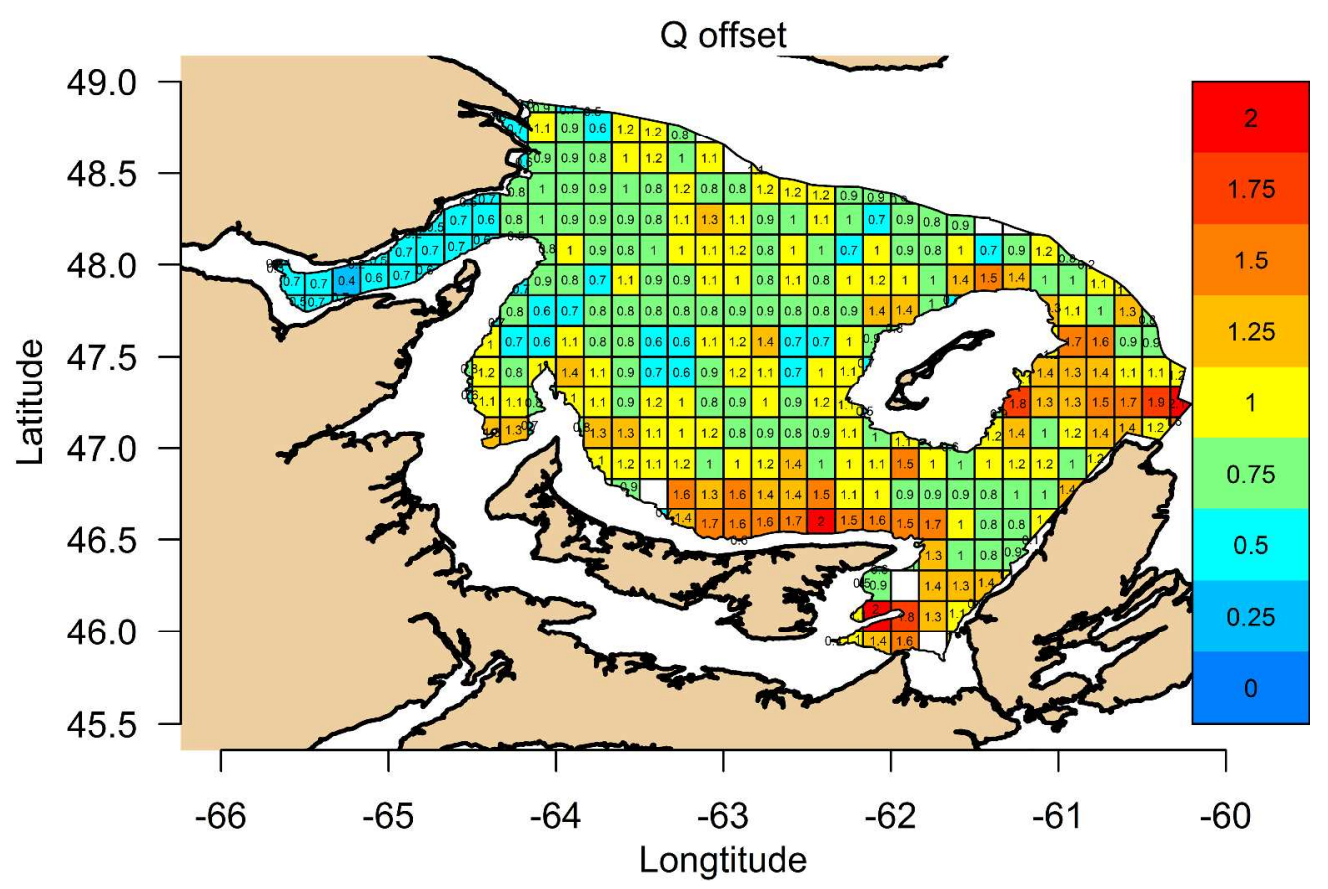

Spatial effects in snow crab catchability. Colors of spatial grids correspond catchability effects, as indicated in the legend on the right hand side and directly on the figure. Darkest red grids indicate effects $>95^{\text {th }}$ percentile. 


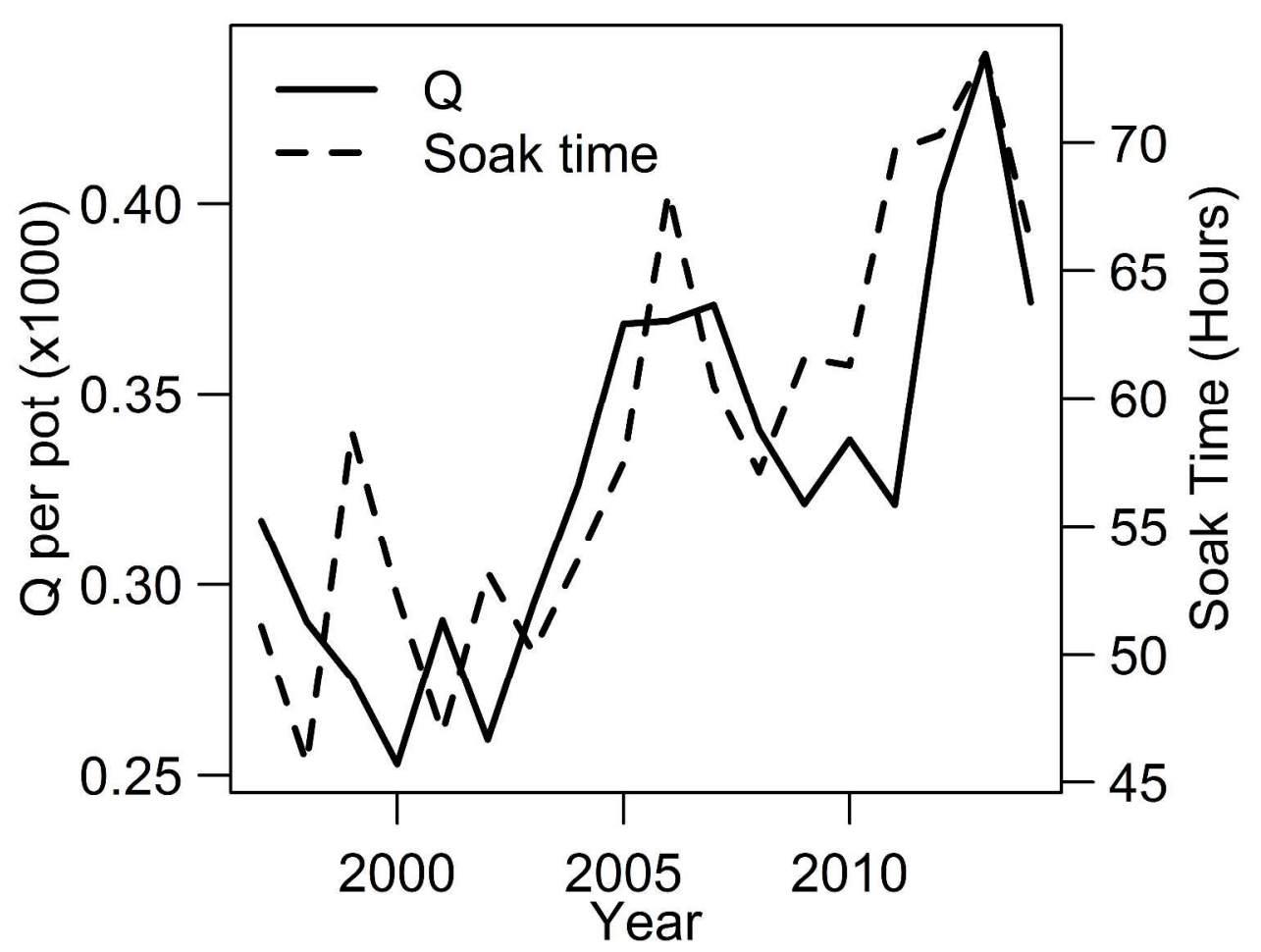

Pot catchability (solid lines) and soak time (dashed lines) versus year. Catchability is effort-weighted across grid cells and standardized by grid area. 


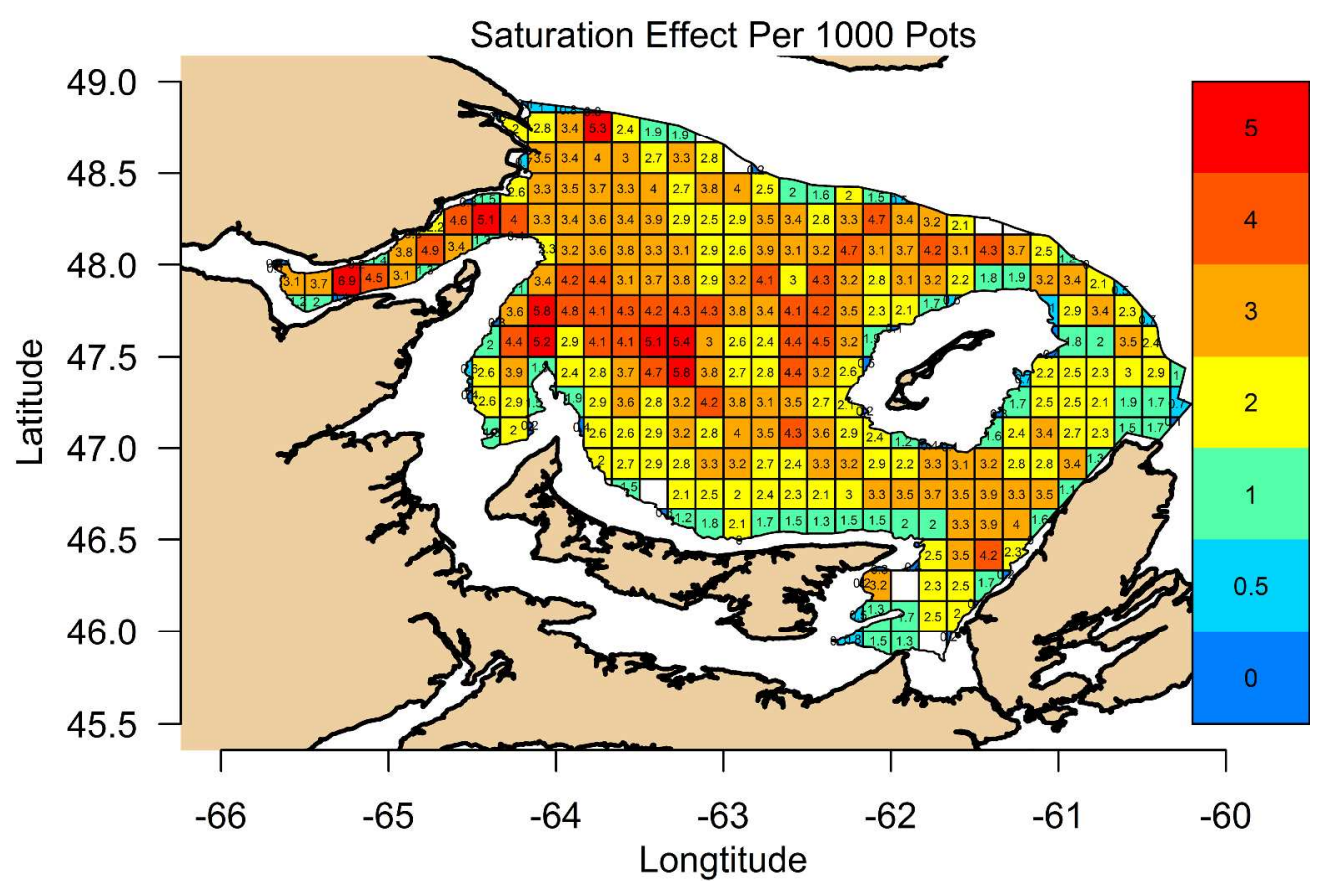

Annual average maximum effective effort (pots $x$ 1000). Colors of spatial grids correspond to effort, as indicated in the legend on the right hand side and directly on the figure. Darkest red grids indicate effort $>$ 5000 pots. 


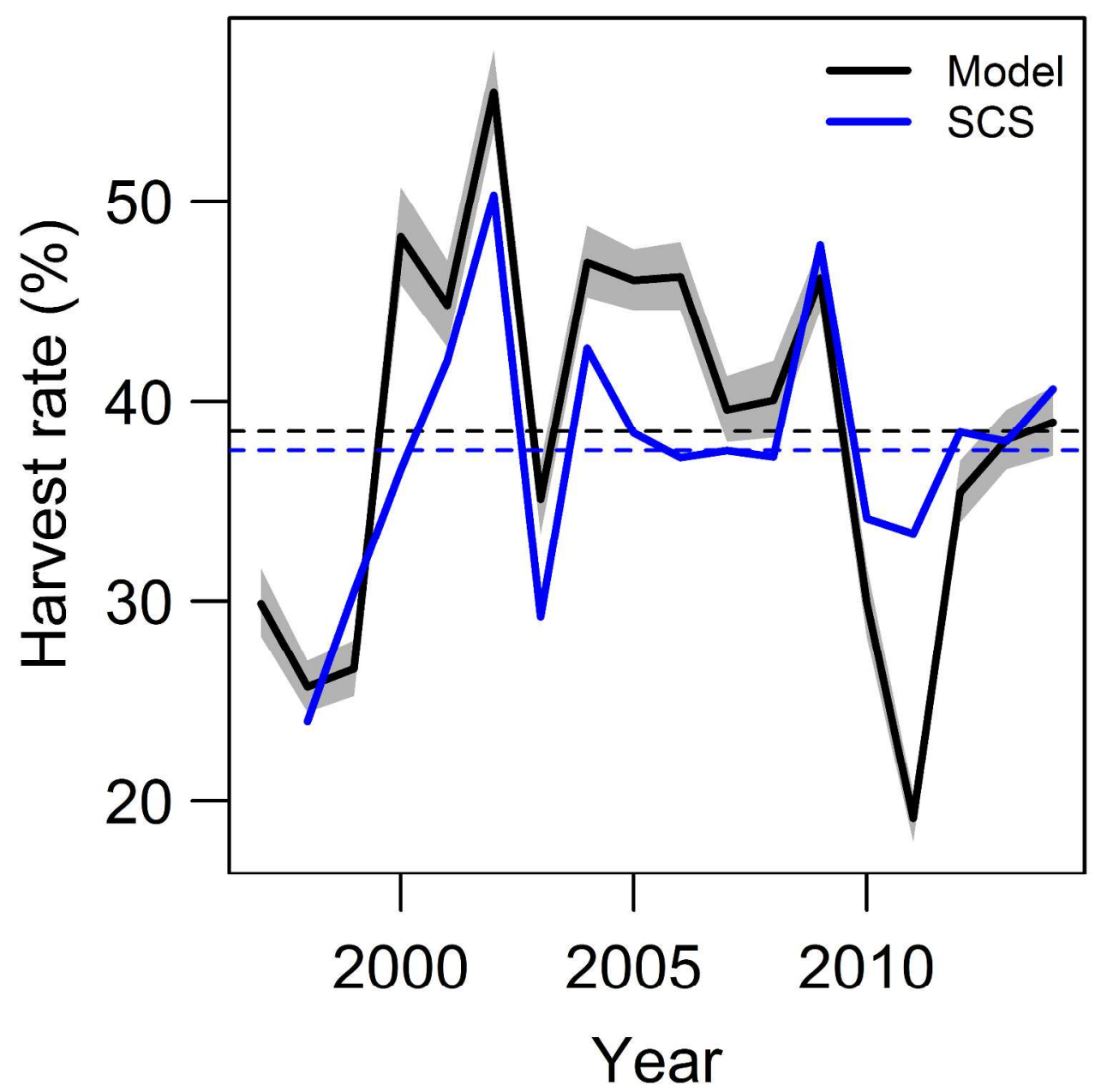

Harvest rate estimates from the M4 model and values based on projected stock assessment estimates of snow crab survey (SCS) commercial biomass. Colors correspond to sources and shaded regions indicate $95 \%$ confidence intervals. Horizontal lines indicate the series average. 


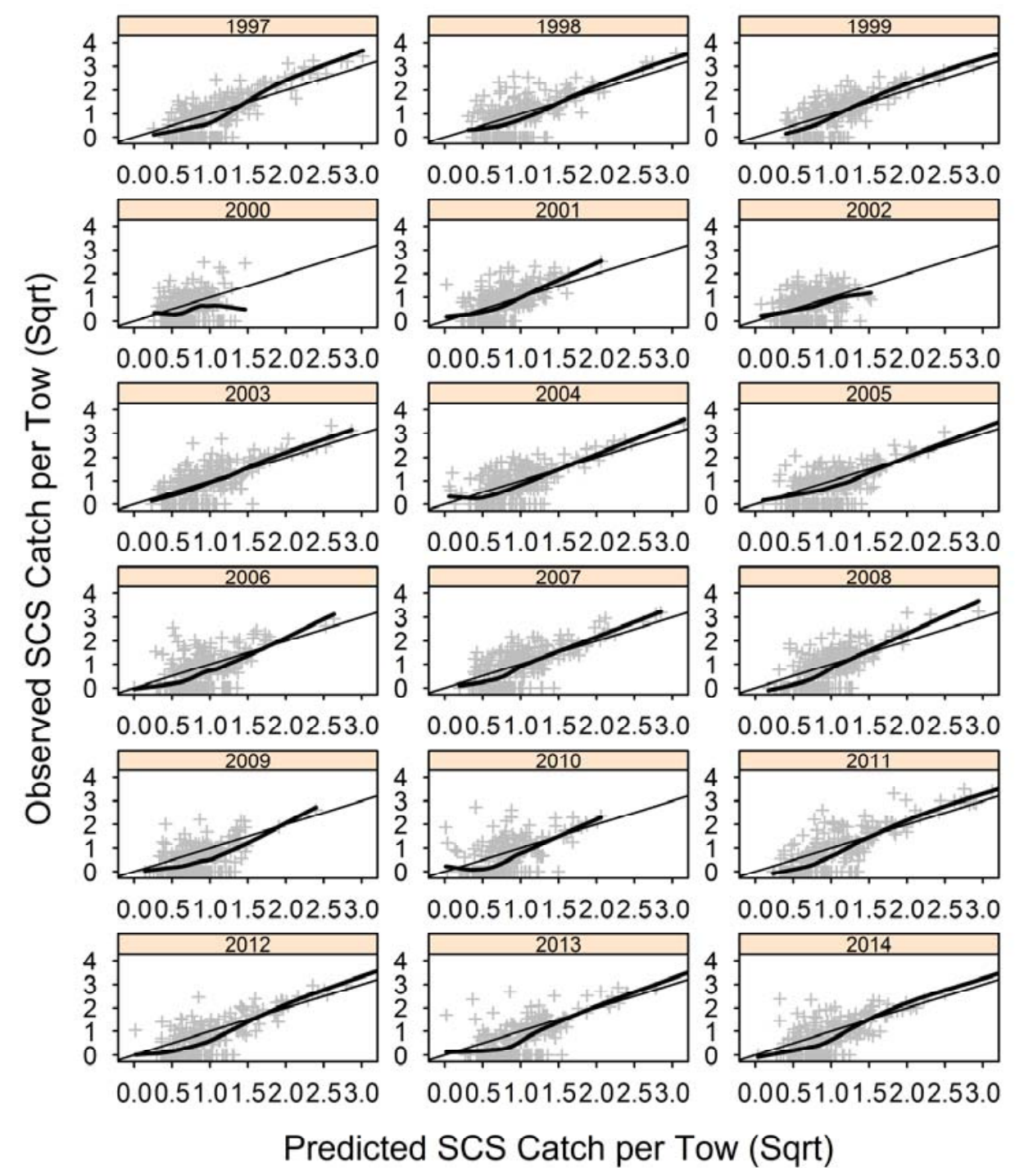

Figure S1. Observed versus predicted snow crab survey exploitable biomass catch per tow (grey +'s). Panels correspond to years. Predicted biomass values were obtained from model M4 (see Table 2). Thin solid lines indicates 1:1 lines, and thick solid lines indicate smoothers. 


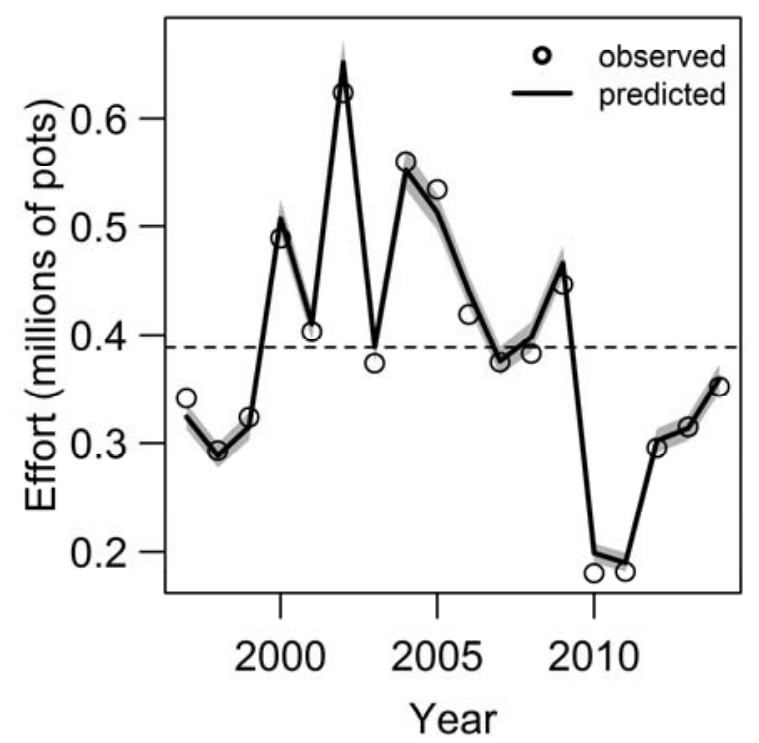

Figure S2. Observed and predicted total annual effort. Shaded regions indicate 95\% confidence intervals. Predicted values were obtained from model M4 (see Table 2). 


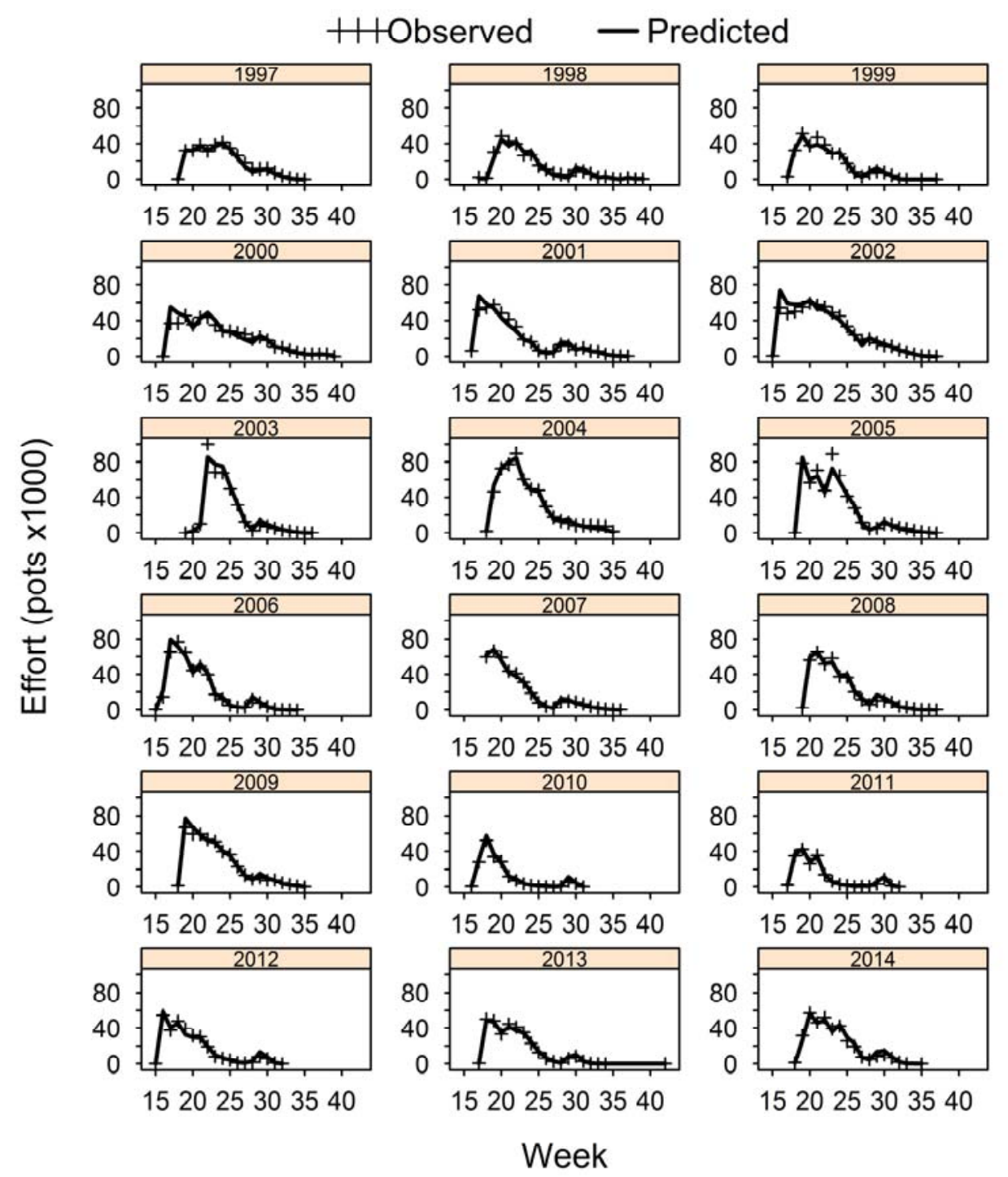

Figure S3. Observed versus predicted total weekly effort. Panels correspond to years. Predicted values were obtained from model M4 (see Table 2). 


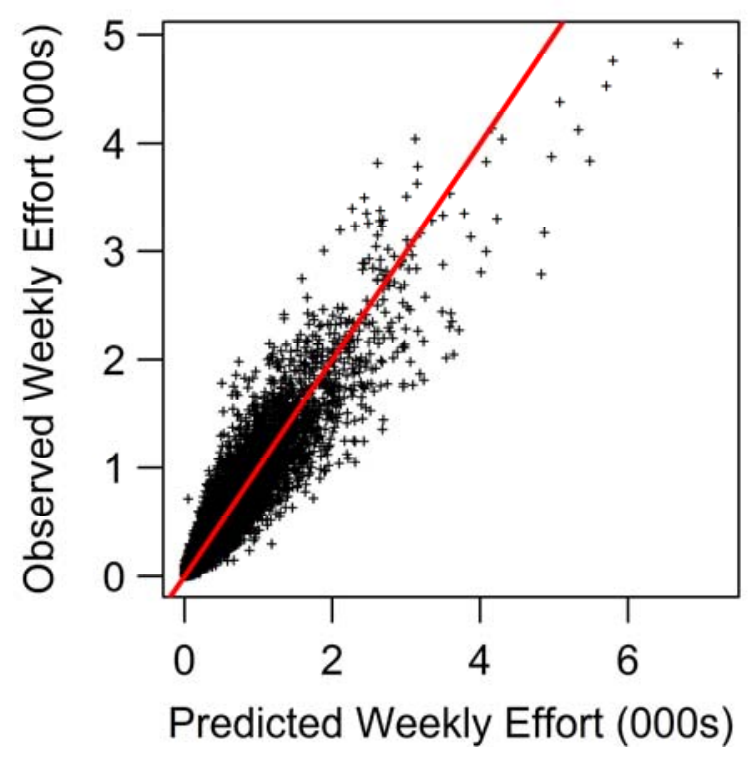

Figure S4. Observed versus predicted effort. Predicted values were obtained from model M4 (see Table 2). The 1:1 line is shown in red. 


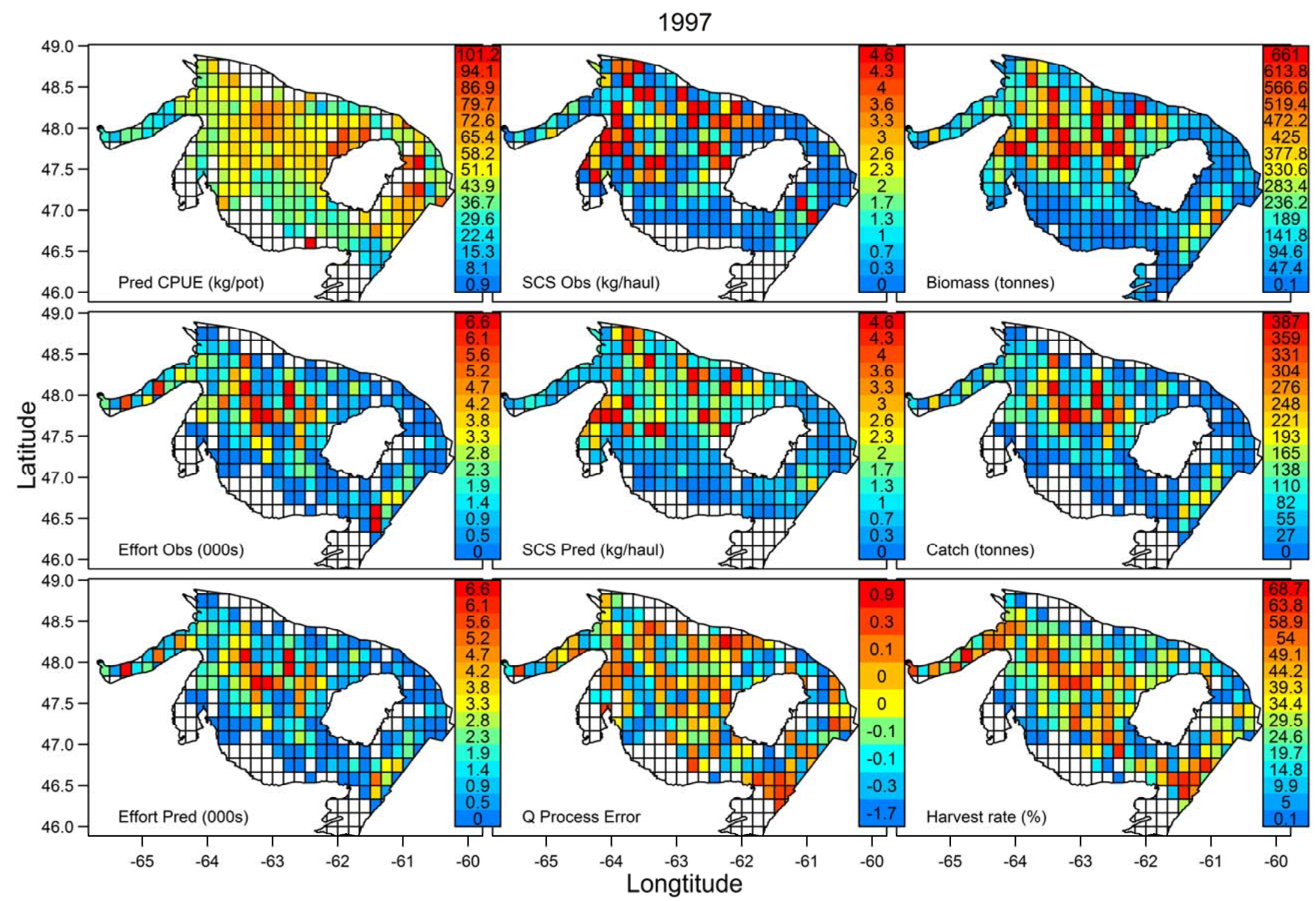

Figure S5. Model M4 (see Table 2) spatial results for 1997. Catch and effort are aggregated across weeks, and CPUE is the ratio of aggregated catch divided by aggregated effort. Q process errors are averaged over weeks. White cells indicate no observations. 


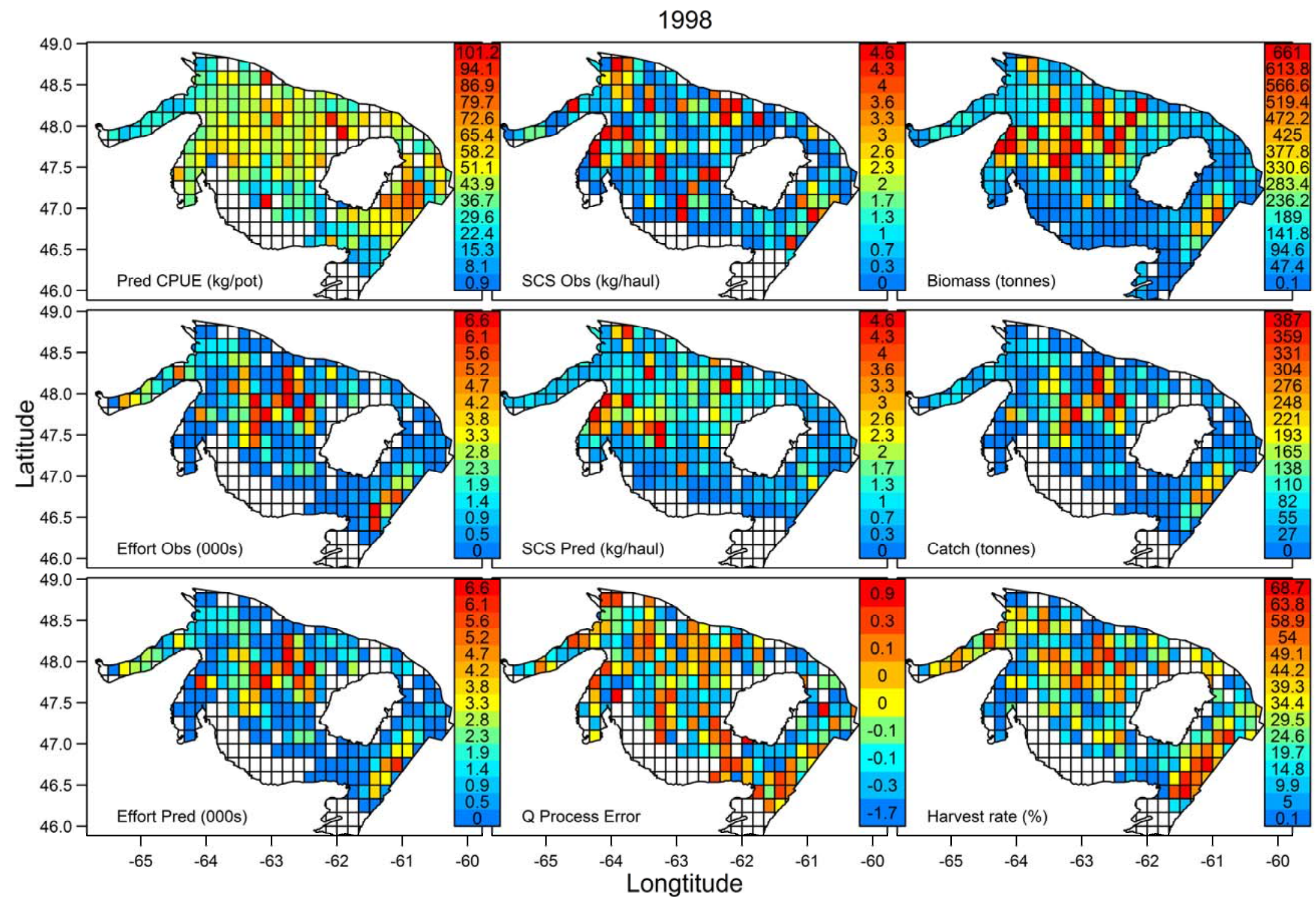

Figure S6. Model M4 (see Table 2) spatial results for 1998. Catch and effort are aggregated across weeks, and CPUE is the ratio of aggregated catch divided by aggregated effort. Q process errors are averaged over weeks. White cells indicate no observations. 


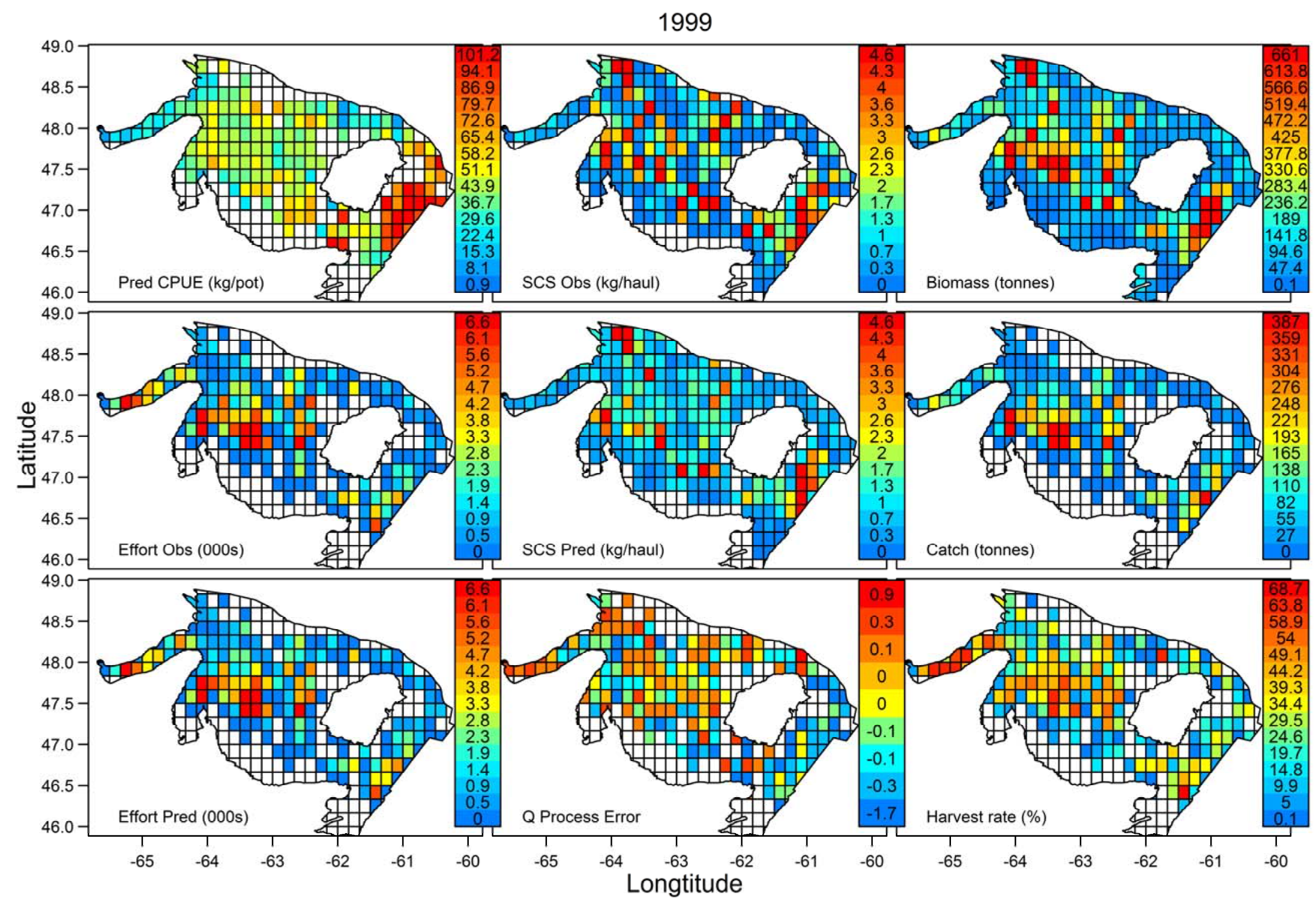

Figure S7. Model M4 (see Table 2) spatial results for 1999. Catch and effort are aggregated across weeks, and CPUE is the ratio of aggregated catch divided by aggregated effort. Q process errors are averaged over weeks. White cells indicate no observations. 


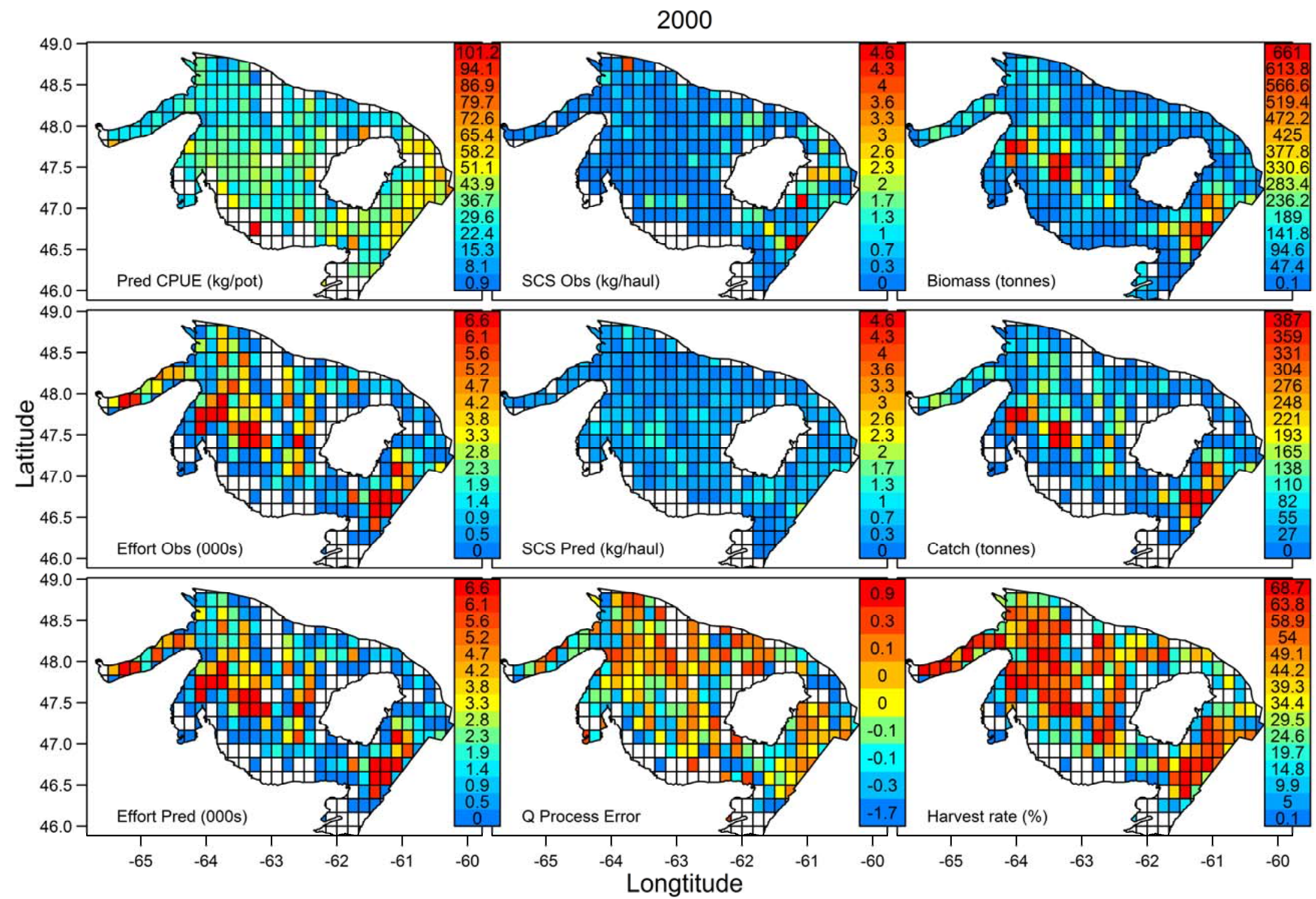

Figure S8. Model M4 (see Table 2) spatial results for 2000. Catch and effort are aggregated across weeks, and CPUE is the ratio of aggregated catch divided by aggregated effort. Q process errors are averaged over weeks. White cells indicate no observations. 


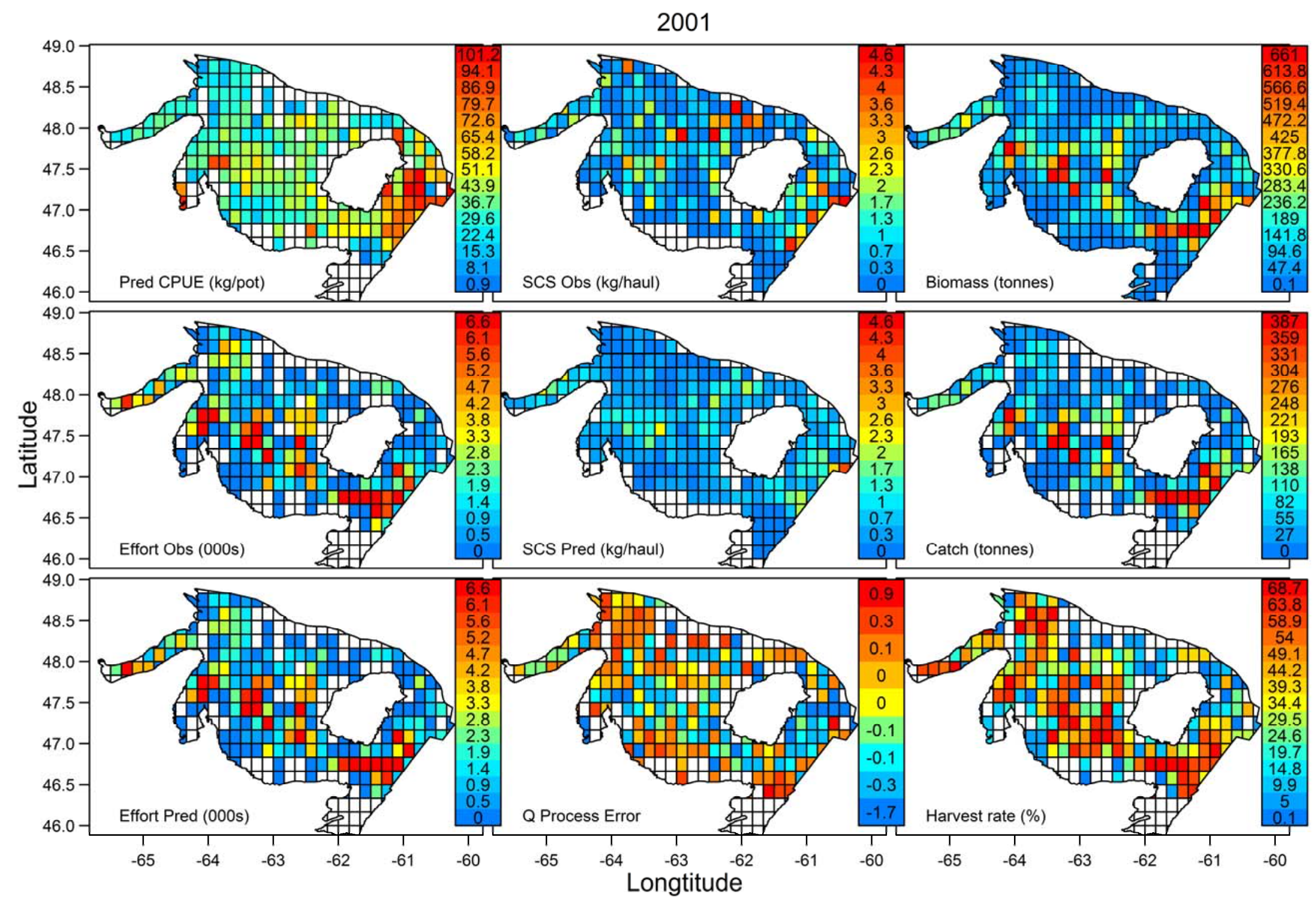

Figure S9. Model M4 (see Table 2) spatial results for 2001. Catch and effort are aggregated across weeks, and CPUE is the ratio of aggregated catch divided by aggregated effort. Q process errors are averaged over weeks. White cells indicate no observations. 


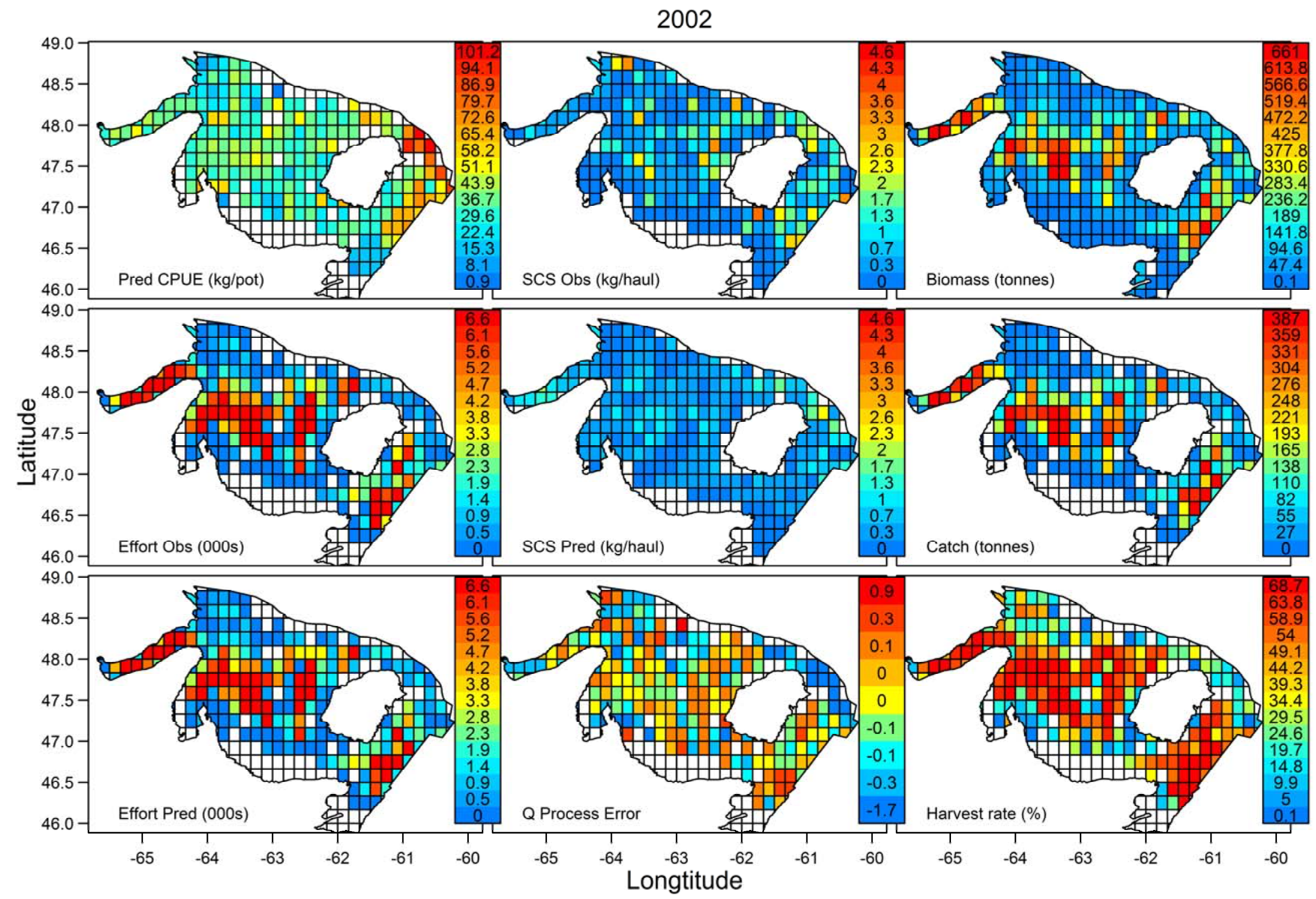

Figure S10. Model M4 (see Table 2) spatial results for 2002. Catch and effort are aggregated across weeks, and CPUE is the ratio of aggregated catch divided by aggregated effort. Q process errors are averaged over weeks. White cells indicate no observations. 


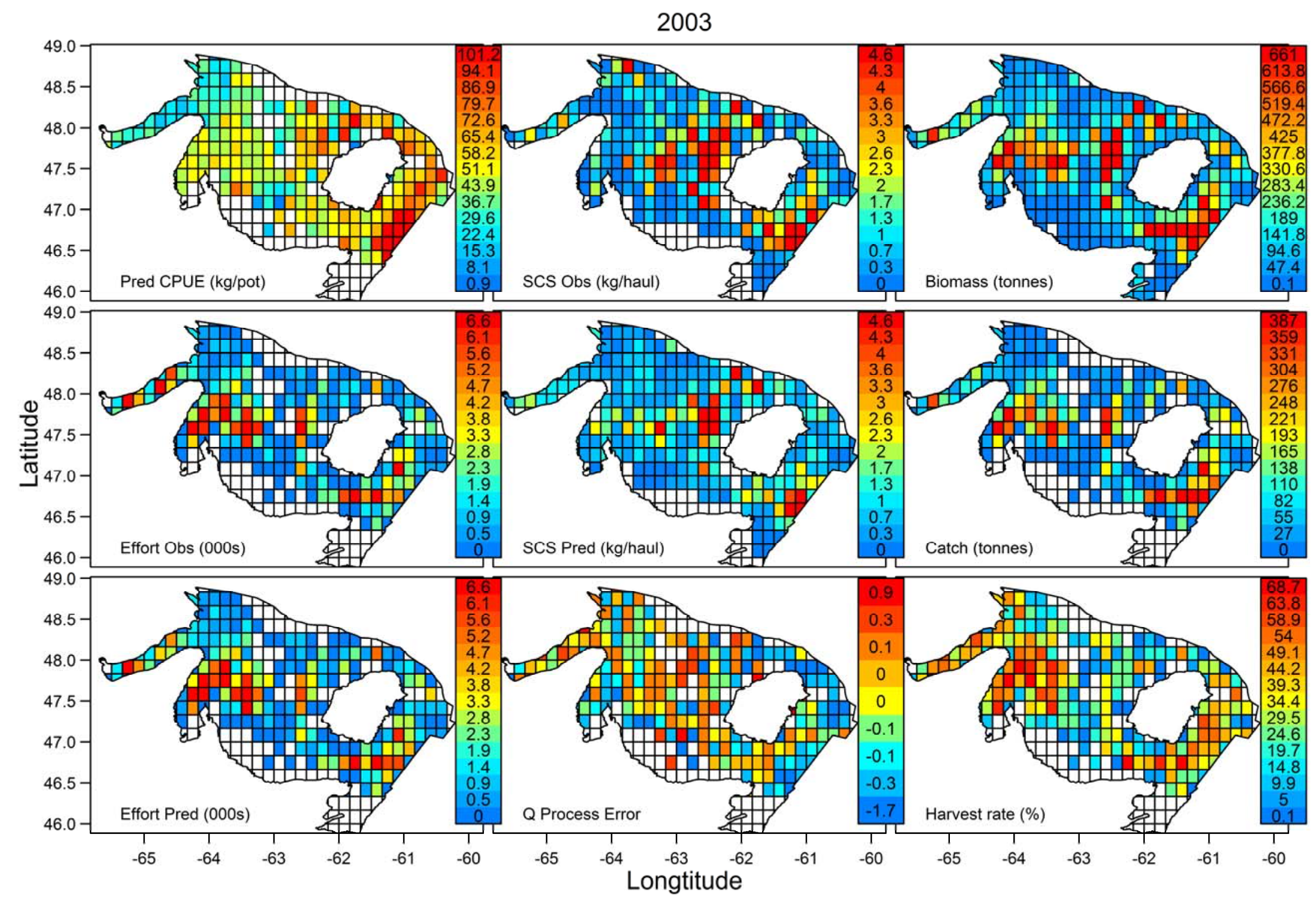

Figure S11. Model M4 (see Table 2) spatial results for 2003. Catch and effort are aggregated across weeks, and CPUE is the ratio of aggregated catch divided by aggregated effort. Q process errors are averaged over weeks. White cells indicate no observations. 


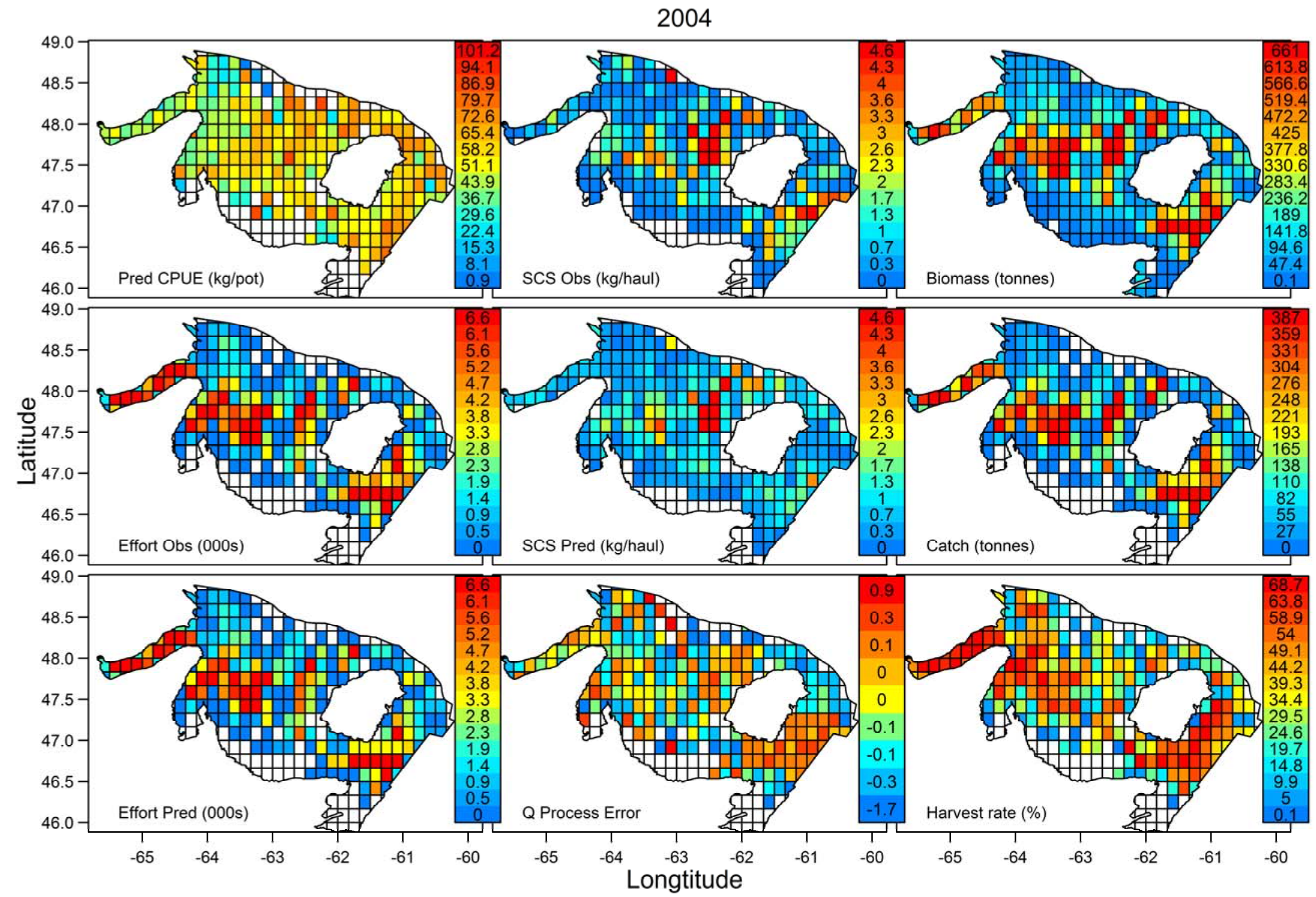

Figure S12. Model M4 (see Table 2) spatial results for 2004. Catch and effort are aggregated across weeks, and CPUE is the ratio of aggregated catch divided by aggregated effort. Q process errors are averaged over weeks. White cells indicate no observations. 


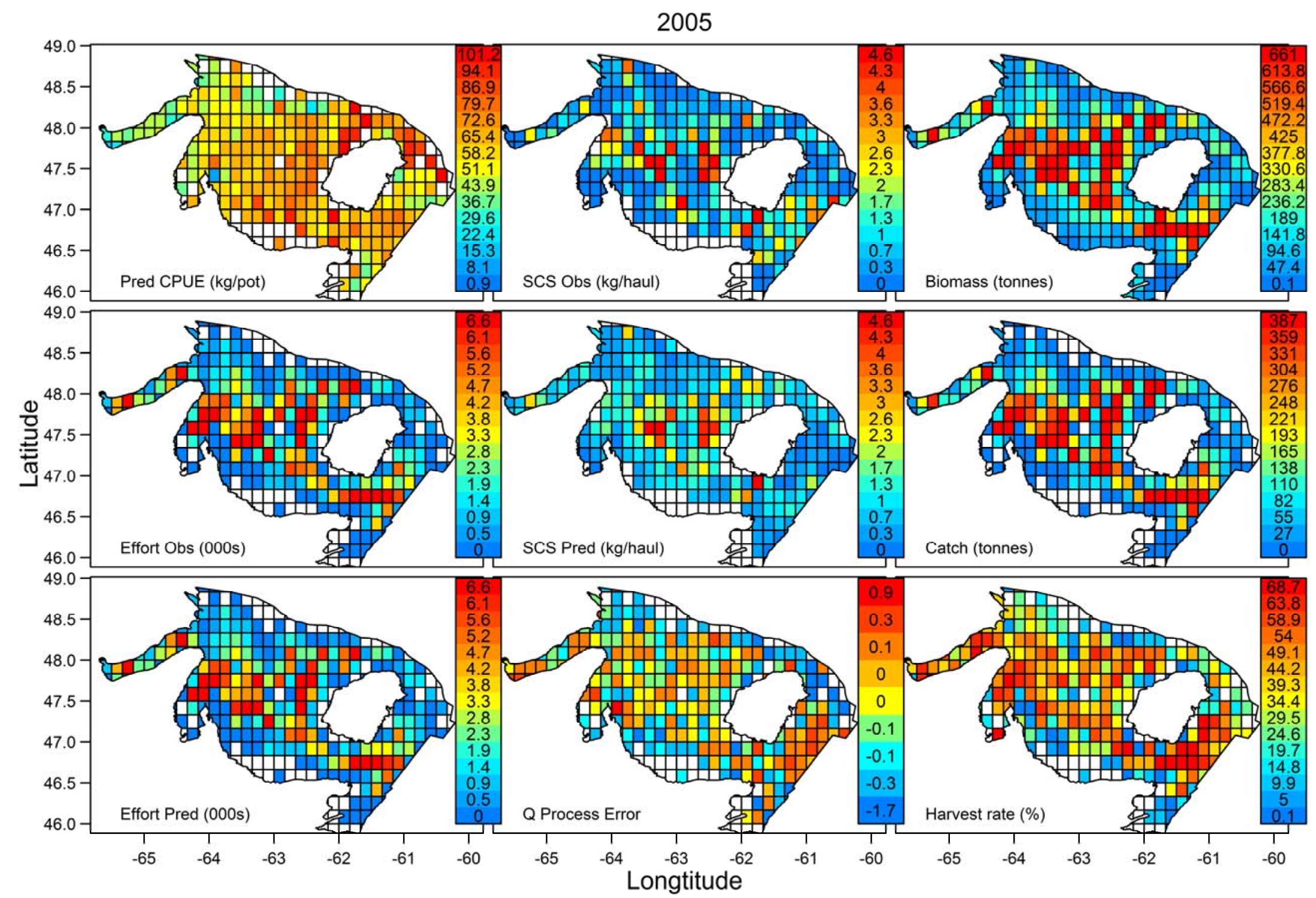

Figure S13. Model M4 (see Table 2) spatial results for 2005. Catch and effort are aggregated across weeks, and CPUE is the ratio of aggregated catch divided by aggregated effort. Q process errors are averaged over weeks. White cells indicate no observations. 


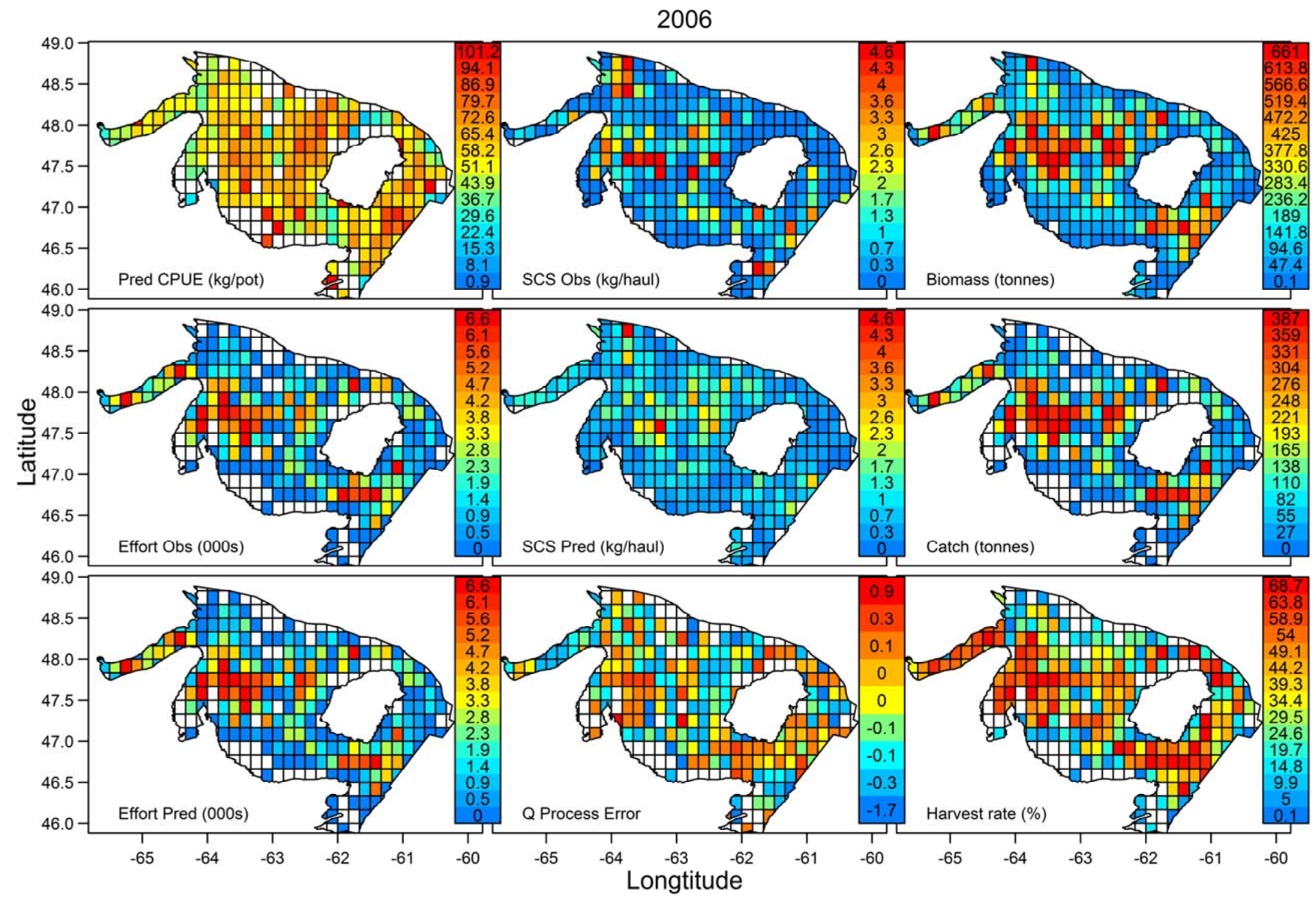

Figure S14. Model M4 (see Table 2) spatial results for 2006. Catch and effort are aggregated across weeks, and CPUE is the ratio of aggregated catch divided by aggregated effort. Q process errors are averaged over weeks. White cells indicate no observations. 


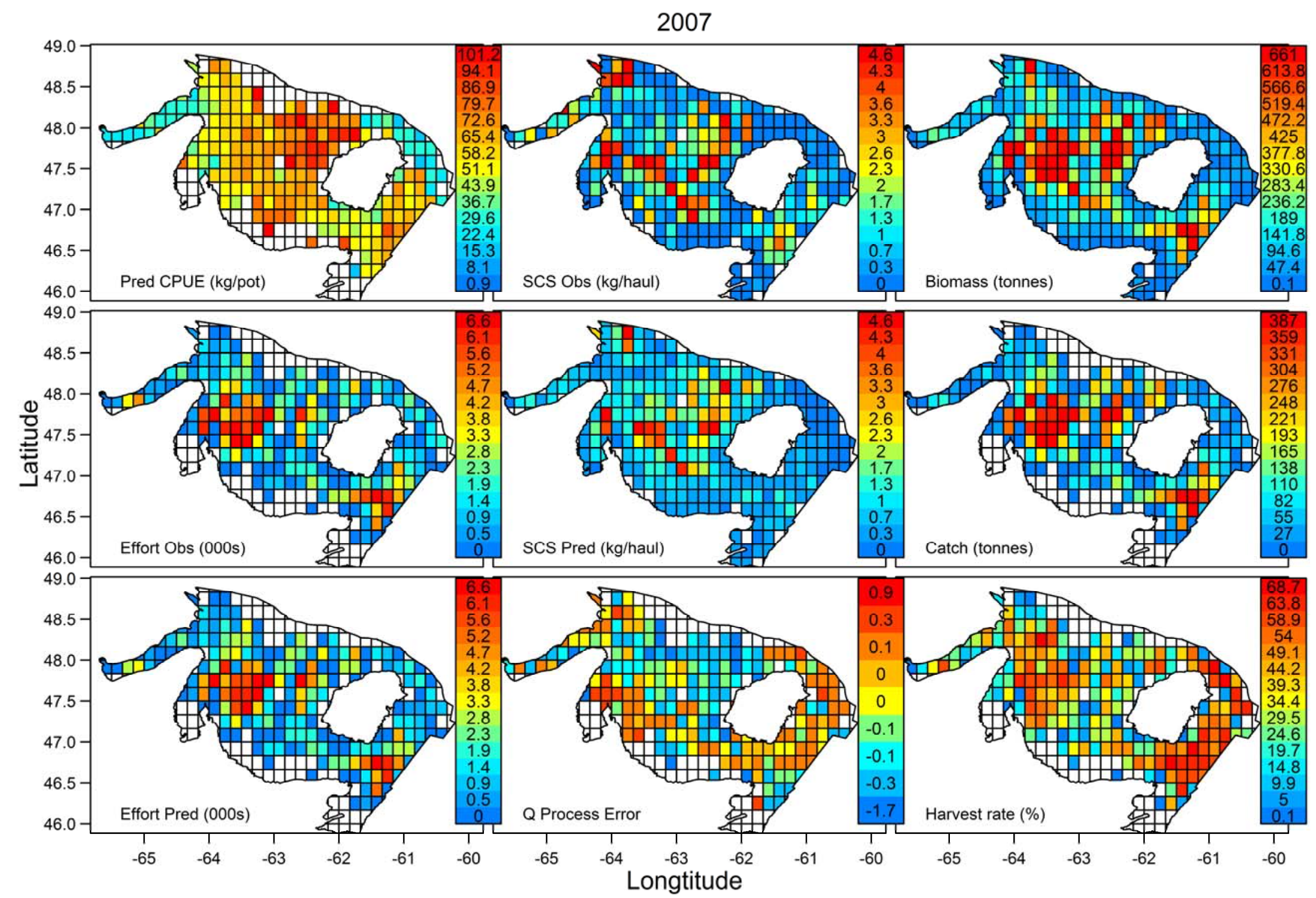

Figure S15. Model M4 (see Table 2) spatial results for 2007. Catch and effort are aggregated across weeks, and CPUE is the ratio of aggregated catch divided by aggregated effort. Q process errors are averaged over weeks. White cells indicate no observations. 


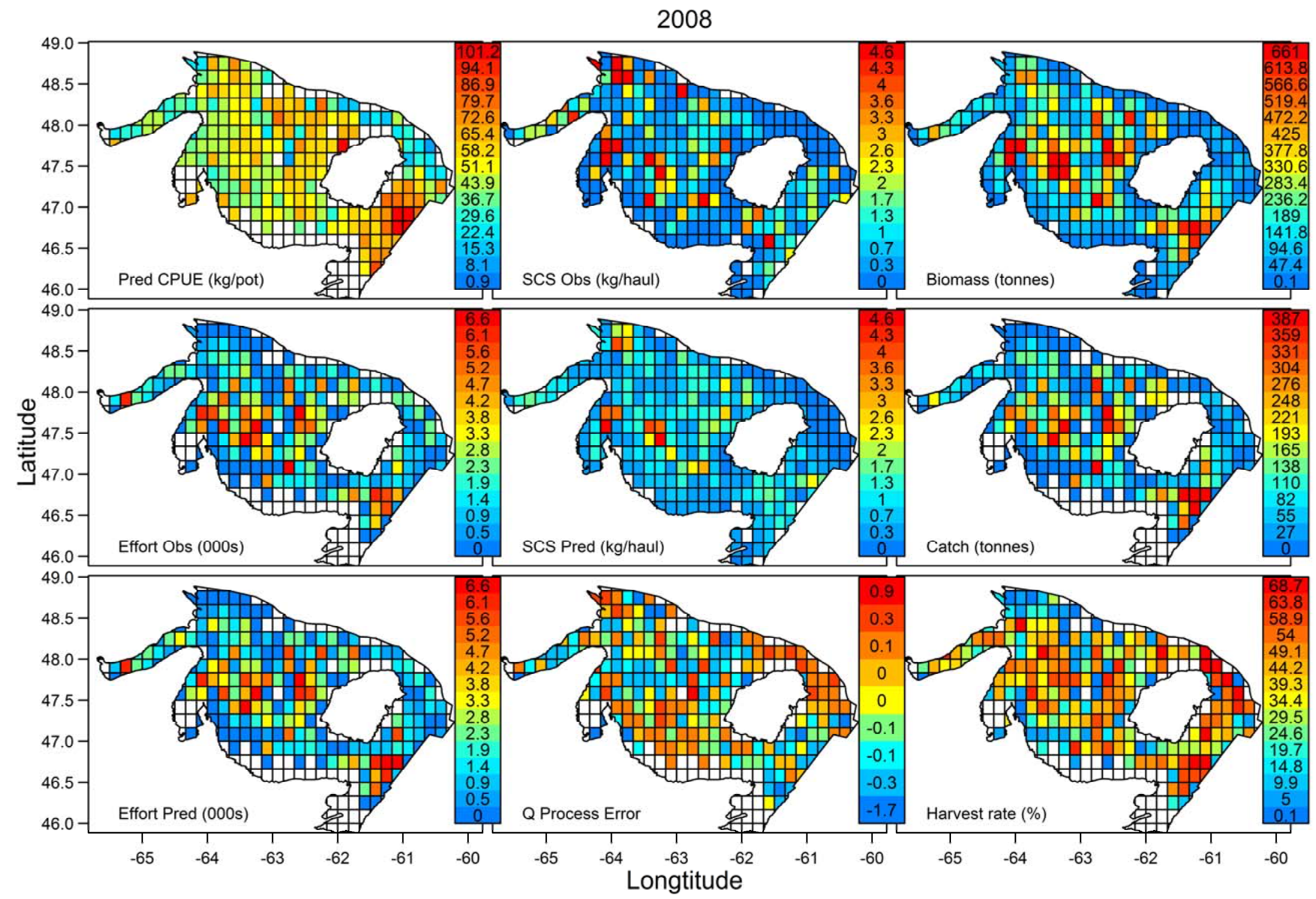

Figure S16. Model M4 (see Table 2) spatial results for 2008. Catch and effort are aggregated across weeks, and CPUE is the ratio of aggregated catch divided by aggregated effort. Q process errors are averaged over weeks. White cells indicate no observations. 


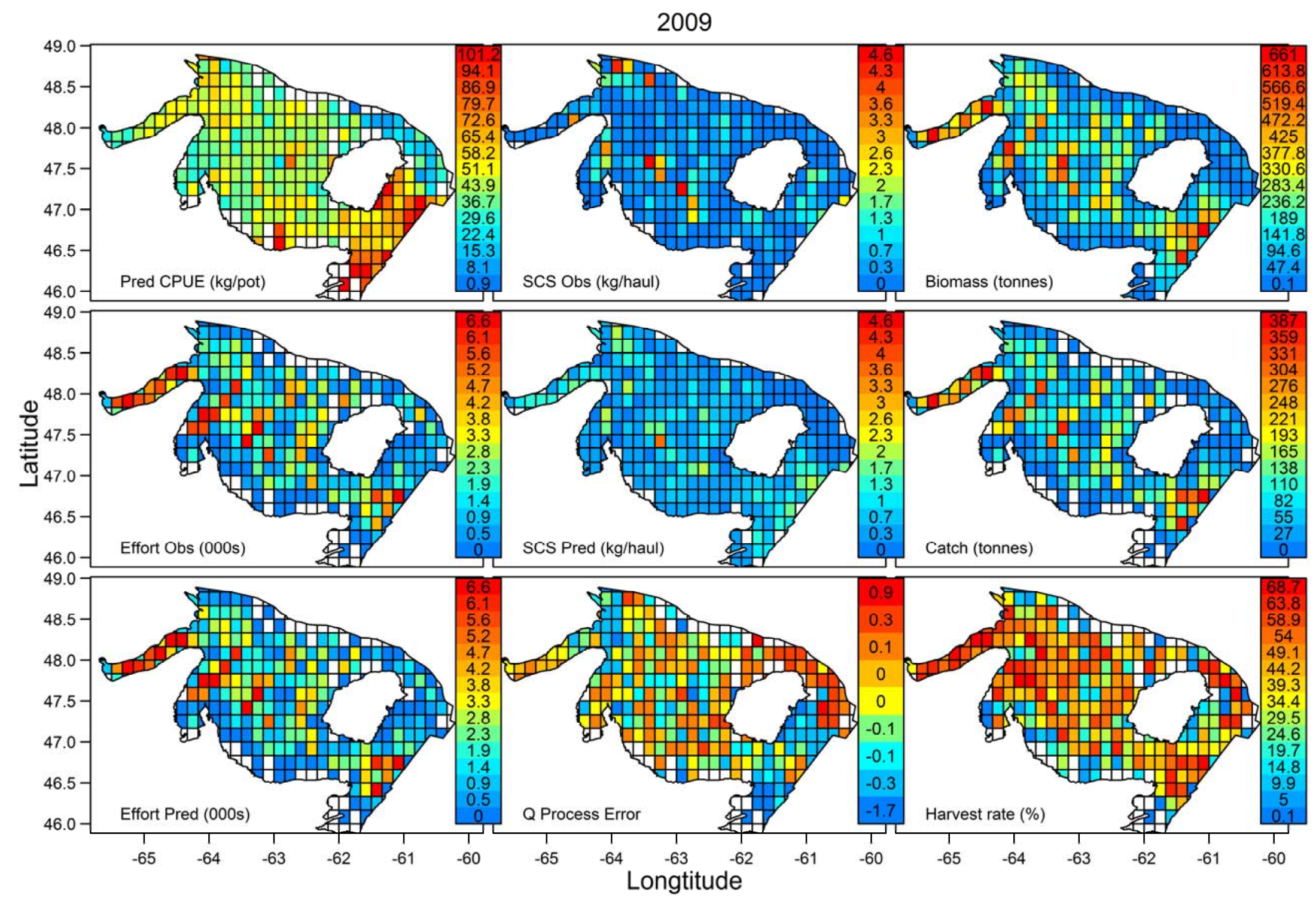

Figure S17. Model M4 (see Table 2) spatial results for 2009. Catch and effort are aggregated across weeks, and CPUE is the ratio of aggregated catch divided by aggregated effort. Q process errors are averaged over weeks. White cells indicate no observations. 


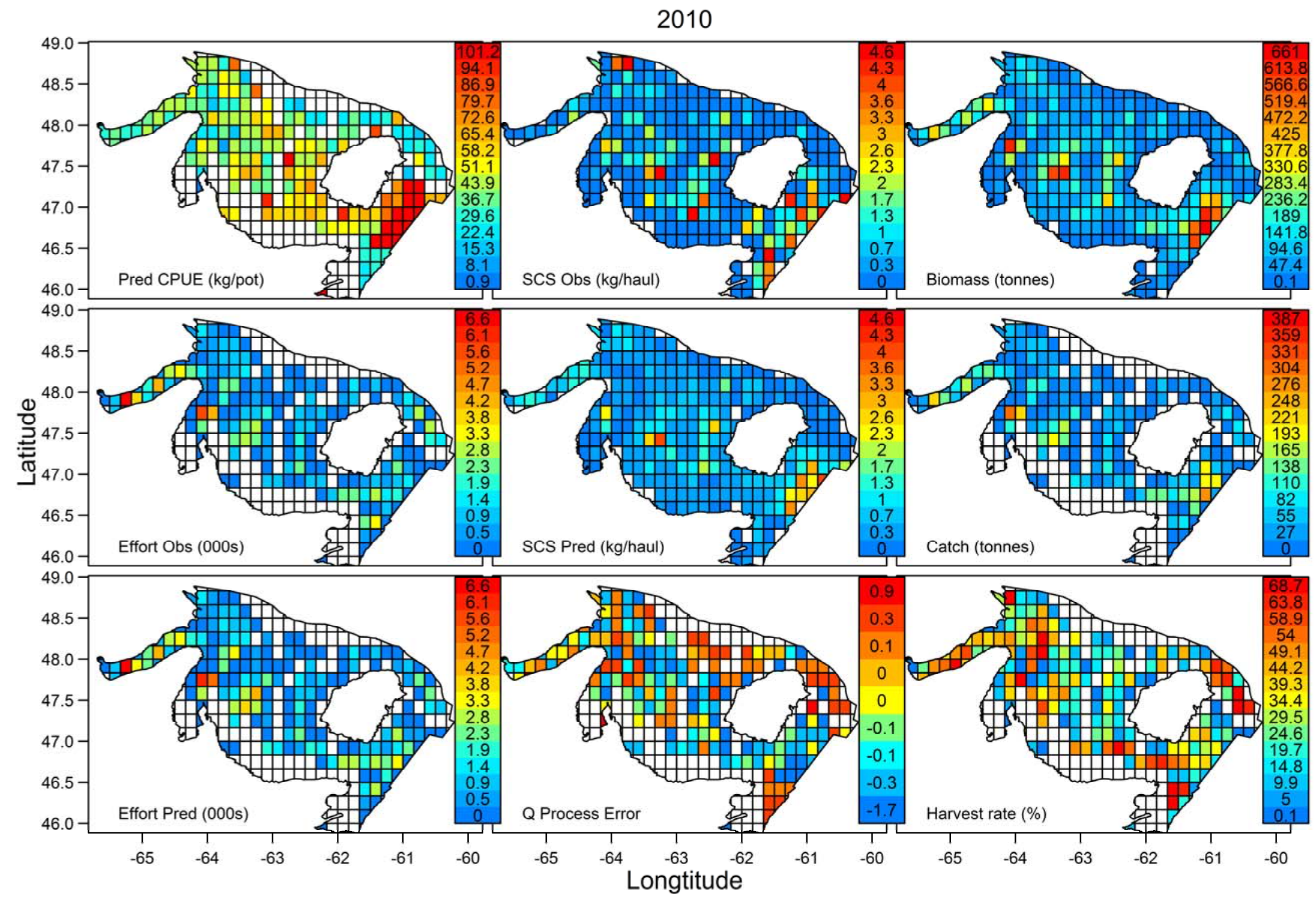

Figure S18. Model M4 (see Table 2) spatial results for 2010. Catch and effort are aggregated across weeks, and CPUE is the ratio of aggregated catch divided by aggregated effort. Q process errors are averaged over weeks. White cells indicate no observations. 


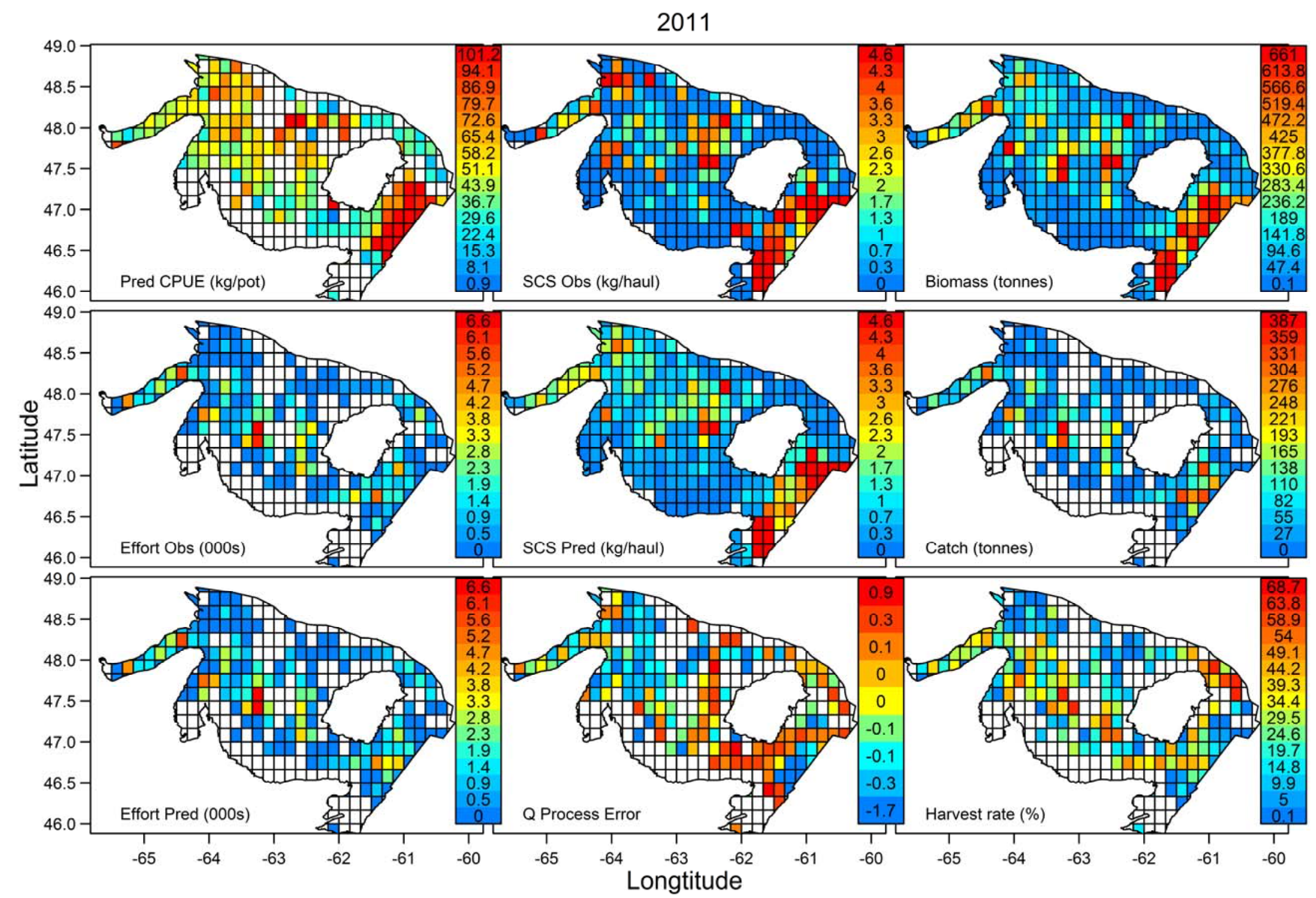

Figure S19. Model M4 (see Table 2) spatial results for 2011. Catch and effort are aggregated across weeks, and CPUE is the ratio of aggregated catch divided by aggregated effort. Q process errors are averaged over weeks. White cells indicate no observations. 


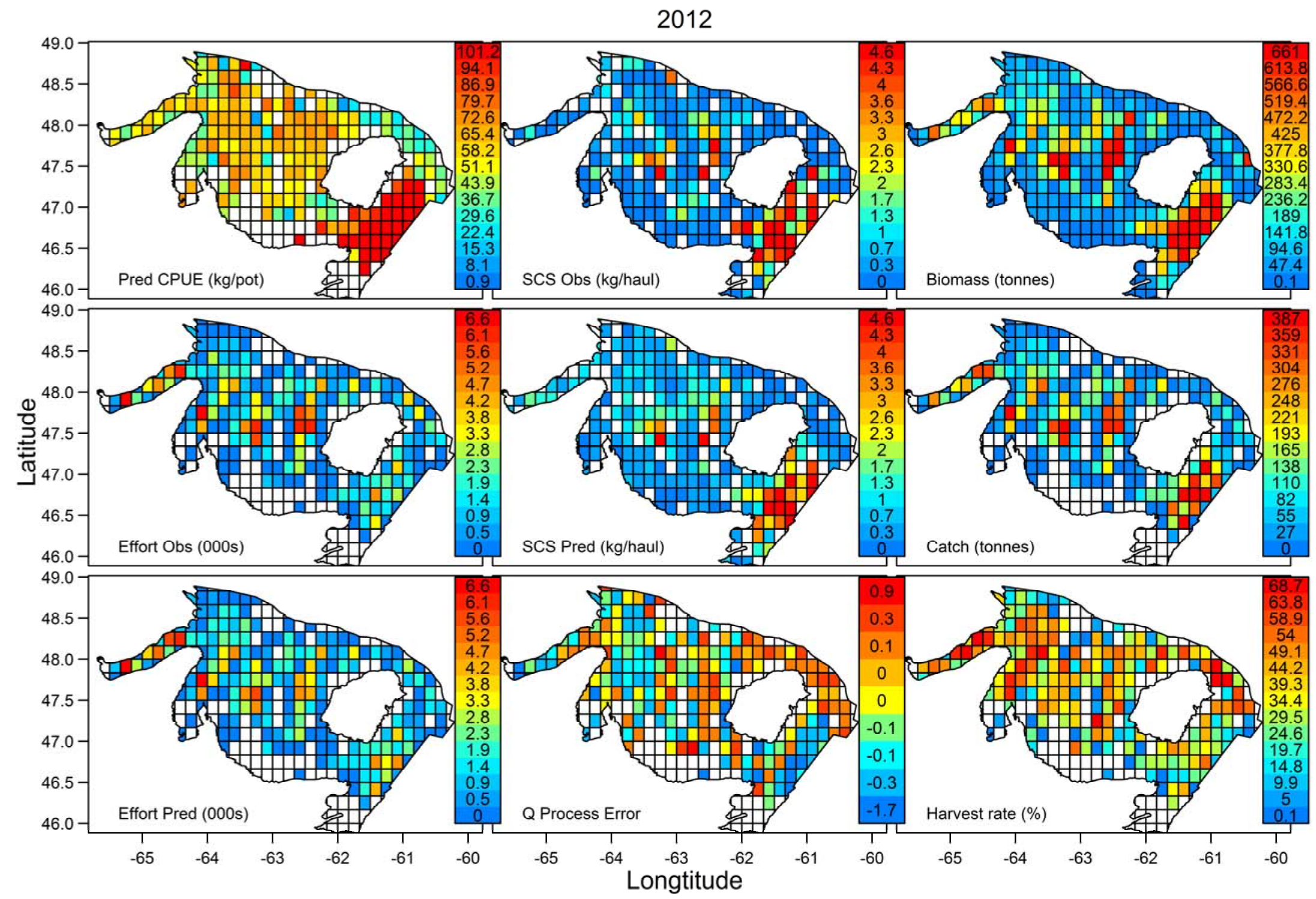

Figure S20. Model M4 (see Table 2) spatial results for 2012. Catch and effort are aggregated across weeks, and CPUE is the ratio of aggregated catch divided by aggregated effort. Q process errors are averaged over weeks. White cells indicate no observations. 


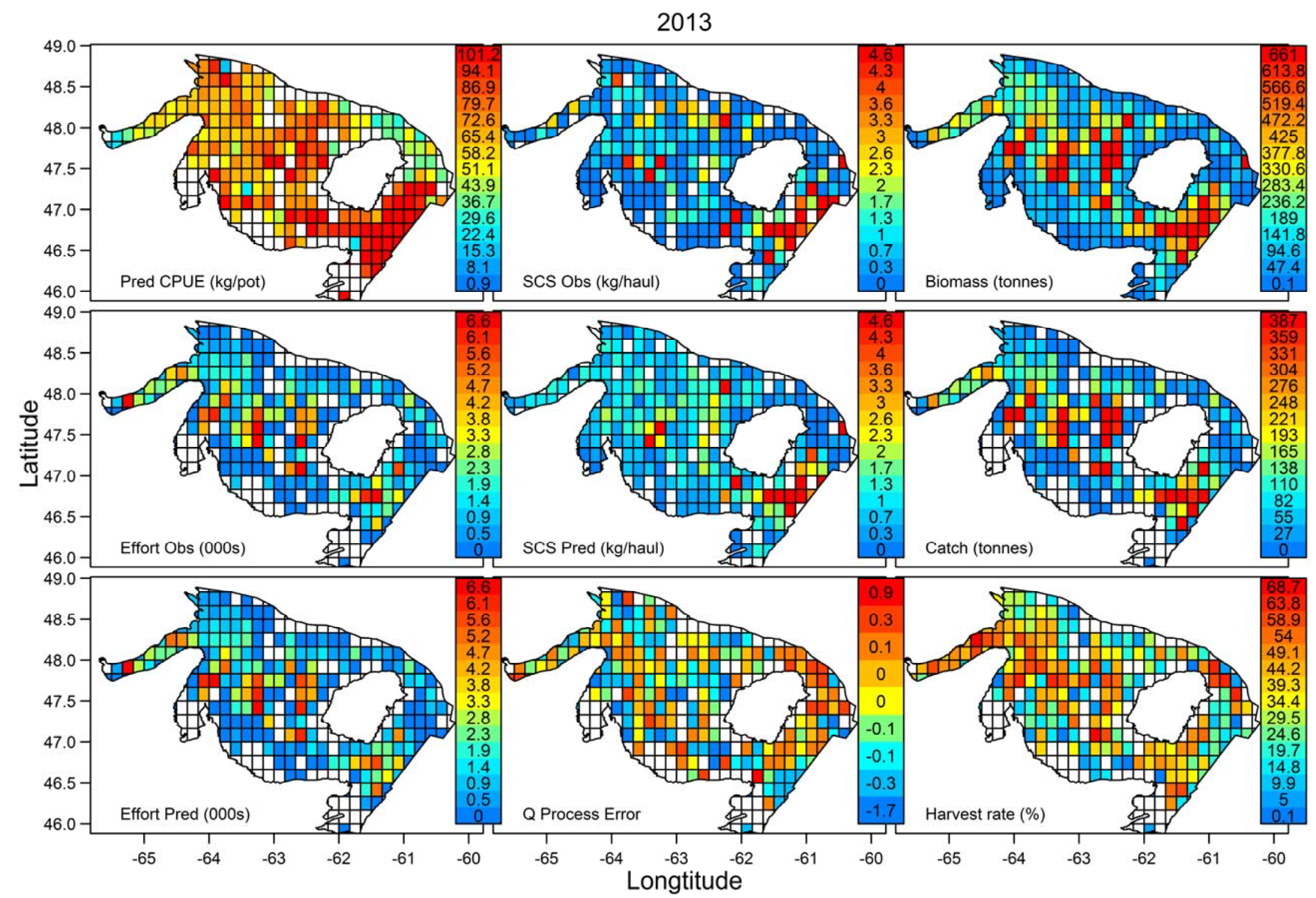

Figure S21. Model M4 (see Table 2) spatial results for 2013. Catch and effort are aggregated across weeks, and CPUE is the ratio of aggregated catch divided by aggregated effort. Q process errors are averaged over weeks. White cells indicate no observations. 


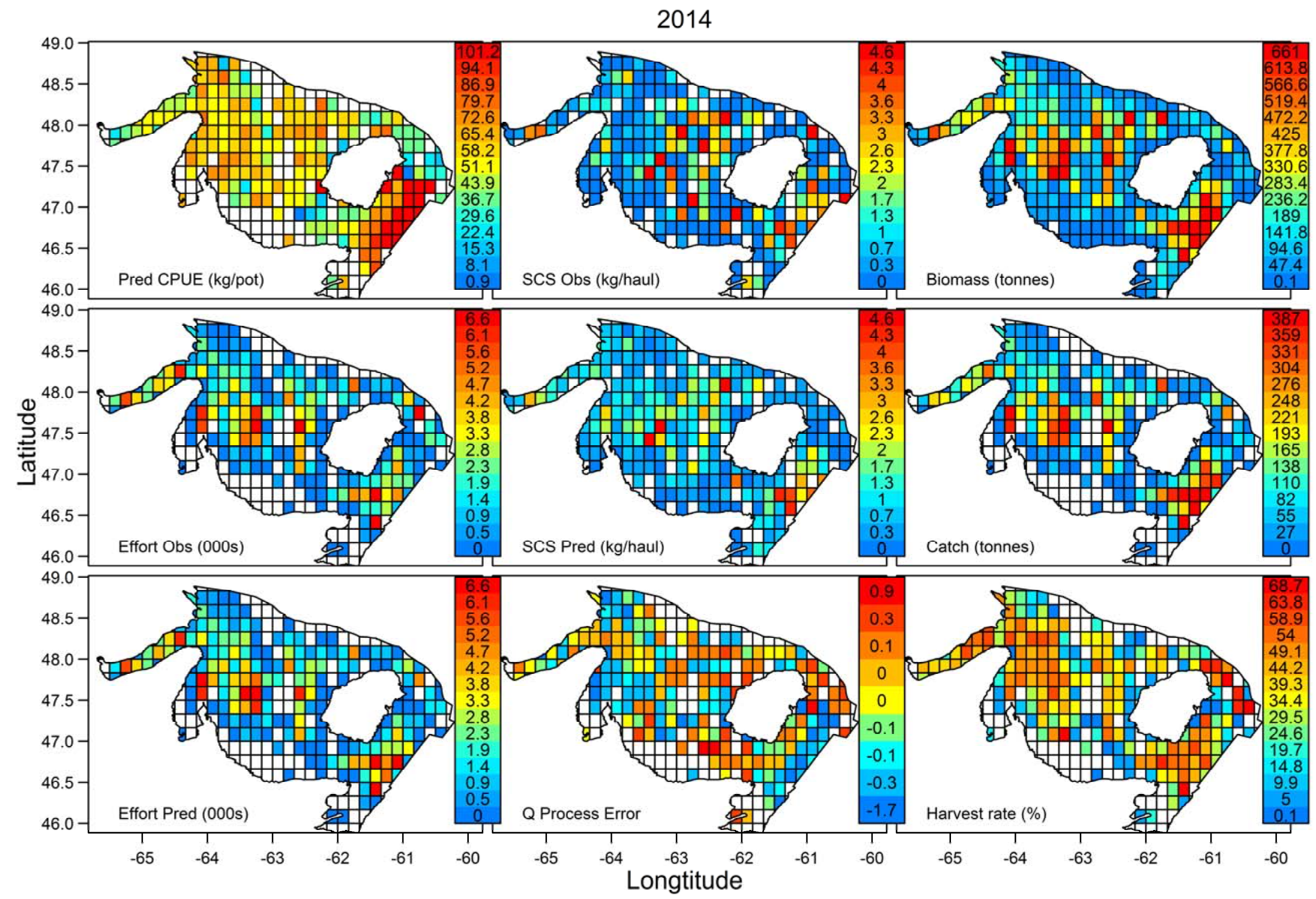

Figure S22. Model M4 (see Table 2) spatial results for 2014. Catch and effort are aggregated across weeks, and CPUE is the ratio of aggregated catch divided by aggregated effort. Q process errors are averaged over weeks. White cells indicate no observations. 
Total annual average $=20991$ tonnes, 346 cells; cell average $=1092.01$ tonnes,
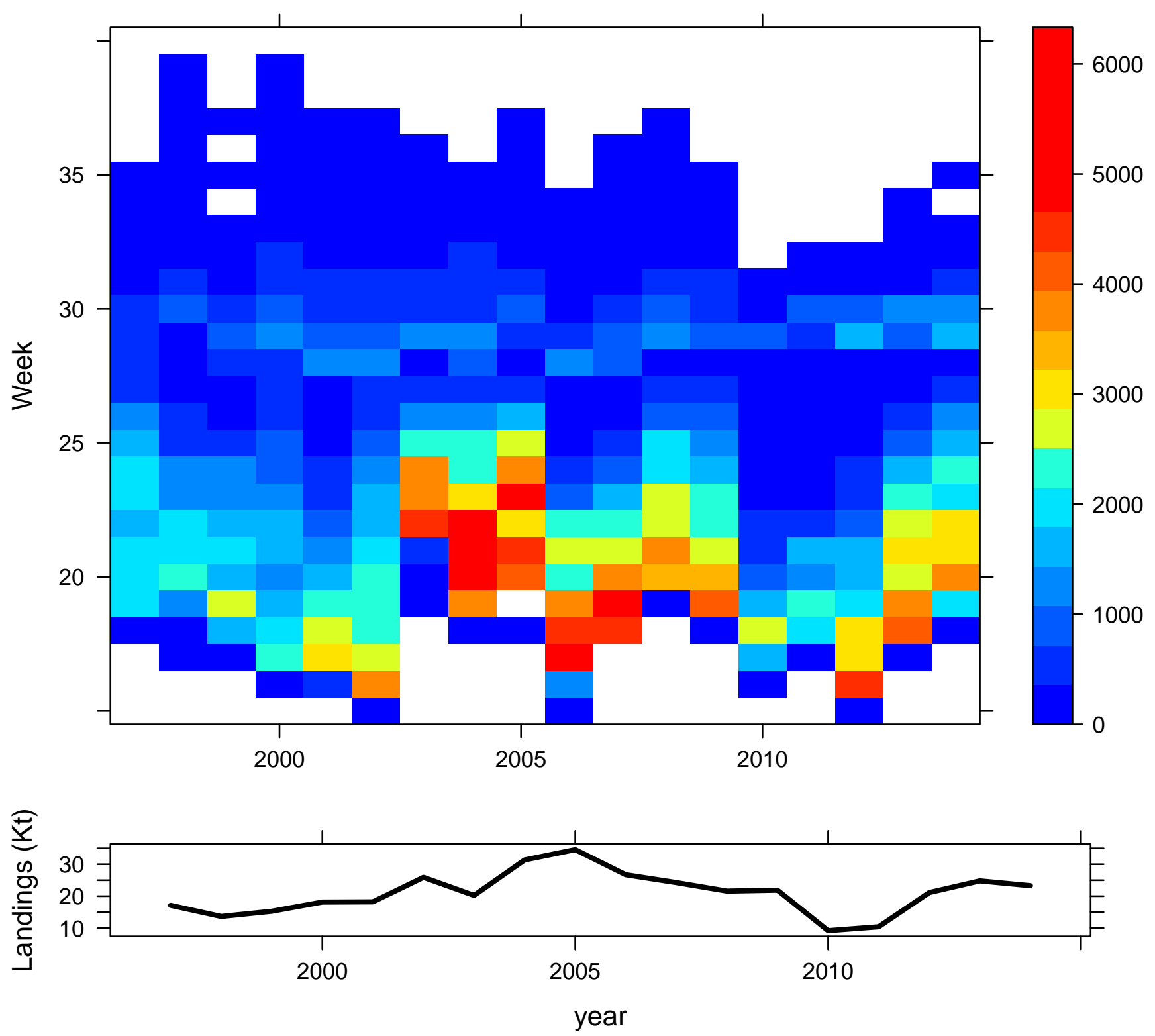

Figure SC.1. Top panel: Total catch (tonnes) of snow crab each week (rows) and year (columns). Colors correspond to catch levels, as indicated in the legend on the right-hand side. Darkest red grids indicate catch $>98$ th percentile. Bottom panel: Total catch (000 tonnes) each year for all weeks. 


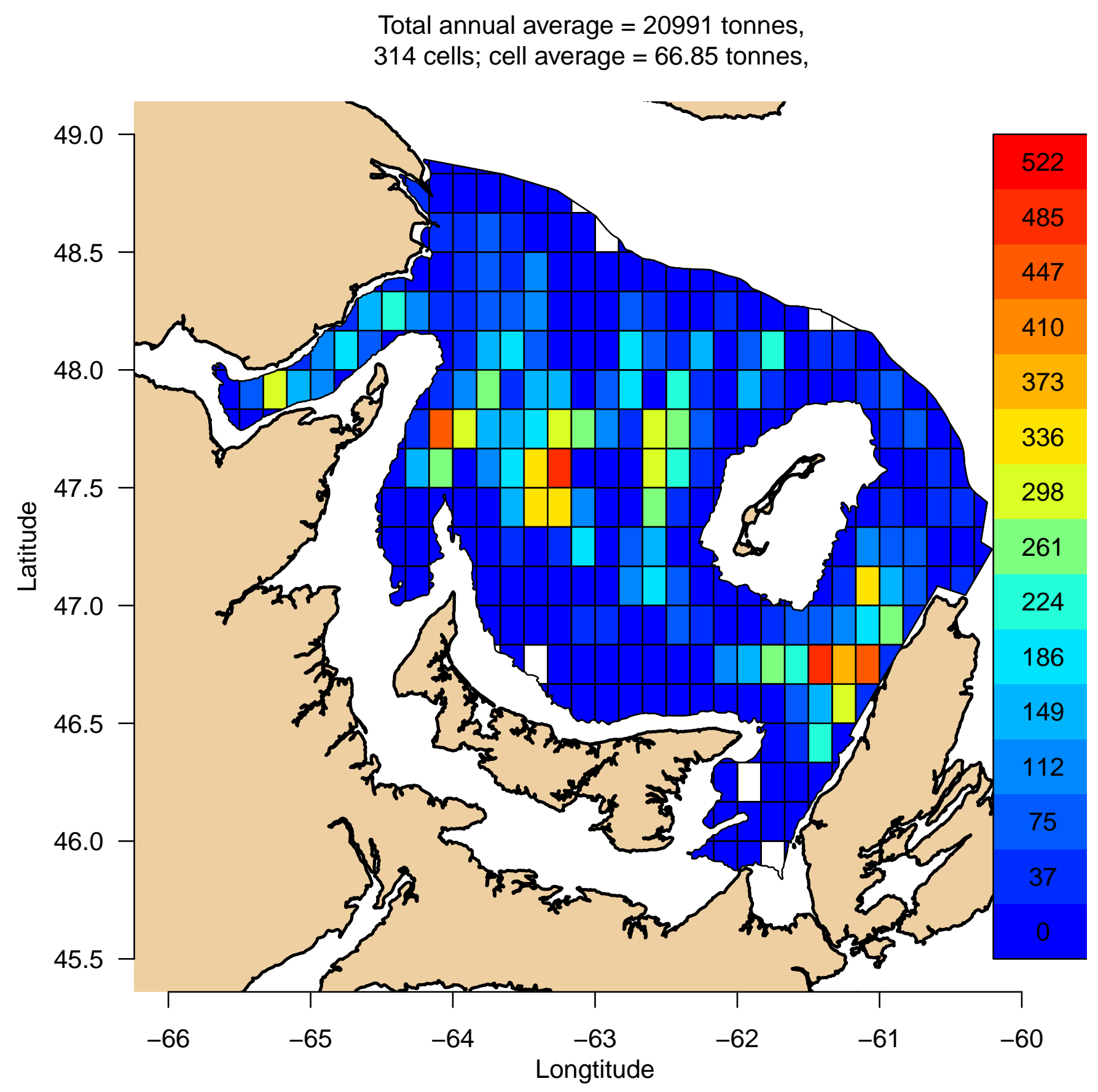

Figure SC.2. Total annual catch (tonnes) of snow crab in each grid cell, averaged for 1997-2014. Colors correspond to catch levels, as indicated in the legend on the right-hand side. Darkest red grids indicate catch $>$ 98th percentile. 


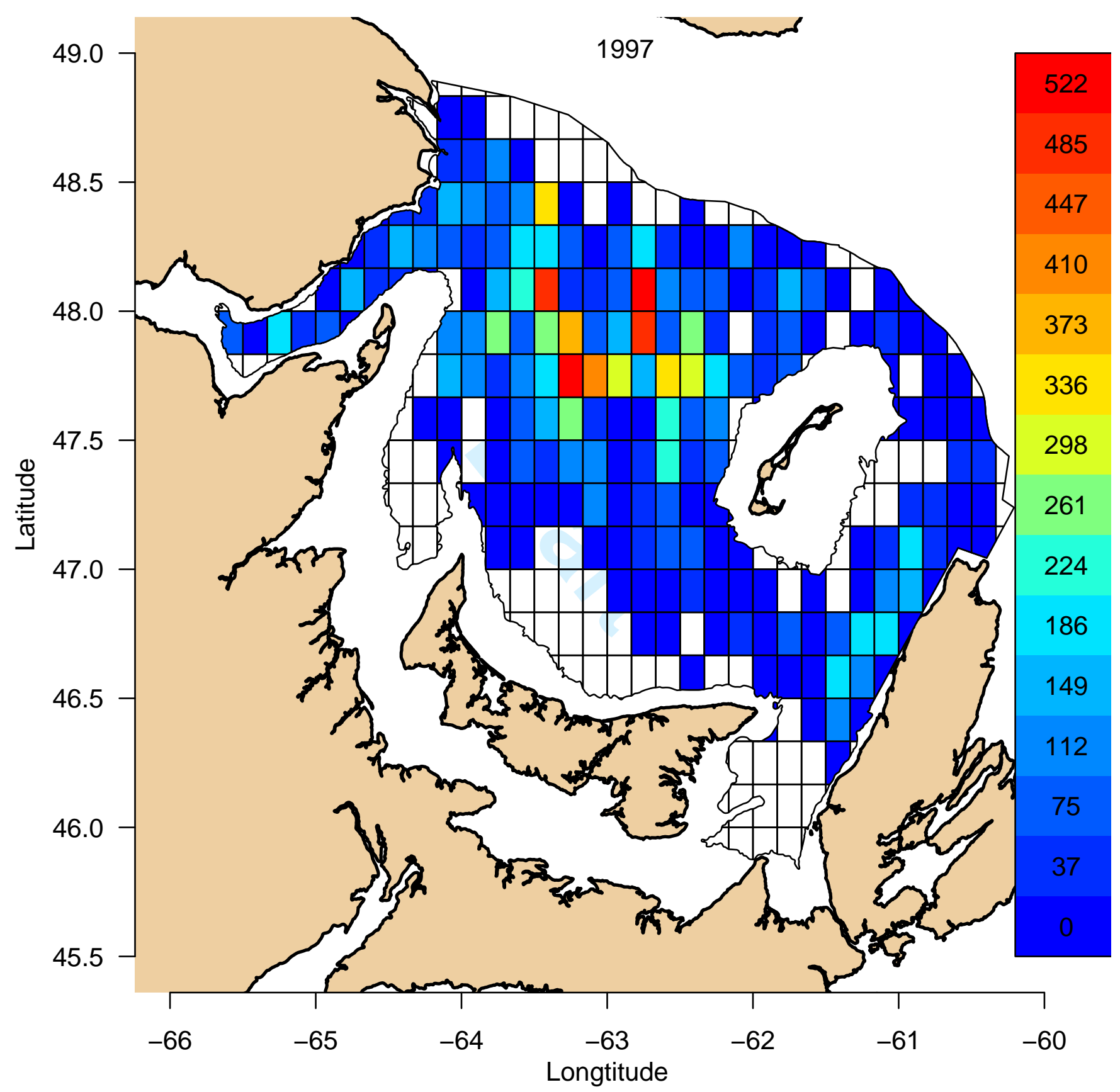

Figure SC.3a. Total annual catch (tonnes) of snow crab in each grid cell in 1997. Colors correspond to catch levels, as indicated in the legend on the right-hand side. Darkest red grids indicate catch $>98$ th percentile. 
Total $=17133$ tonnes, 1997
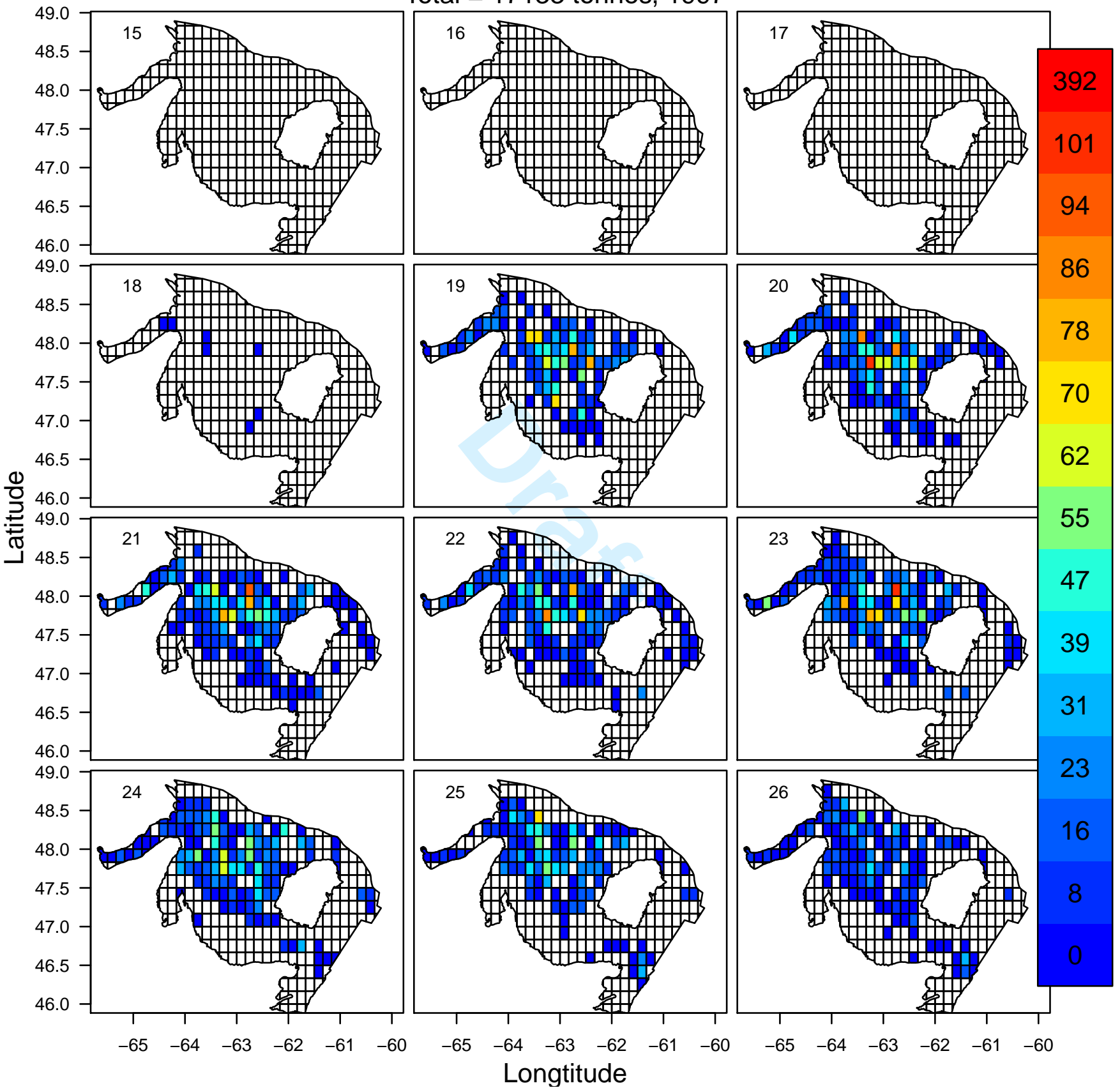

Figure SC.3b. Catch (tonnes) of snow crab in each week and grid cell in 1997. The week number is indicated in the top left-hand corner. Colors correspond to catch levels, as indicated in the legend on the right-hand side. Darkest red grids indicate catch $>98$ th percentile. 


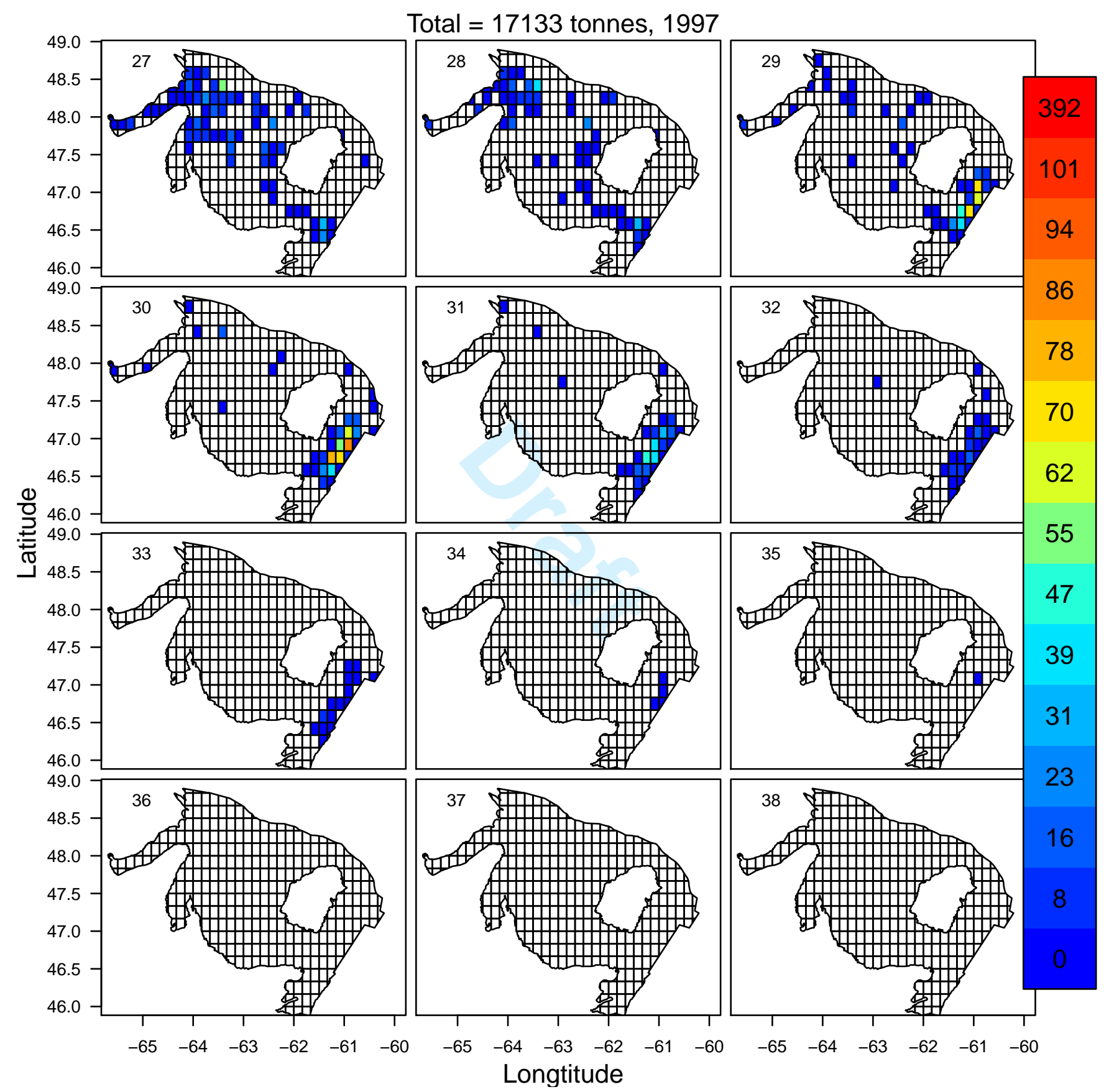

Figure SC.3c. Catch (tonnes) of snow crab in each week and grid cell in 1997. The week number is indicated in the top left-hand corner. Colors correspond to catch levels, as indicated in the legend on the right-hand side. Darkest red grids indicate catch $>98$ th percentile. 


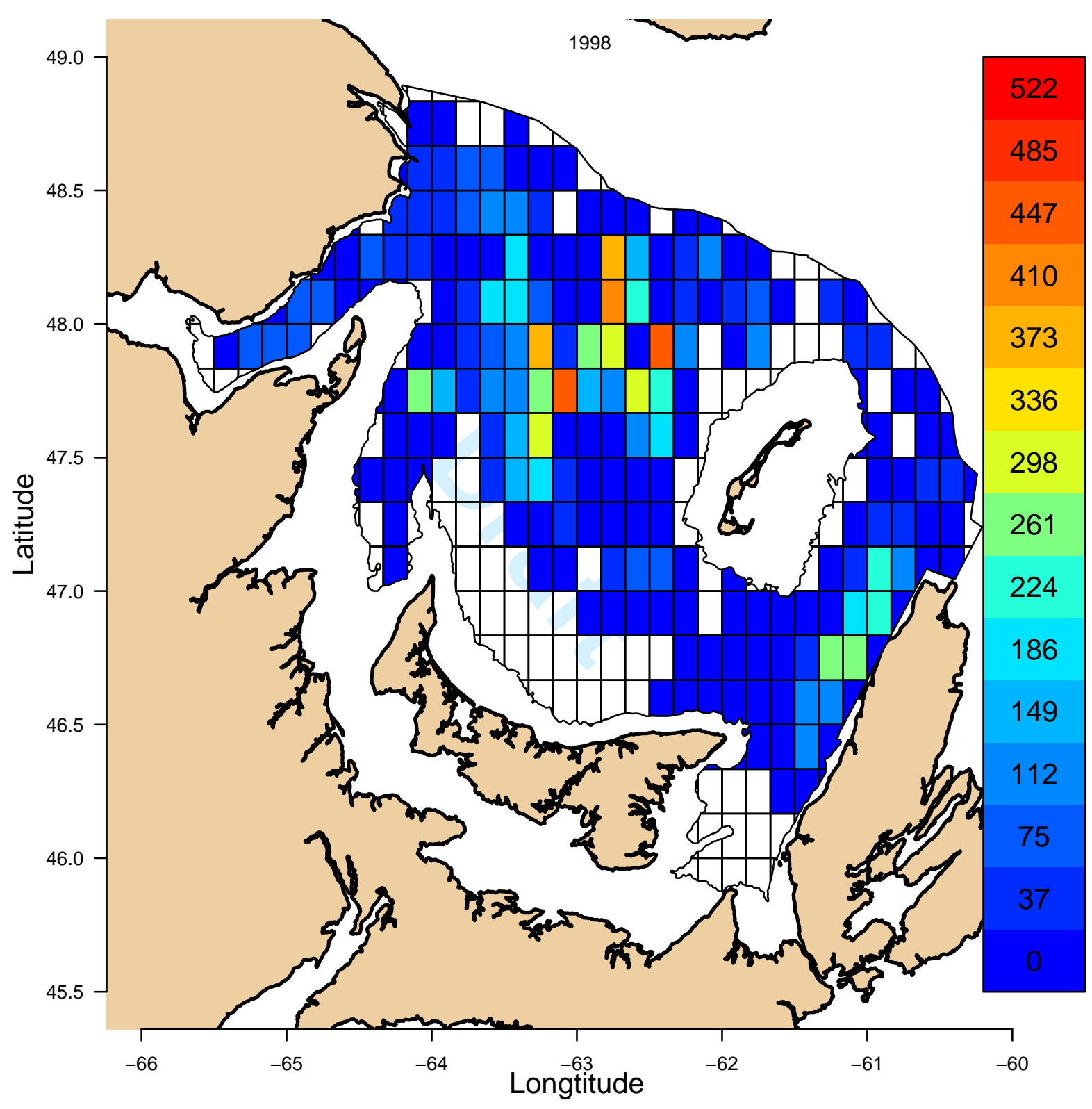

Figure SC.4a. Total annual catch (tonnes) of snow crab in each grid cell in 1998. Colors correspond to catch levels, as indicated in the legend on the right-hand side. Darkest red grids indicate catch $>98$ th percentile. 


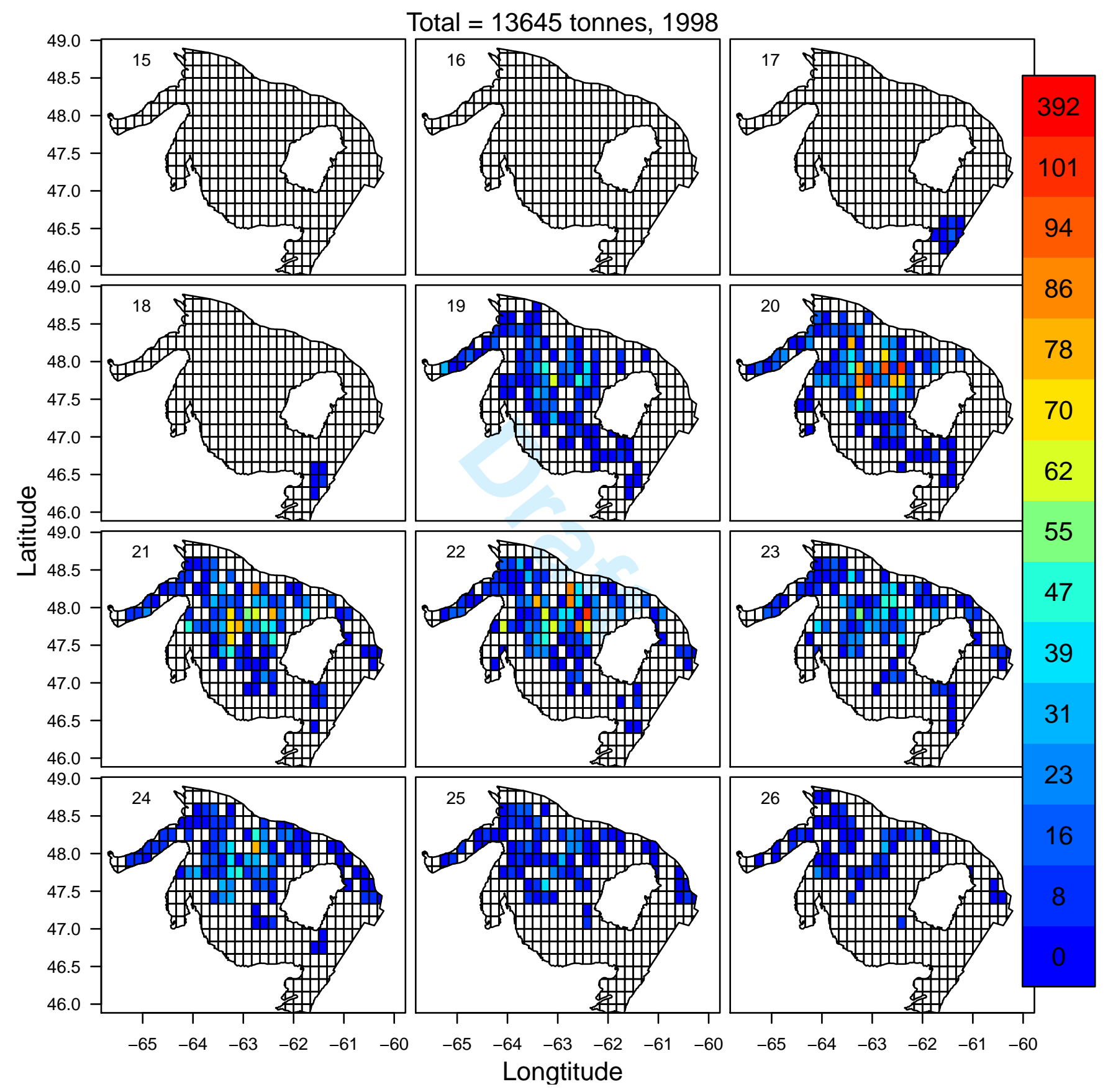

Figure SC.4b. Catch (tonnes) of snow crab in each week and grid cell in 1998. The week number is indicated in the top left-hand corner. Colors correspond to catch levels, as indicated in the legend on the right-hand side. Darkest red grids indicate catch $>98$ th percentile. 
Total $=13645$ tonnes, 1998
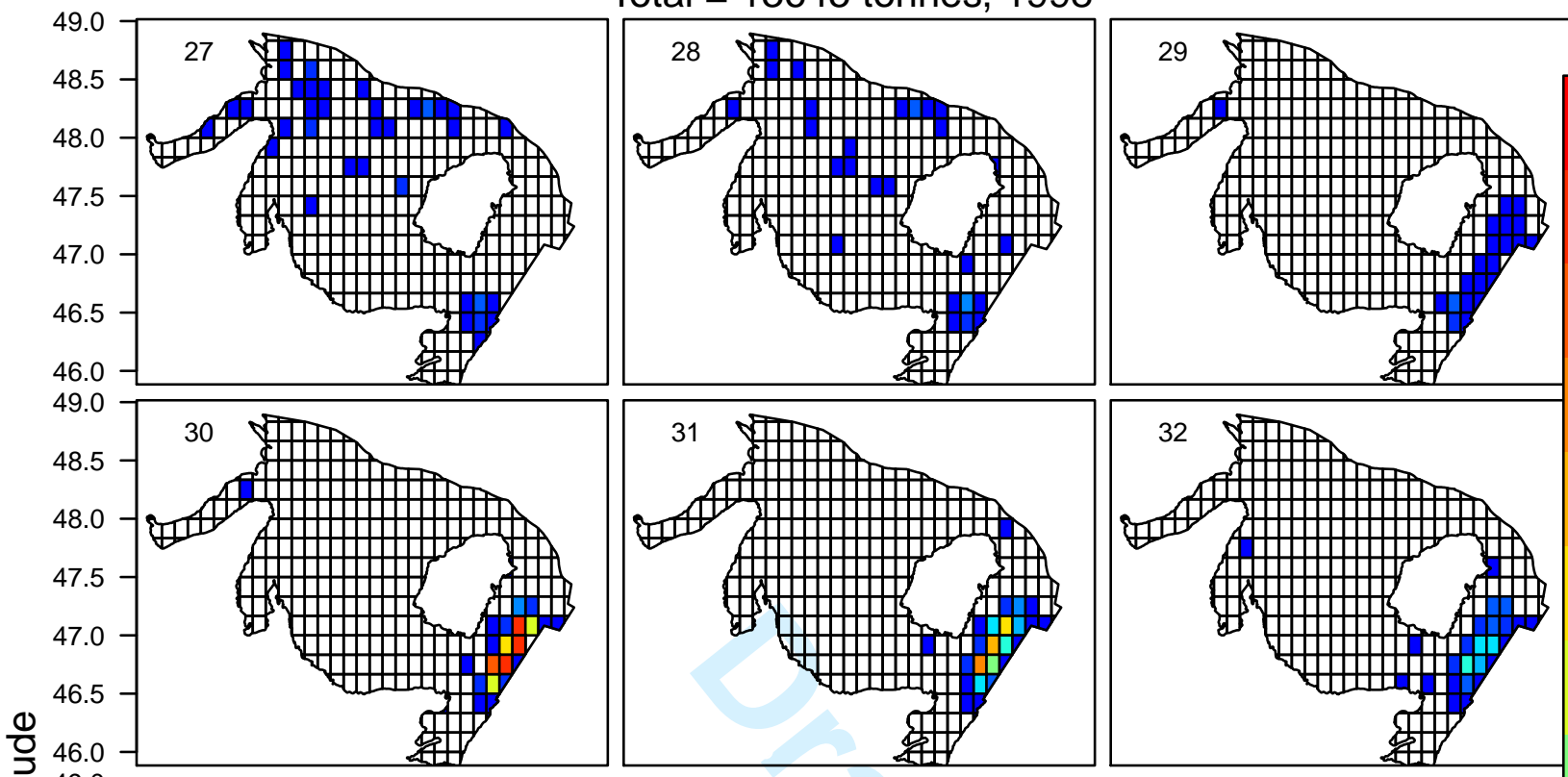

䒕
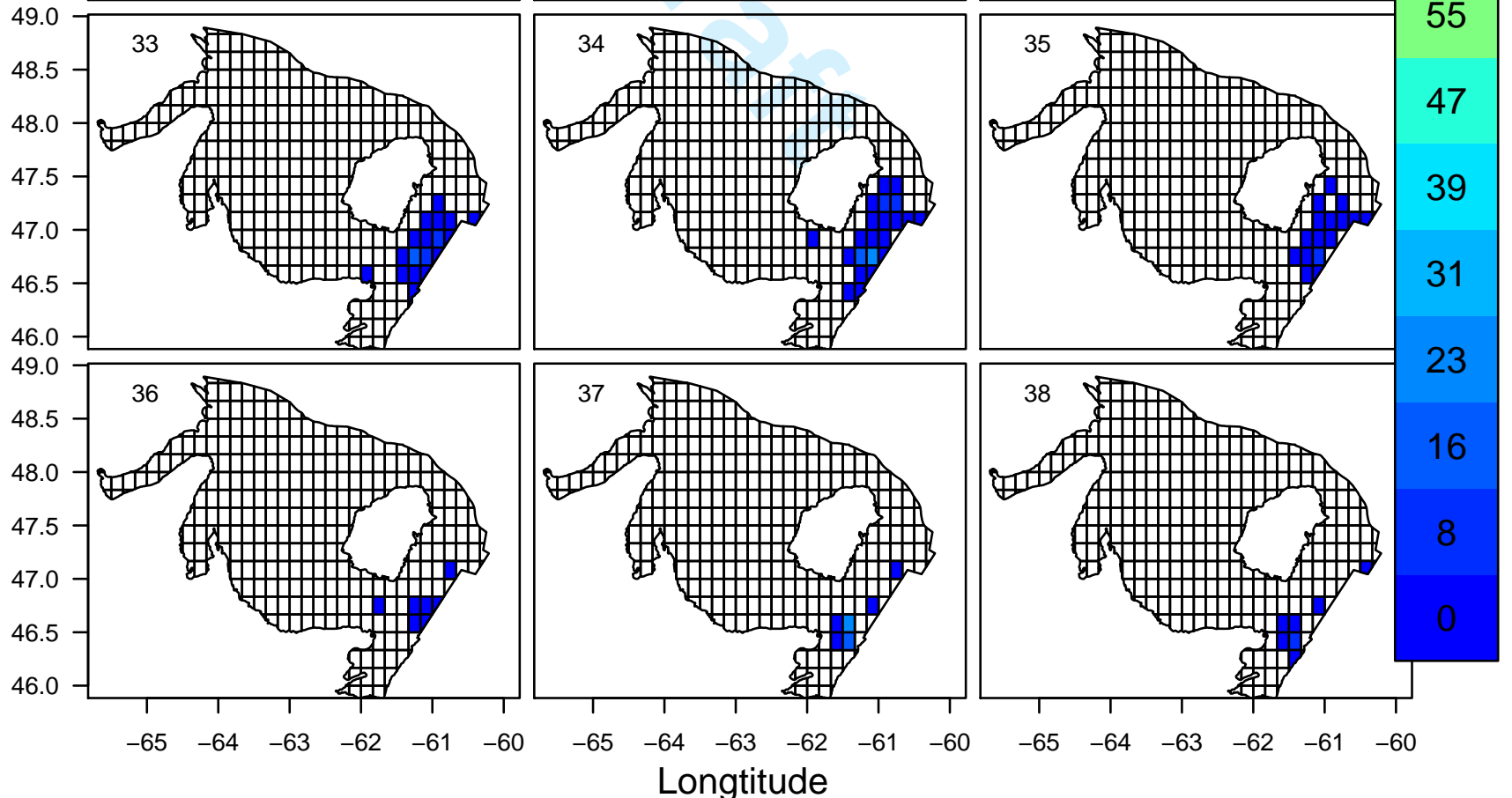

Figure SC.4c. Catch (tonnes) of snow crab in each week and grid cell in 1998. The week number is indicated in the top left-hand corner. Colors correspond to catch levels, as indicated in the legend on the right-hand side. Darkest red grids indicate catch $>98$ th percentile. 


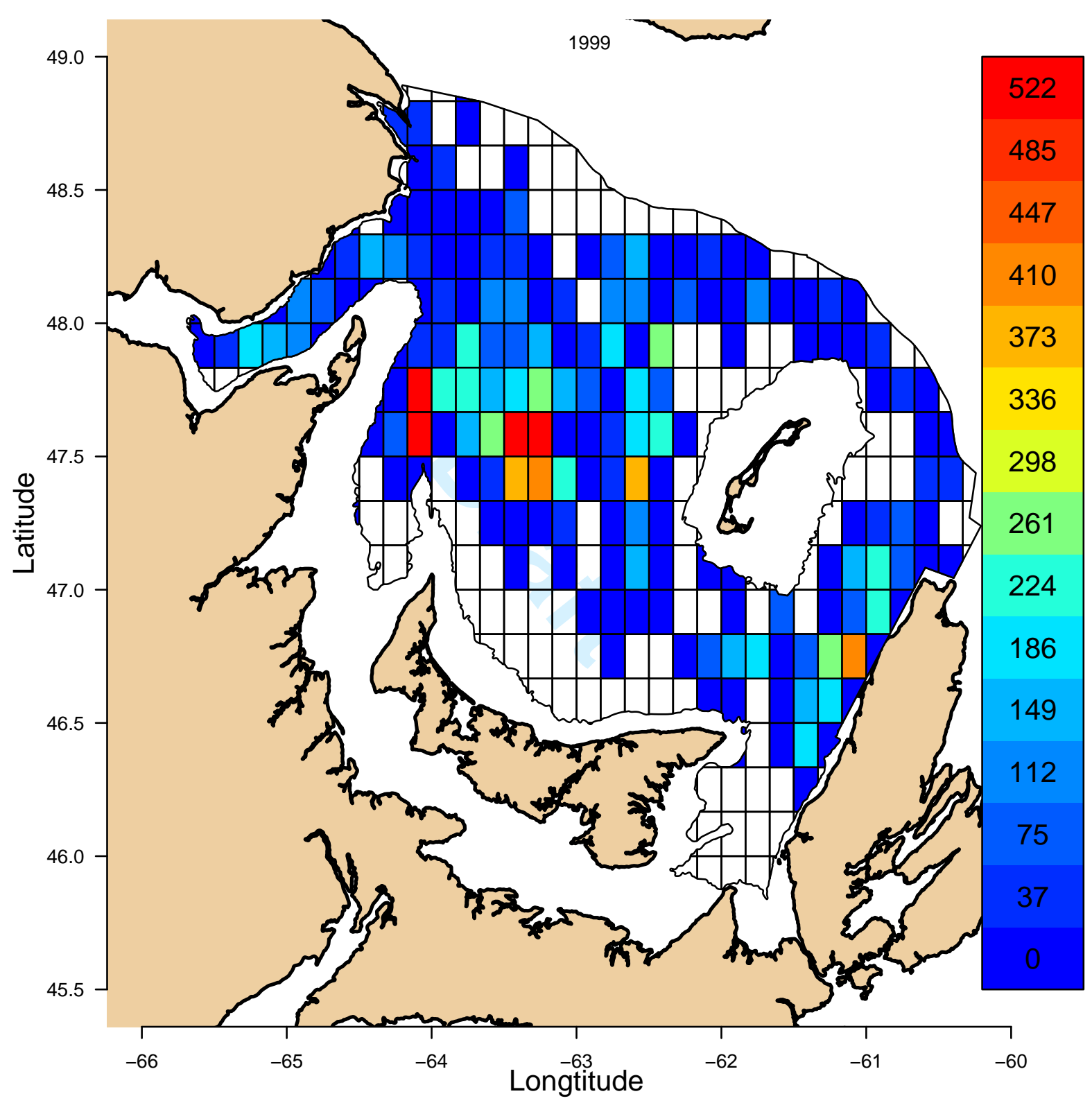

Figure SC.5a. Total annual catch (tonnes) of snow crab in each grid cell in 1999. Colors correspond to catch levels, as indicated in the legend on the right-hand side. Darkest red grids indicate catch $>98$ th percentile. 
Total $=15276$ tonnes, 1999
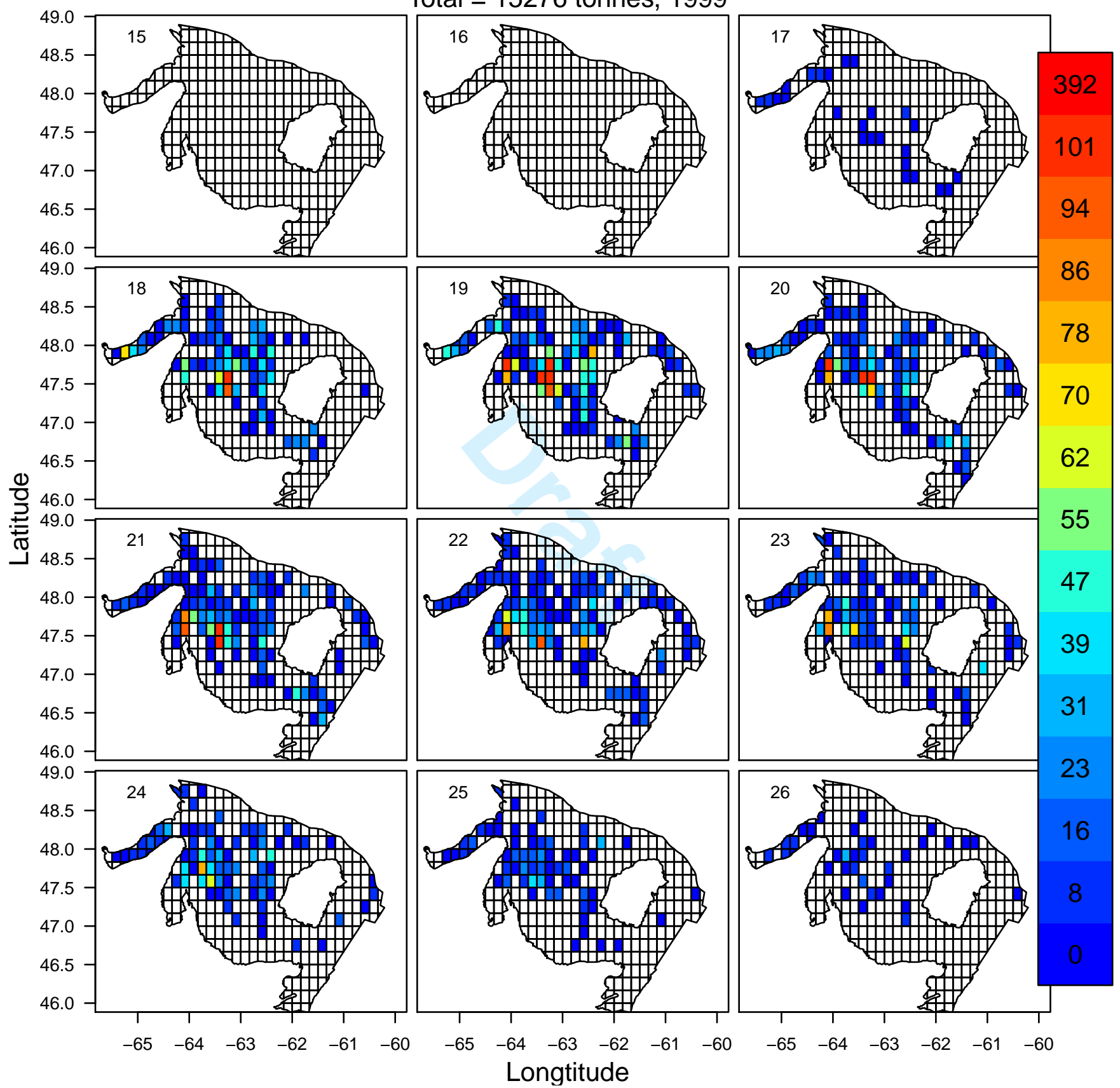

Figure SC.5b. Catch (tonnes) of snow crab in each week and grid cell in 1999. The week number is indicated in the top left-hand corner. Colors correspond to catch levels, as indicated in the legend on the right-hand side. Darkest red grids indicate catch $>98$ th percentile. 


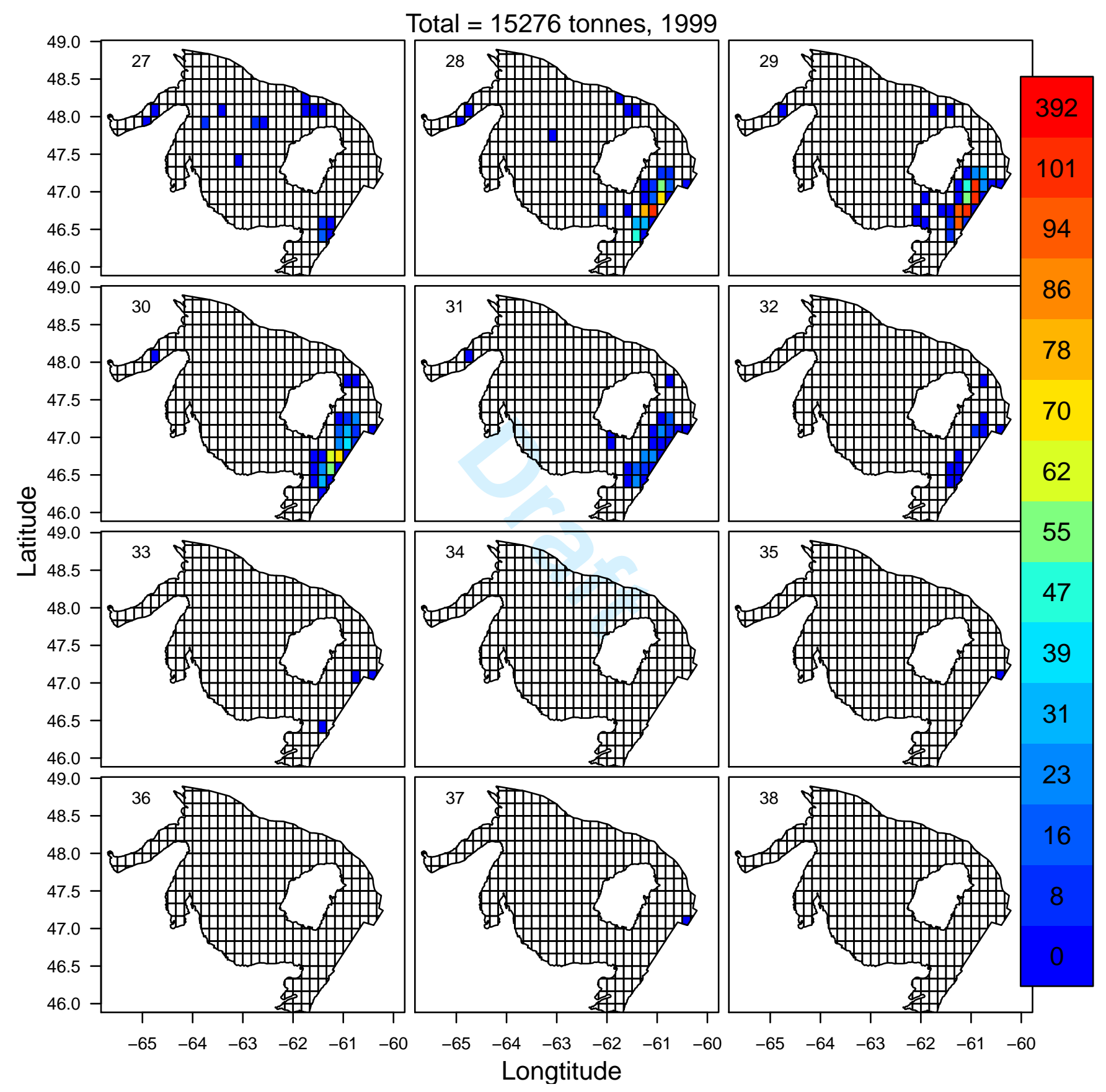

Figure SC.5c. Catch (tonnes) of snow crab in each week and grid cell in 1999. The week number is indicated in the top left-hand corner. Colors correspond to catch levels, as indicated in the legend on the right-hand side. Darkest red grids indicate catch $>98$ th percentile. 


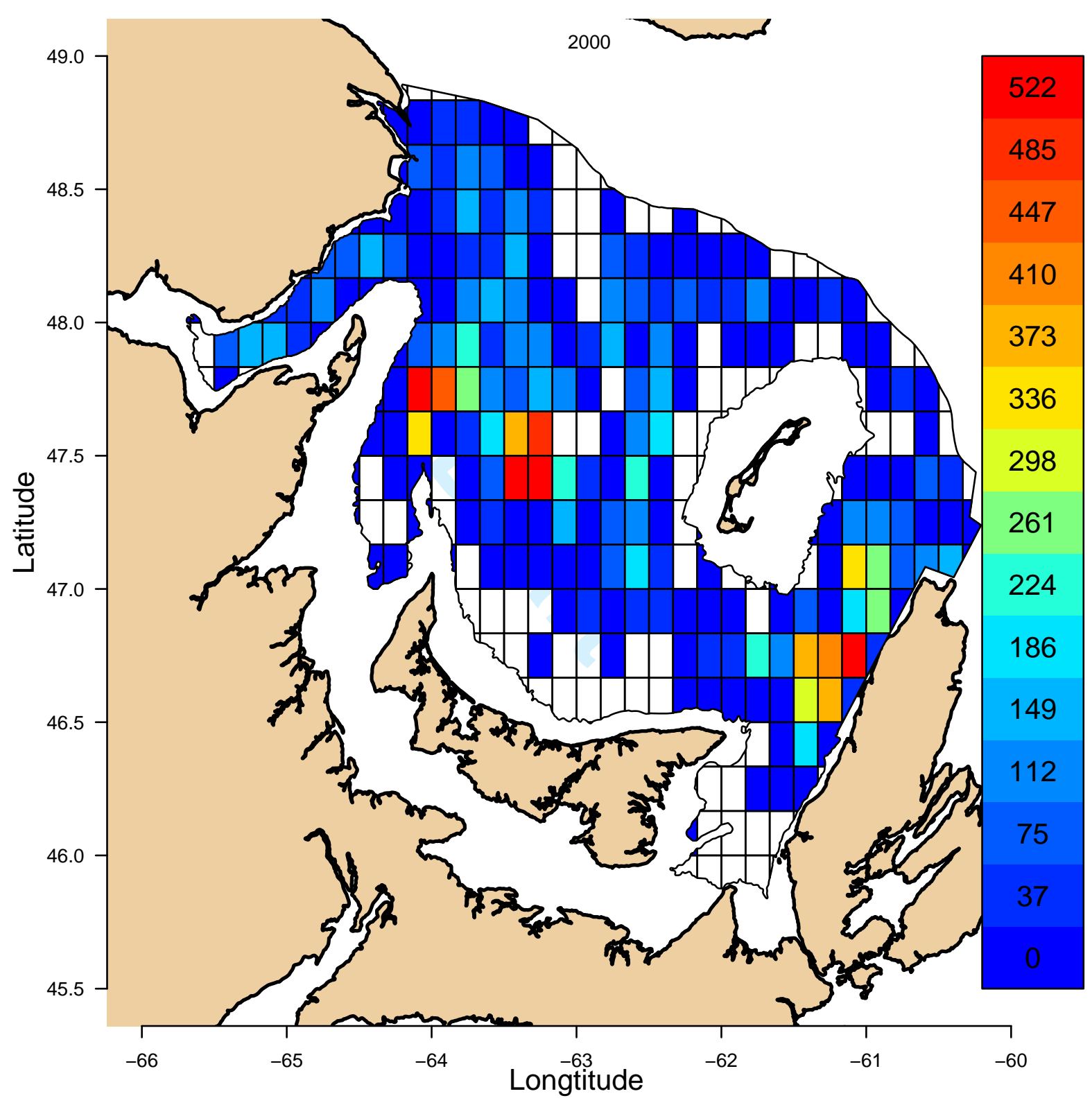

Figure SC.6a. Total annual catch (tonnes) of snow crab in each grid cell in 2000. Colors correspond to catch levels, as indicated in the legend on the right-hand side. Darkest red grids indicate catch $>98$ th percentile. 


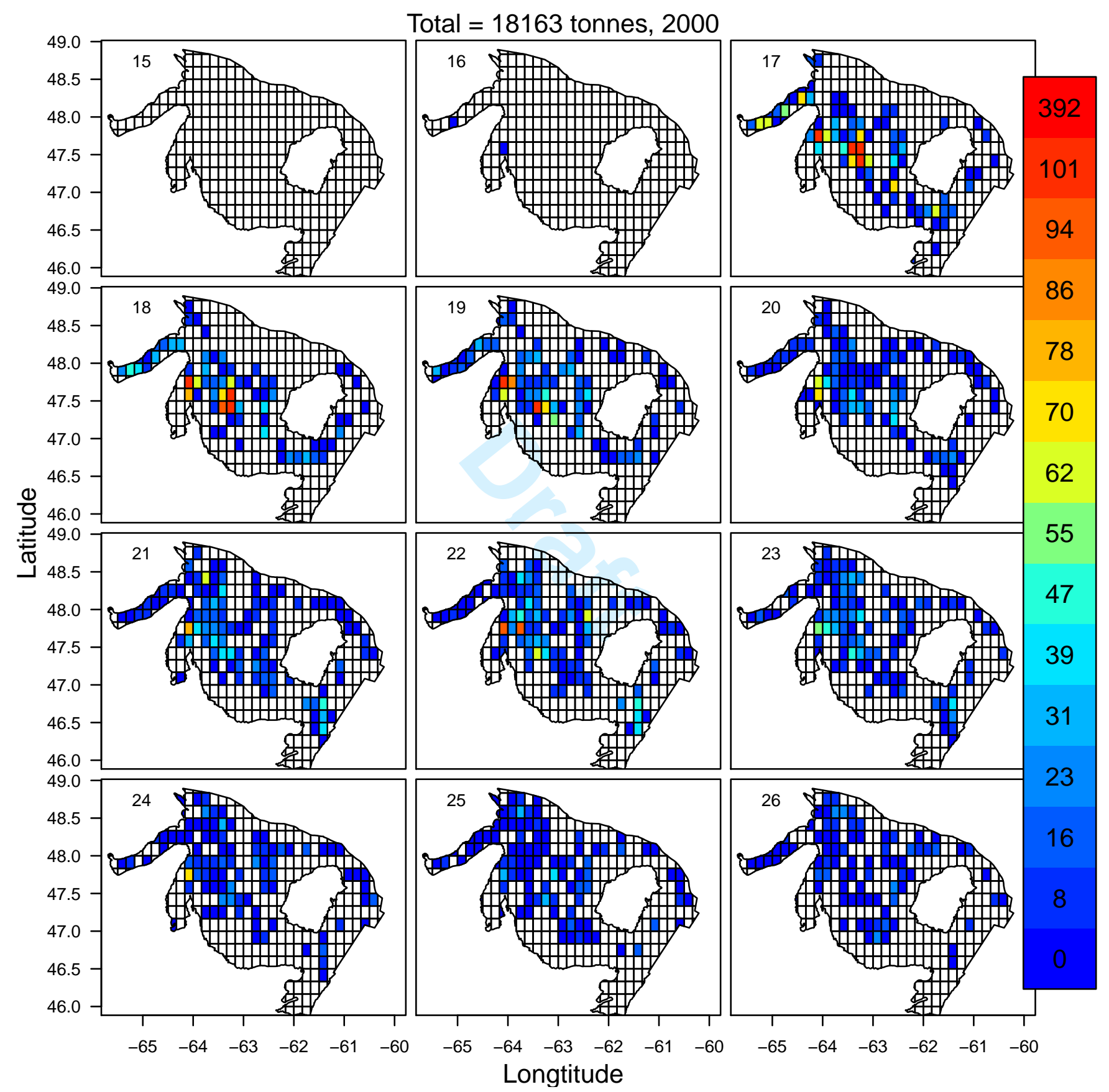

Figure SC.6b. Catch (tonnes) of snow crab in each week and grid cell in 2000. The week number is indicated in the top left-hand corner. Colors correspond to catch levels, as indicated in the legend on the right-hand side. Darkest red grids indicate catch $>98$ th percentile. 
Total $=18163$ tonnes, 2000
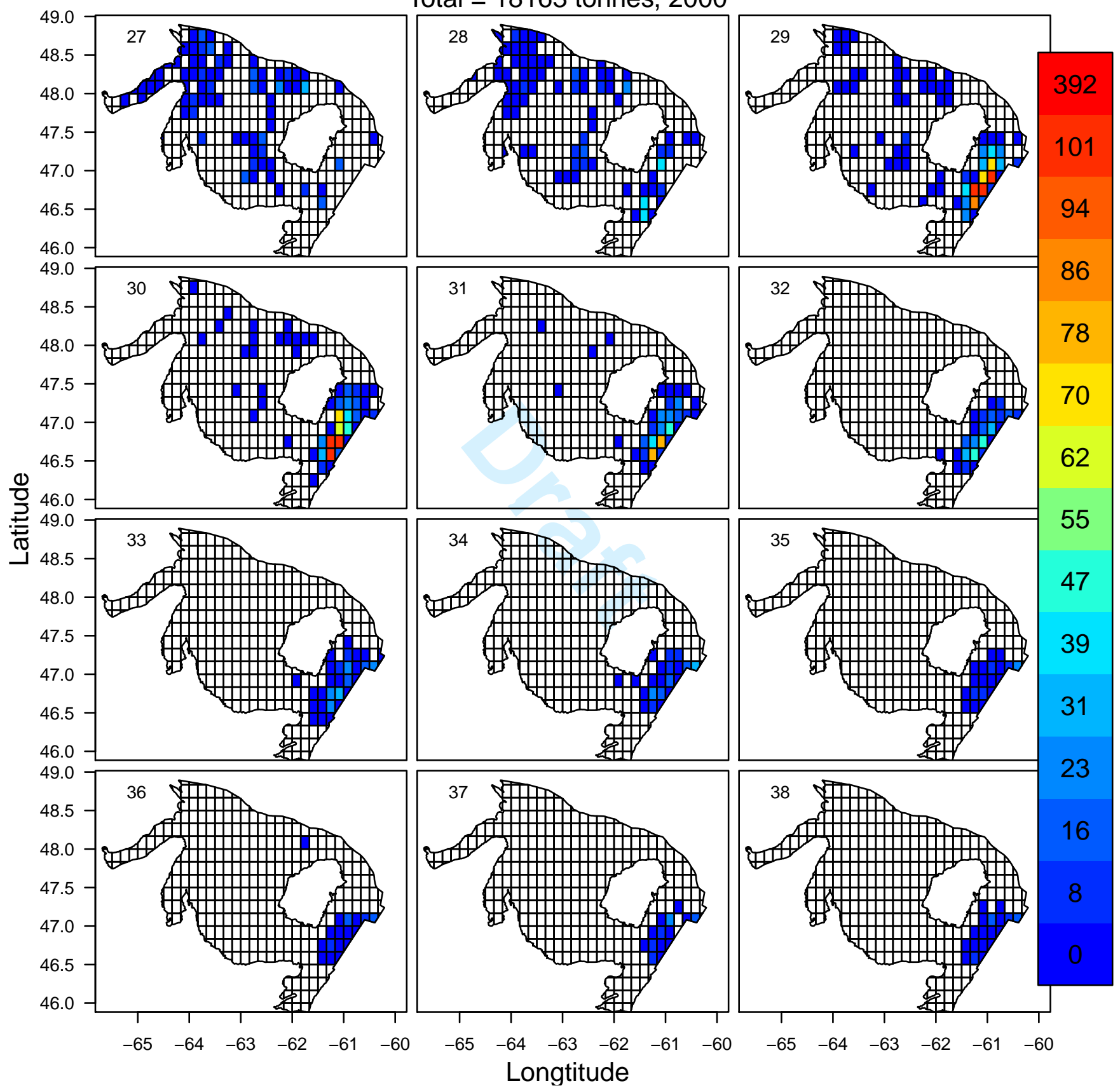

Figure SC.6c. Catch (tonnes) of snow crab in each week and grid cell in 2000. The week number is indicated in the top left-hand corner. Colors correspond to catch levels, as indicated in the legend on the right-hand side. Darkest red grids indicate catch $>98$ th percentile. 


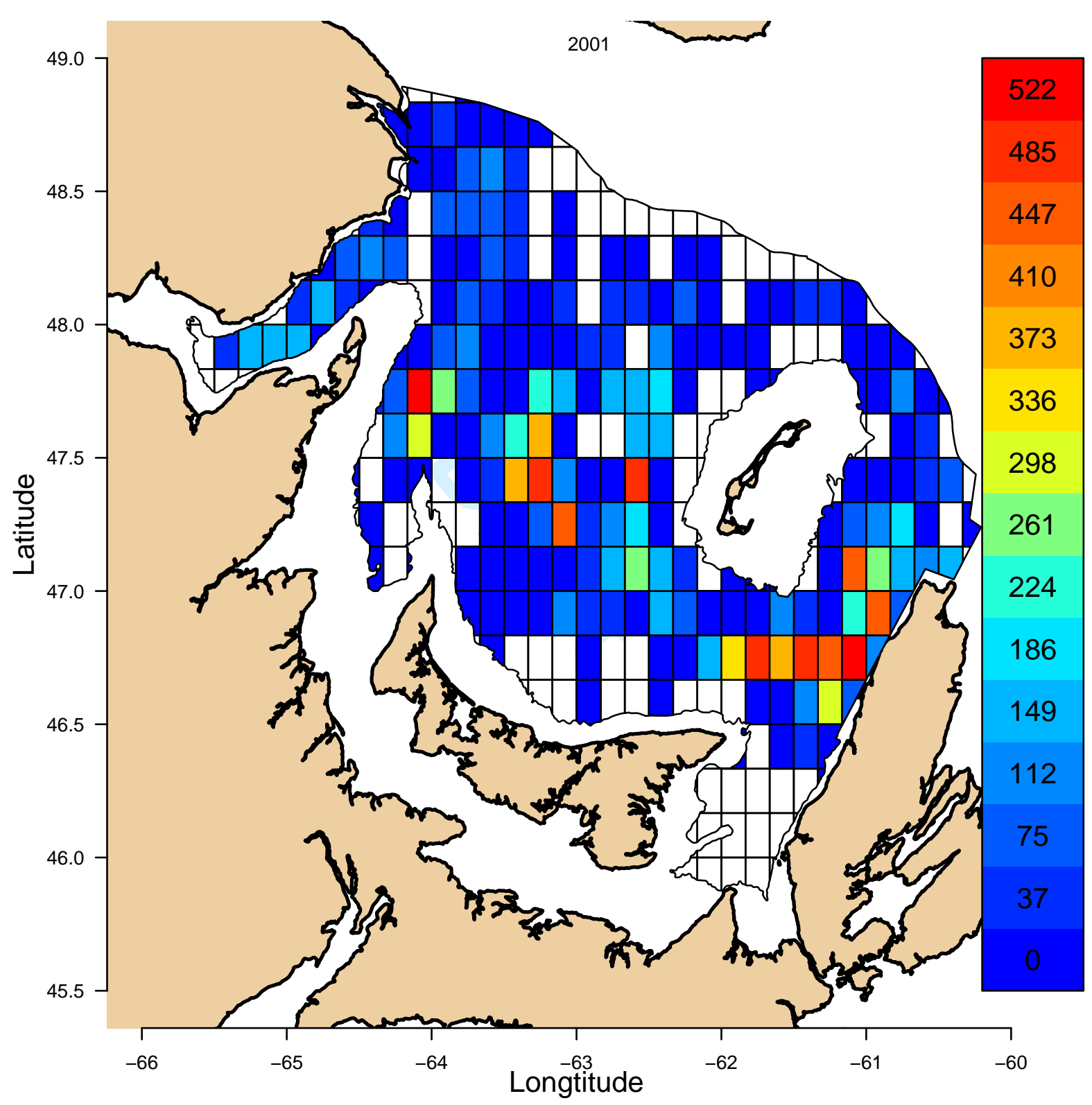

Figure SC.7a. Total annual catch (tonnes) of snow crab in each grid cell in 2001. Colors correspond to catch levels, as indicated in the legend on the right-hand side. Darkest red grids indicate catch $>98$ th percentile. 
Total $=18235$ tonnes, 2001
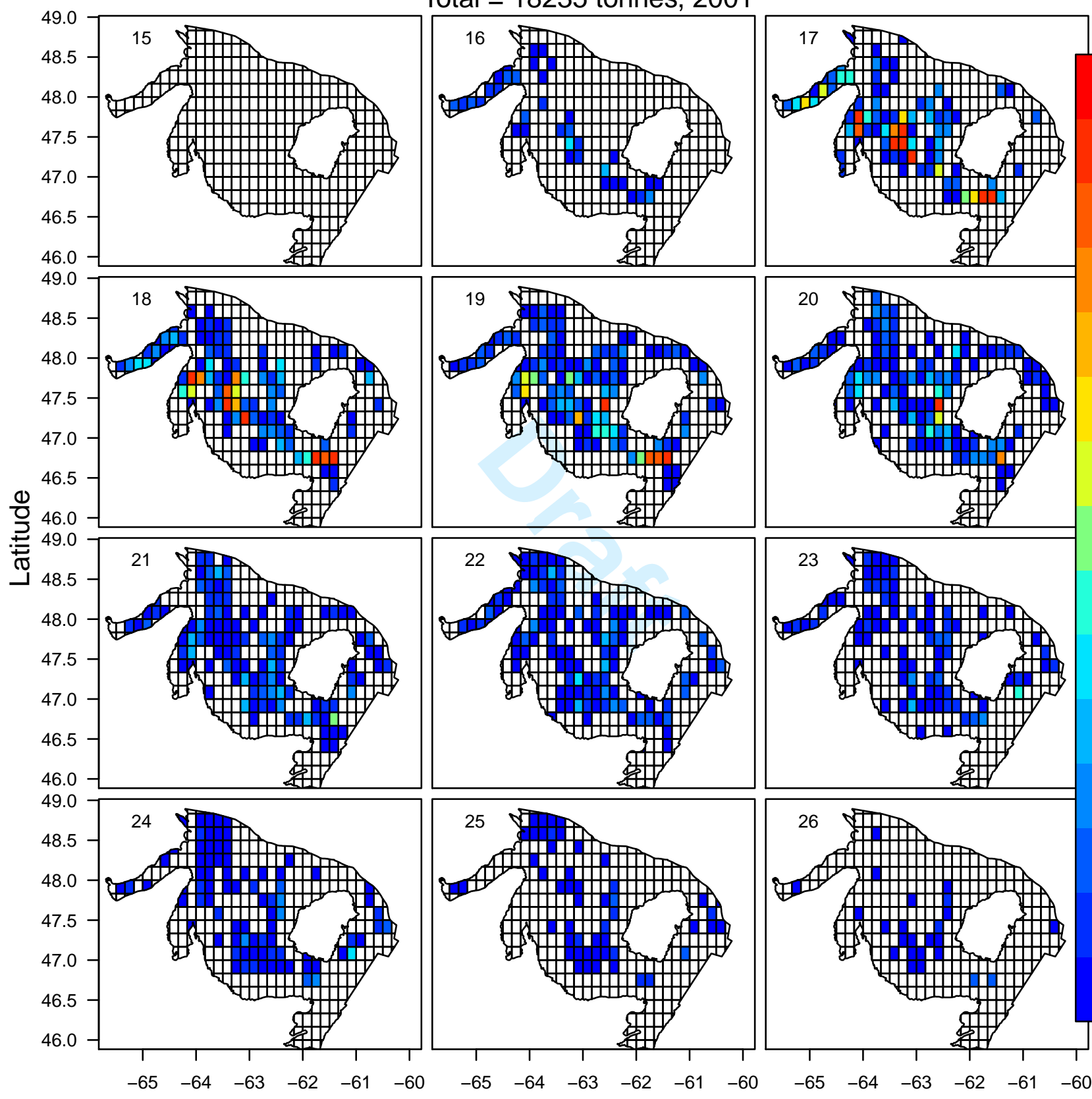

86
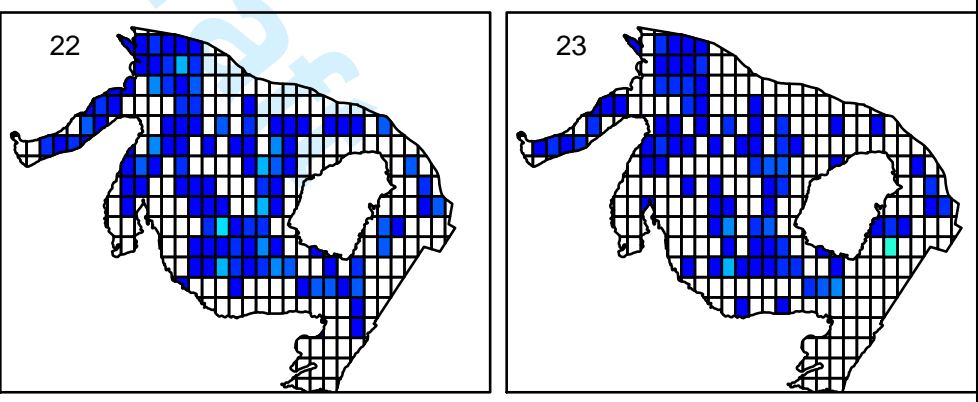

55
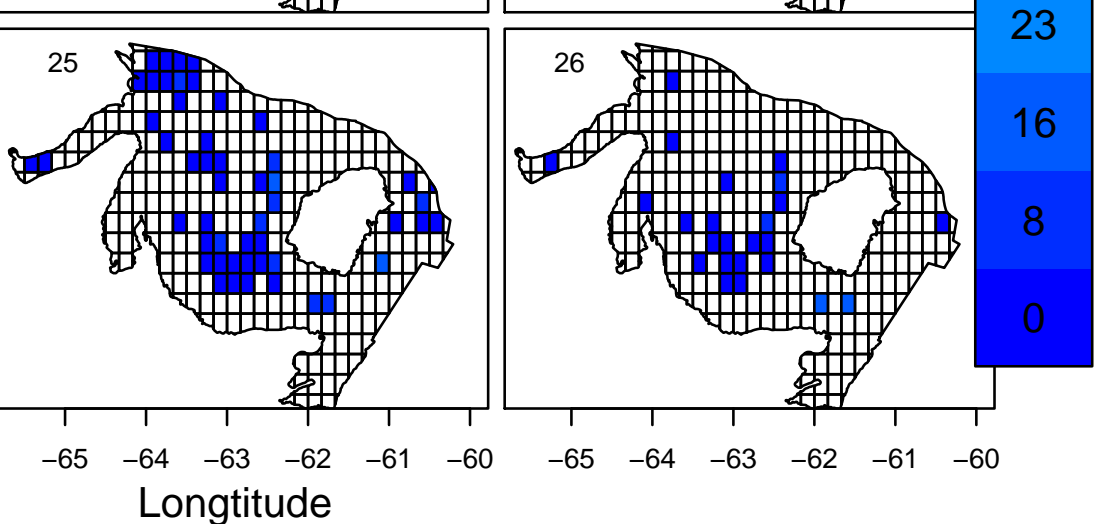

Figure SC.7b. Catch (tonnes) of snow crab in each week and grid cell in 2001. The week number is indicated in the top left-hand corner. Colors correspond to catch levels, as indicated in the legend on the right-hand side. Darkest red grids indicate catch $>98$ th percentile. 


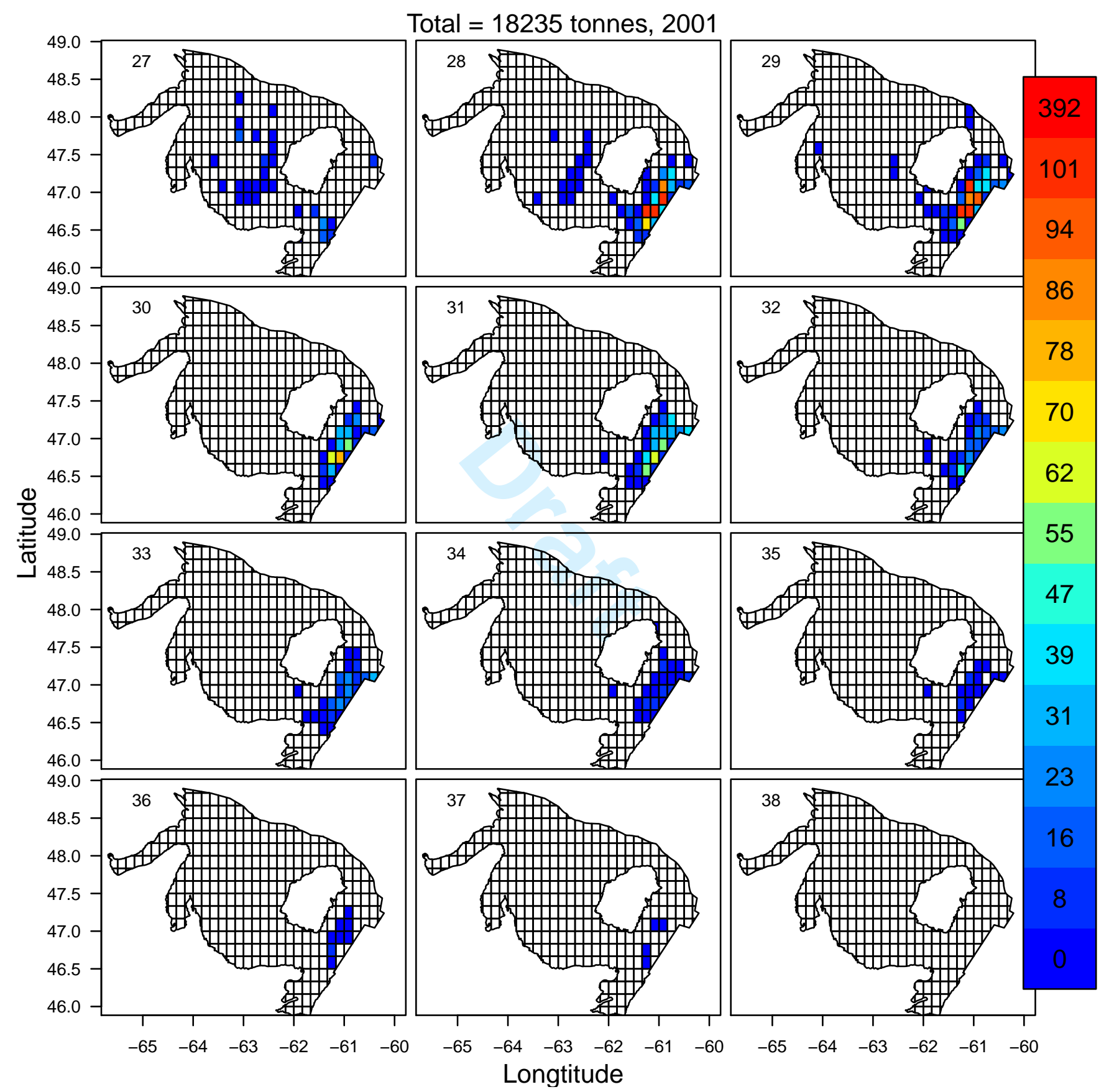

Figure SC.7c. Catch (tonnes) of snow crab in each week and grid cell in 2001. The week number is indicated in the top left-hand corner. Colors correspond to catch levels, as indicated in the legend on the right-hand side. Darkest red grids indicate catch $>$ 98th percentile. 


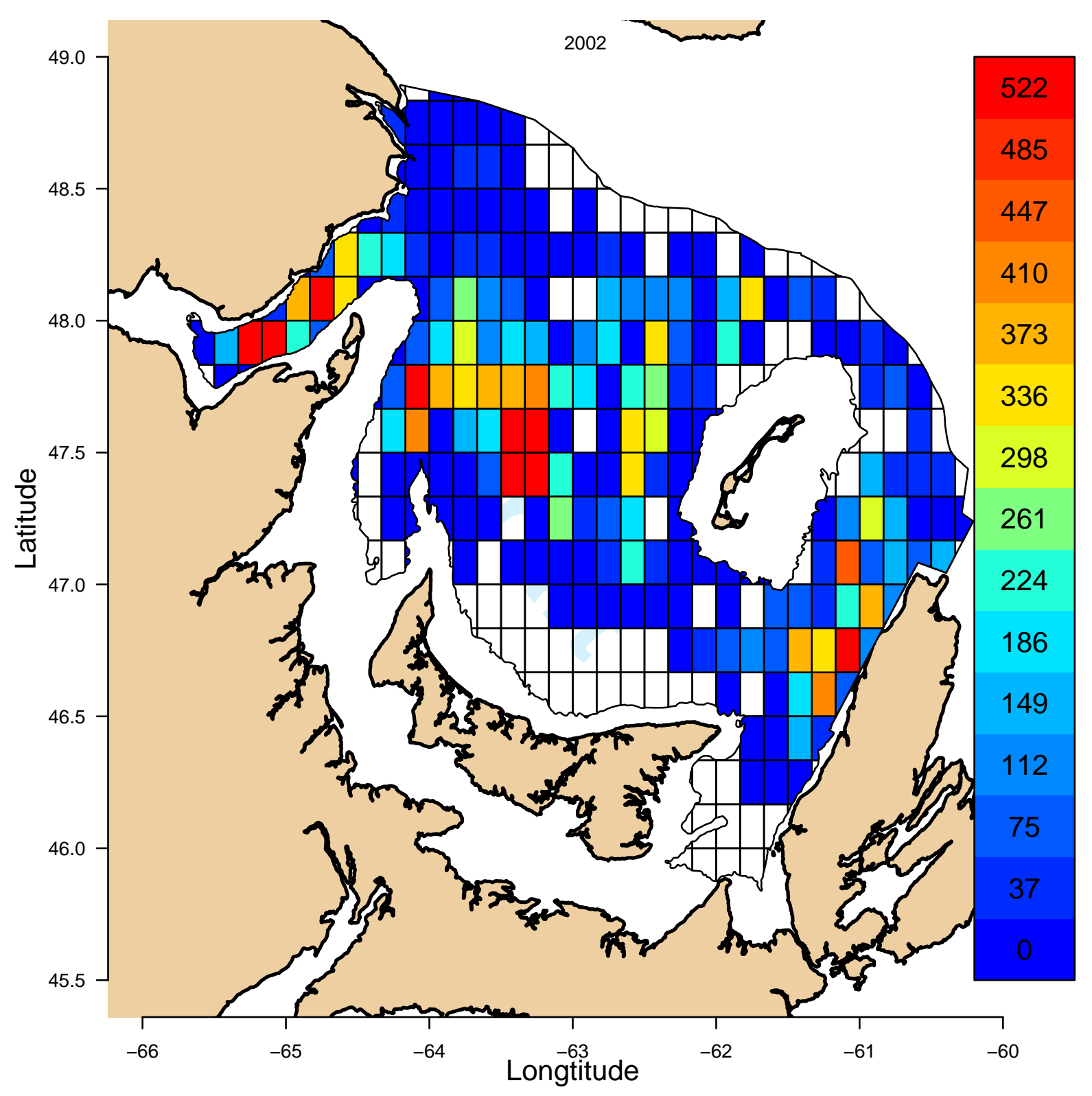

Figure SC.8a. Total annual catch (tonnes) of snow crab in each grid cell in 2002. Colors correspond to catch levels, as indicated in the legend on the right-hand side. Darkest red grids indicate catch $>98$ th percentile. 


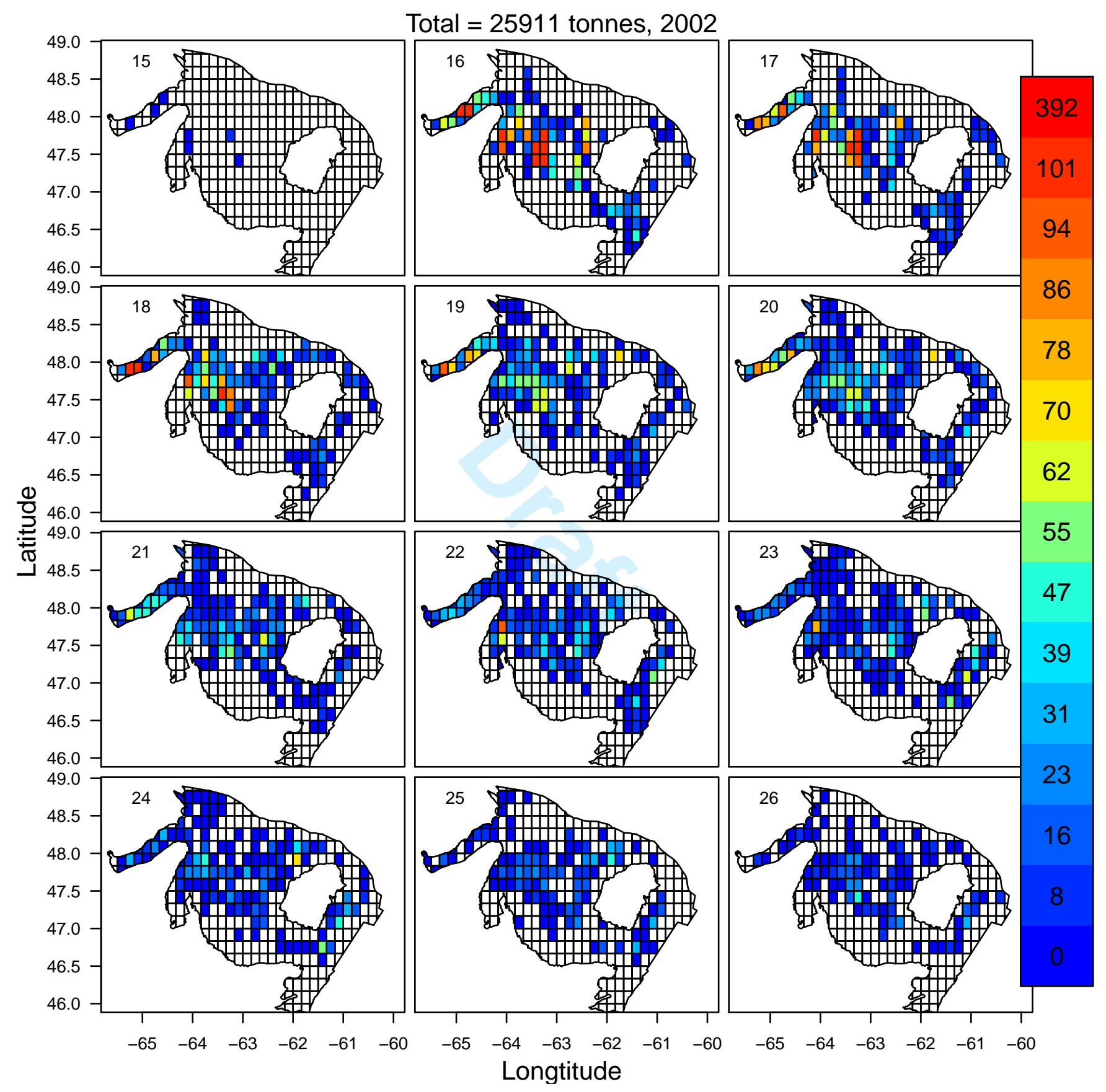

Figure SC.8b. Catch (tonnes) of snow crab in each week and grid cell in 2002. The week number is indicated in the top left-hand corner. Colors correspond to catch levels, as indicated in the legend on the right-hand side. Darkest red grids indicate catch $>98$ th percentile. 
Total $=25911$ tonnes, 2002
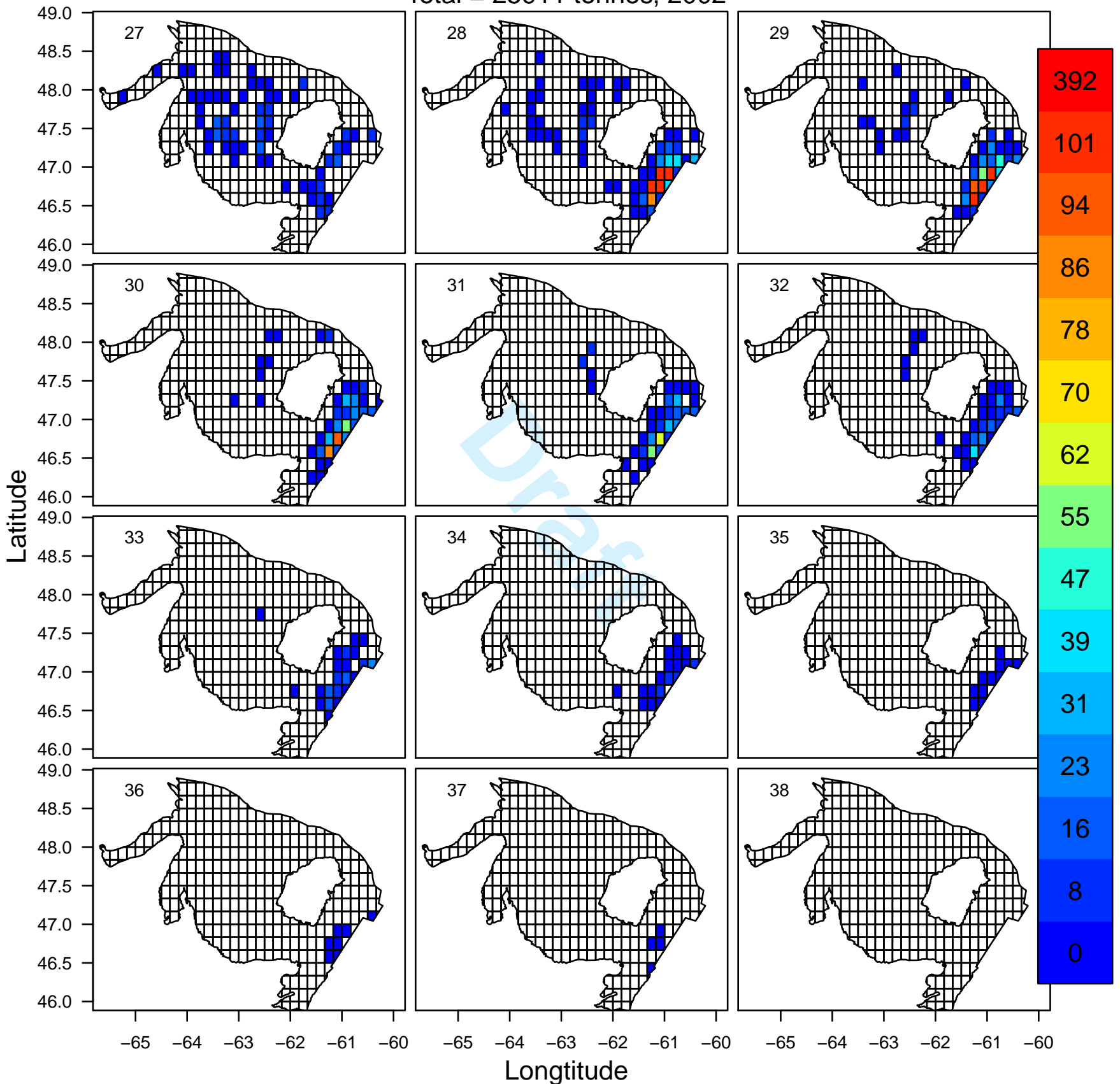

Figure SC.8c. Catch (tonnes) of snow crab in each week and grid cell in 2002. The week number is indicated in the top left-hand corner. Colors correspond to catch levels, as indicated in the legend on the right-hand side. Darkest red grids indicate catch $>98$ th percentile. 


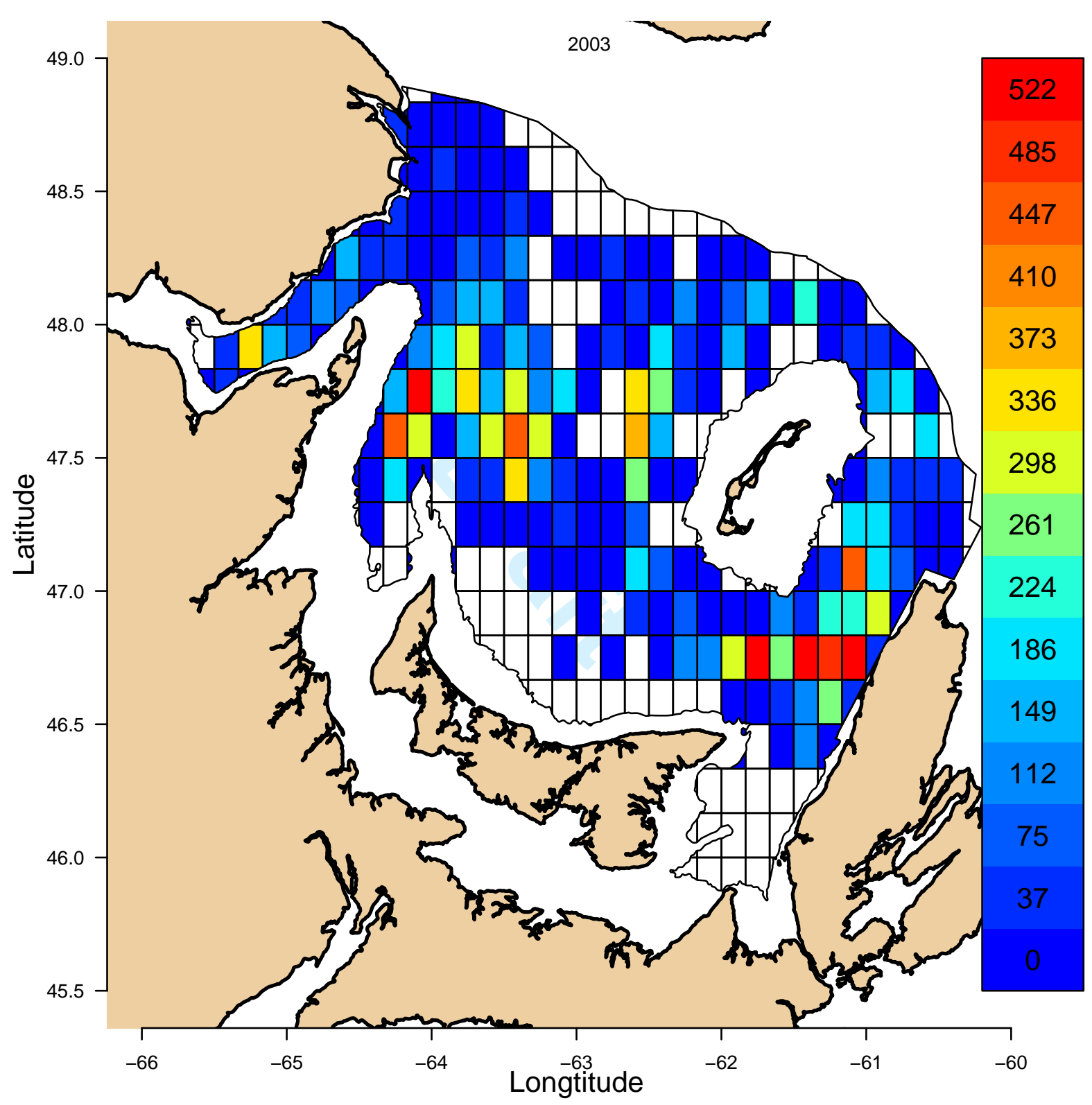

Figure SC.9a. Total annual catch (tonnes) of snow crab in each grid cell in 2003. Colors correspond to catch levels, as indicated in the legend on the right-hand side. Darkest red grids indicate catch $>98$ th percentile. 
Total $=20246$ tonnes, 2003
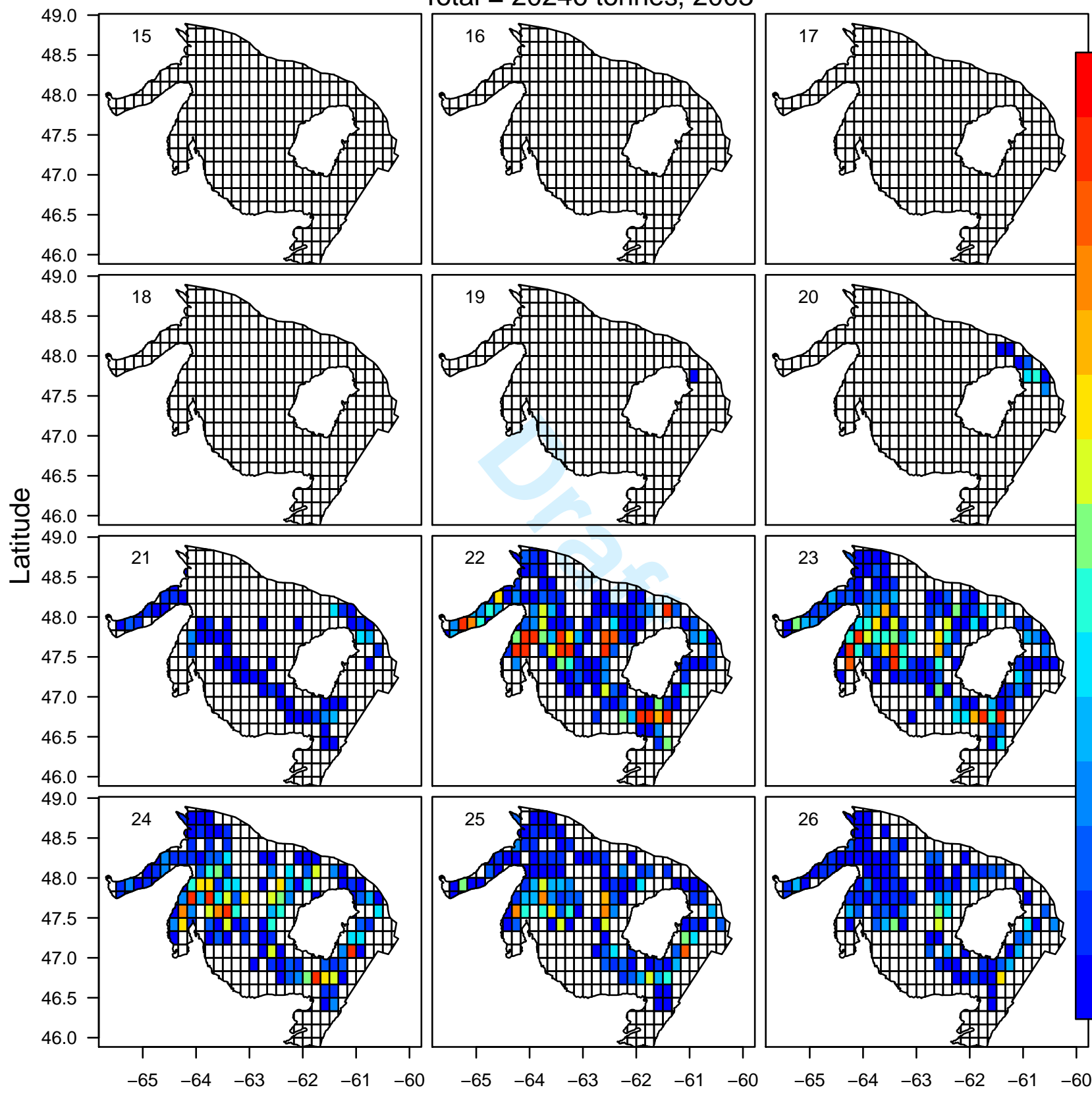

86
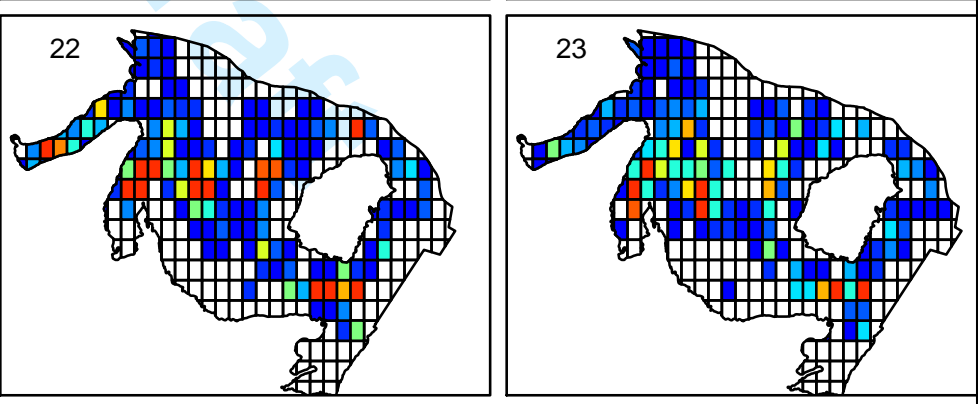

55
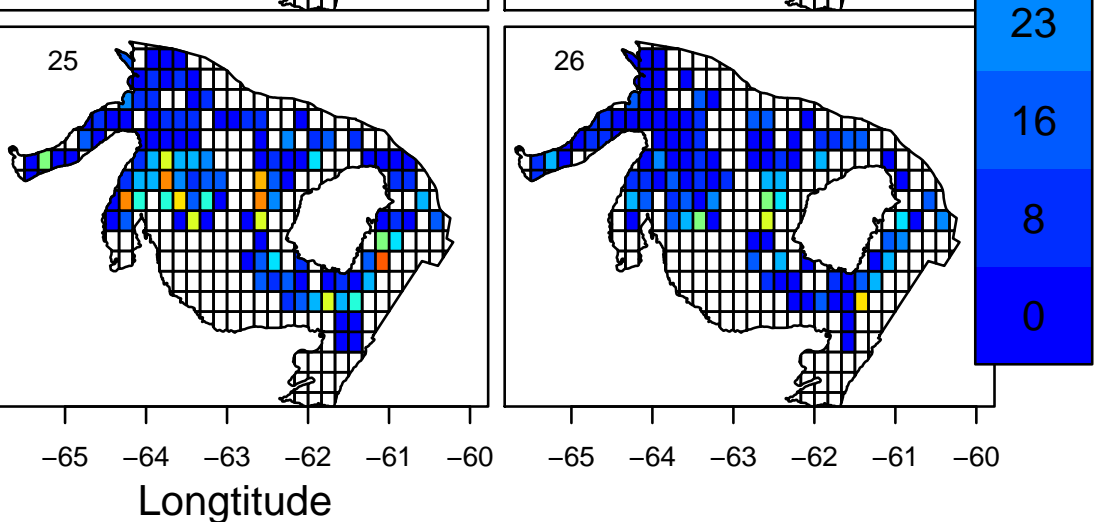

Figure SC.9b. Catch (tonnes) of snow crab in each week and grid cell in 2003. The week number is indicated in the top left-hand corner. Colors correspond to catch levels, as indicated in the legend on the right-hand side. Darkest red grids indicate catch $>98$ th percentile. 


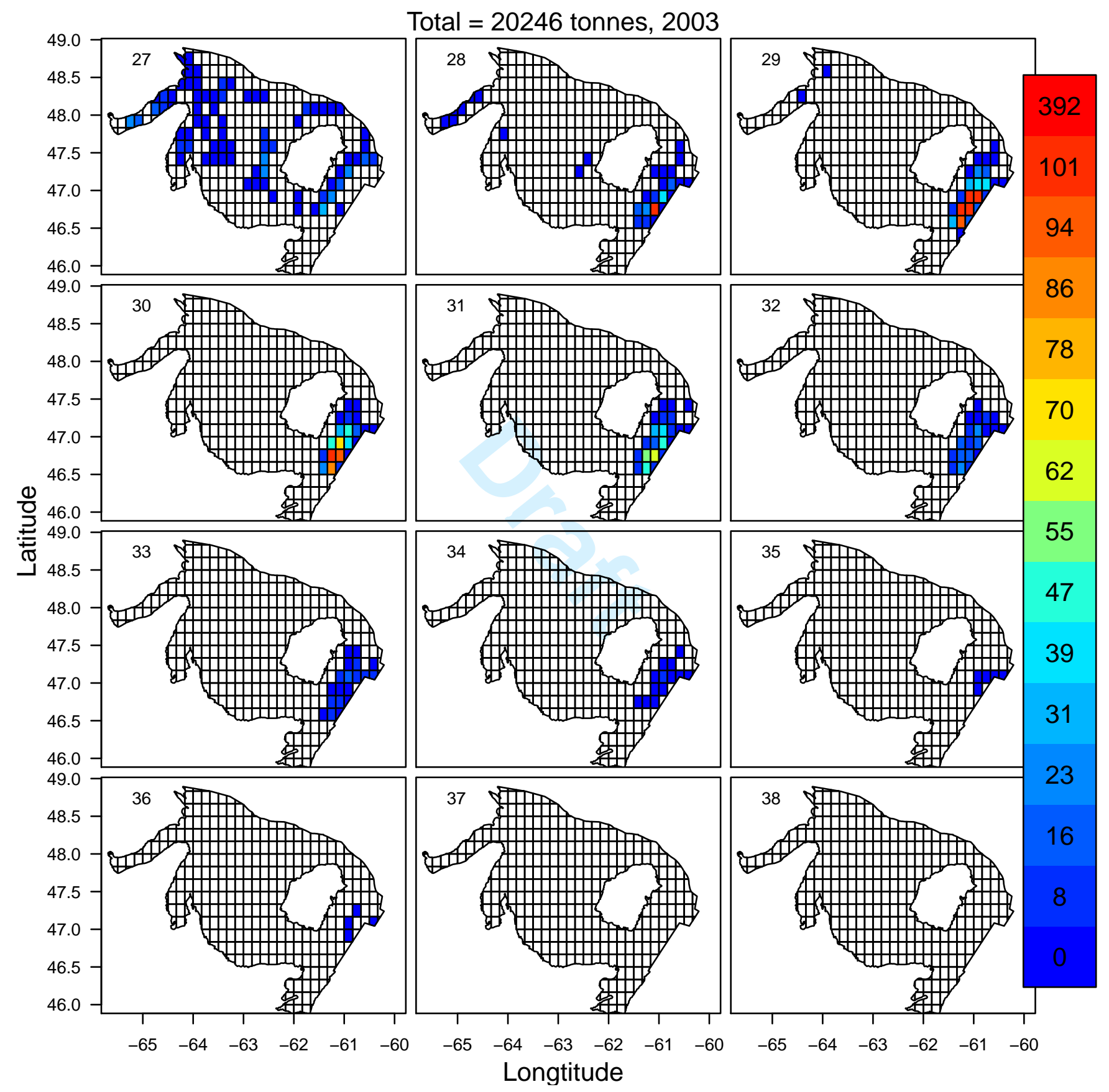

Figure SC.9c. Catch (tonnes) of snow crab in each week and grid cell in 2003. The week number is indicated in the top left-hand corner. Colors correspond to catch levels, as indicated in the legend on the right-hand side. Darkest red grids indicate catch $>98$ th percentile. 


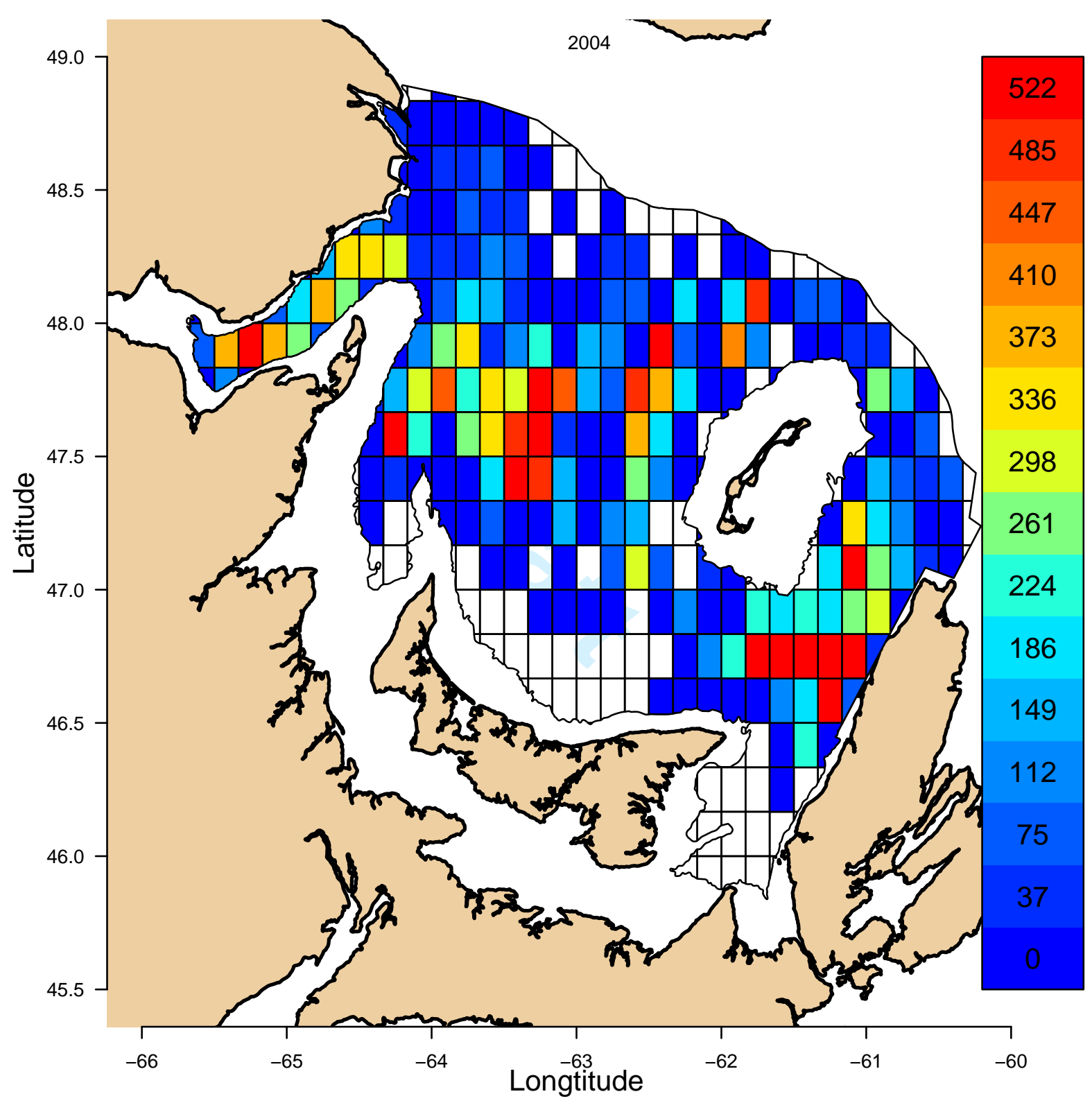

Figure SC.10a. Total annual catch (tonnes) of snow crab in each grid cell in 2004. Colors correspond to catch levels, as indicated in the legend on the right-hand side. Darkest red grids indicate catch $>98$ th percentile. 


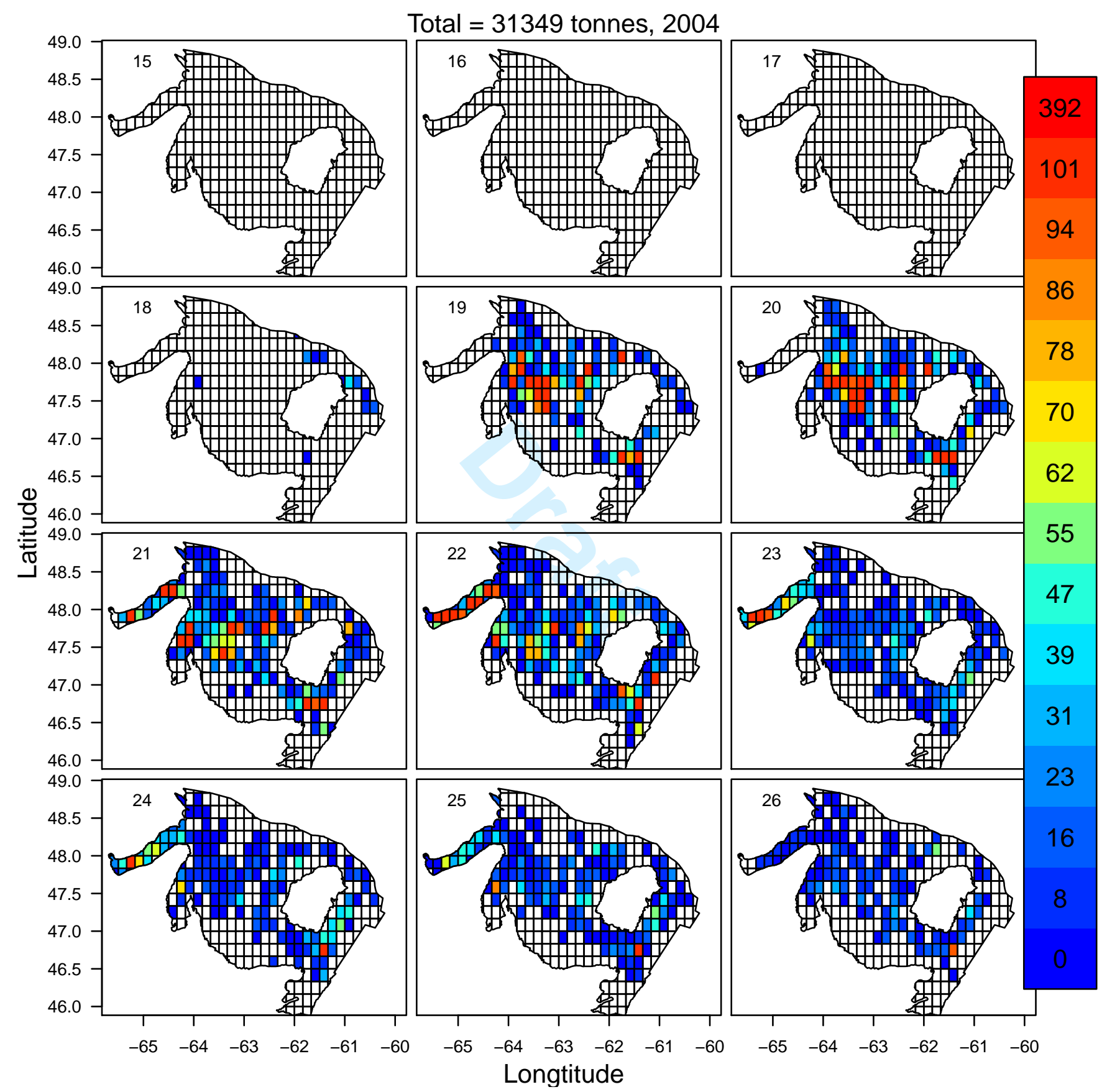

Figure SC.10b. Catch (tonnes) of snow crab in each week and grid cell in 2004 . The week number is indicated in the top left-hand corner. Colors correspond to catch levels, as indicated in the legend on the right-hand side. Darkest red grids indicate catch $>98$ th percentile. 
Total $=31349$ tonnes, 2004
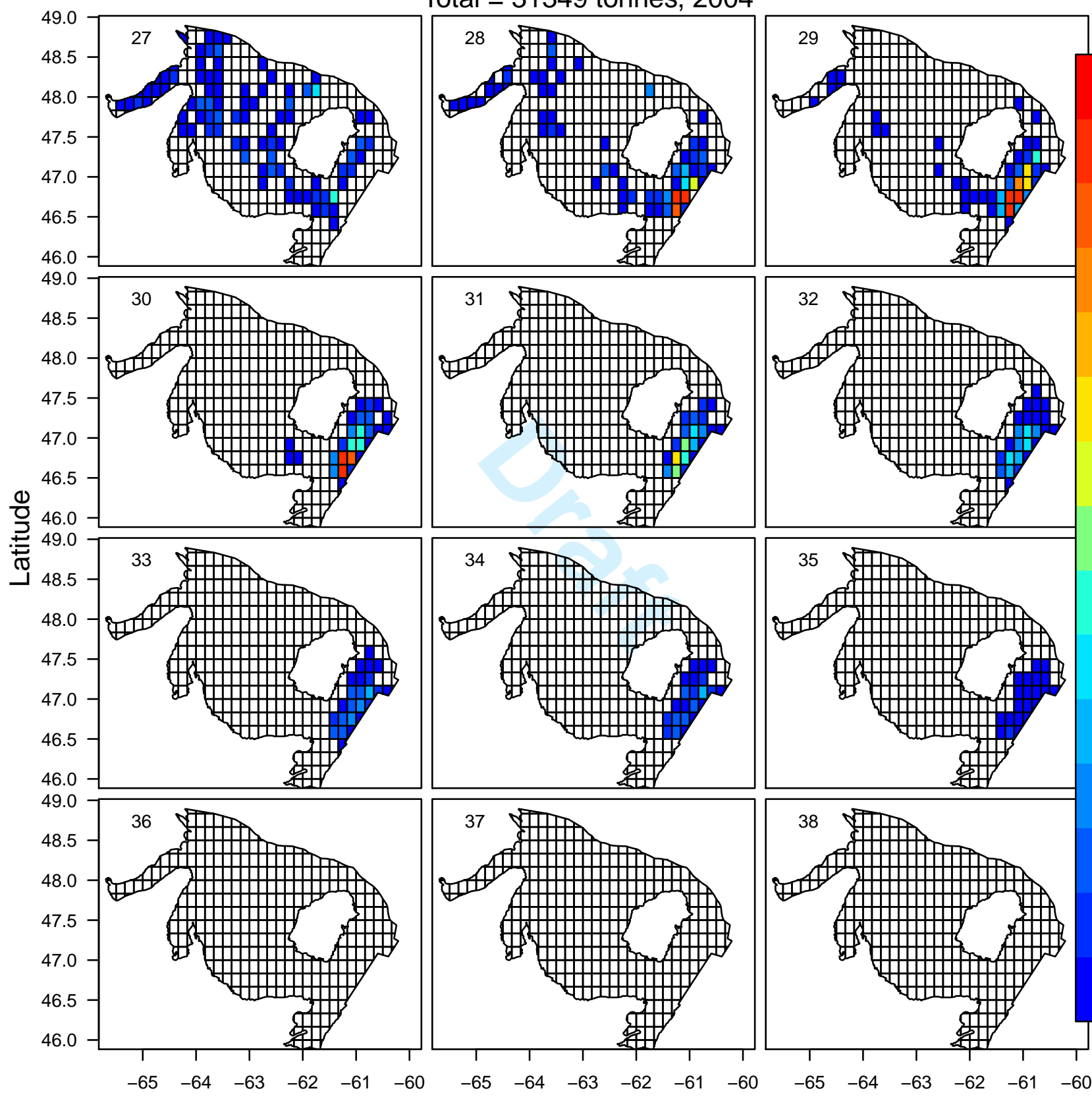

86
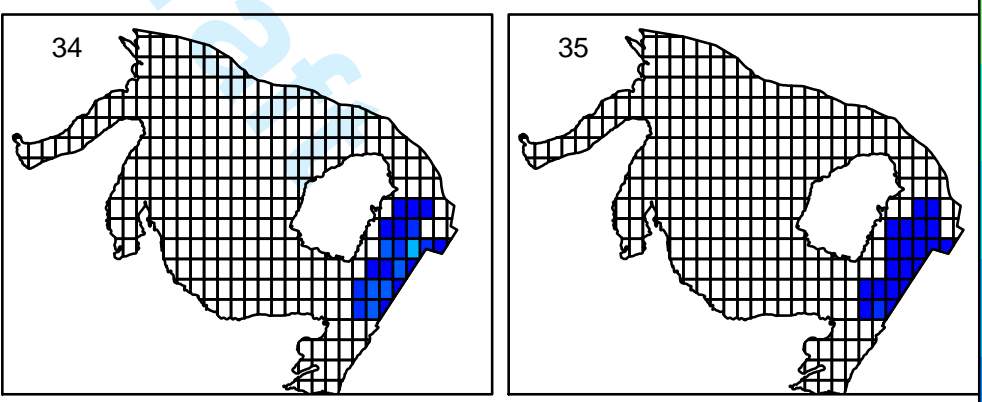

55
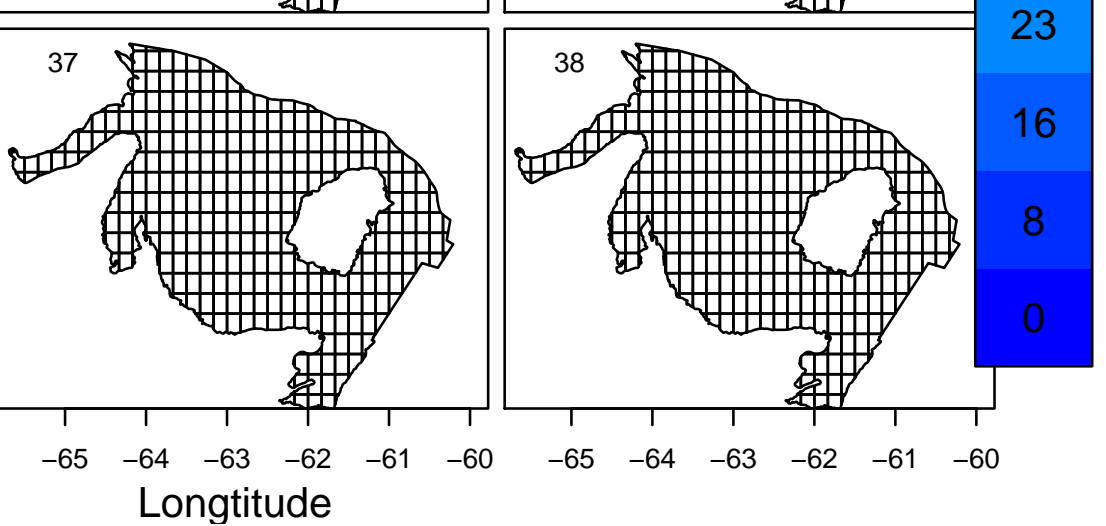

Figure SC.10c. Catch (tonnes) of snow crab in each week and grid cell in 2004. The week number is indicated in the top left-hand corner. Colors correspond to catch levels, as indicated in the legend on the right-hand side. Darkest red grids indicate catch $>98$ th percentile. 


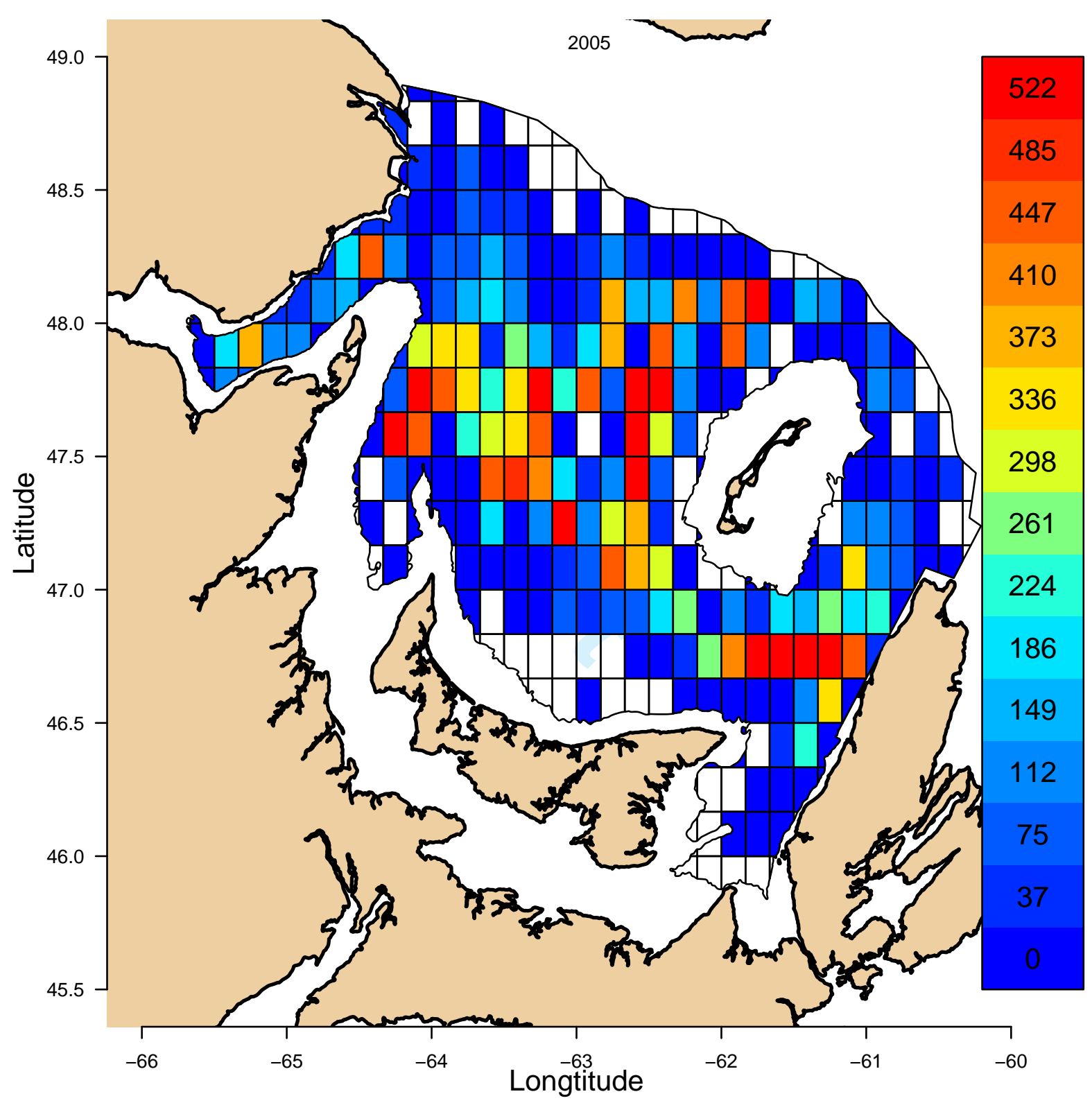

Figure SC.11a. Total annual catch (tonnes) of snow crab in each grid cell in 2005. Colors correspond to catch levels, as indicated in the legend on the right-hand side. Darkest red grids indicate catch $>98$ th percentile. 
Total $=34586$ tonnes, 2005
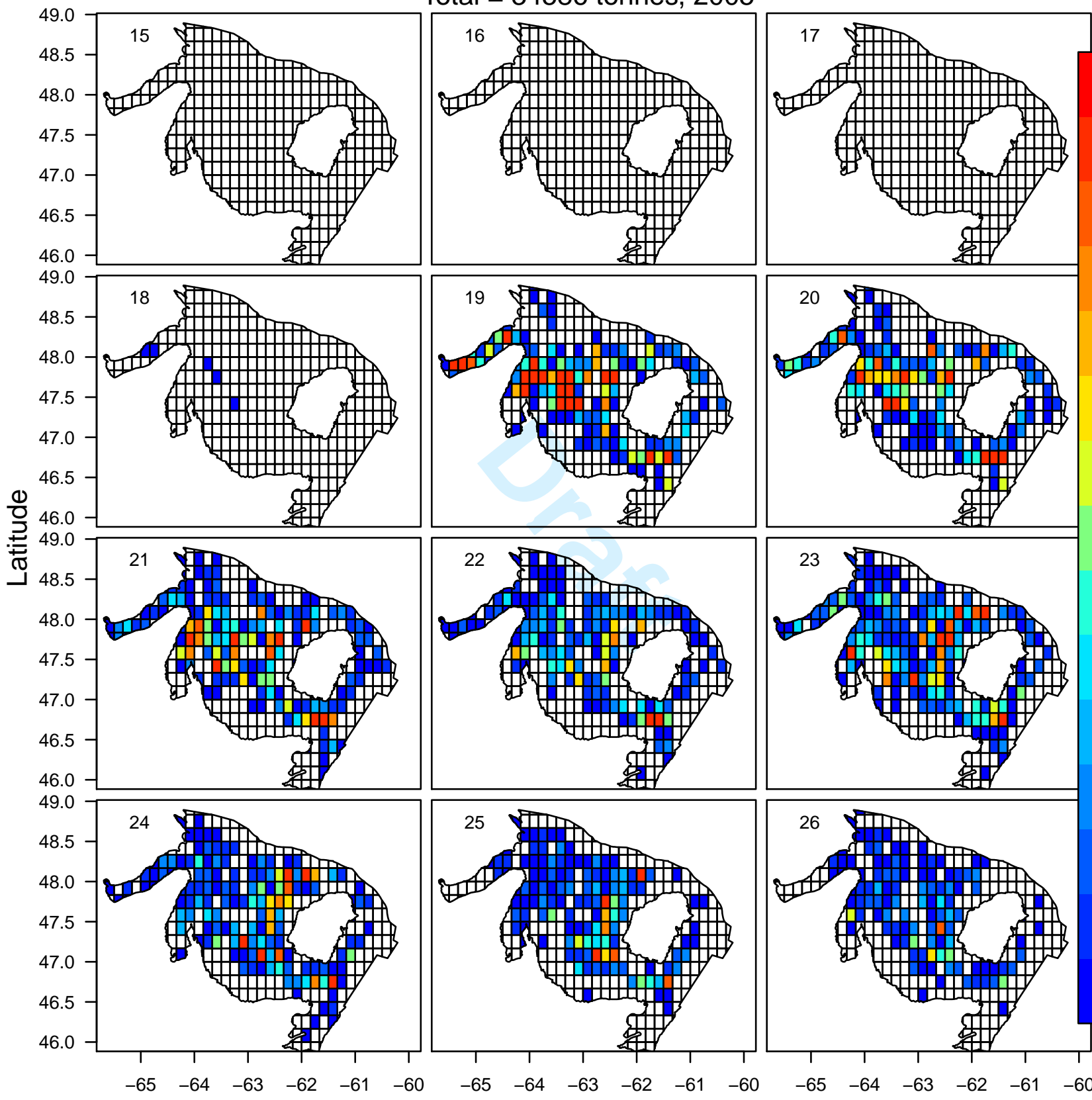

86
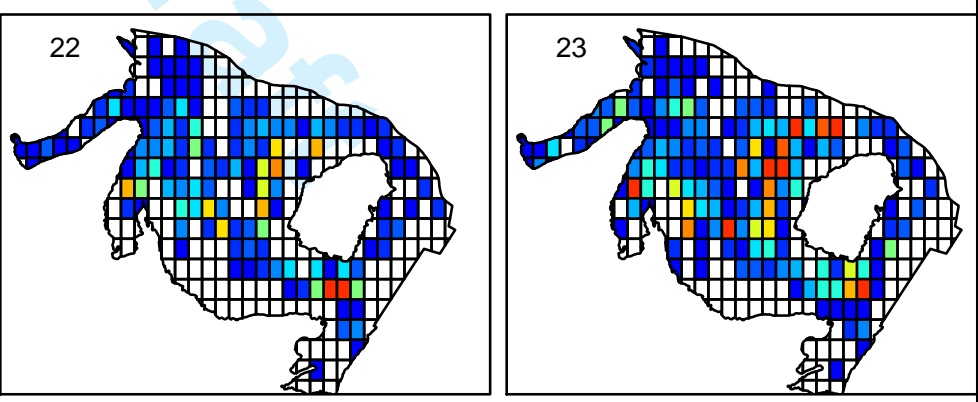

55
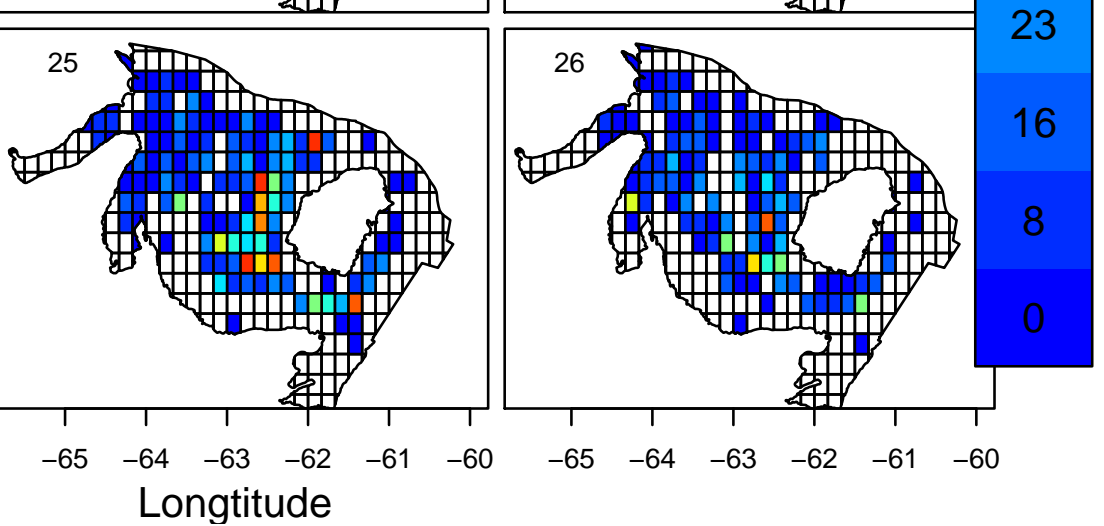

Figure SC.11b. Catch (tonnes) of snow crab in each week and grid cell in 2005. The week number is indicated in the top left-hand corner. Colors correspond to catch levels, as indicated in the legend on the righthand side. Darkest red grids indicate catch $>98$ th percentile. 


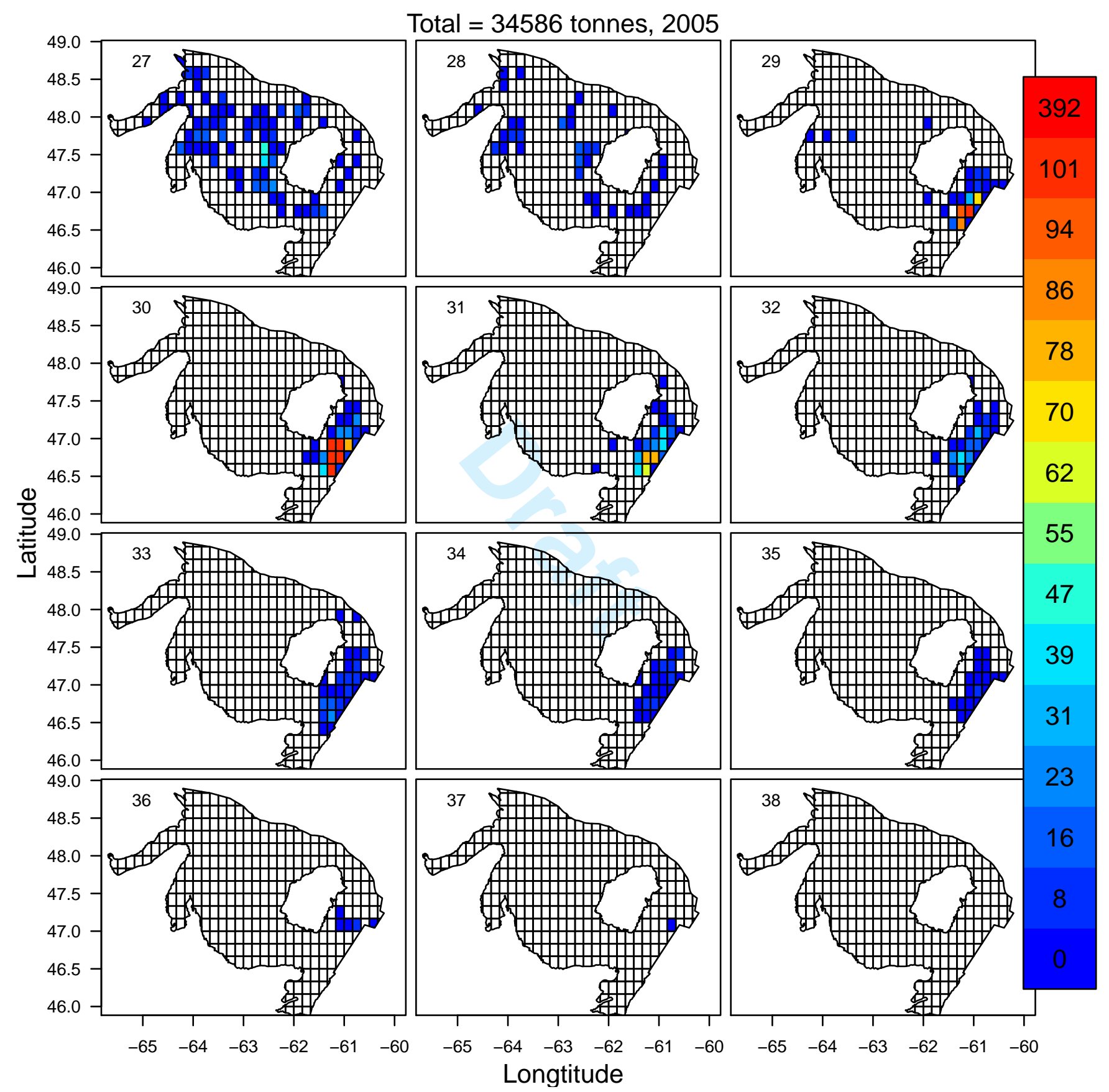

Figure SC.11c. Catch (tonnes) of snow crab in each week and grid cell in 2005. The week number is indicated in the top left-hand corner. Colors correspond to catch levels, as indicated in the legend on the right-hand side. Darkest red grids indicate catch $>98$ th percentile. 


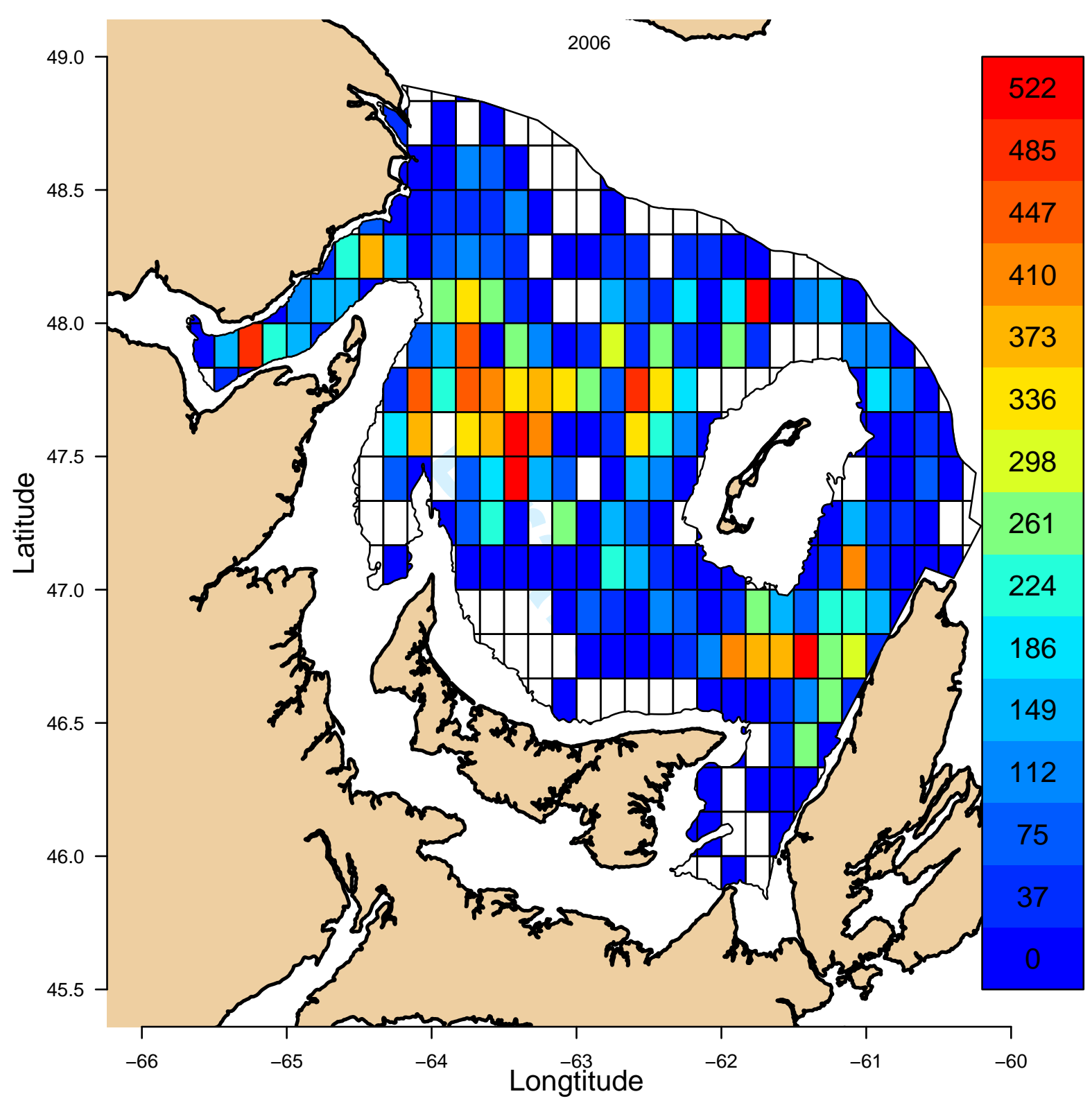

Figure SC.12a. Total annual catch (tonnes) of snow crab in each grid cell in 2006. Colors correspond to catch levels, as indicated in the legend on the right-hand side. Darkest red grids indicate catch $>98$ th percentile. 


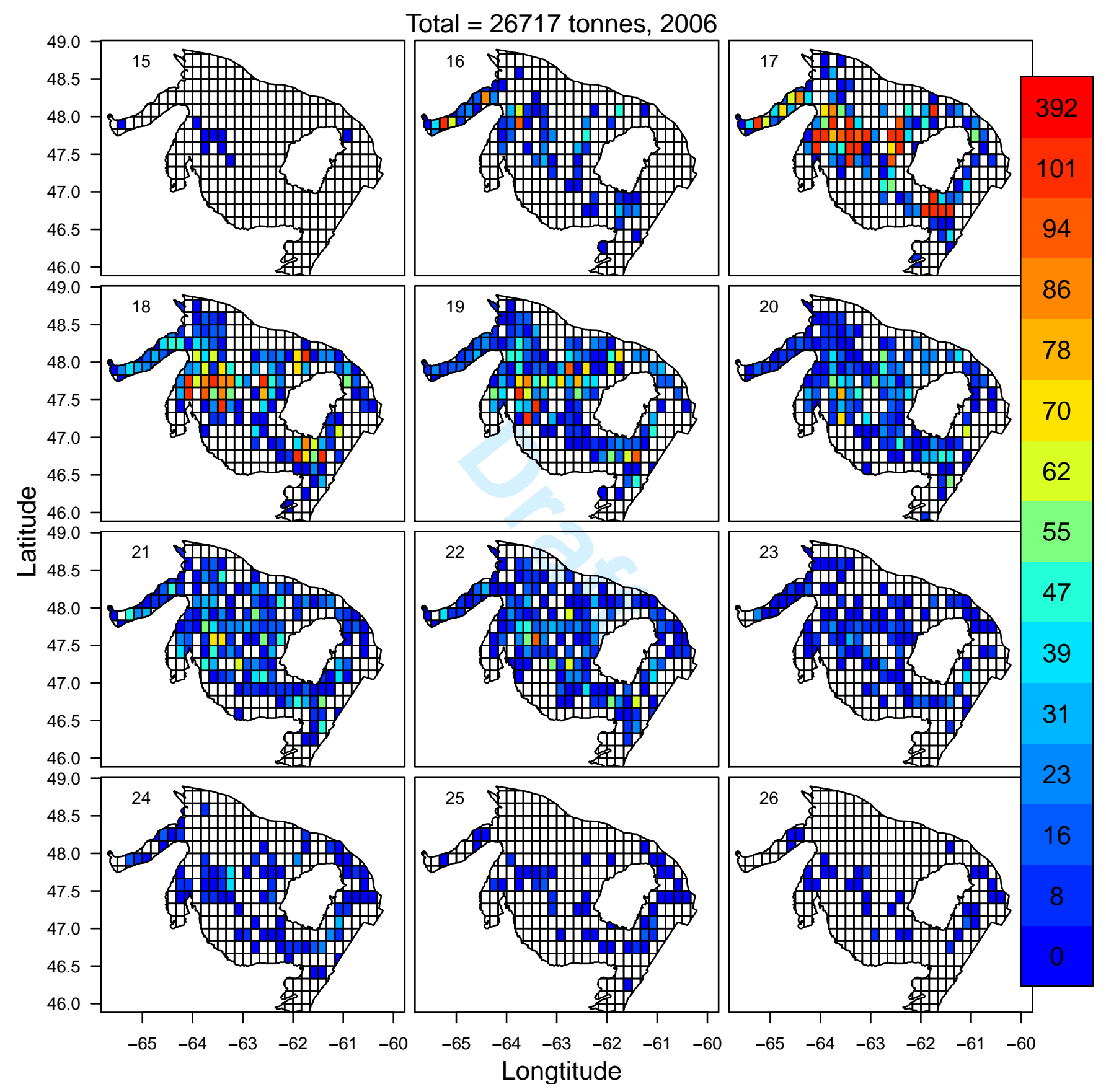

Figure SC.12b. Catch (tonnes) of snow crab in each week and grid cell in 2006. The week number is indicated in the top left-hand corner. Colors correspond to catch levels, as indicated in the legend on the right-hand side. Darkest red grids indicate catch $>98$ th percentile. 
Total $=26717$ tonnes, 2006
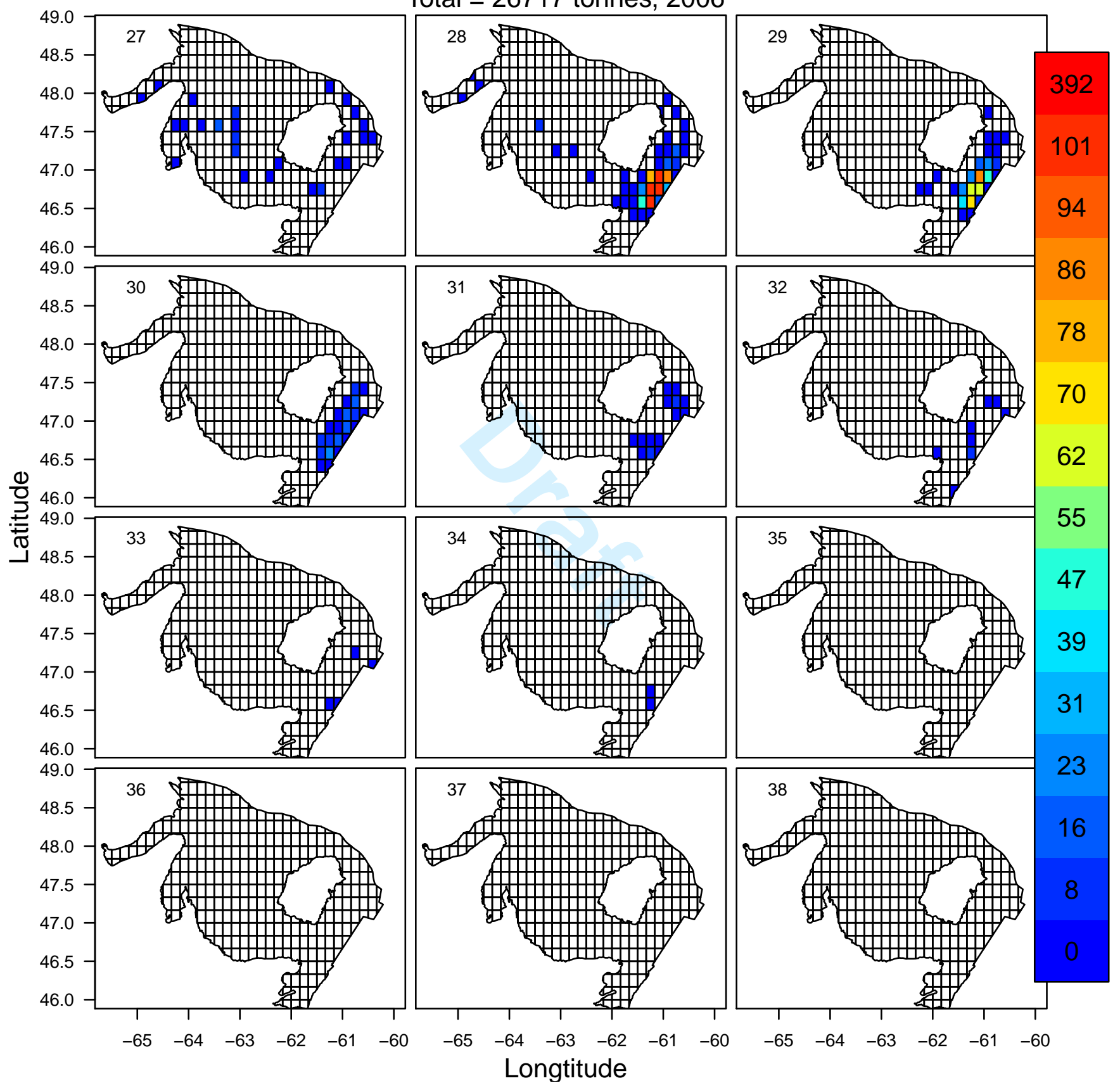

Figure SC.12c. Catch (tonnes) of snow crab in each week and grid cell in 2006. The week number is indicated in the top left-hand corner. Colors correspond to catch levels, as indicated in the legend on the right-hand side. Darkest red grids indicate catch $>98$ th percentile. 


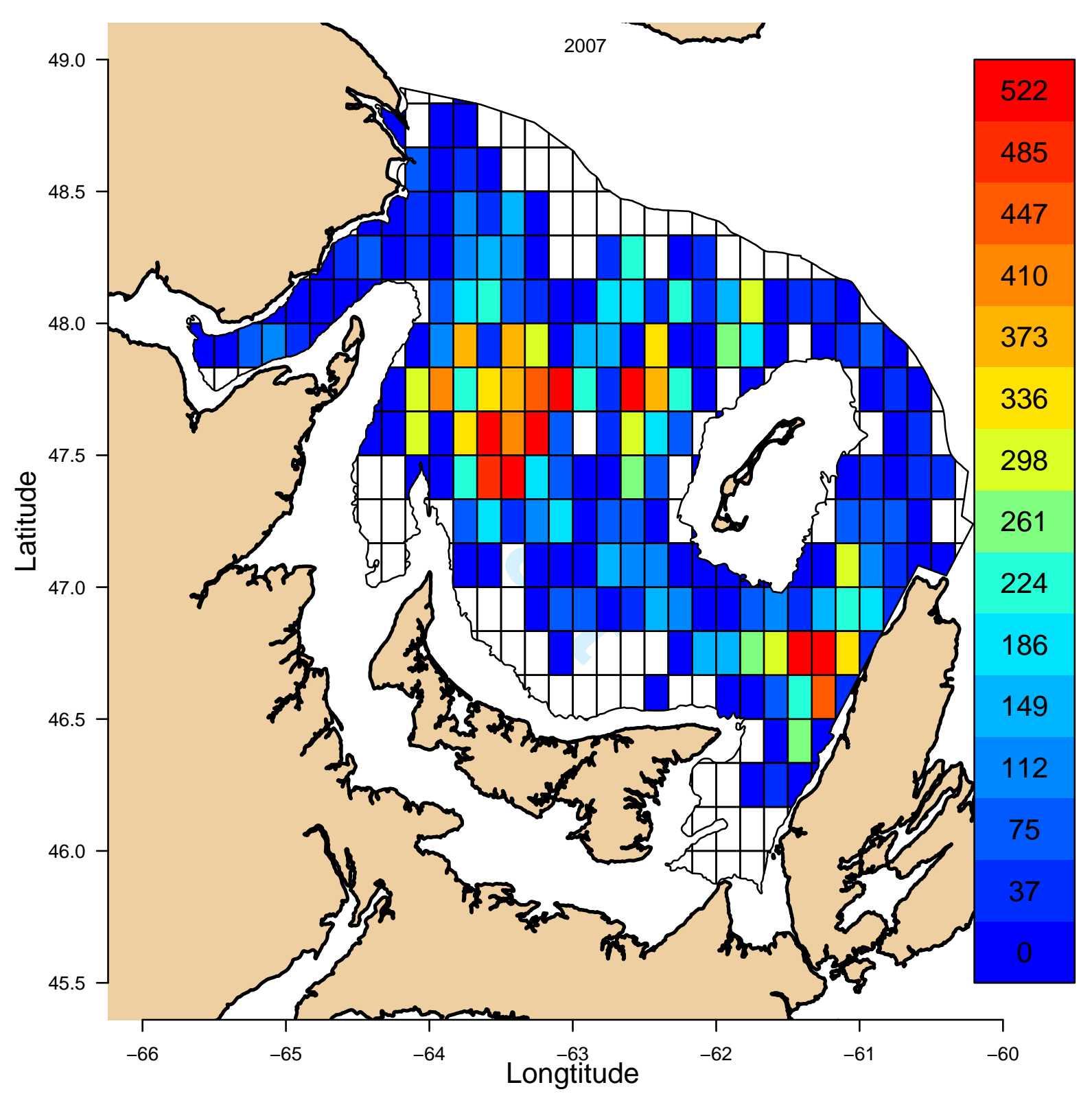

Figure SC.13a. Total annual catch (tonnes) of snow crab in each grid cell in 2007. Colors correspond to catch levels, as indicated in the legend on the right-hand side. Darkest red grids indicate catch $>98$ th percentile. 
Total $=24264$ tonnes, 2007
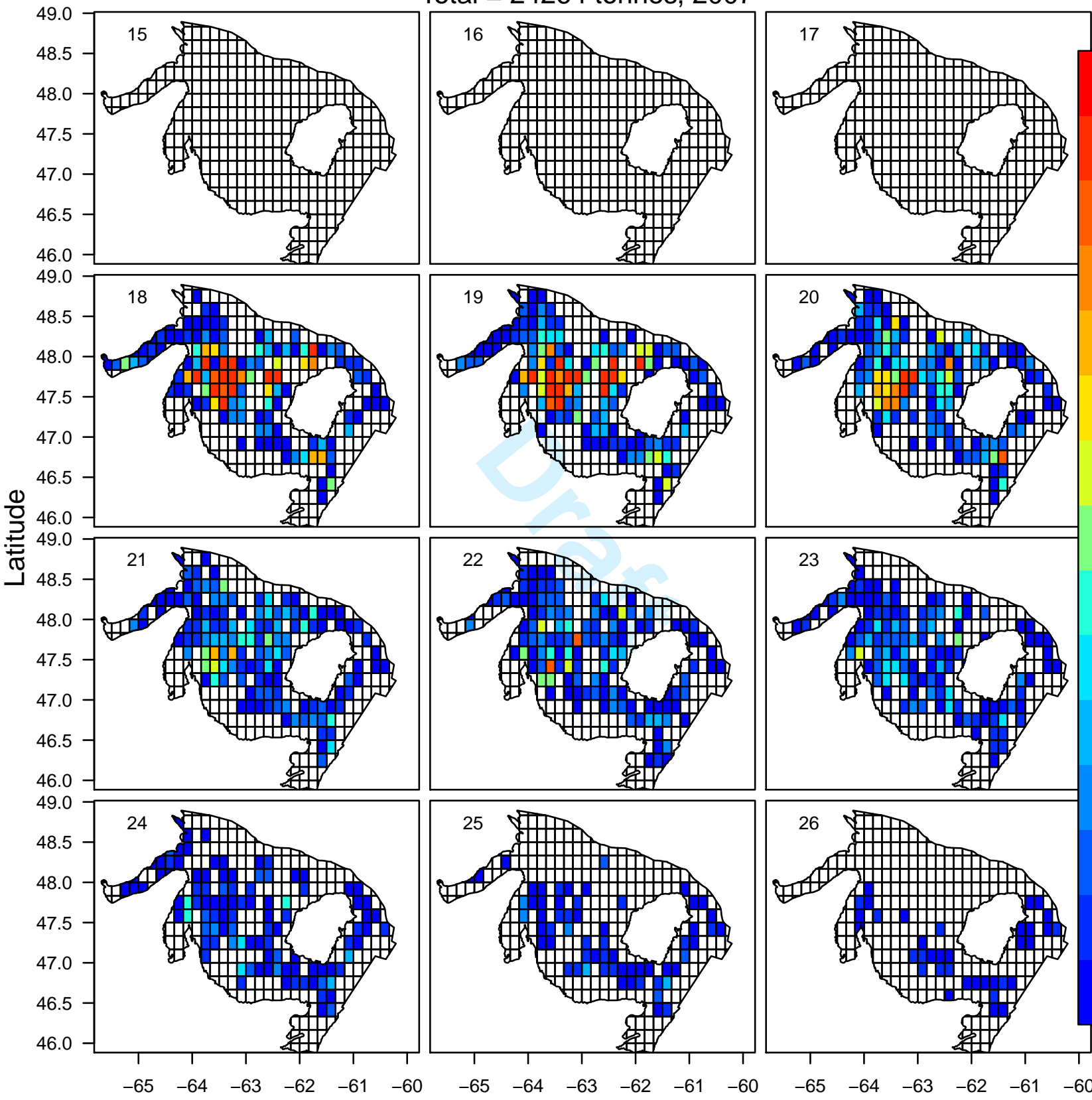

86
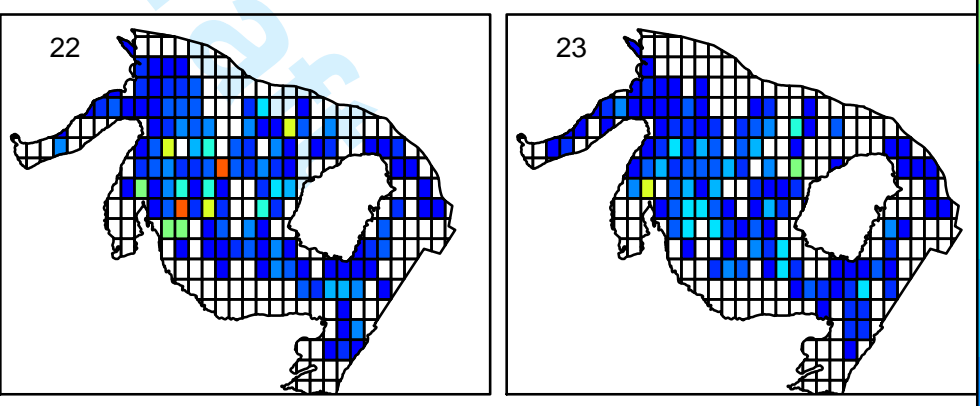

55
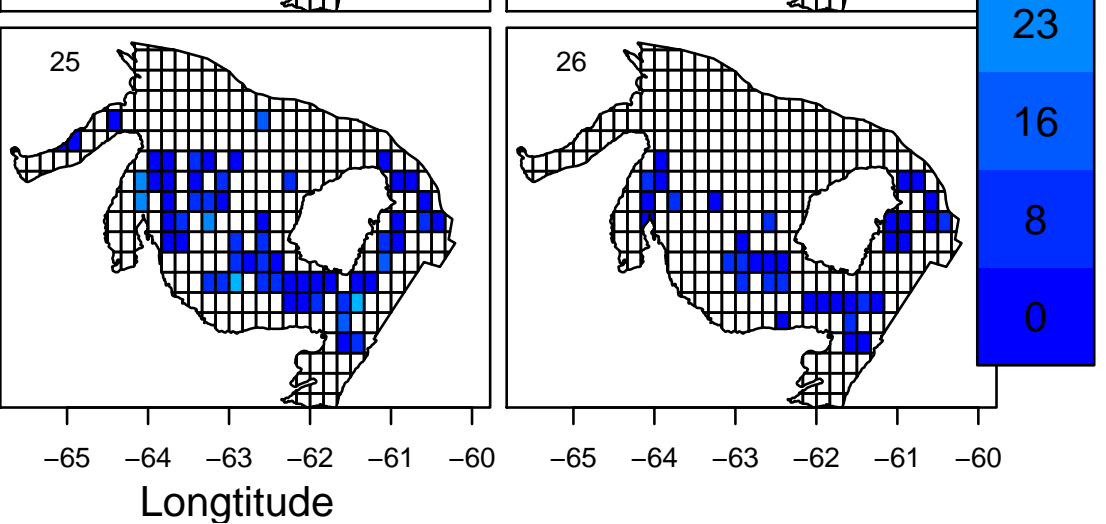

Figure SC.13b. Catch (tonnes) of snow crab in each week and grid cell in 2007. The week number is indicated in the top left-hand corner. Colors correspond to catch levels, as indicated in the legend on the right-hand side. Darkest red grids indicate catch $>98$ th percentile. 
Total $=24264$ tonnes, 2007
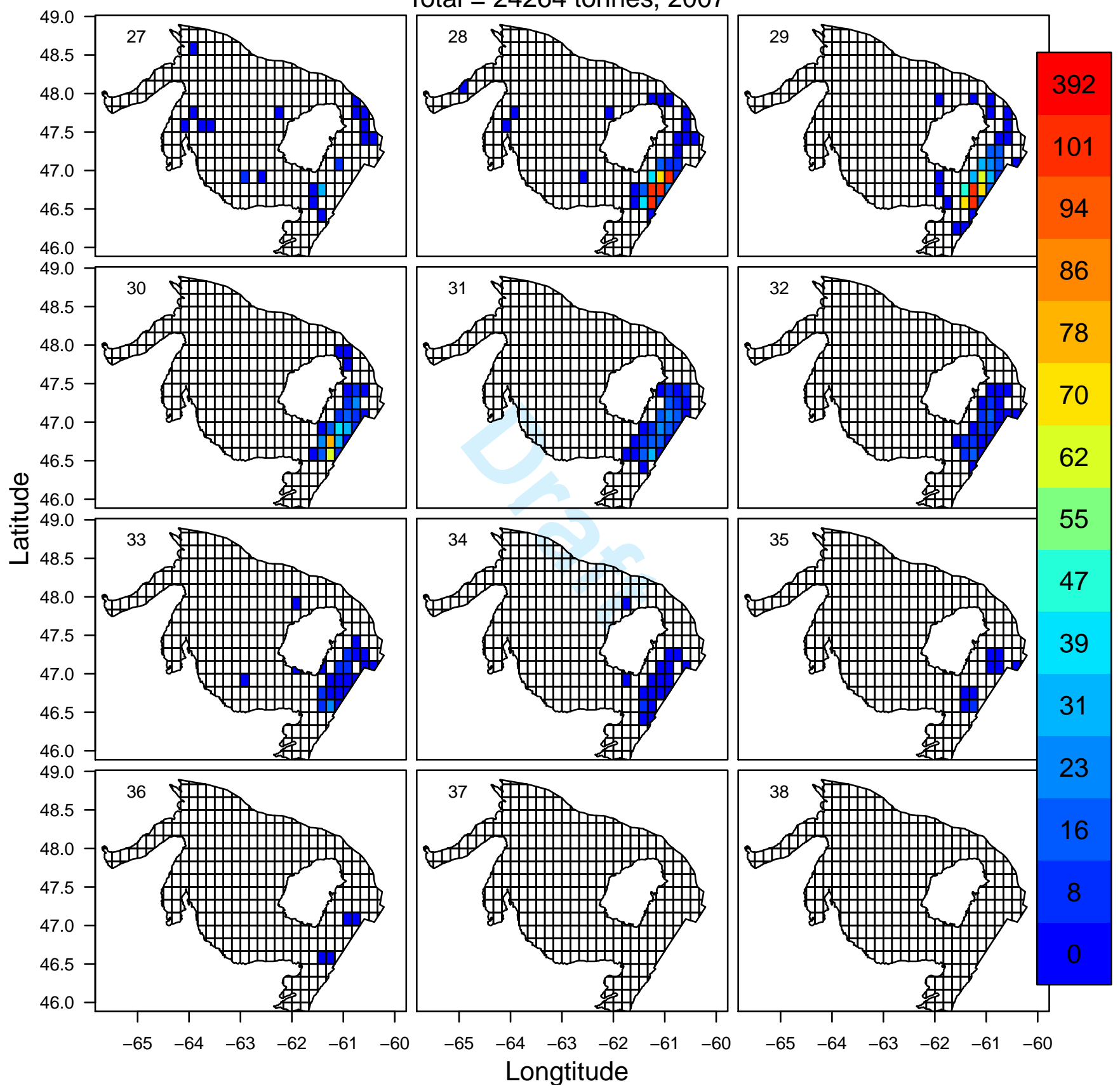

Figure SC.13c. Catch (tonnes) of snow crab in each week and grid cell in 2007. The week number is indicated in the top left-hand corner. Colors correspond to catch levels, as indicated in the legend on the right-hand side. Darkest red grids indicate catch $>98$ th percentile. 


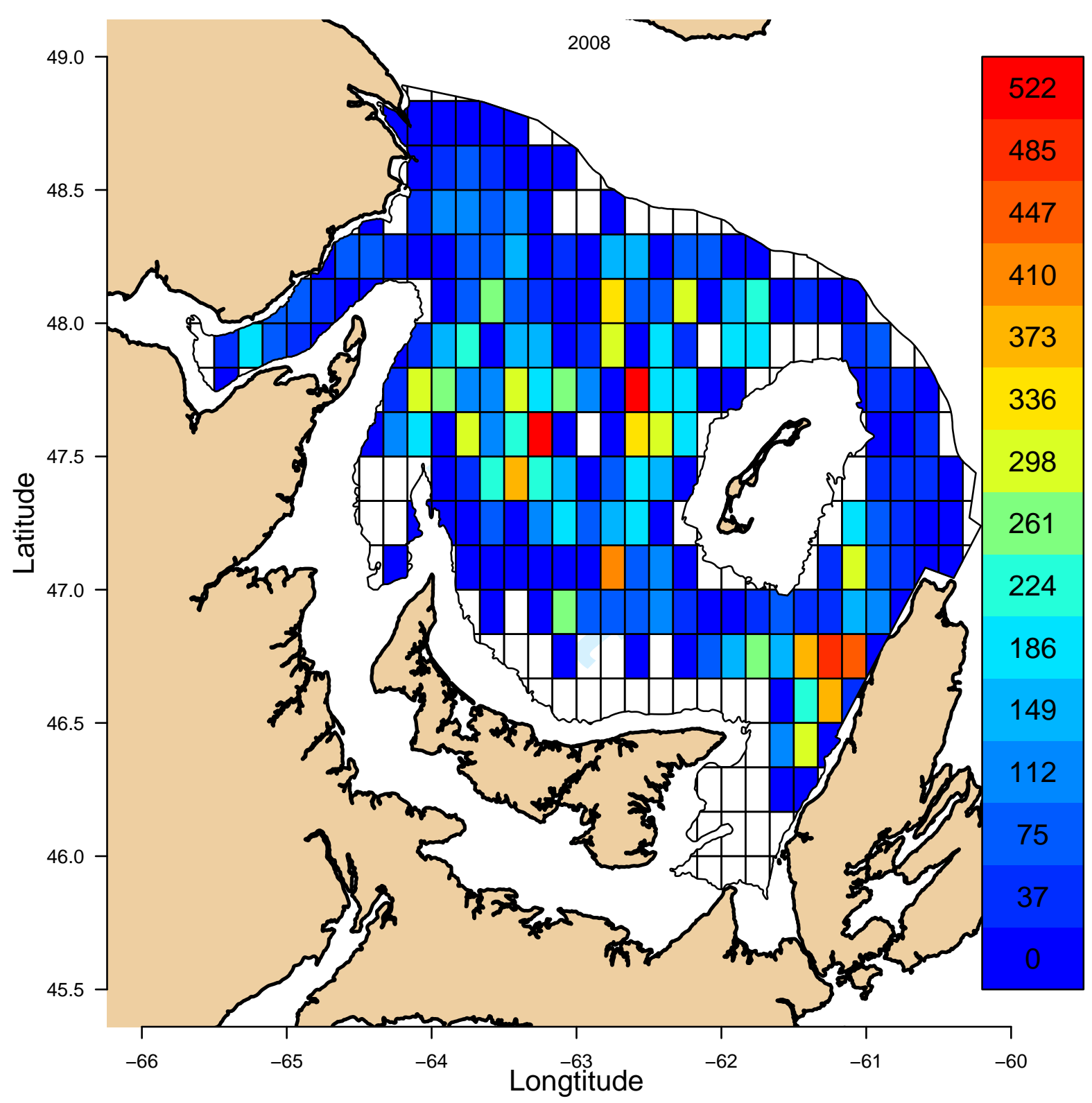

Figure SC.14a. Total annual catch (tonnes) of snow crab in each grid cell in 2008. Colors correspond to catch levels, as indicated in the legend on the right-hand side. Darkest red grids indicate catch $>98$ th percentile. 


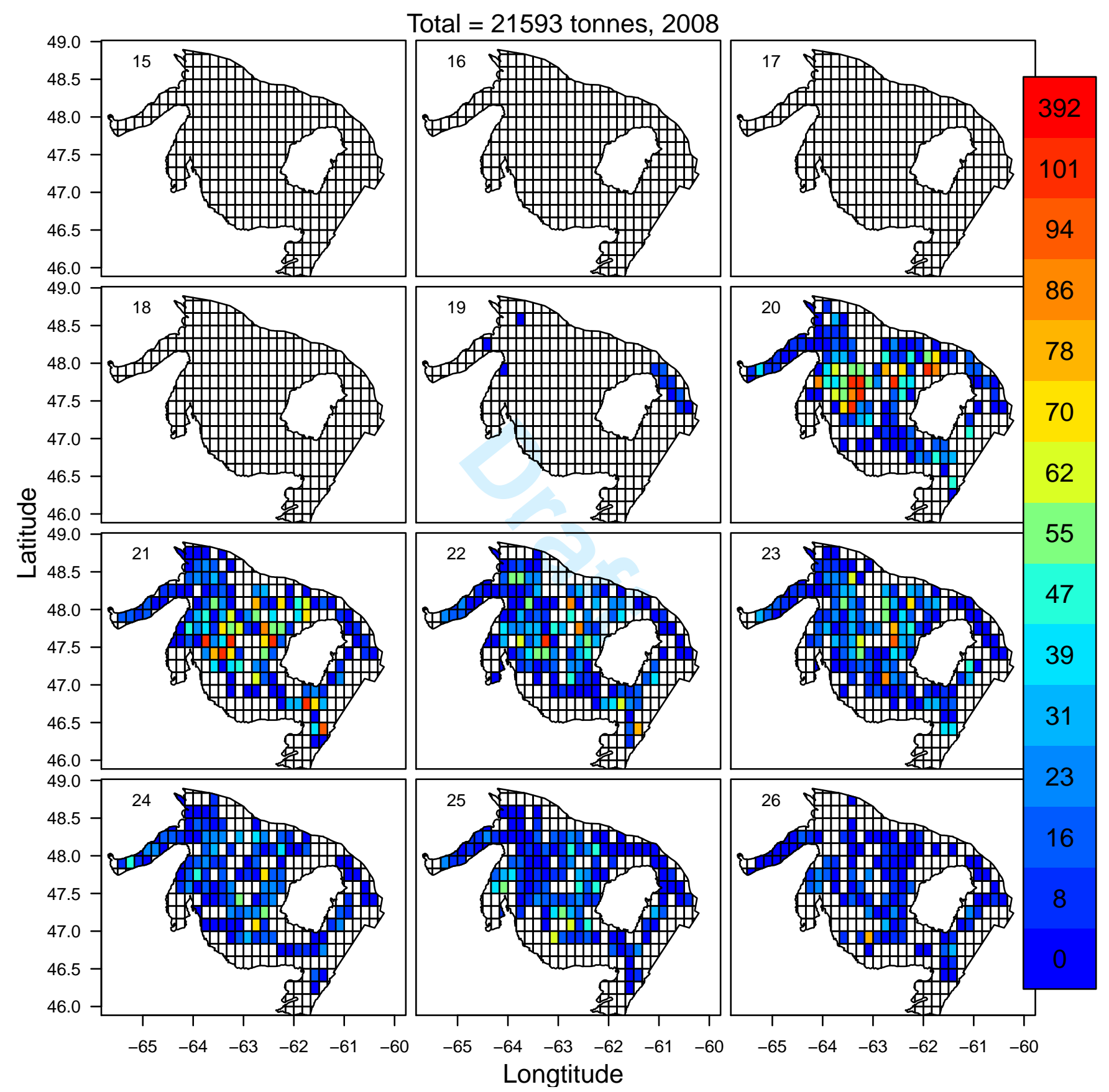

Figure SC.14b. Catch (tonnes) of snow crab in each week and grid cell in 2008. The week number is indicated in the top left-hand corner. Colors correspond to catch levels, as indicated in the legend on the right-hand side. Darkest red grids indicate catch $>98$ th percentile. 
Total $=21593$ tonnes, 2008
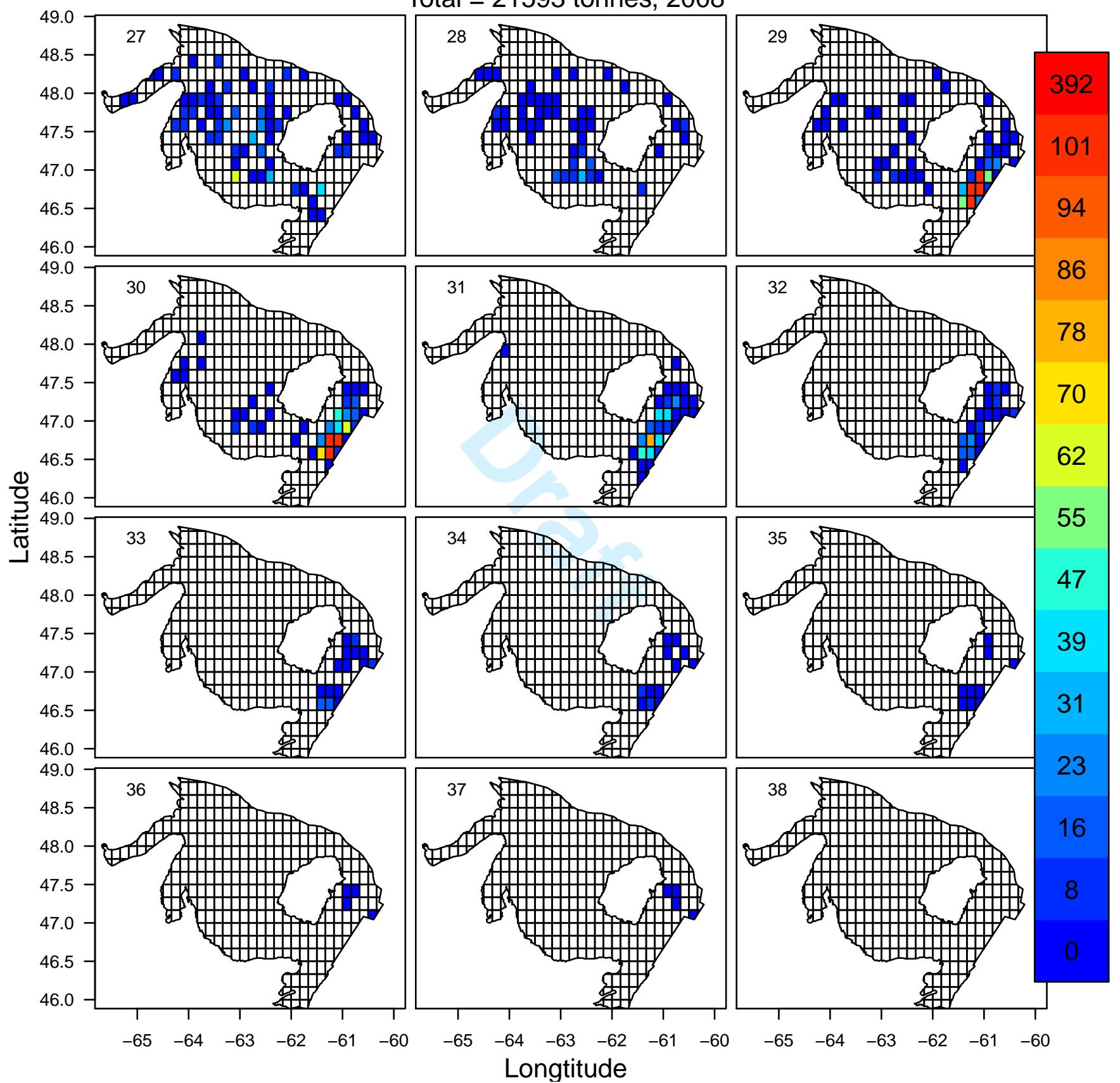

Figure SC.14c. Catch (tonnes) of snow crab in each week and grid cell in 2008. The week number is indicated in the top left-hand corner. Colors correspond to catch levels, as indicated in the legend on the right-hand side. Darkest red grids indicate catch $>98$ th percentile. 


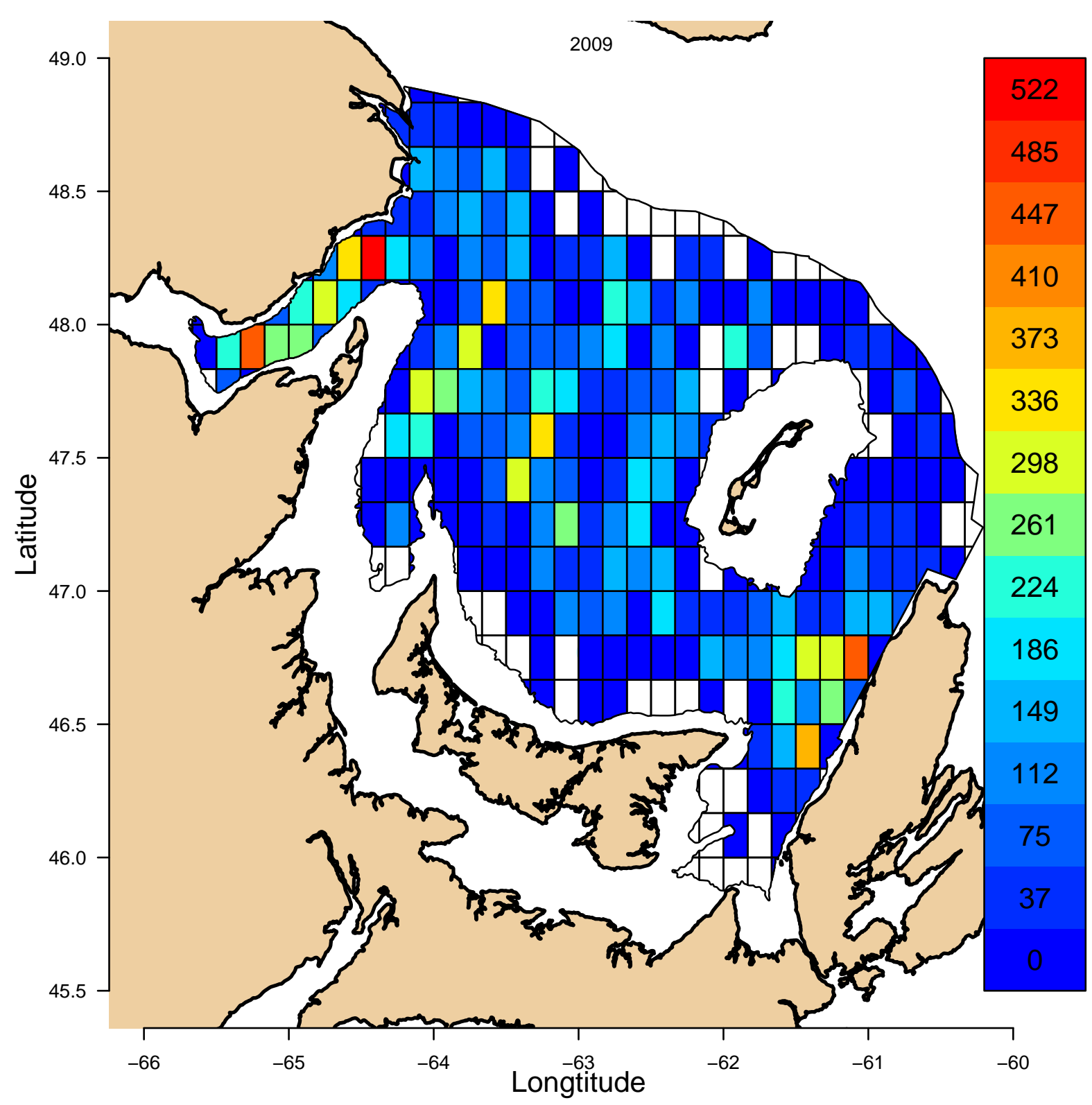

Figure SC.15a. Total annual catch (tonnes) of snow crab in each grid cell in 2009. Colors correspond to catch levels, as indicated in the legend on the right-hand side. Darkest red grids indicate catch $>98$ th percentile. 
Total $=21884$ tonnes, 2009
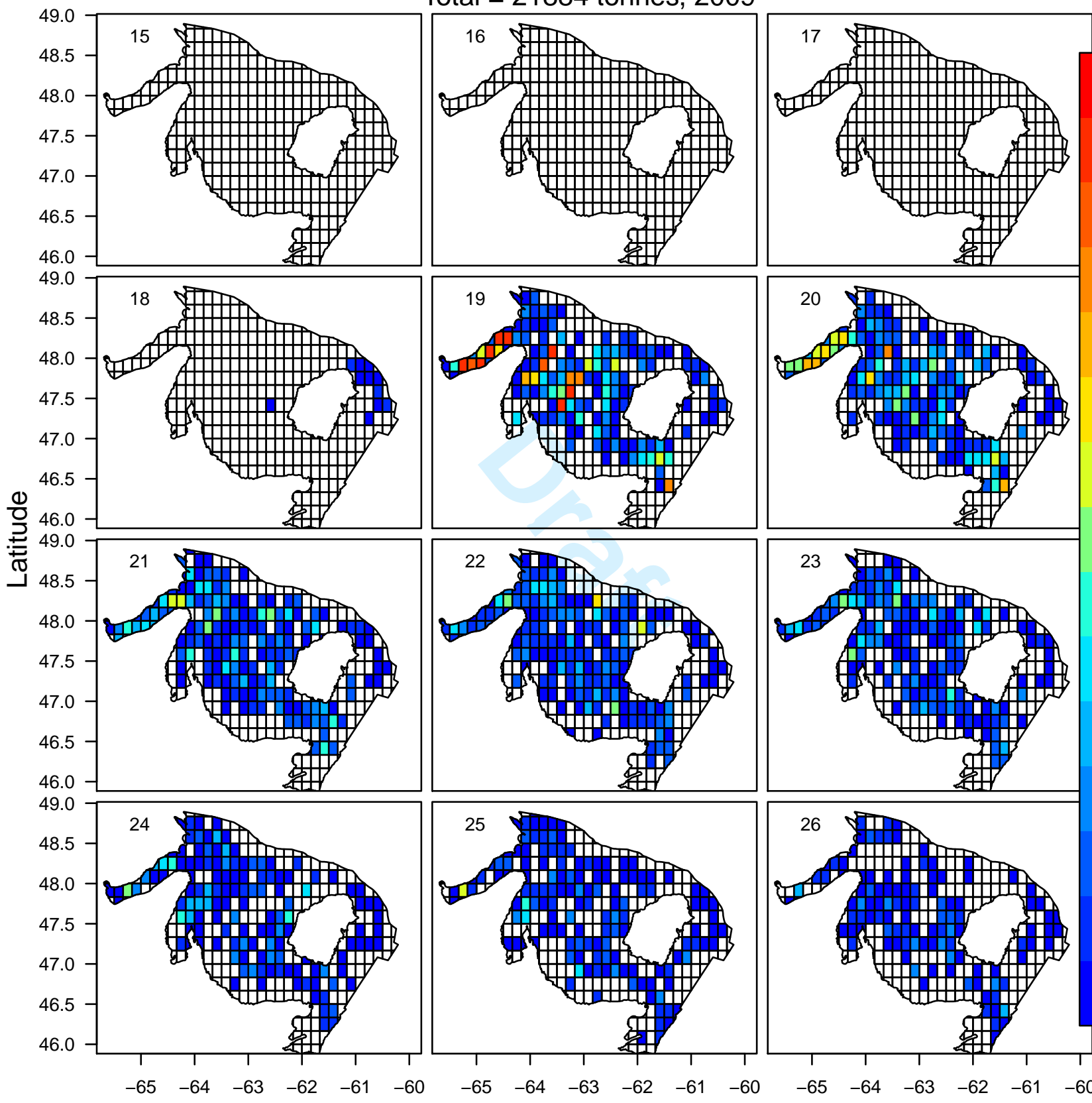

86
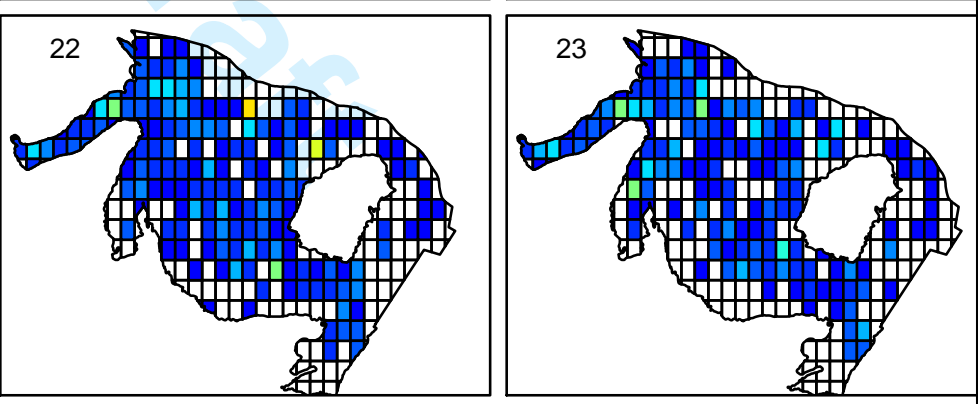

55
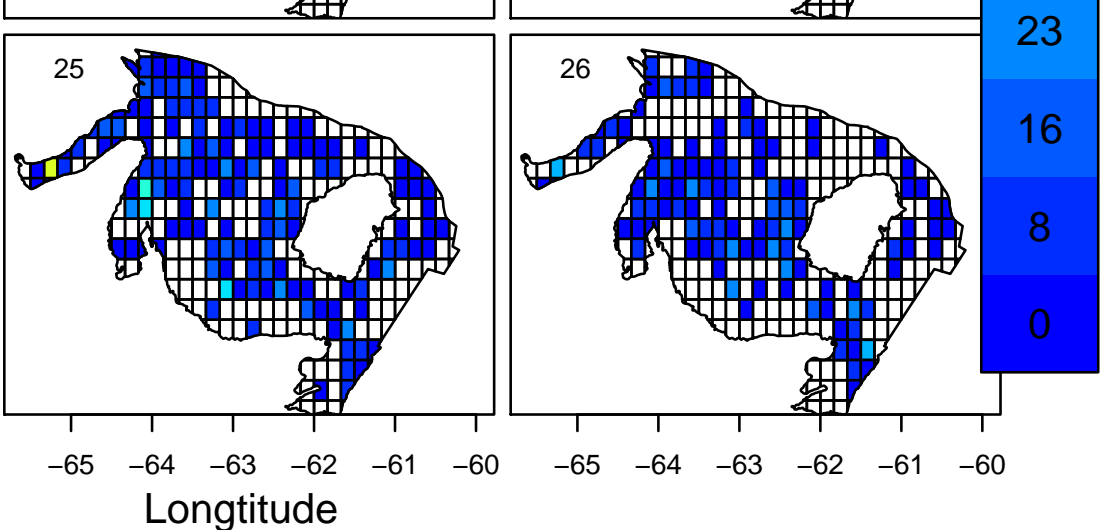

Figure SC.15b. Catch (tonnes) of snow crab in each week and grid cell in 2009. The week number is indicated in the top left-hand corner. Colors correspond to catch levels, as indicated in the legend on the righthand side. Darkest red grids indicate catch $>98$ th percentile. 


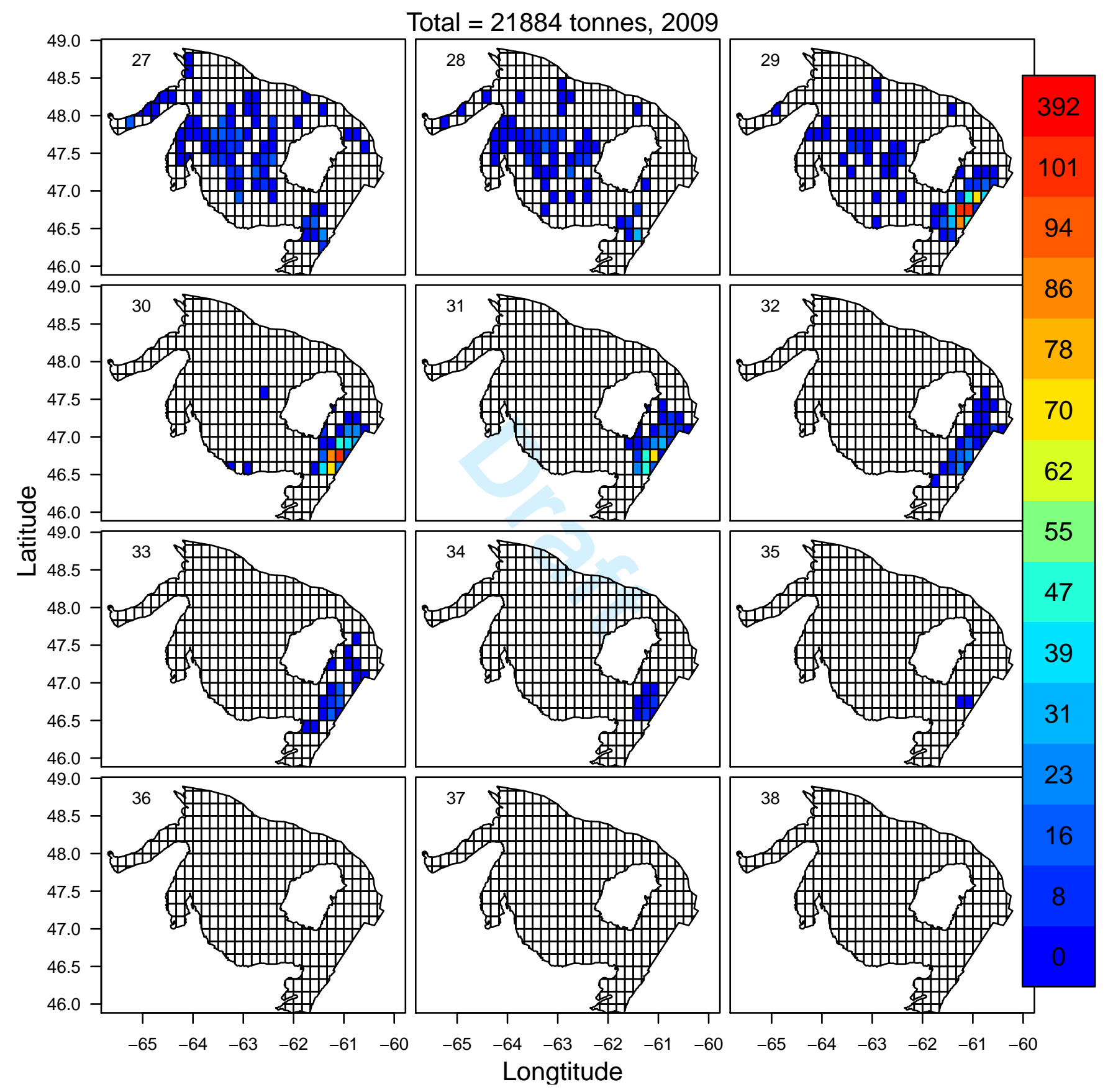

Figure SC.15c. Catch (tonnes) of snow crab in each week and grid cell in 2009. The week number is indicated in the top left-hand corner. Colors correspond to catch levels, as indicated in the legend on the right-hand side. Darkest red grids indicate catch $>98$ th percentile. 


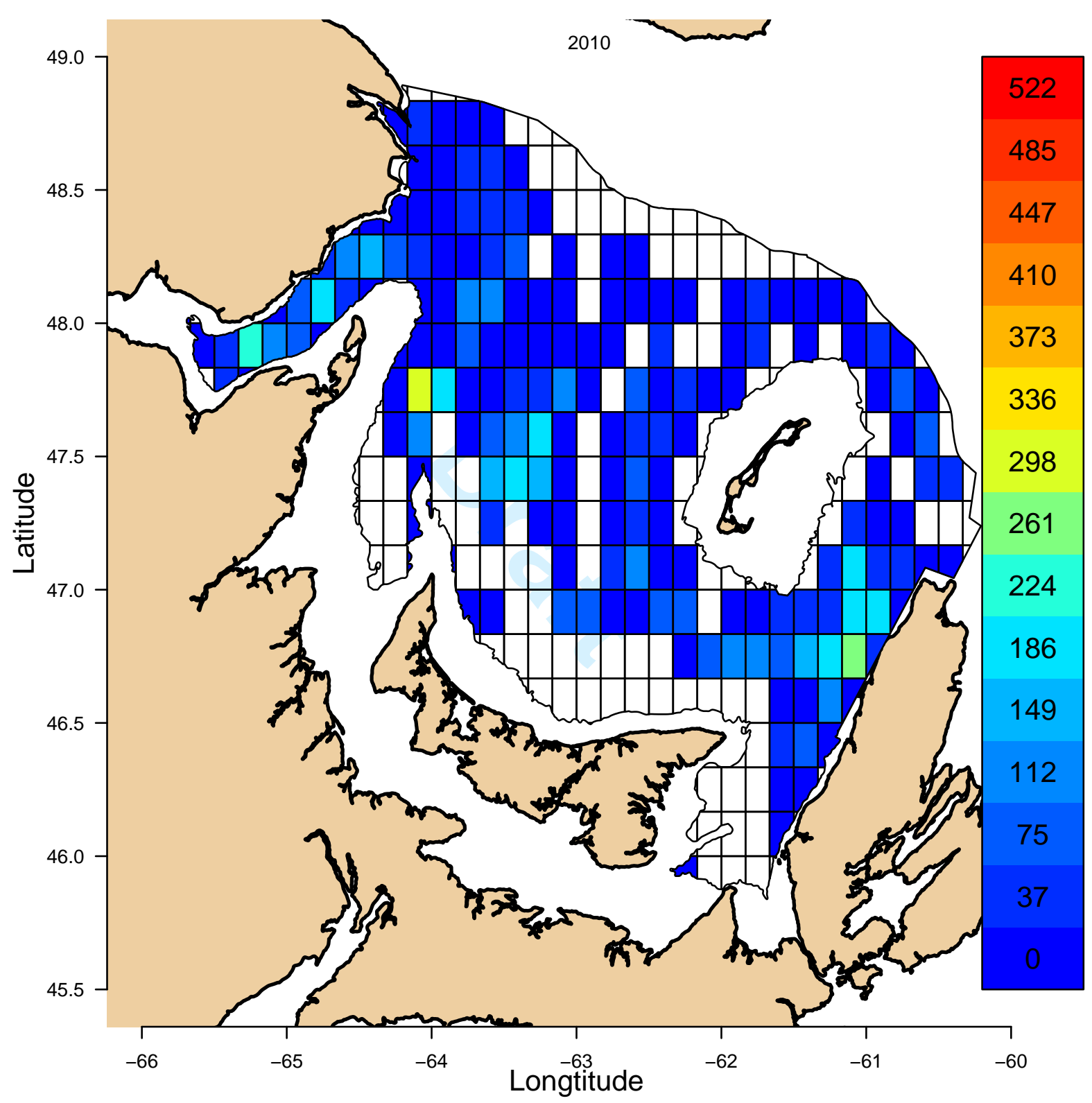

Figure SC.16a. Total annual catch (tonnes) of snow crab in each grid cell in 2010. Colors correspond to catch levels, as indicated in the legend on the right-hand side. Darkest red grids indicate catch $>98$ th percentile. 


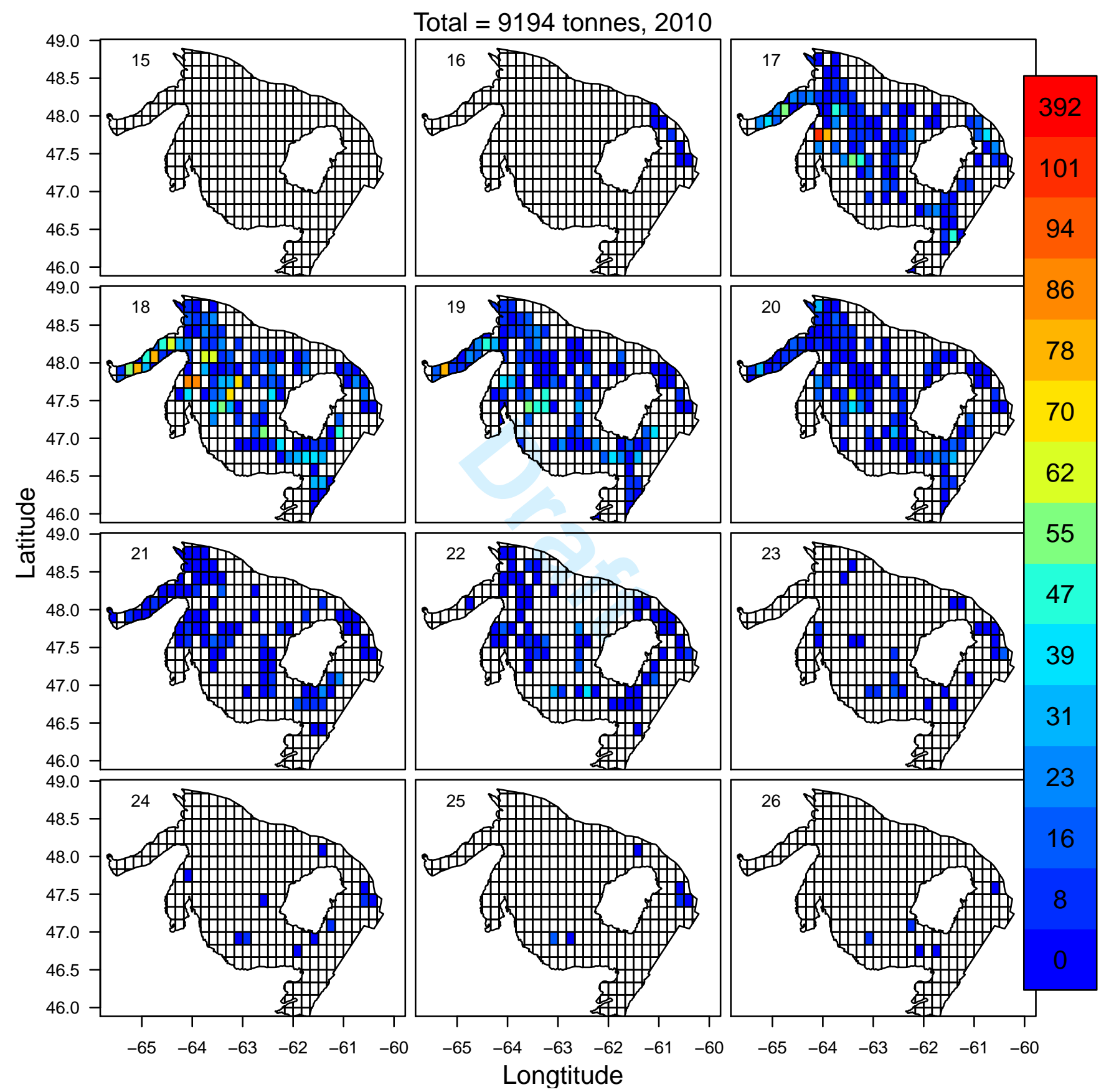

Figure SC.16b. Catch (tonnes) of snow crab in each week and grid cell in 2010. The week number is indicated in the top left-hand corner. Colors correspond to catch levels, as indicated in the legend on the right-hand side. Darkest red grids indicate catch $>98$ th percentile. 
Total $=9194$ tonnes, 2010
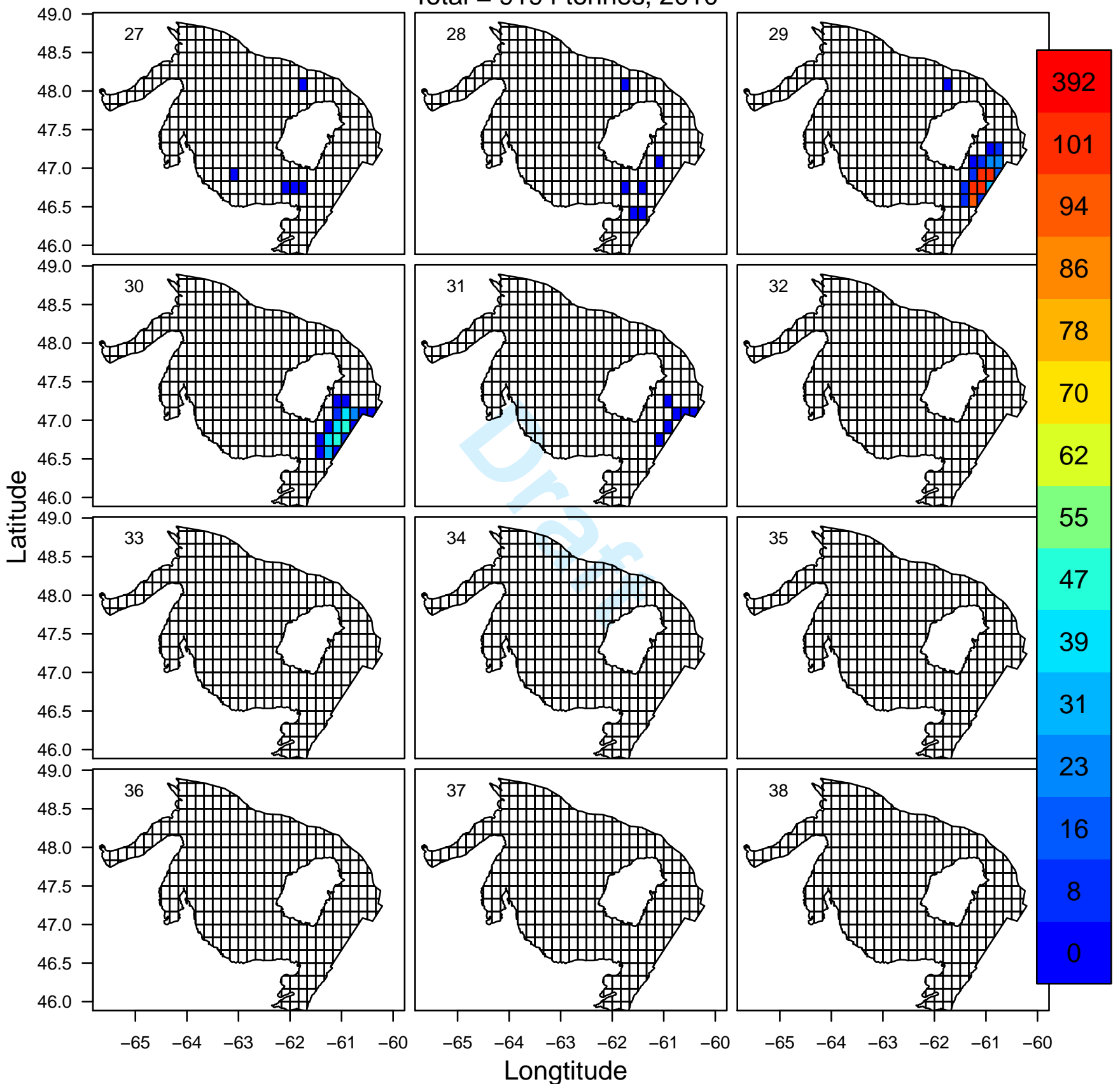

Figure SC.16c. Catch (tonnes) of snow crab in each week and grid cell in 2010. The week number is indicated in the top left-hand corner. Colors correspond to catch levels, as indicated in the legend on the righthand side. Darkest red grids indicate catch $>98$ th percentile. 


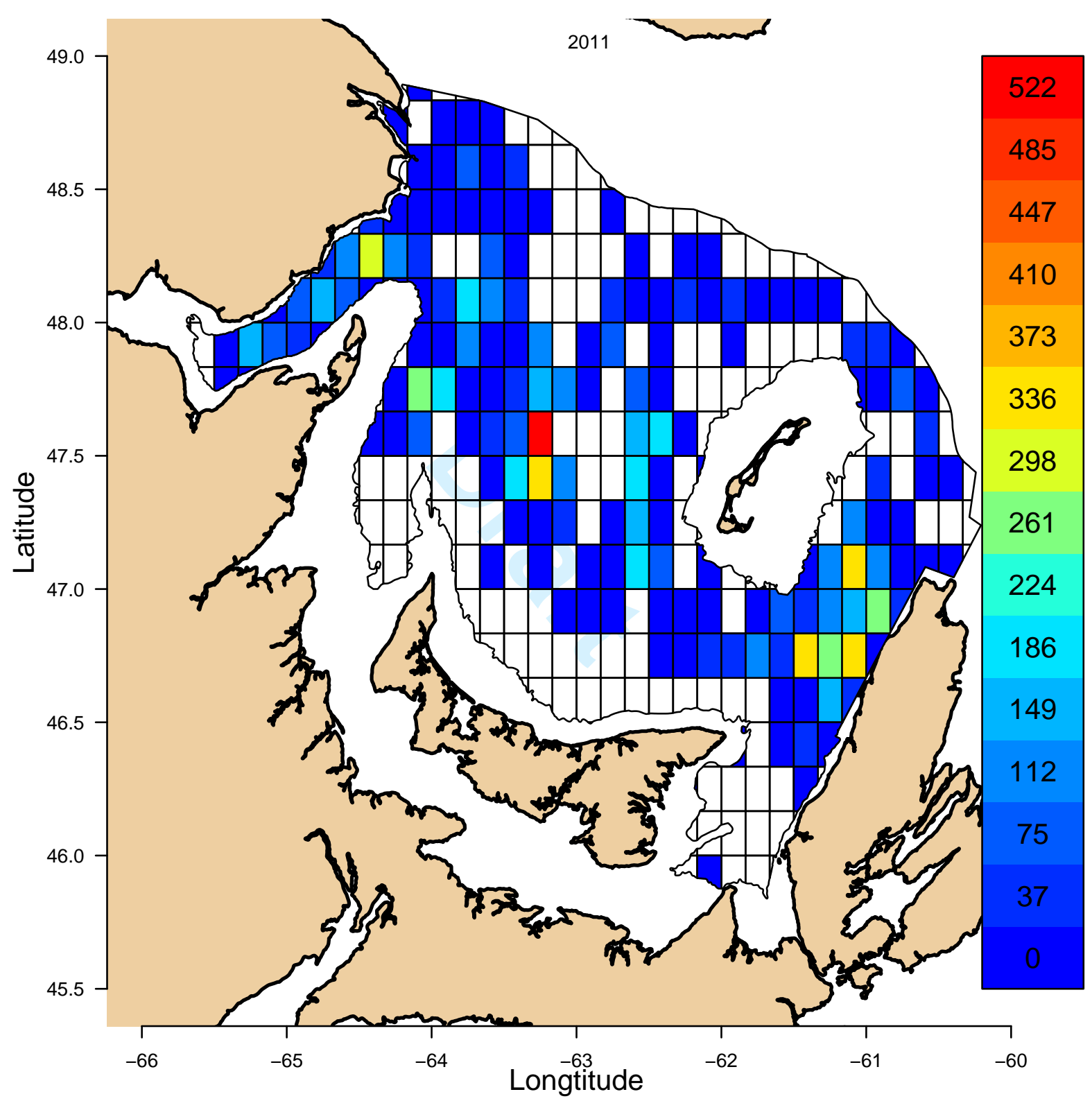

Figure SC.17a. Total annual catch (tonnes) of snow crab in each grid cell in 2011. Colors correspond to catch levels, as indicated in the legend on the right-hand side. Darkest red grids indicate catch $>98$ th percentile. 
Total $=10408$ tonnes, 2011
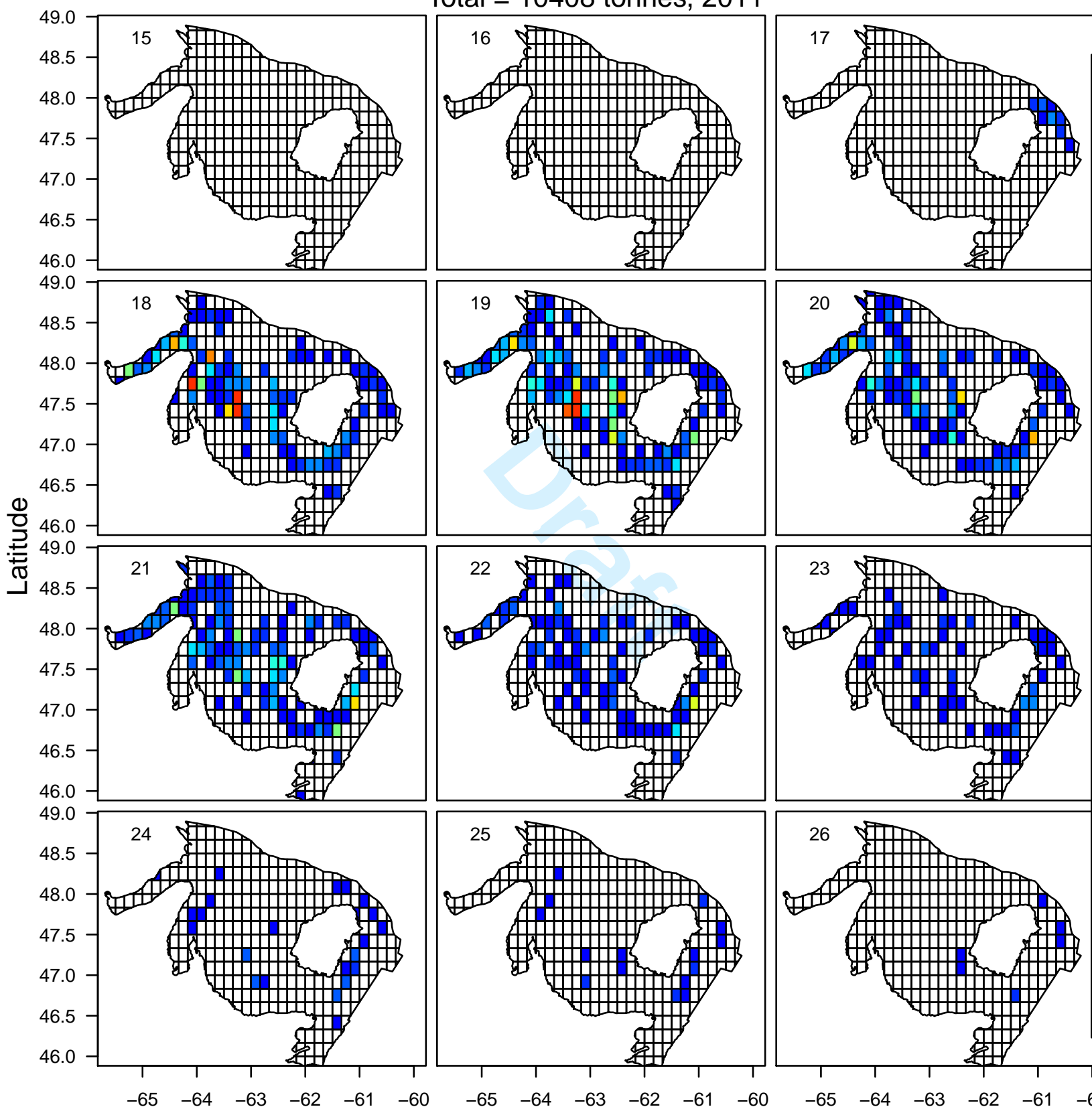

55
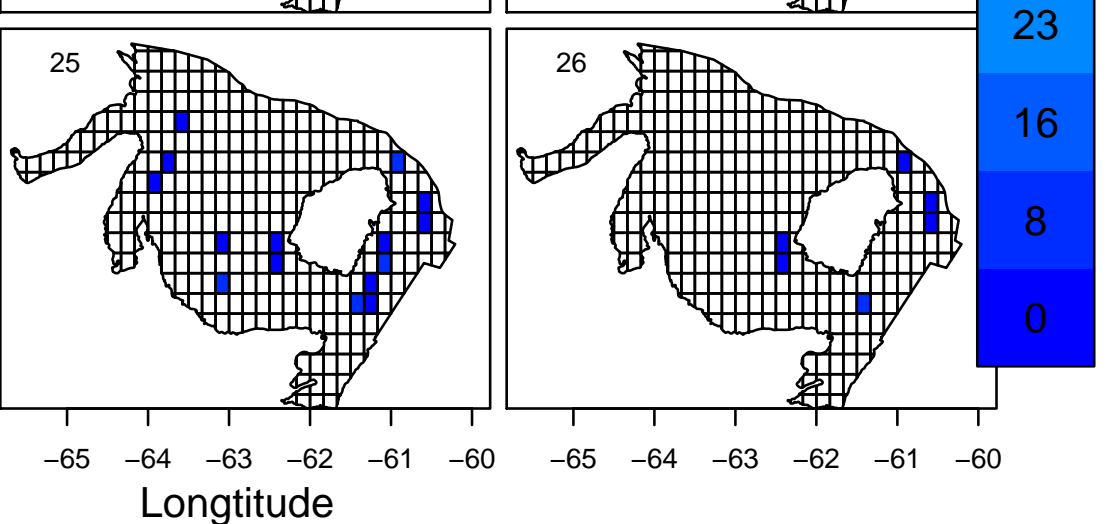

Figure SC.17b. Catch (tonnes) of snow crab in each week and grid cell in 2011. The week number is indicated in the top left-hand corner. Colors correspond to catch levels, as indicated in the legend on the right-hand side. Darkest red grids indicate catch $>98$ th percentile. 


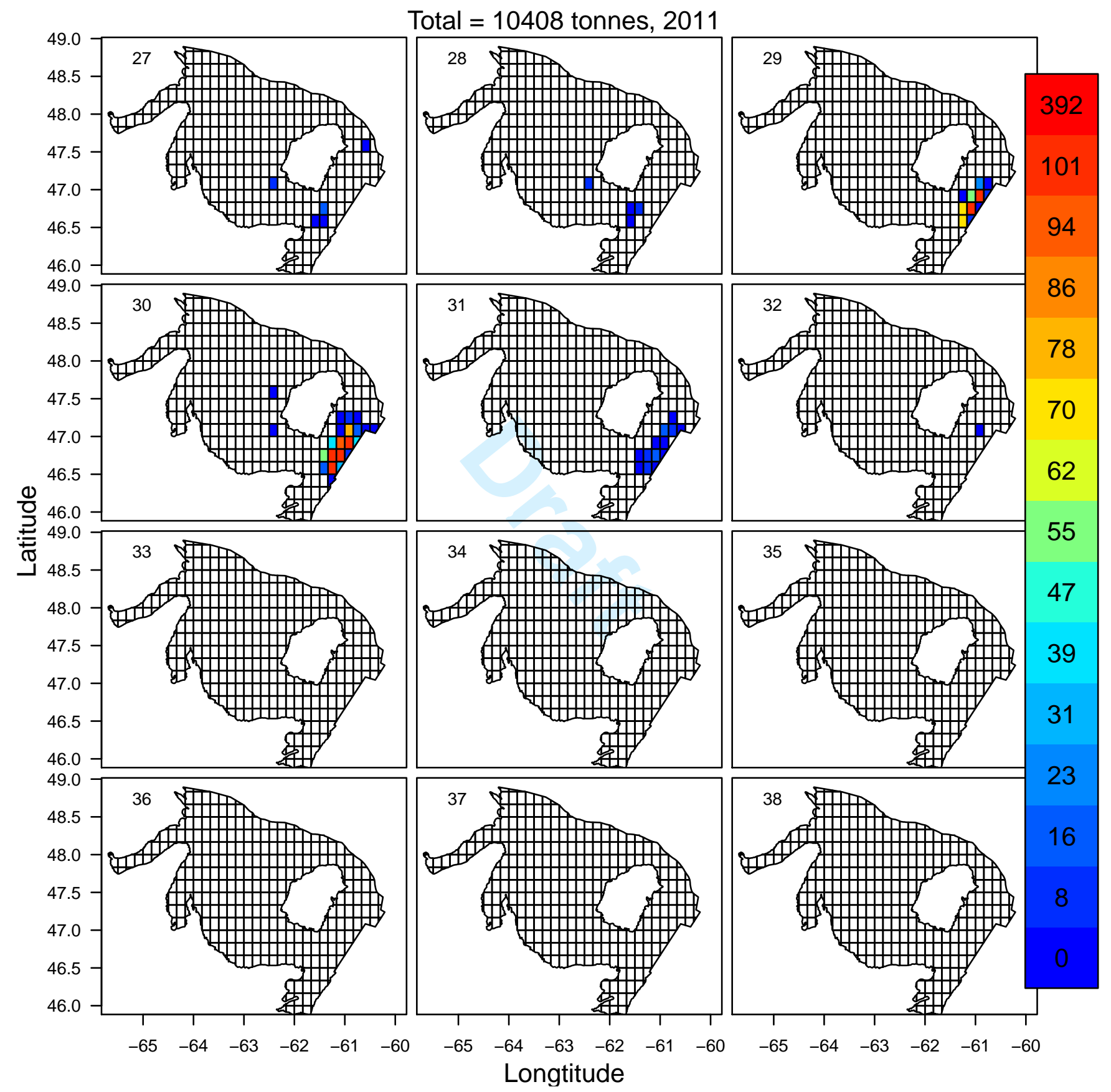

Figure SC.17c. Catch (tonnes) of snow crab in each week and grid cell in 2011. The week number is indicated in the top left-hand corner. Colors correspond to catch levels, as indicated in the legend on the right-hand side. Darkest red grids indicate catch $>98$ th percentile. 


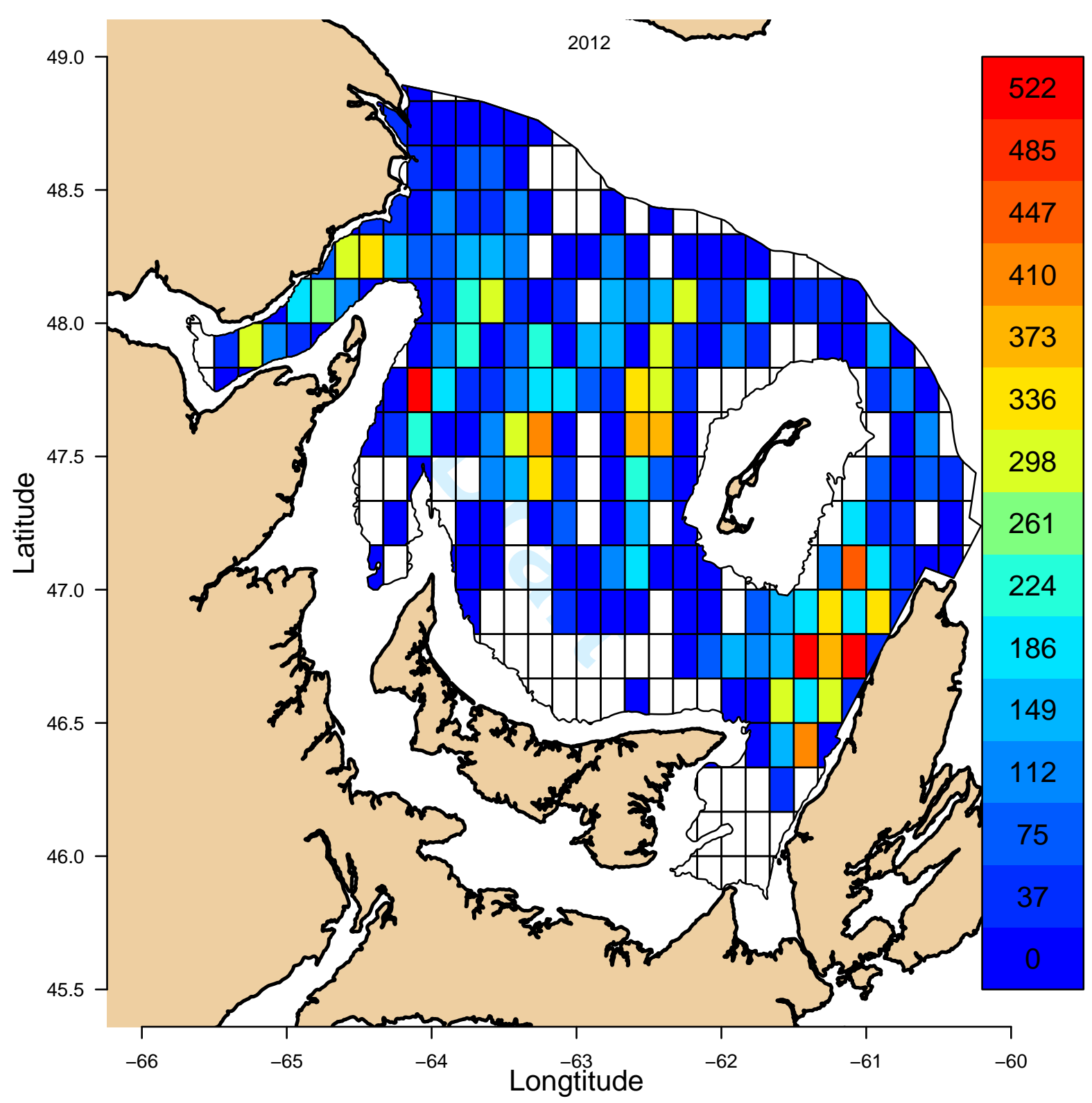

Figure SC.18a. Total annual catch (tonnes) of snow crab in each grid cell in 2012. Colors correspond to catch levels, as indicated in the legend on the right-hand side. Darkest red grids indicate catch $>98$ th percentile. 


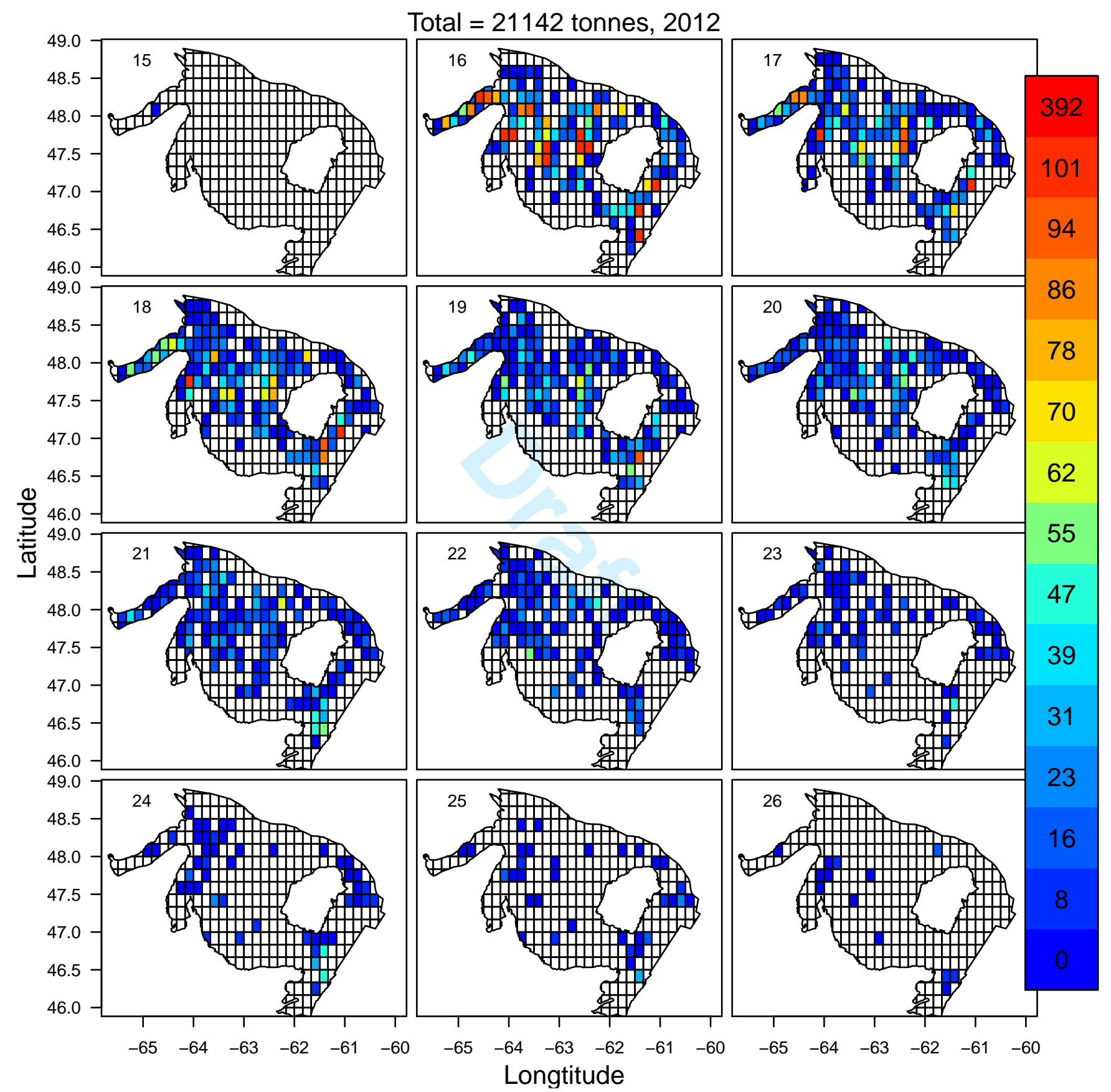

Figure SC.18b. Catch (tonnes) of snow crab in each week and grid cell in 2012. The week number is indicated in the top left-hand corner. Colors correspond to catch levels, as indicated in the legend on the right-hand side. Darkest red grids indicate catch $>98$ th percentile. 
Total $=21142$ tonnes, 2012
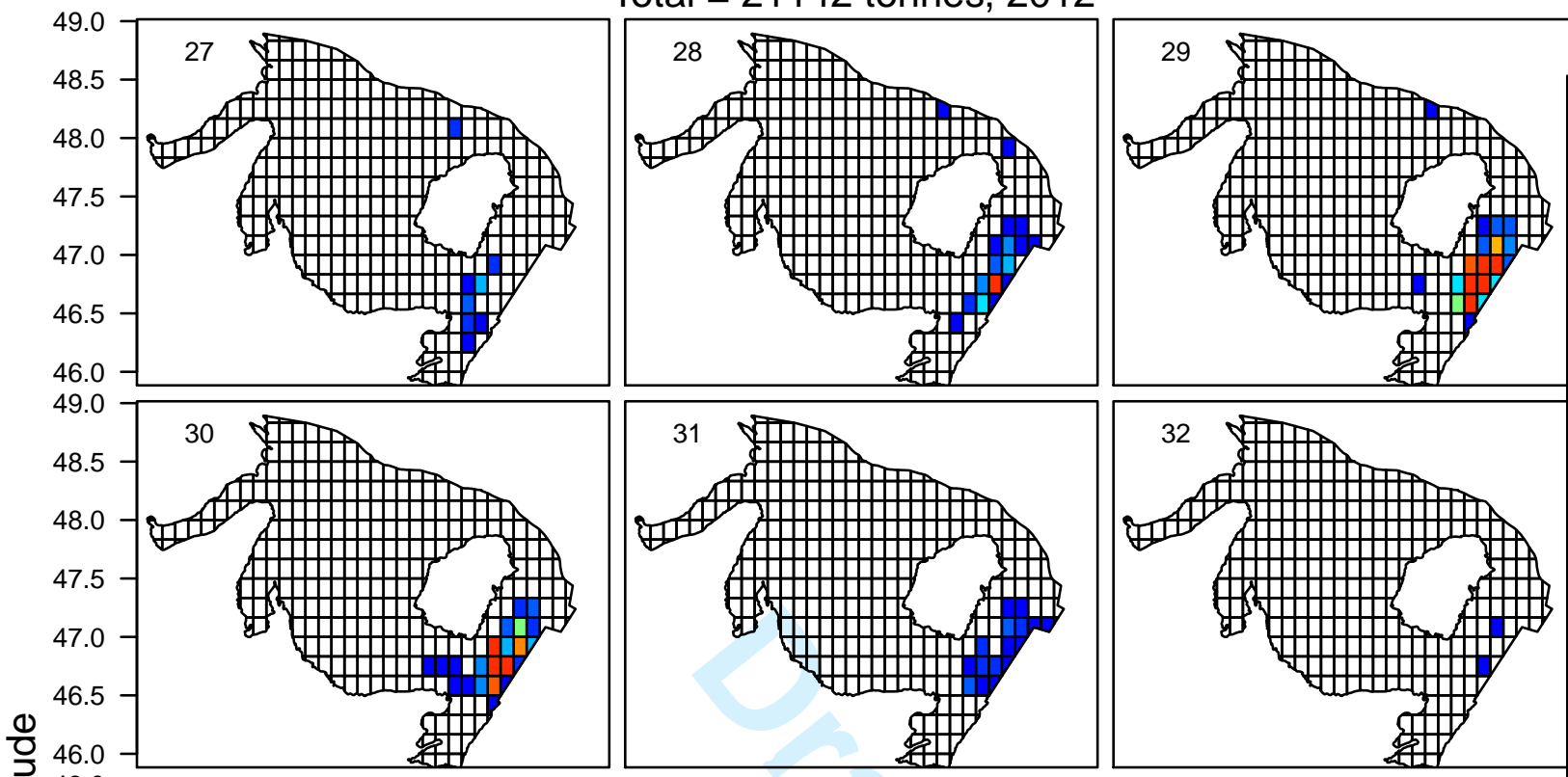

86

䒕
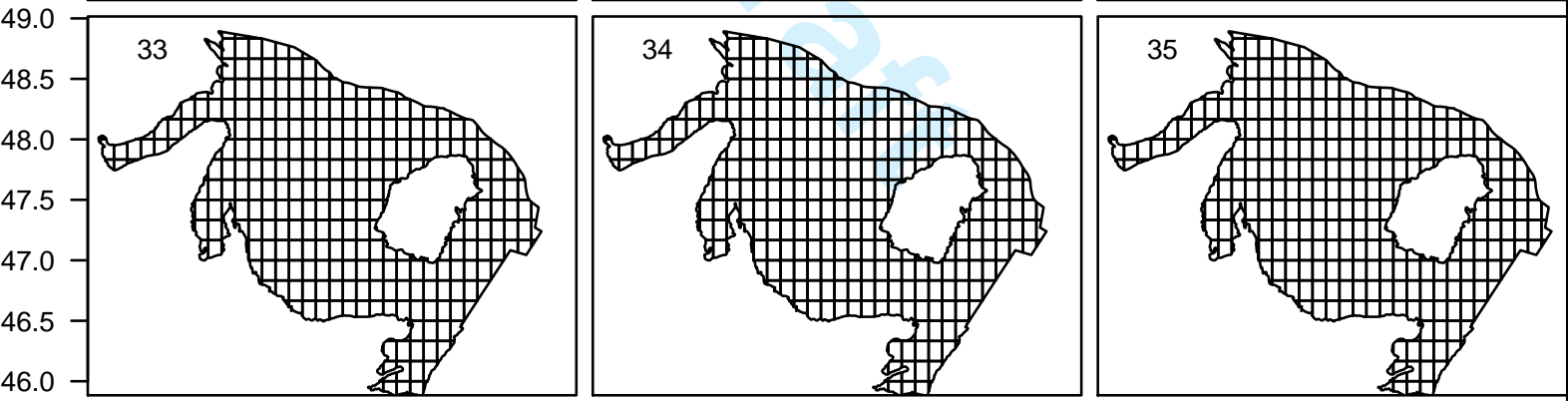

55
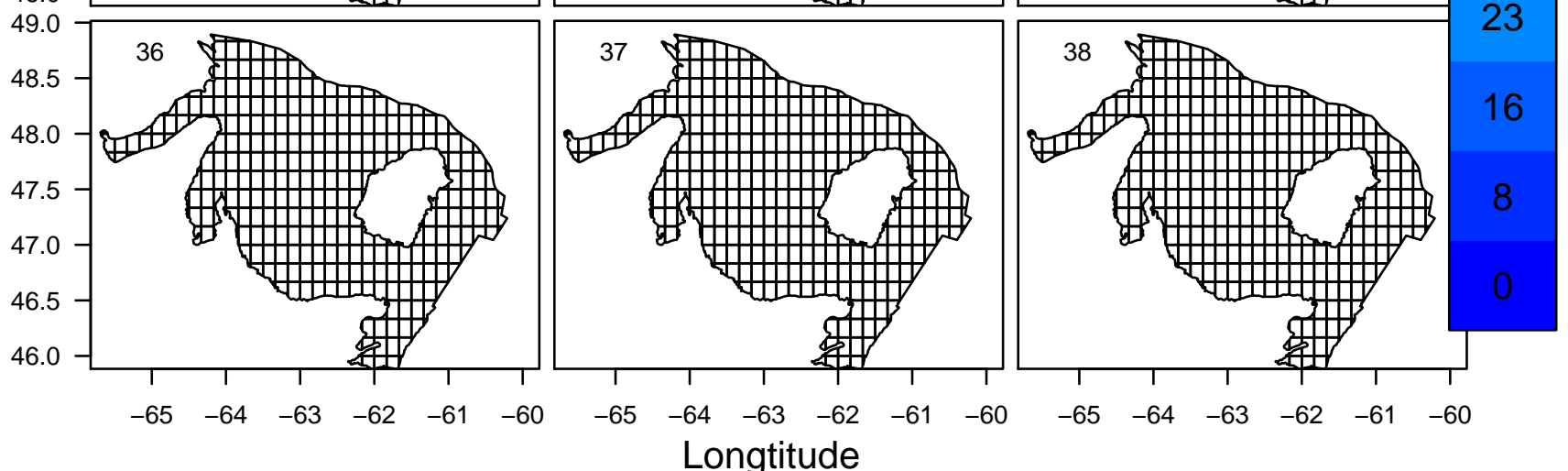

Figure SC.18c. Catch (tonnes) of snow crab in each week and grid cell in 2012. The week number is indicated in the top left-hand corner. Colors correspond to catch levels, as indicated in the legend on the right-hand side. Darkest red grids indicate catch $>98$ th percentile. 


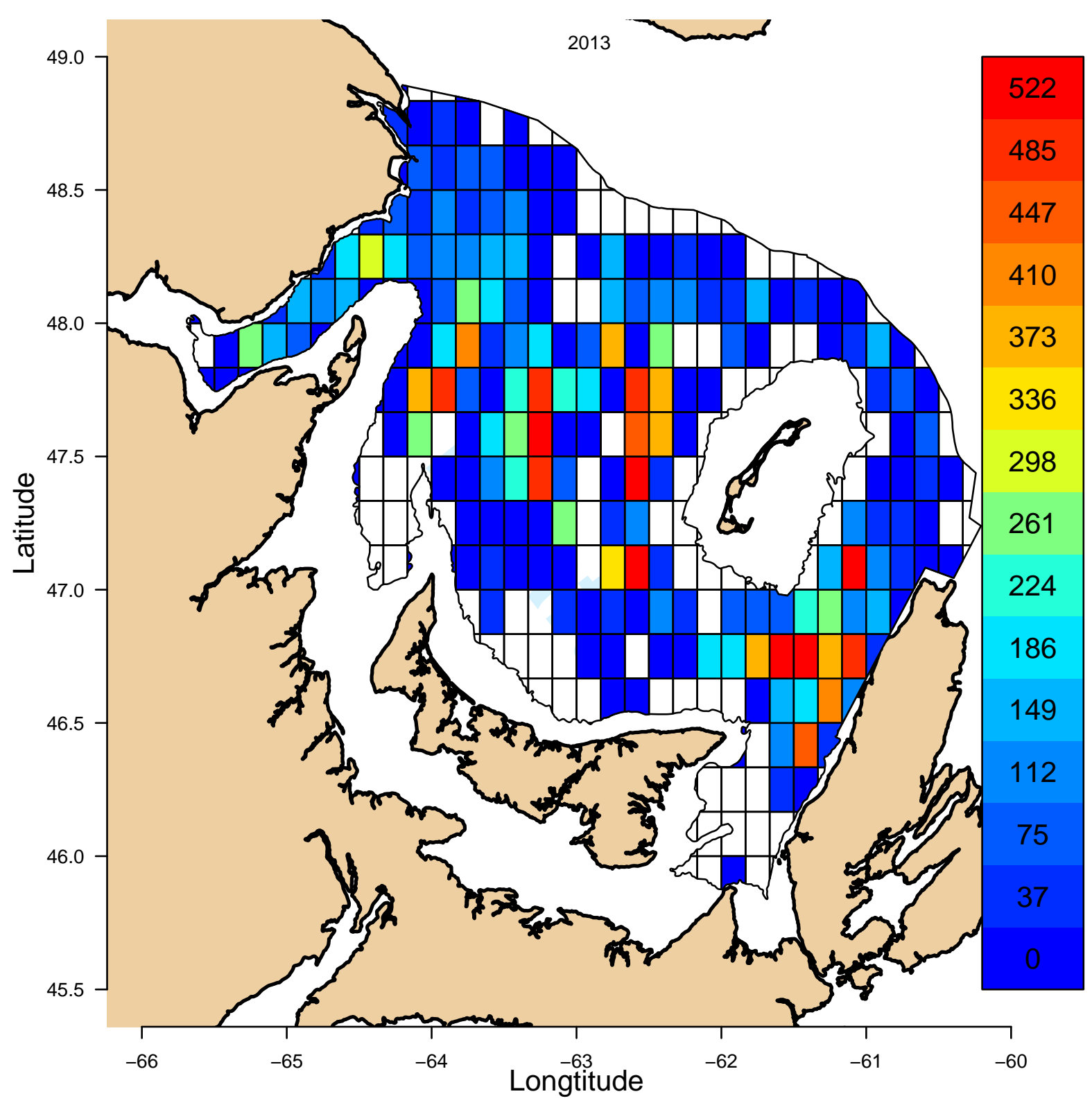

Figure SC.19a. Total annual catch (tonnes) of snow crab in each grid cell in 2013. Colors correspond to catch levels, as indicated in the legend on the right-hand side. Darkest red grids indicate catch $>98$ th percentile. 
Total $=24799$ tonnes, 2013
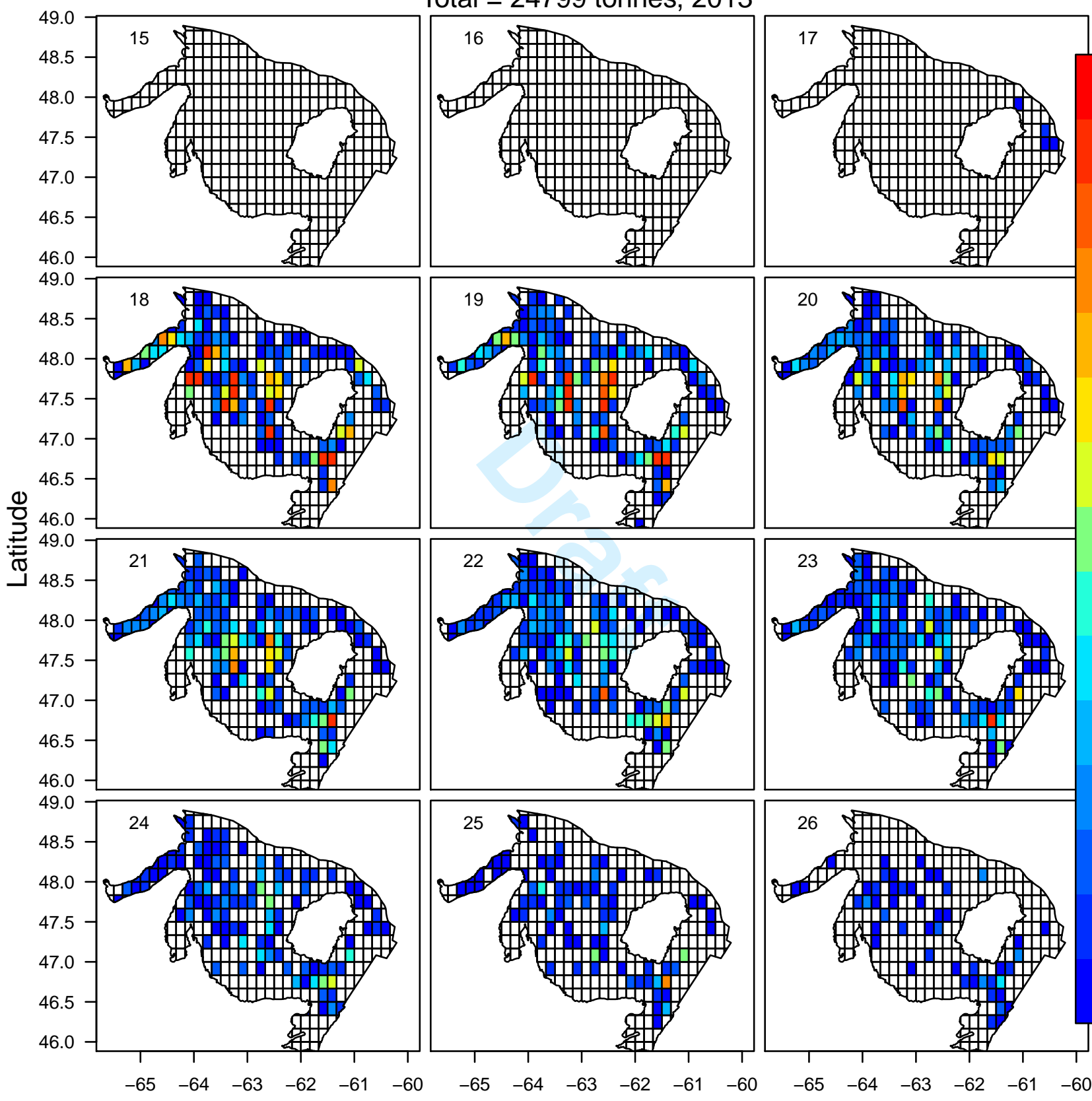

86
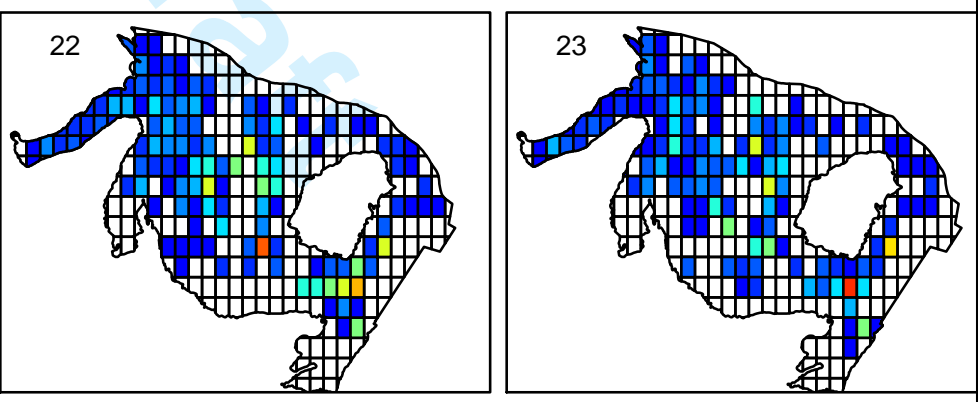

55
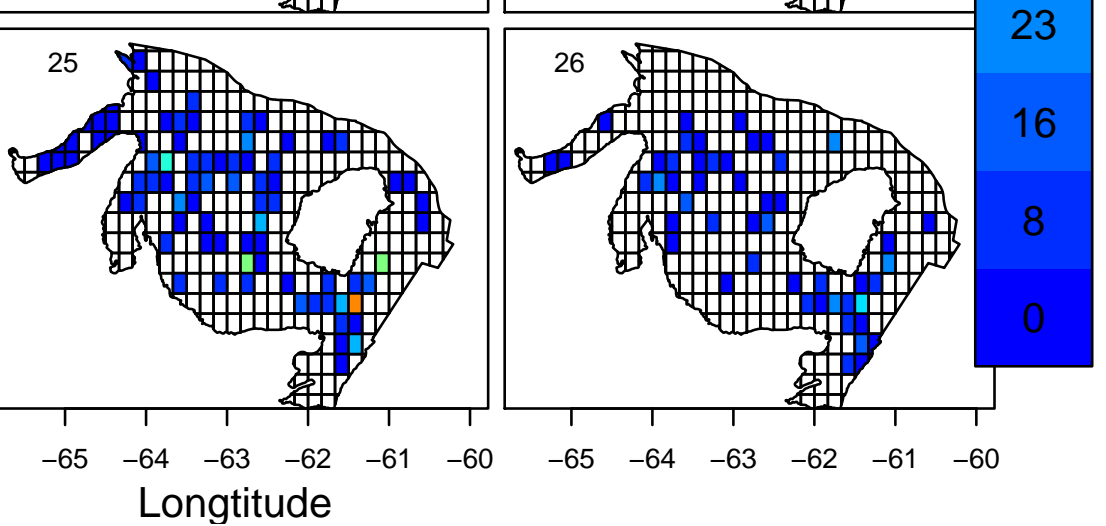

Figure SC.19b. Catch (tonnes) of snow crab in each week and grid cell in 2013. The week number is indicated in the top left-hand corner. Colors correspond to catch levels, as indicated in the legend on the right-hand side. Darkest red grids indicate catch $>98$ th percentile. 


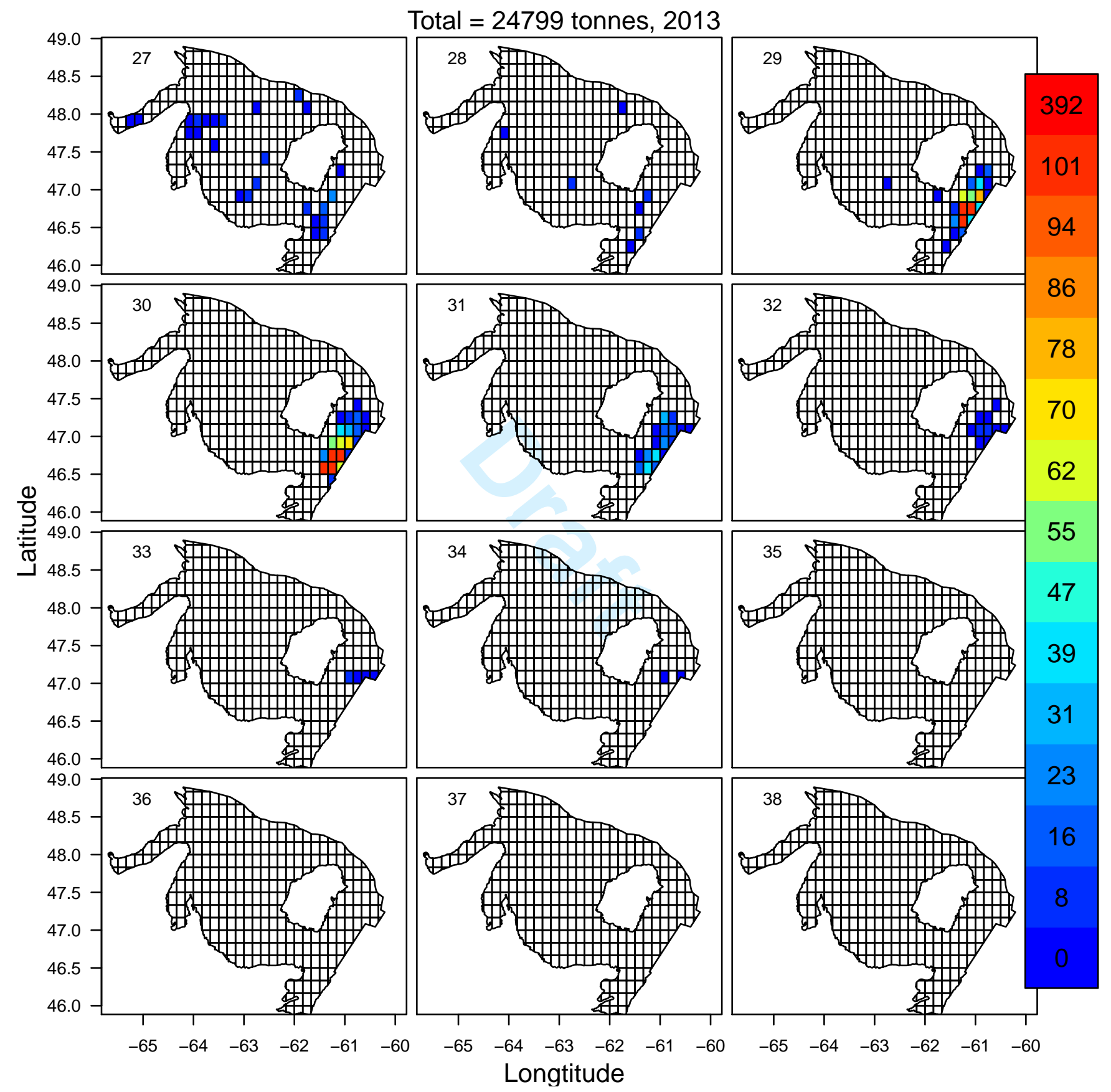

Figure SC.19c. Catch (tonnes) of snow crab in each week and grid cell in 2013. The week number is indicated in the top left-hand corner. Colors correspond to catch levels, as indicated in the legend on the right-hand side. Darkest red grids indicate catch $>98$ th percentile. 


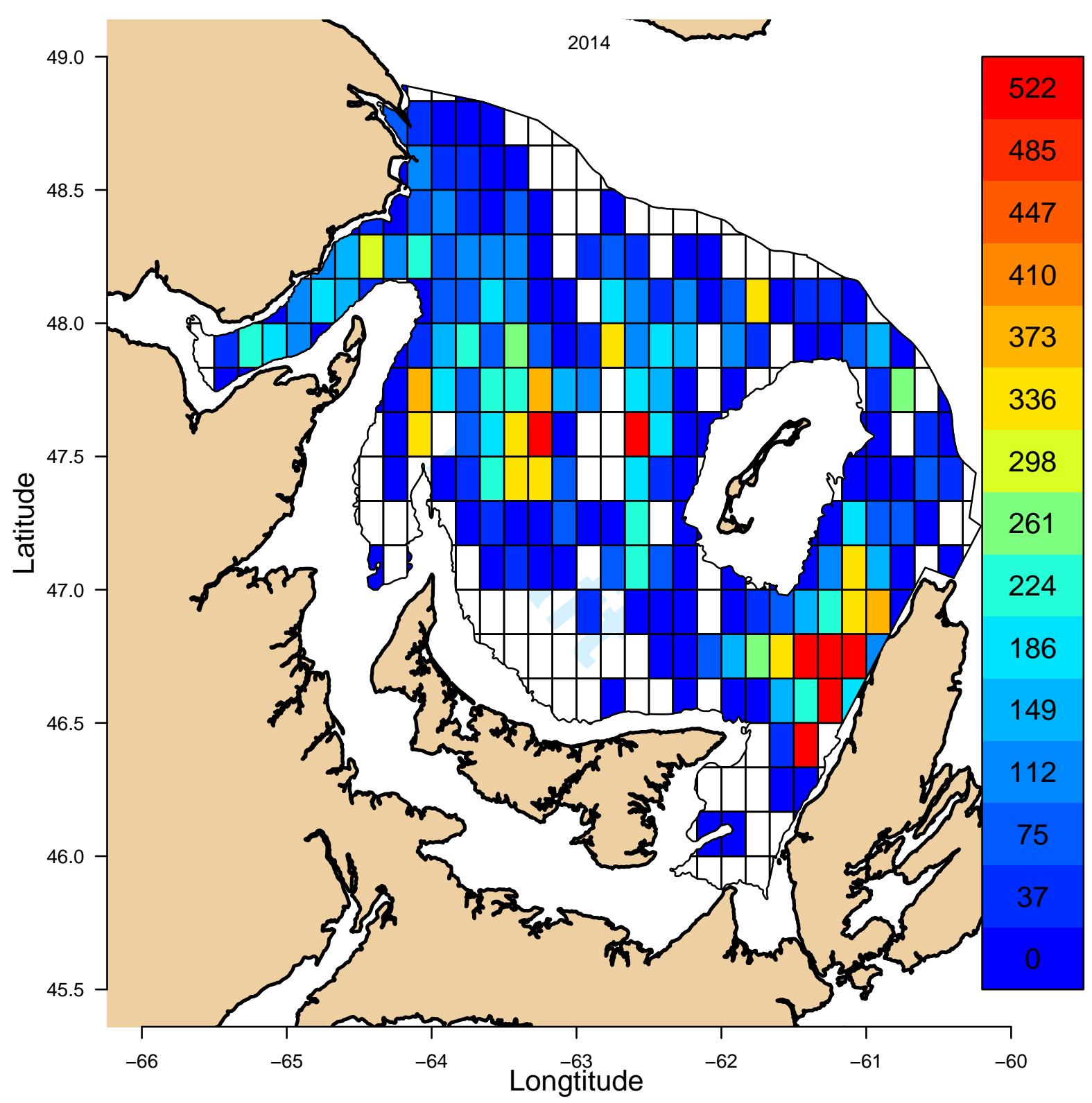

Figure SC.20a. Total annual catch (tonnes) of snow crab in each grid cell in 2014. Colors correspond to catch levels, as indicated in the legend on the right-hand side. Darkest red grids indicate catch $>98$ th percentile. 


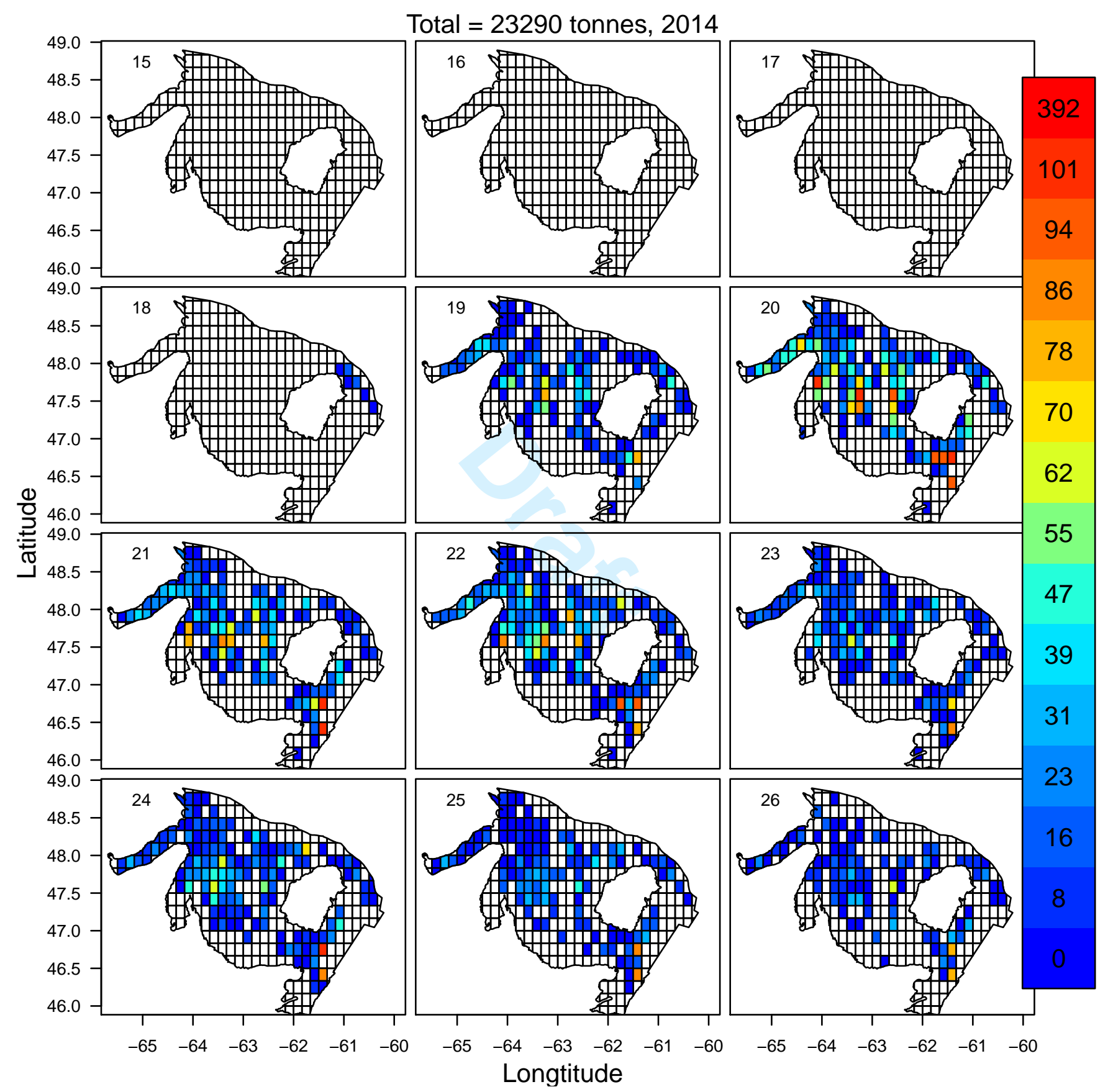

Figure SC.20b. Catch (tonnes) of snow crab in each week and grid cell in 2014. The week number is indicated in the top left-hand corner. Colors correspond to catch levels, as indicated in the legend on the right-hand side. Darkest red grids indicate catch $>98$ th percentile. 
Total $=23290$ tonnes, 2014
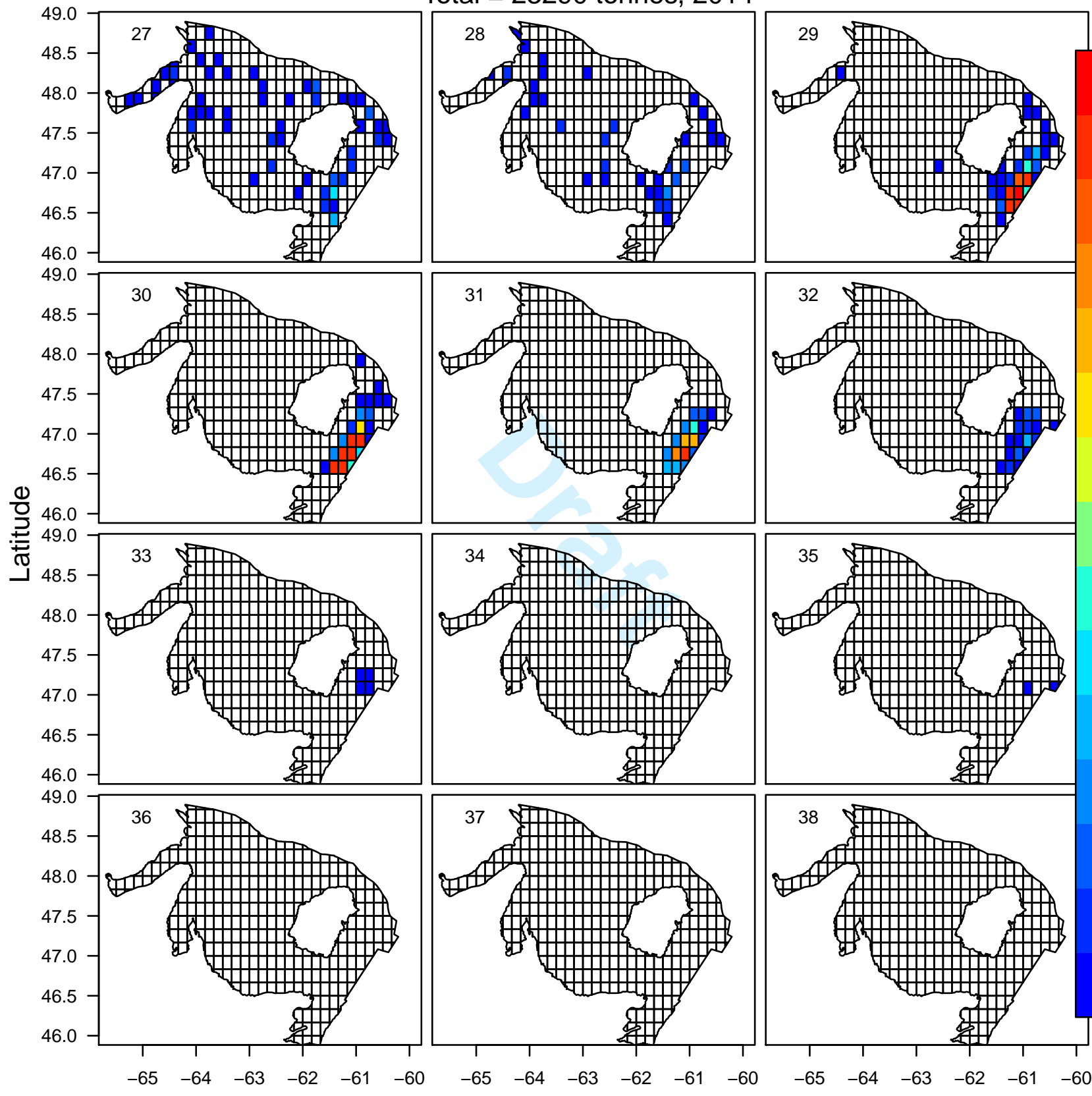

86
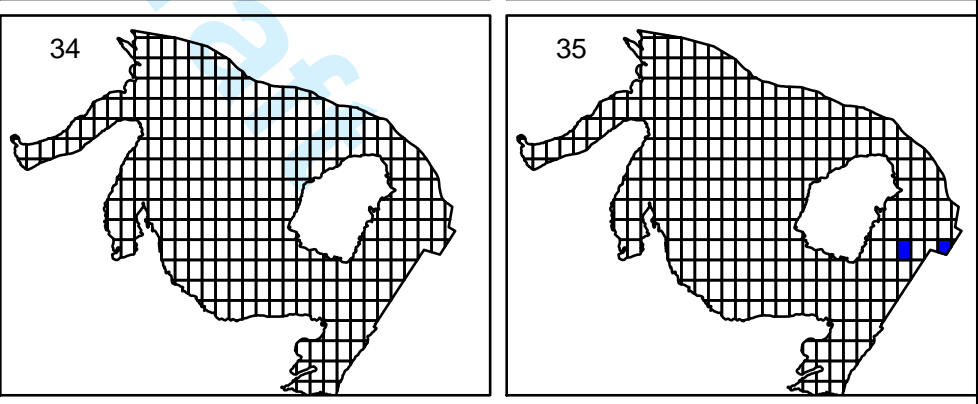

55
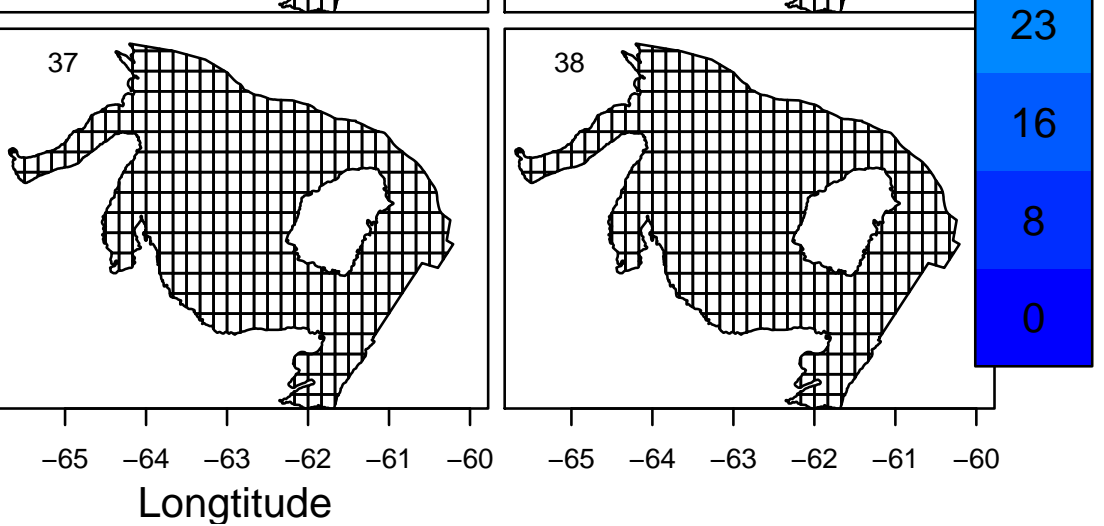

Figure SC.20c. Catch (tonnes) of snow crab in each week and grid cell in 2014. The week number is indicated in the top left-hand corner. Colors correspond to catch levels, as indicated in the legend on the righthand side. Darkest red grids indicate catch $>98$ th percentile. 
Total annual average $=382770$ pots, 346 cells; cell average $=19912.67$ pots,

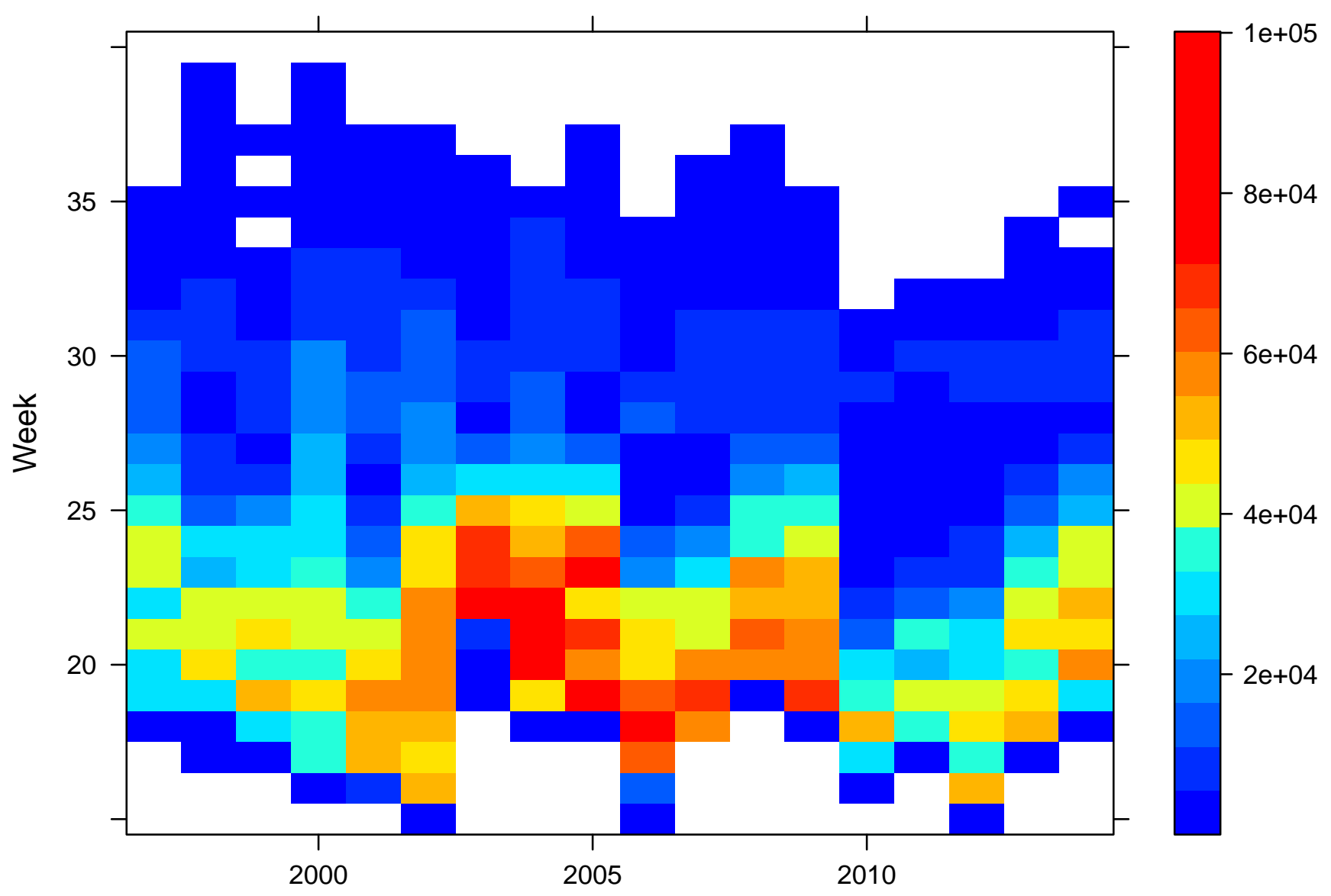

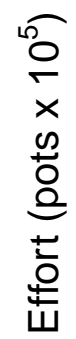

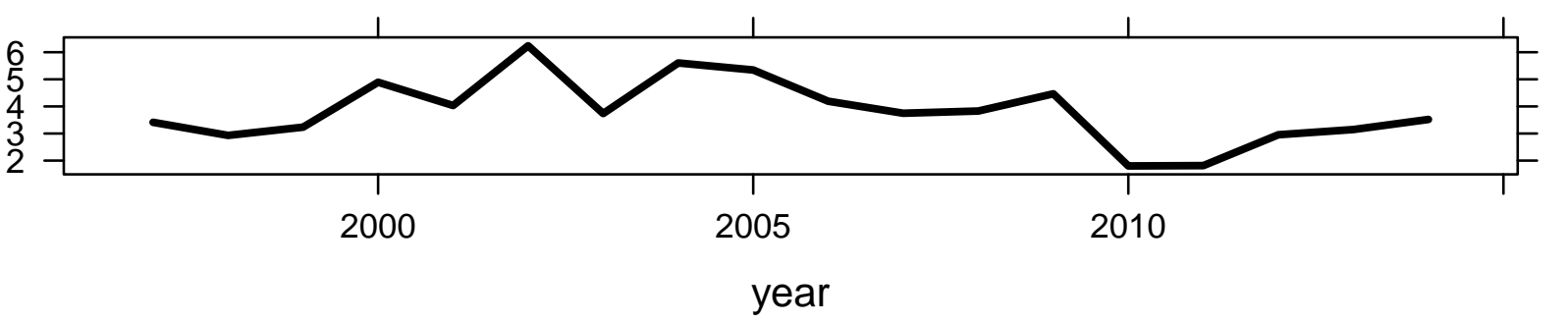

Figure SE.1. Top panel: Total effort (number of pots) of snow crab each week (rows) and year (columns). Colors correspond to effort levels, as indicated in the legend on the right-hand side. Darkest red grids indicate effort $>$ 98th percentile. Bottom panel: Total efforteach year for all weeks. 


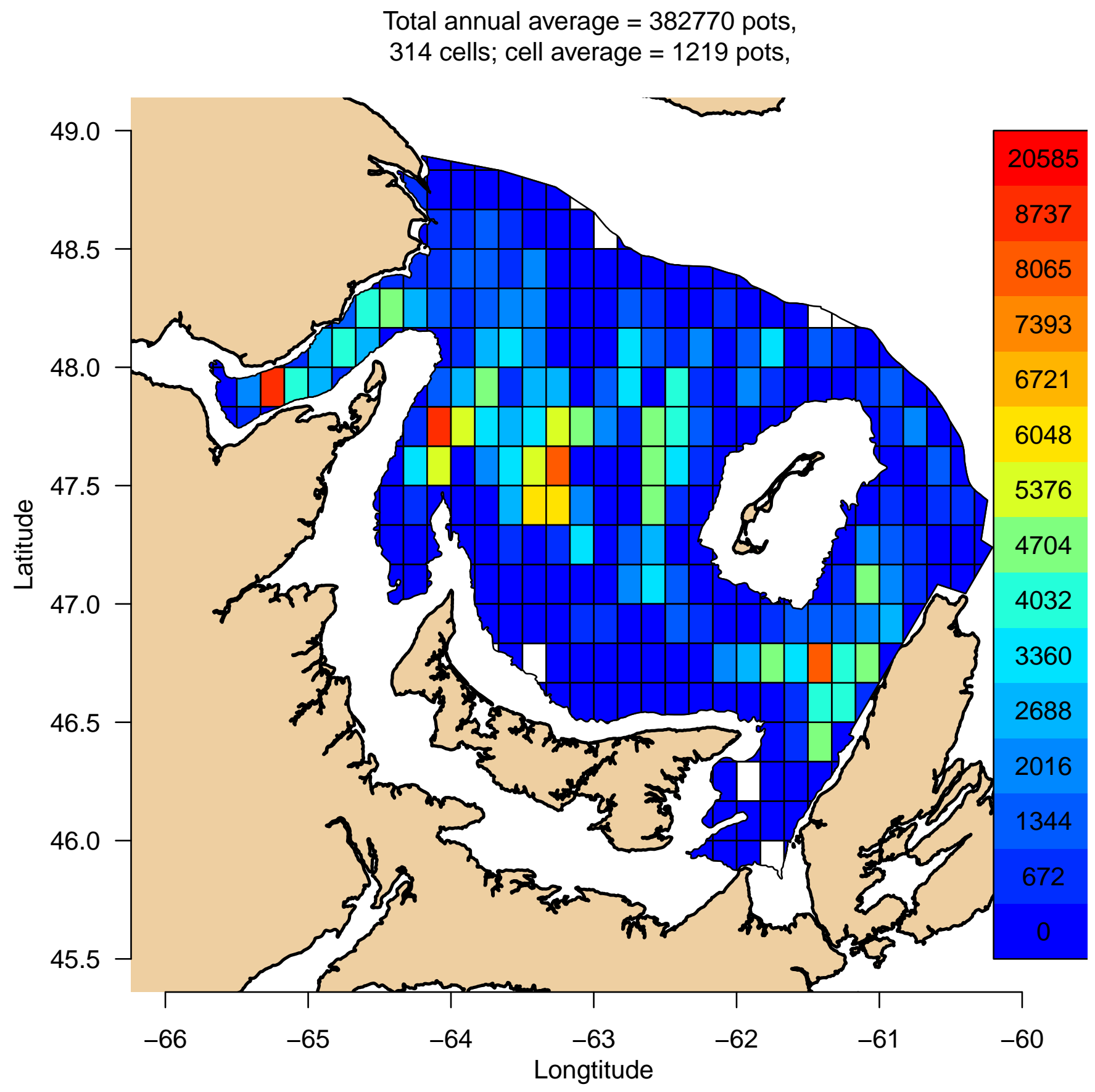

Figure SE.2. Total annual effort (number of pots) for snow crab in each grid cell, averaged for 1997-2014. Colors correspond to effort levels, as indicated in the legend on the right-hand side. Darkest red grids indicate effort $>98$ th percentile. 


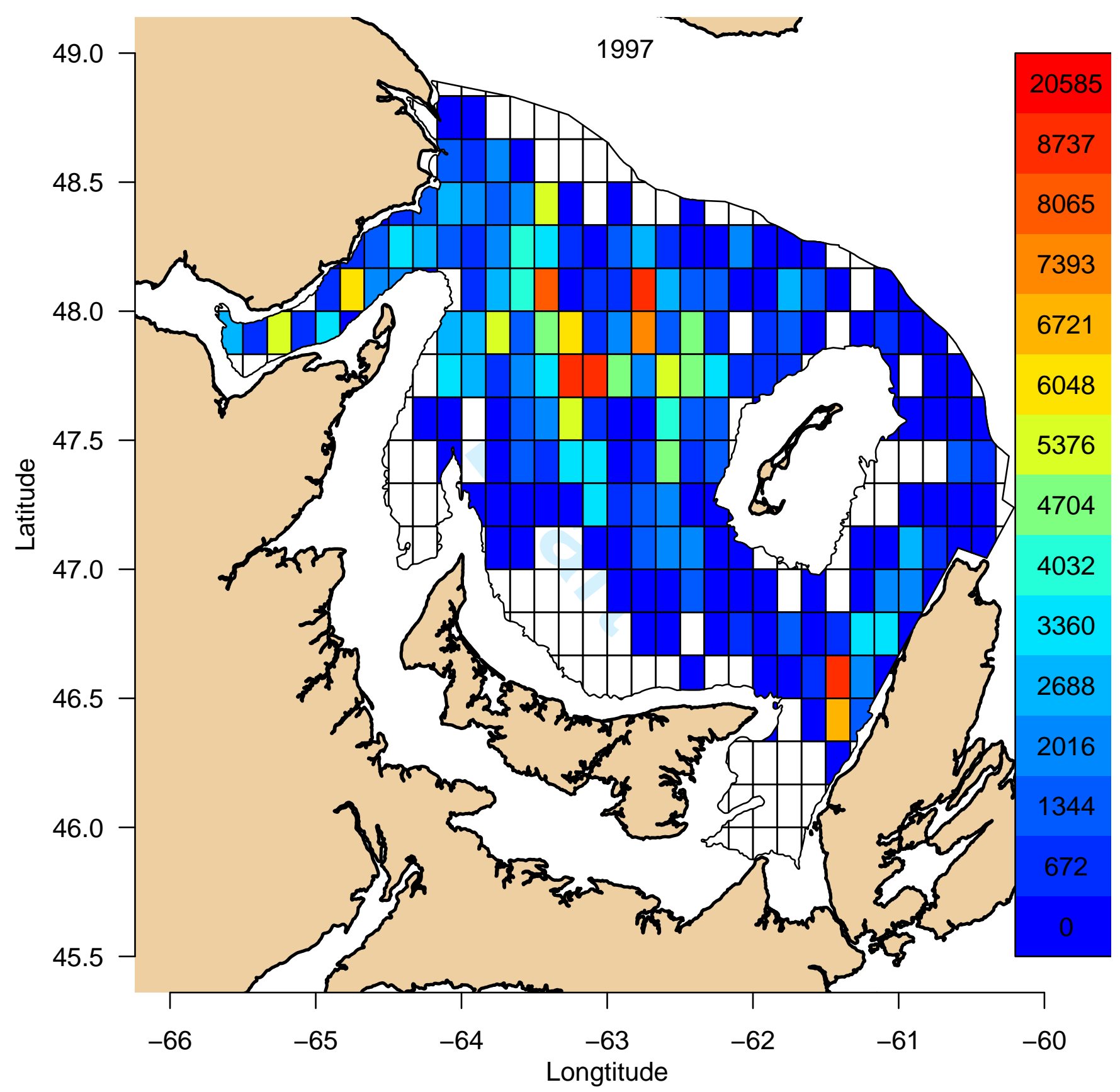

Figure SE.3a. Total annual effort (number of pots) for snow crab in each grid cell in 1997. Colors correspond to effort levels, as indicated in the legend on the right-hand side. Darkest red grids indicate effort $>98$ th percentile. 
Total $=341331$ pots, 1997
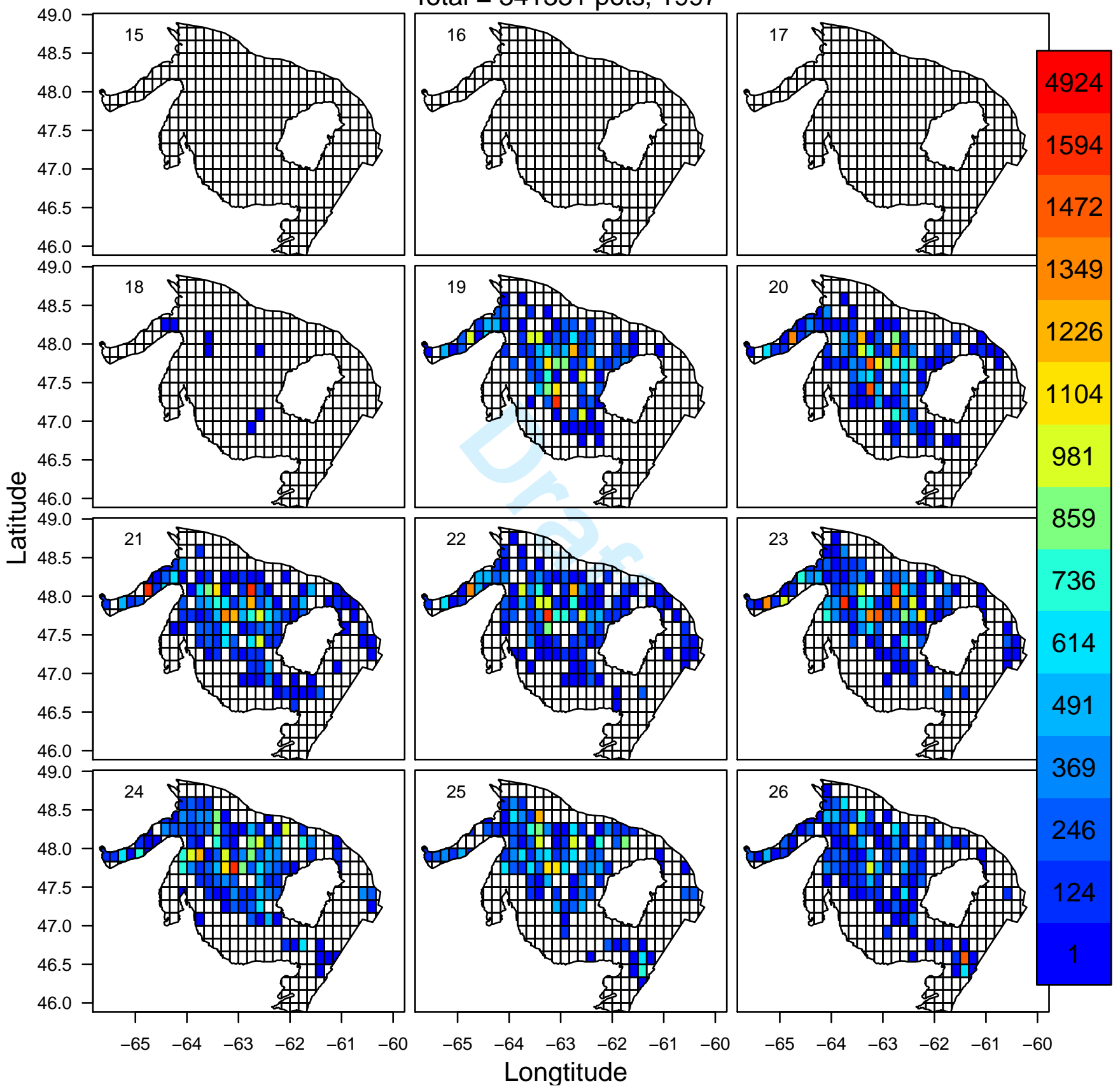

Figure SE.3b. Effort (number of pots) for snow crab in each week and grid cell in 1997. The week number is indicated in the top left-hand corner. Colors correspond to effort levels, as indicated in the legend on the right-hand side. Darkest red grids indicate effort $>98$ th percentile. 


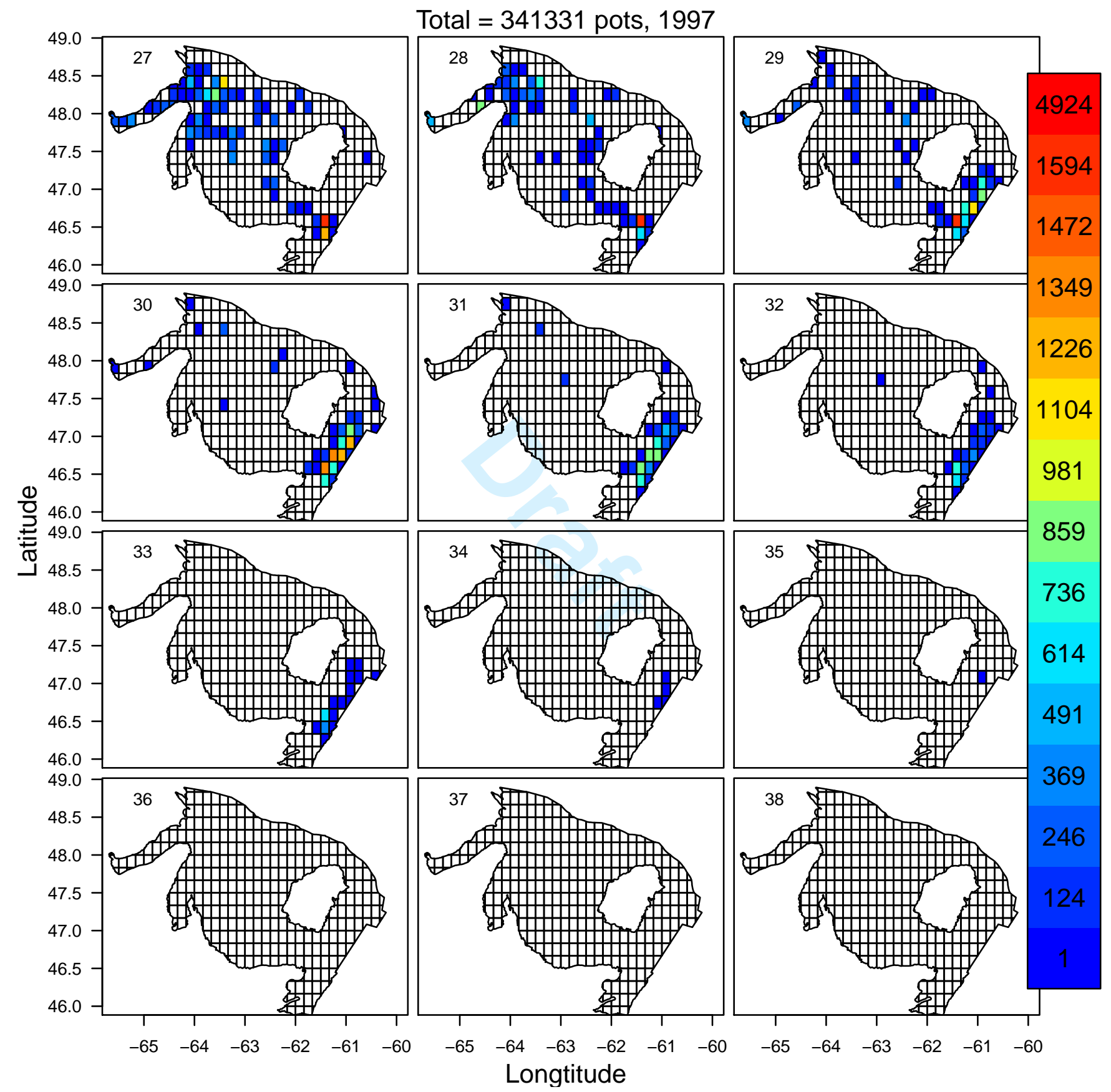

Figure SE.3c. Effort (number of pots) for snow crab in each week and grid cell in 1997. The week number is indicated in the top left-hand corner. Colors correspond to effort levels, as indicated in the legend on the righthand side. Darkest red grids indicate effort $>98$ th percentile. 


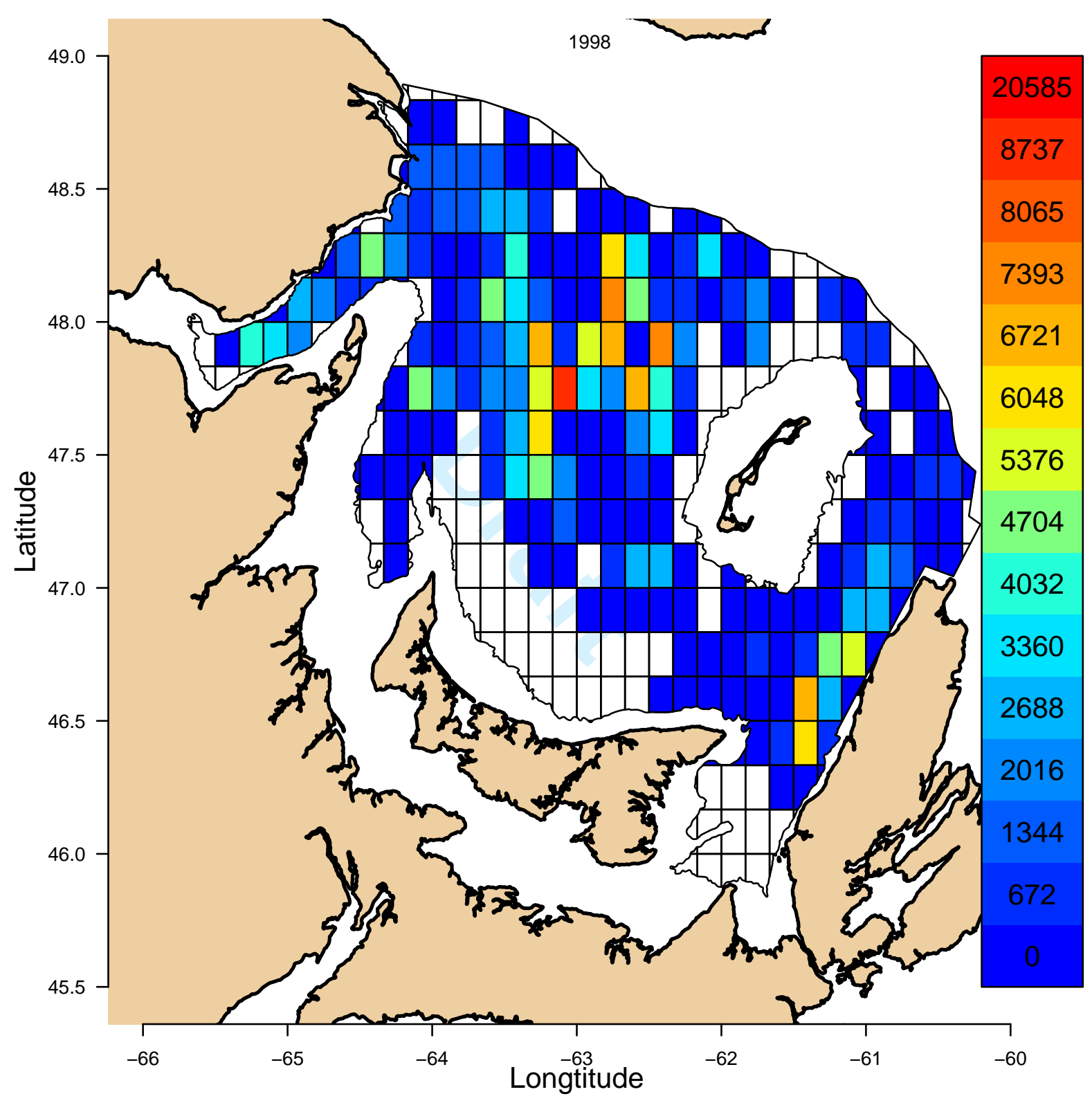

Figure SE.4a. Total annual effort (number of pots) for snow crab in each grid cell in 1998. Colors correspond to effort levels, as indicated in the legend on the right-hand side. Darkest red grids indicate effort $>98$ th percentile. 


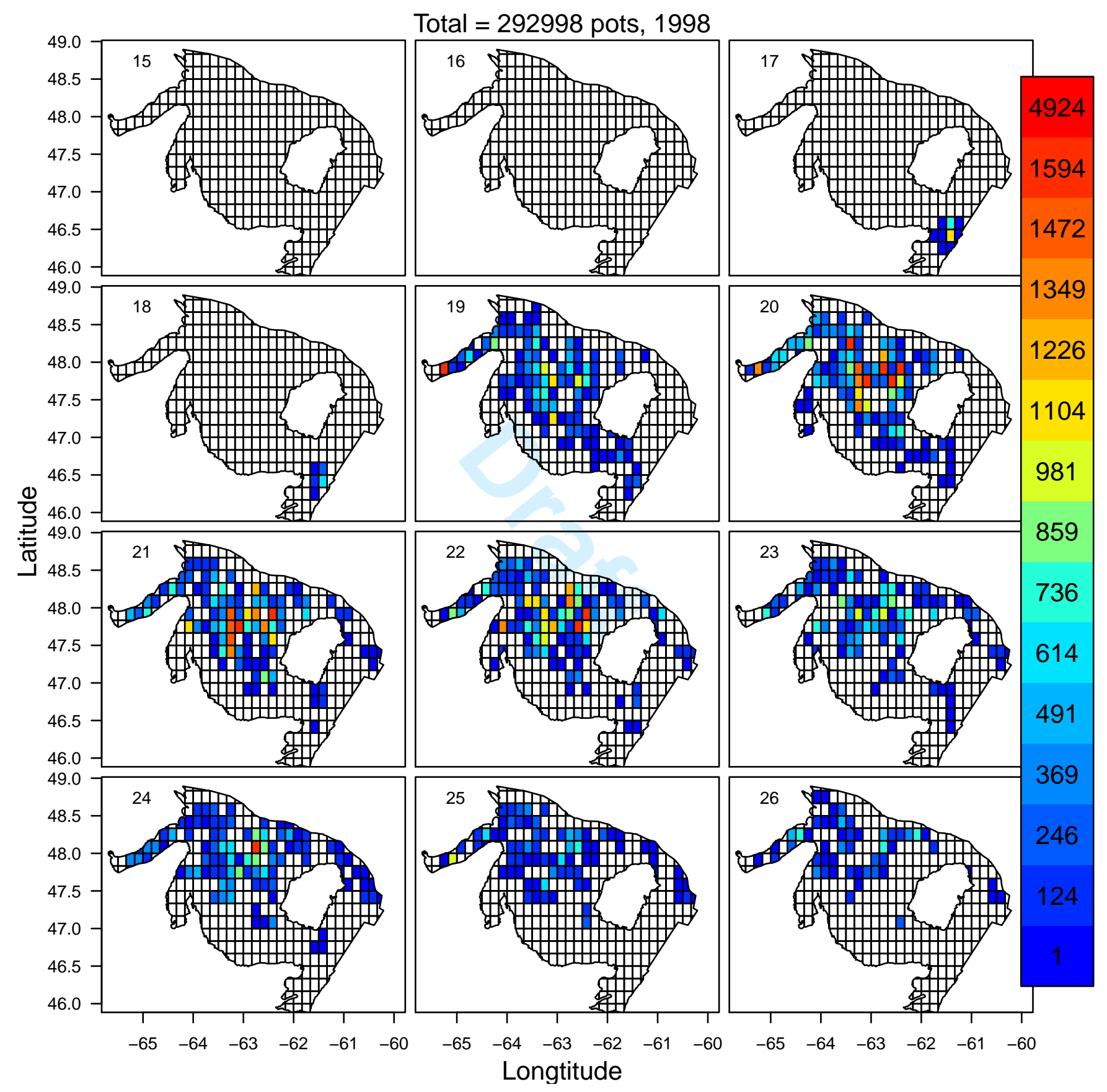

Figure SE.4b. Effort (number of pots) for snow crab in each week and grid cell in 1998. The week number is indicated in the top left-hand corner. Colors correspond to effort levels, as indicated in the legend on the righthand side. Darkest red grids indicate effort $>98$ th percentile. 
Total $=292998$ pots, 1998
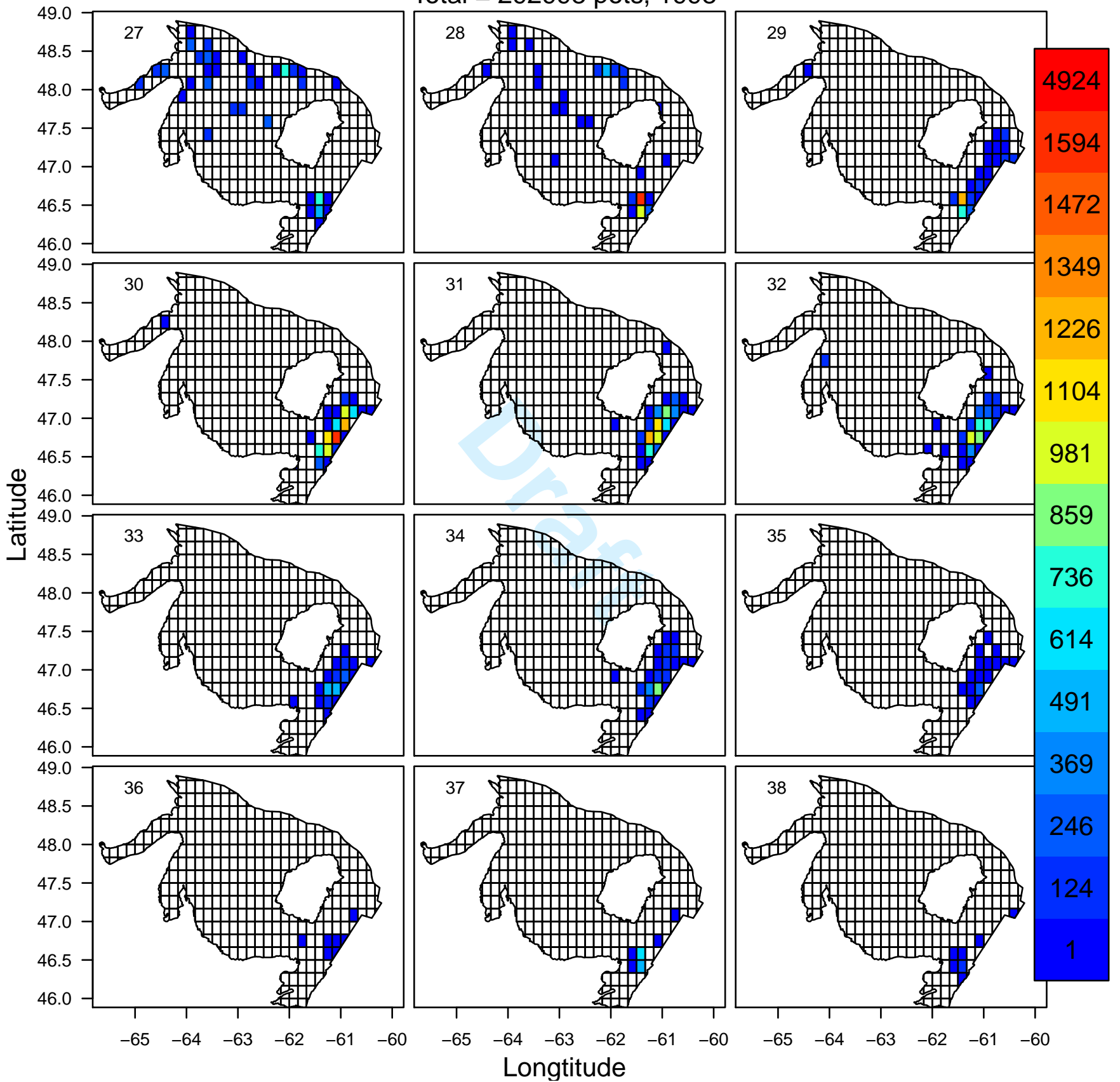

Figure SE.4c. Effort (number of pots) for snow crab in each week and grid cell in 1998. The week number is indicated in the top left-hand corner. Colors correspond to effort levels, as indicated in the legend on the right-hand side. Darkest red grids indicate effort $>98$ th percentile. 


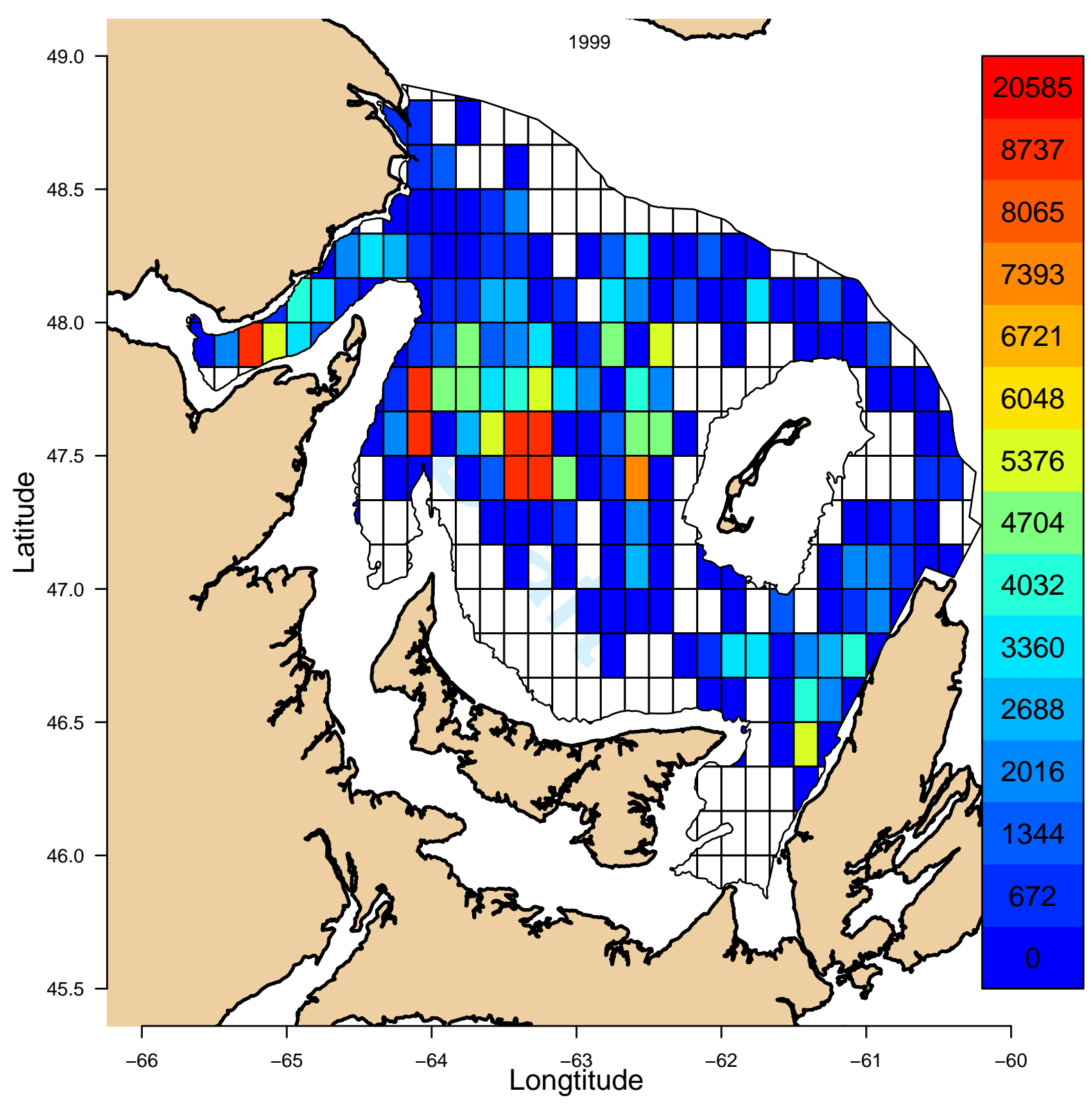

Figure SE.5a. Total annual effort (number of pots) for snow crab in each grid cell in 1999. Colors correspond to effort levels, as indicated in the legend on the right-hand side. Darkest red grids indicate effort $>98$ th percentile. 
Total $=323519$ pots, 1999
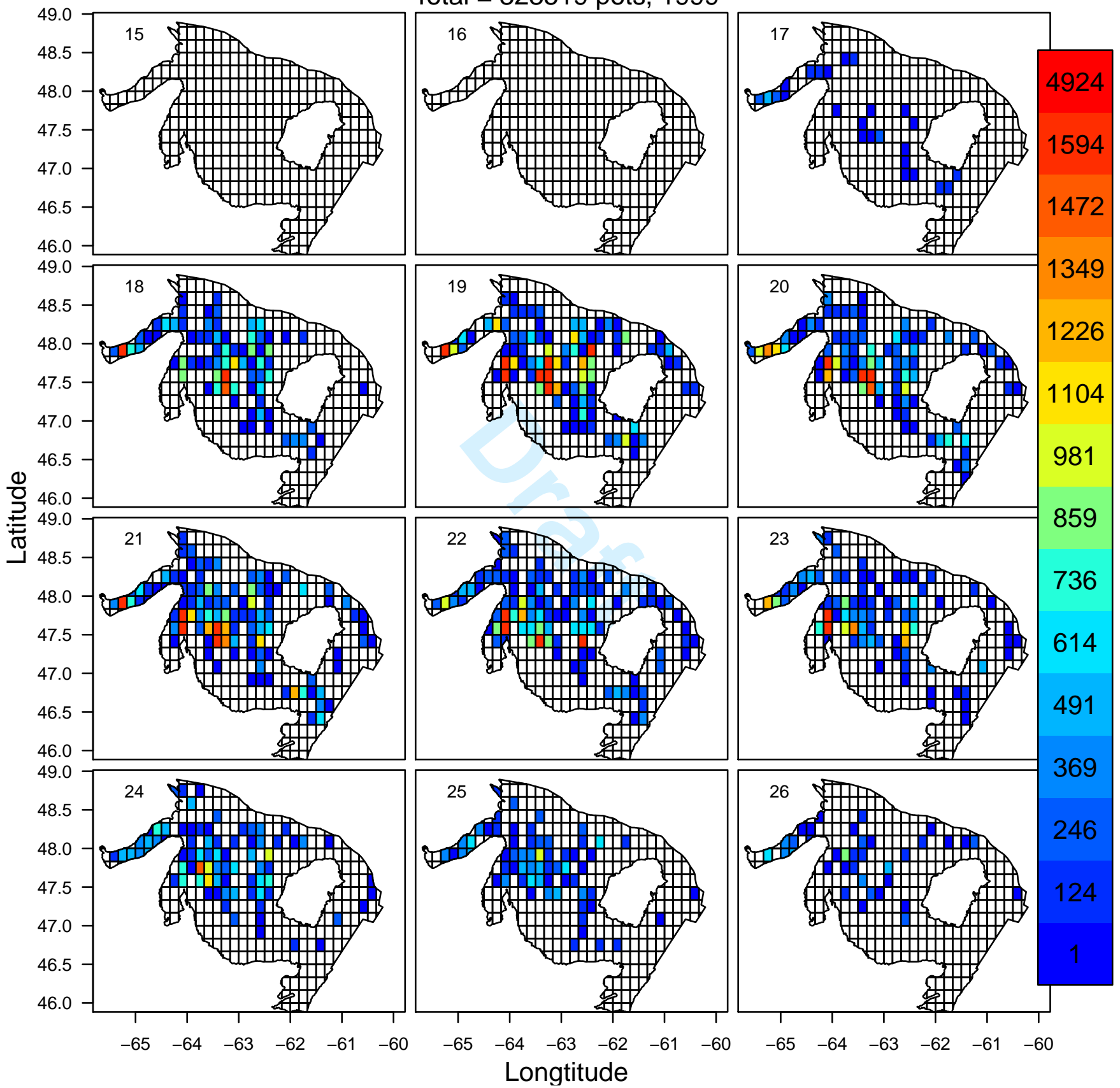

Figure SE.5b. Effort (number of pots) for snow crab in each week and grid cell in 1999. The week number is indicated in the top left-hand corner. Colors correspond to effort levels, as indicated in the legend on the right-hand side. Darkest red grids indicate effort $>98$ th percentile. 


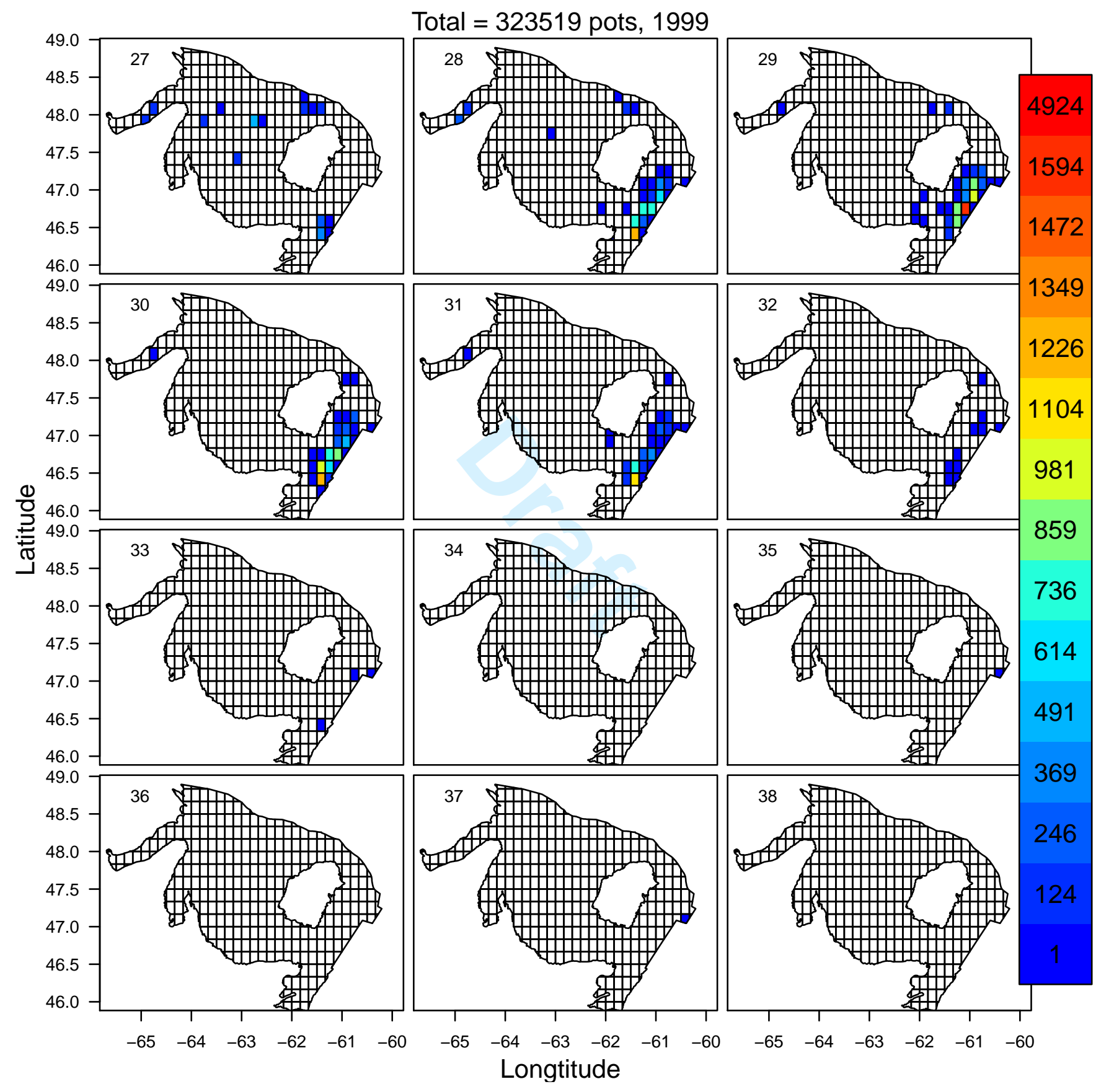

Figure SE.5c. Effort (number of pots) for snow crab in each week and grid cell in 1999. The week number is indicated in the top left-hand corner. Colors correspond to effort levels, as indicated in the legend on the right-hand side. Darkest red grids indicate effort $>98$ th percentile. 


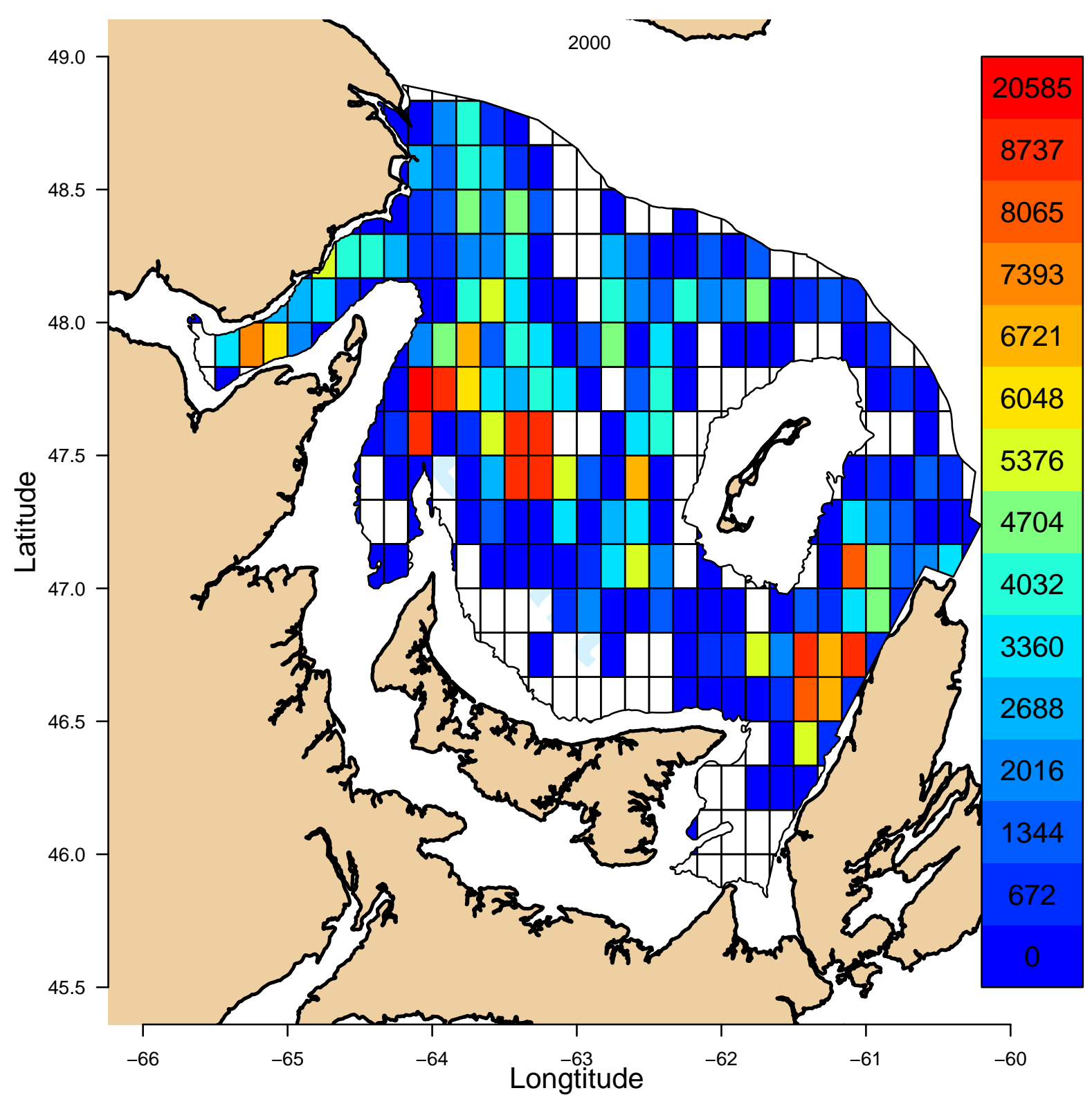

Figure SE.6a. Total annual effort (number of pots) for snow crab in each grid cell in 2000. Colors correspond to effort levels, as indicated in the legend on the right-hand side. Darkest red grids indicate effort $>98$ th percentile. 


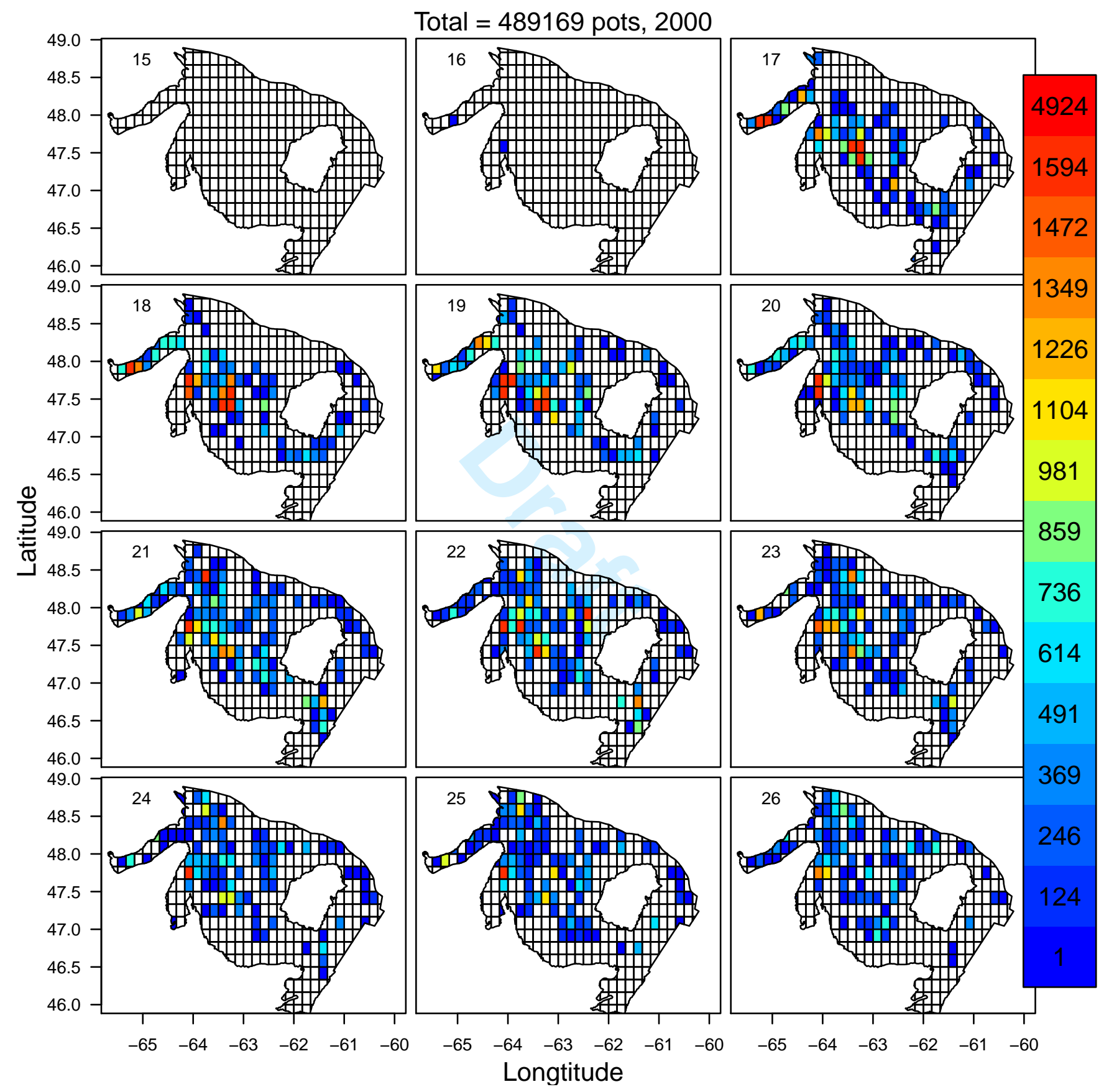

Figure SE.6b. Effort (number of pots) for snow crab in each week and grid cell in 2000. The week number is indicated in the top left-hand corner. Colors correspond to effort levels, as indicated in the legend on the right-hand side. Darkest red grids indicate effort $>98$ th percentile. 
Total $=489169$ pots, 2000
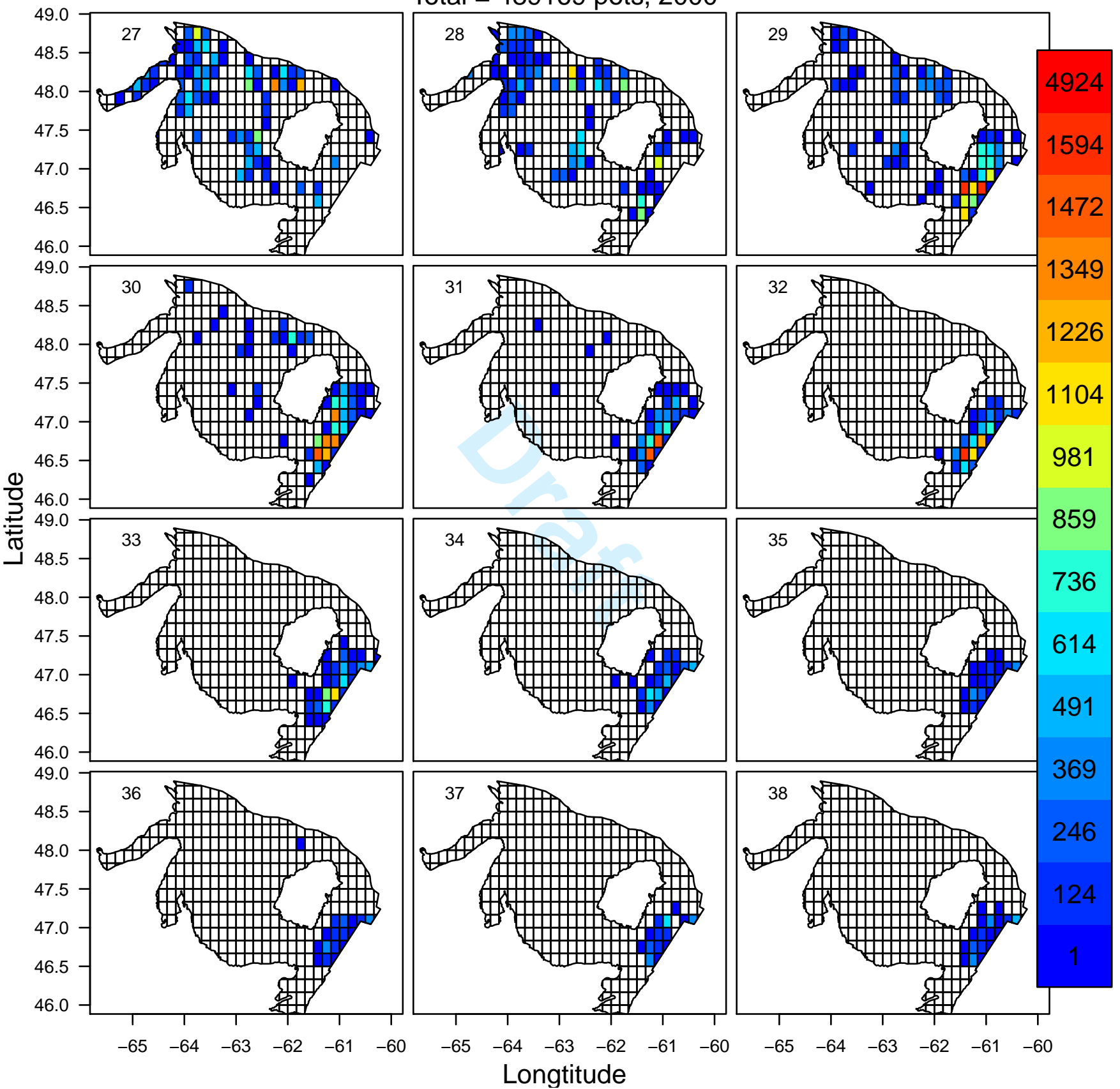

Figure SE.6c. Effort (number of pots) for snow crab in each week and grid cell in 2000. The week number is indicated in the top left-hand corner. Colors correspond to effort levels, as indicated in the legend on the right-hand side. Darkest red grids indicate effort $>$ 98th percentile. 


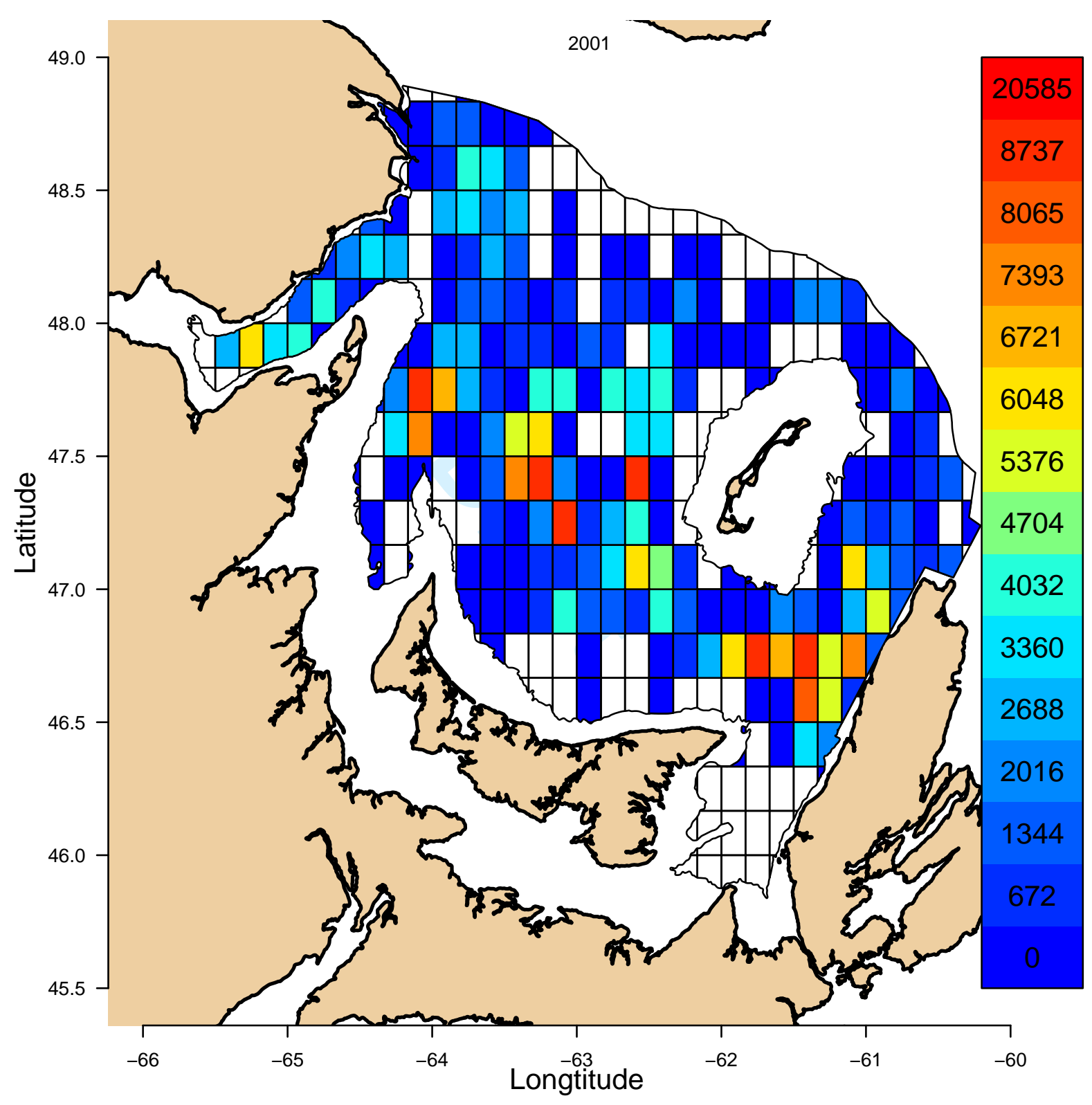

Figure SE.7a. Total annual effort (number of pots) for snow crab in each grid cell in 2001. Colors correspond to effort levels, as indicated in the legend on the right-hand side. Darkest red grids indicate effort $>98$ th percentile. 
Total $=403328$ pots, 2001
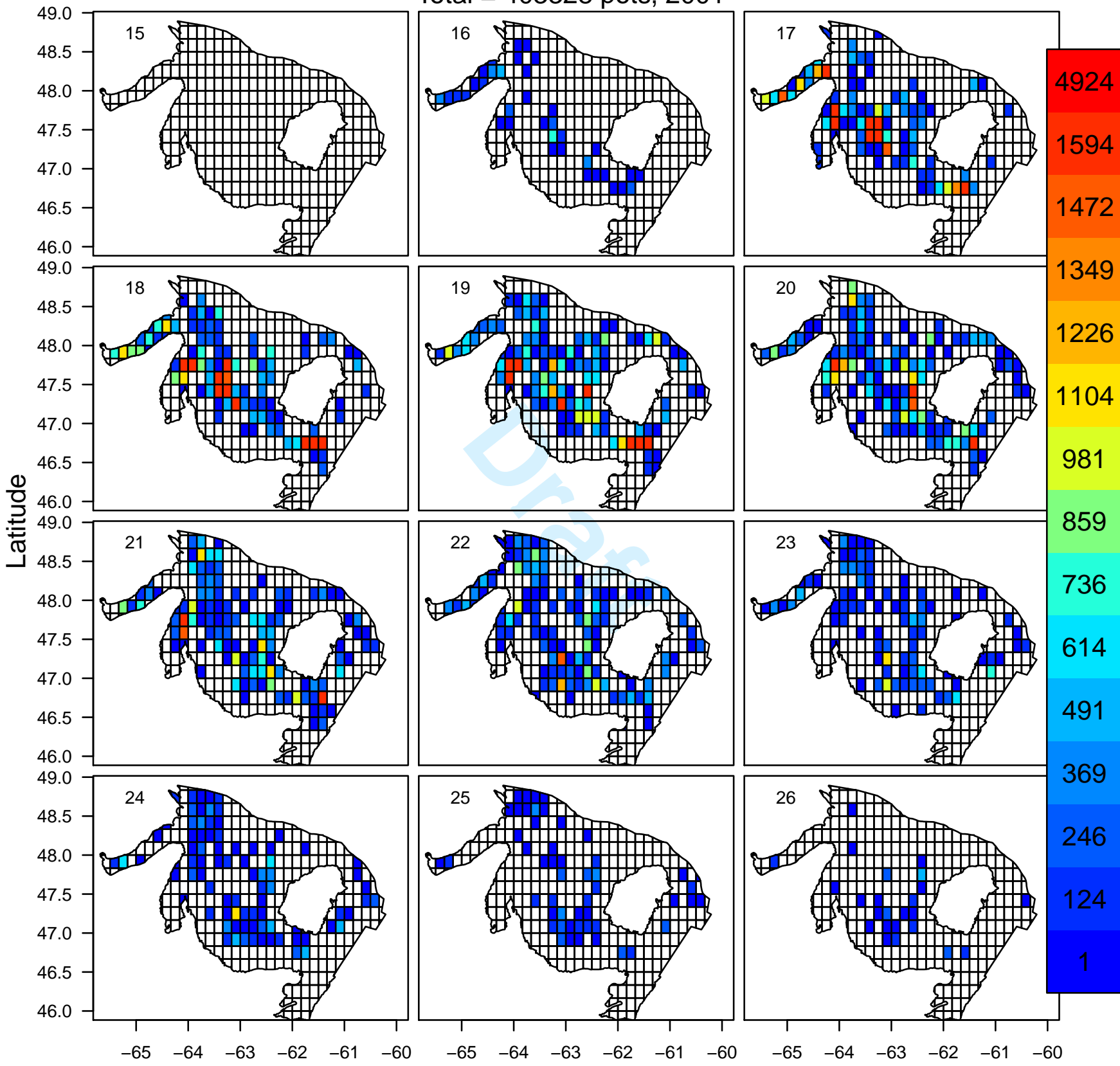

1226

1104

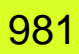

859
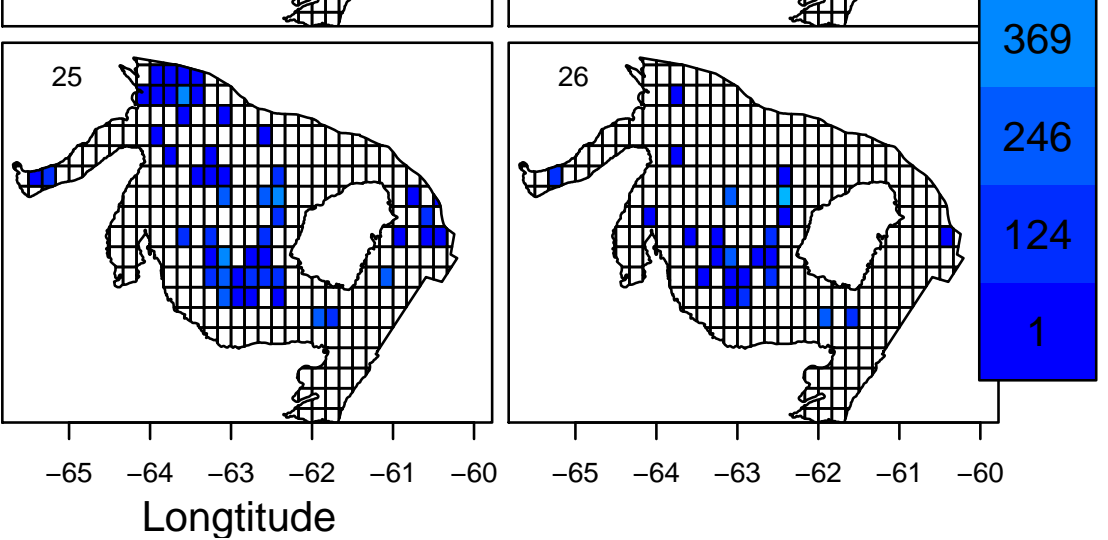

Figure SE.7b. Effort (number of pots) for snow crab in each week and grid cell in 2001. The week number is indicated in the top left-hand corner. Colors correspond to effort levels, as indicated in the legend on the right-hand side. Darkest red grids indicate effort $>98$ th percentile. 


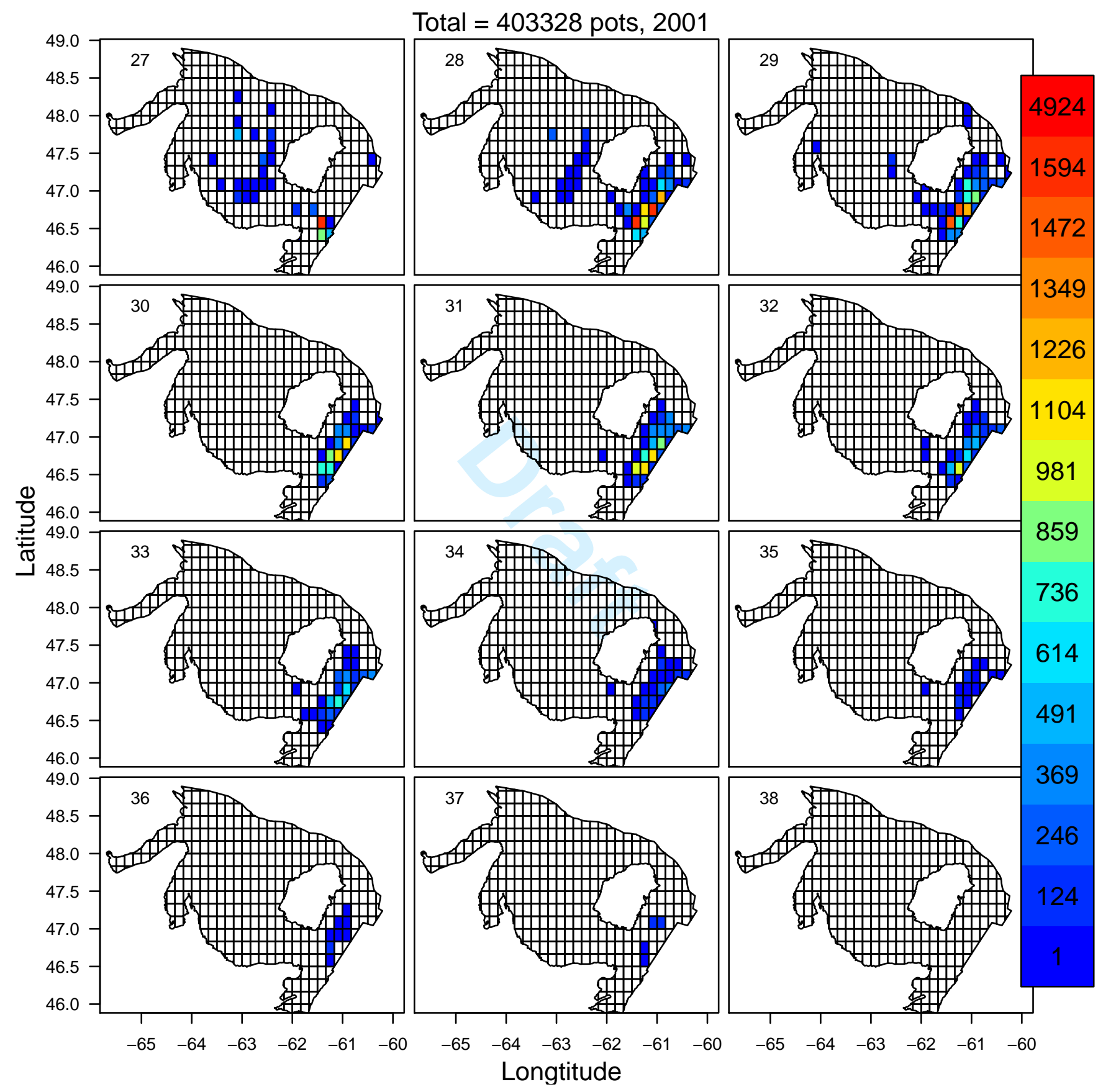

Figure SE.7c. Effort (number of pots) for snow crab in each week and grid cell in 2001. The week number is indicated in the top left-hand corner. Colors correspond to effort levels, as indicated in the legend on the right-hand side. Darkest red grids indicate effort $>98$ th percentile. 


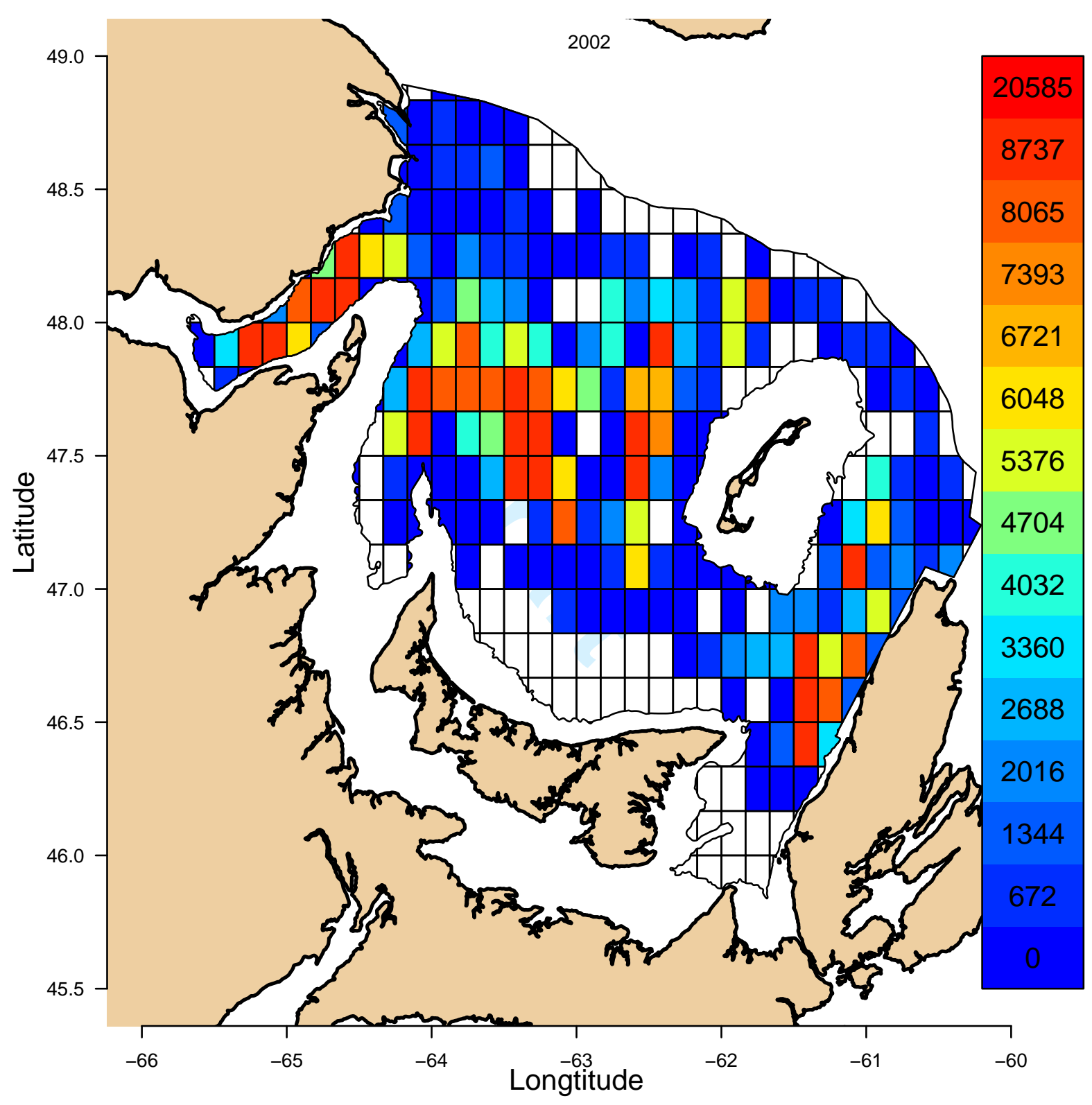

Figure SE.8a. Total annual effort (number of pots) for snow crab in each grid cell in 2002. Colors correspond to effort levels, as indicated in the legend on the right-hand side. Darkest red grids indicate effort $>98$ th percentile. 


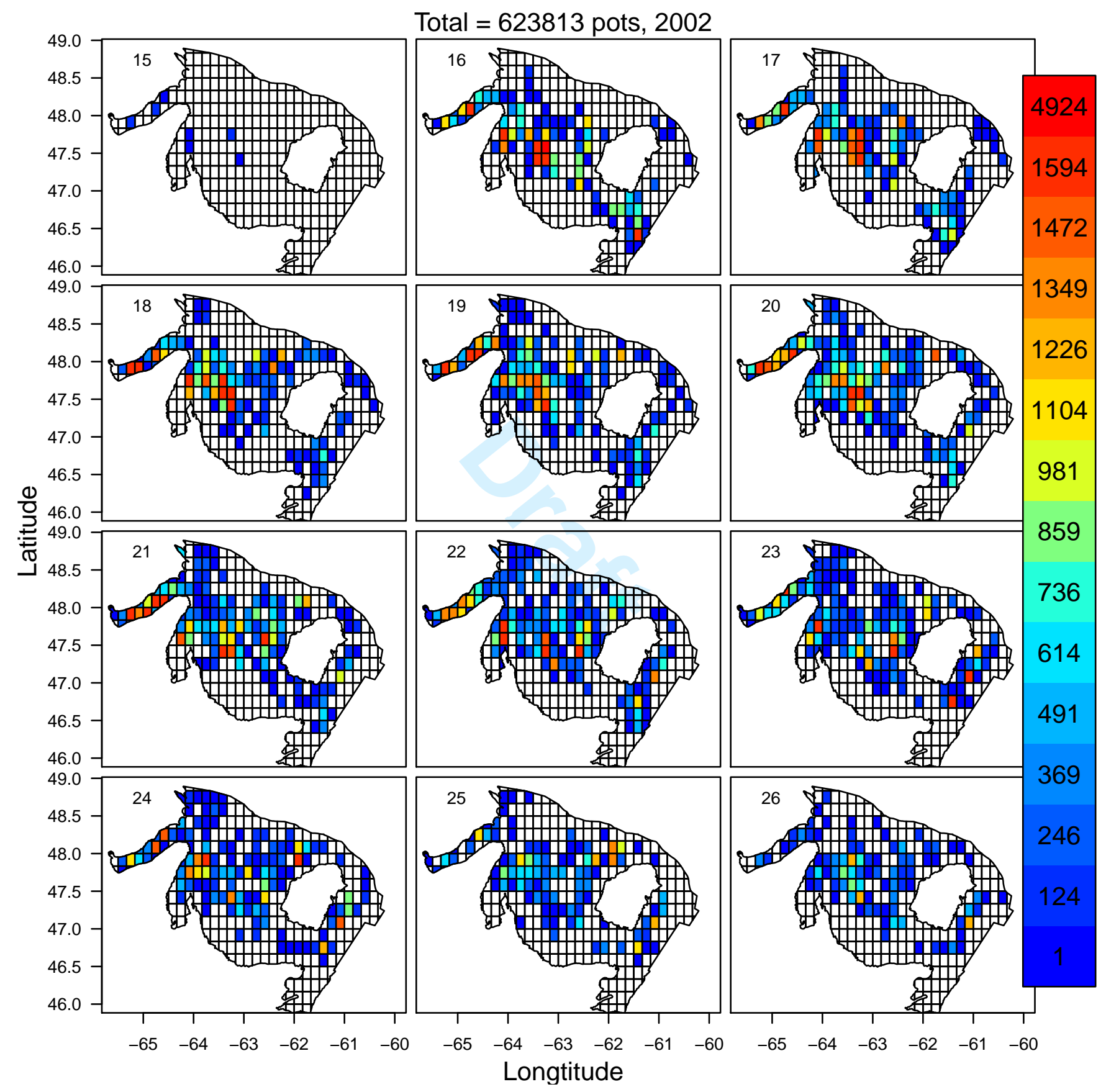

Figure SE.8b. Effort (number of pots) for snow crab in each week and grid cell in 2002. The week number is indicated in the top left-hand corner. Colors correspond to effort levels, as indicated in the legend on the right-hand side. Darkest red grids indicate effort $>98$ th percentile. 
Total $=623813$ pots, 2002
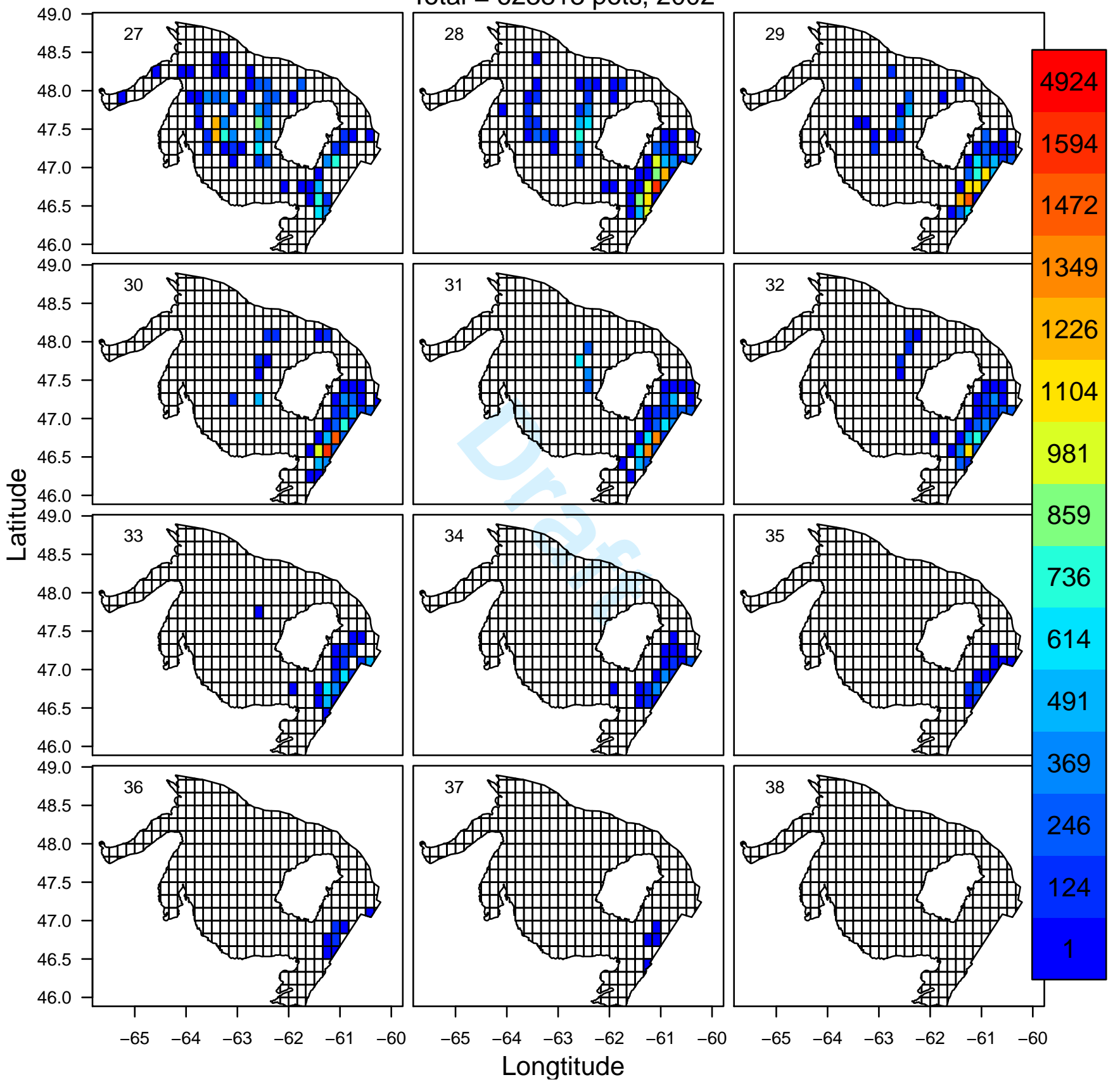

Figure SE.8c. Effort (number of pots) for snow crab in each week and grid cell in 2002. The week number is indicated in the top left-hand corner. Colors correspond to effort levels, as indicated in the legend on the right-hand side. Darkest red grids indicate effort $>98$ th percentile. 


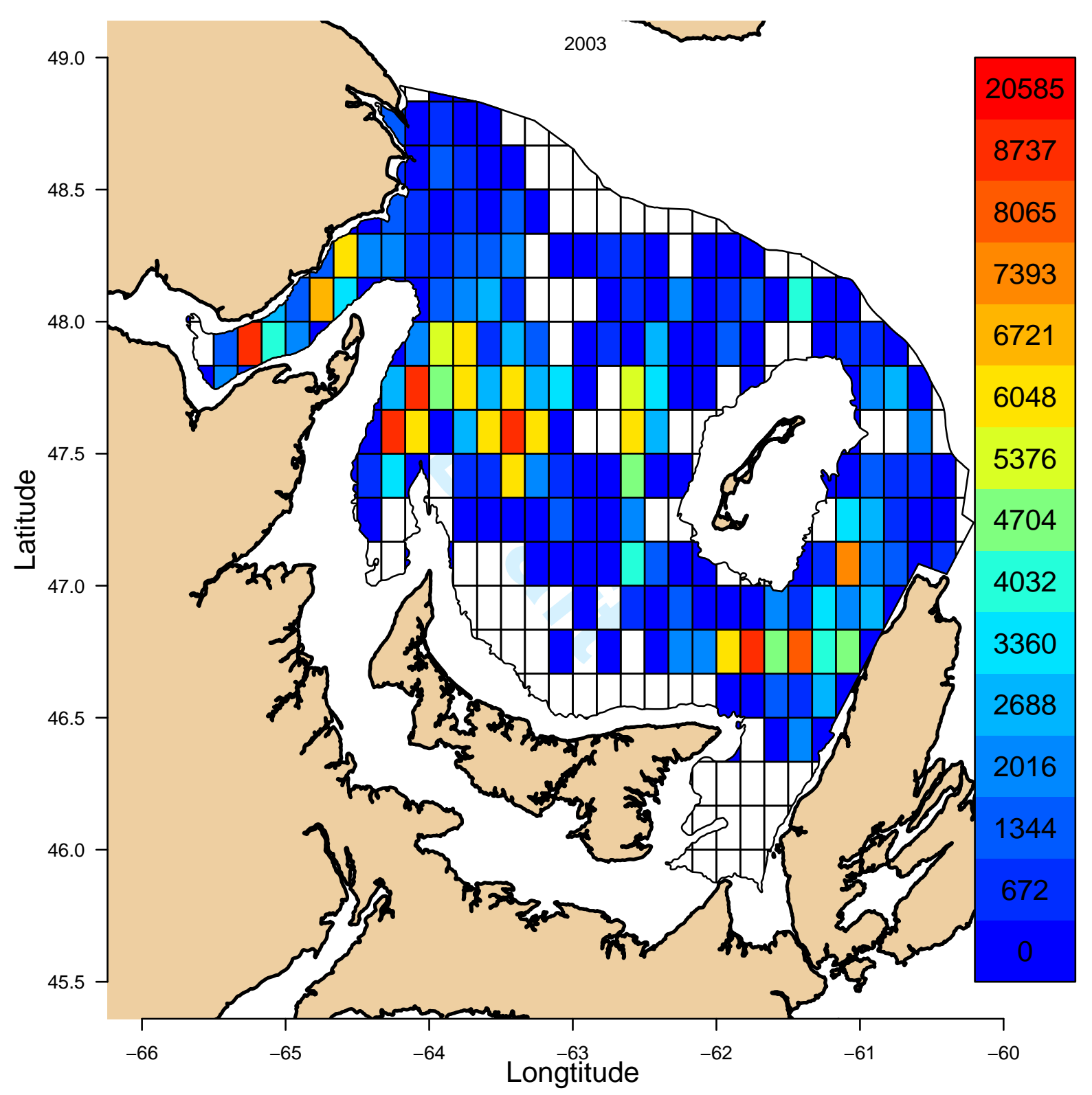

Figure SE.9a. Total annual effort (number of pots) for snow crab in each grid cell in 2003. Colors correspond to effort levels, as indicated in the legend on the right-hand side. Darkest red grids indicate effort $>98$ th percentile. 
Total $=373744$ pots, 2003
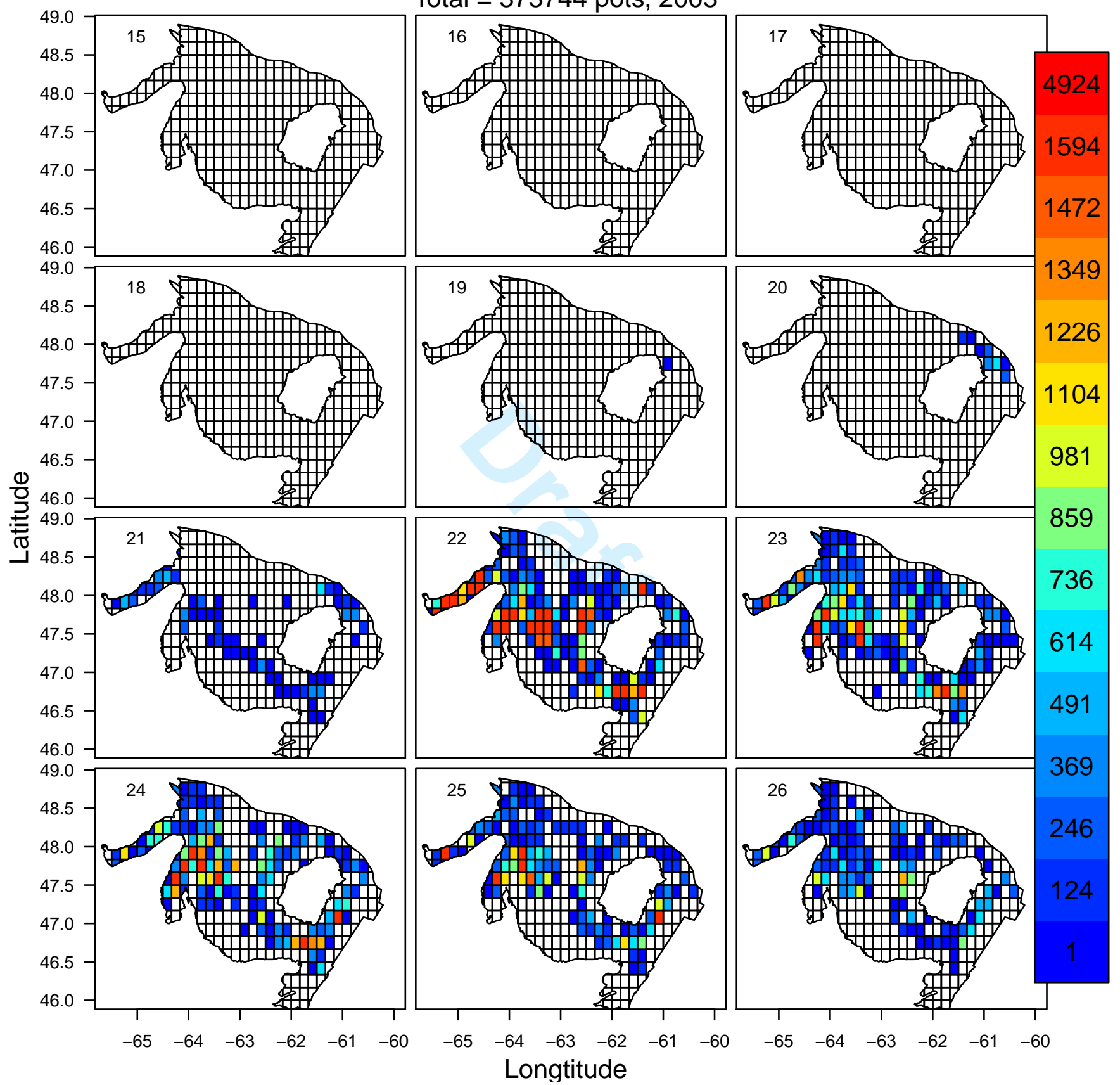

Figure SE.9b. Effort (number of pots) for snow crab in each week and grid cell in 2003. The week number is indicated in the top left-hand corner. Colors correspond to effort levels, as indicated in the legend on the right-hand side. Darkest red grids indicate effort $>98$ th percentile. 


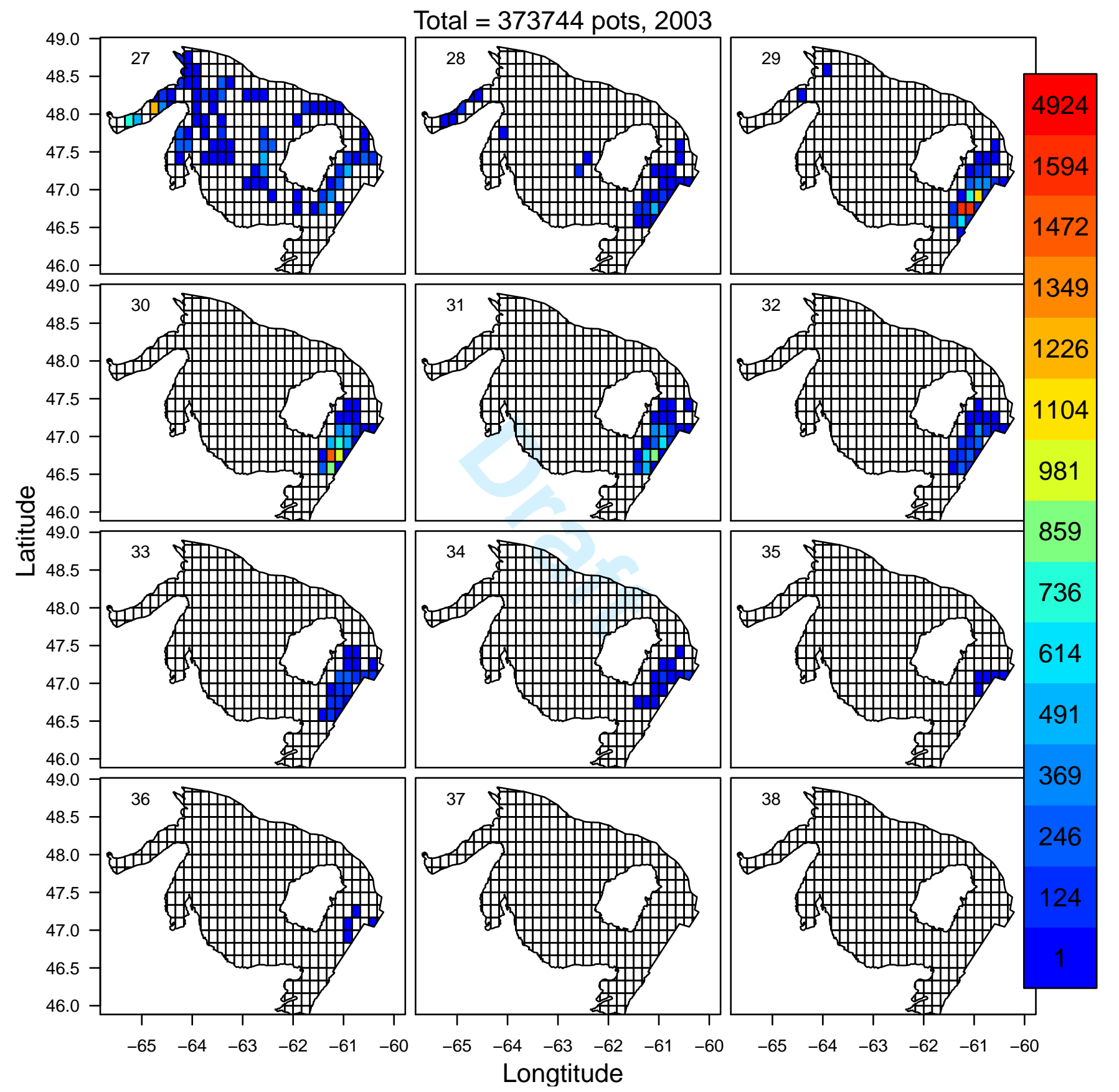

Figure SE.9c. Effort (number of pots) for snow crab in each week and grid cell in 2003. The week number is indicated in the top left-hand corner. Colors correspond to effort levels, as indicated in the legend on the right-hand side. Darkest red grids indicate effort $>98$ th percentile. 


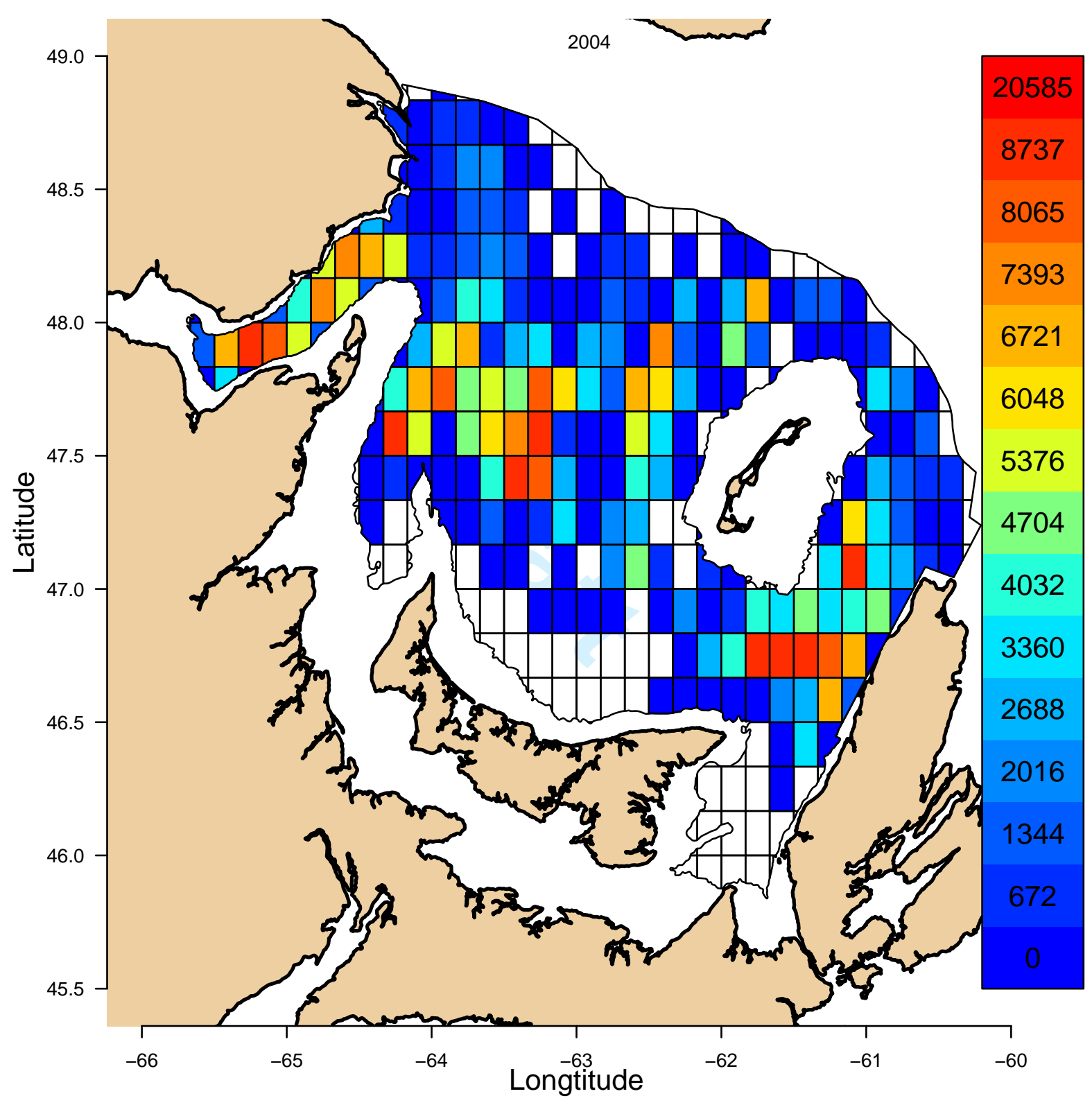

Figure SE.10a. Total annual effort (number of pots) for snow crab in each grid cell in 2004. Colors correspond to effort levels, as indicated in the legend on the right-hand side. Darkest red grids indicate effort $>98$ th percentile. 


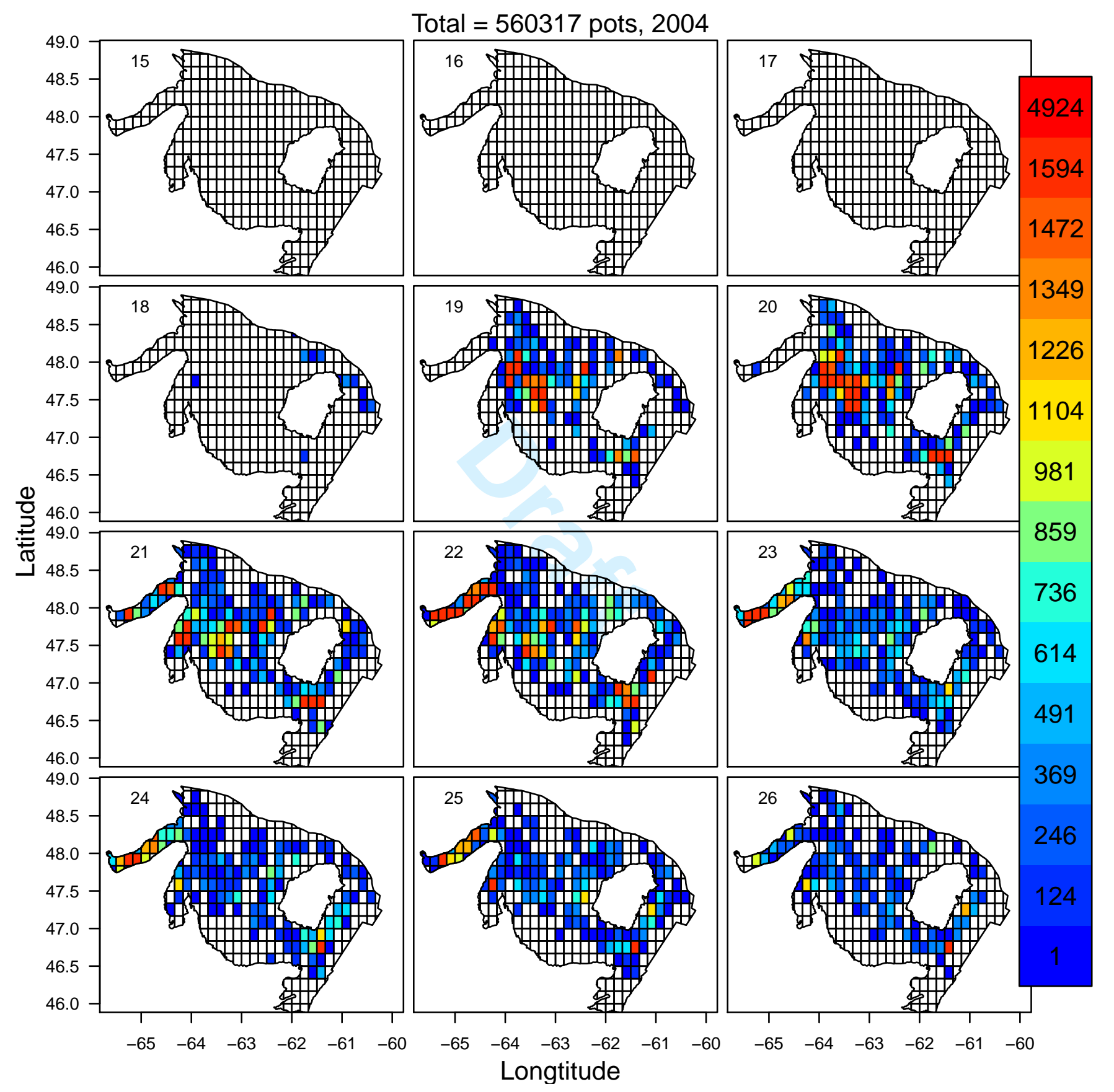

Figure SE.10b. Effort (number of pots) for snow crab in each week and grid cell in 2004. The week number is indicated in the top left-hand corner. Colors correspond to effort levels, as indicated in the legend on the right-hand side. Darkest red grids indicate effort $>98$ th percentile. 
Total $=560317$ pots, 2004
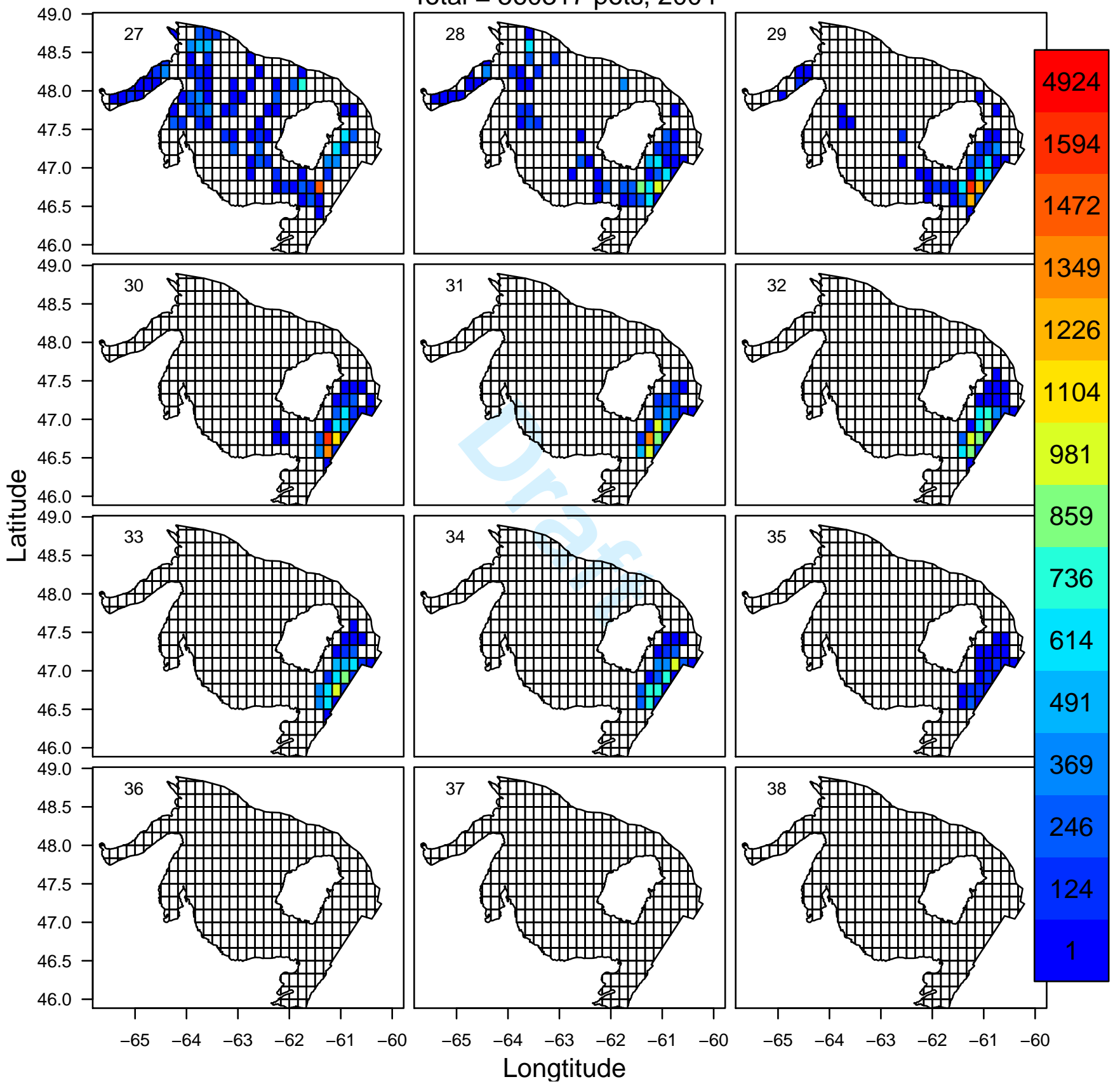

Figure SE.10c. Effort (number of pots) for snow crab in each week and grid cell in 2004. The week number is indicated in the top left-hand corner. Colors correspond to effort levels, as indicated in the legend on the right-hand side. Darkest red grids indicate effort $>98$ th percentile. 


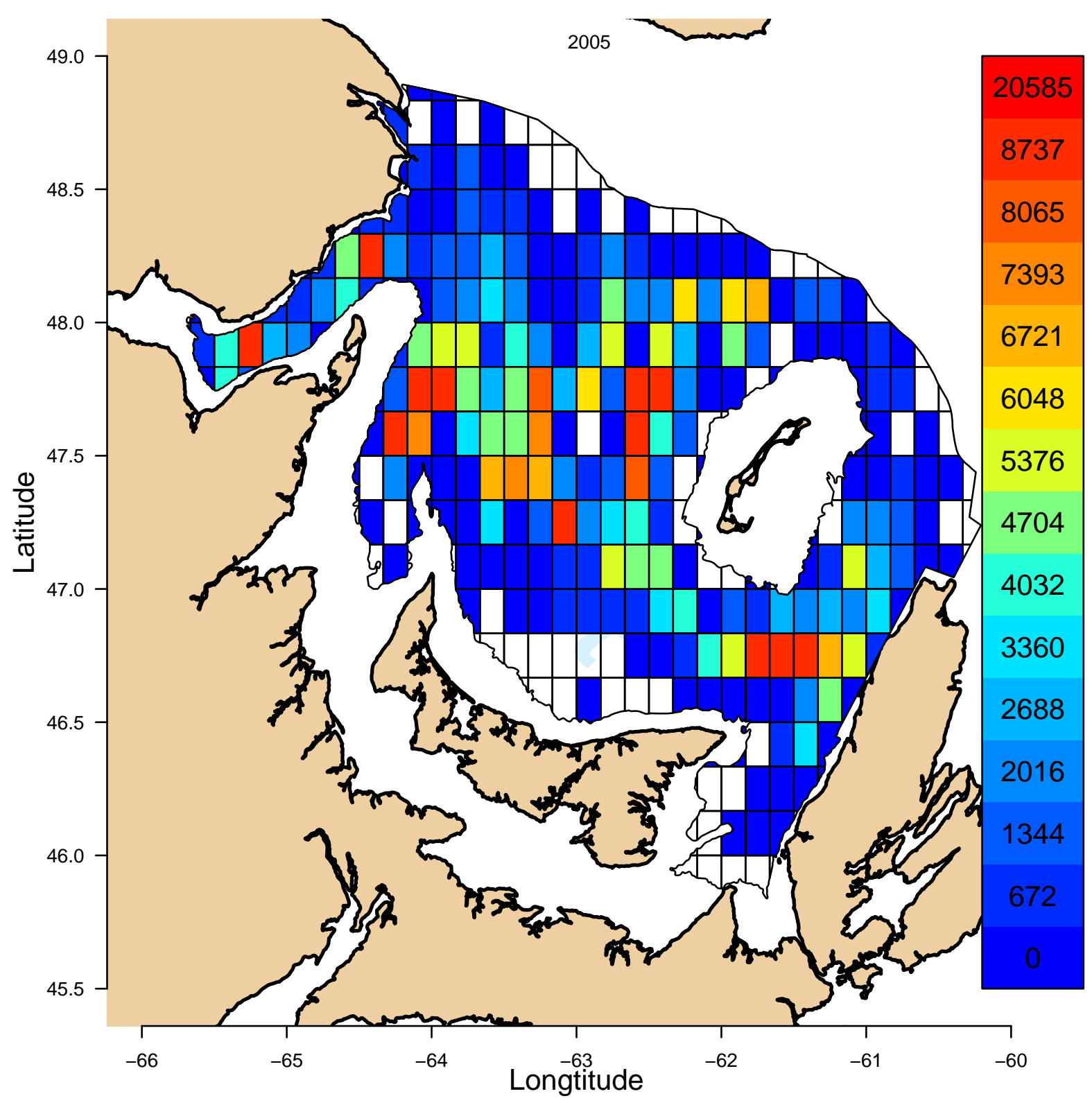

Figure SE.11a. Total annual effort (number of pots) for snow crab in each grid cell in 2005. Colors correspond to effort levels, as indicated in the legend on the right-hand side. Darkest red grids indicate effort $>98$ th percentile. 
Total $=534410$ pots, 2005
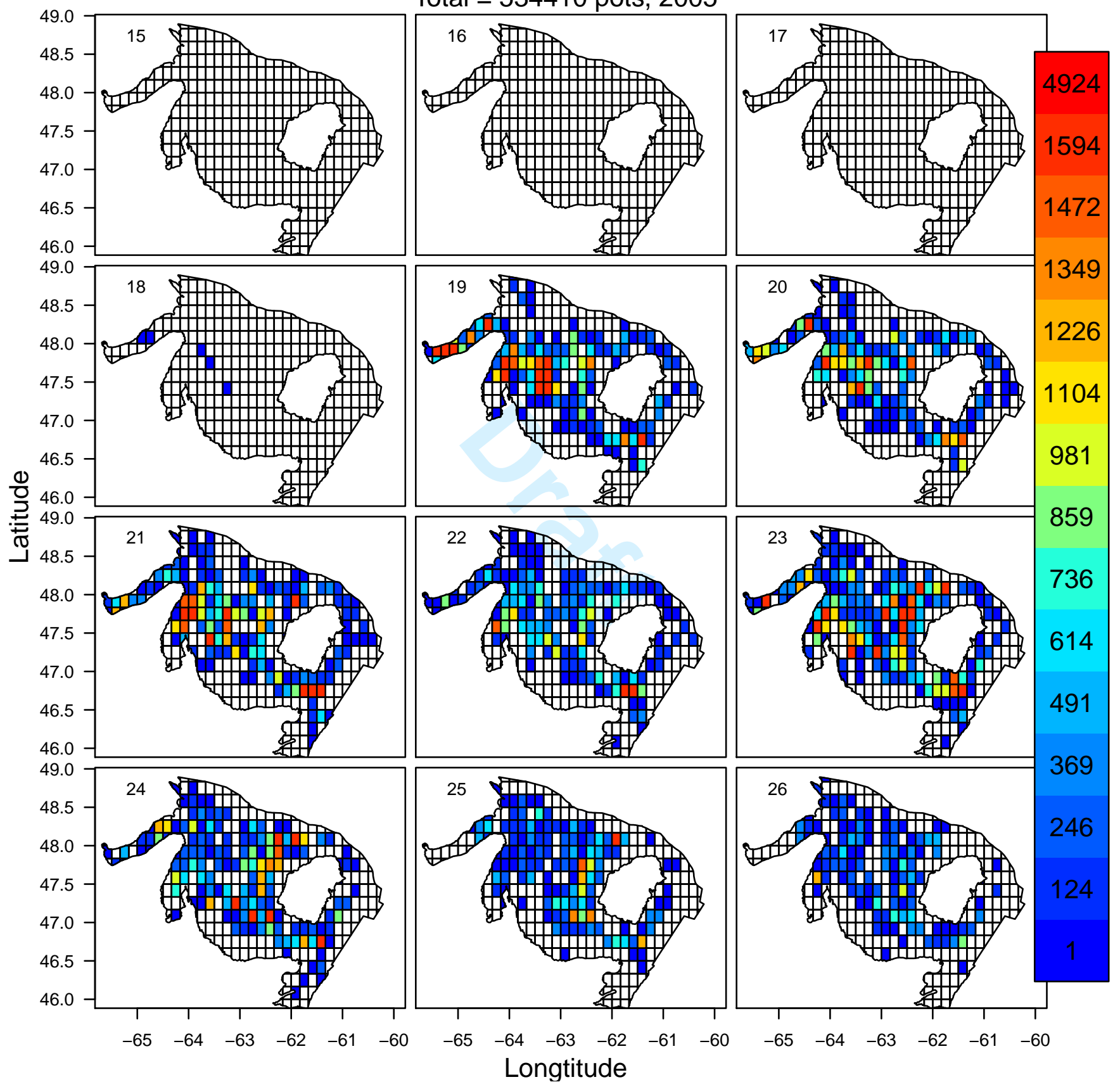

Figure SE.11b. Effort (number of pots) for snow crab in each week and grid cell in 2005 . The week number is indicated in the top left-hand corner. Colors correspond to effort levels, as indicated in the legend on the right-hand side. Darkest red grids indicate effort $>98$ th percentile. 


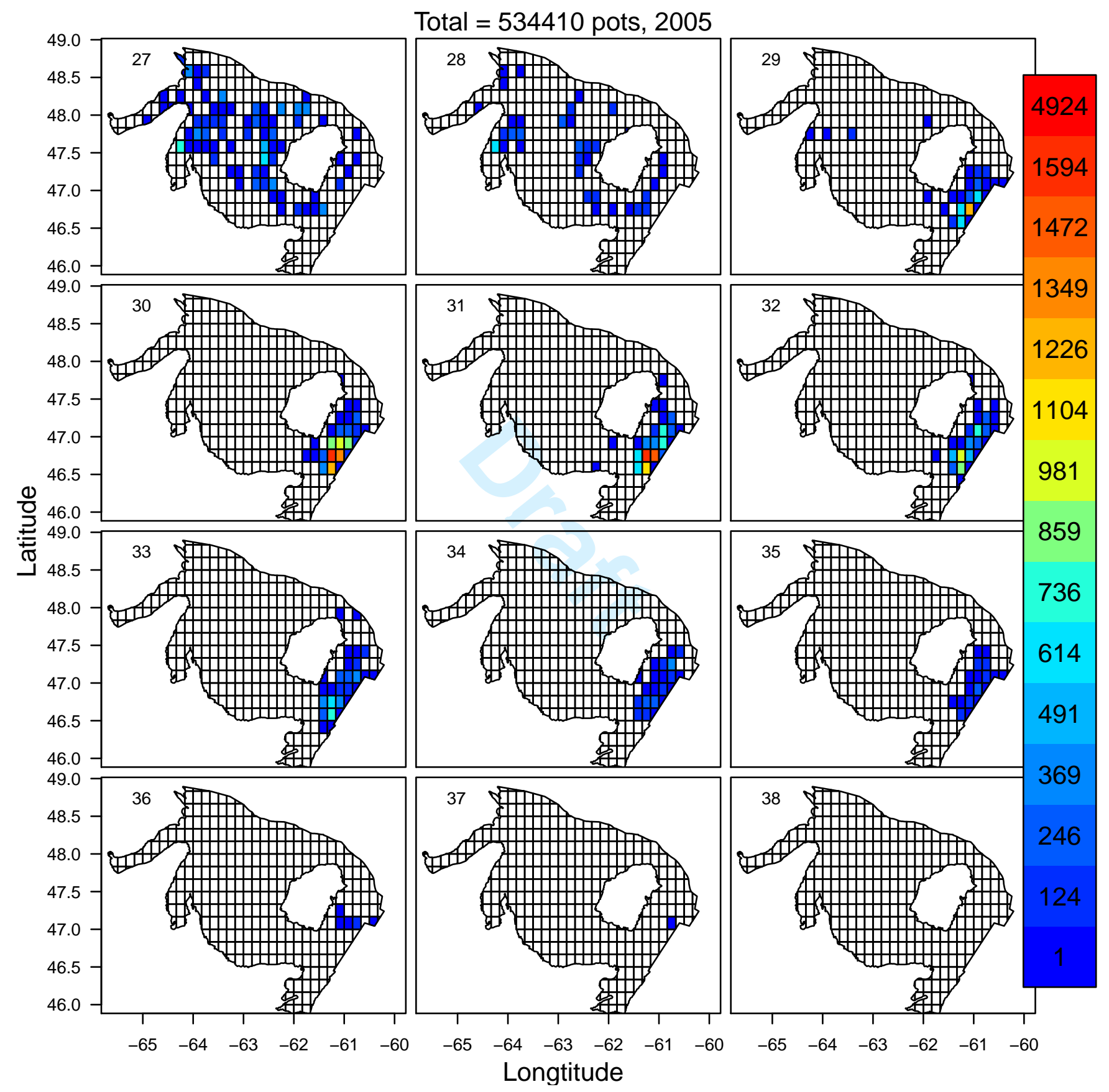

Figure SE.11c. Effort (number of pots) for snow crab in each week and grid cell in 2005. The week number is indicated in the top left-hand corner. Colors correspond to effort levels, as indicated in the legend on the right-hand side. Darkest red grids indicate effort $>98$ th percentile. 


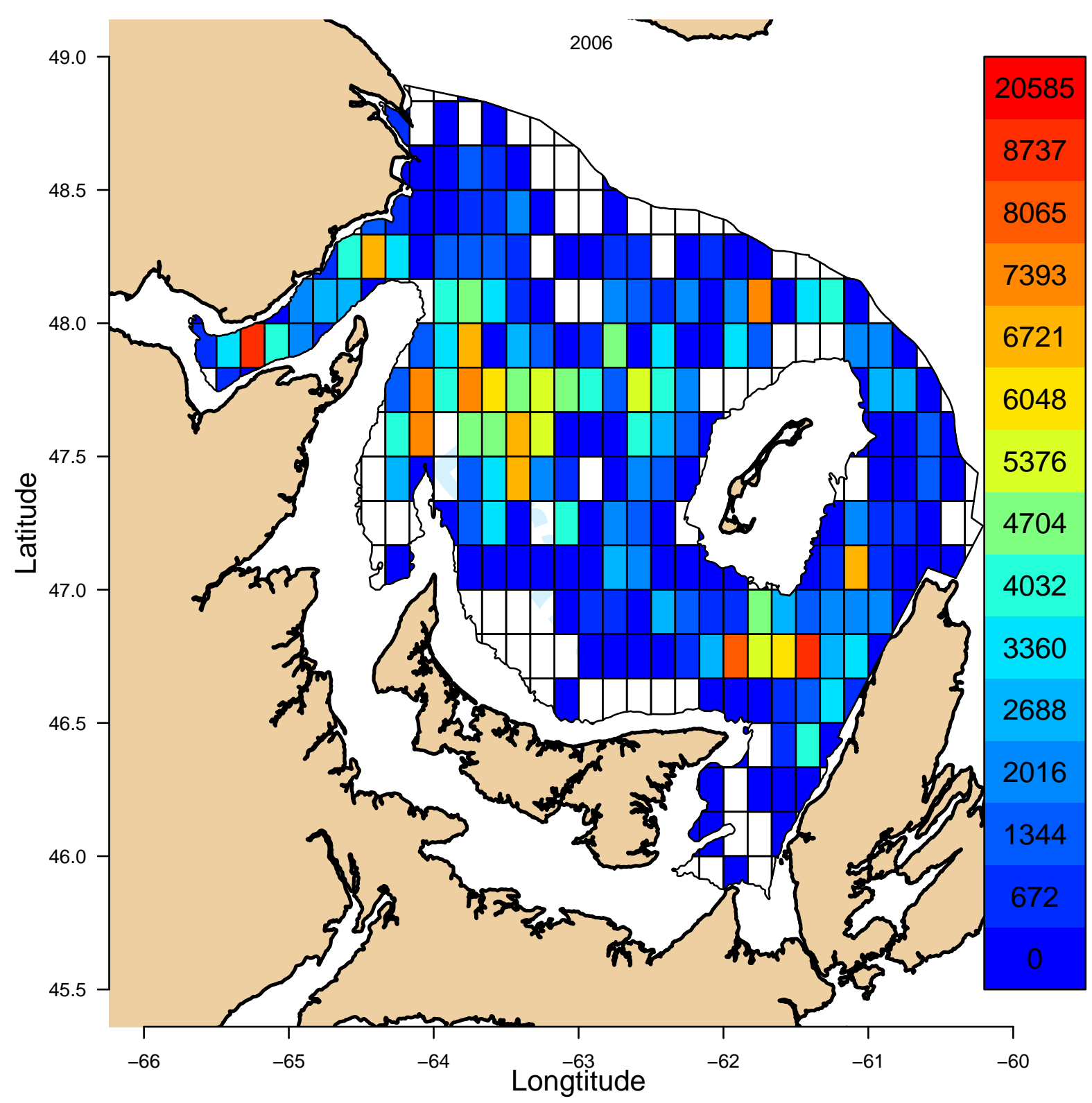

Figure SE.12a. Total annual effort (number of pots) for snow crab in each grid cell in 2006. Colors correspond to effort levels, as indicated in the legend on the right-hand side. Darkest red grids indicate effort $>98$ th percentile. 


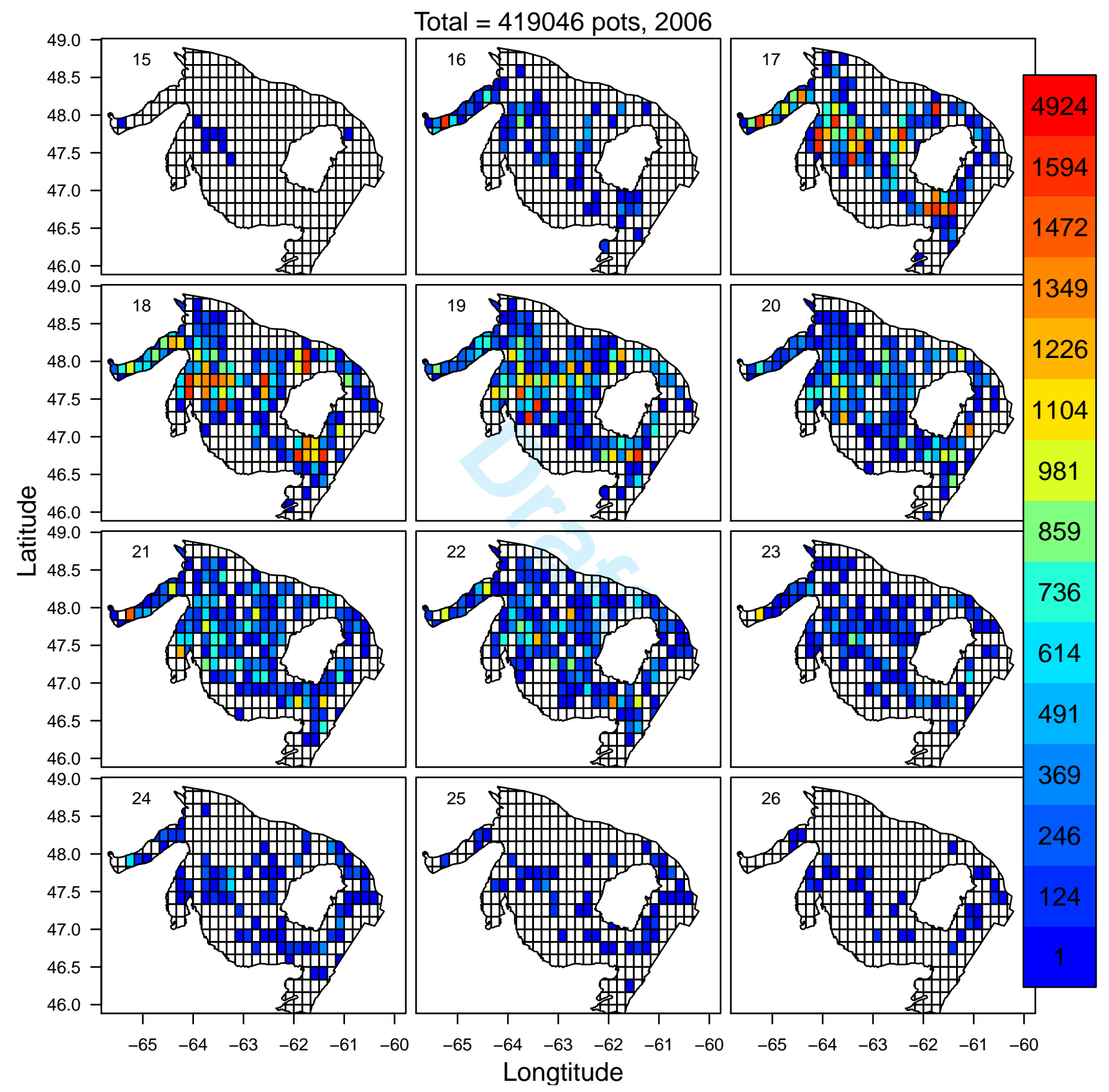

Figure SE.12b. Effort (number of pots) for snow crab in each week and grid cell in 2006. The week number is indicated in the top left-hand corner. Colors correspond to effort levels, as indicated in the legend on the right-hand side. Darkest red grids indicate effort $>$ 98th percentile. 
Total $=419046$ pots, 2006
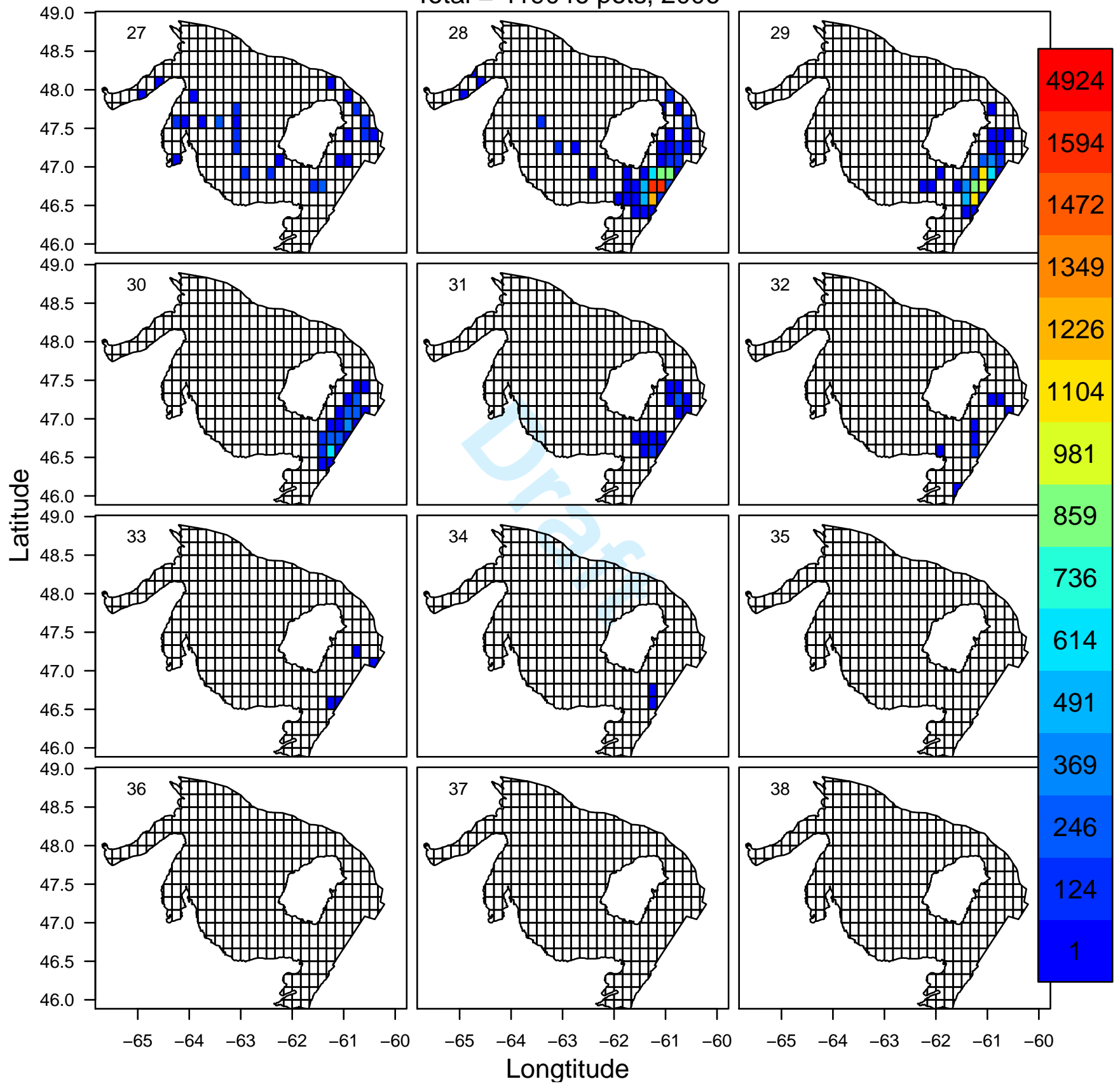

Figure SE.12c. Effort (number of pots) for snow crab in each week and grid cell in 2006. The week number is indicated in the top left-hand corner. Colors correspond to effort levels, as indicated in the legend on the righthand side. Darkest red grids indicate effort $>98$ th percentile. 


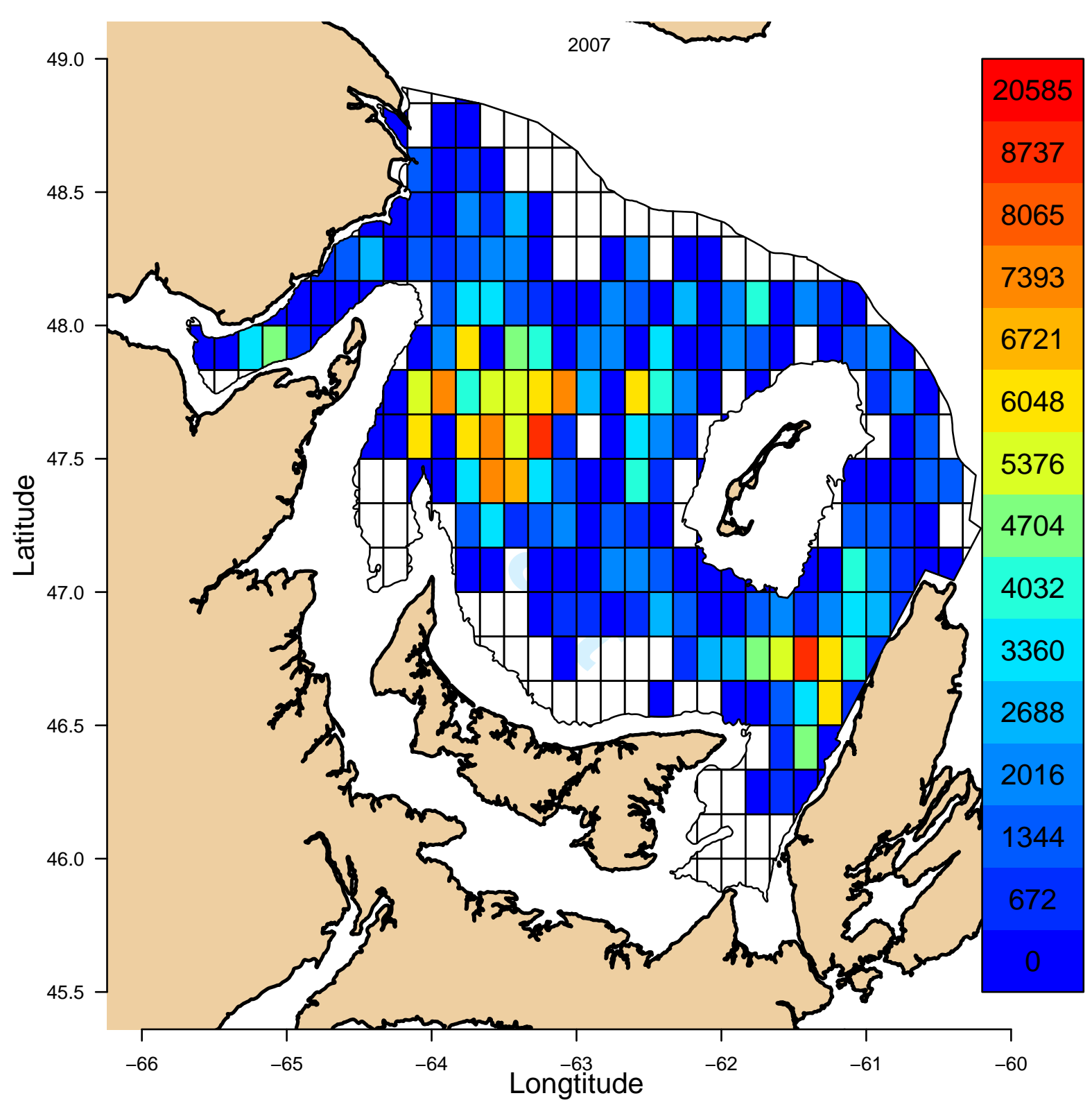

Figure SE.13a. Total annual effort (number of pots) for snow crab in each grid cell in 2007. Colors correspond to effort levels, as indicated in the legend on the right-hand side. Darkest red grids indicate effort $>98$ th percentile. 
Total $=374842$ pots, 2007
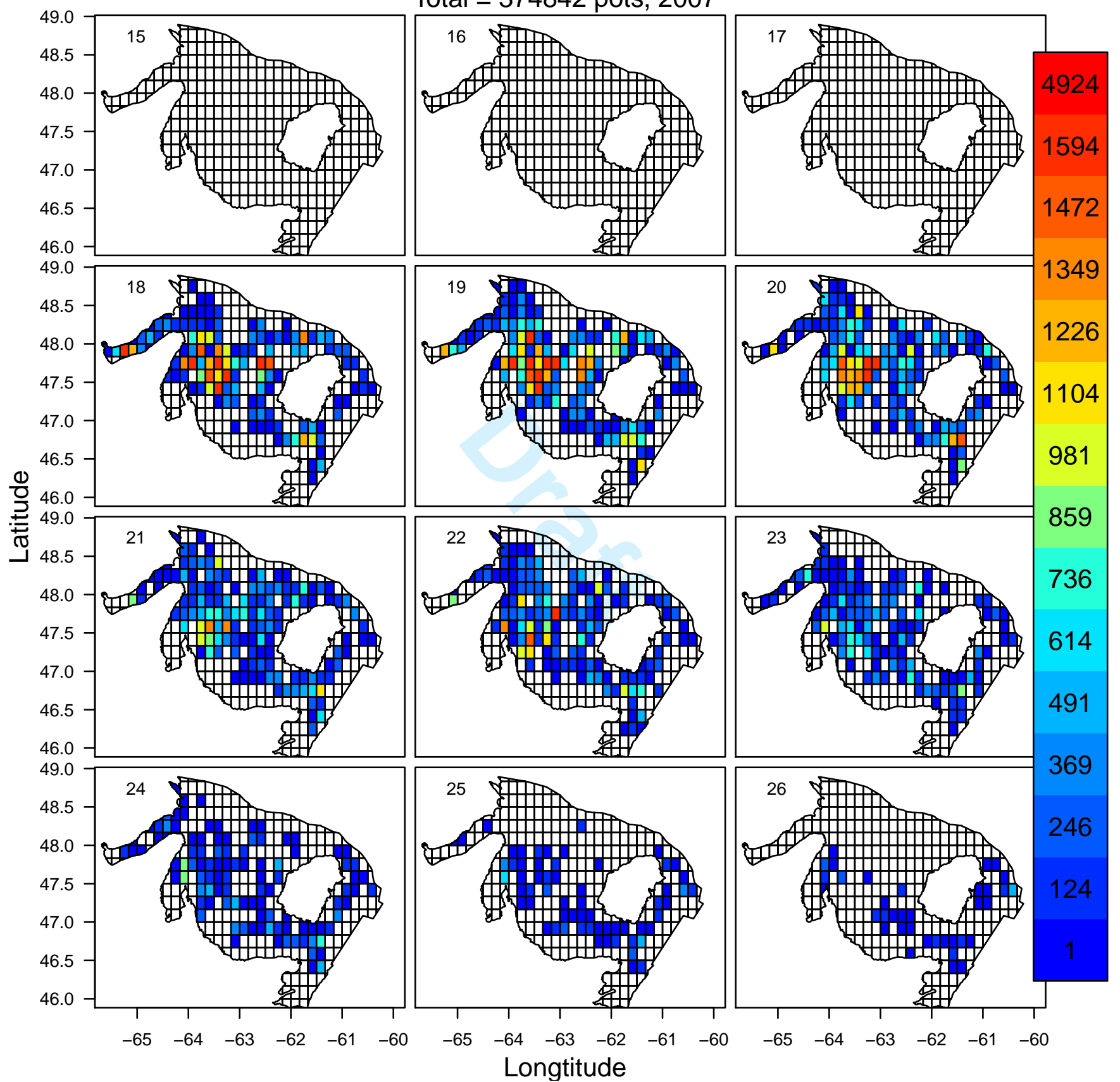

Figure SE.13b. Effort (number of pots) for snow crab in each week and grid cell in 2007. The week number is indicated in the top left-hand corner. Colors correspond to effort levels, as indicated in the legend on the righthand side. Darkest red grids indicate effort $>98$ th percentile. 


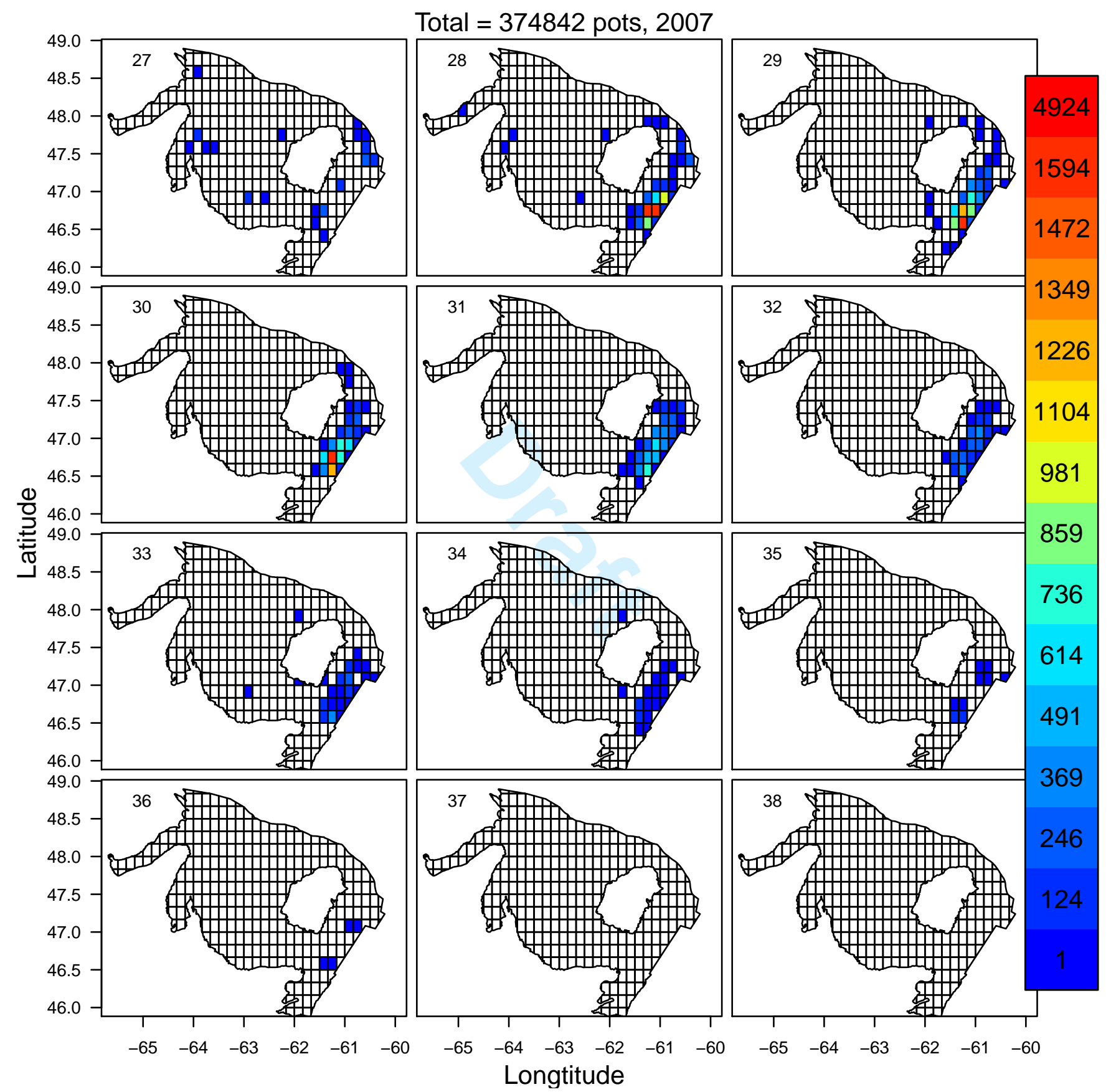

Figure SE.13c. Effort (number of pots) for snow crab in each week and grid cell in 2007. The week number is indicated in the top left-hand corner. Colors correspond to effort levels, as indicated in the legend on the right-hand side. Darkest red grids indicate effort $>98$ th percentile. 


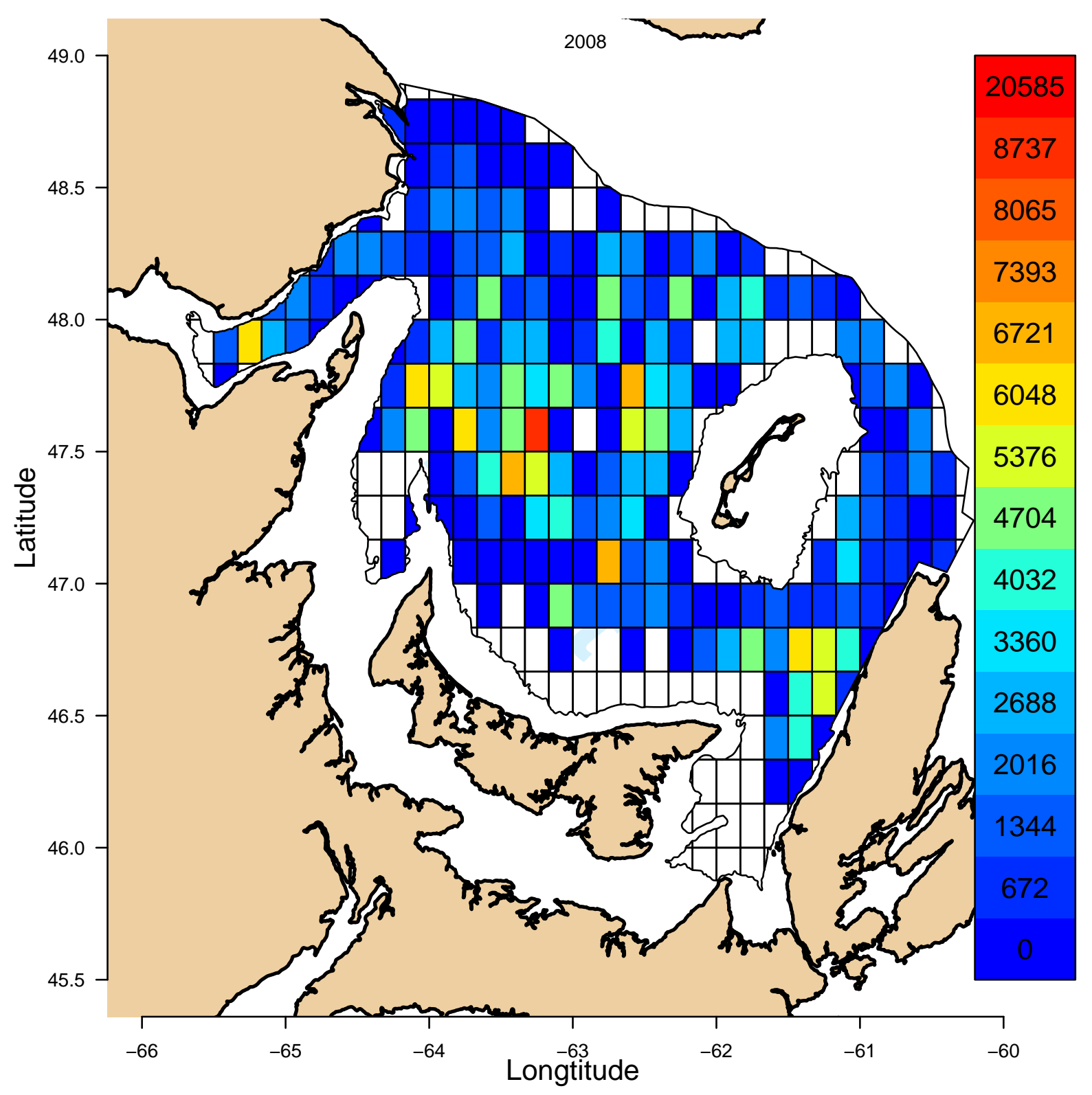

Figure SE.14a. Total annual effort (number of pots) for snow crab in each grid cell in 2008. Colors correspond to effort levels, as indicated in the legend on the right-hand side. Darkest red grids indicate effort $>98$ th percentile. 


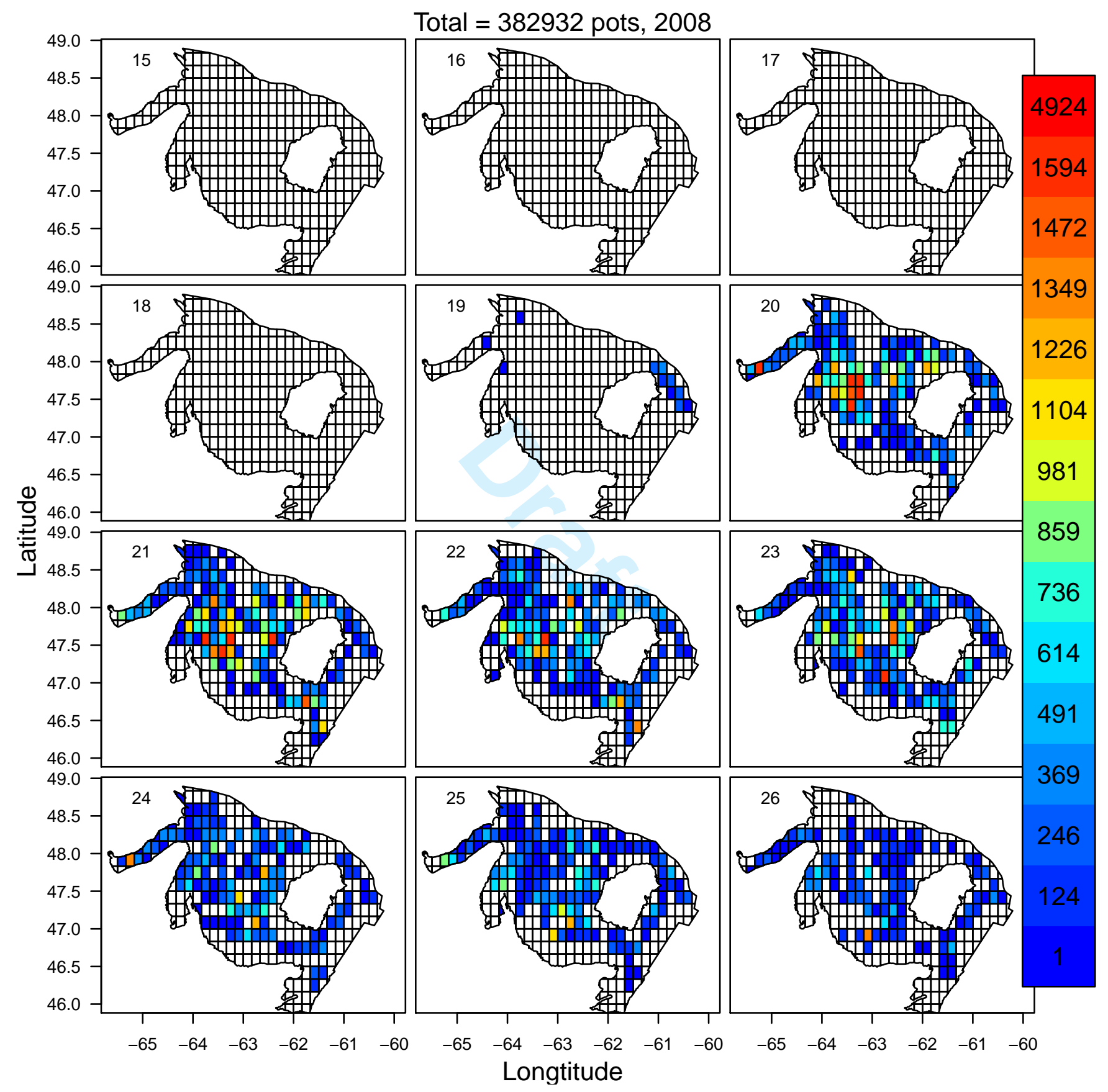

Figure SE.14b. Effort (number of pots) for snow crab in each week and grid cell in 2008. The week number is indicated in the top left-hand corner. Colors correspond to effort levels, as indicated in the legend on the right-hand side. Darkest red grids indicate effort $>98$ th percentile. 
Total $=382932$ pots, 2008
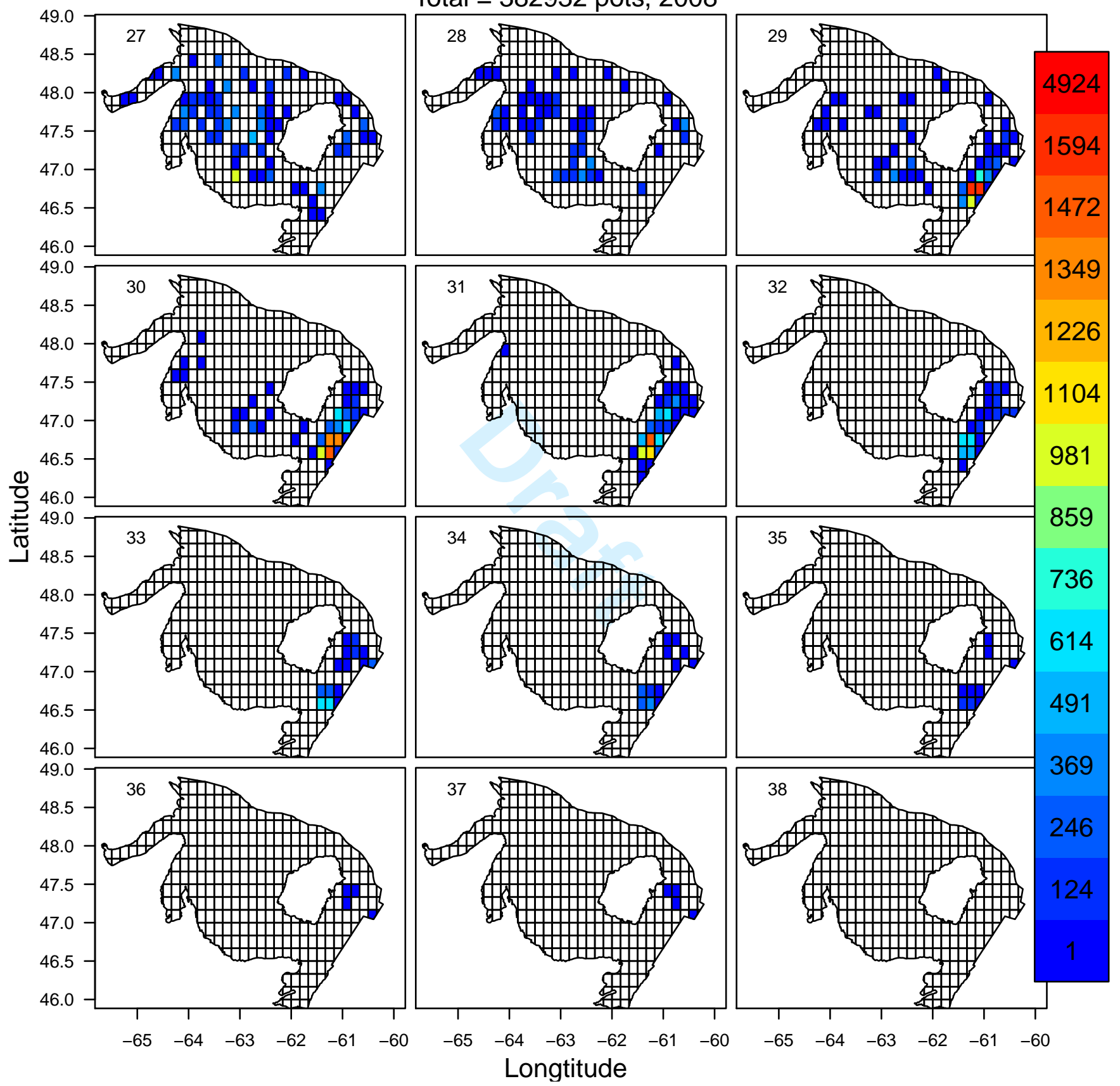

Figure SE.14c. Effort (number of pots) for snow crab in each week and grid cell in 2008. The week number is indicated in the top left-hand corner. Colors correspond to effort levels, as indicated in the legend on the right-hand side. Darkest red grids indicate effort $>98$ th percentile. 


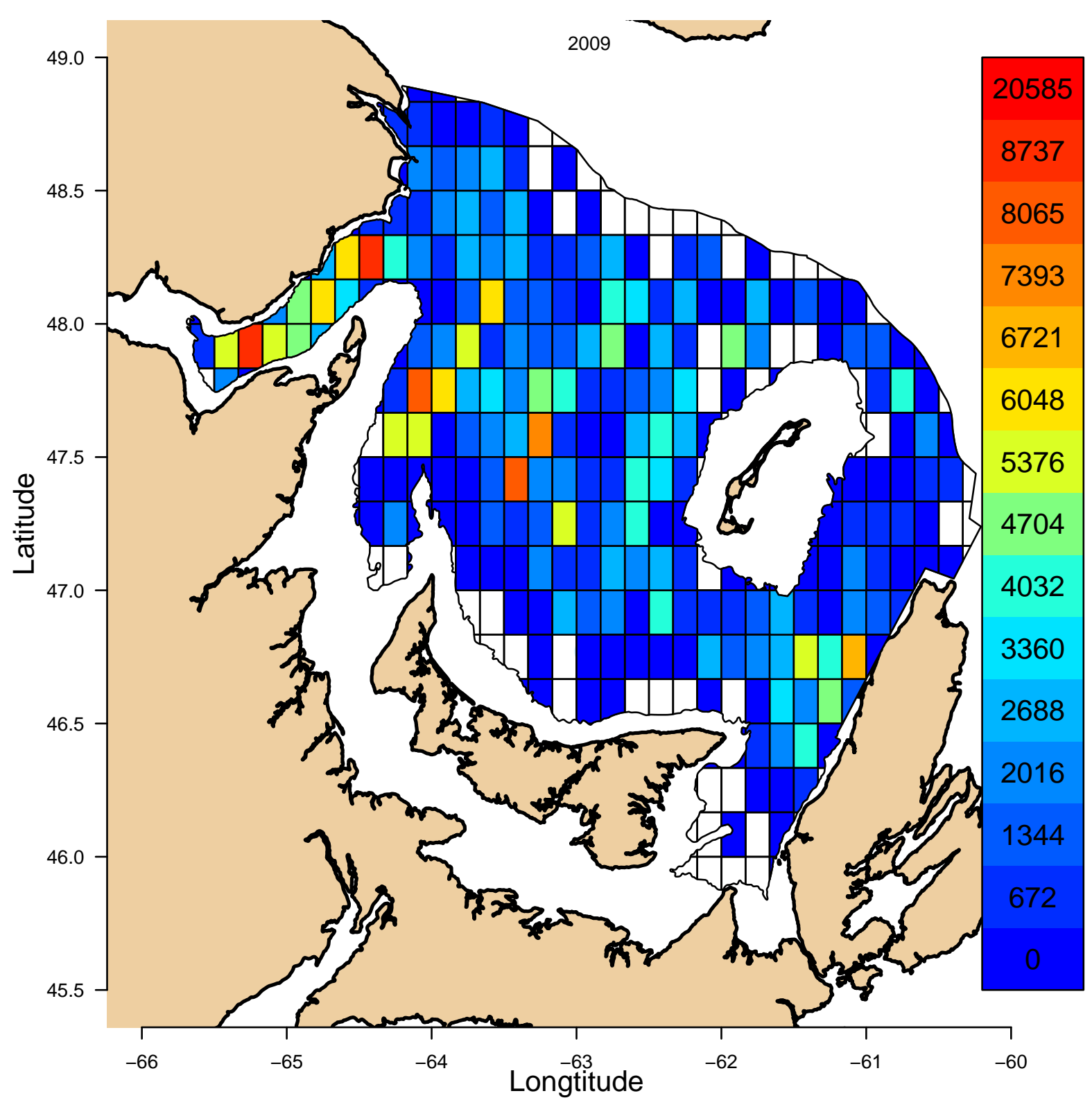

Figure SE.15a. Total annual effort (number of pots) for snow crab in each grid cell in 2009. Colors correspond to effort levels, as indicated in the legend on the right-hand side. Darkest red grids indicate effort $>98$ th percentile. 
Total $=446522$ pots, 2009
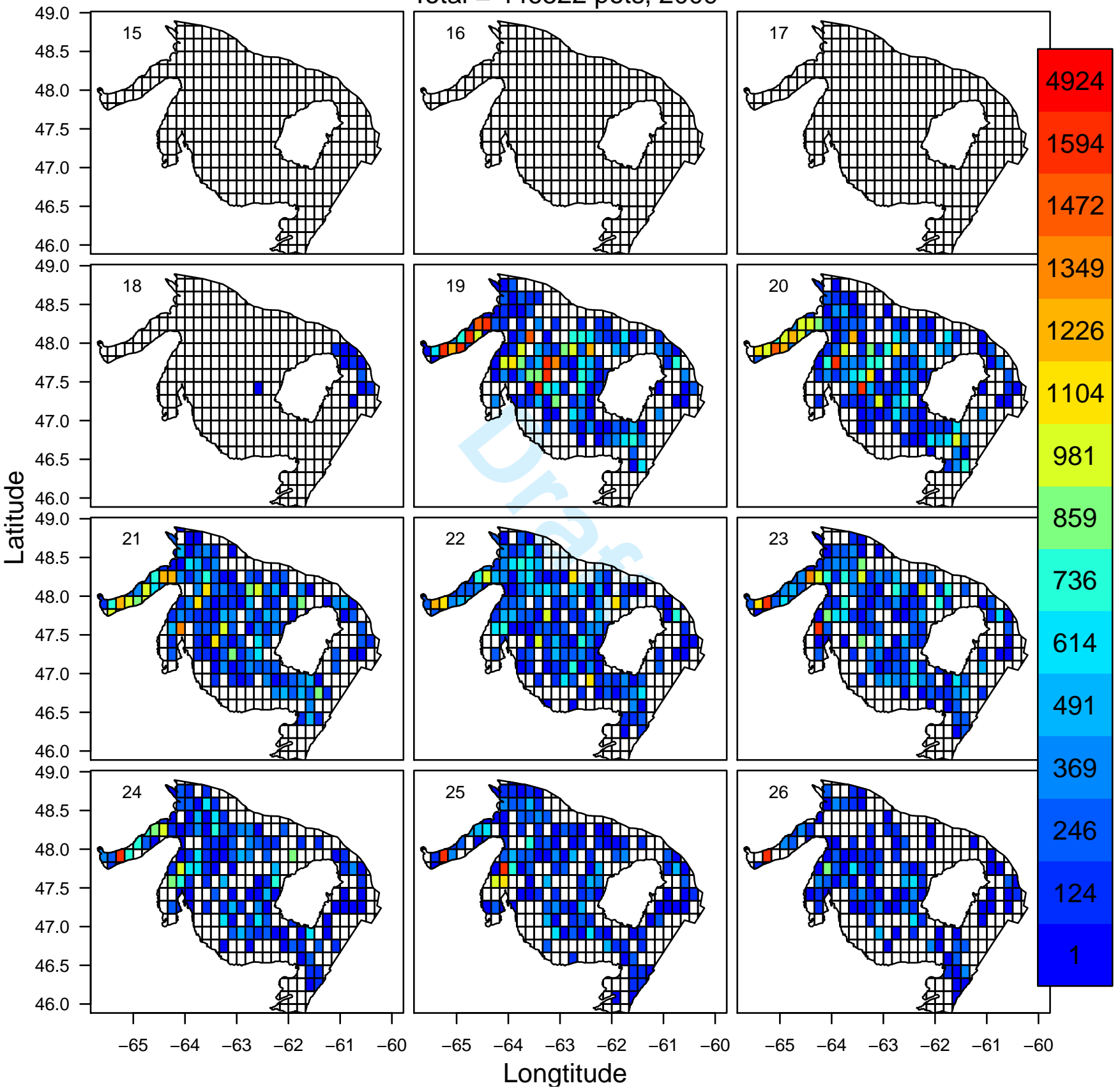

Figure SE.15b. Effort (number of pots) for snow crab in each week and grid cell in 2009. The week number is indicated in the top left-hand corner. Colors correspond to effort levels, as indicated in the legend on the right-hand side. Darkest red grids indicate effort $>$ 98th percentile. 


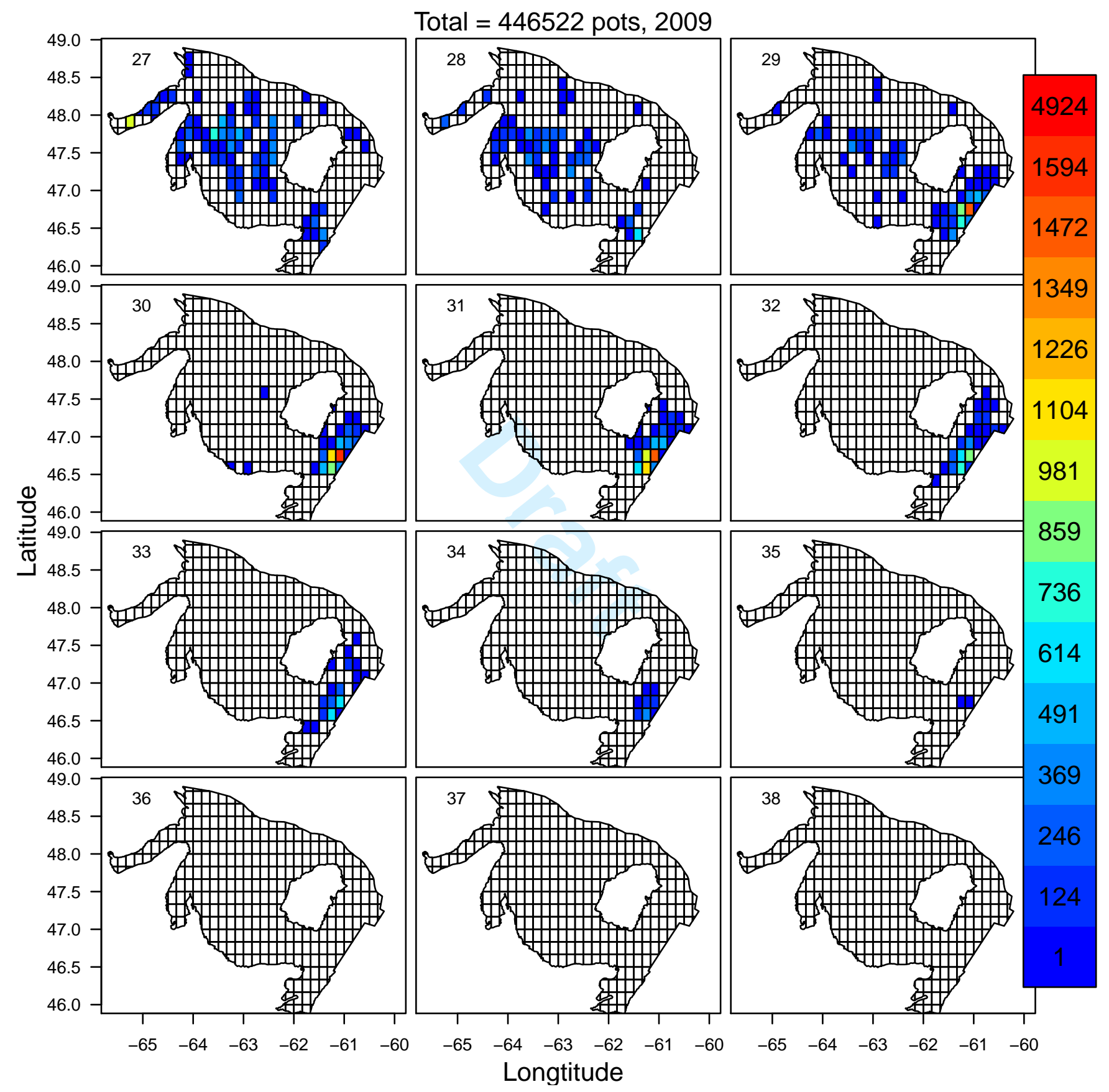

Figure SE.15c. Effort (number of pots) for snow crab in each week and grid cell in 2009. The week number is indicated in the top left-hand corner. Colors correspond to effort levels, as indicated in the legend on the right-hand side. Darkest red grids indicate effort $>98$ th percentile. 


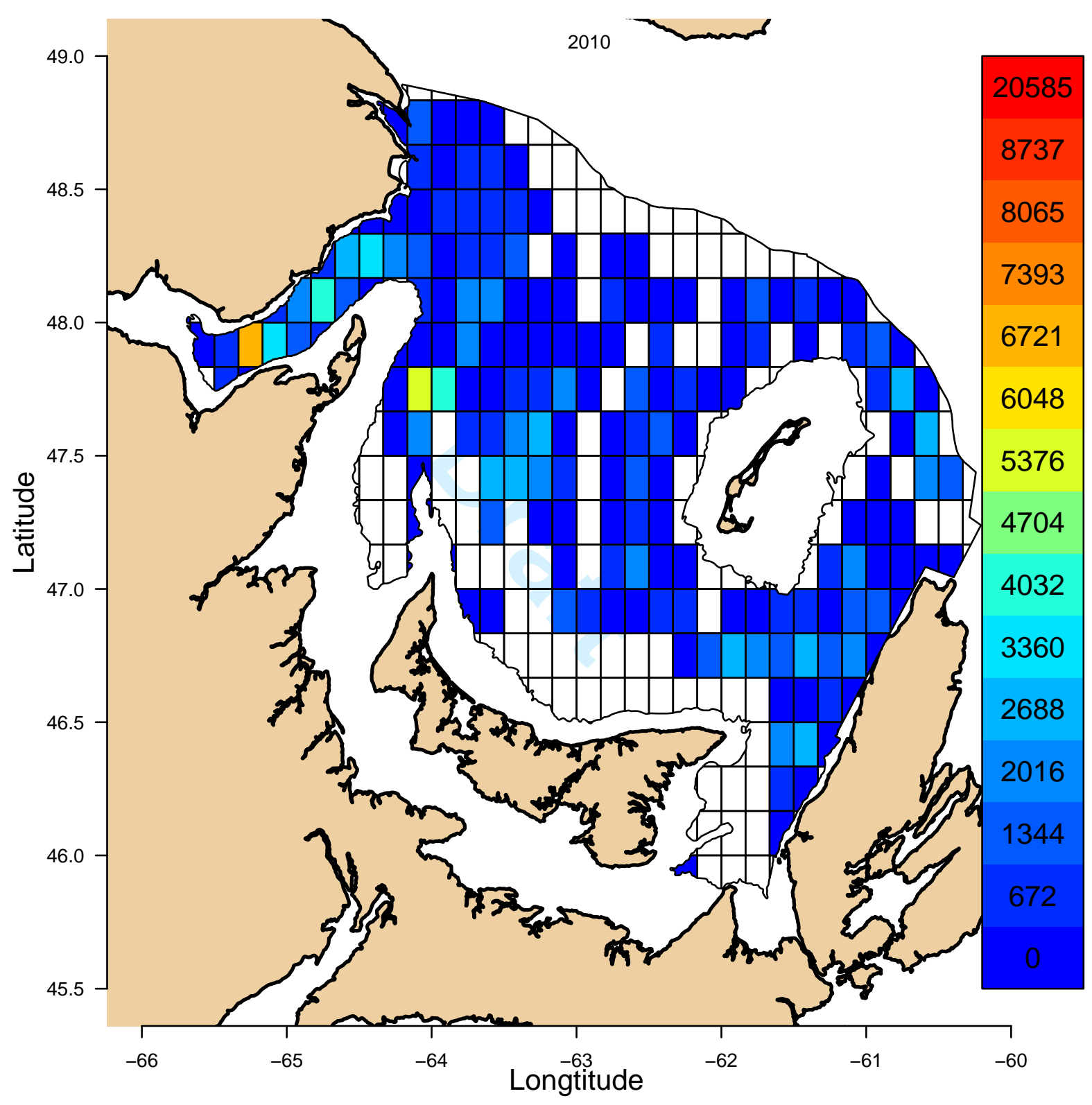

Figure SE.16a. Total annual effort (number of pots) for snow crab in each grid cell in 2010. Colors correspond to effort levels, as indicated in the legend on the right-hand side. Darkest red grids indicate effort $>98$ th percentile. 


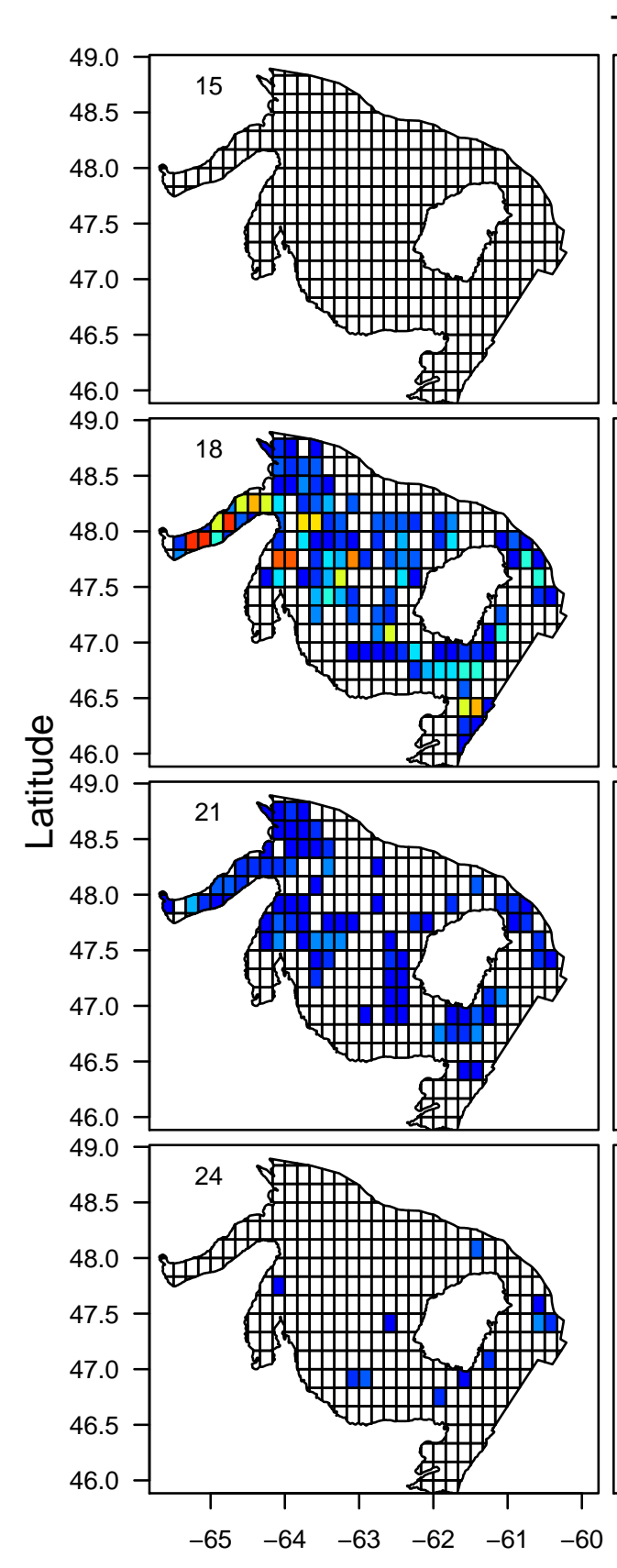

Total $=180326$ pots, 2010
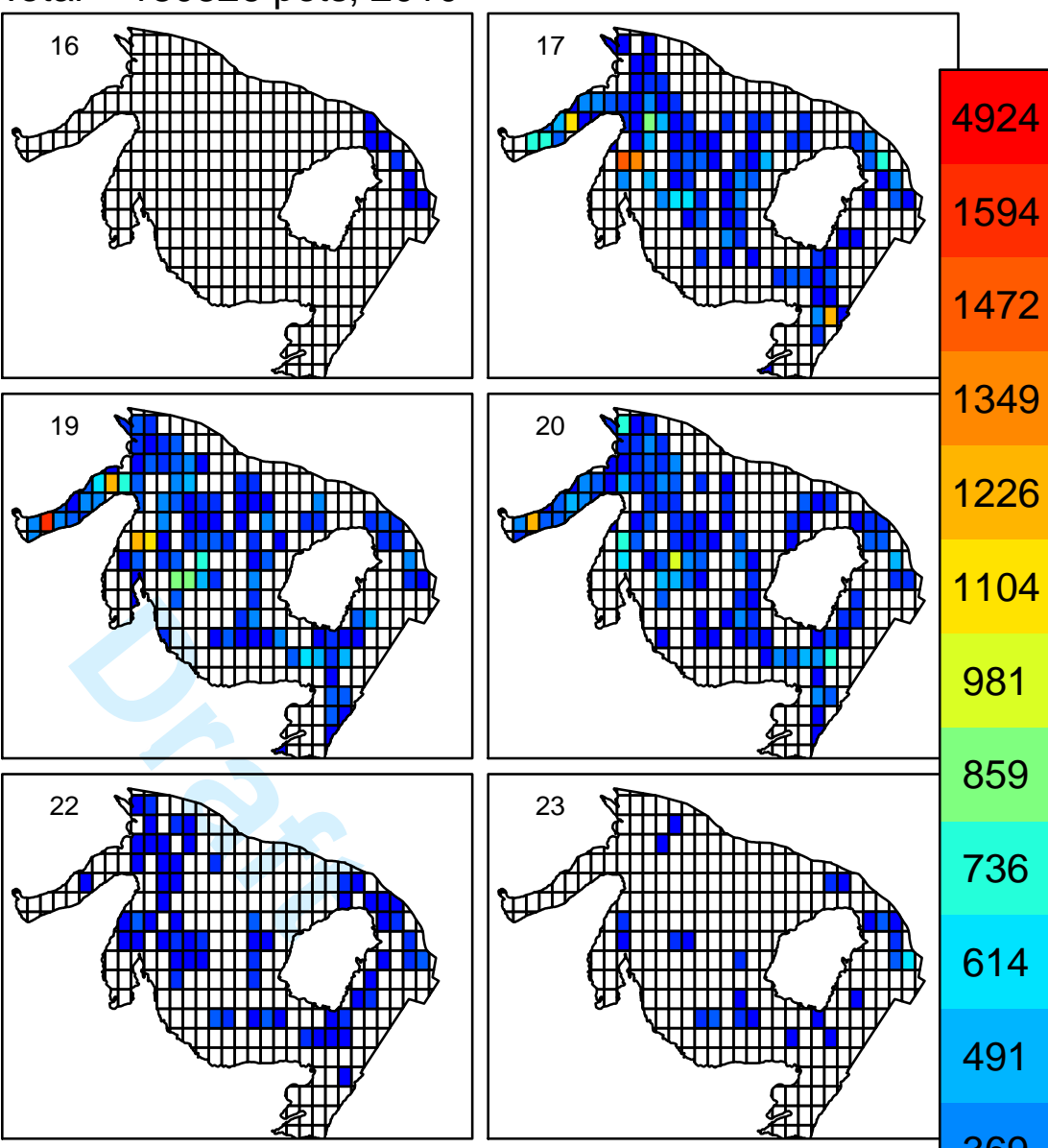

1226

1104

981

859
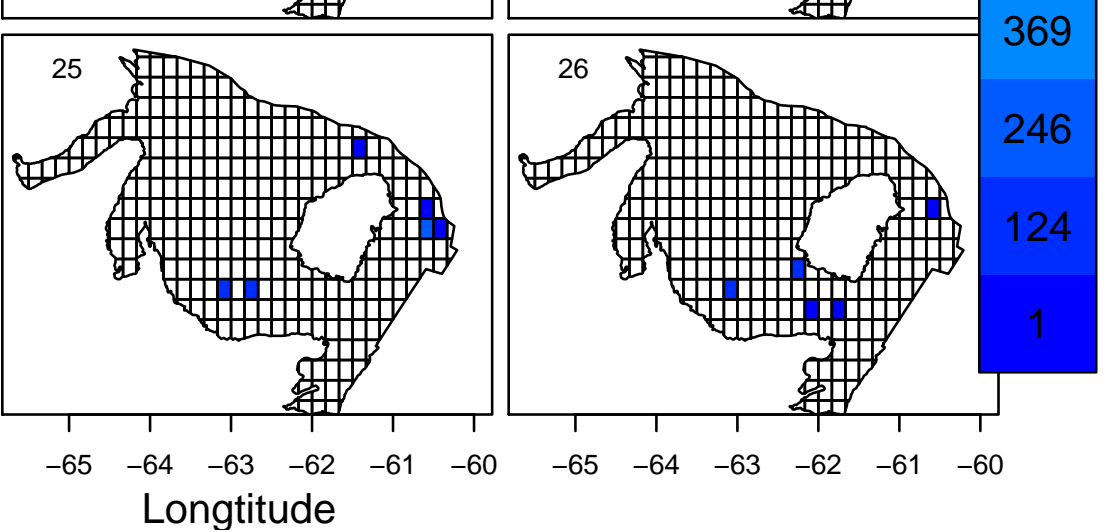

Figure SE.16b. Effort (number of pots) for snow crab in each week and grid cell in 2010. The week number is indicated in the top left-hand corner. Colors correspond to effort levels, as indicated in the legend on the right-hand side. Darkest red grids indicate effort $>98$ th percentile. 
Total $=180326$ pots, 2010
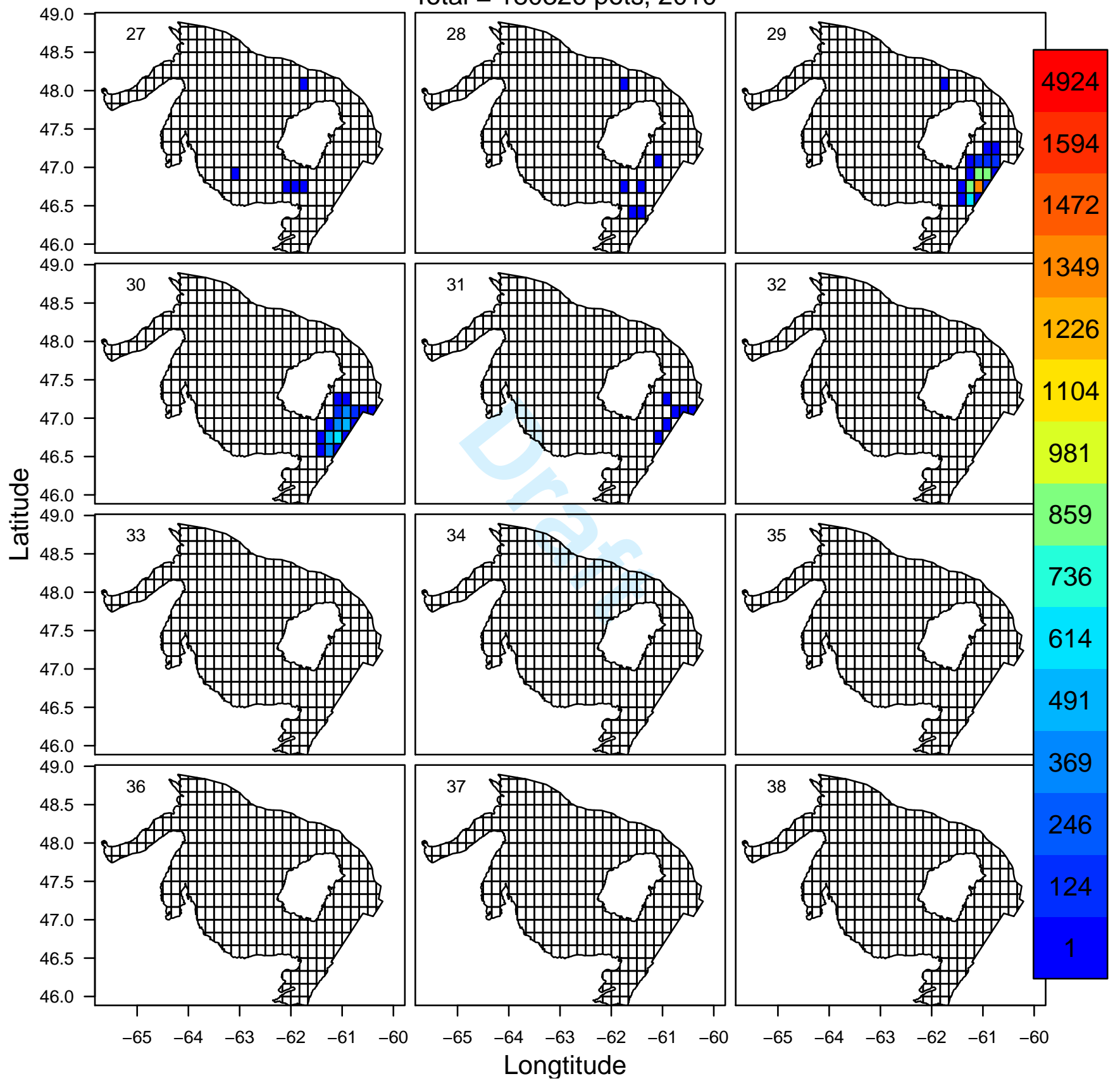

Figure SE.16c. Effort (number of pots) for snow crab in each week and grid cell in 2010. The week number is indicated in the top left-hand corner. Colors correspond to effort levels, as indicated in the legend on the right-hand side. Darkest red grids indicate effort $>98$ th percentile. 


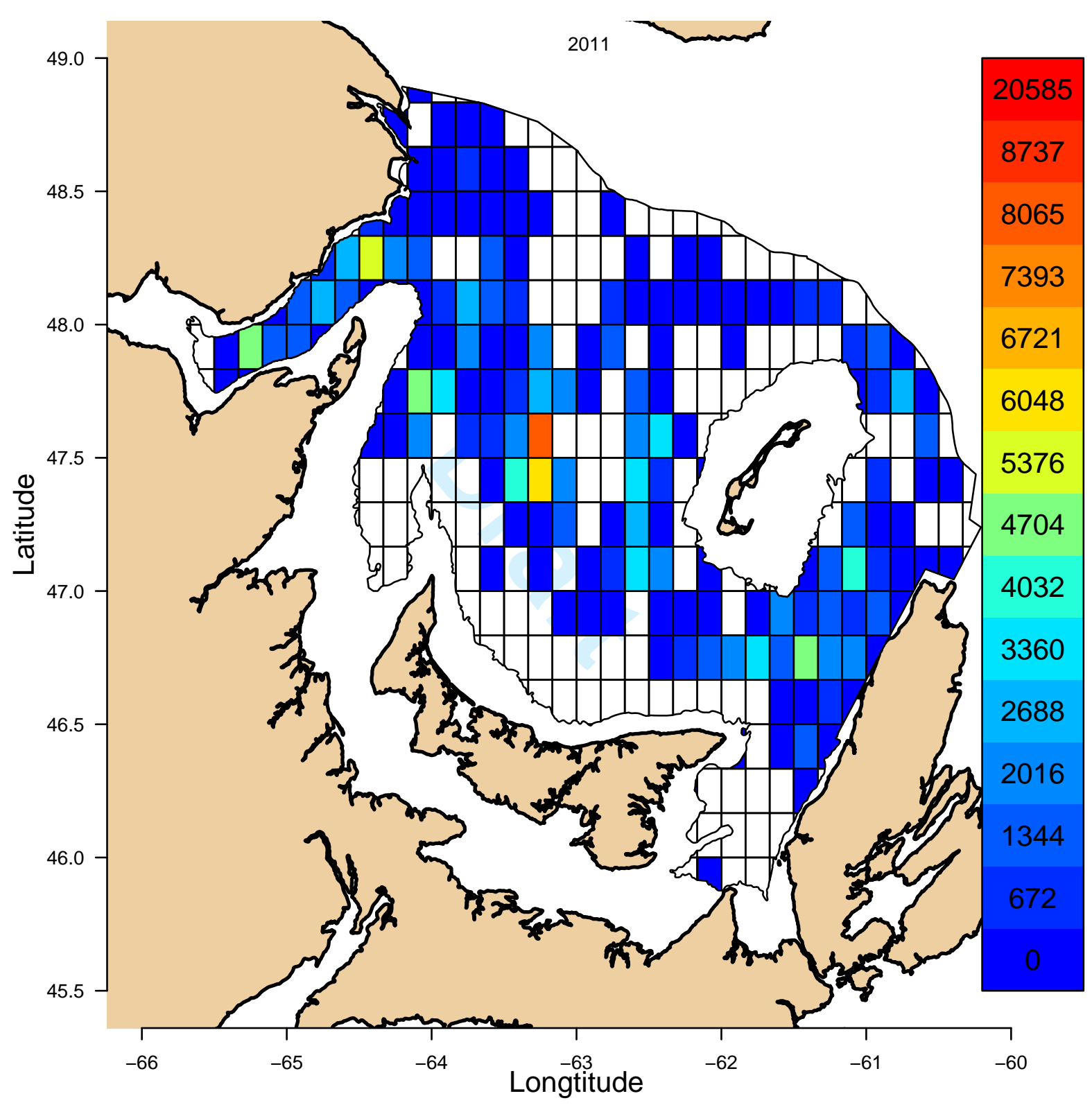

Figure SE.17a. Total annual effort (number of pots) for snow crab in each grid cell in 2011. Colors correspond to effort levels, as indicated in the legend on the right-hand side. Darkest red grids indicate effort $>98$ th percentile. 
Total $=181597$ pots, 2011
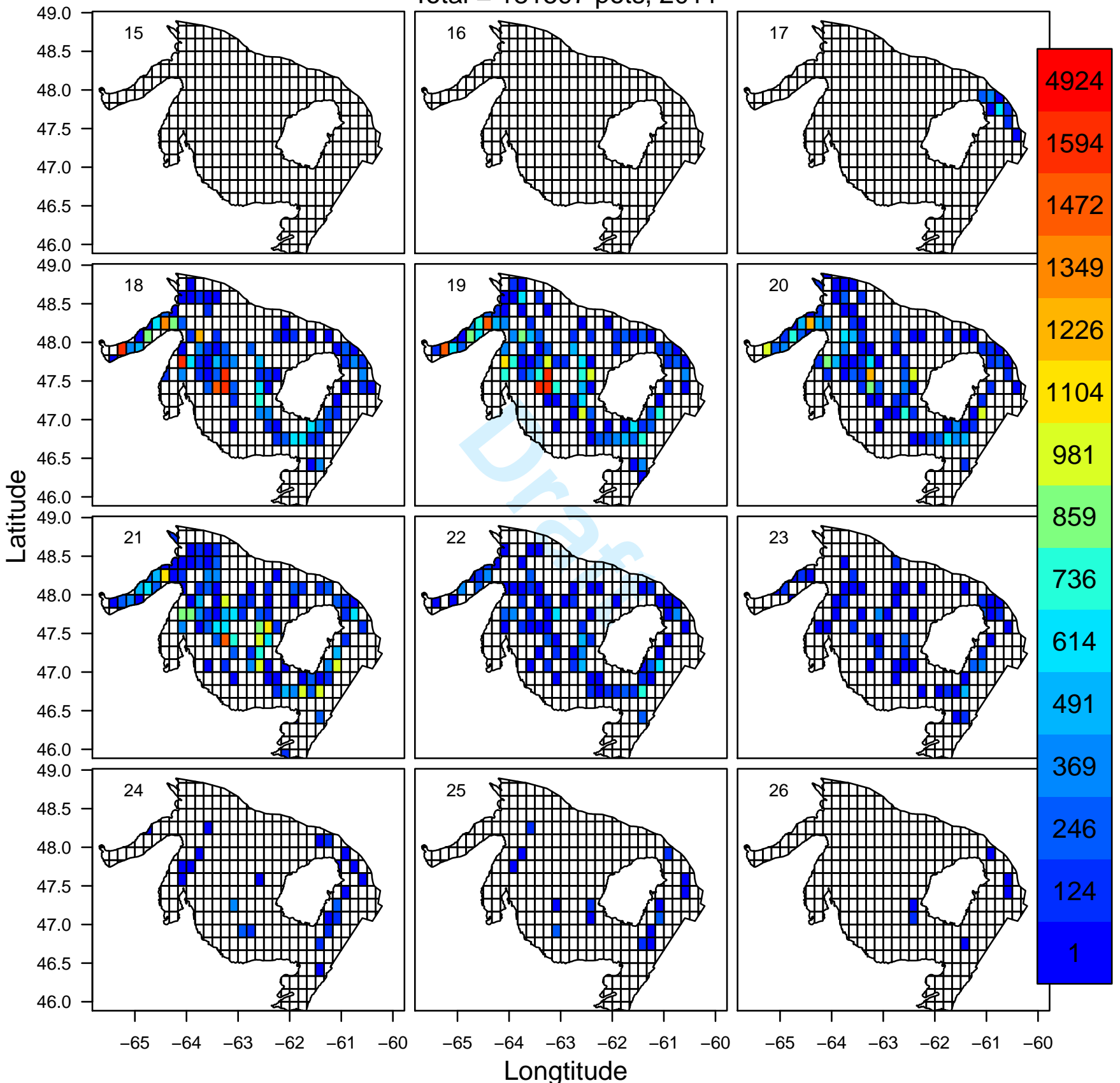

Figure SE.17b. Effort (number of pots) for snow crab in each week and grid cell in 2011. The week number is indicated in the top left-hand corner. Colors correspond to effort levels, as indicated in the legend on the right-hand side. Darkest red grids indicate effort $>98$ th percentile. 


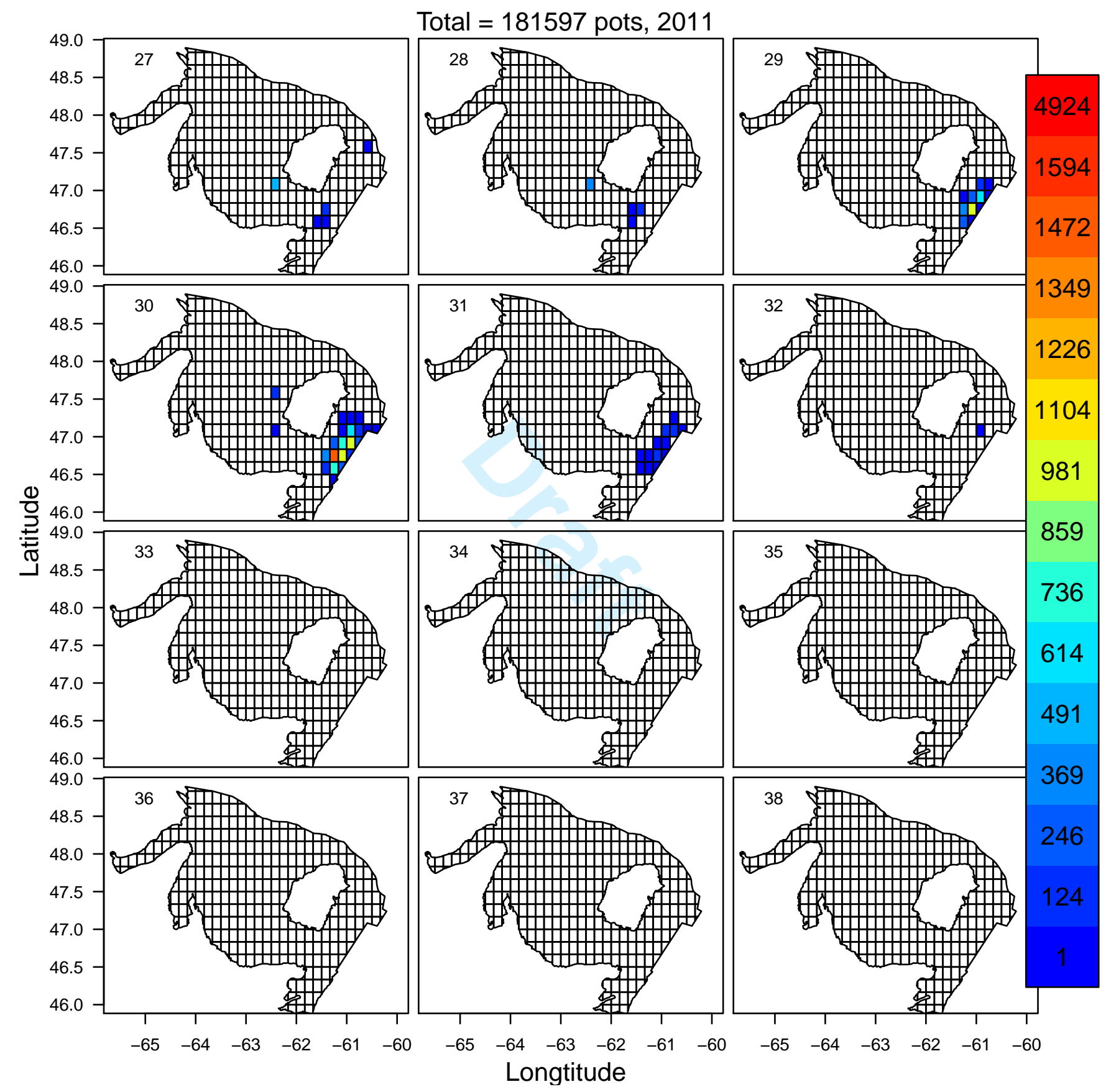

Figure SE.17c. Effort (number of pots) for snow crab in each week and grid cell in 2011. The week number is indicated in the top left-hand corner. Colors correspond to effort levels, as indicated in the legend on the right-hand side. Darkest red grids indicate effort $>98$ th percentile. 


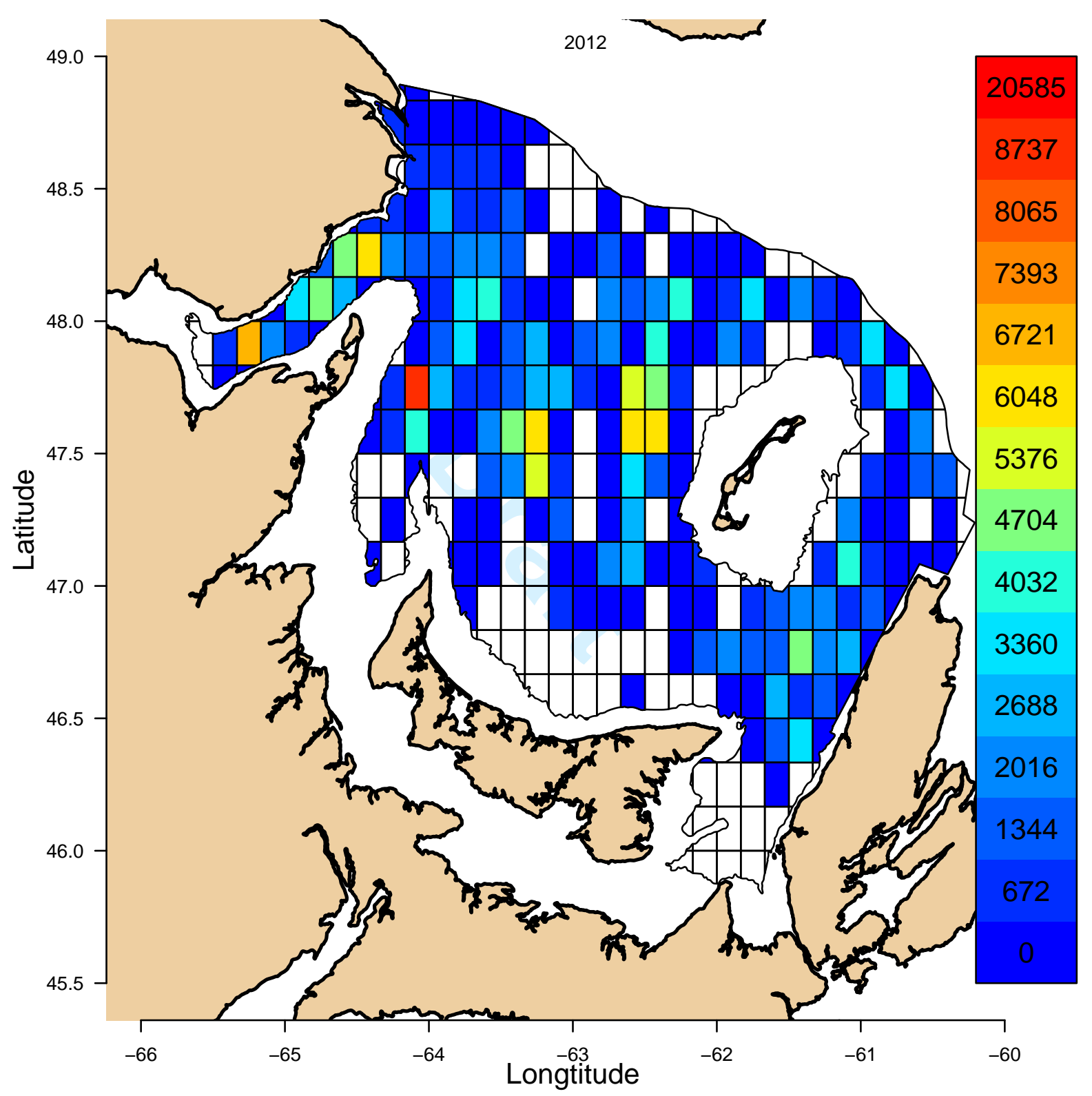

Figure SE.18a. Total annual effort (number of pots) for snow crab in each grid cell in 2012. Colors correspond to effort levels, as indicated in the legend on the right-hand side. Darkest red grids indicate effort $>98$ th percentile. 


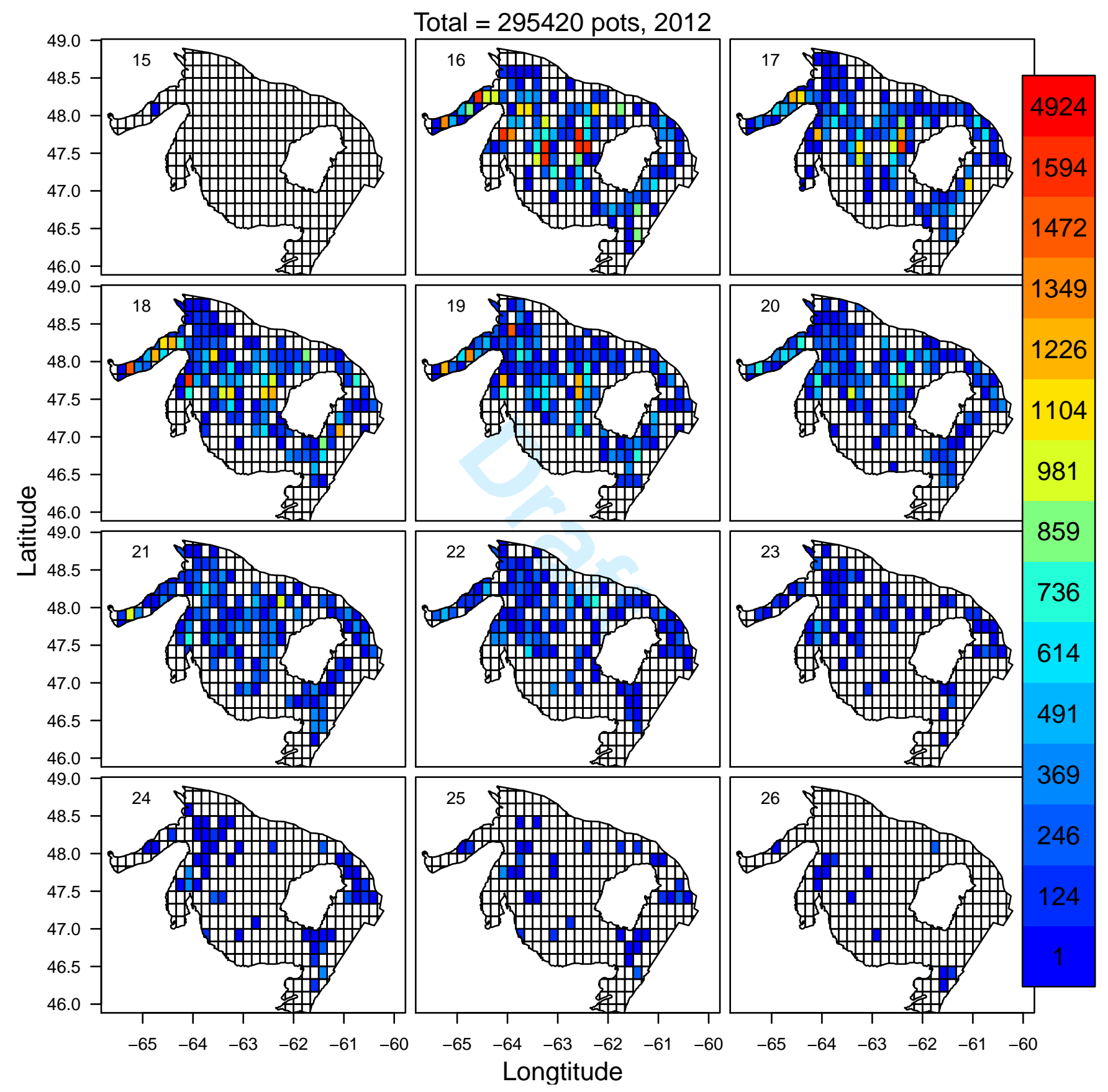

Figure SE.18b. Effort (number of pots) for snow crab in each week and grid cell in 2012. The week number is indicated in the top left-hand corner. Colors correspond to effort levels, as indicated in the legend on the right-hand side. Darkest red grids indicate effort $>98$ th percentile. 
Total $=295420$ pots, 2012
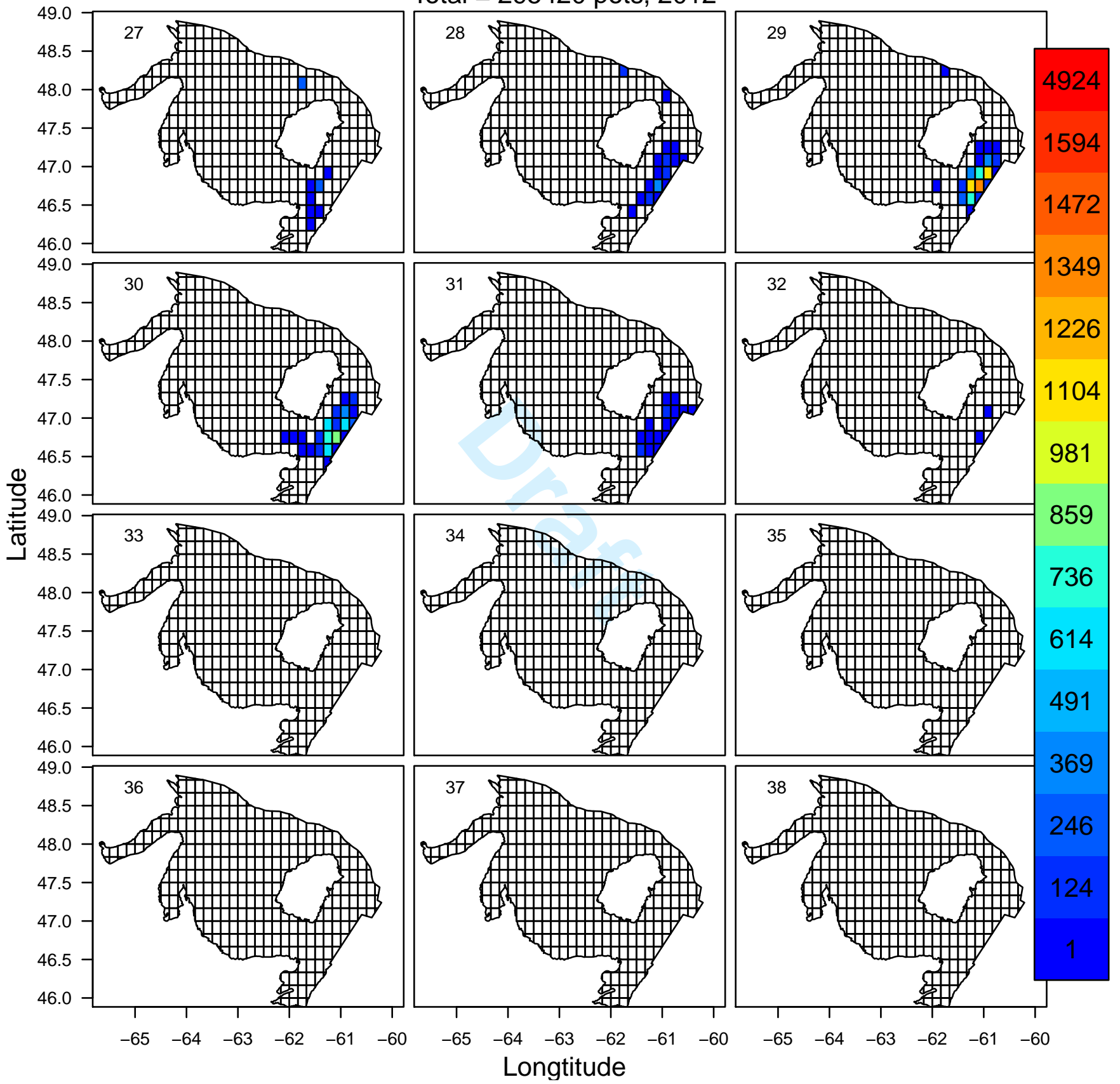

Figure SE.18c. Effort (number of pots) for snow crab in each week and grid cell in 2012. The week number is indicated in the top left-hand corner. Colors correspond to effort levels, as indicated in the legend on the right-hand side. Darkest red grids indicate effort $>98$ th percentile. 


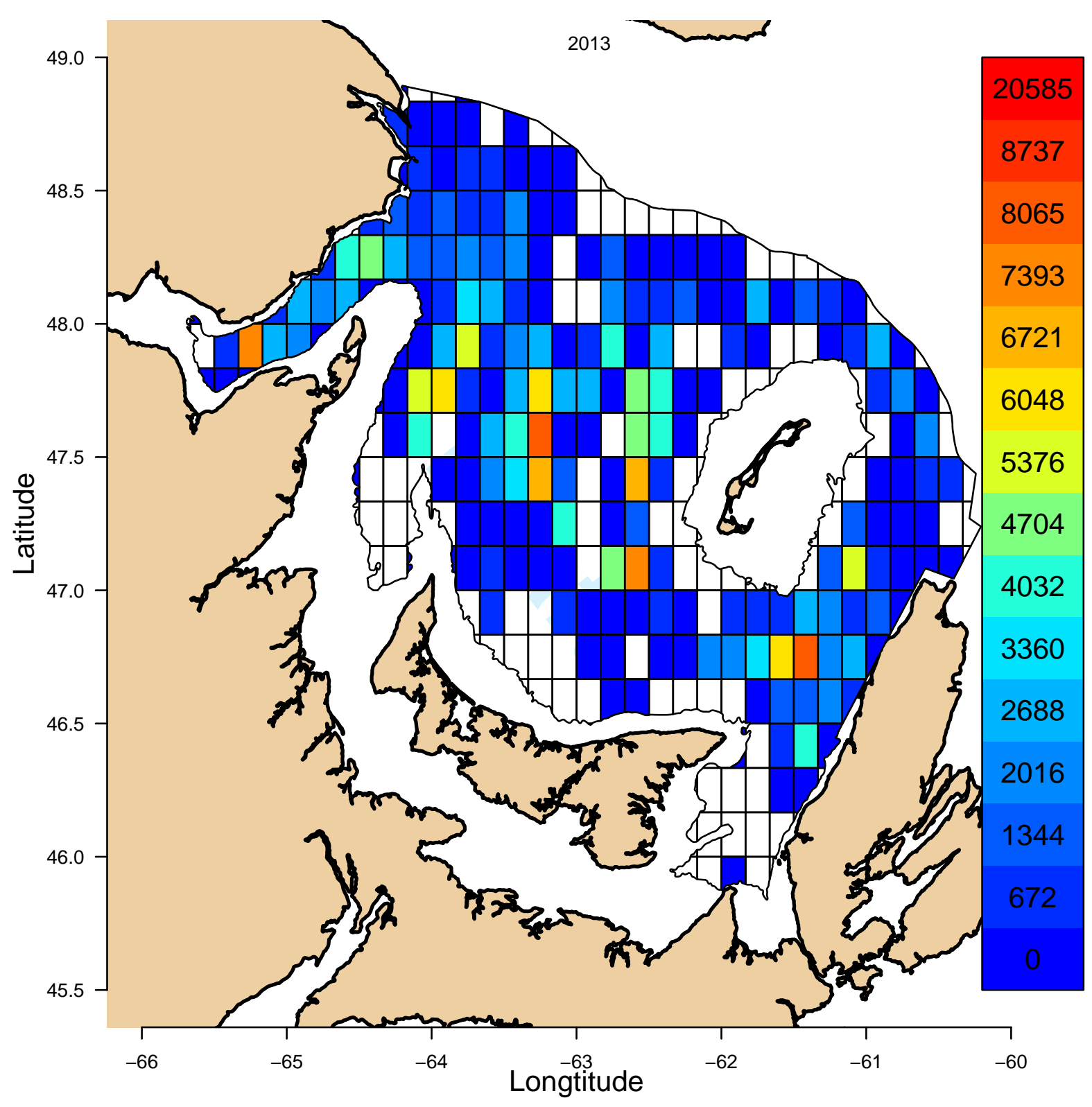

Figure SE.19a. Total annual effort (number of pots) for snow crab in each grid cell in 2013. Colors correspond to effort levels, as indicated in the legend on the right-hand side. Darkest red grids indicate effort $>98$ th percentile. 
Total $=314712$ pots, 2013
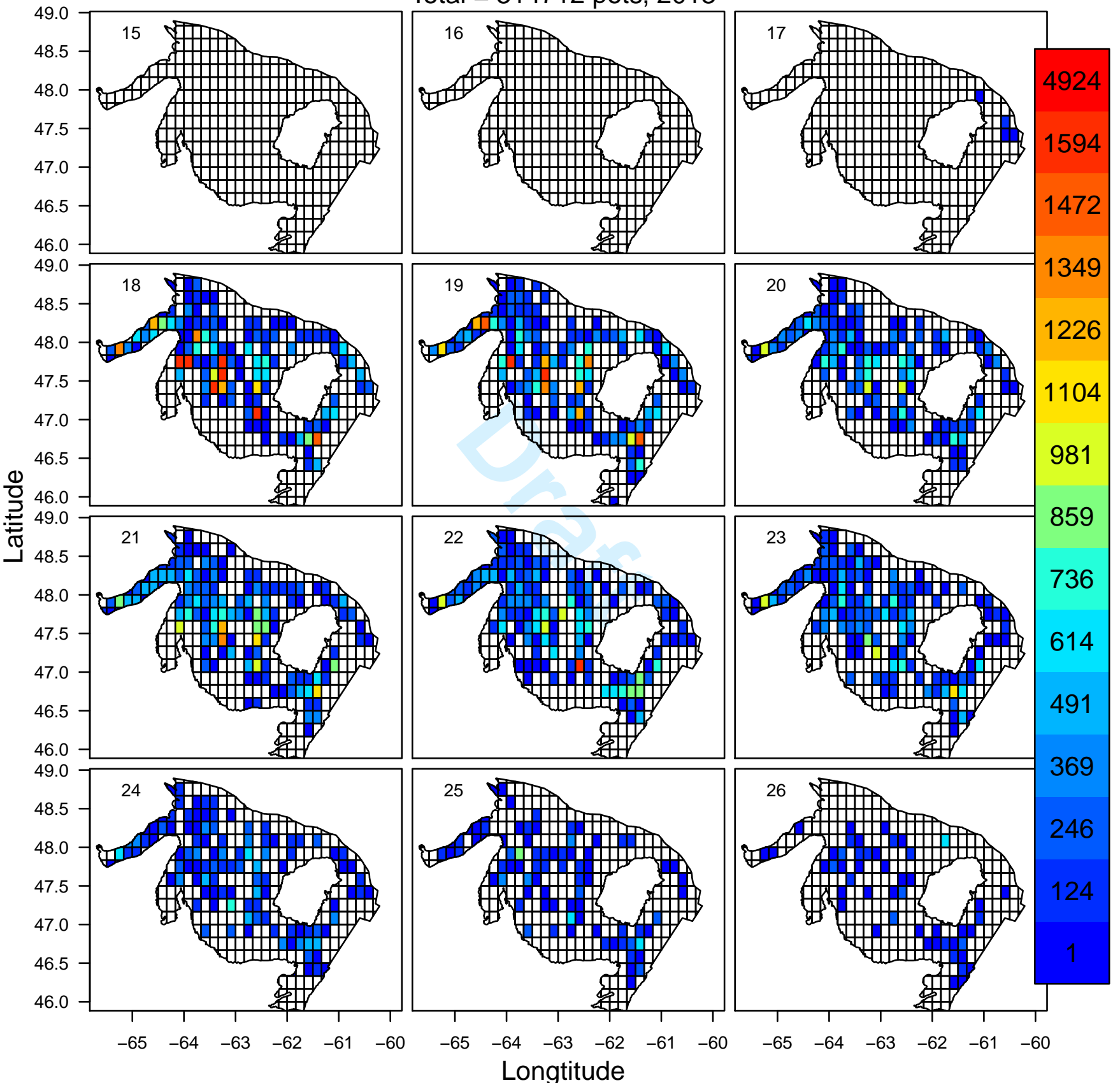

Figure SE.19b. Effort (number of pots) for snow crab in each week and grid cell in 2013. The week number is indicated in the top left-hand corner. Colors correspond to effort levels, as indicated in the legend on the righthand side. Darkest red grids indicate effort $>98$ th percentile. 


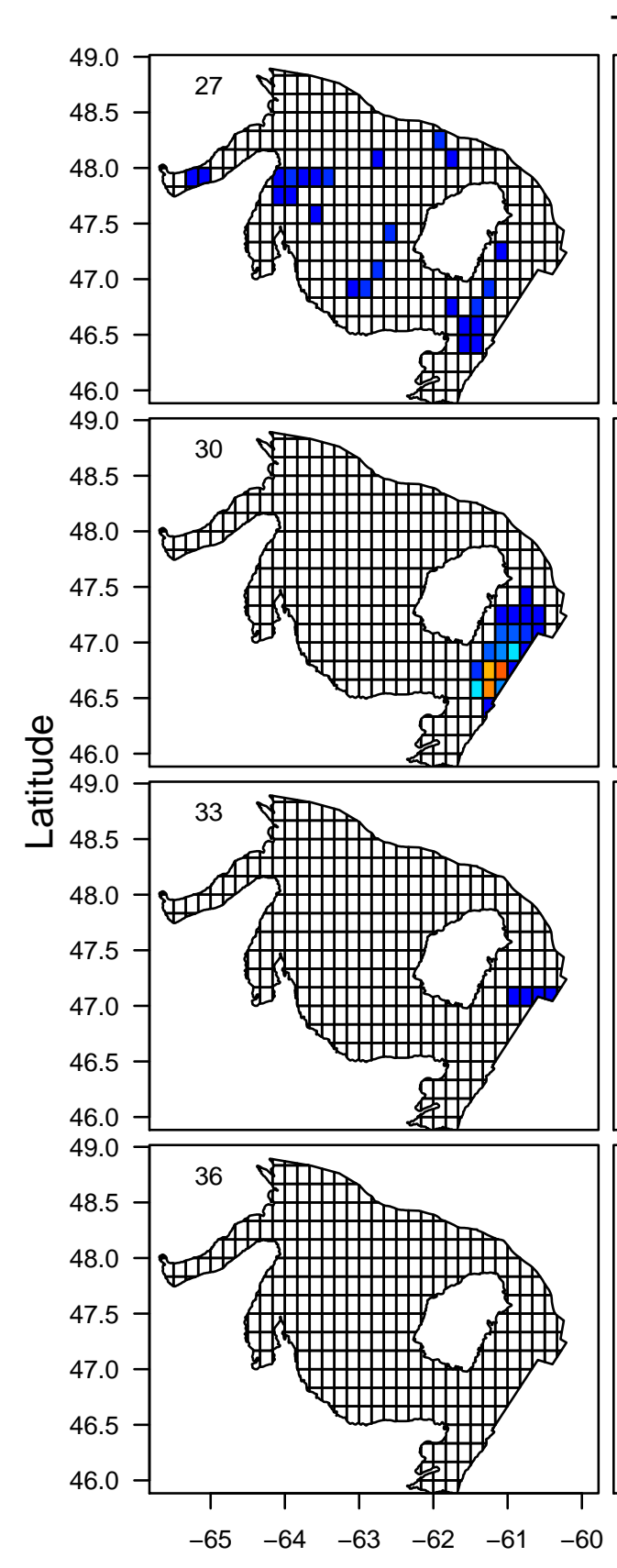

Total $=314712$ pots, 2013
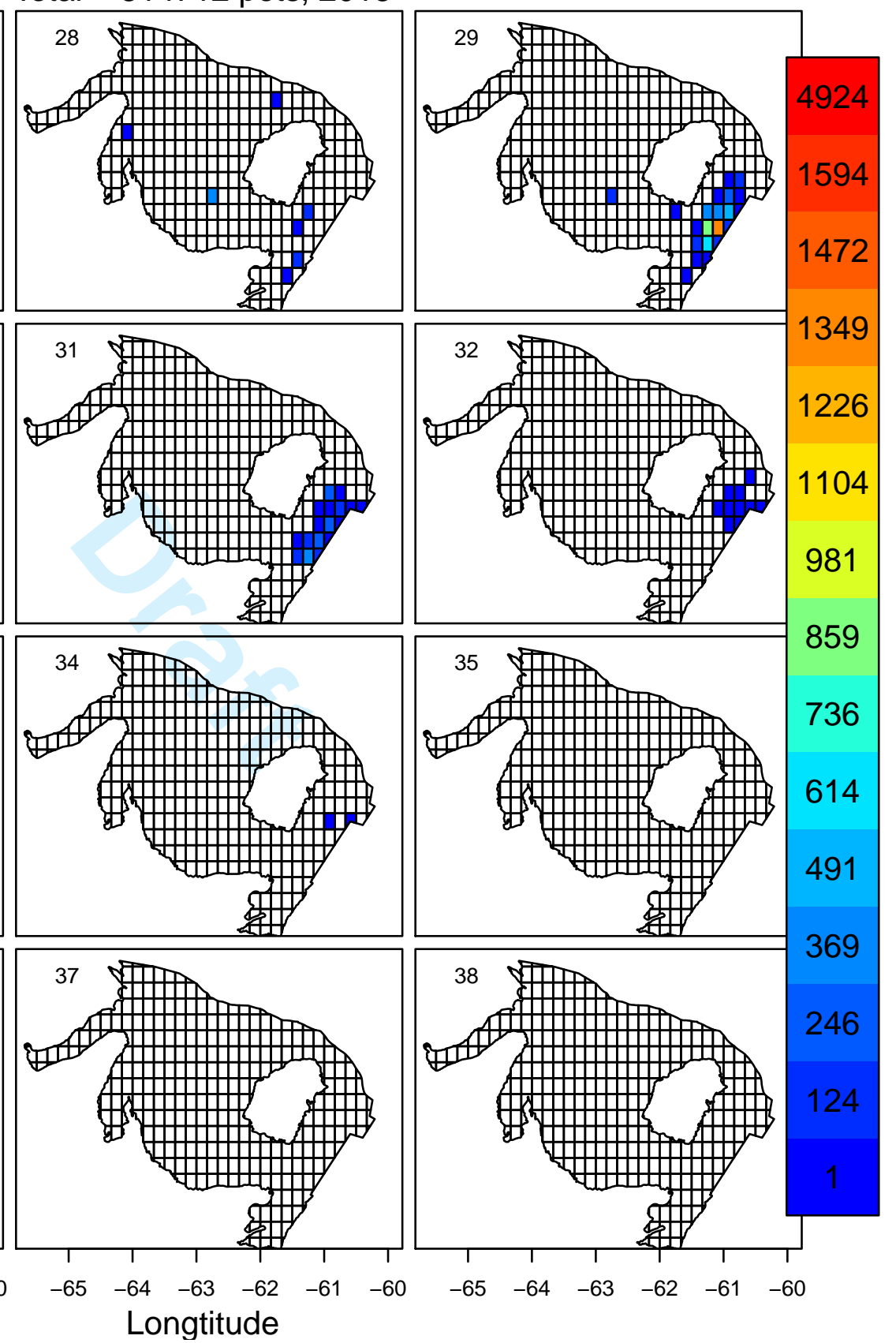

Figure SE.19c. Effort (number of pots) for snow crab in each week and grid cell in 2013. The week number is indicated in the top left-hand corner. Colors correspond to effort levels, as indicated in the legend on the right-hand side. Darkest red grids indicate effort $>98$ th percentile. 


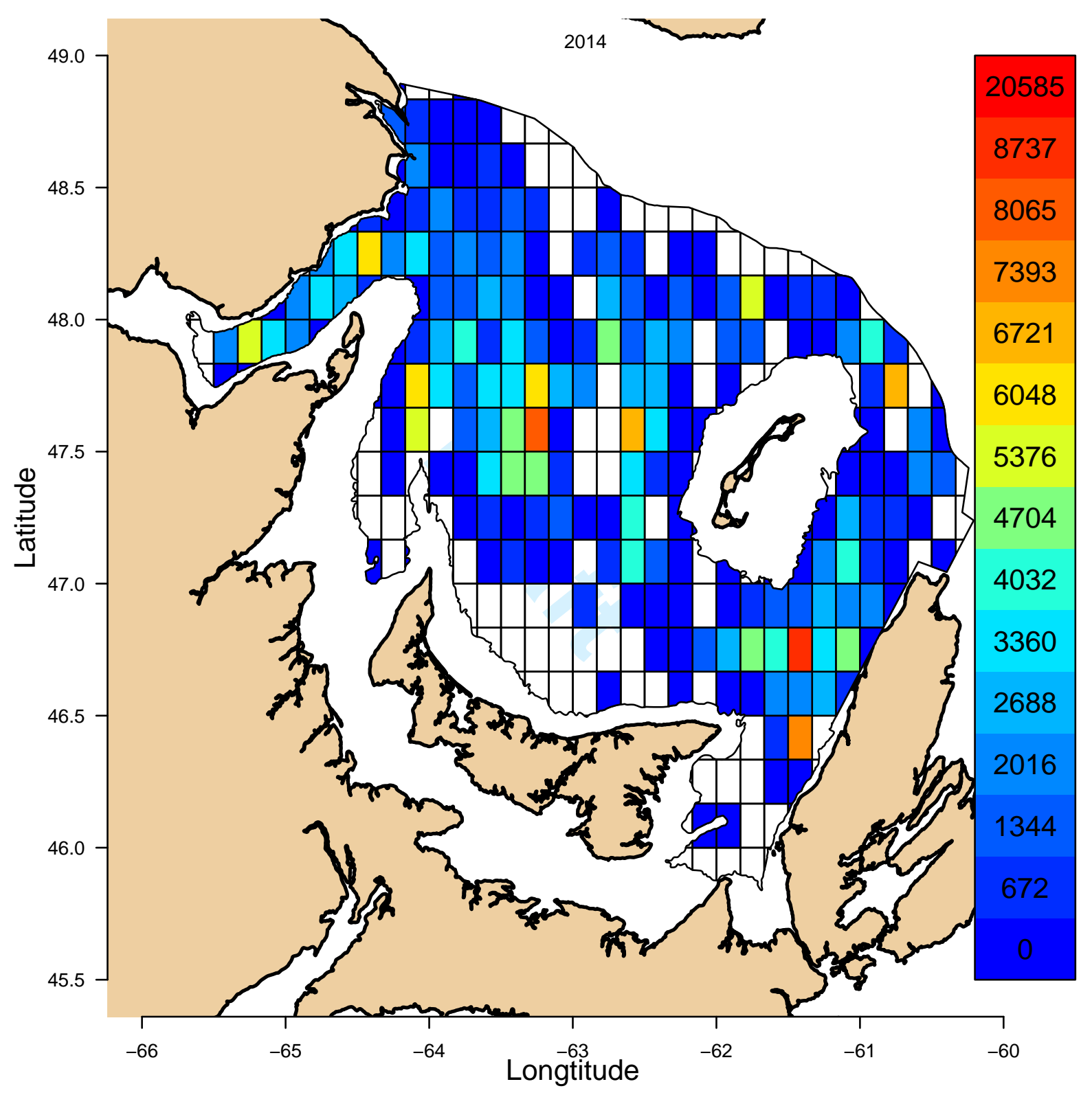

Figure SE.20a. Total annual effort (number of pots) for snow crab in each grid cell in 2014. Colors correspond to effort levels, as indicated in the legend on the right-hand side. Darkest red grids indicate effort $>98$ th percentile. 


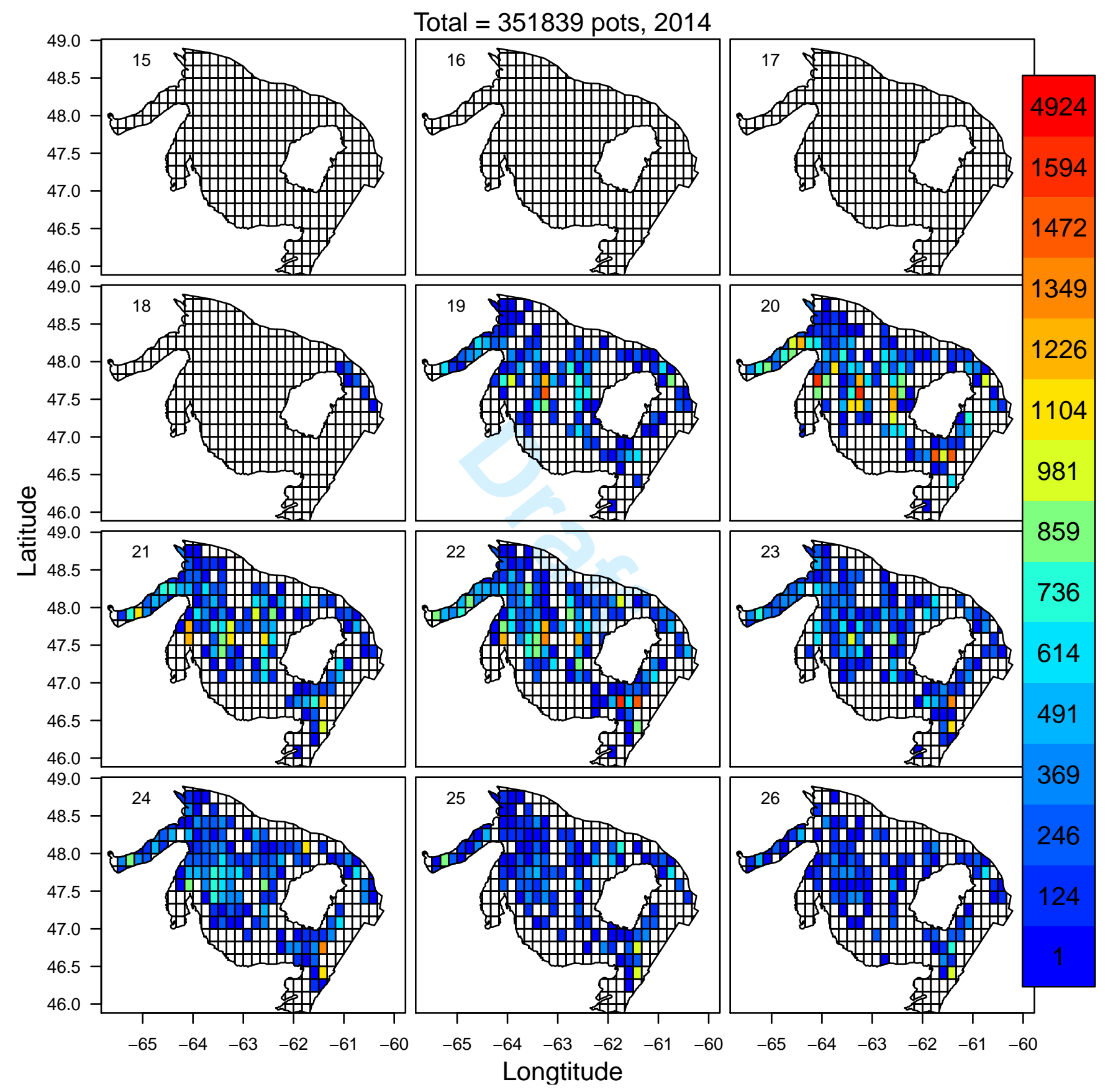

Figure SE.20b. Effort (number of pots) for snow crab in each week and grid cell in 2014. The week number is indicated in the top left-hand corner. Colors correspond to effort levels, as indicated in the legend on the right-hand side. Darkest red grids indicate effort $>98$ th percentile. 
Total $=351839$ pots, 2014
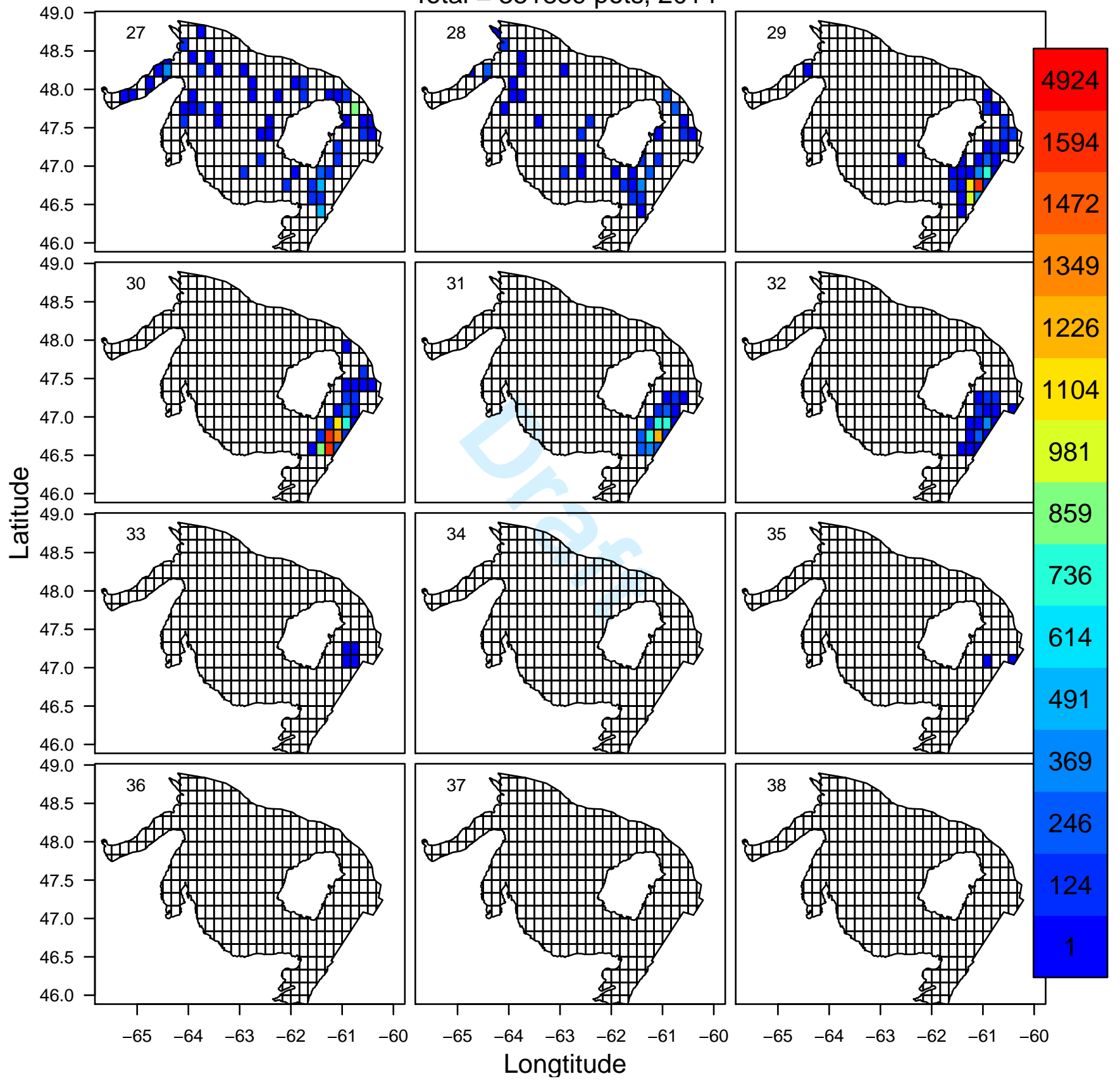

Figure SE.20c. Effort (number of pots) for snow crab in each week and grid cell in 2014. The week number is indicated in the top left-hand corner. Colors correspond to effort levels, as indicated in the legend on the right-hand side. Darkest red grids indicate effort $>98$ th percentile. 
Total annual average $=55(\mathrm{~kg} / \mathrm{pot})$, 346 cells; cell average $=56.96(\mathrm{~kg} / \mathrm{pot})$,

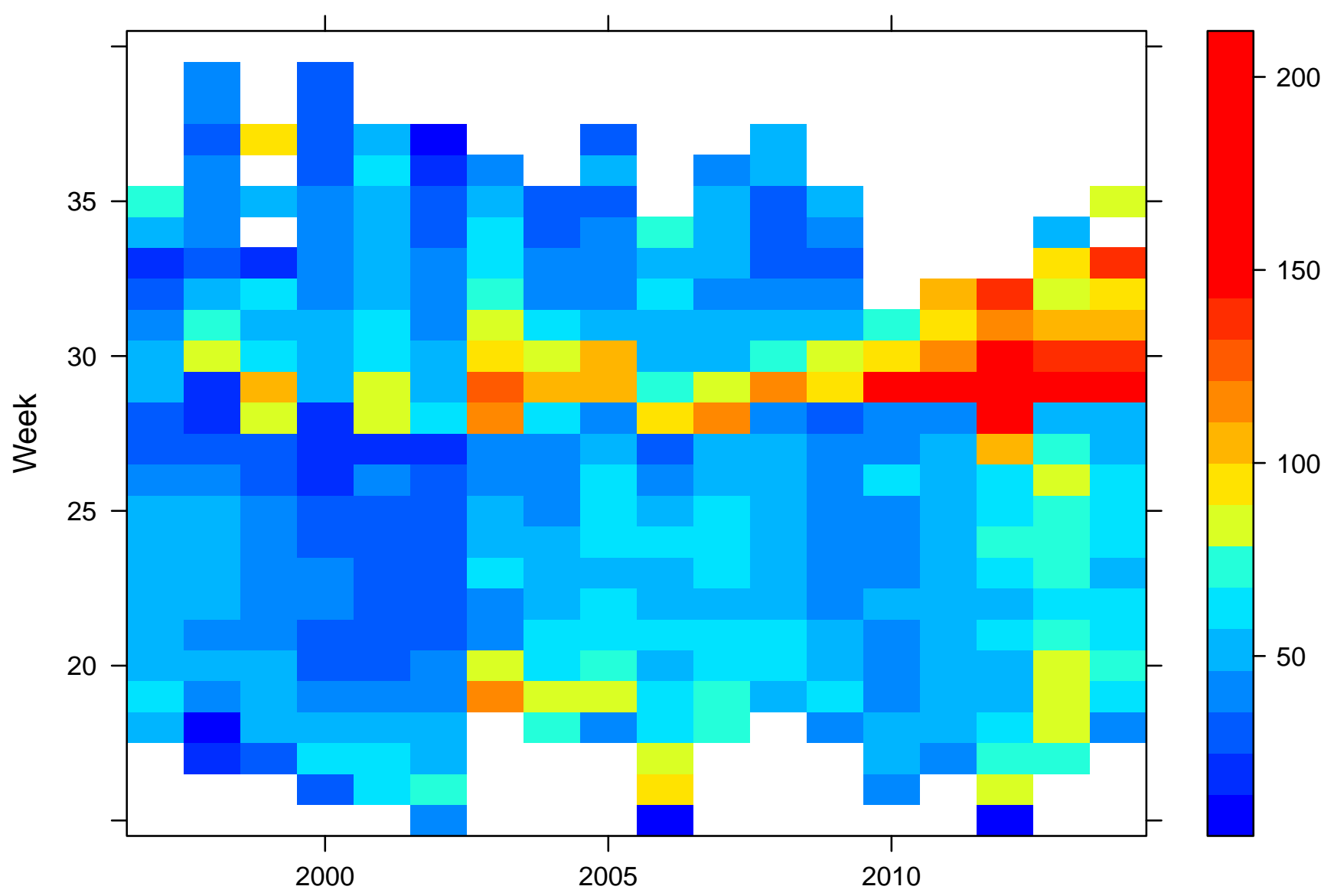

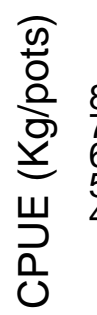

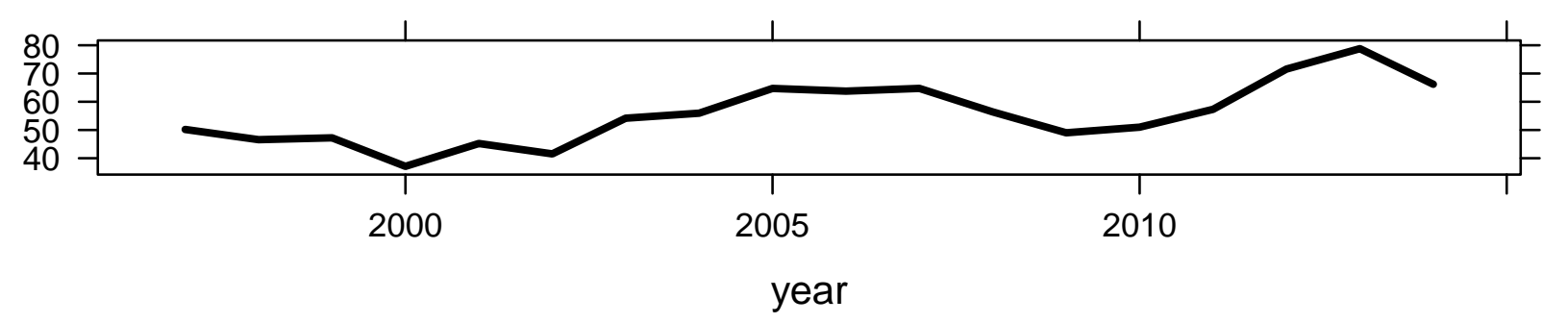

Figure SX.1. Top panel: Total CPUE of snow crab each week (rows) and year (columns). Colors correspond to CPUE levels, as indicated in the legend on the right-hand side. Darkest red grids indicate CPUE > 98th percentile. Bottom panel: Total CPUE each year for all weeks. 


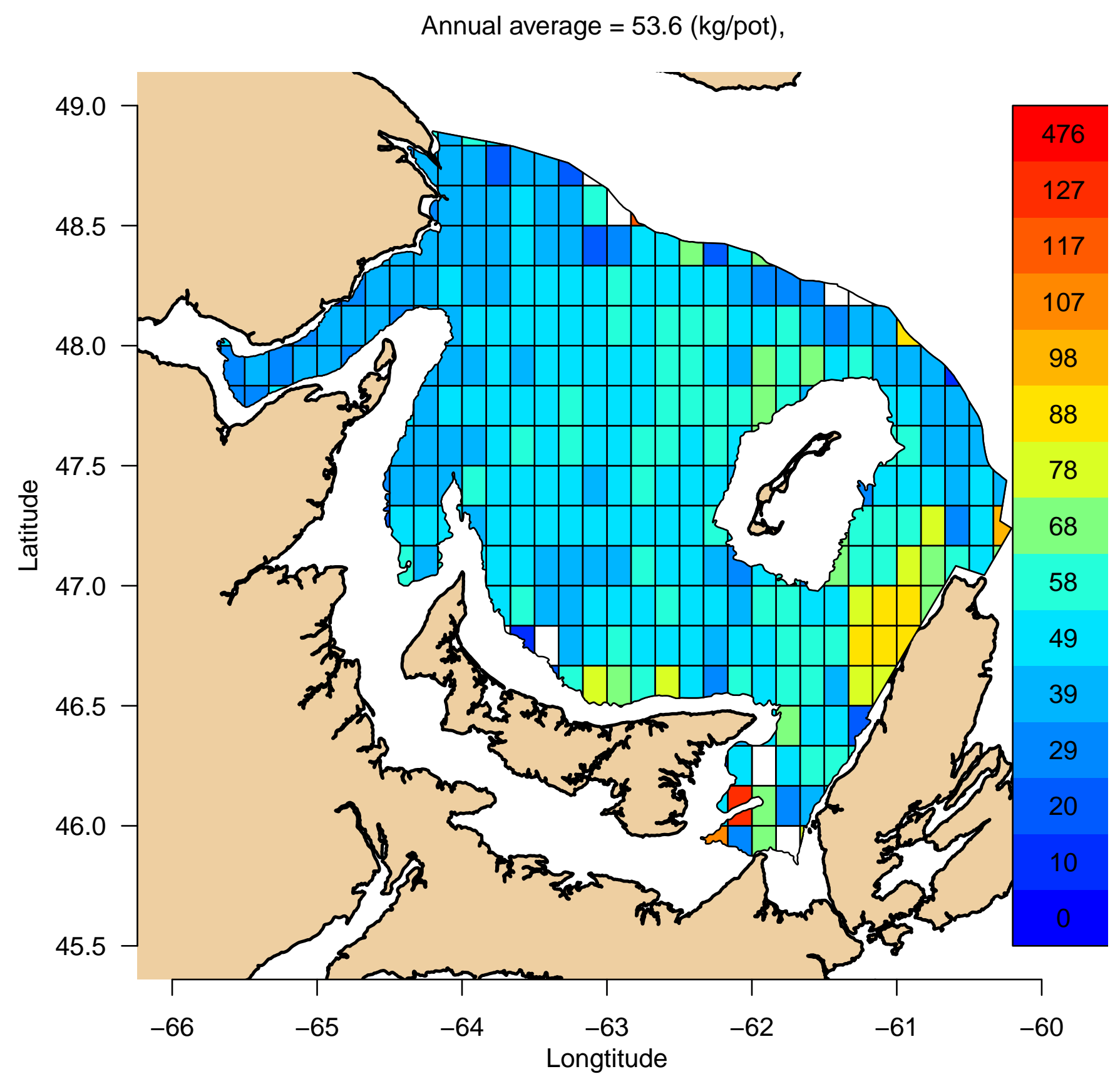

Figure SX.2. Total annual CPUE of snow crab in each grid cell, averaged for 1997-2014. Colors correspond to CPUE levels, as indicated in the legend on the right-hand side. Darkest red grids indicate CPUE > 98th percentile. 


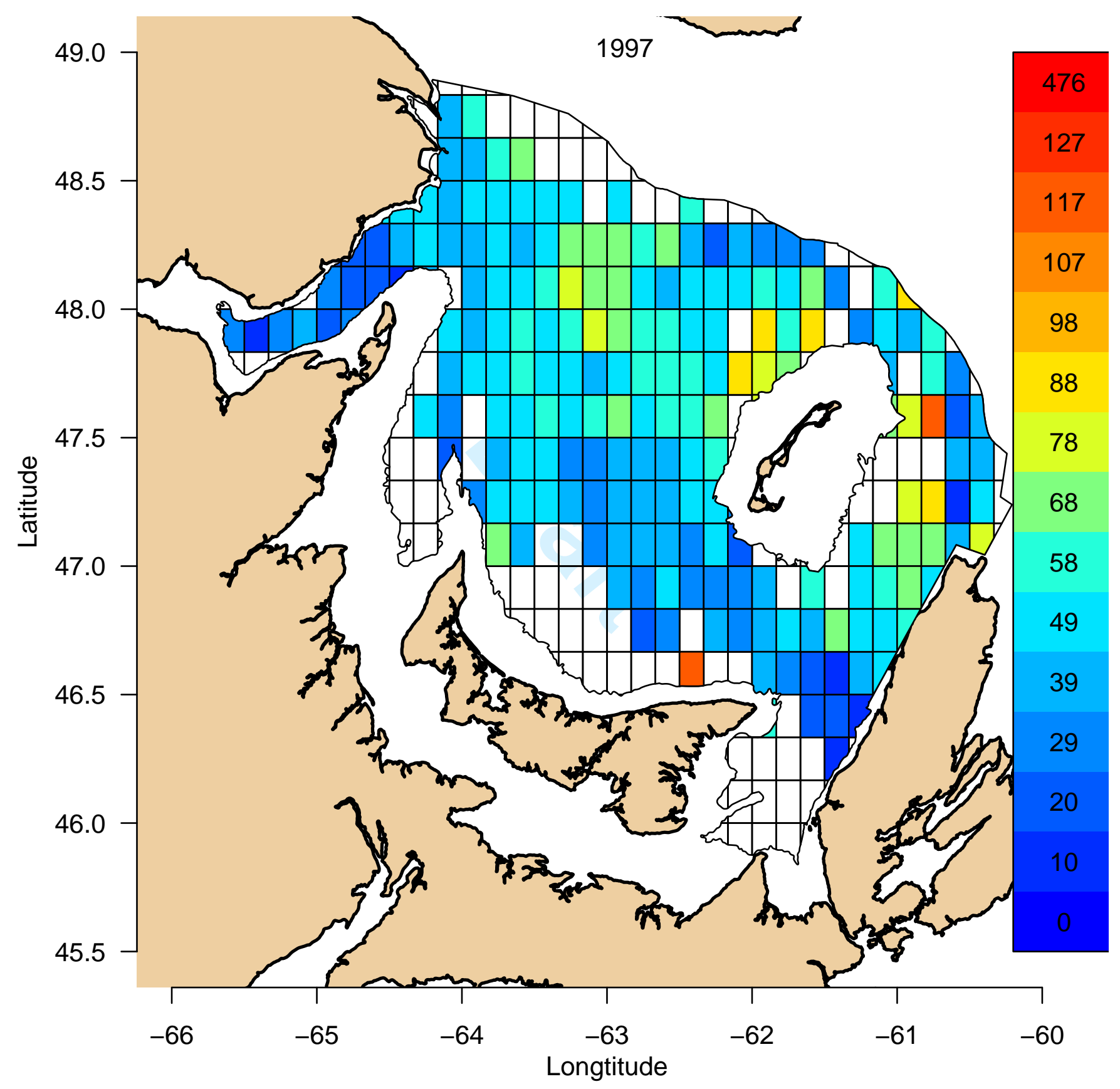

Figure SX.3a. Total annual CPUE of snow crab in each grid cell in 1997. Colors correspond to CPUE levels, as indicated in the legend on the right-hand side. Darkest red grids indicate CPUE > 98th percentile. 


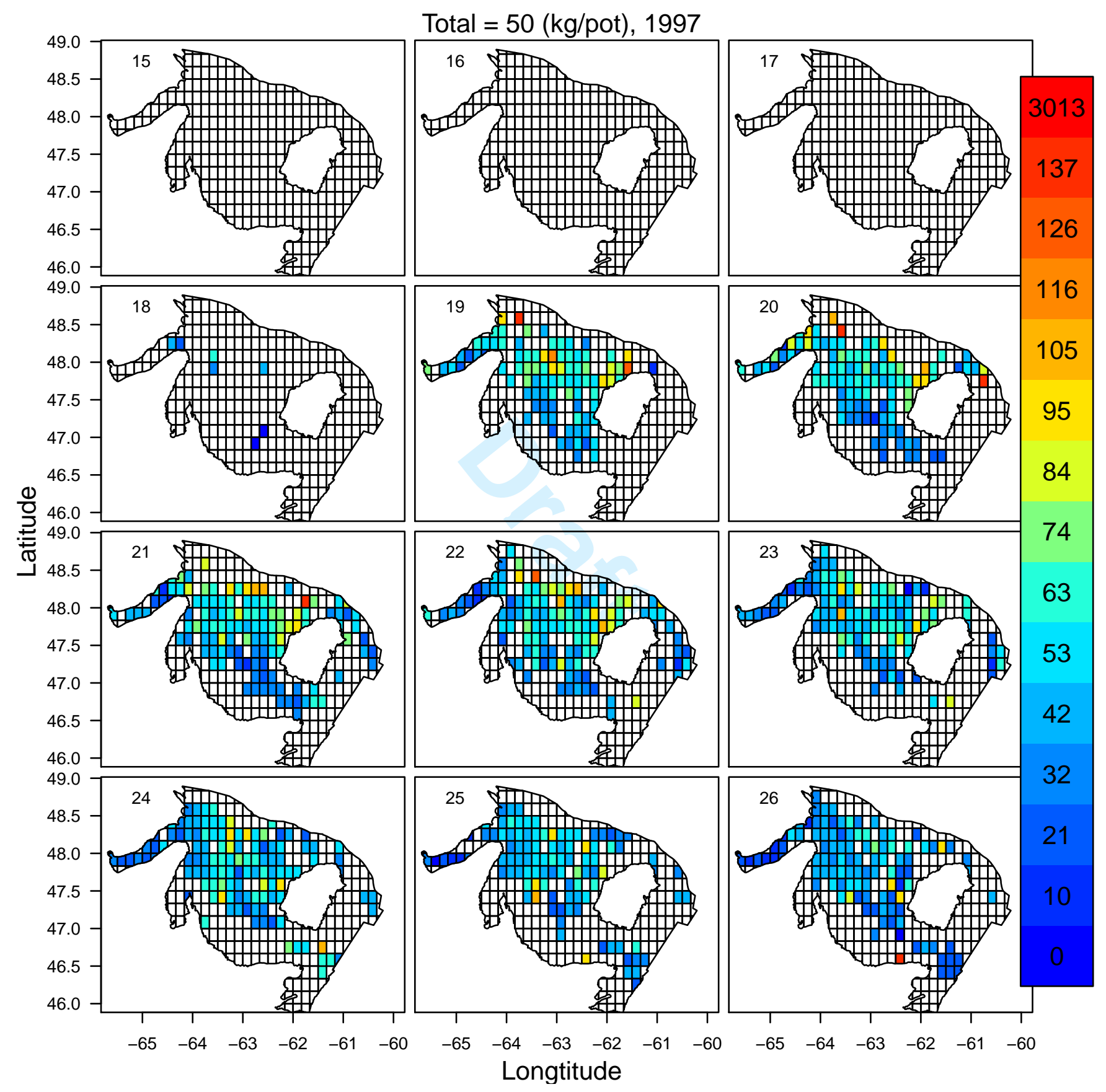

Figure SX.3b. CPUE of snow crab in each week and grid cell in 1997. The week number is indicated in the top left-hand corner. Colors correspond to CPUE levels, as indicated in the legend on the right-hand side. Darkest red grids indicate CPUE $>$ 98th percentile. 


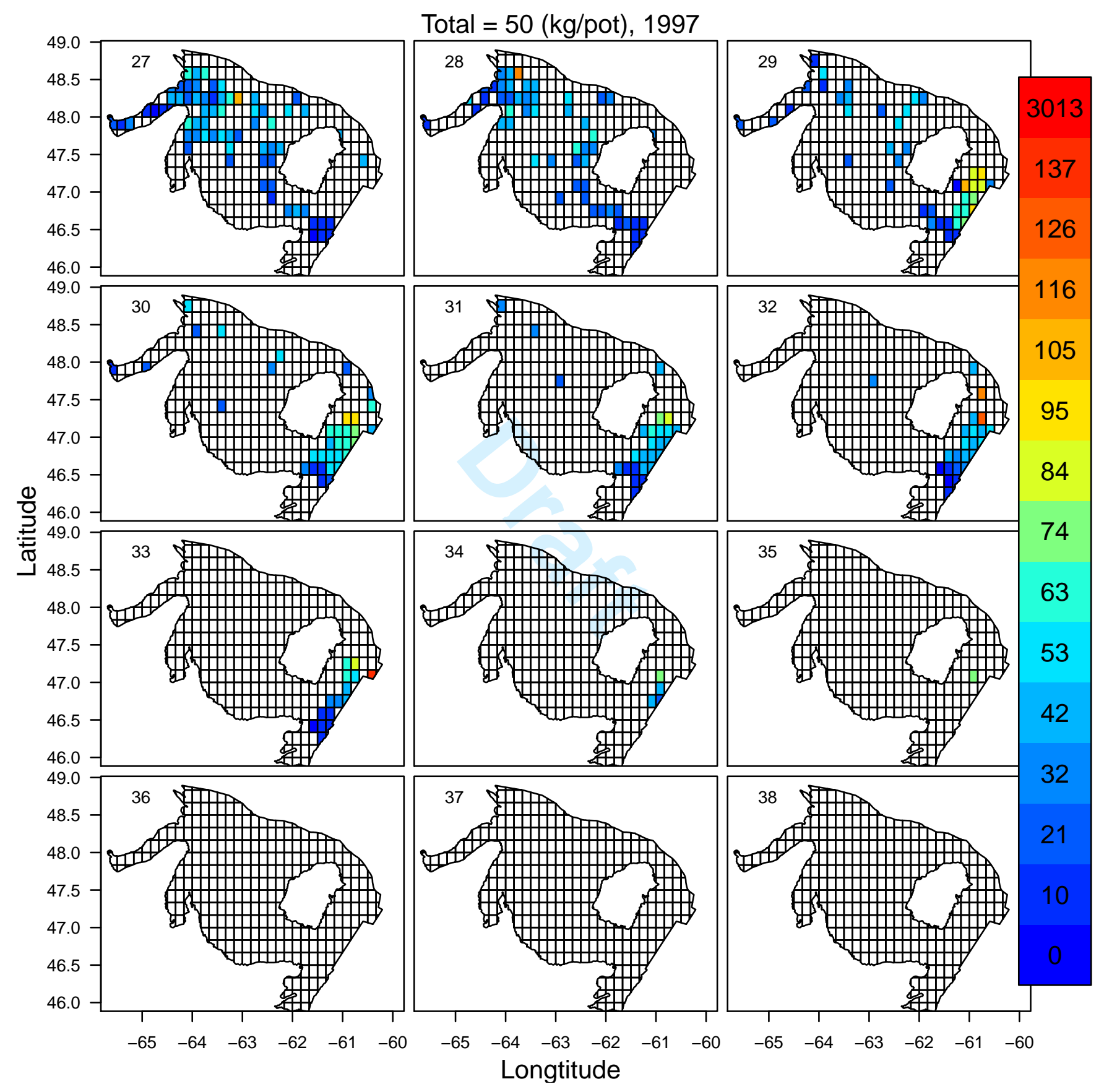

Figure SX.3c. CPUE of snow crab in each week and grid cell in 1997. The week number is indicated in the top left-hand corner. Colors correspond to CPUE levels, as indicated in the legend on the right-hand side. Darkest red grids indicate CPUE $>$ 98th percentile. 


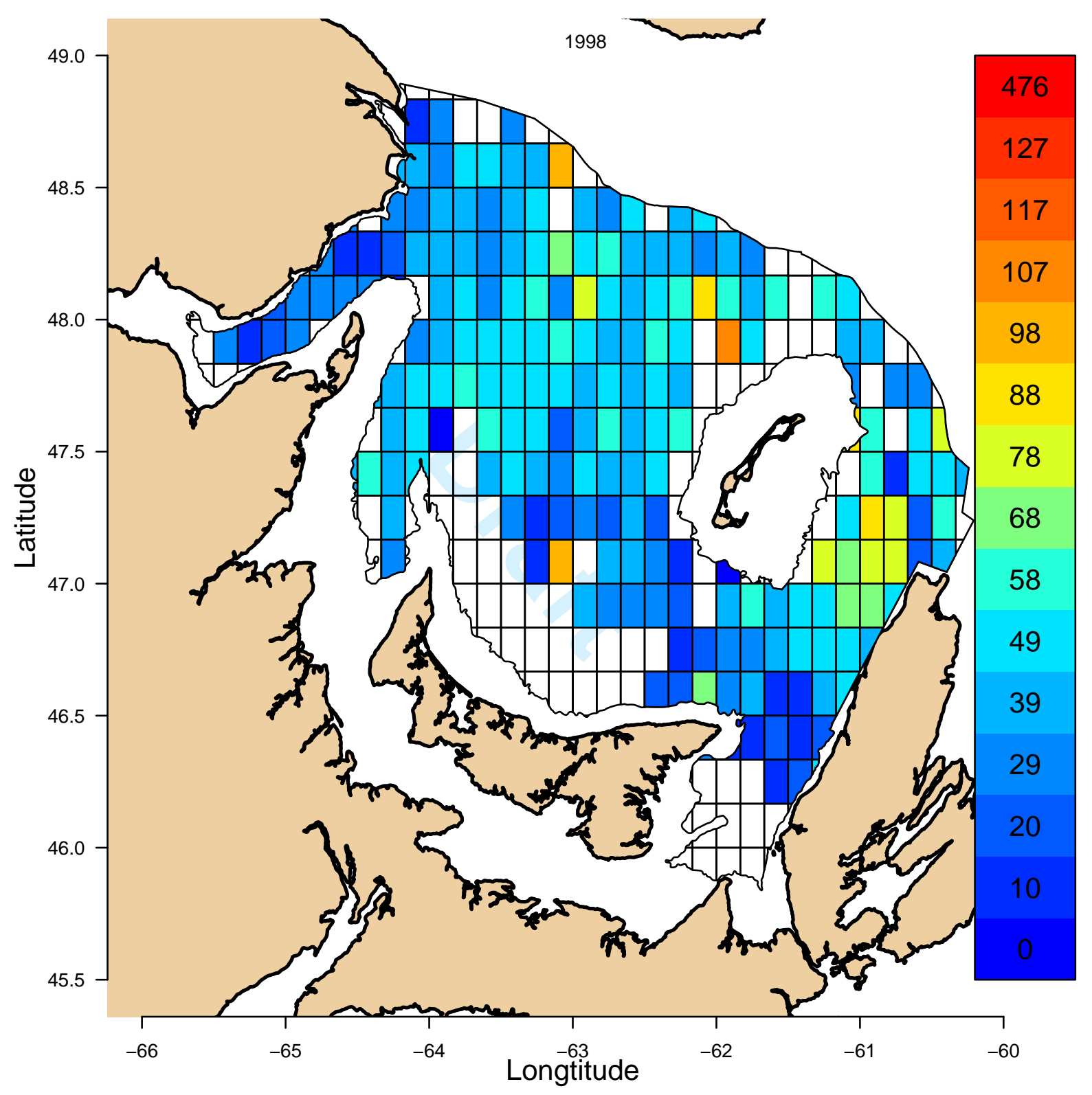

Figure SX.4a. Total annual CPUE of snow crab in each grid cell in 1998. Colors correspond to CPUE levels, as indicated in the legend on the right-hand side. Darkest red grids indicate CPUE $>$ 98th percentile. 


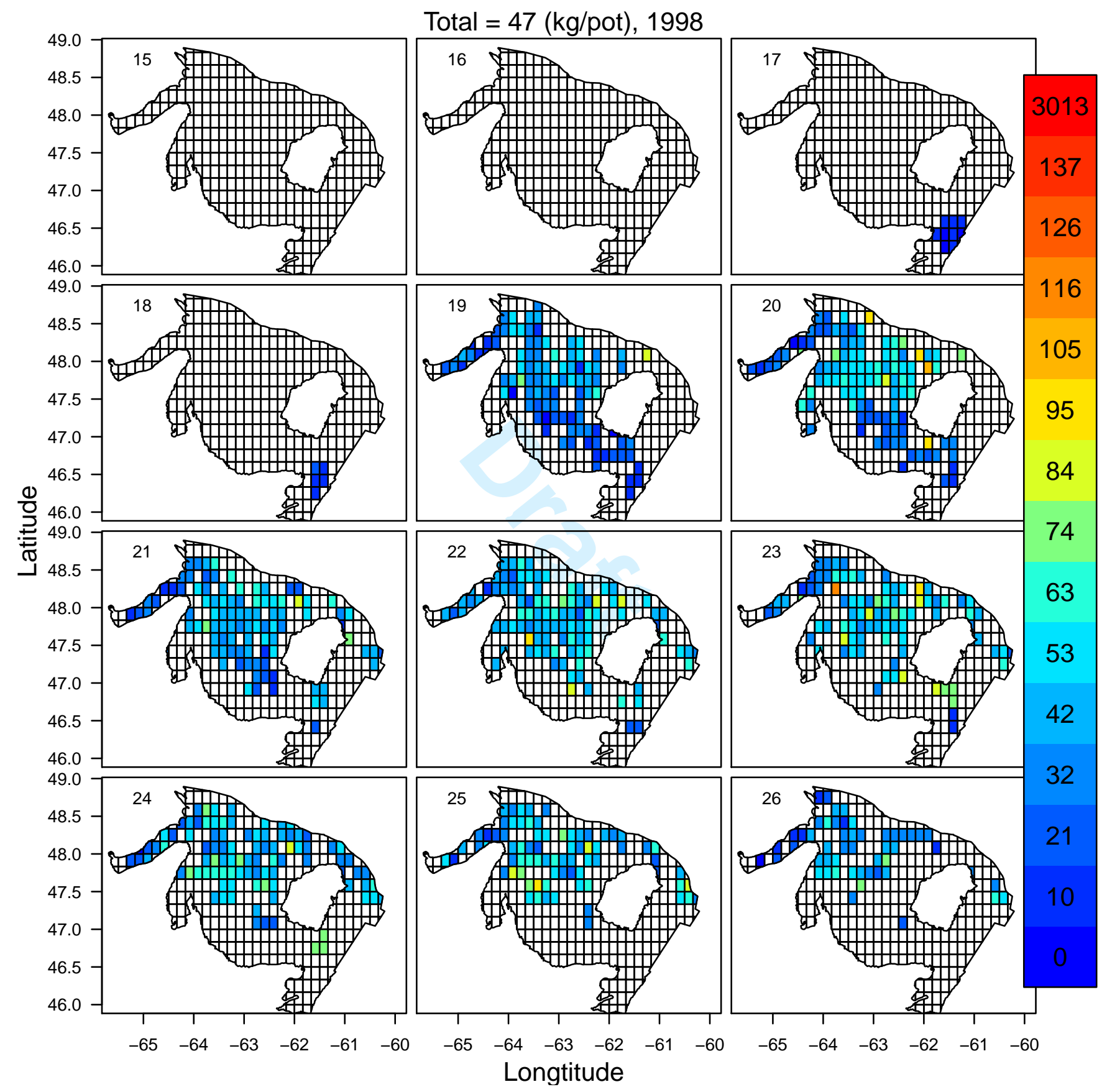

Figure SX.4b. CPUE of snow crab in each week and grid cell in 1998. The week number is indicated in the top left-hand corner. Colors correspond to CPUE levels, as indicated in the legend on the right-hand side. Darkest red grids indicate CPUE $>$ 98th percentile. 


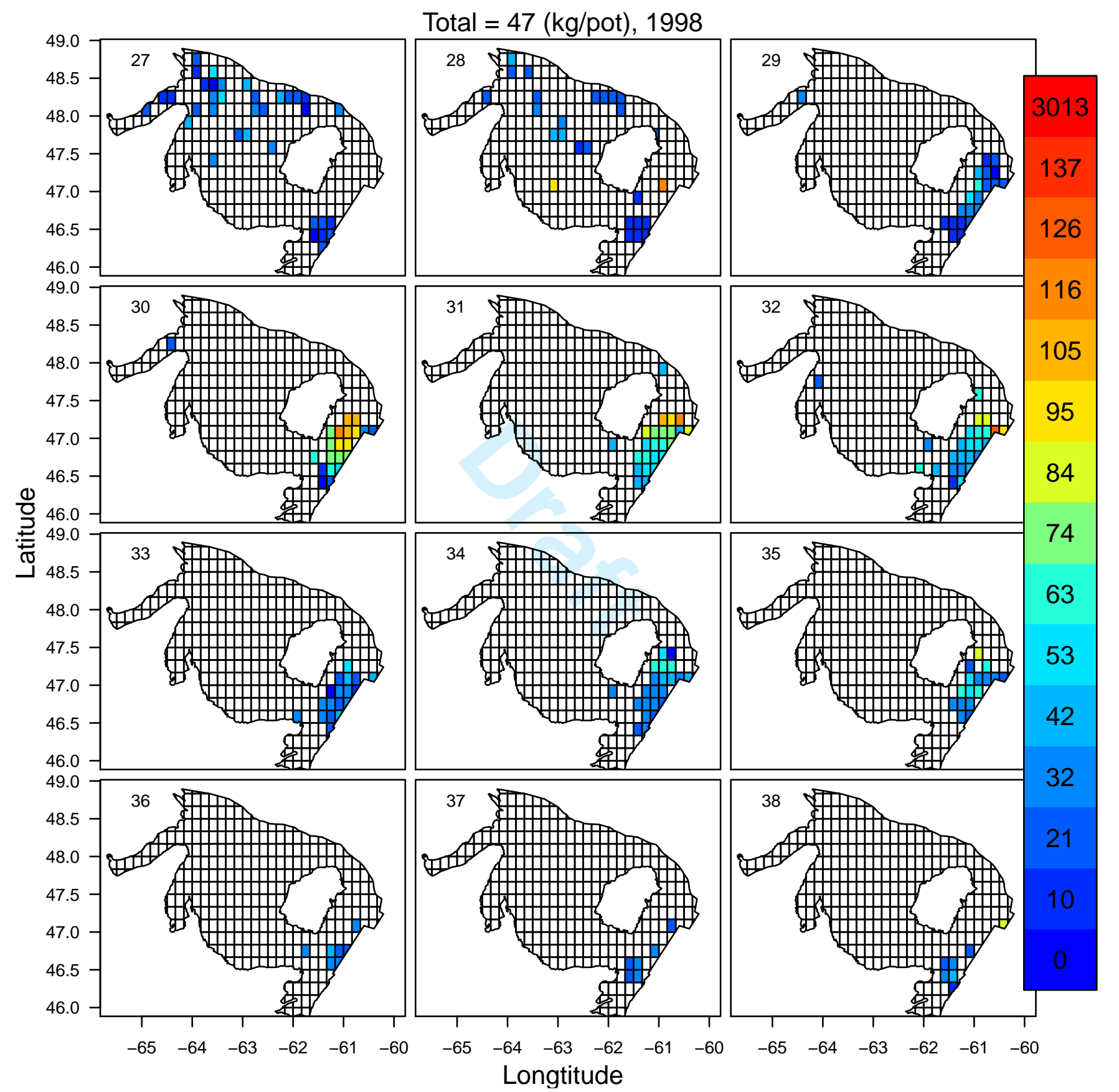

Figure SX.4c. CPUE of snow crab in each week and grid cell in 1998. The week number is indicated in the top left-hand corner. Colors correspond to CPUE levels, as indicated in the legend on the right-hand side. Darkest red grids indicate CPUE $>$ 98th percentile. 


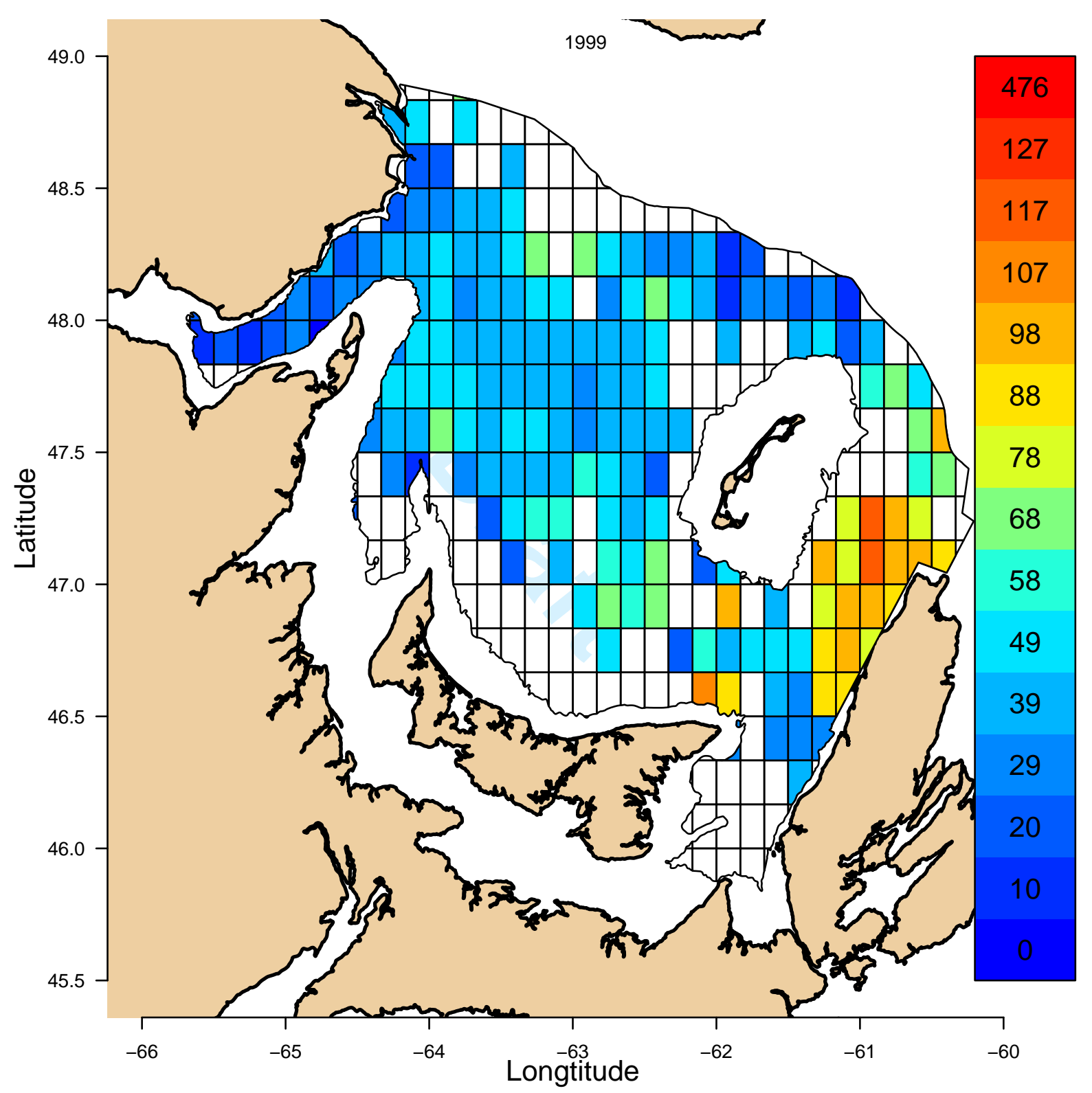

Figure SX.5a. Total annual CPUE of snow crab in each grid cell in 1999. Colors correspond to CPUE levels, as indicated in the legend on the right-hand side. Darkest red grids indicate CPUE > 98th percentile. 


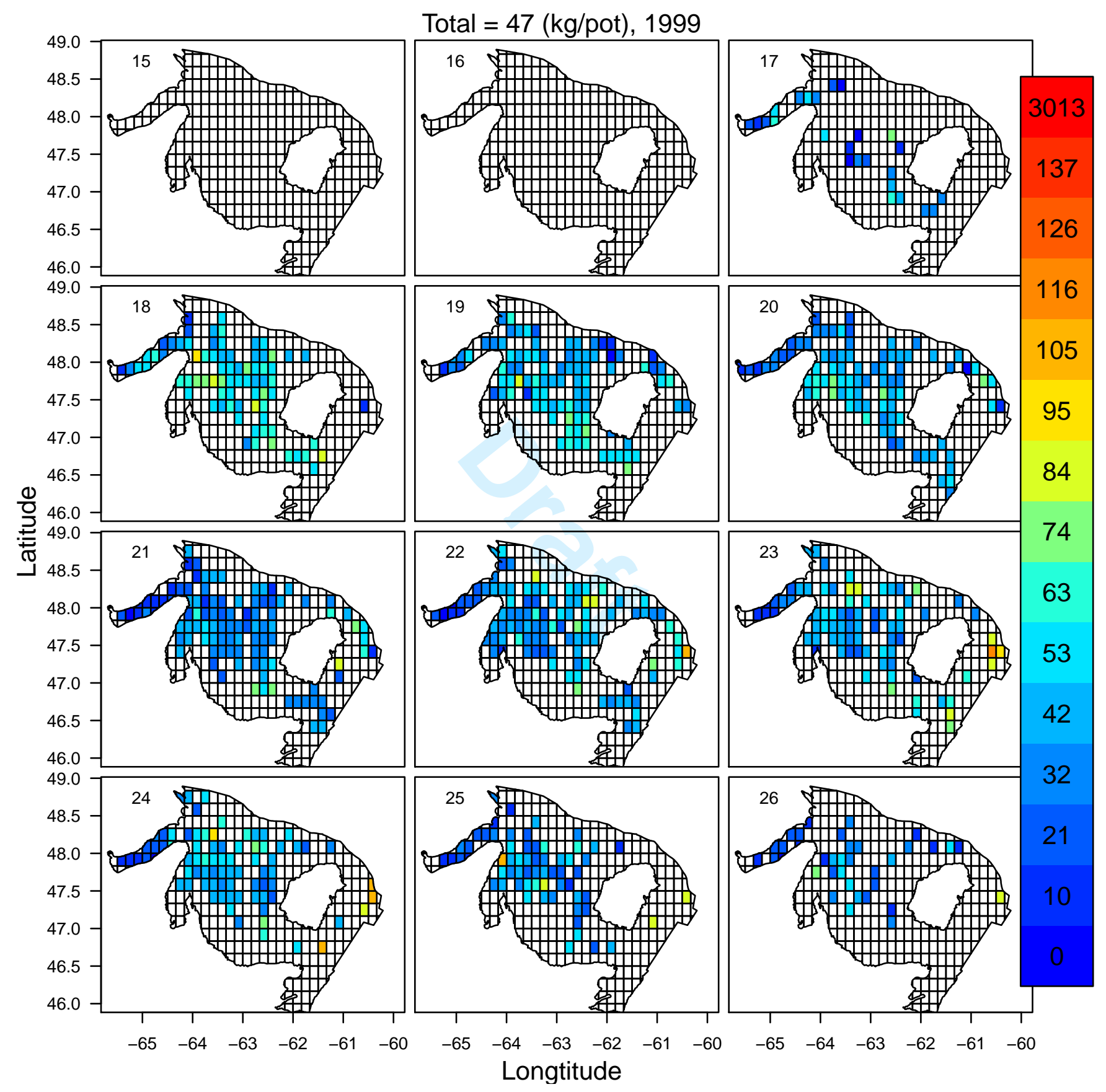

Figure SX.5b. CPUE of snow crab in each week and grid cell in 1999. The week number is indicated in the top left-hand corner. Colors correspond to CPUE levels, as indicated in the legend on the right-hand side. Darkest red grids indicate CPUE $>$ 98th percentile. 


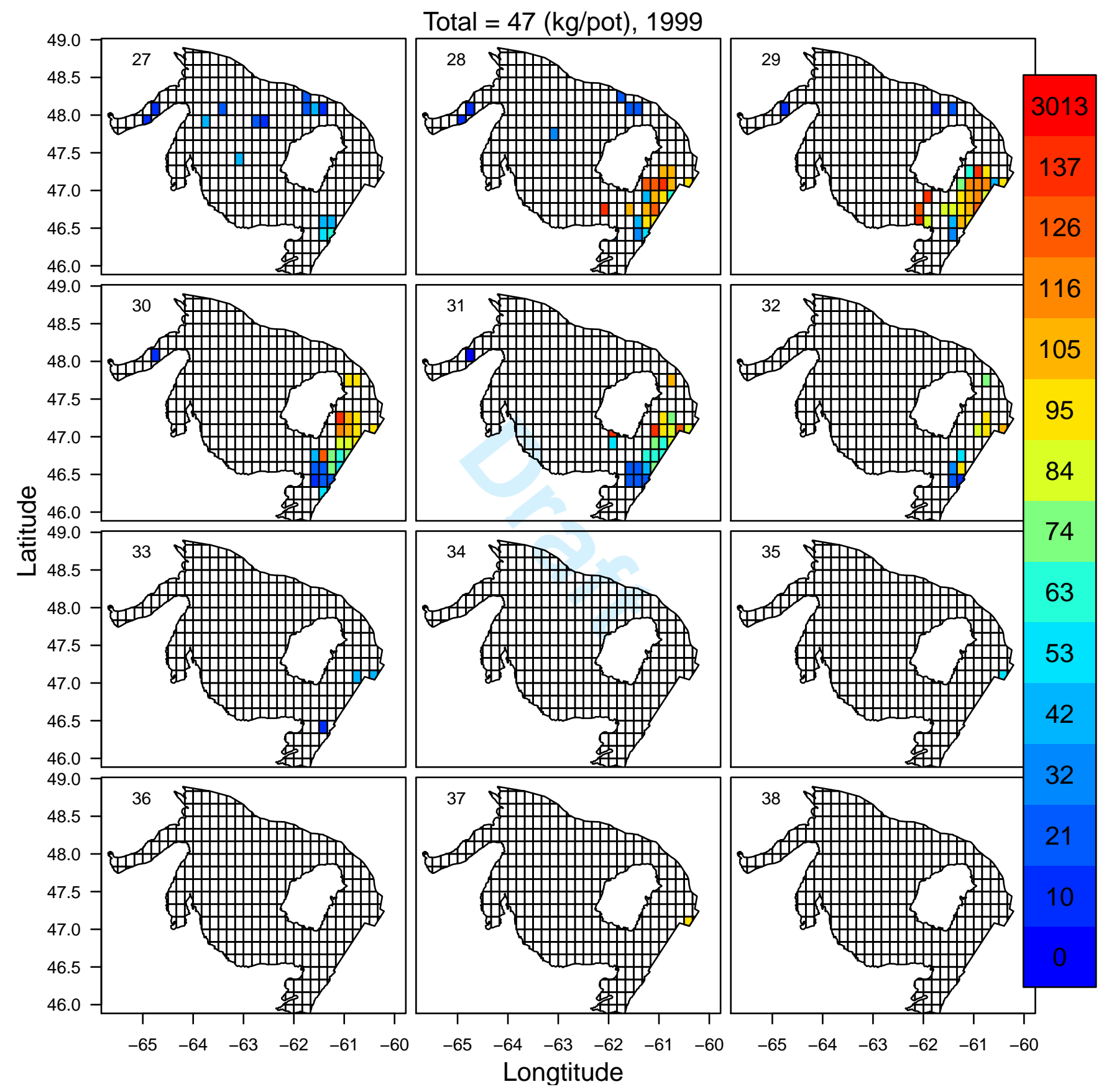

Figure SX.5c. CPUE of snow crab in each week and grid cell in 1999. The week number is indicated in the top left-hand corner. Colors correspond to CPUE levels, as indicated in the legend on the right-hand side. Darkest red grids indicate CPUE $>$ 98th percentile. 


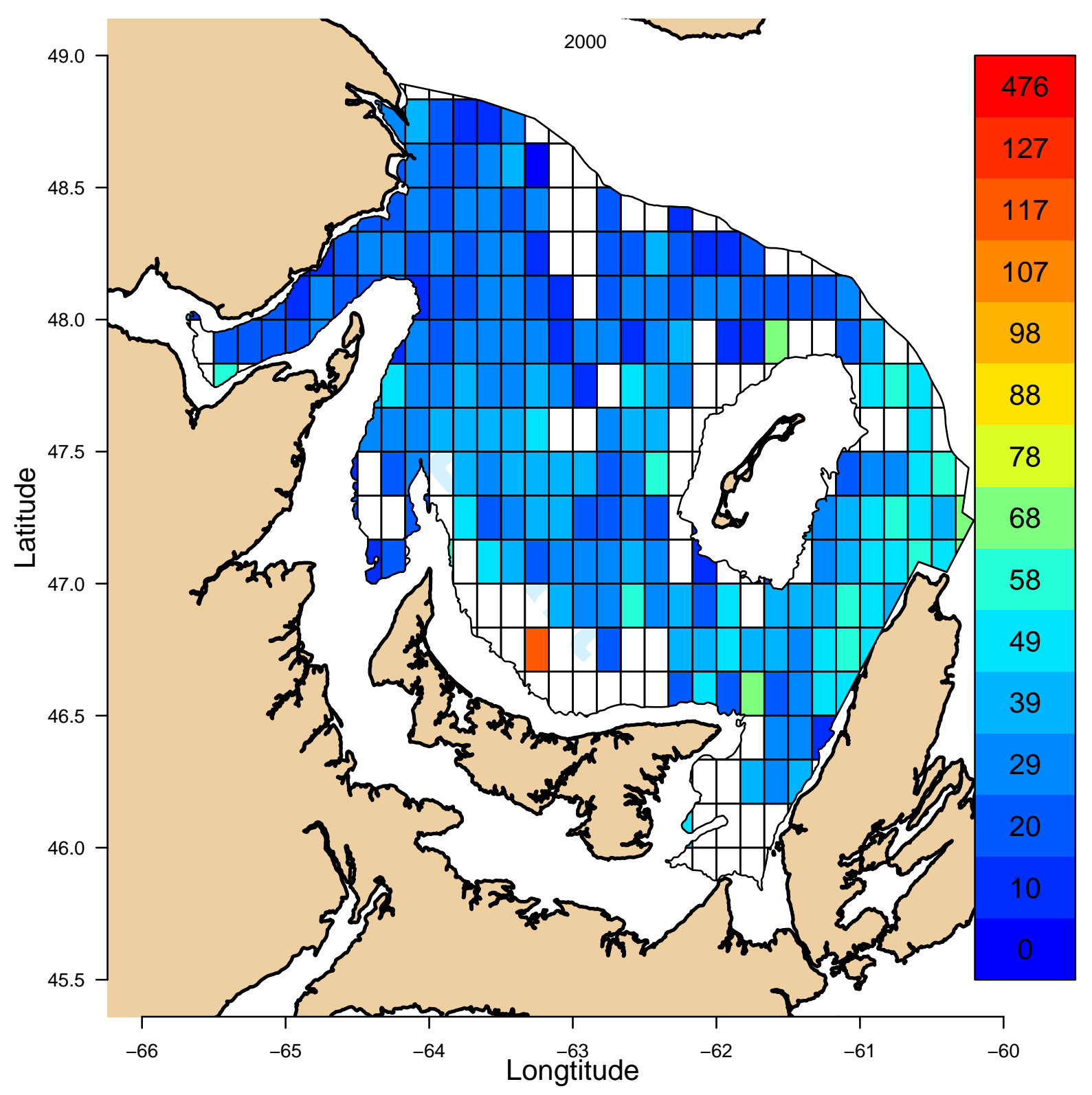

Figure SX.6a. Total annual CPUE of snow crab in each grid cell in 2000. Colors correspond to CPUE levels, as indicated in the legend on the right-hand side. Darkest red grids indicate CPUE > 98th percentile. 


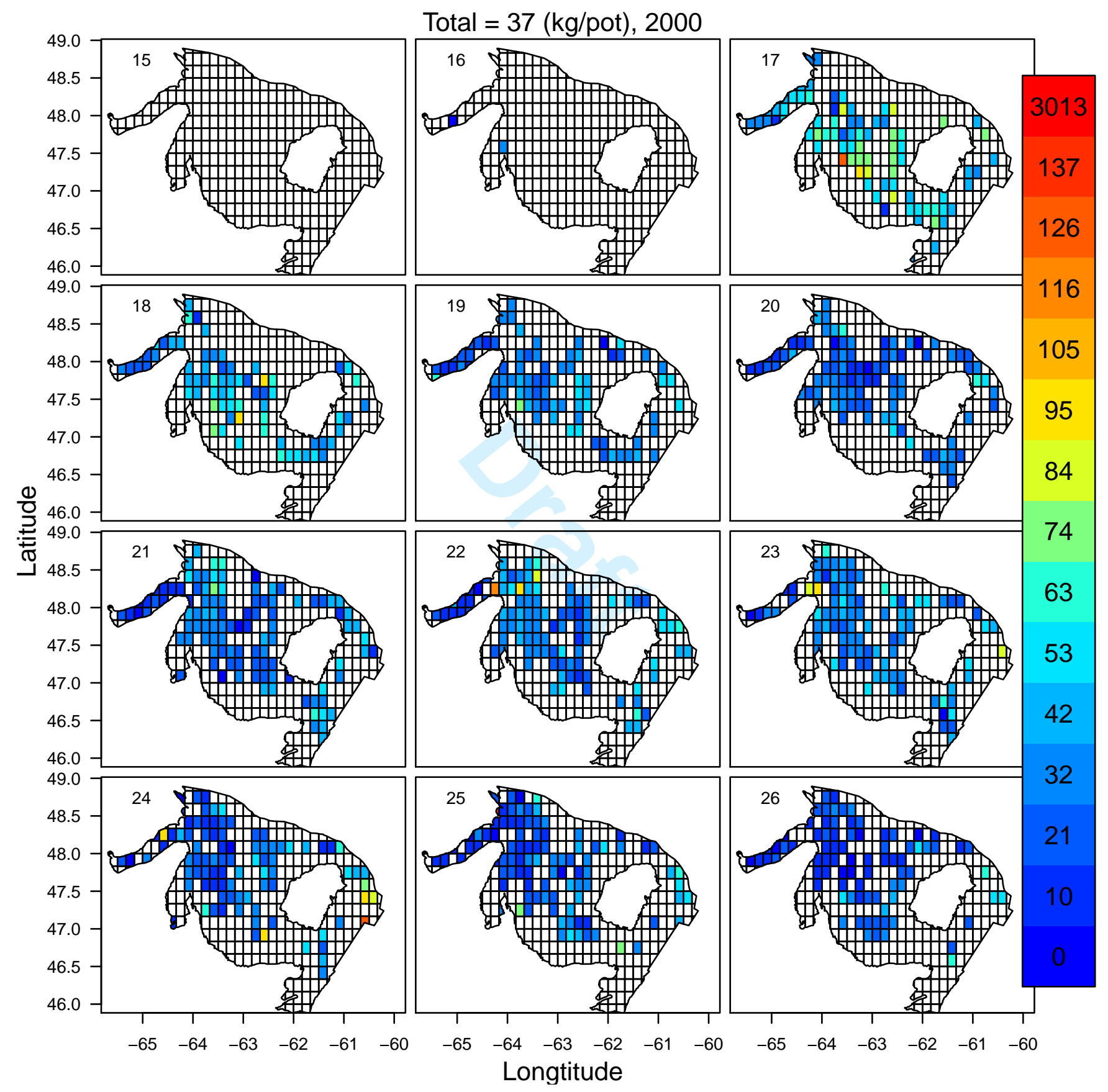

Figure SX.6b. CPUE of snow crab in each week and grid cell in 2000. The week number is indicated in the top left-hand corner. Colors correspond to CPUE levels, as indicated in the legend on the right-hand side. Darkest red grids indicate CPUE $>$ 98th percentile. 


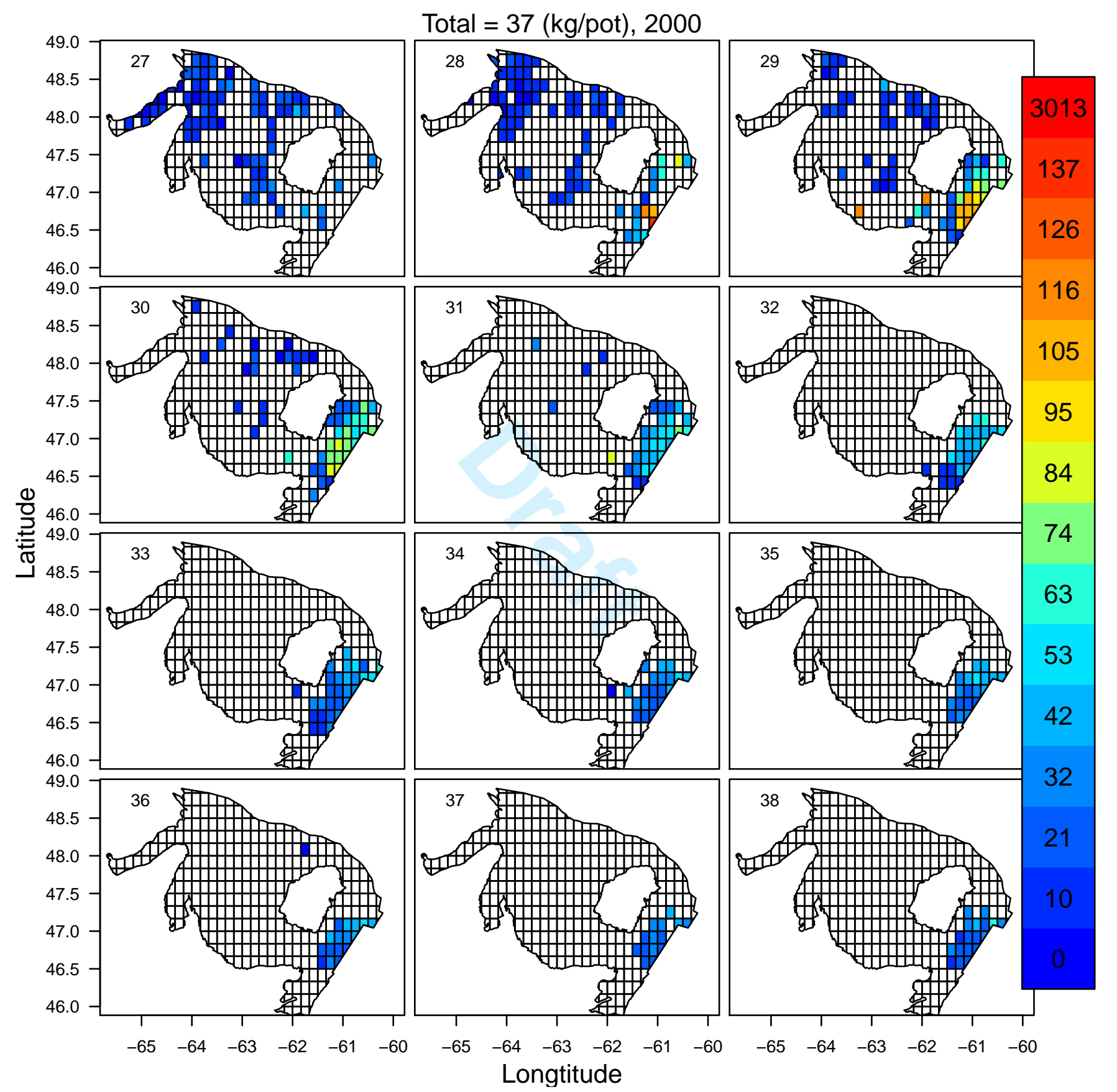

Figure SX.6c. CPUE of snow crab in each week and grid cell in 2000. The week number is indicated in the top left-hand corner. Colors correspond to CPUE levels, as indicated in the legend on the right-hand side. Darkest red grids indicate CPUE $>$ 98th percentile. 


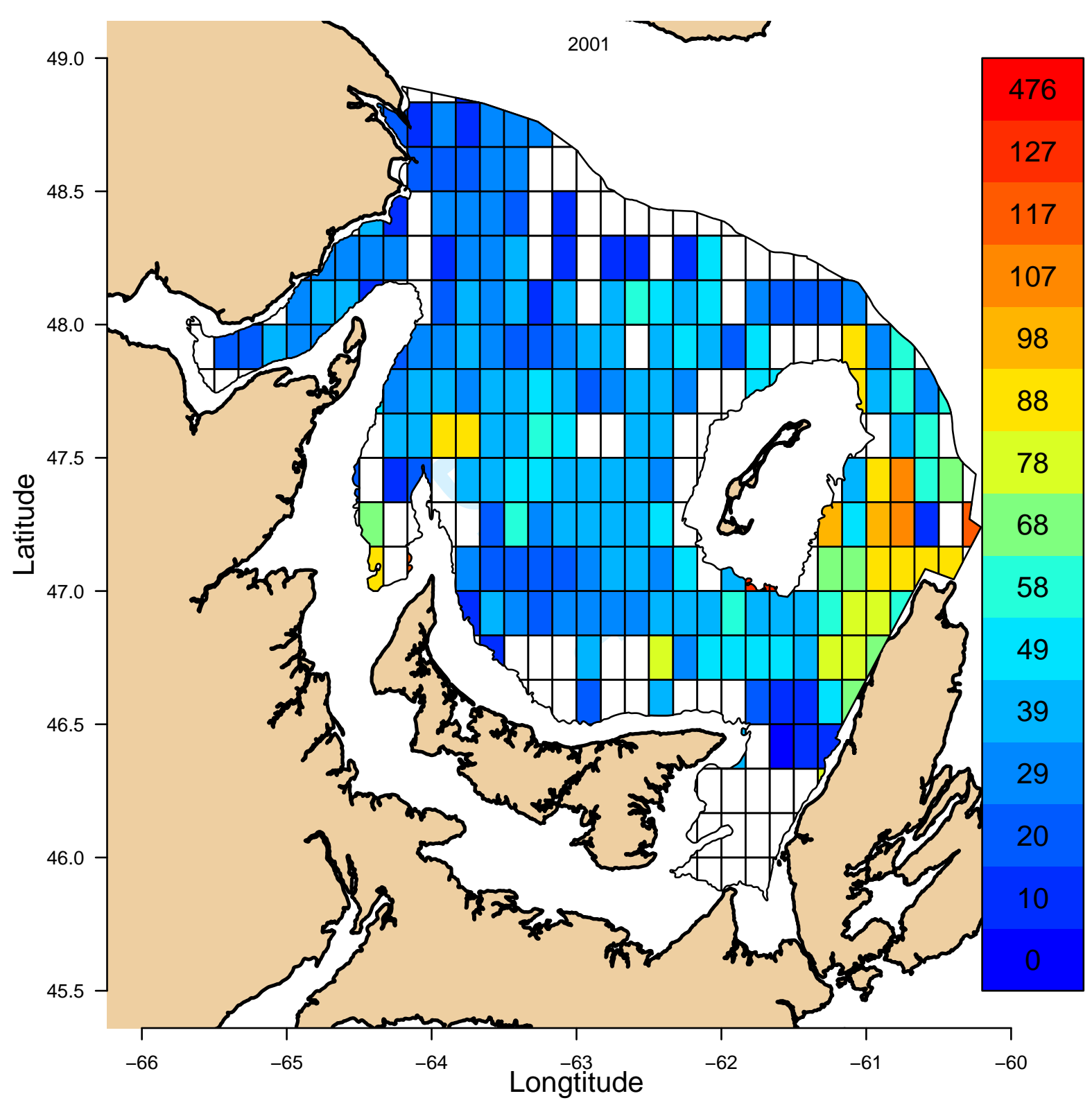

Figure SX.7a. Total annual CPUE of snow crab in each grid cell in 2001. Colors correspond to CPUE levels, as indicated in the legend on the right-hand side. Darkest red grids indicate CPUE > 98th percentile. 


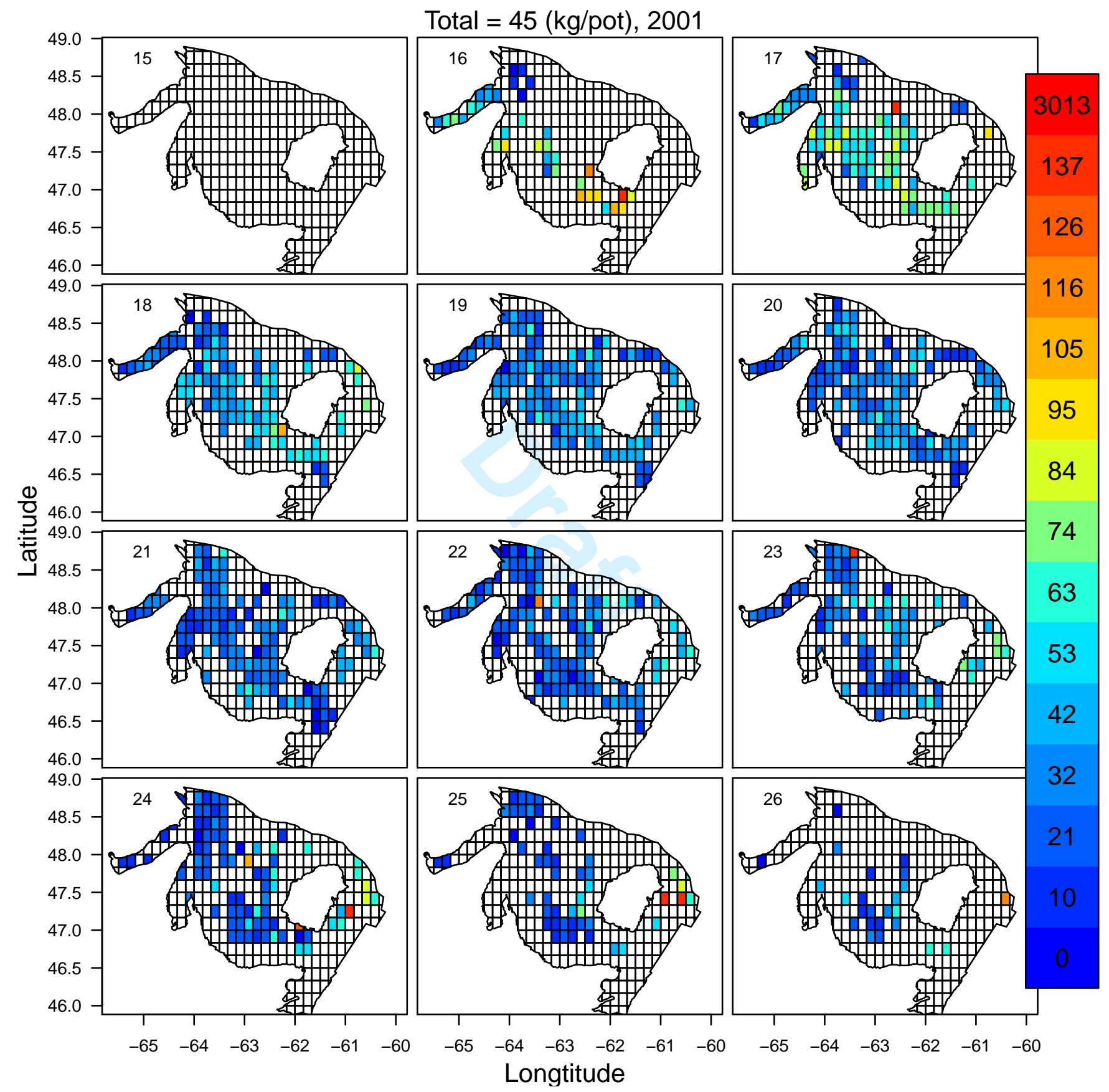

Figure SX.7b. CPUE of snow crab in each week and grid cell in 2001. The week number is indicated in the top left-hand corner. Colors correspond to CPUE levels, as indicated in the legend on the right-hand side. Darkest red grids indicate CPUE $>$ 98th percentile. 


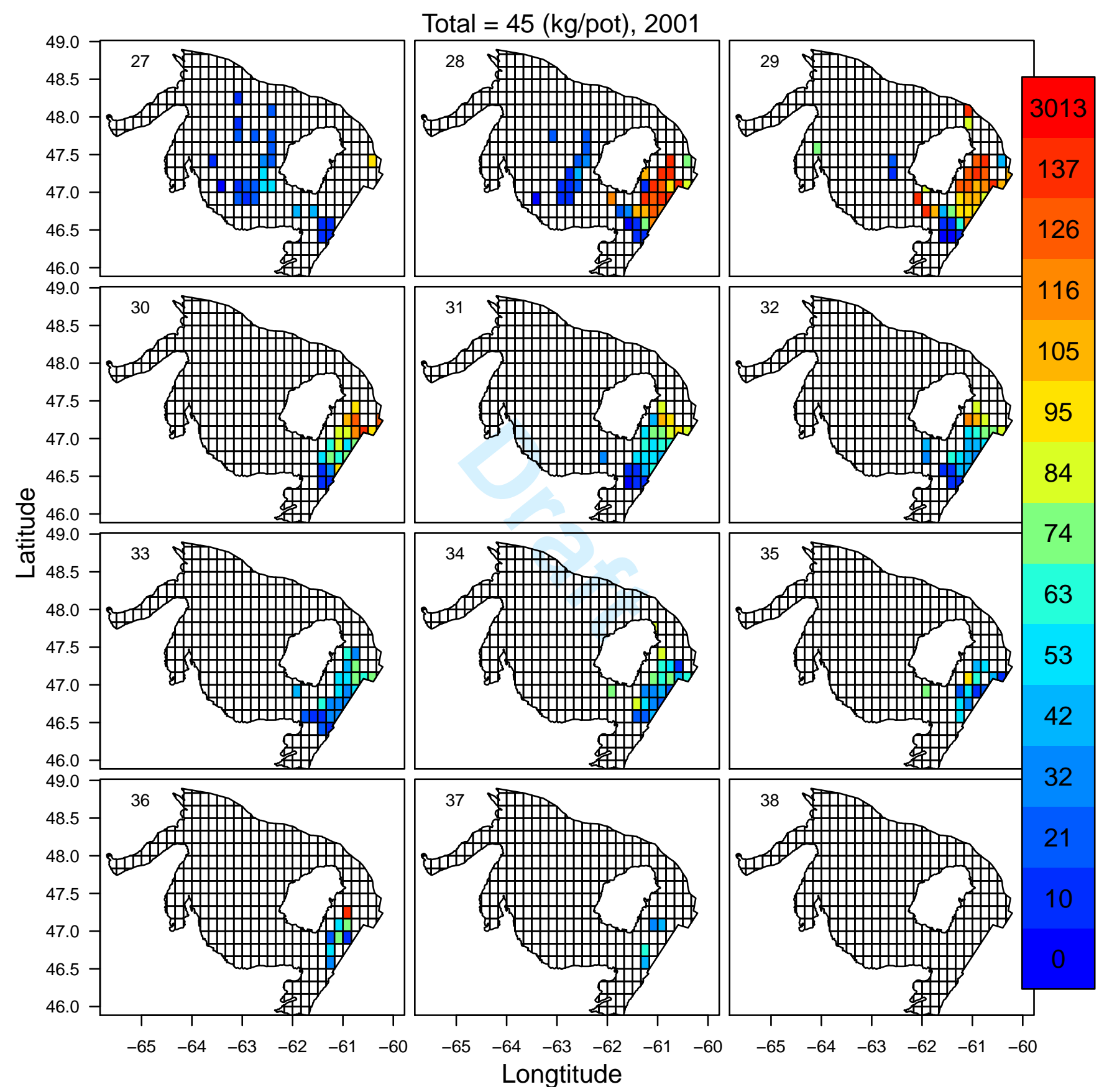

Figure SX.7c. CPUE of snow crab in each week and grid cell in 2001. The week number is indicated in the top left-hand corner. Colors correspond to CPUE levels, as indicated in the legend on the right-hand side. Darkest red grids indicate CPUE $>$ 98th percentile. 


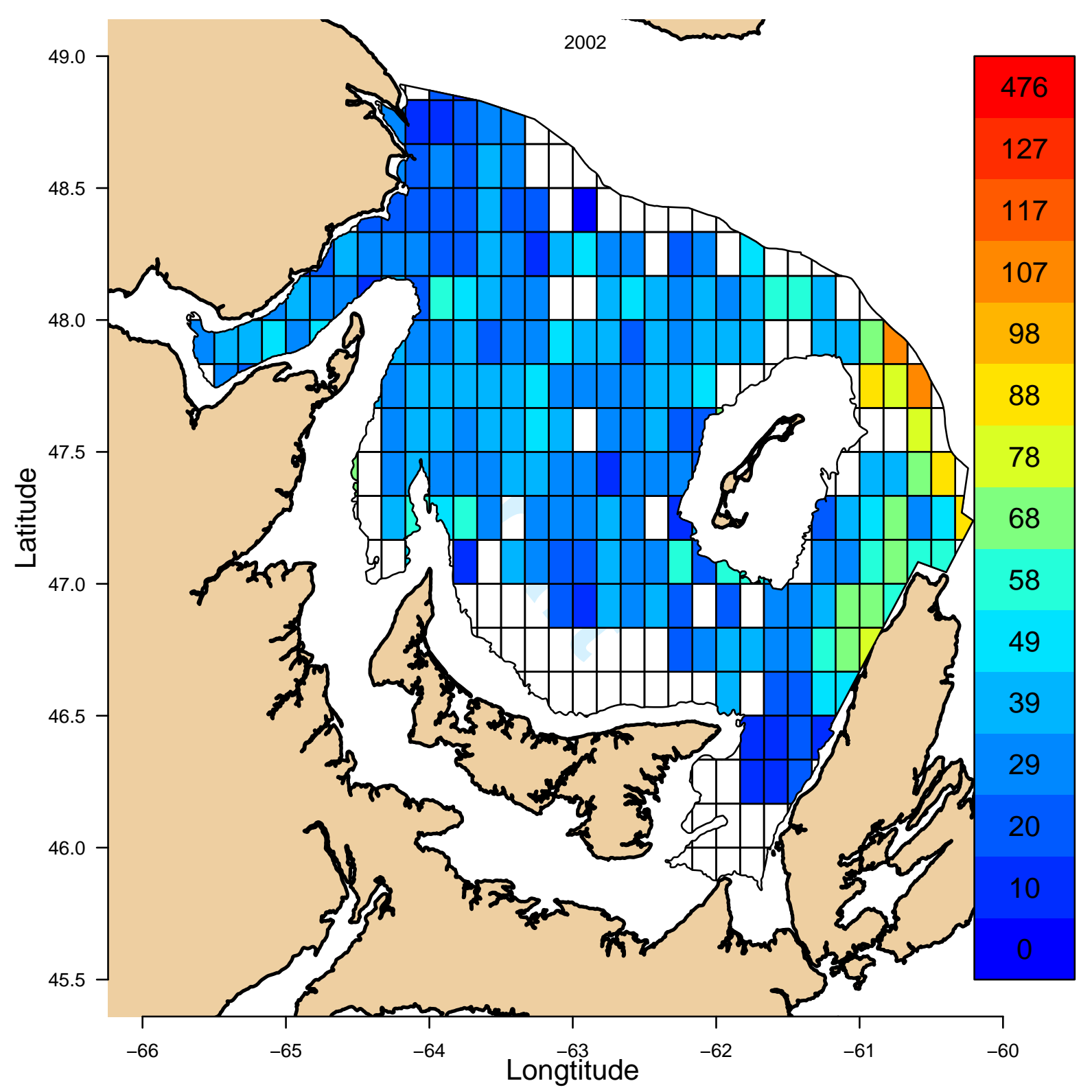

Figure SX.8a. Total annual CPUE of snow crab in each grid cell in 2002. Colors correspond to CPUE levels, as indicated in the legend on the right-hand side. Darkest red grids indicate CPUE > 98th percentile. 


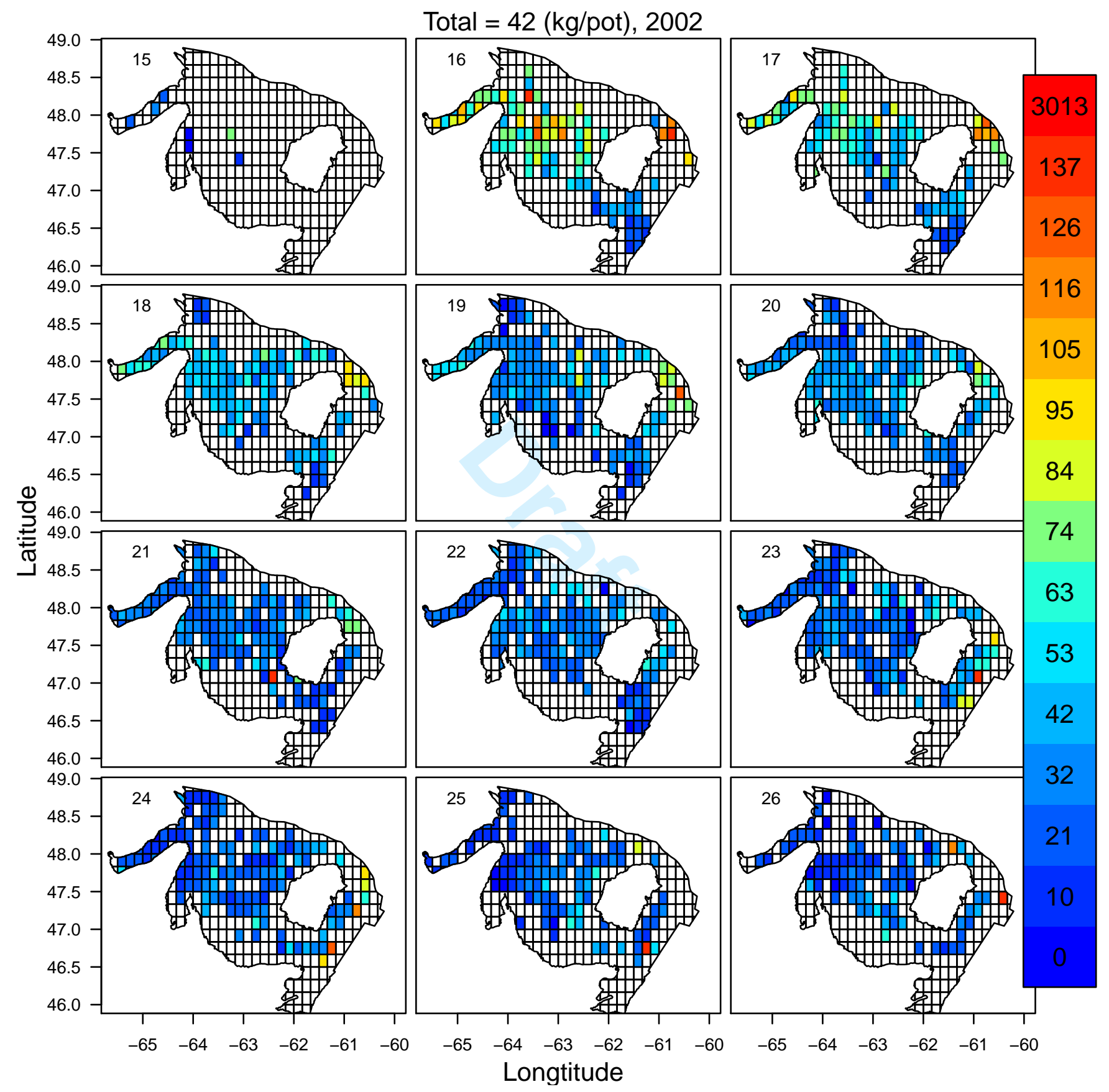

Figure SX.8b. CPUE of snow crab in each week and grid cell in 2002. The week number is indicated in the top left-hand corner. Colors correspond to CPUE levels, as indicated in the legend on the right-hand side. Darkest red grids indicate CPUE $>98$ th percentile 


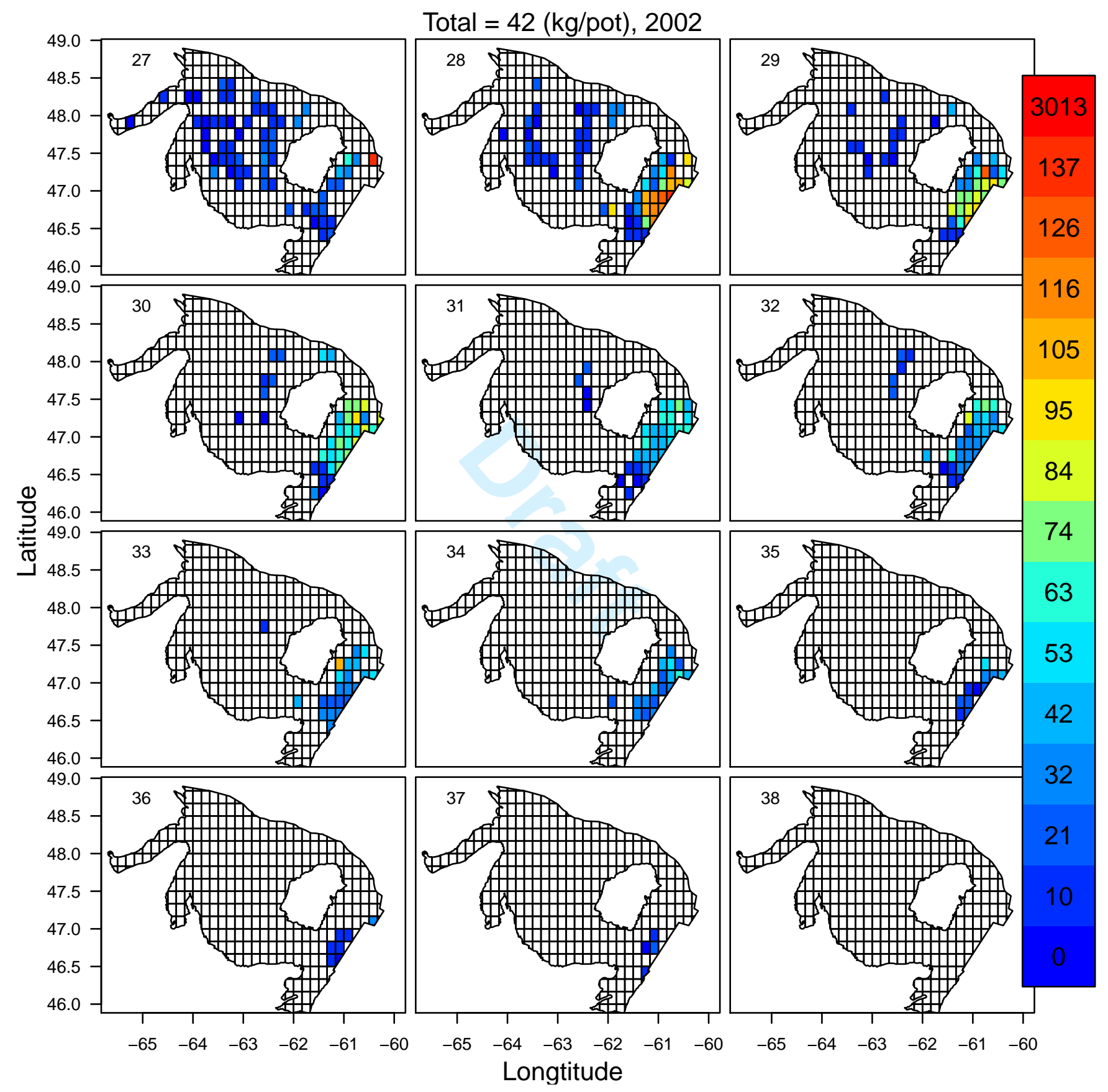

Figure SX.8c. CPUE of snow crab in each week and grid cell in 2002. The week number is indicated in the top left-hand corner. Colors correspond to CPUE levels, as indicated in the legend on the right-hand side. Darkest red grids indicate CPUE $>$ 98th percentile. 


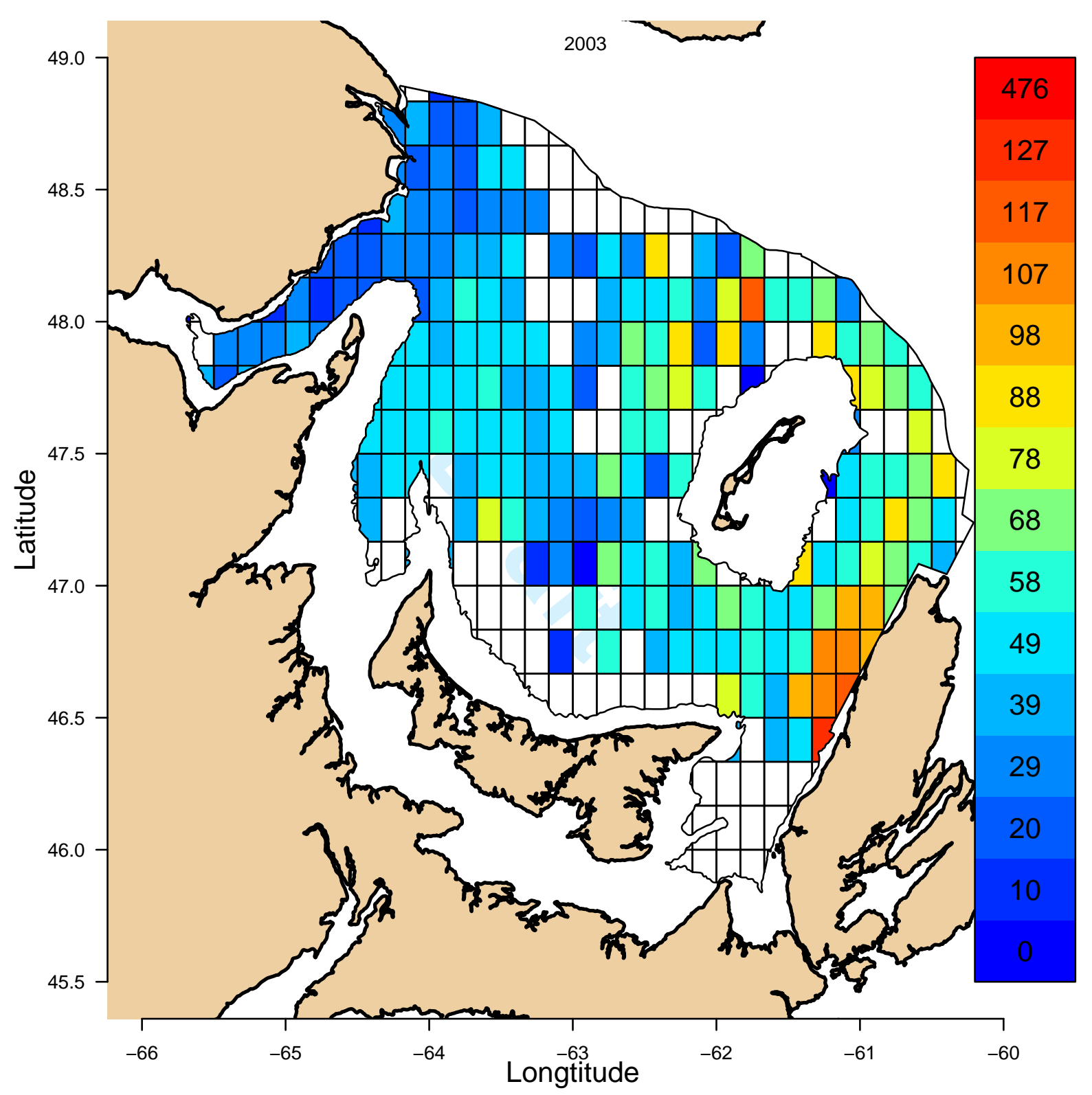

Figure SX.9a. Total annual CPUE of snow crab in each grid cell in 2003. Colors correspond to CPUE levels, as indicated in the legend on the right-hand side. Darkest red grids indicate CPUE > 98th percentile. 


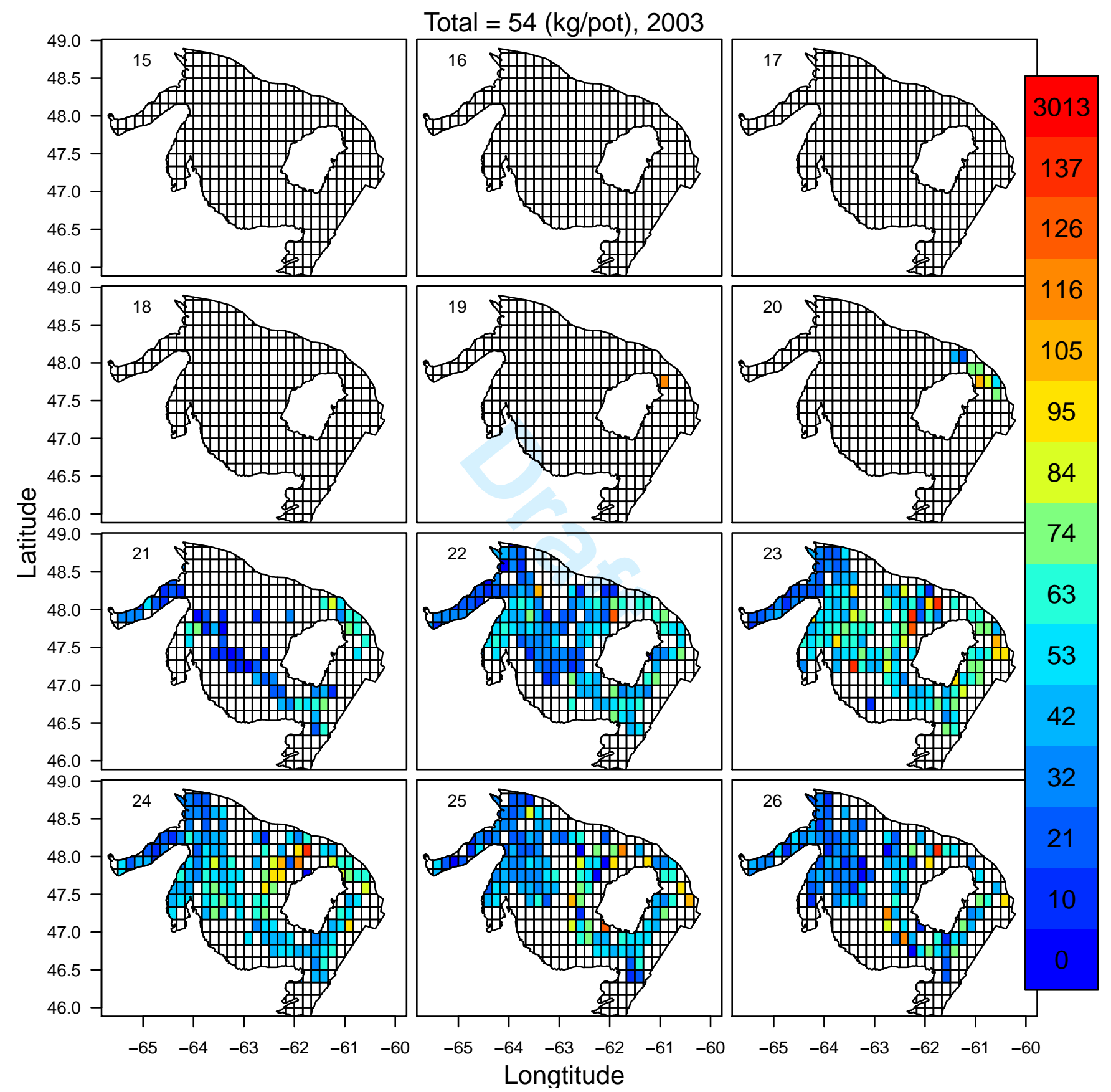

Figure SX.9b. CPUE of snow crab in each week and grid cell in 2003. The week number is indicated in the top left-hand corner. Colors correspond to CPUE levels, as indicated in the legend on the right-hand side. Darkest red grids indicate CPUE $>$ 98th percentile. 


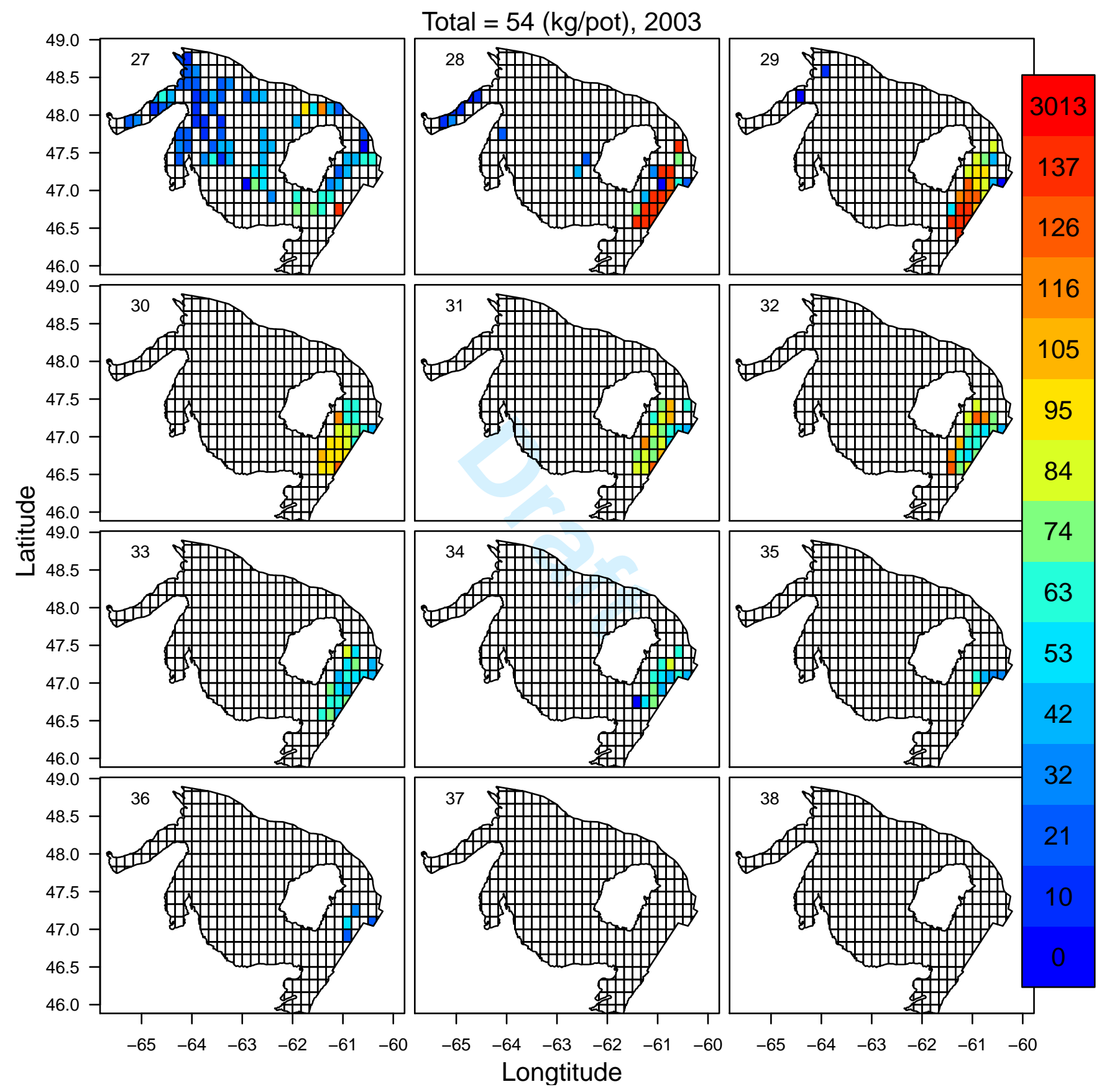

Figure SX.9c. CPUE of snow crab in each week and grid cell in 2003. The week number is indicated in the top left-hand corner. Colors correspond to CPUE levels, as indicated in the legend on the right-hand side. Darkest red grids indicate CPUE $>$ 98th percentile. 


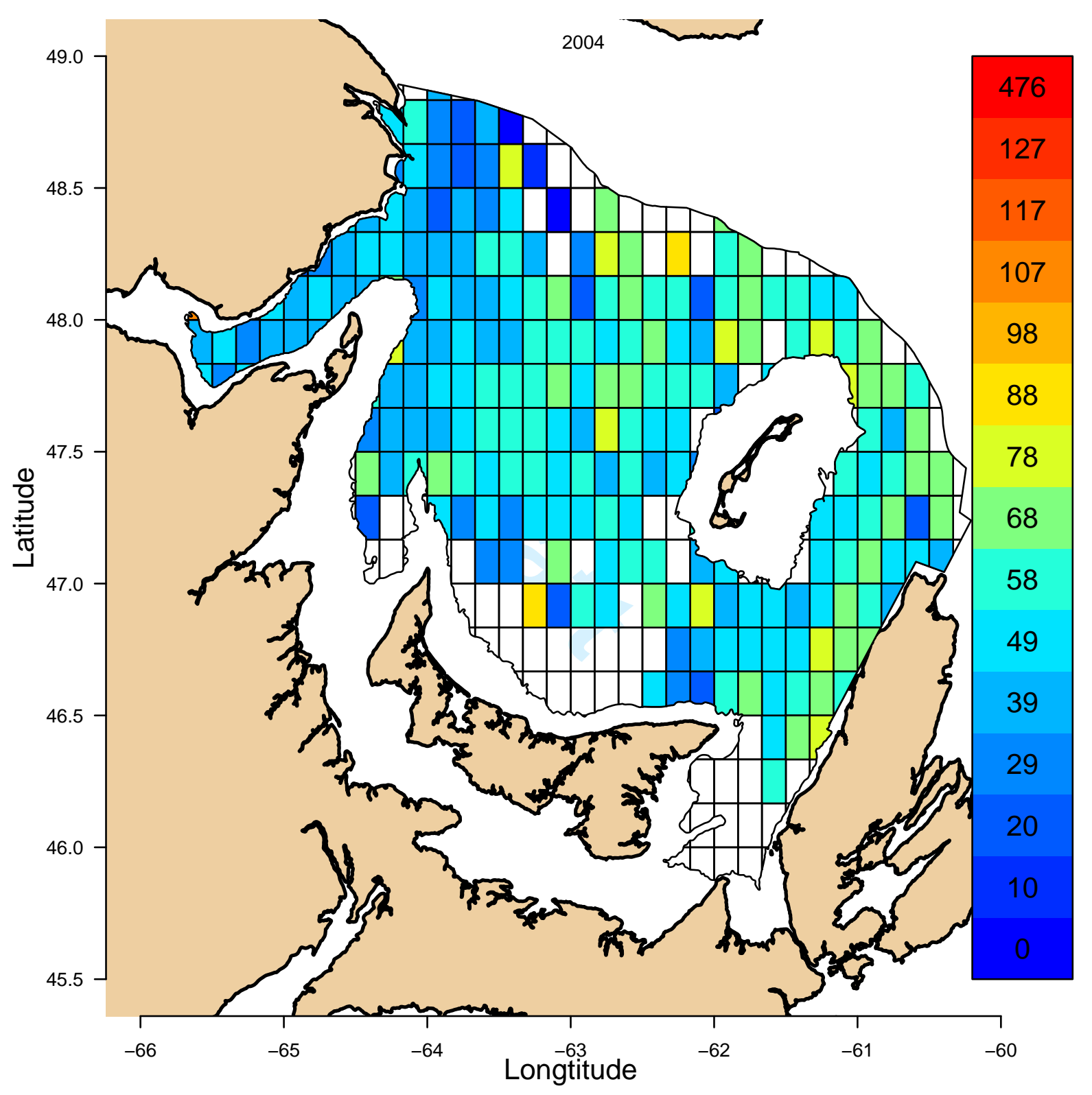

Figure SX.10a. Total annual CPUE of snow crab in each grid cell in 2004. Colors correspond to CPUE levels, as indicated in the legend on the right-hand side. Darkest red grids indicate CPUE > 98th percentile. 


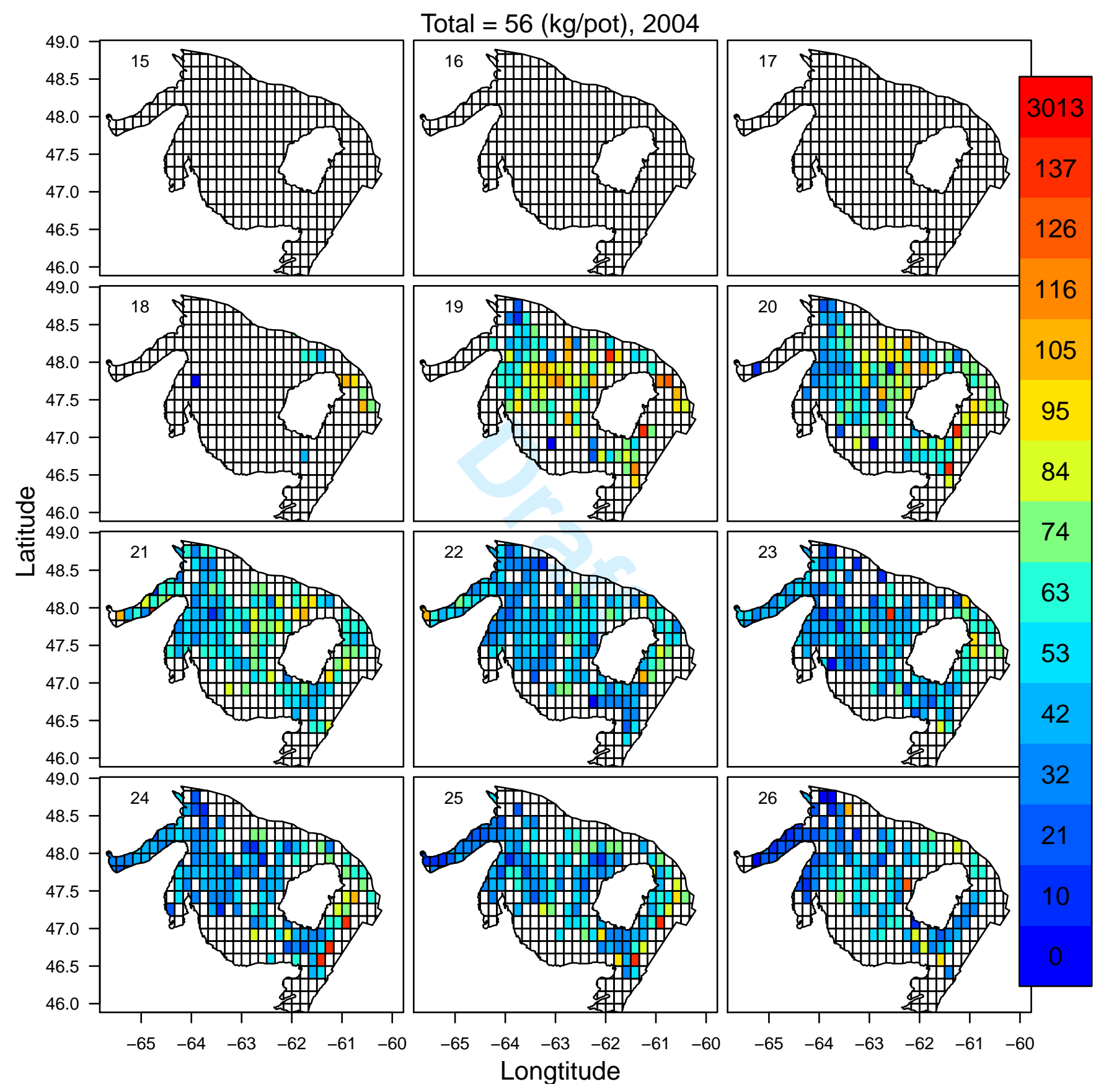

Figure SX.10b. CPUE of snow crab in each week and grid cell in 2004. The week number is indicated in the top left-hand corner. Colors correspond to CPUE levels, as indicated in the legend on the right-hand side. Darkest red grids indicate CPUE $>98$ th percentile. 


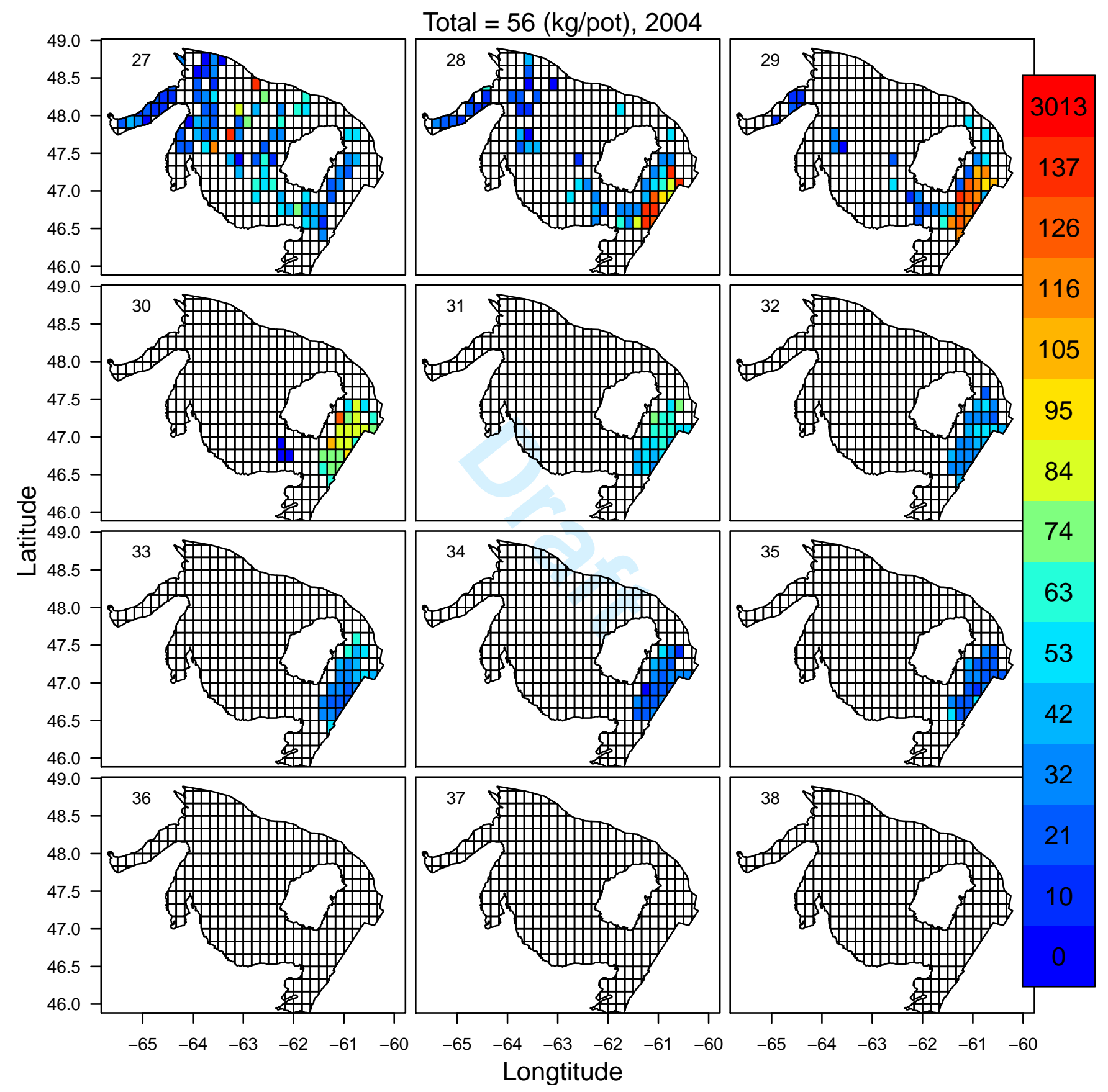

Figure SX.10c. CPUE of snow crab in each week and grid cell in 2004. The week number is indicated in the top left-hand corner. Colors correspond to CPUE levels, as indicated in the legend on the right-hand side. Darkest red grids indicate CPUE $>$ 98th percentile. 


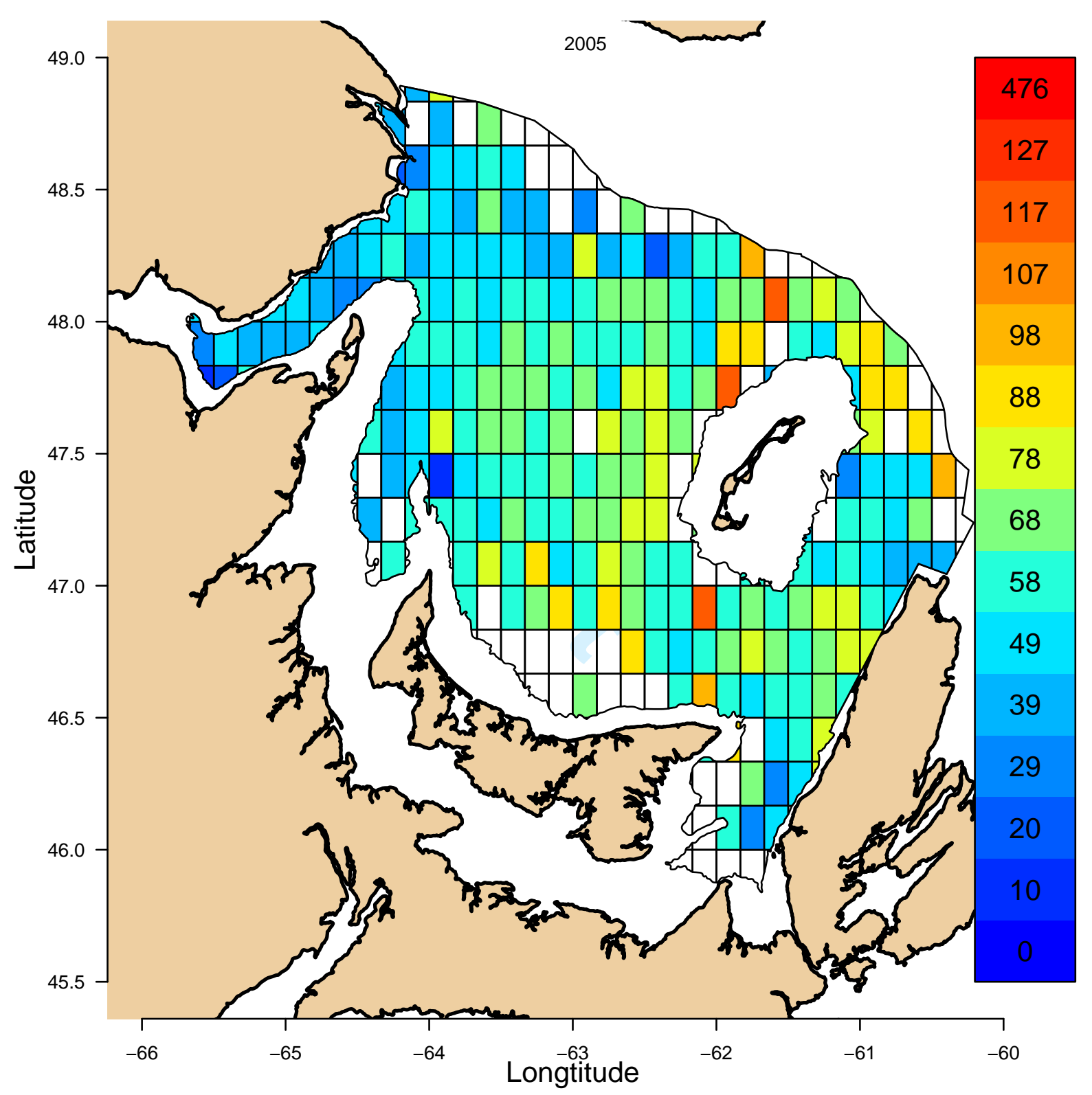

Figure SX.11a. Total annual CPUE of snow crab in each grid cell in 2005. Colors correspond to CPUE levels, as indicated in the legend on the right-hand side. Darkest red grids indicate CPUE > 98th percentile. 


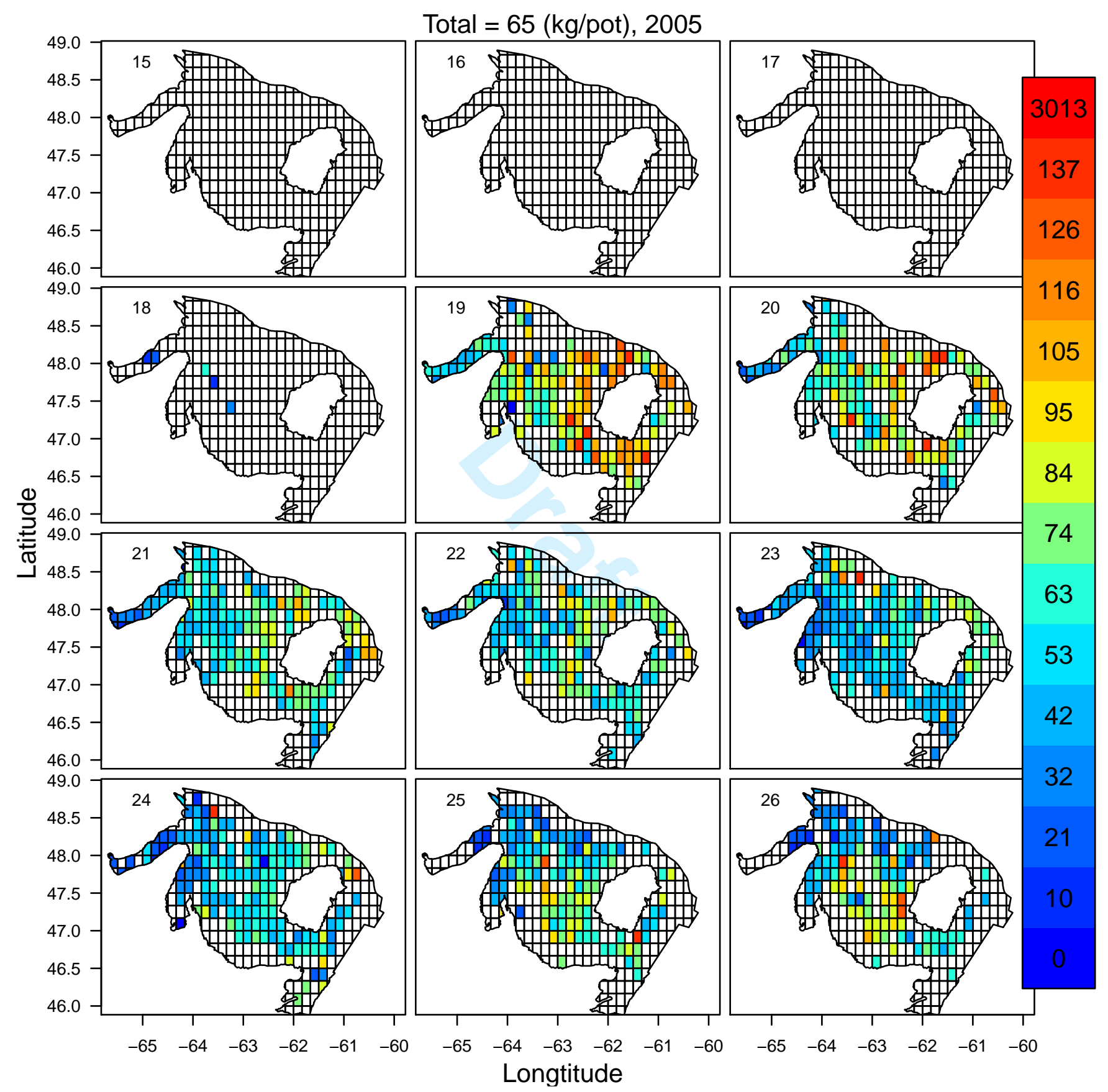

Figure SX.11b. CPUE of snow crab in each week and grid cell in 2005 . The week number is indicated in the top left-hand corner. Colors correspond to CPUE levels, as indicated in the legend on the right-hand side. Darkest red grids indicate CPUE $>$ 98th percentile. 


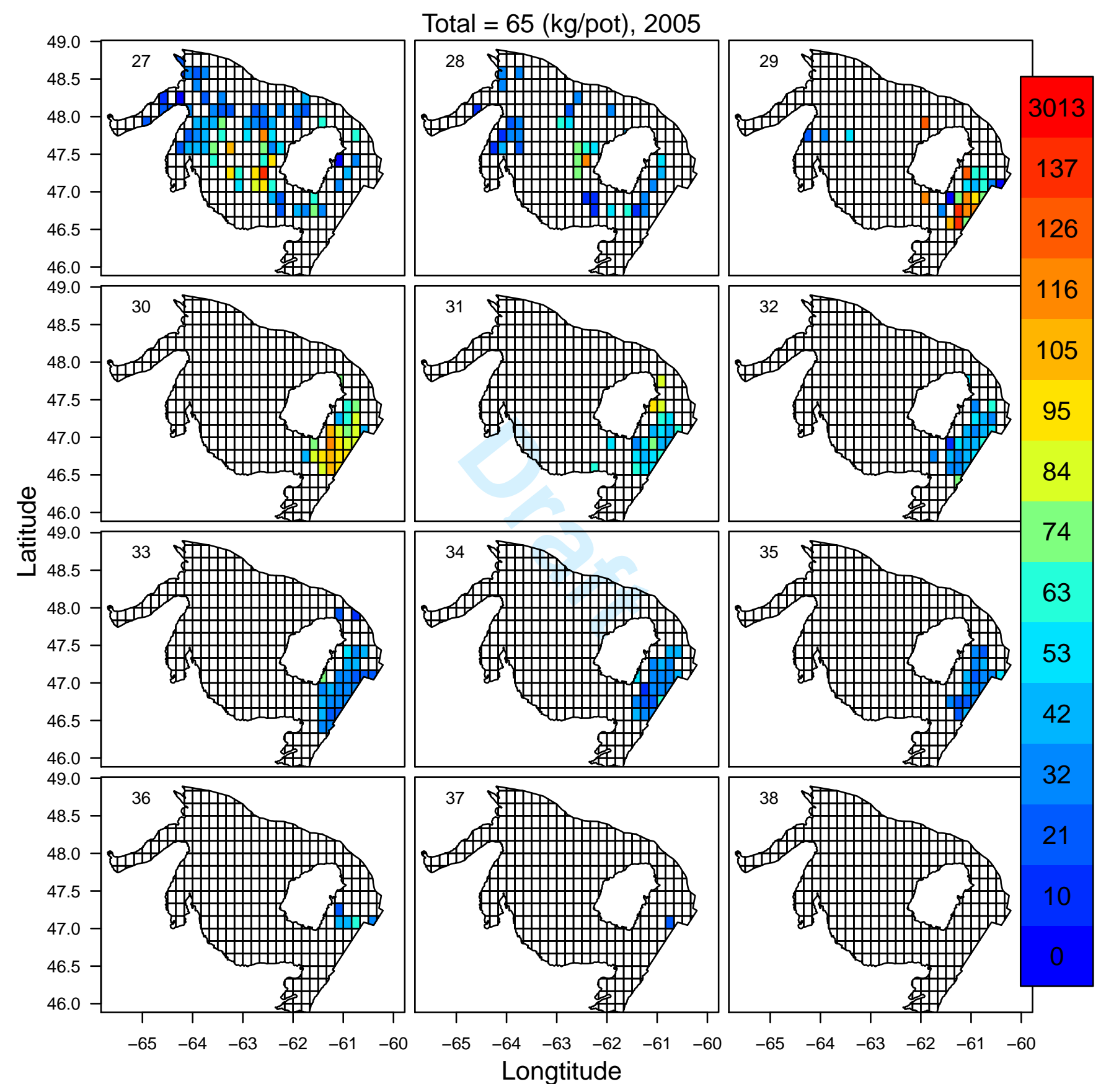

Figure SX.11c. CPUE of snow crab in each week and grid cell in 2005. The week number is indicated in the top left-hand corner. Colors correspond to CPUE levels, as indicated in the legend on the right-hand side. Darkest red grids indicate CPUE $>98$ th percentile. 


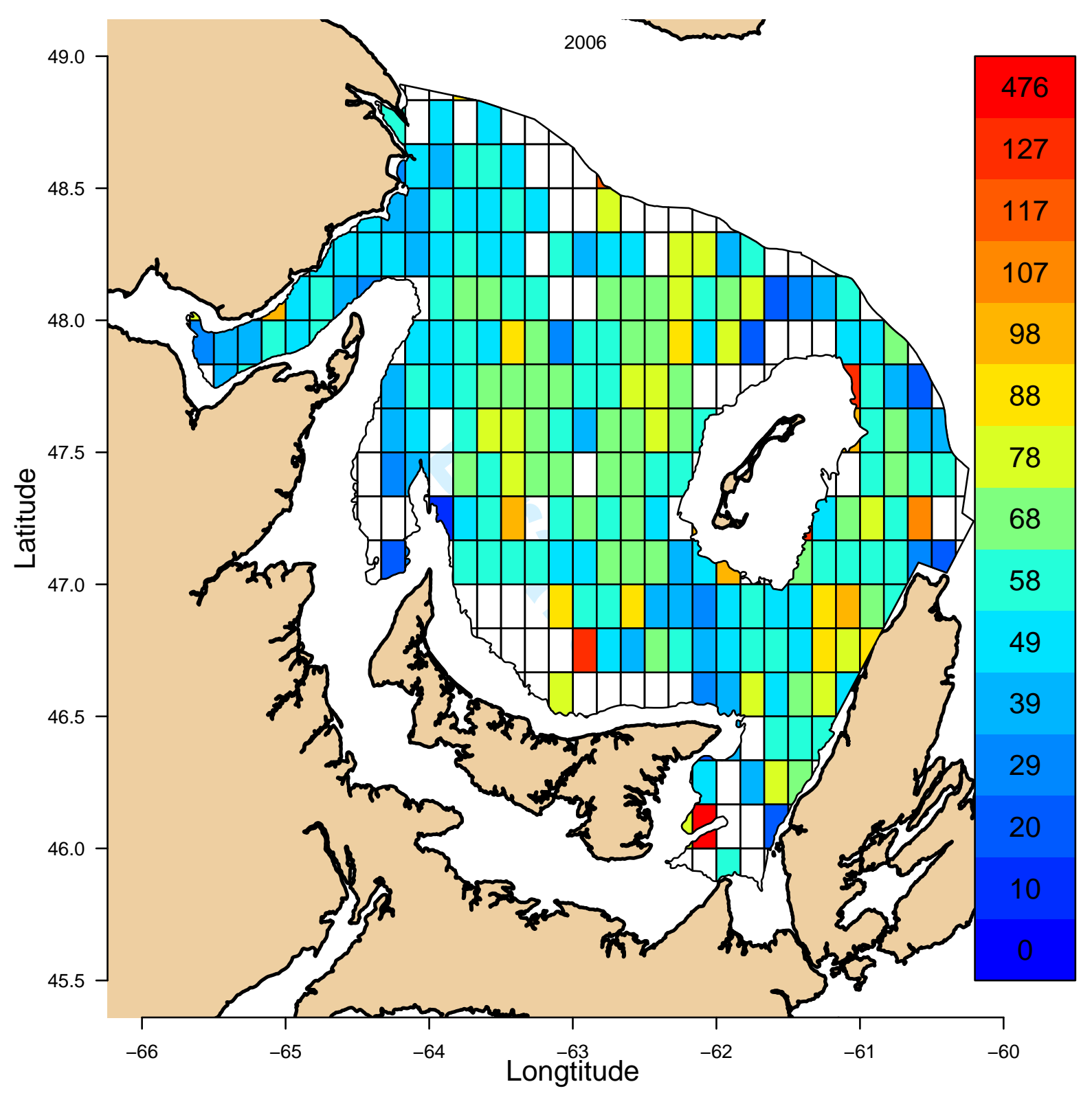

Figure SX.12a. Total annual CPUE of snow crab in each grid cell in 2006. Colors correspond to CPUE levels, as indicated in the legend on the right-hand side. Darkest red grids indicate CPUE > 98th percentile. 


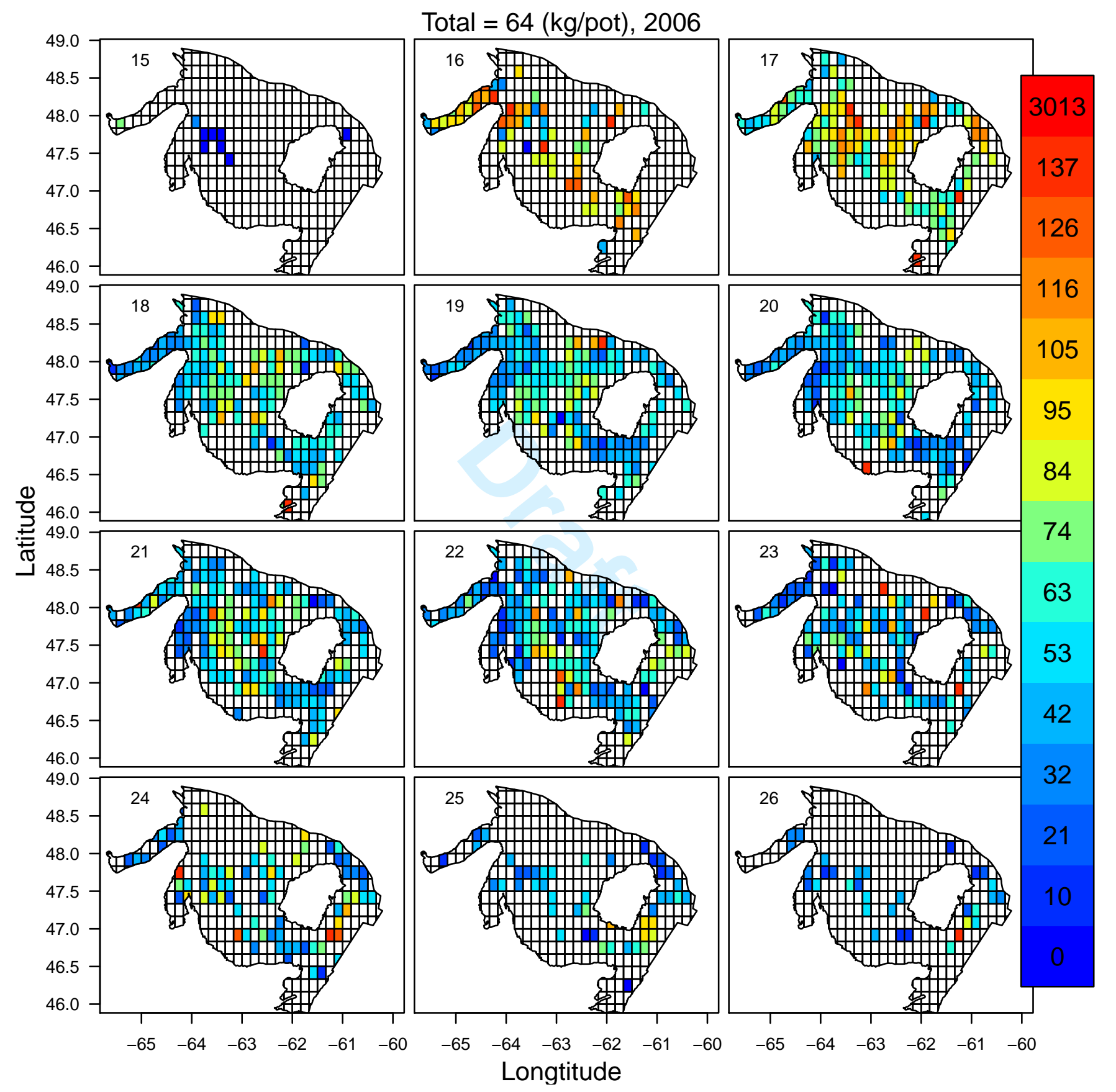

Figure SX.12b. CPUE of snow crab in each week and grid cell in 2006. The week number is indicated in the top left-hand corner. Colors correspond to CPUE levels, as indicated in the legend on the right-hand side. Darkest red grids indicate CPUE $>$ 98th percentile. 


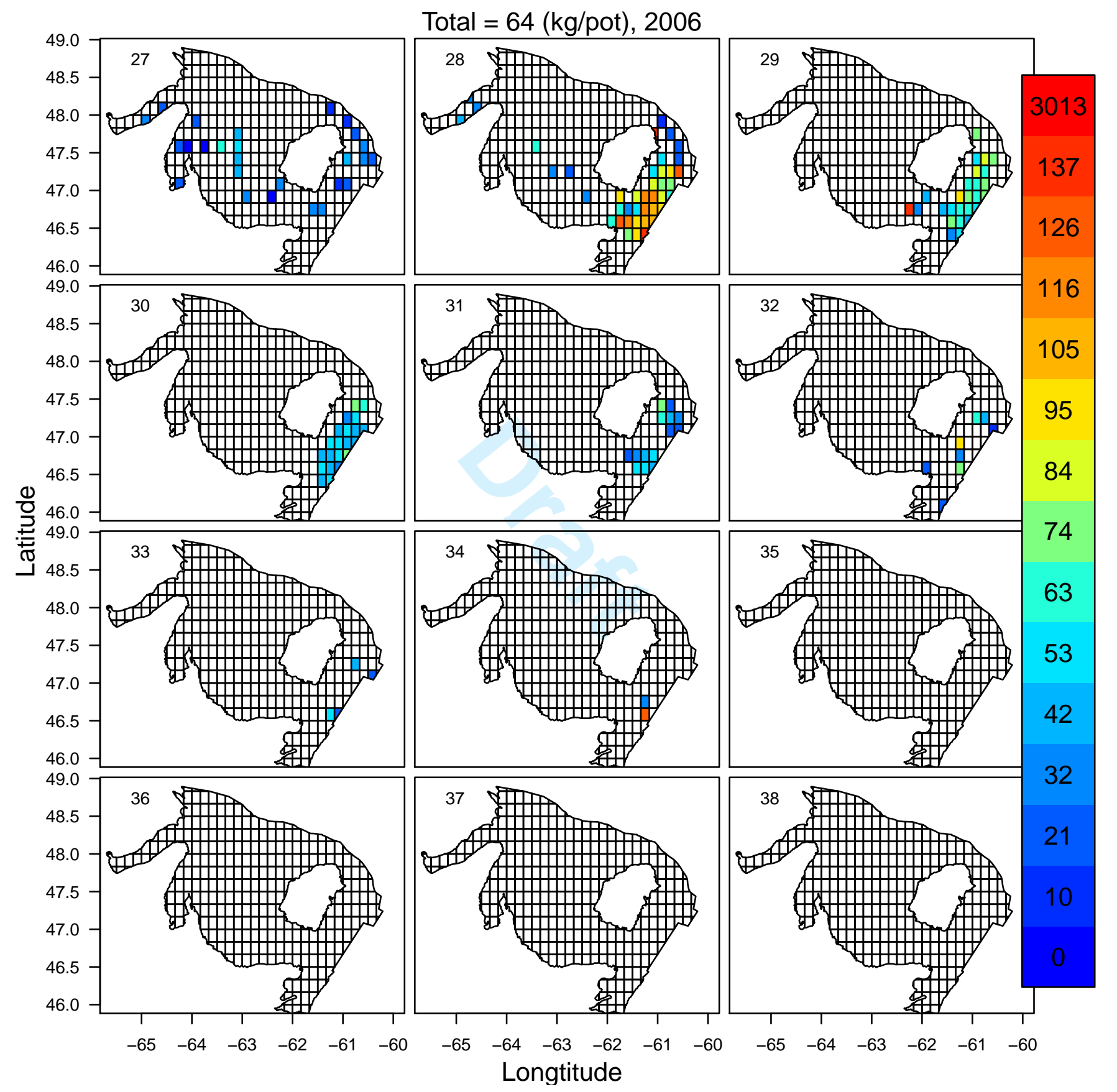

Figure SX.12c. CPUE of snow crab in each week and grid cell in 2006. The week number is indicated in the top left-hand corner. Colors correspond to CPUE levels, as indicated in the legend on the right-hand side. Darkest red grids indicate CPUE $>98$ th percentile. 


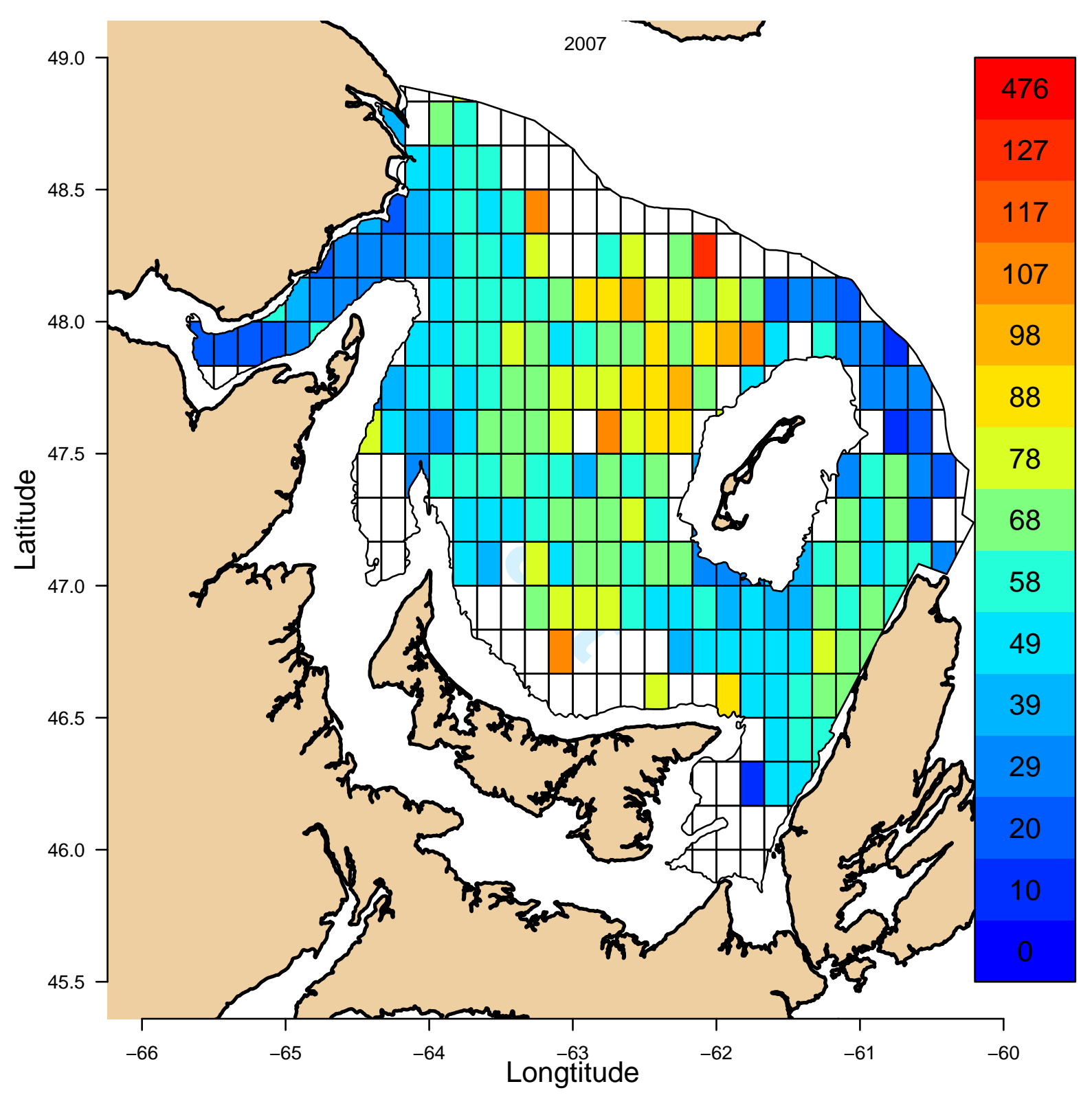

Figure SX.13a. Total annual CPUE of snow crab in each grid cell in 2007. Colors correspond to CPUE levels, as indicated in the legend on the right-hand side. Darkest red grids indicate CPUE $>$ 98th percentile. 


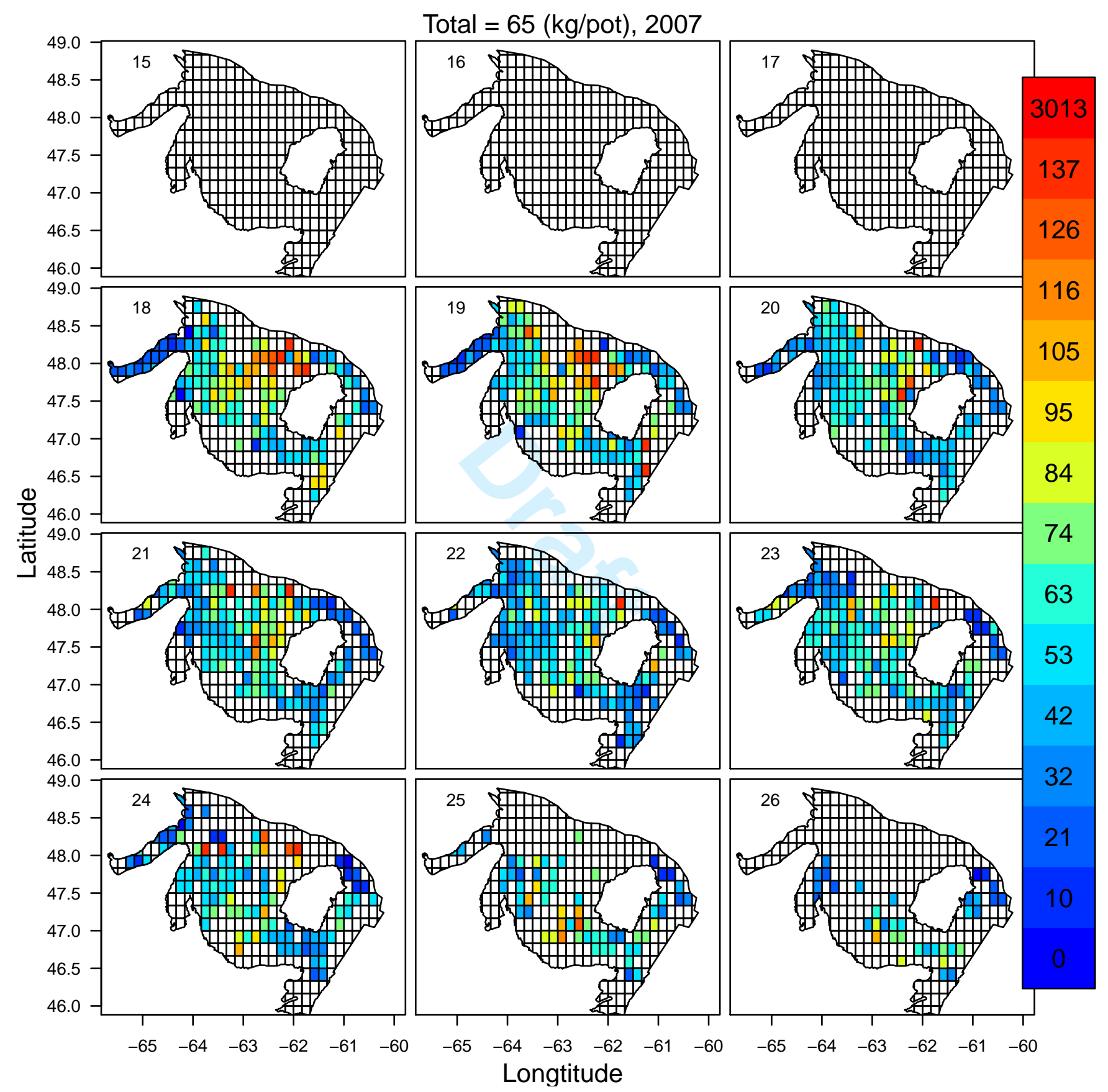

Figure SX.13b. CPUE of snow crab in each week and grid cell in 2007. The week number is indicated in the top left-hand corner. Colors correspond to CPUE levels, as indicated in the legend on the right-hand side. Darkest red grids indicate CPUE $>$ 98th percentile. 


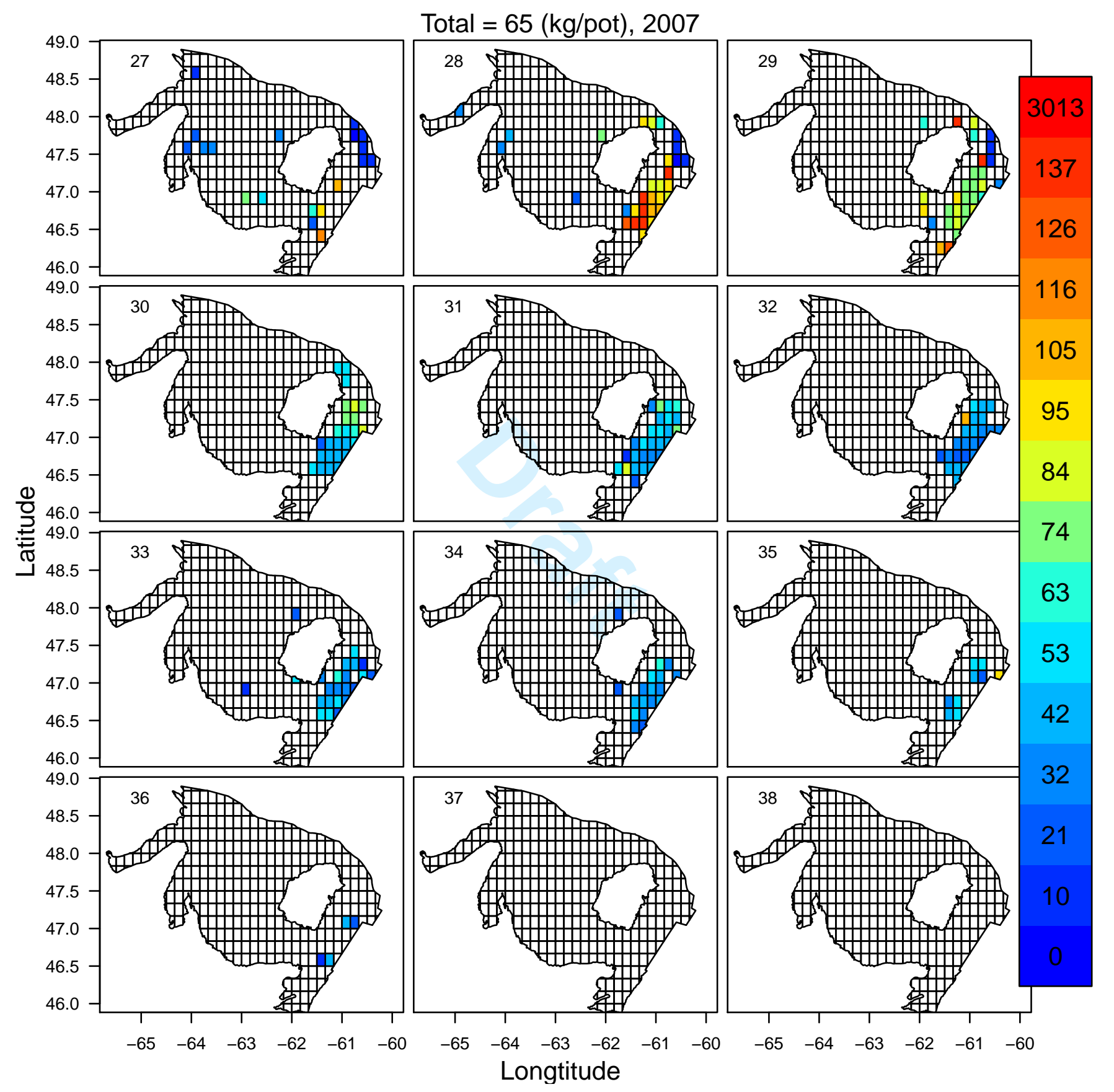

Figure SX.13c. CPUE of snow crab in each week and grid cell in 2007. The week number is indicated in the top left-hand corner. Colors correspond to CPUE levels, as indicated in the legend on the right-hand side. Darkest red grids indicate CPUE $>$ 98th percentile. 


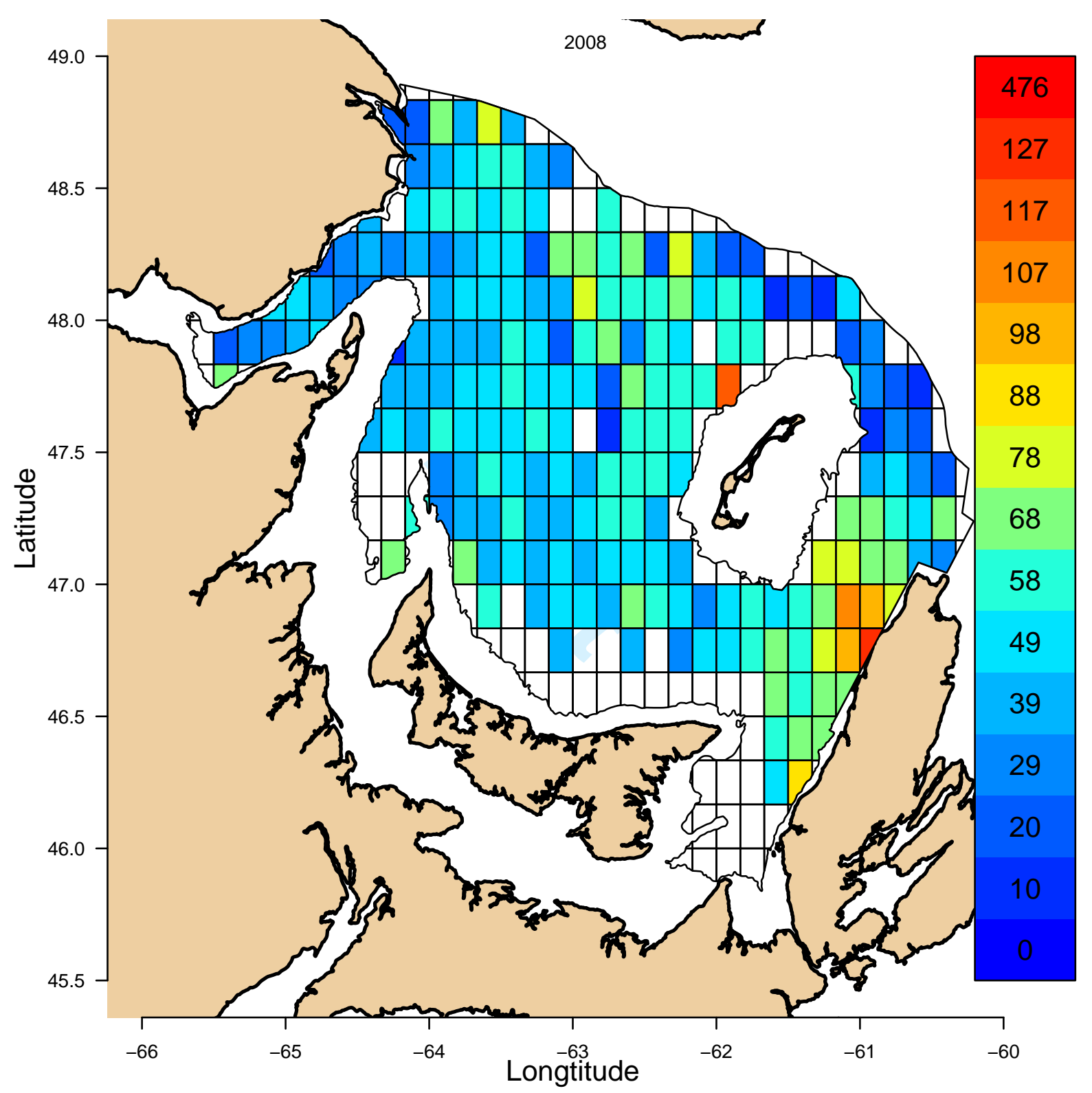

Figure SX.14a. Total annual CPUE of snow crab in each grid cell in 2008. Colors correspond to CPUE levels, as indicated in the legend on the right-hand side. Darkest red grids indicate CPUE $>$ 98th percentile. 


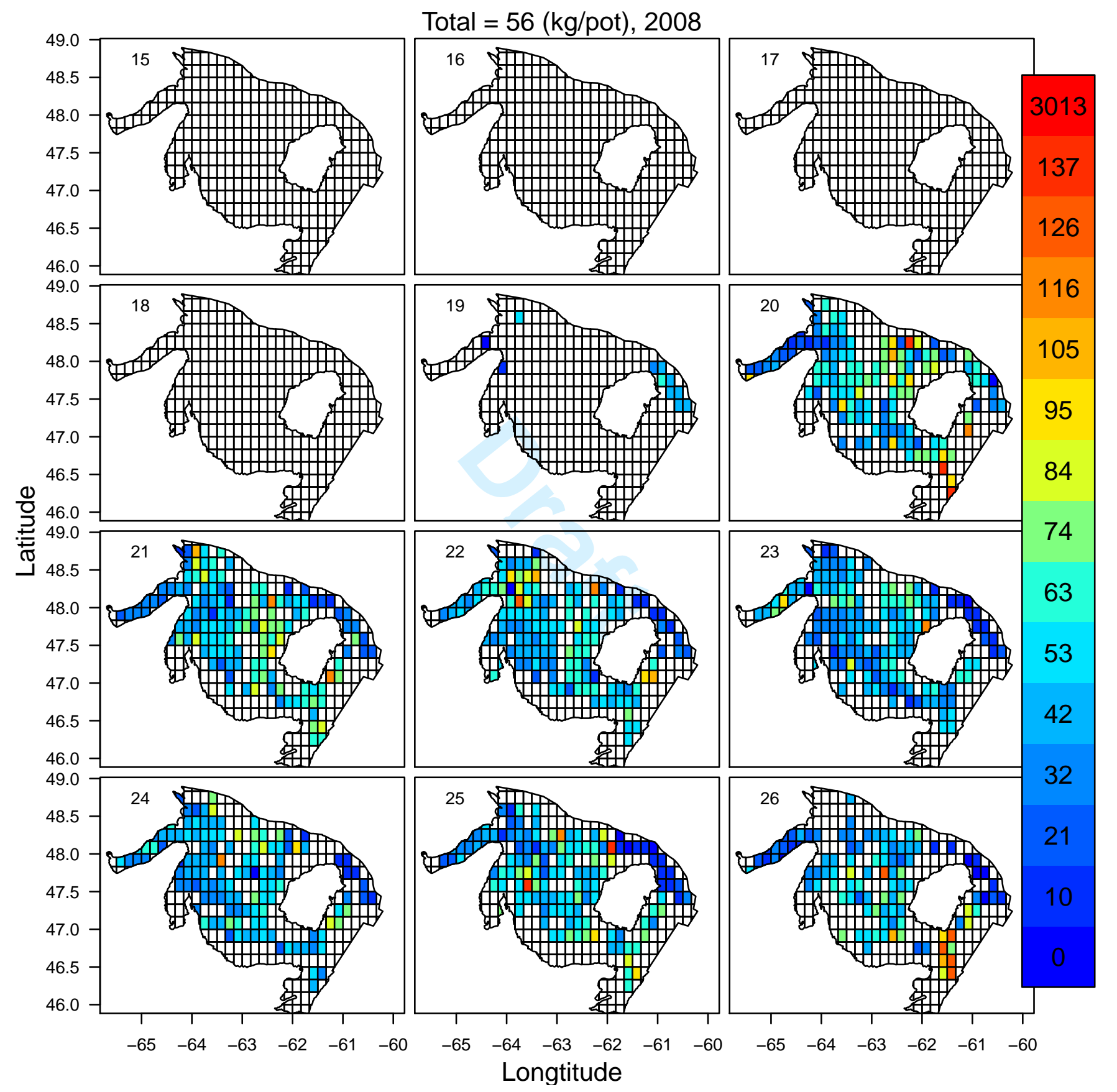

Figure SX.14b. CPUE of snow crab in each week and grid cell in 2008. The week number is indicated in the top left-hand corner. Colors correspond to CPUE levels, as indicated in the legend on the right-hand side. Darkest red grids indicate CPUE $>$ 98th percentile. 


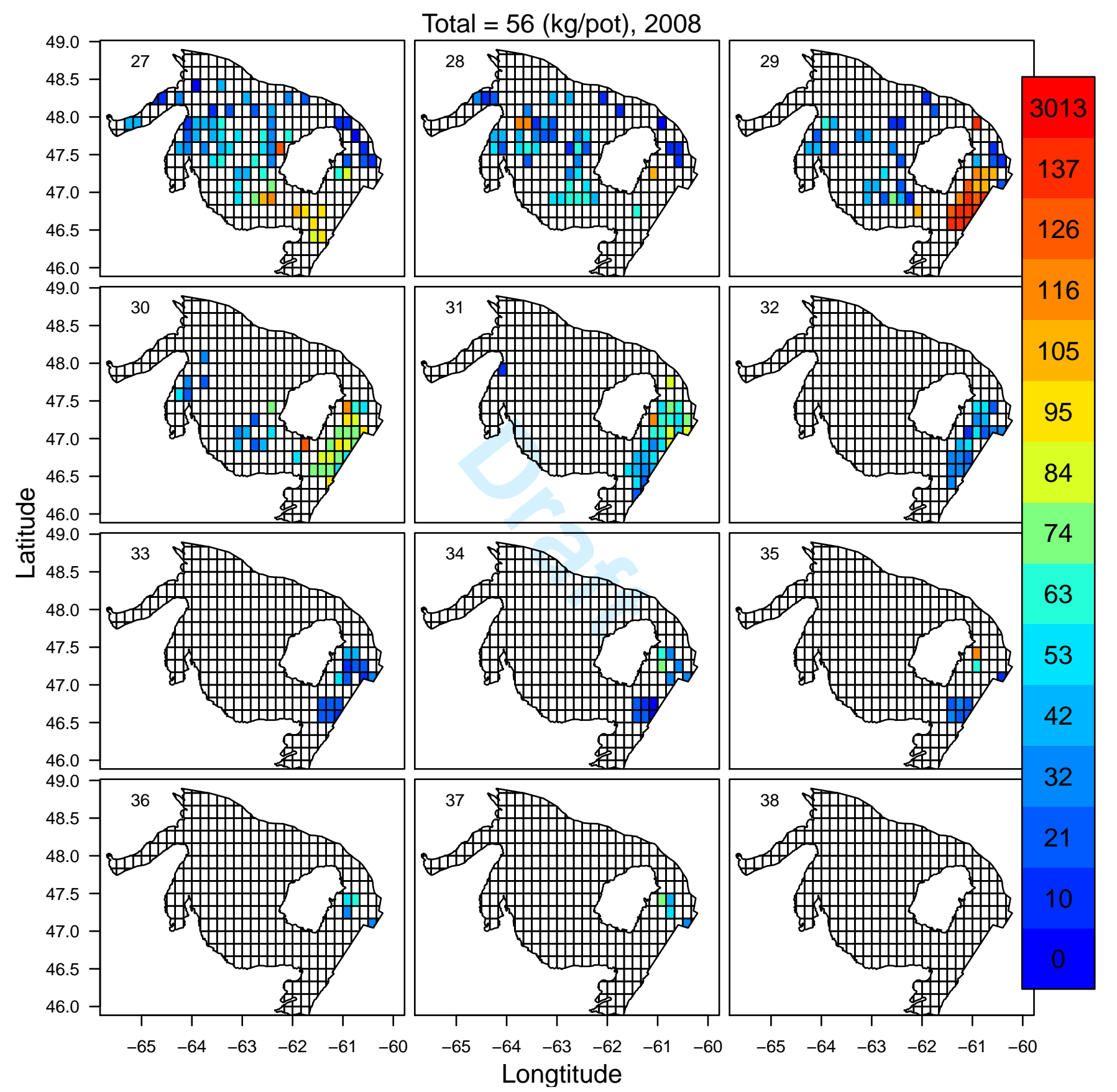

Figure SX.14c. CPUE of snow crab in each week and grid cell in 2008. The week number is indicated in the top left-hand corner. Colors correspond to CPUE levels, as indicated in the legend on the right-hand side. Darkest red grids indicate CPUE $>$ 98th percentile. 


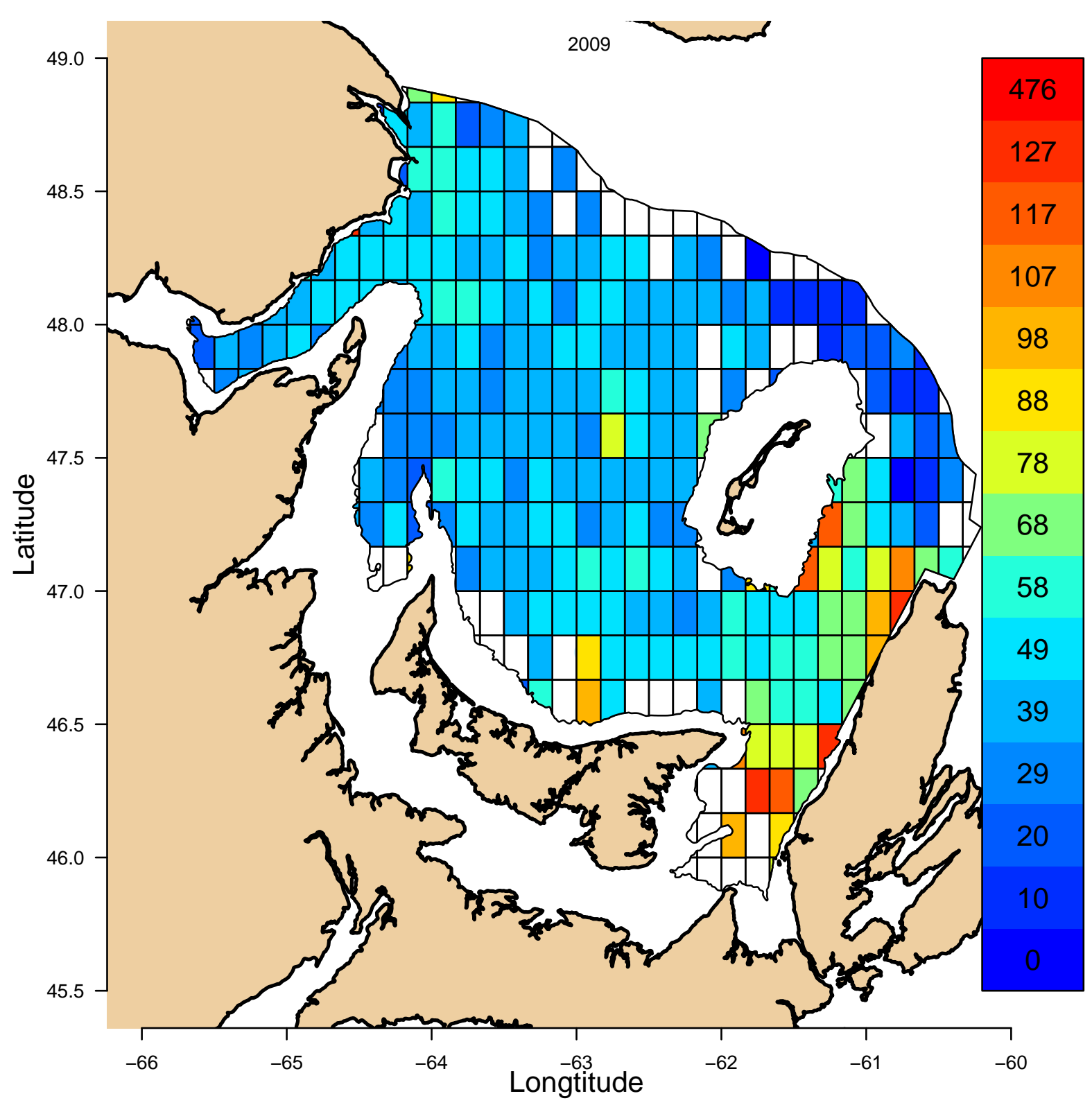

Figure SX.15a. Total annual CPUE of snow crab in each grid cell in 2009. Colors correspond to CPUE levels, as indicated in the legend on the right-hand side. Darkest red grids indicate CPUE > 98th percentile. 


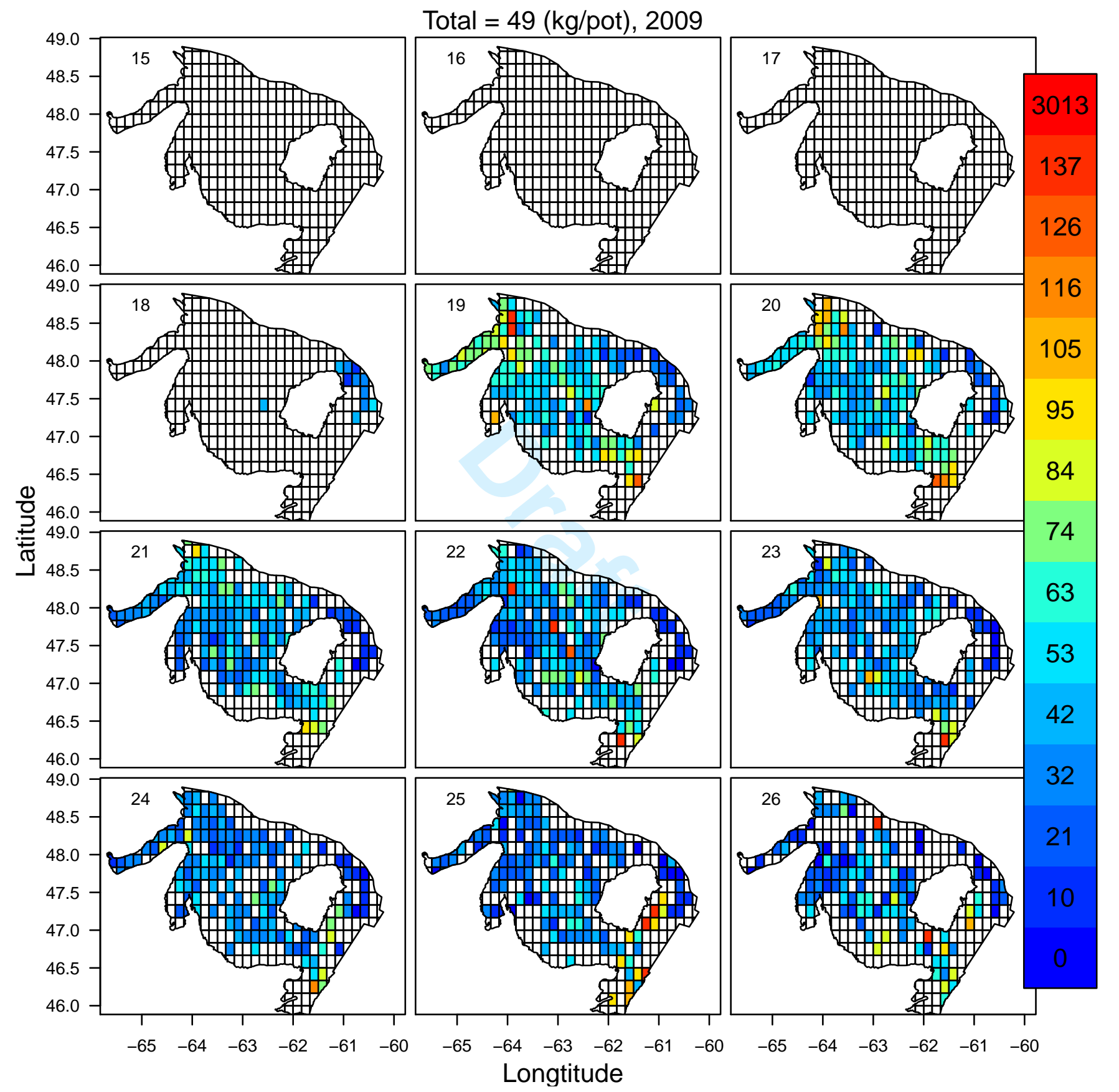

Figure SX.15b. CPUE of snow crab in each week and grid cell in 2009. The week number is indicated in the top left-hand corner. Colors correspond to CPUE levels, as indicated in the legend on the right-hand side. Darkest red grids indicate CPUE $>$ 98th percentile. 


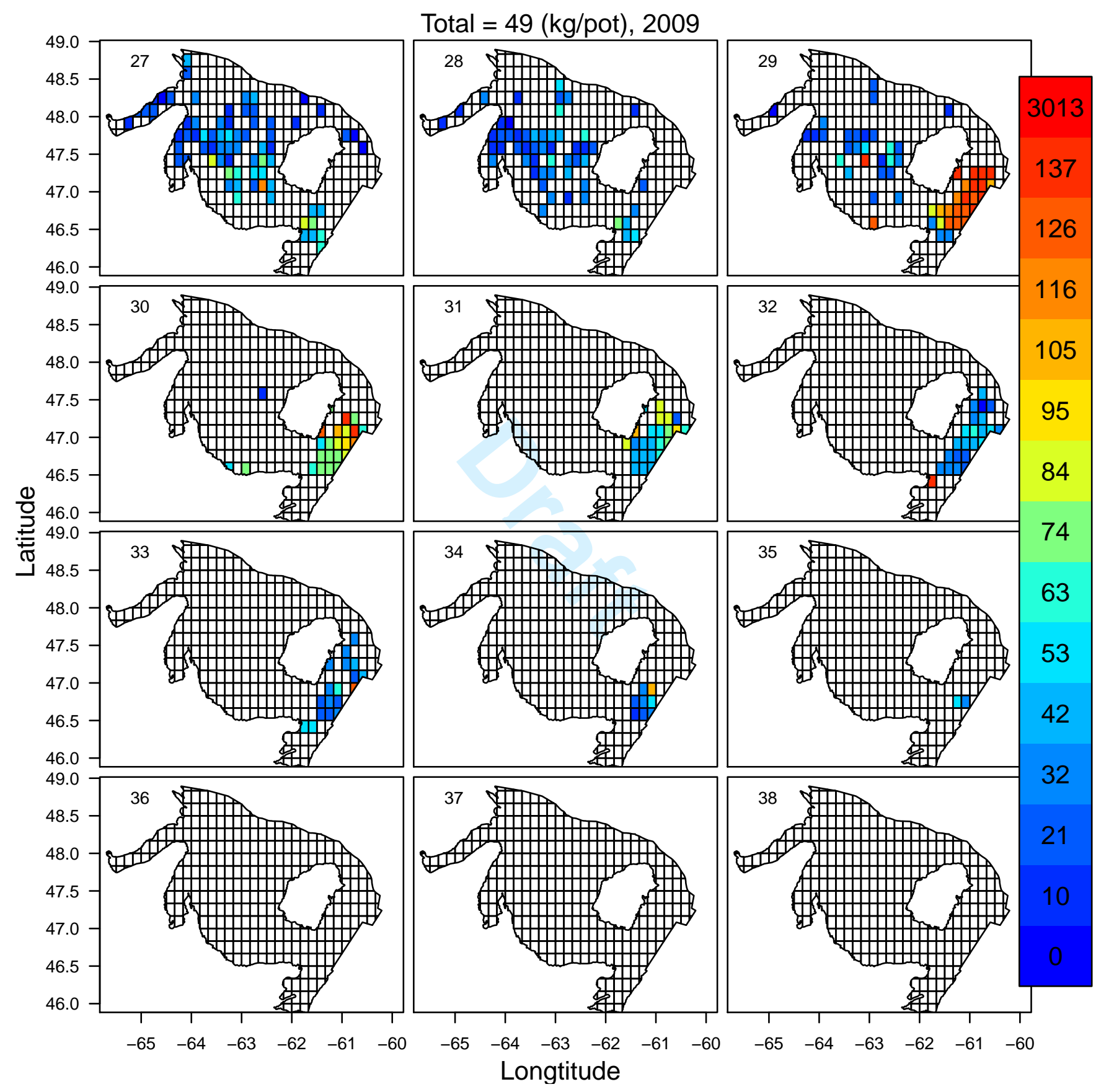

Figure SX.15c. CPUE of snow crab in each week and grid cell in 2009. The week number is indicated in the top left-hand corner. Colors correspond to CPUE levels, as indicated in the legend on the right-hand side. Darkest red grids indicate CPUE $>$ 98th percentile. 


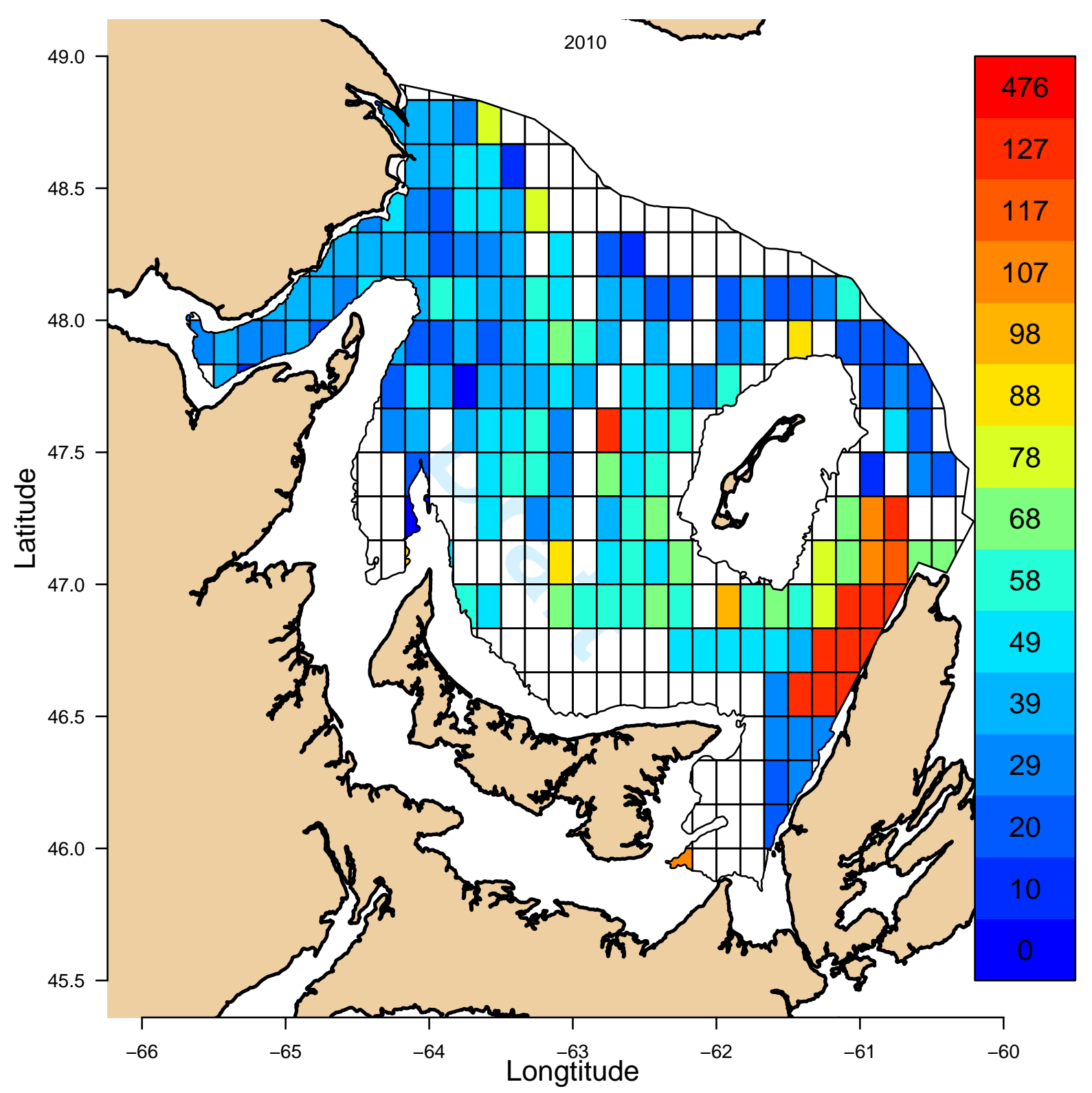

Figure SX.16a. Total annual CPUE of snow crab in each grid cell in 2010. Colors correspond to CPUE levels, as indicated in the legend on the right-hand side. Darkest red grids indicate CPUE > 98th percentile. 


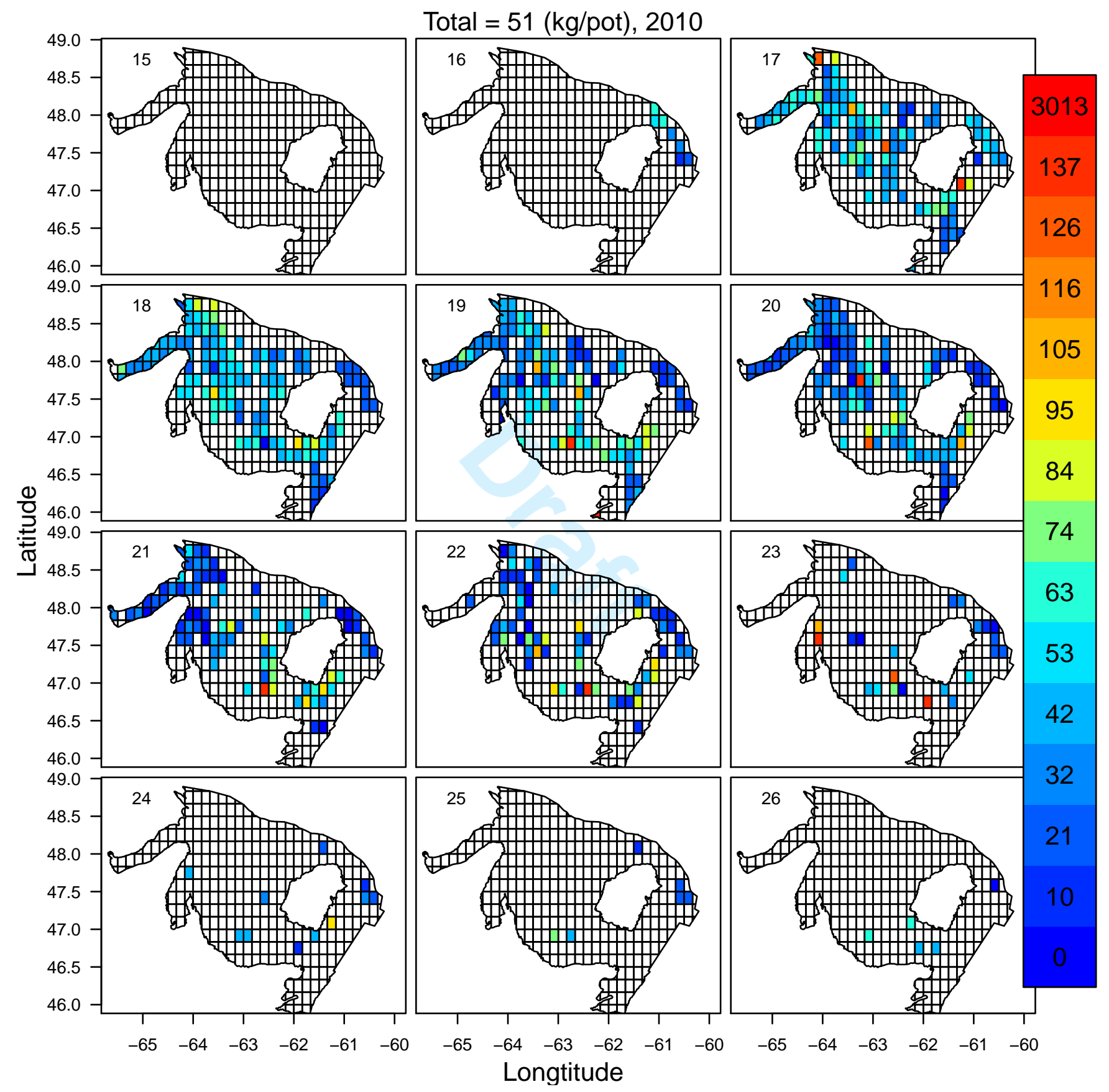

Figure SX.16b. CPUE of snow crab in each week and grid cell in 2010. The week number is indicated in the top left-hand corner. Colors correspond to CPUE levels, as indicated in the legend on the right-hand side. Darkest red grids indicate CPUE $>$ 98th percentile. 


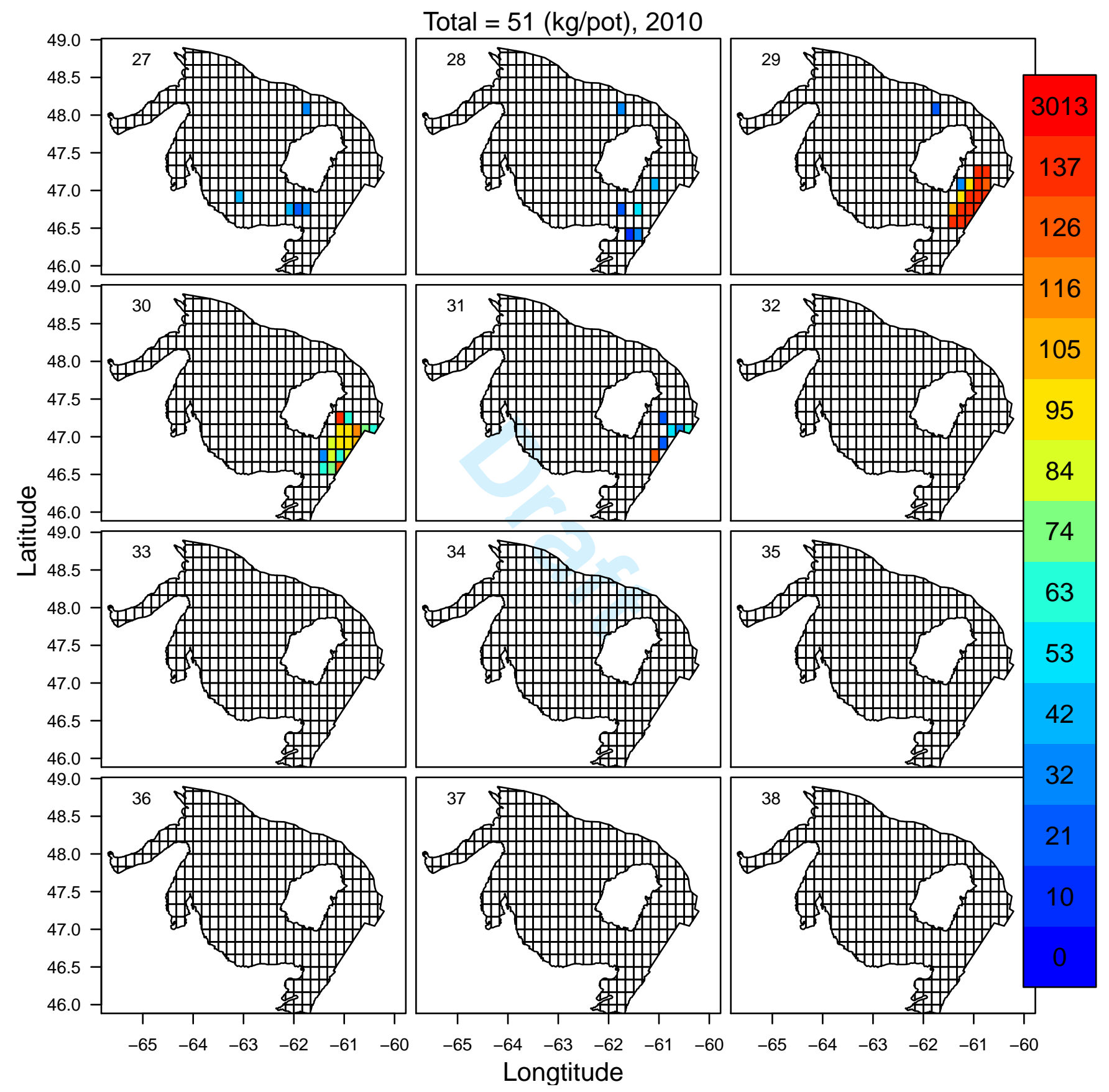

Figure SX.16c. CPUE of snow crab in each week and grid cell in 2010. The week number is indicated in the top left-hand corner. Colors correspond to CPUE levels, as indicated in the legend on the right-hand side. Darkest red grids indicate CPUE $>$ 98th percentile. 


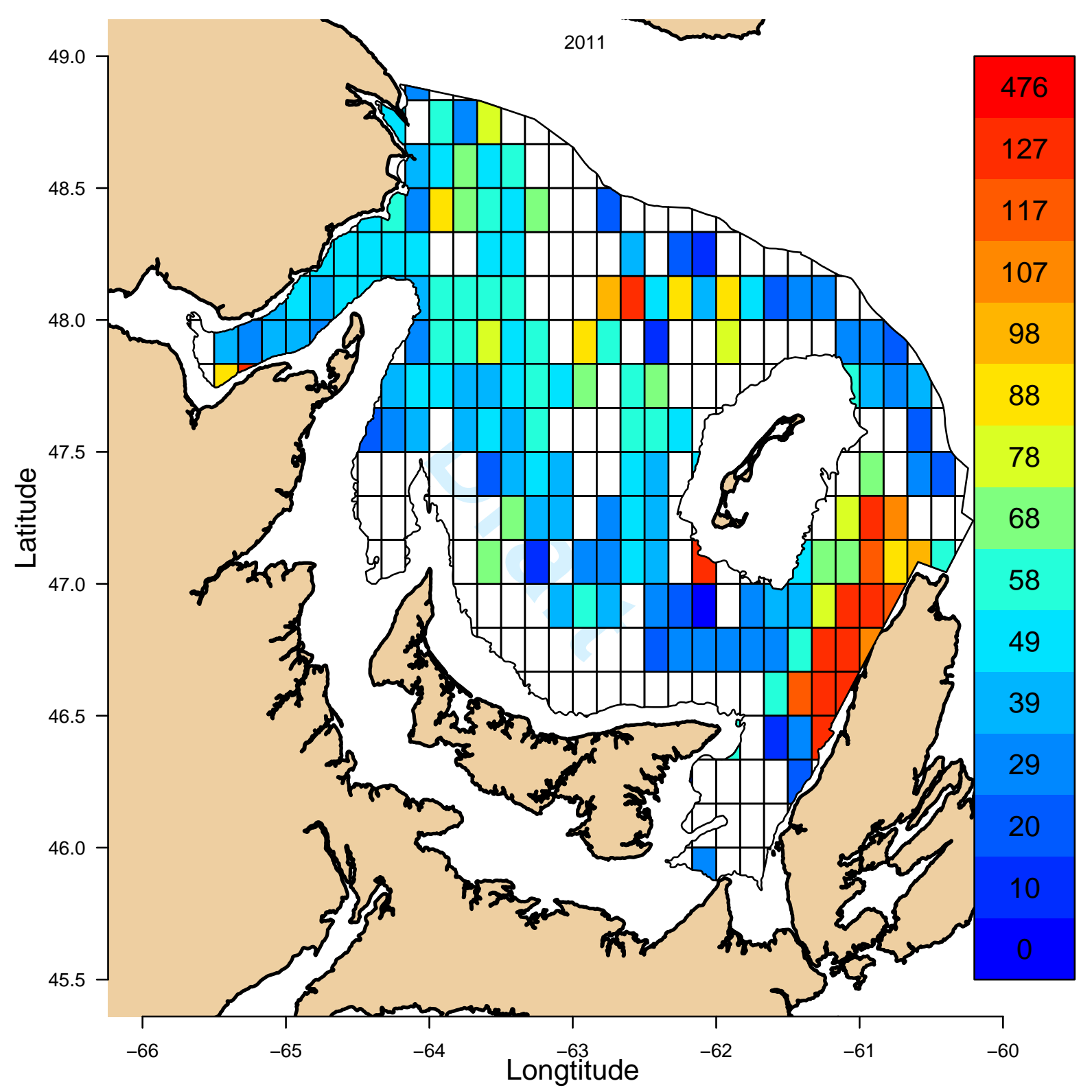

Figure SX.17a. Total annual CPUE of snow crab in each grid cell in 2011. Colors correspond to CPUE levels, as indicated in the legend on the right-hand side. Darkest red grids indicate CPUE > 98th percentile. 


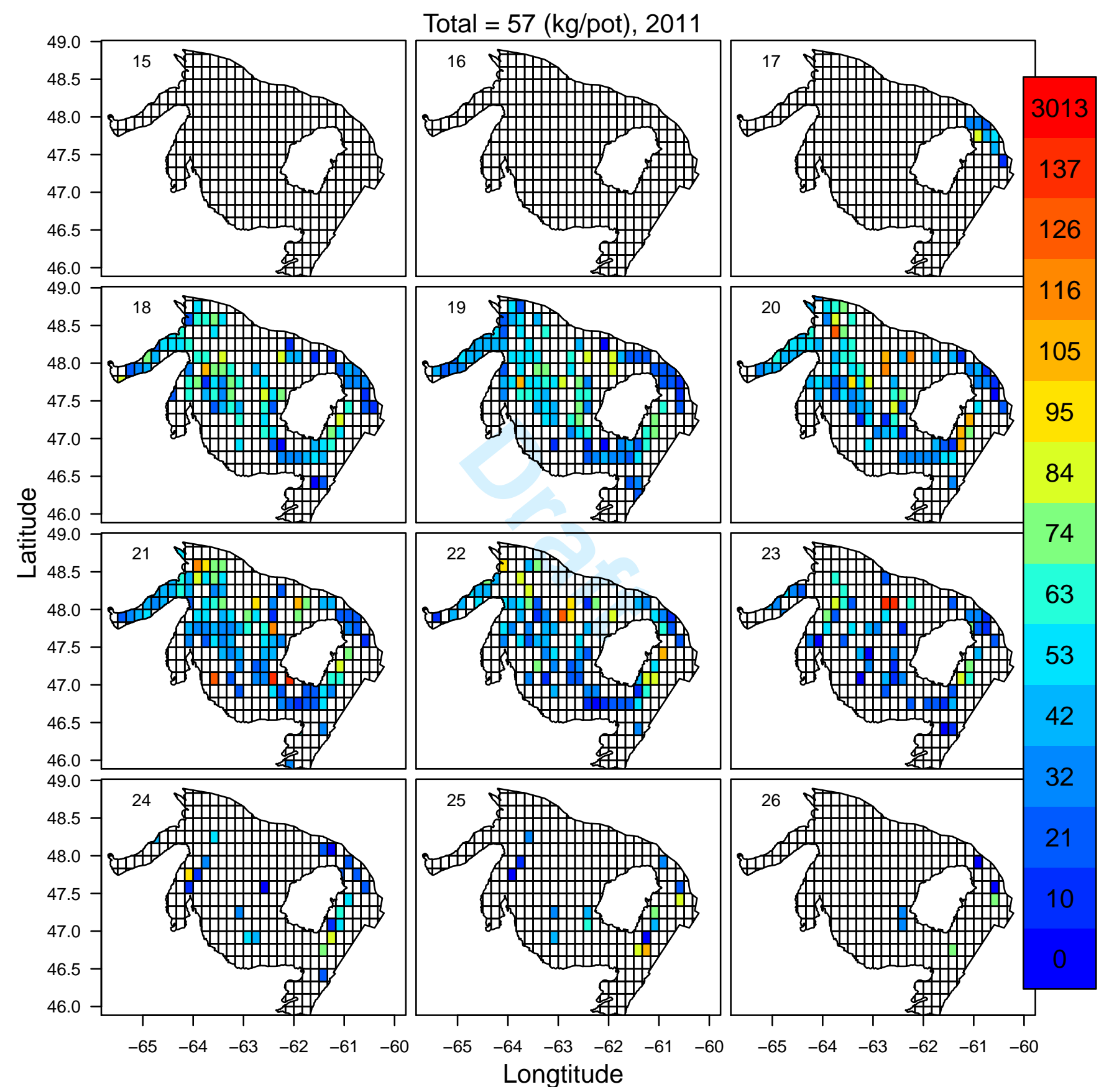

Figure SX.17b. CPUE of snow crab in each week and grid cell in 2011. The week number is indicated in the top left-hand corner. Colors correspond to CPUE levels, as indicated in the legend on the right-hand side. Darkest red grids indicate CPUE $>$ 98th percentile. 


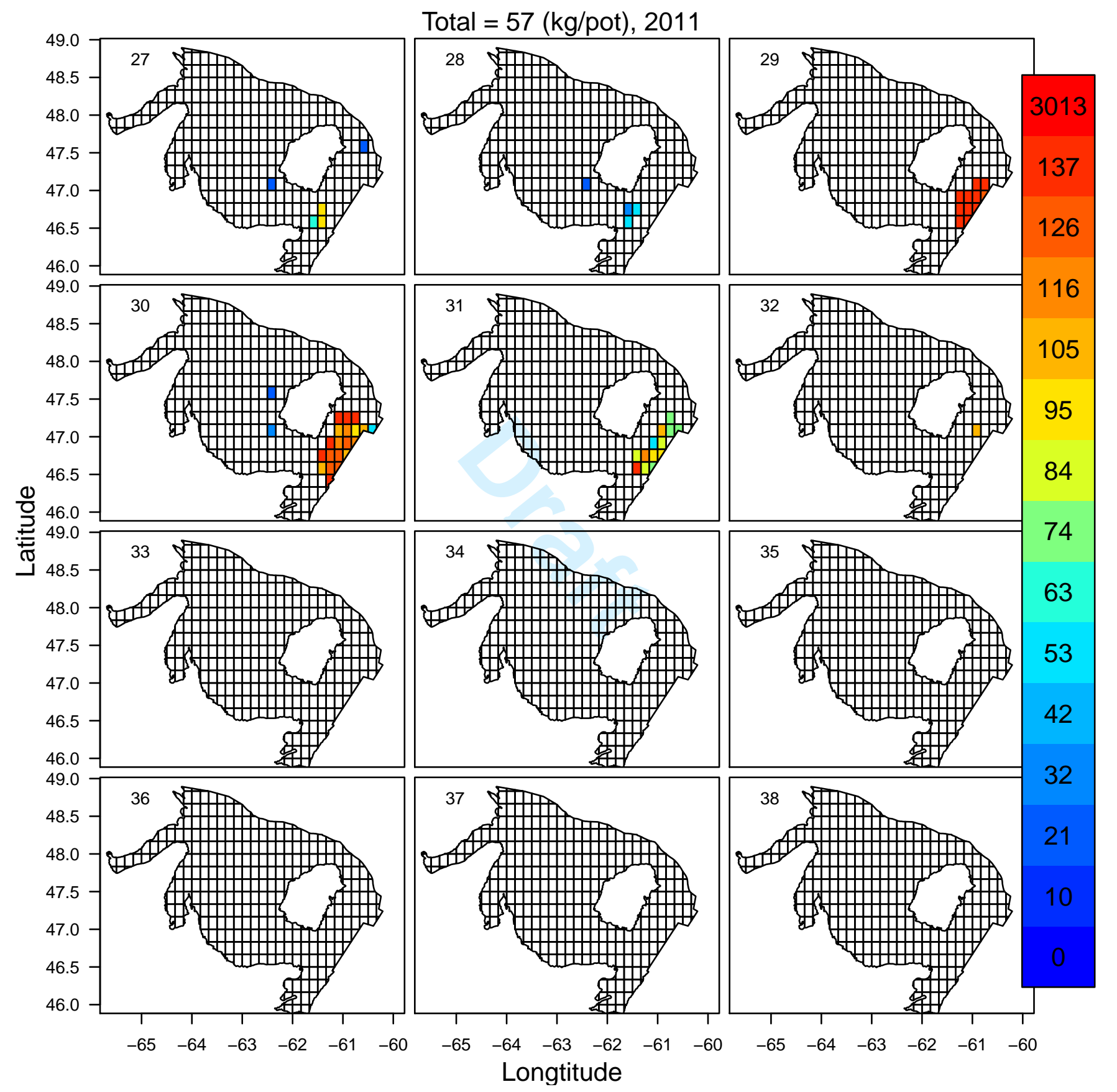

Figure SX.17c. CPUE of snow crab in each week and grid cell in 2011. The week number is indicated in the top left-hand corner. Colors correspond to CPUE levels, as indicated in the legend on the right-hand side. Darkest red grids indicate CPUE $>$ 98th percentile. 


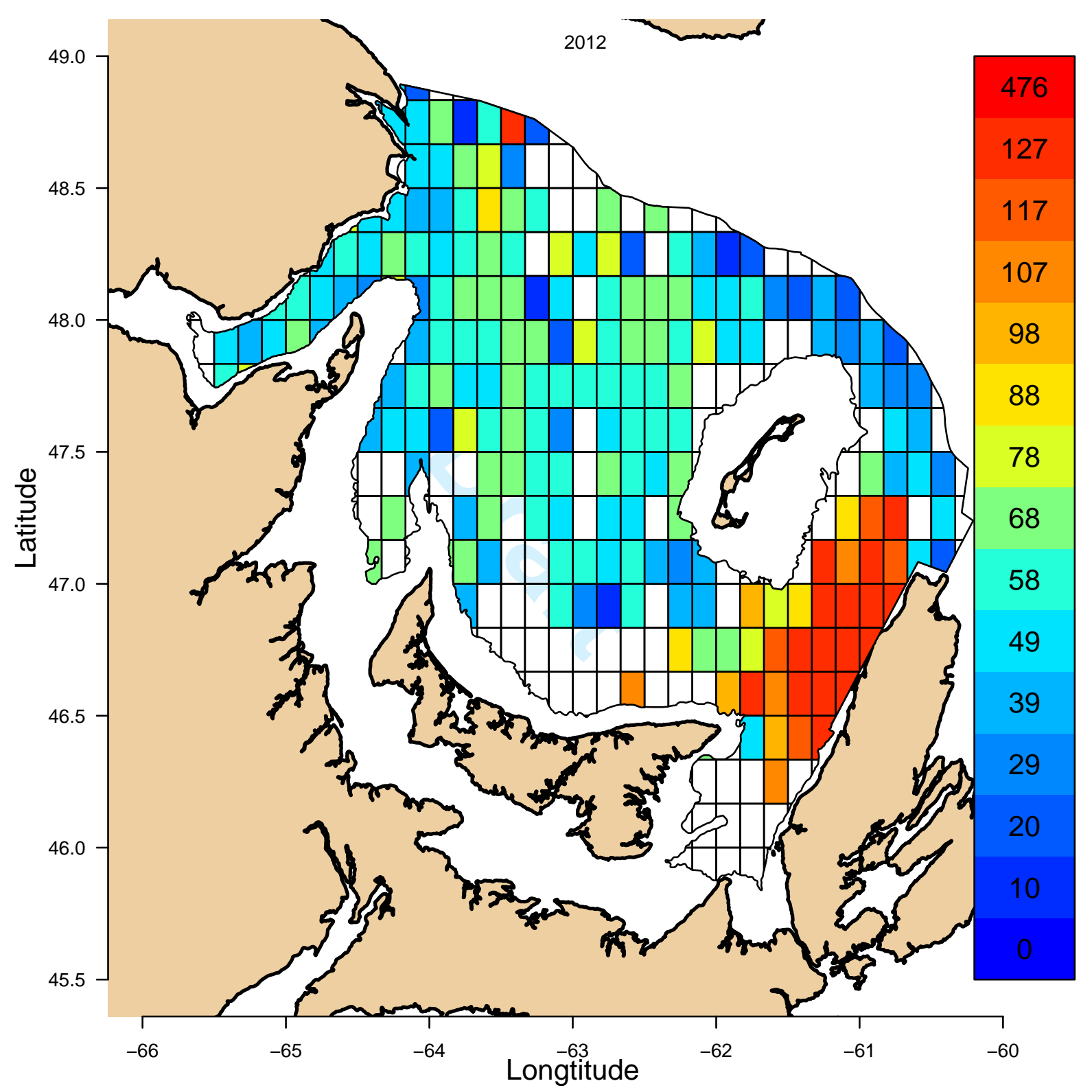

Figure SX.18a. Total annual CPUE of snow crab in each grid cell in 2012. Colors correspond to CPUE levels, as indicated in the legend on the right-hand side. Darkest red grids indicate CPUE $>98$ th percentile. 


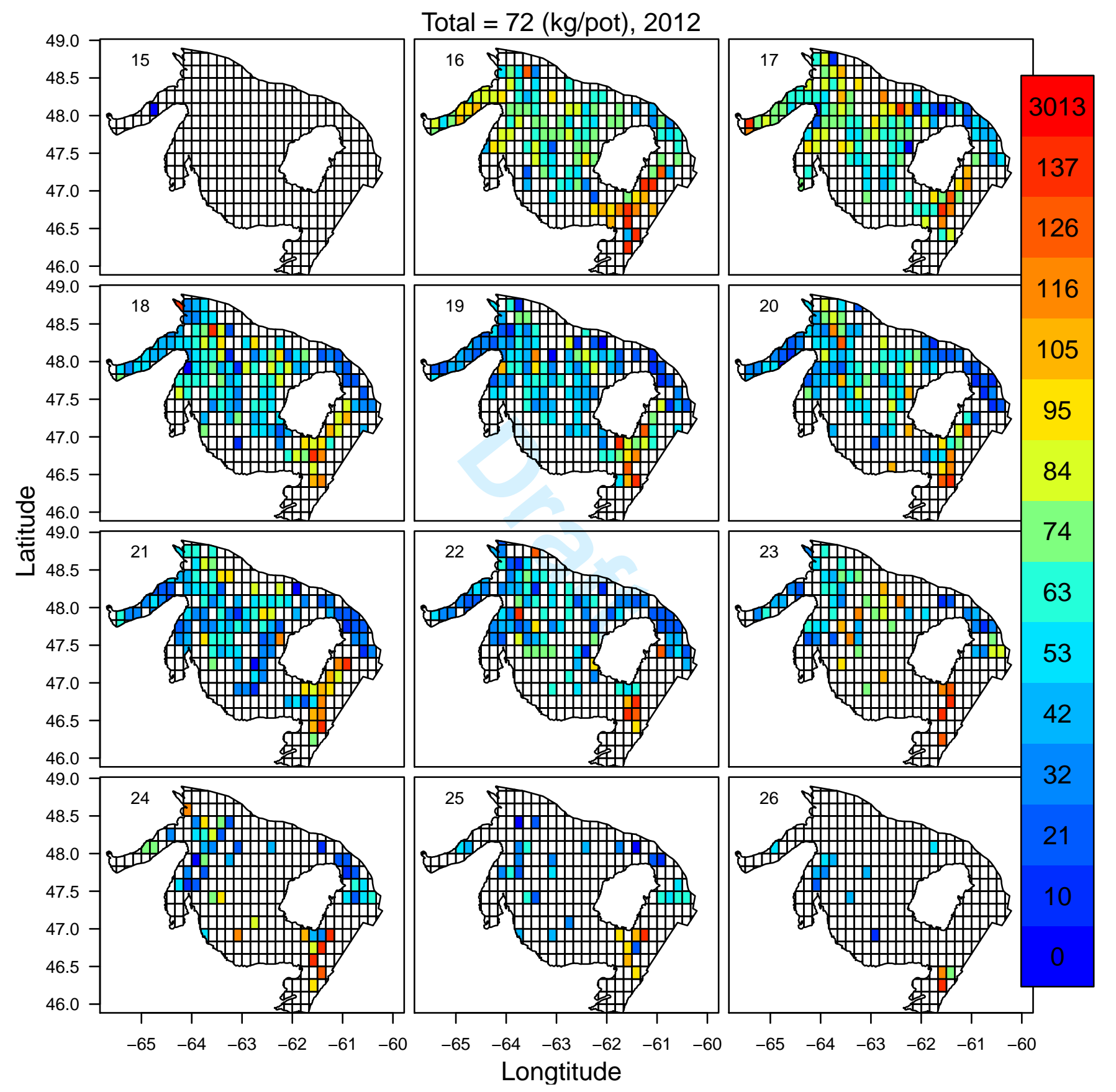

Figure SX.18b. CPUE of snow crab in each week and grid cell in 2012 . The week number is indicated in the top left-hand corner. Colors correspond to CPUE levels, as indicated in the legend on the right-hand side. Darkest red grids indicate CPUE $>$ 98th percentile. 


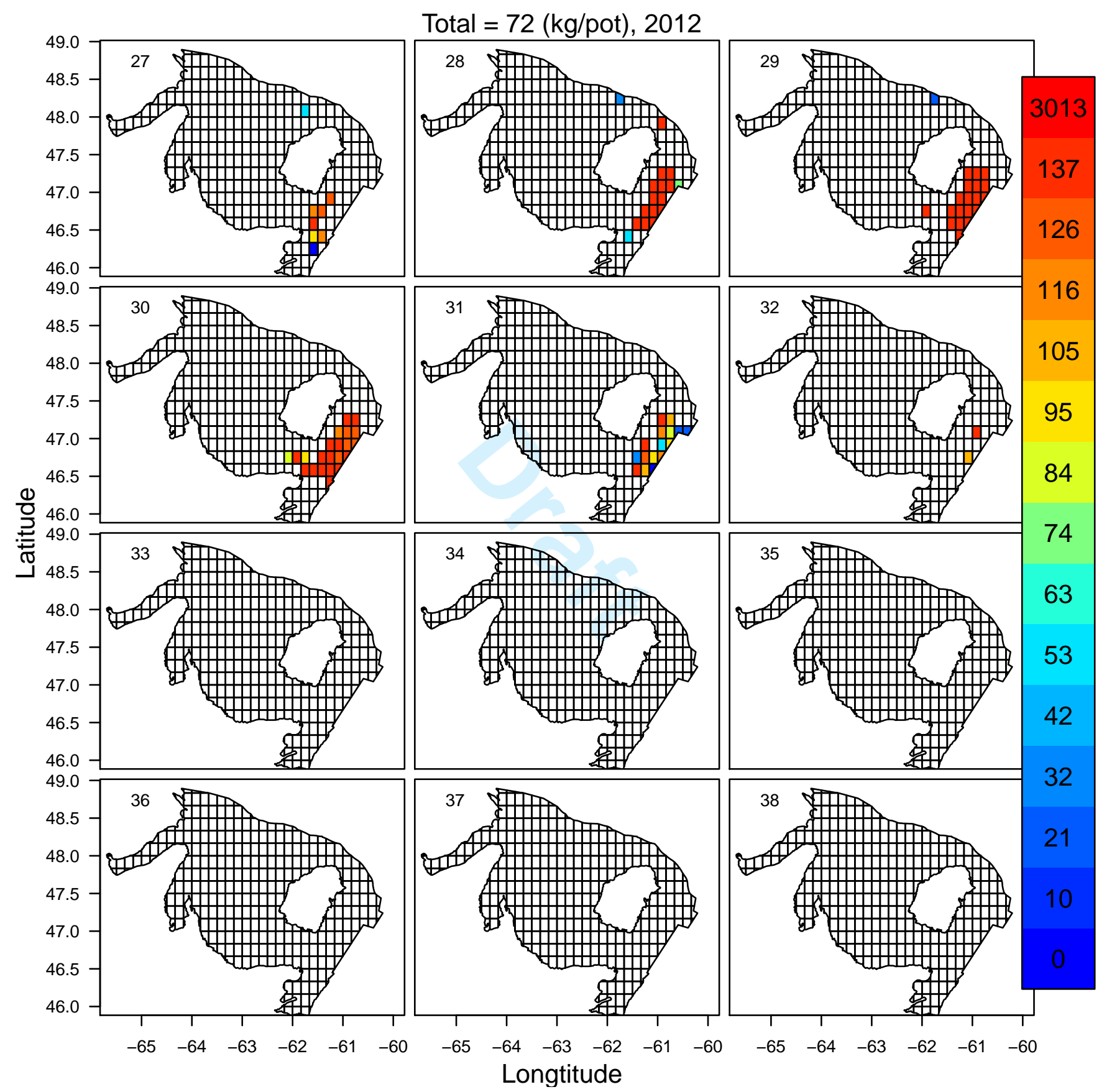

Figure SX.18c. CPUE of snow crab in each week and grid cell in 2012. The week number is indicated in the top left-hand corner. Colors correspond to CPUE levels, as indicated in the legend on the right-hand side. Darkest red grids indicate CPUE $>$ 98th percentile. 


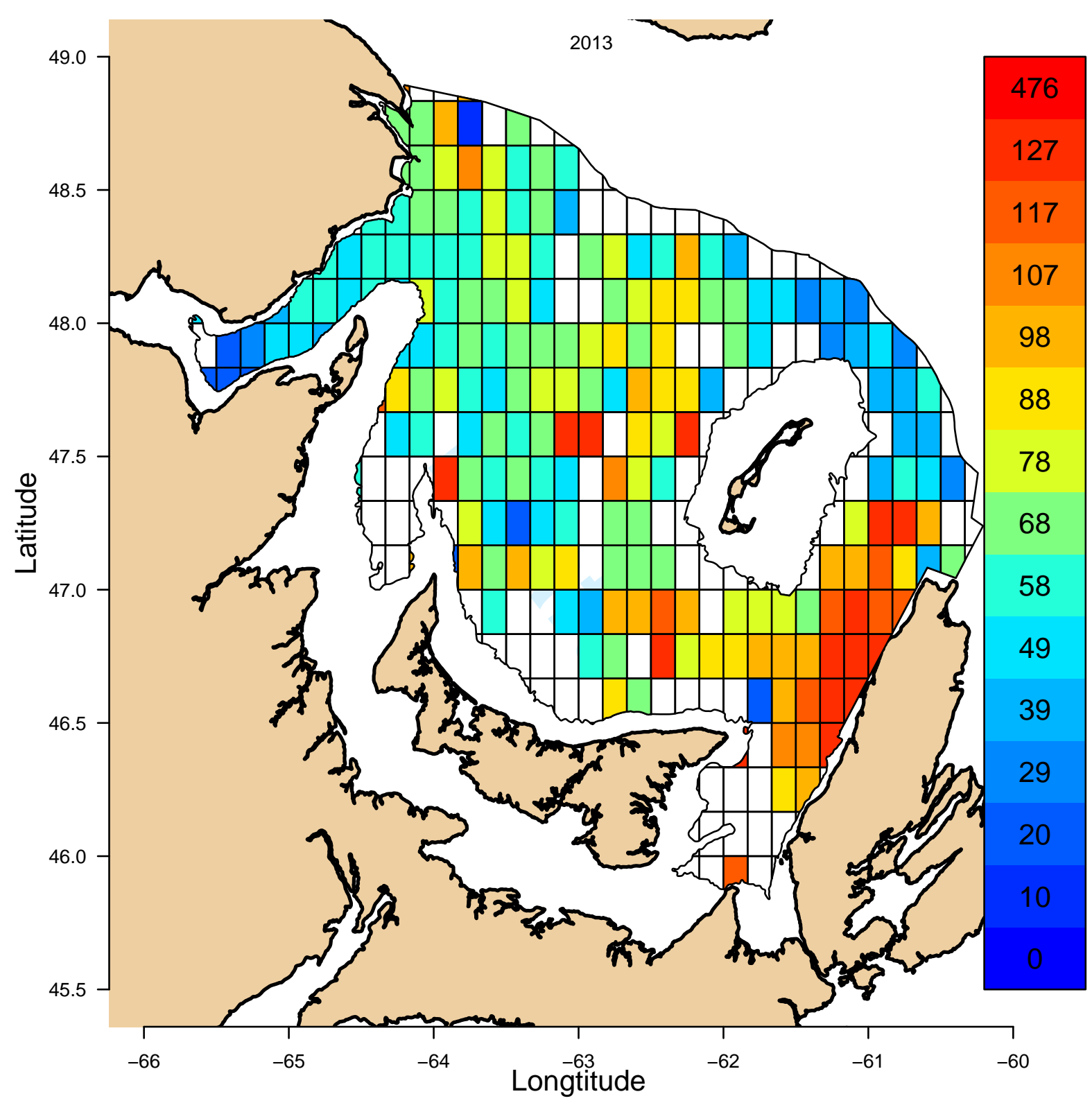

Figure SX.19a. Total annual CPUE of snow crab in each grid cell in 2013. Colors correspond to CPUE levels, as indicated in the legend on the right-hand side. Darkest red grids indicate CPUE > 98th percentile. 


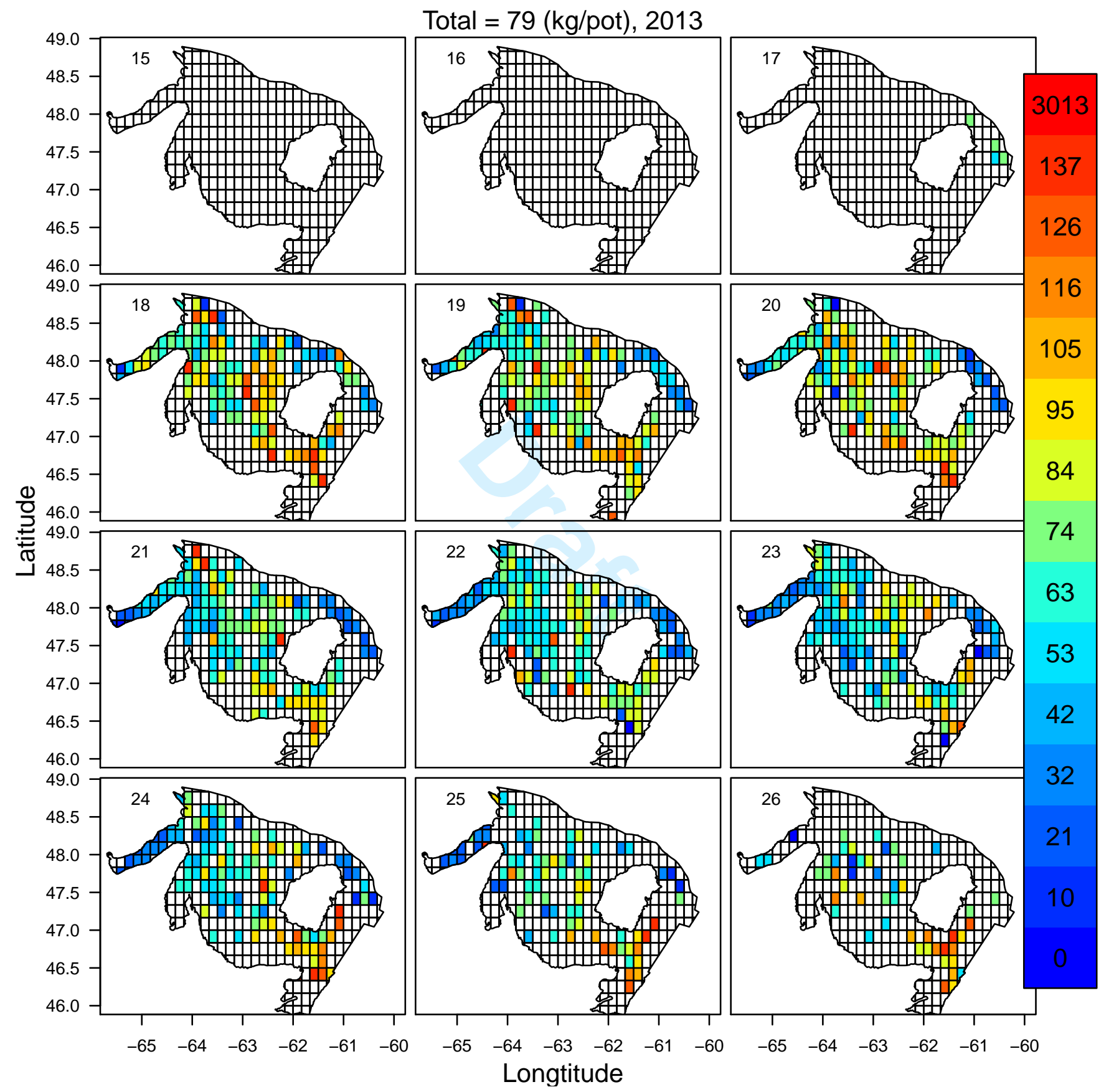

Figure SX.19b. CPUE of snow crab in each week and grid cell in 2013. The week number is indicated in the top left-hand corner. Colors correspond to CPUE levels, as indicated in the legend on the right-hand side. Darkest red grids indicate CPUE $>$ 98th percentile. 


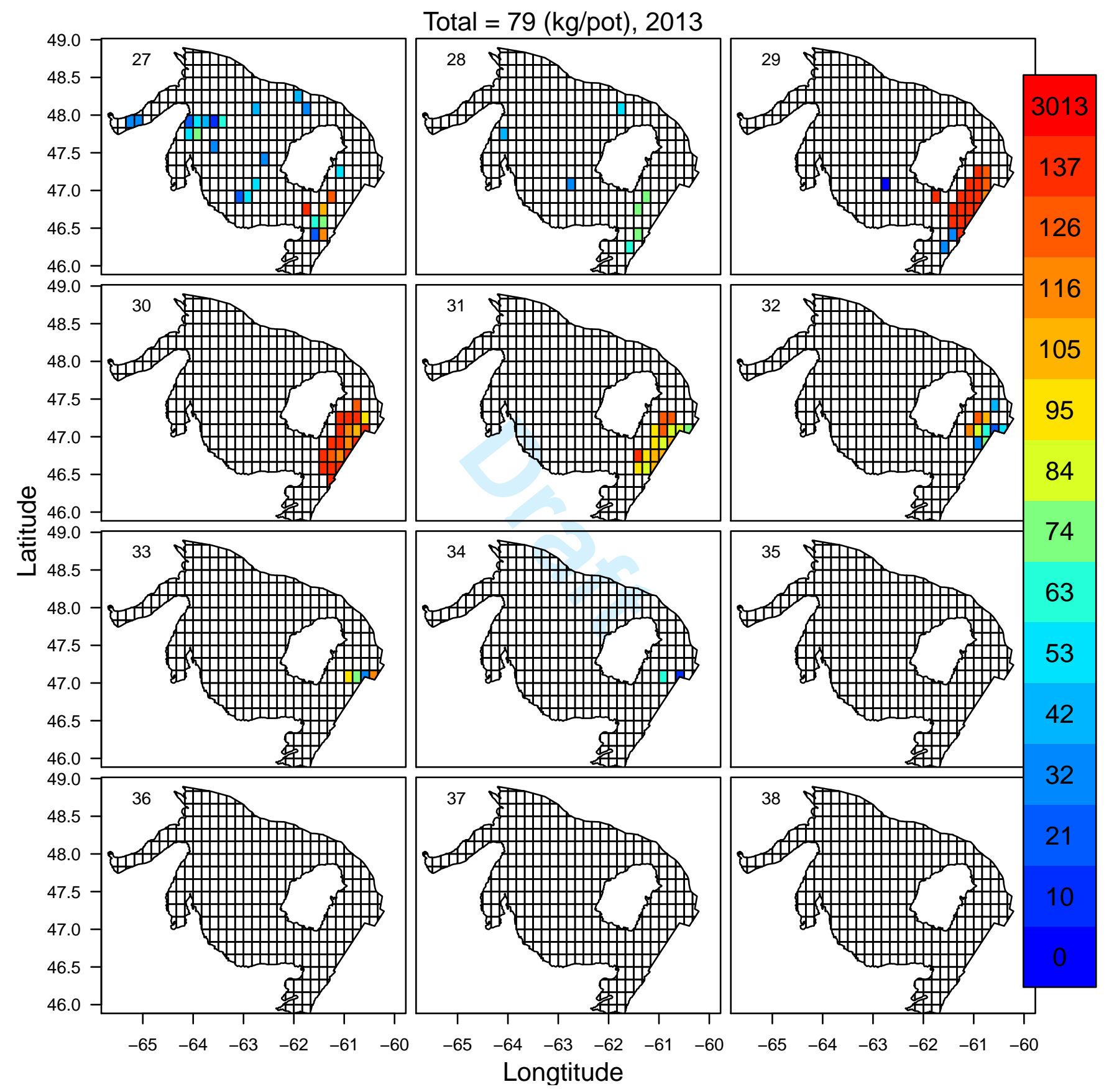

Figure SX.19c. CPUE of snow crab in each week and grid cell in 2013. The week number is indicated in the top left-hand corner. Colors correspond to CPUE levels, as indicated in the legend on the right-hand side. Darkest red grids indicate CPUE $>$ 98th percentile. 


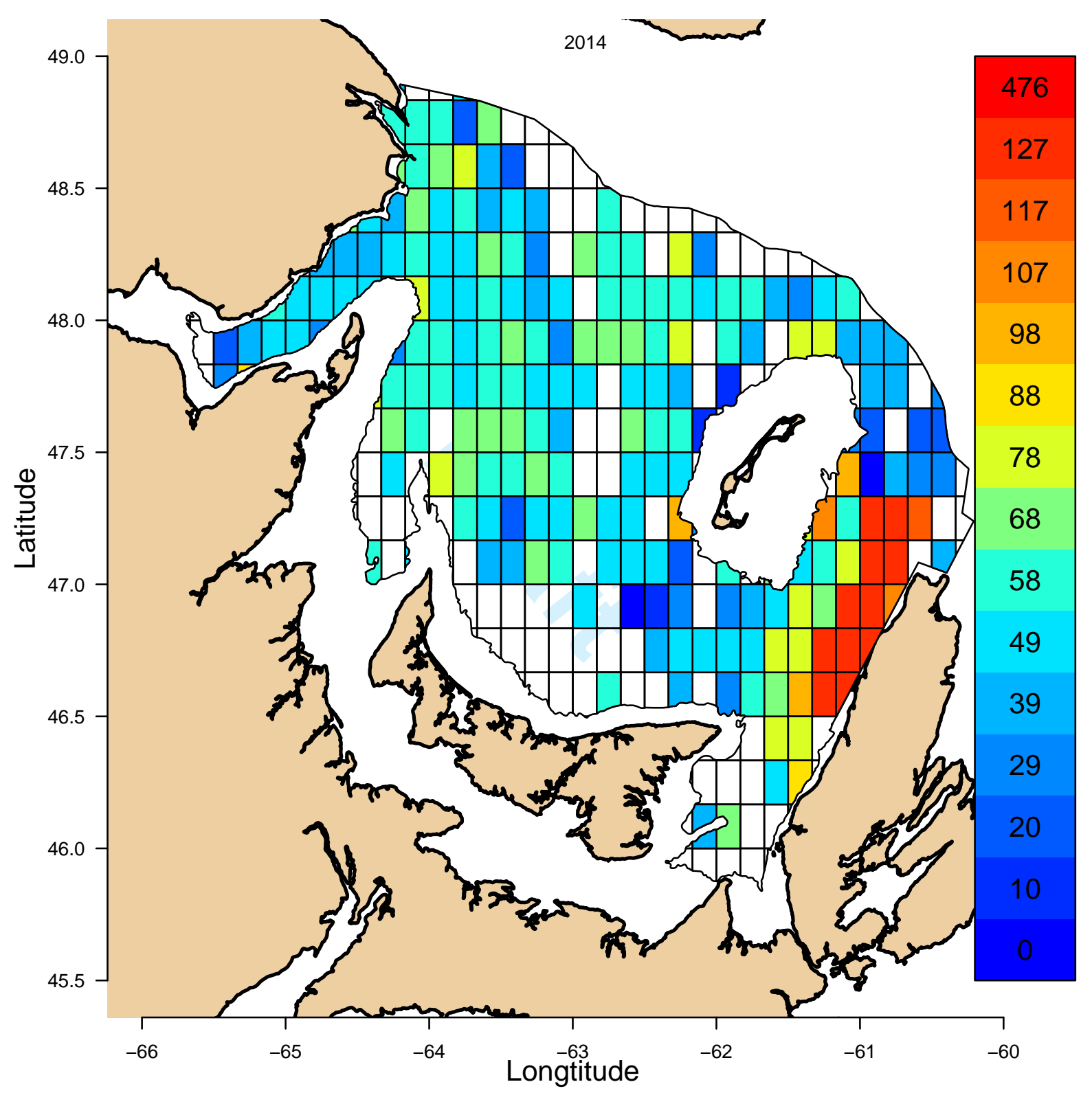

Figure SX.20a. Total annual CPUE of snow crab in each grid cell in 2014. Colors correspond to CPUE levels, as indicated in the legend on the right-hand side. Darkest red grids indicate CPUE > 98th percentile. 


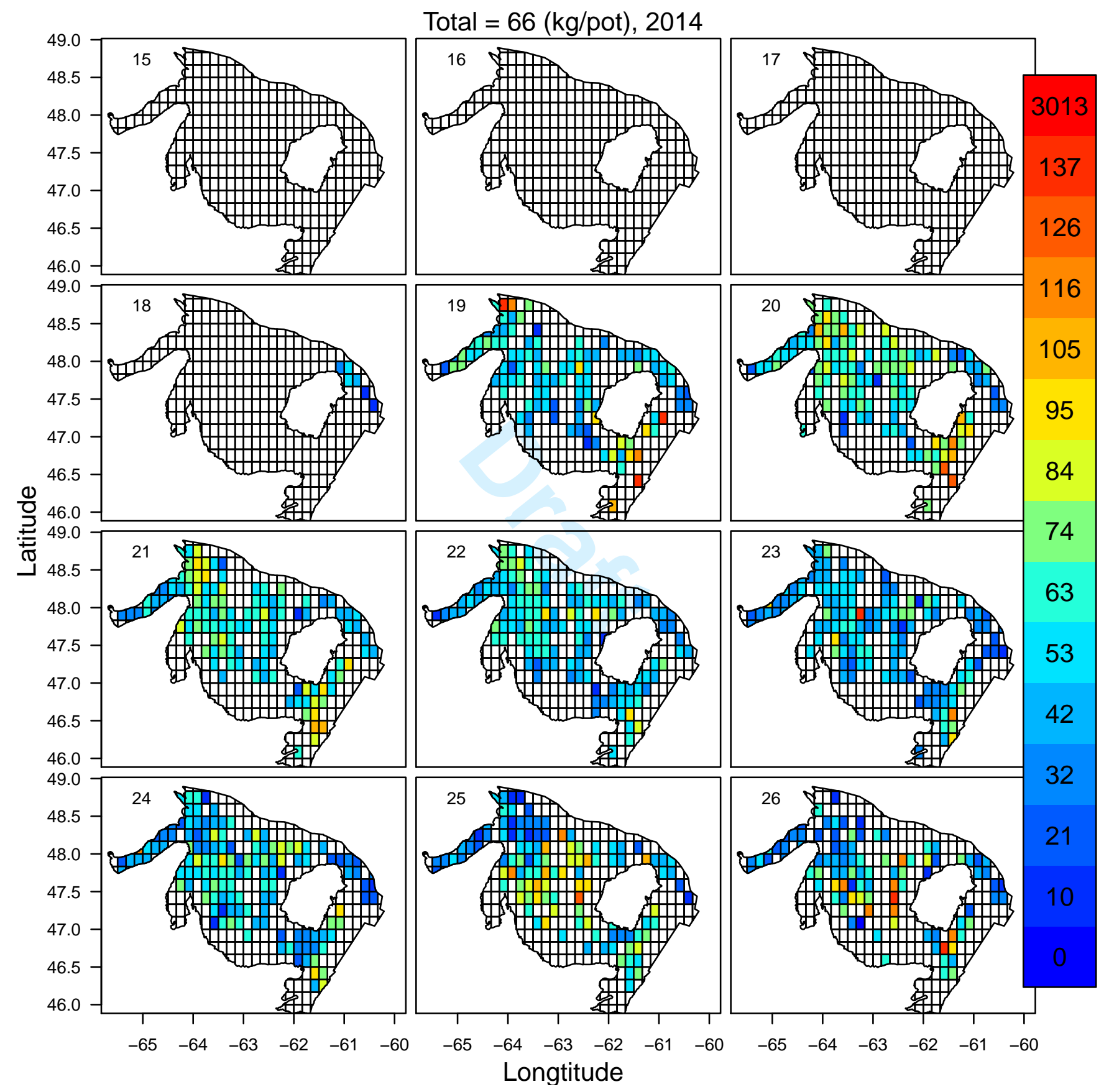

Figure SX.20b. CPUE of snow crab in each week and grid cell in 2014. The week number is indicated in the top left-hand corner. Colors correspond to CPUE levels, as indicated in the legend on the right-hand side. Darkest red grids indicate CPUE $>$ 98th percentile. 


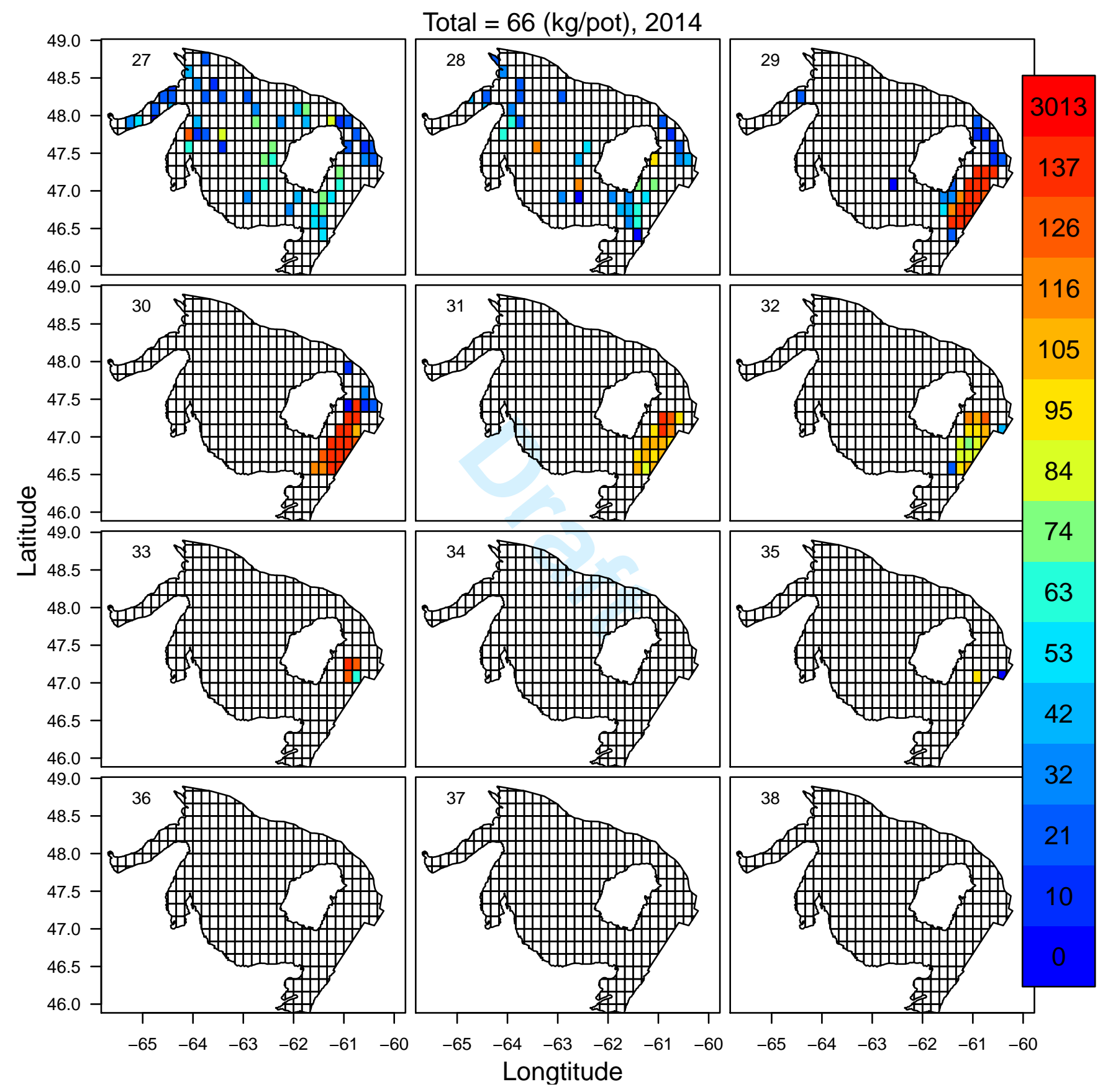

Figure SX.20c. CPUE of snow crab in each week and grid cell in 2014. The week number is indicated in the top left-hand corner. Colors correspond to CPUE levels, as indicated in the legend on the right-hand side. Darkest red grids indicate CPUE $>$ 98th percentile. 


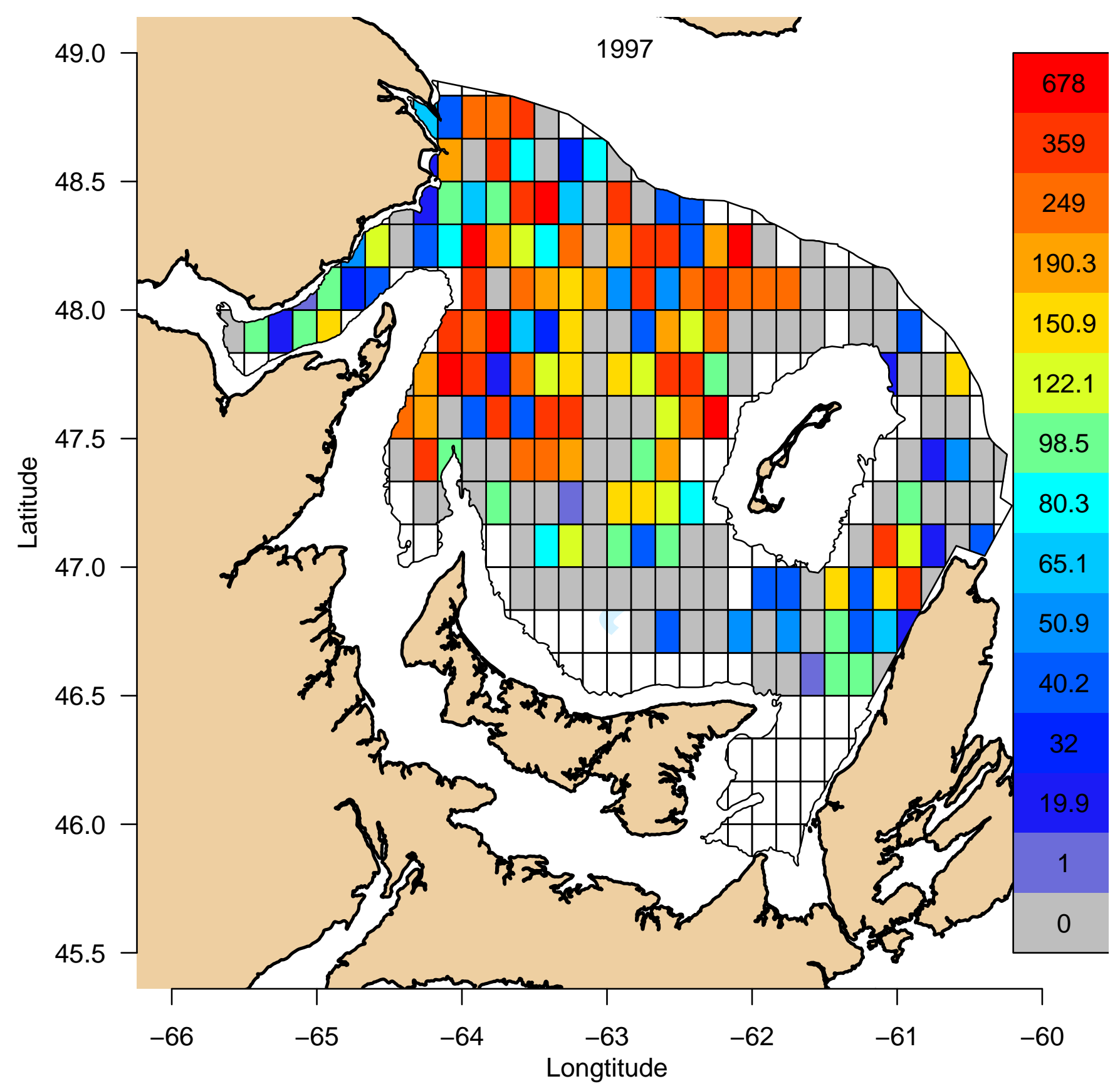

Figure SSEB.1. Snow crab survey exploitable biomass (shell conditions 3-5; tonnes per grid) results for 1997. Colors correspond to biomass levels, as indicated in the legend on the right-hand side. Darkest red grids indicate biomass $>98$ th percentile. 


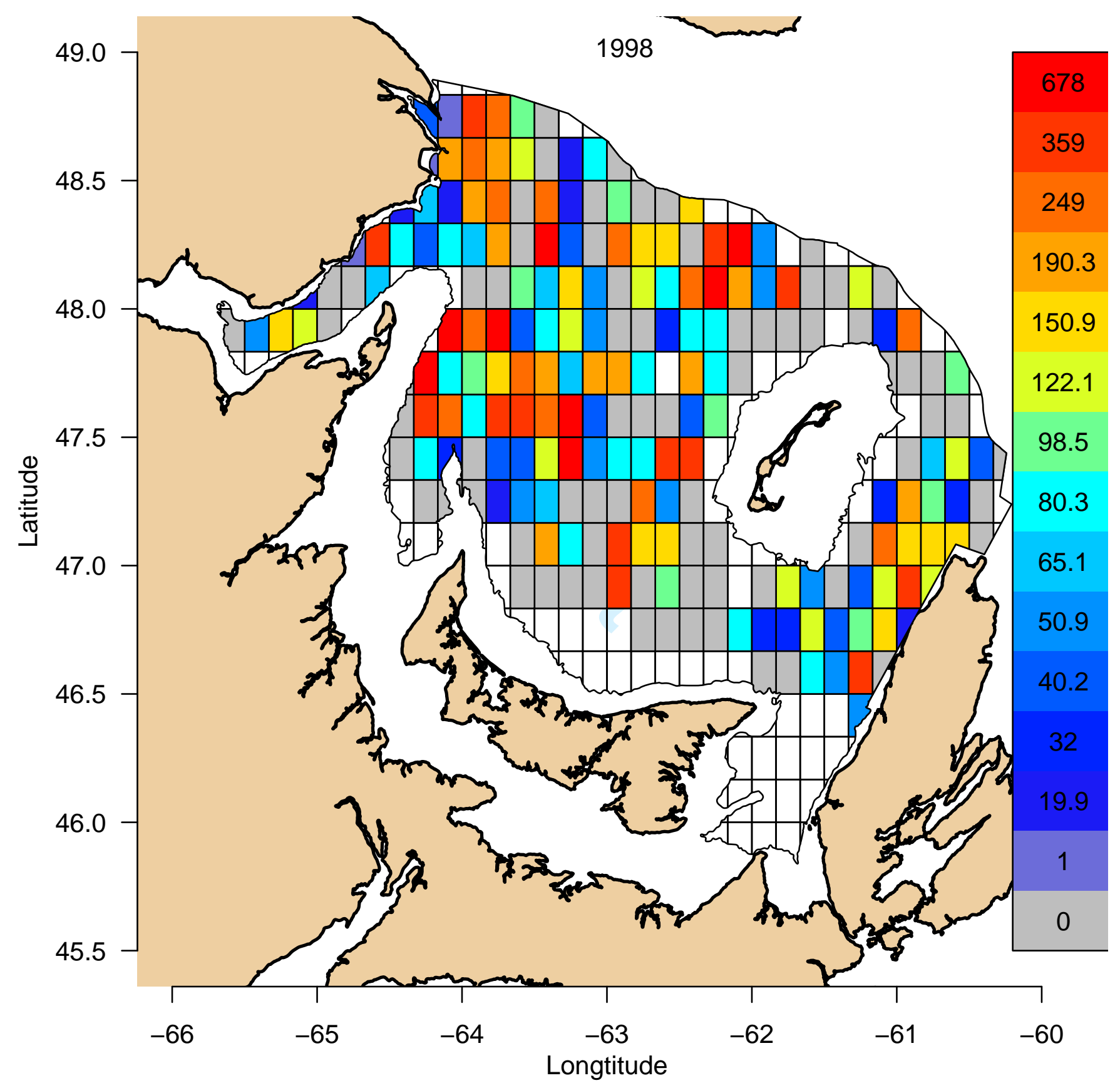

Figure SSEB.2. Snow crab survey exploitable biomass (shell conditions 3-5; tonnes per grid) results for 1998. Colors correspond to biomass levels, as indicated in the legend on the right-hand side. Darkest red grids indicate biomass $>98$ th percentile. 


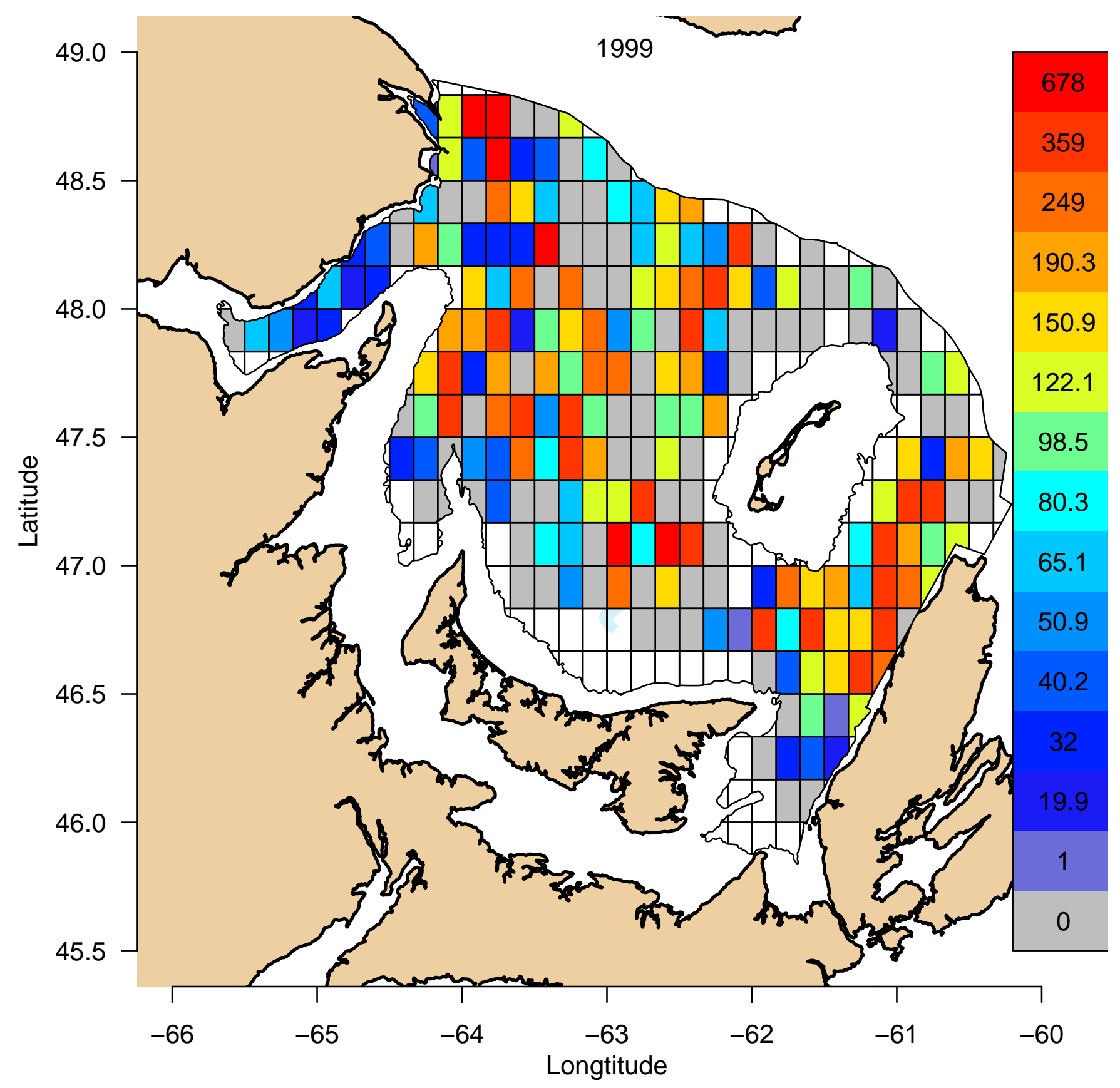

Figure SSEB.3. Snow crab survey exploitable biomass (shell conditions 3-5; tonnes per grid) results for 1999. Colors correspond to biomass levels, as indicated in the legend on the right-hand side. Darkest red grids indicate biomass $>98$ th percentile. 


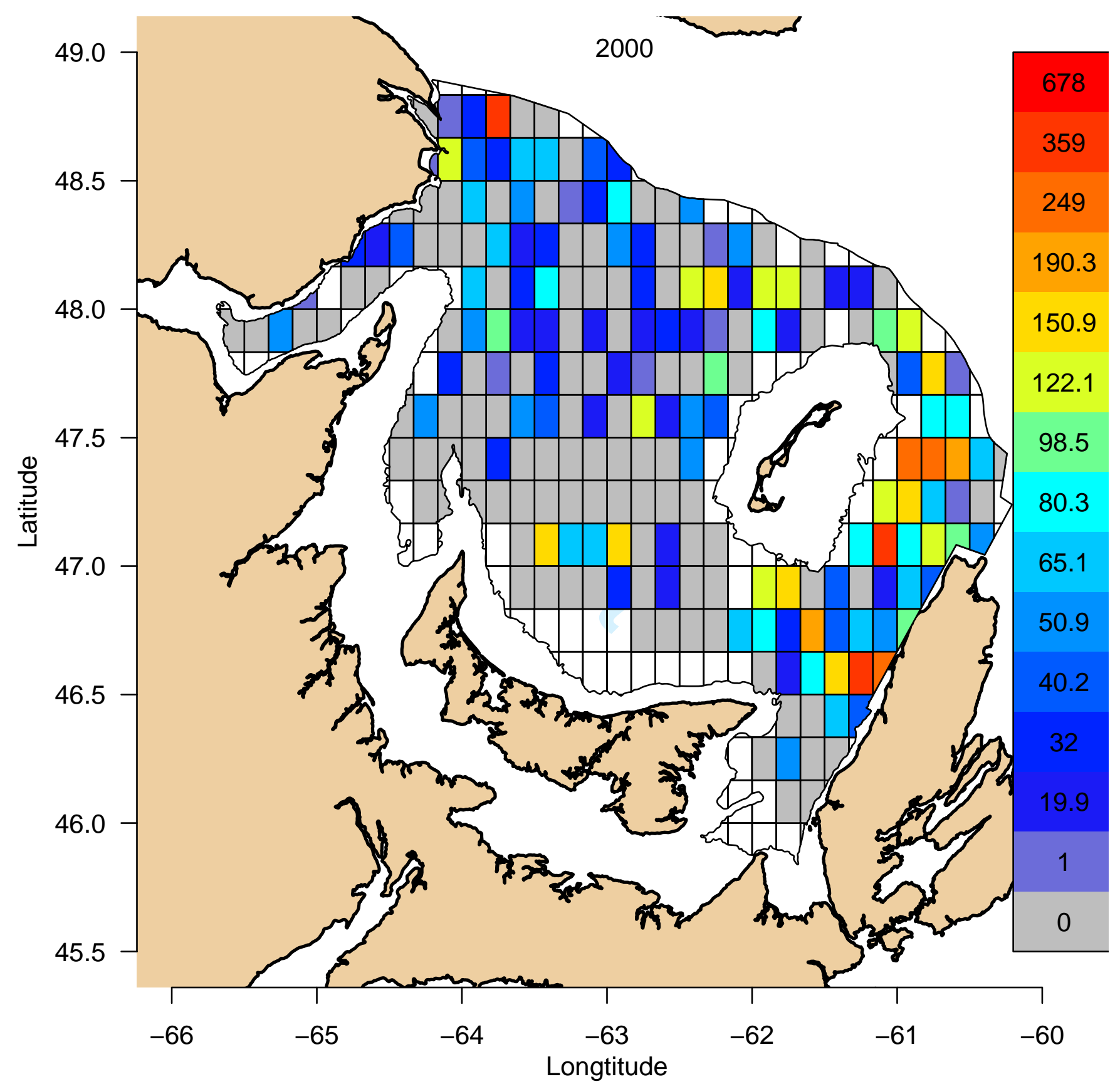

Figure SSEB.4. Snow crab survey exploitable biomass (shell conditions 3-5; tonnes per grid) results for 2000 . Colors correspond to biomass levels, as indicated in the legend on the right-hand side. Darkest red grids indicate biomass $>98$ th percentile. 


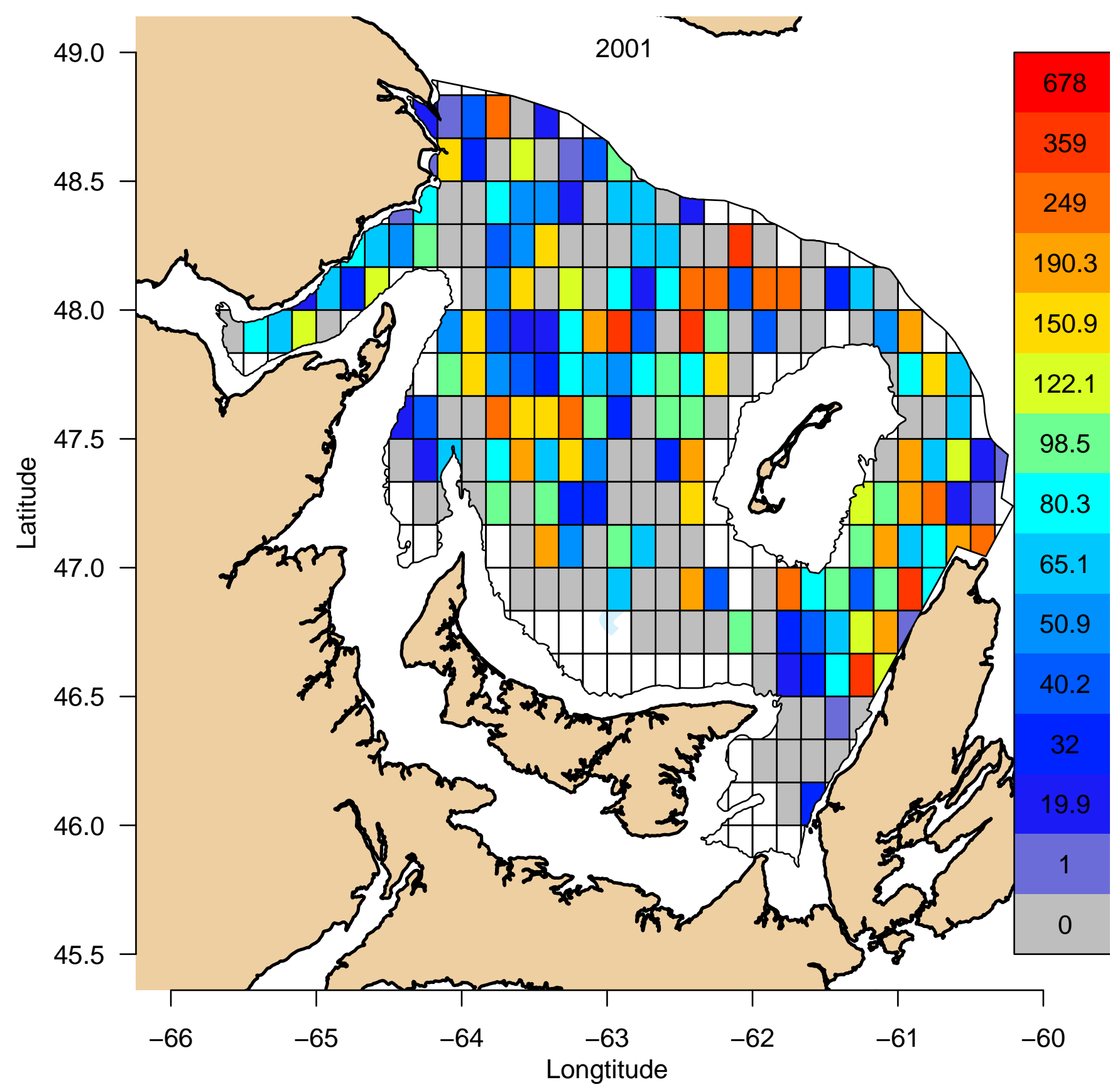

Figure SSEB.5. Snow crab survey exploitable biomass (shell conditions 3-5; tonnes per grid) results for 2001. Colors correspond to biomass levels, as indicated in the legend on the right-hand side. Darkest red grids indicate biomass $>98$ th percentile. 


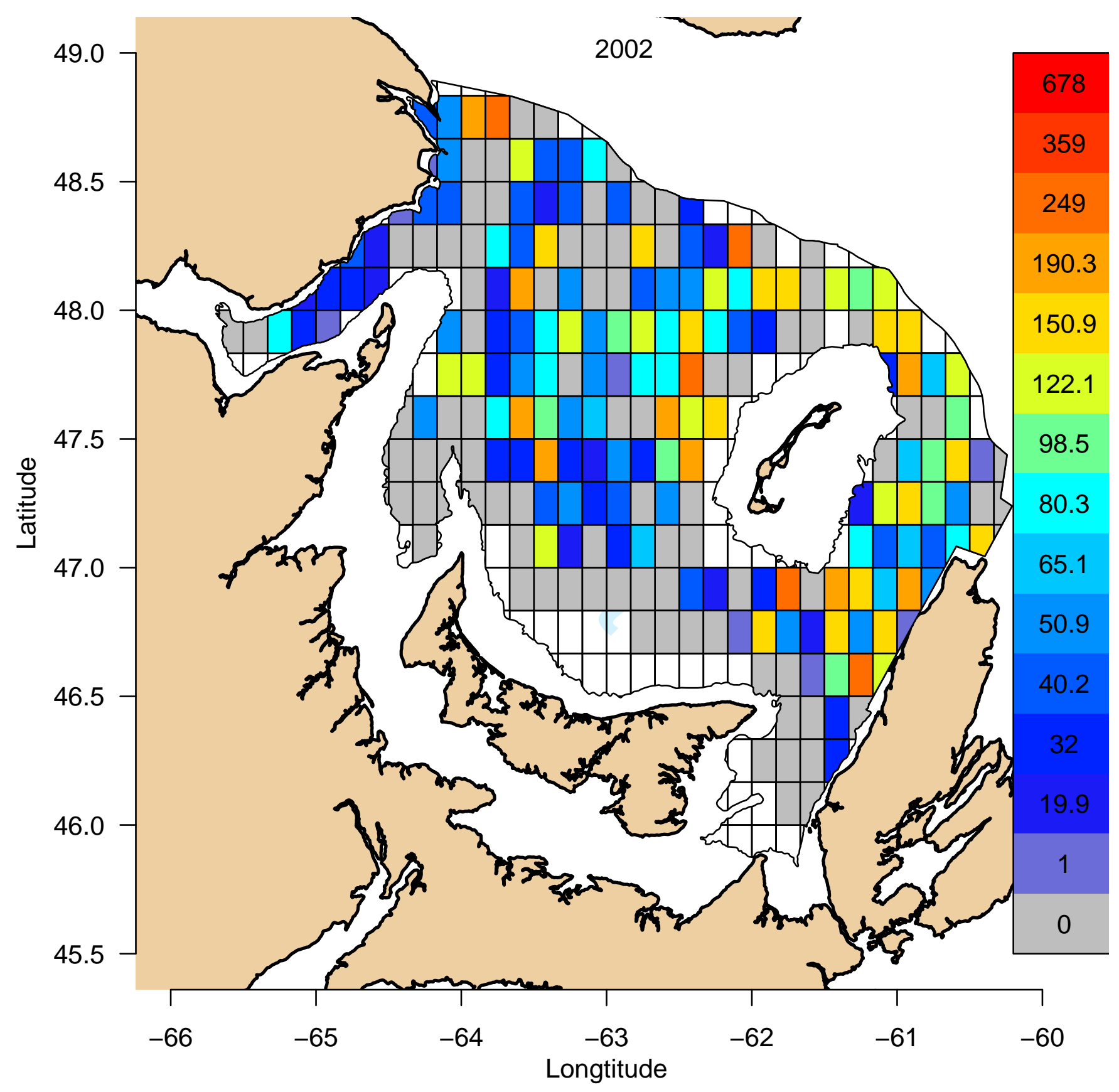

Figure SSEB.6. Snow crab survey exploitable biomass (shell conditions 3-5; tonnes per grid) results for 2002. Colors correspond to biomass levels, as indicated in the legend on the right-hand side. Darkest red grids indicate biomass $>98$ th percentile. 


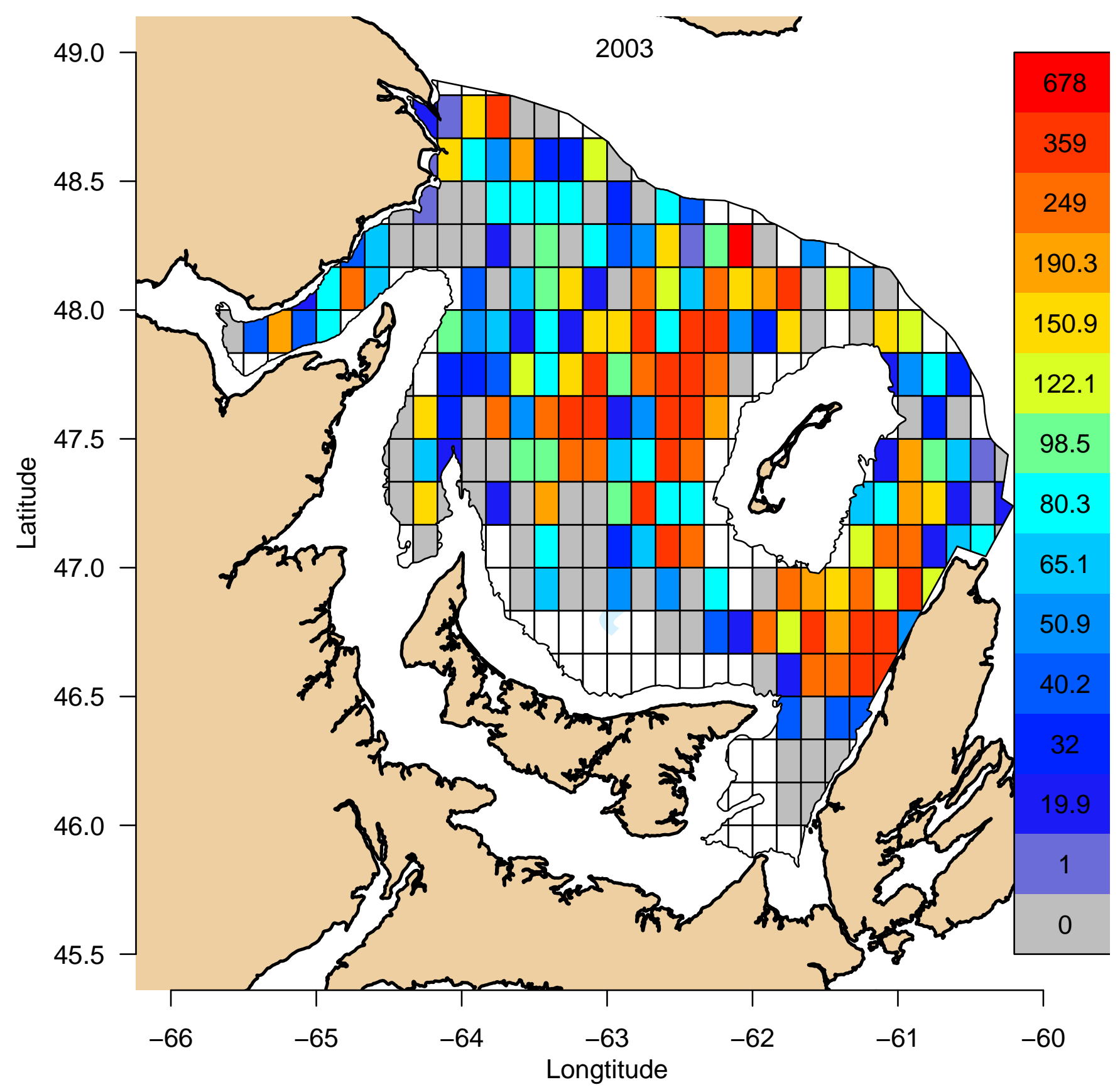

Figure SSEB.7. Snow crab survey exploitable biomass (shell conditions 3-5; tonnes per grid) results for 2003. Colors correspond to biomass levels, as indicated in the legend on the right-hand side. Darkest red grids indicate biomass $>98$ th percentile. 


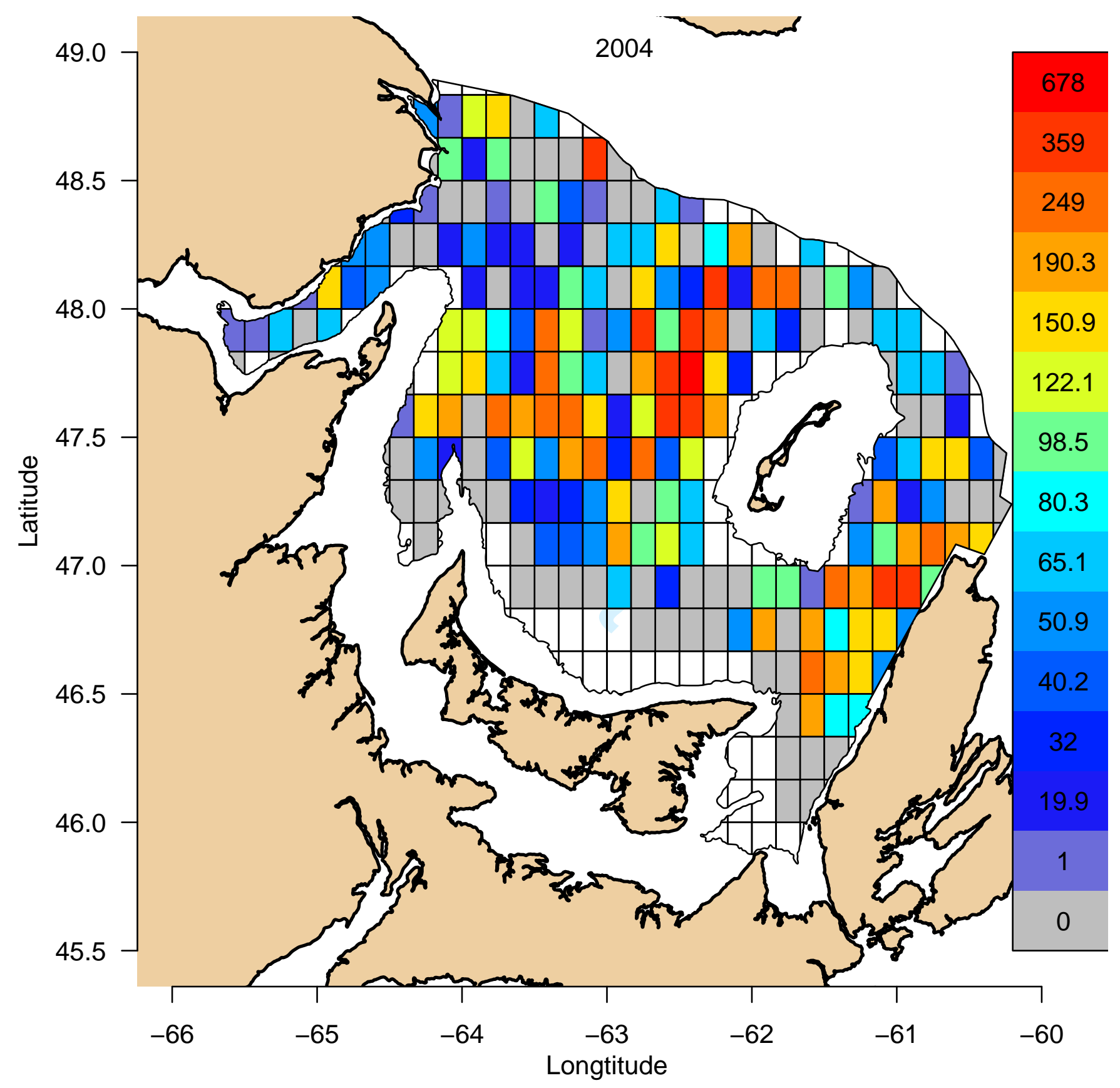

Figure SSEB.8. Snow crab survey exploitable biomass (shell conditions 3-5; tonnes per grid) results for 2004. Colors correspond to biomass levels, as indicated in the legend on the right-hand side. Darkest red grids indicate biomass $>98$ th percentile. 


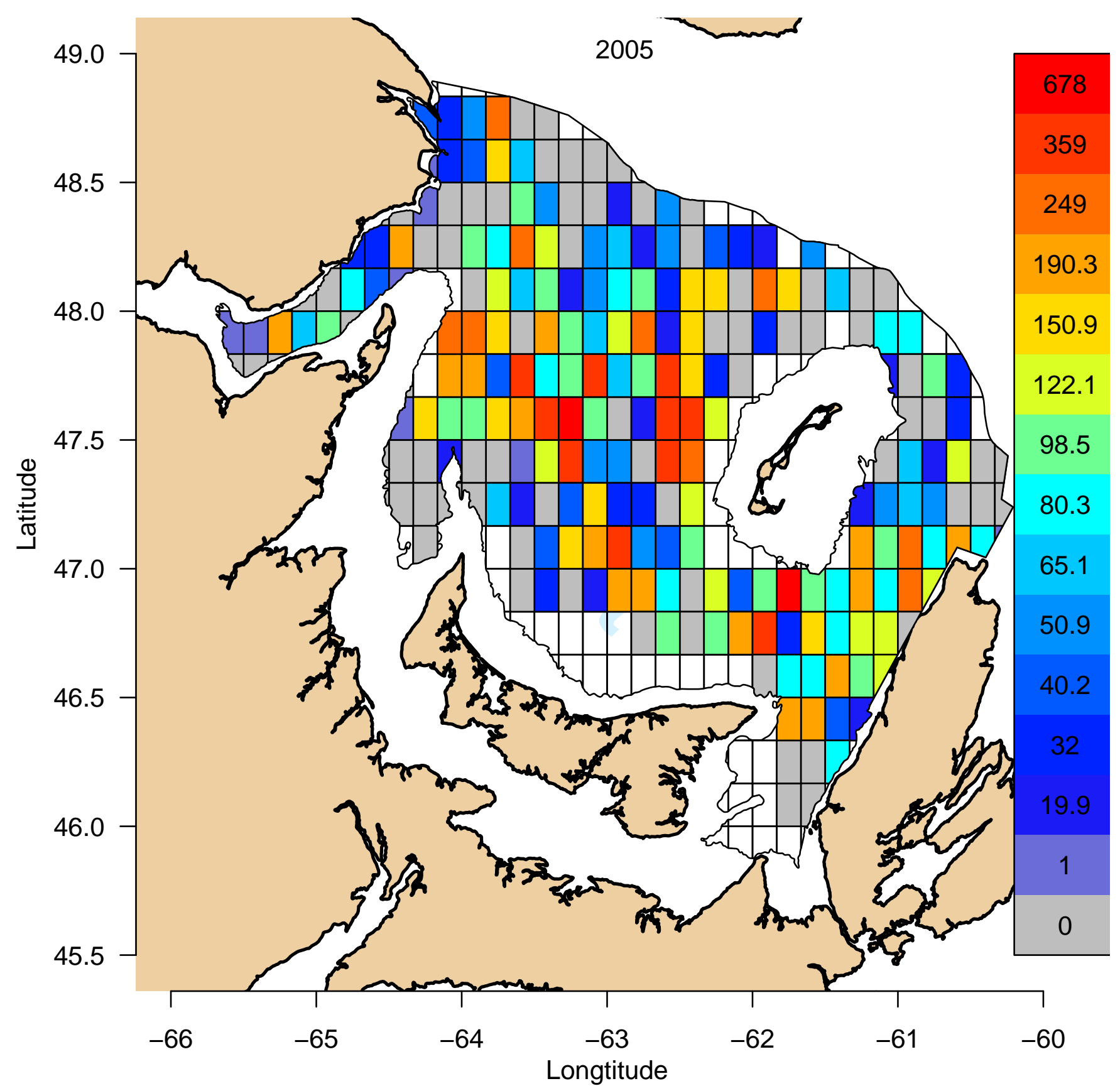

Figure SSEB.9. Snow crab survey exploitable biomass (shell conditions 3-5; tonnes per grid) results for 2005. Colors correspond to biomass levels, as indicated in the legend on the right-hand side. Darkest red grids indicate biomass $>98$ th percentile. 


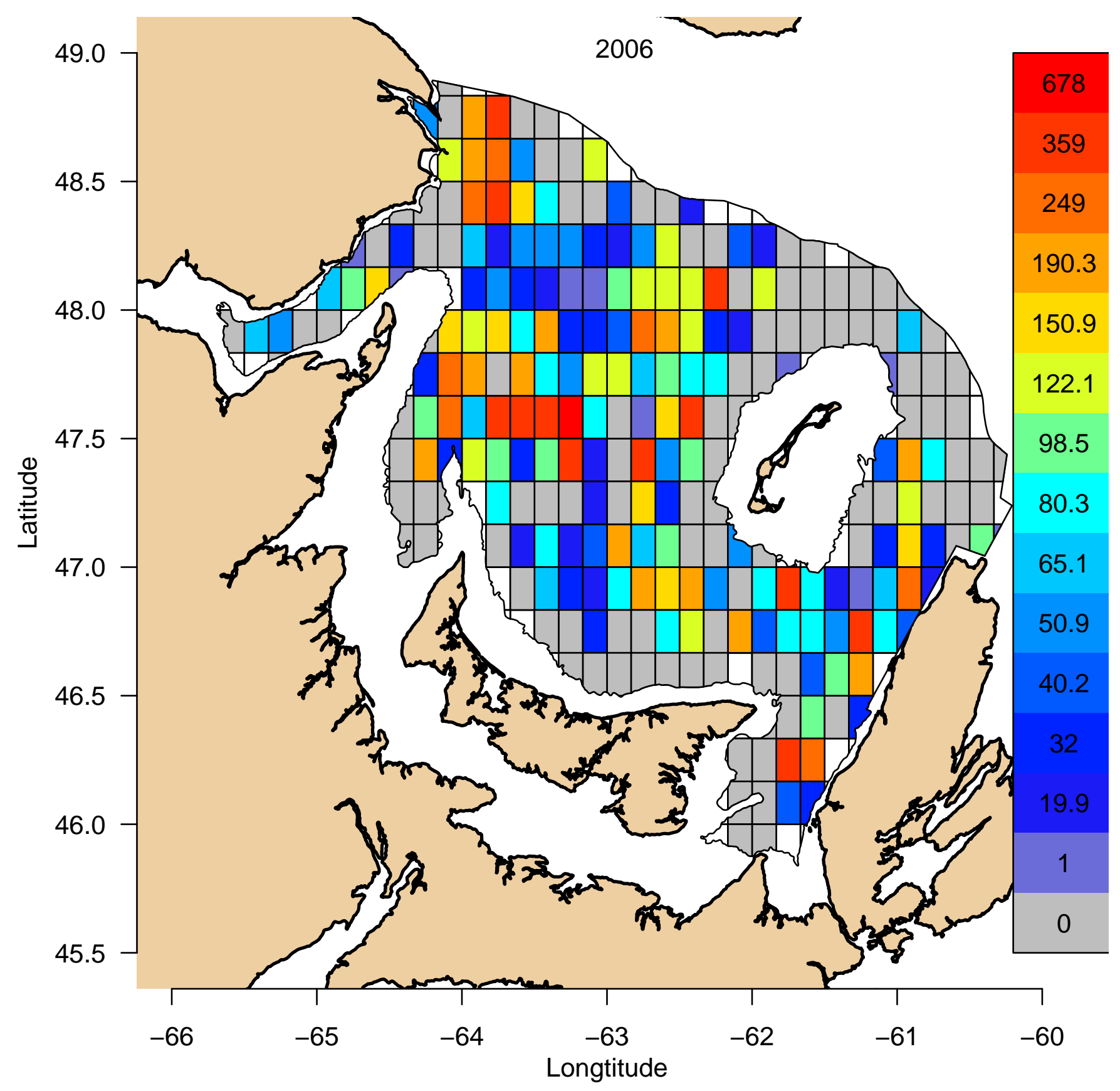

Figure SSEB.10. Snow crab survey exploitable biomass (shell conditions 3-5; tonnes per grid) results for 2006. Colors correspond to biomass levels, as indicated in the legend on the right-hand side. Darkest red grids indicate biomass $>98$ th percentile. 


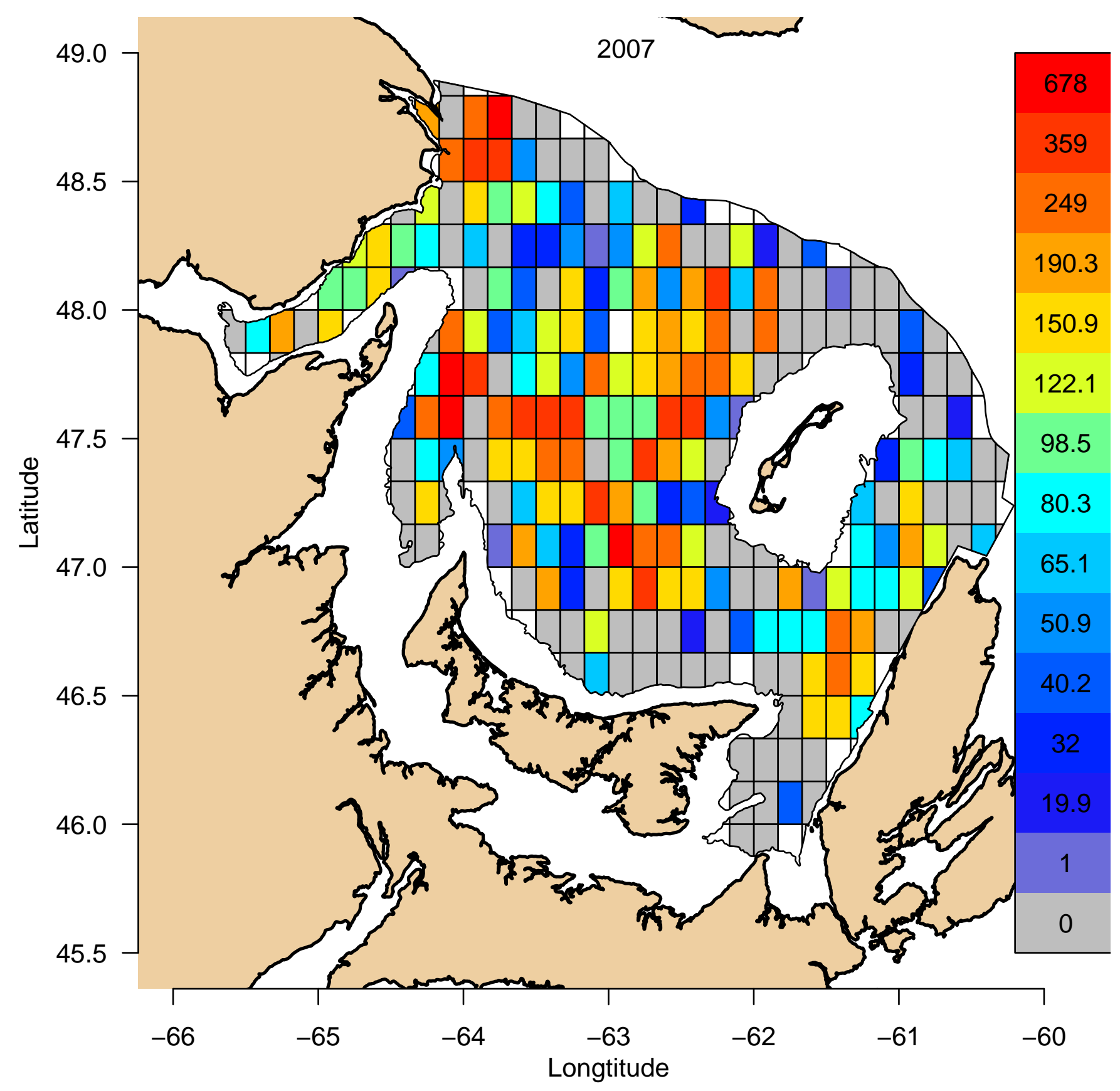

Figure SSEB.11. Snow crab survey exploitable biomass (shell conditions 3-5; tonnes per grid) results for 2007. Colors correspond to biomass levels, as indicated in the legend on the right-hand side. Darkest red grids indicate biomass $>98$ th percentile. 


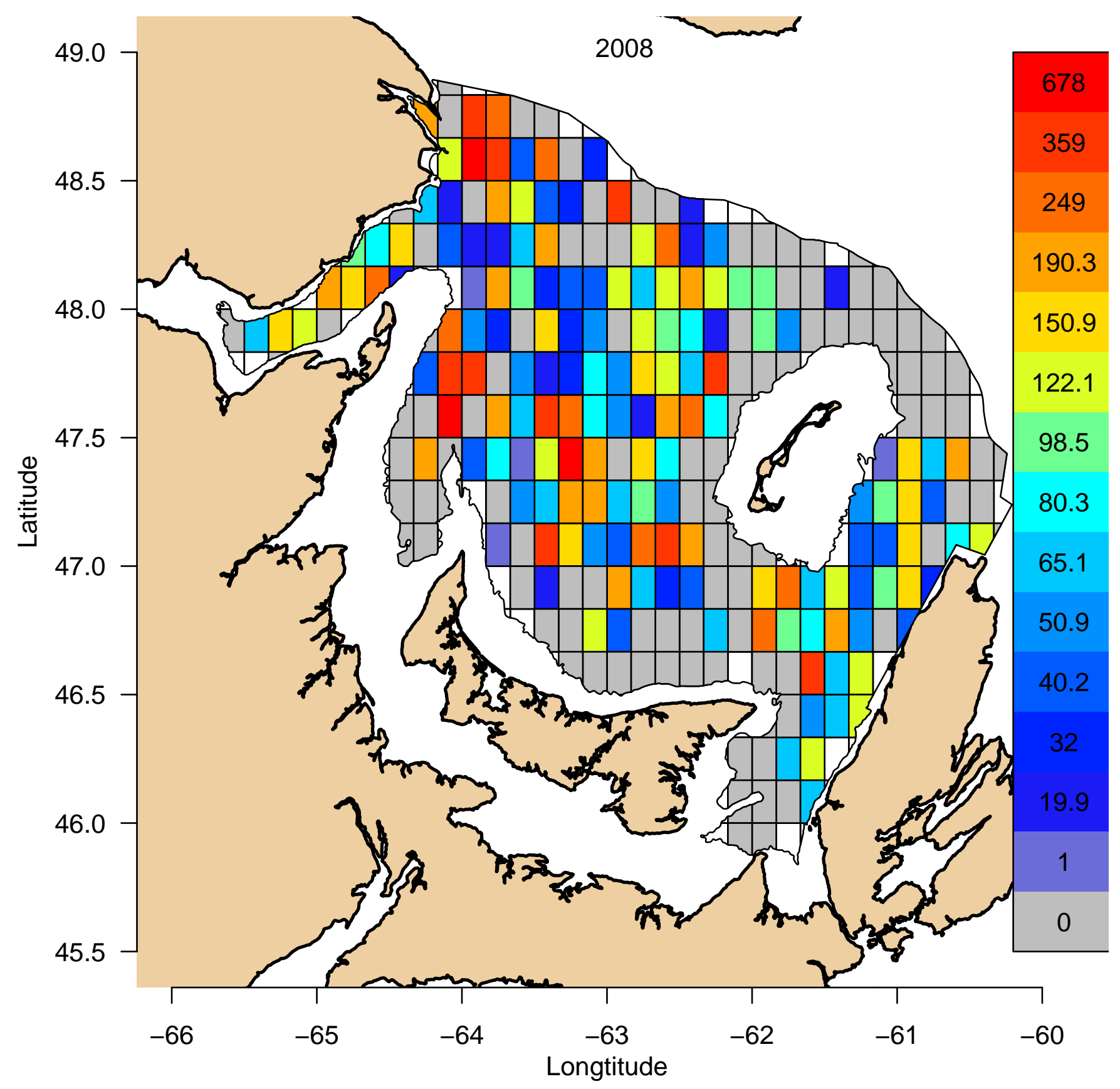

Figure SSEB.12. Snow crab survey exploitable biomass (shell conditions 3-5; tonnes per grid) results for 2008. Colors correspond to biomass levels, as indicated in the legend on the right-hand side. Darkest red grids indicate biomass $>98$ th percentile. 


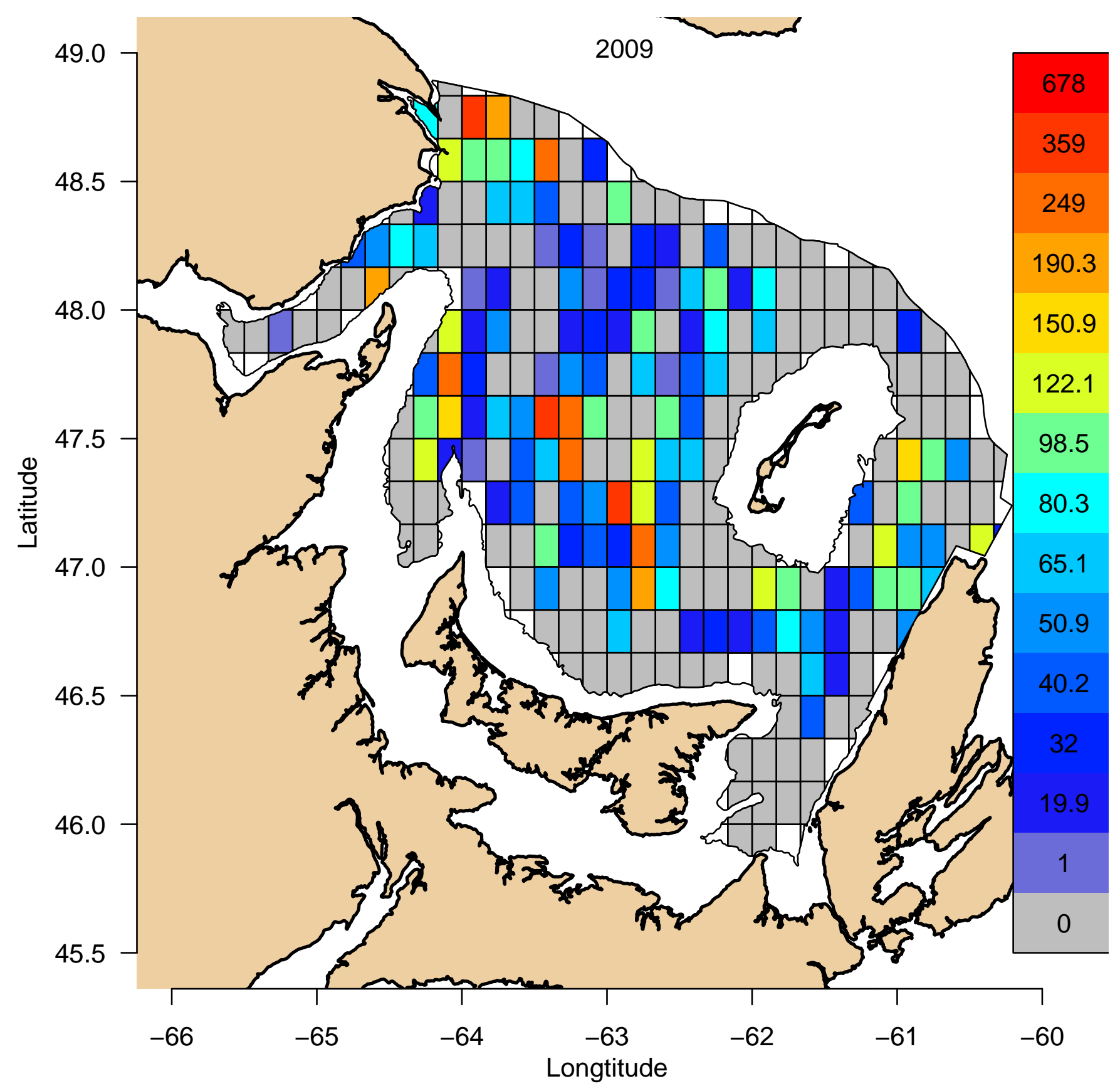

Figure SSEB.13. Snow crab survey exploitable biomass (shell conditions 3-5; tonnes per grid) results for 2009. Colors correspond to biomass levels, as indicated in the legend on the right-hand side. Darkest red grids indicate biomass $>98$ th percentile. 


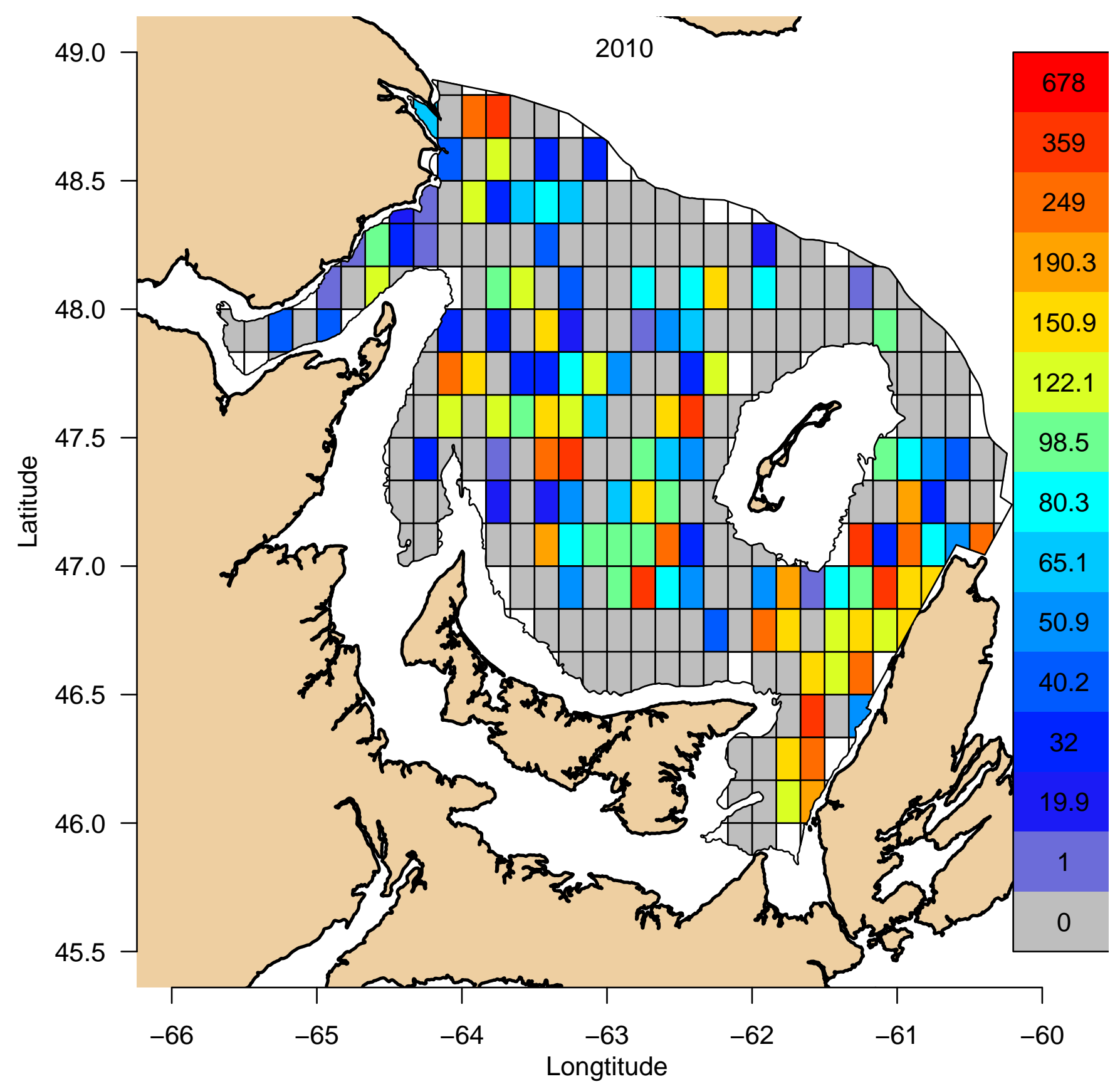

Figure SSEB.14. Snow crab survey exploitable biomass (shell conditions 3-5; tonnes per grid) results for 2010. Colors correspond to biomass levels, as indicated in the legend on the right-hand side. Darkest red grids indicate biomass $>98$ th percentile. 


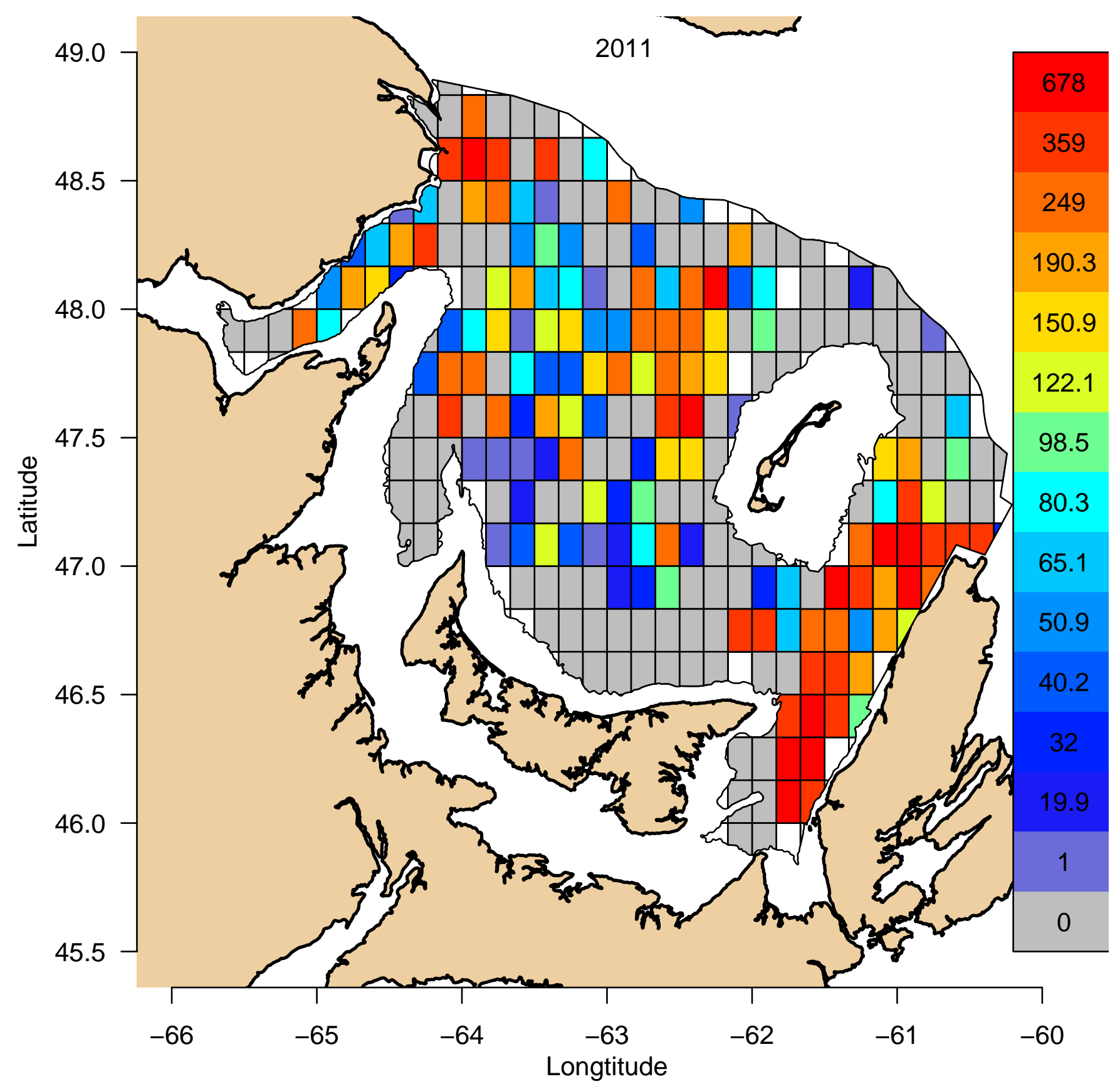

Figure SSEB.15. Snow crab survey exploitable biomass (shell conditions 3-5; tonnes per grid) results for 2011. Colors correspond to biomass levels, as indicated in the legend on the right-hand side. Darkest red grids indicate biomass $>98$ th percentile. 


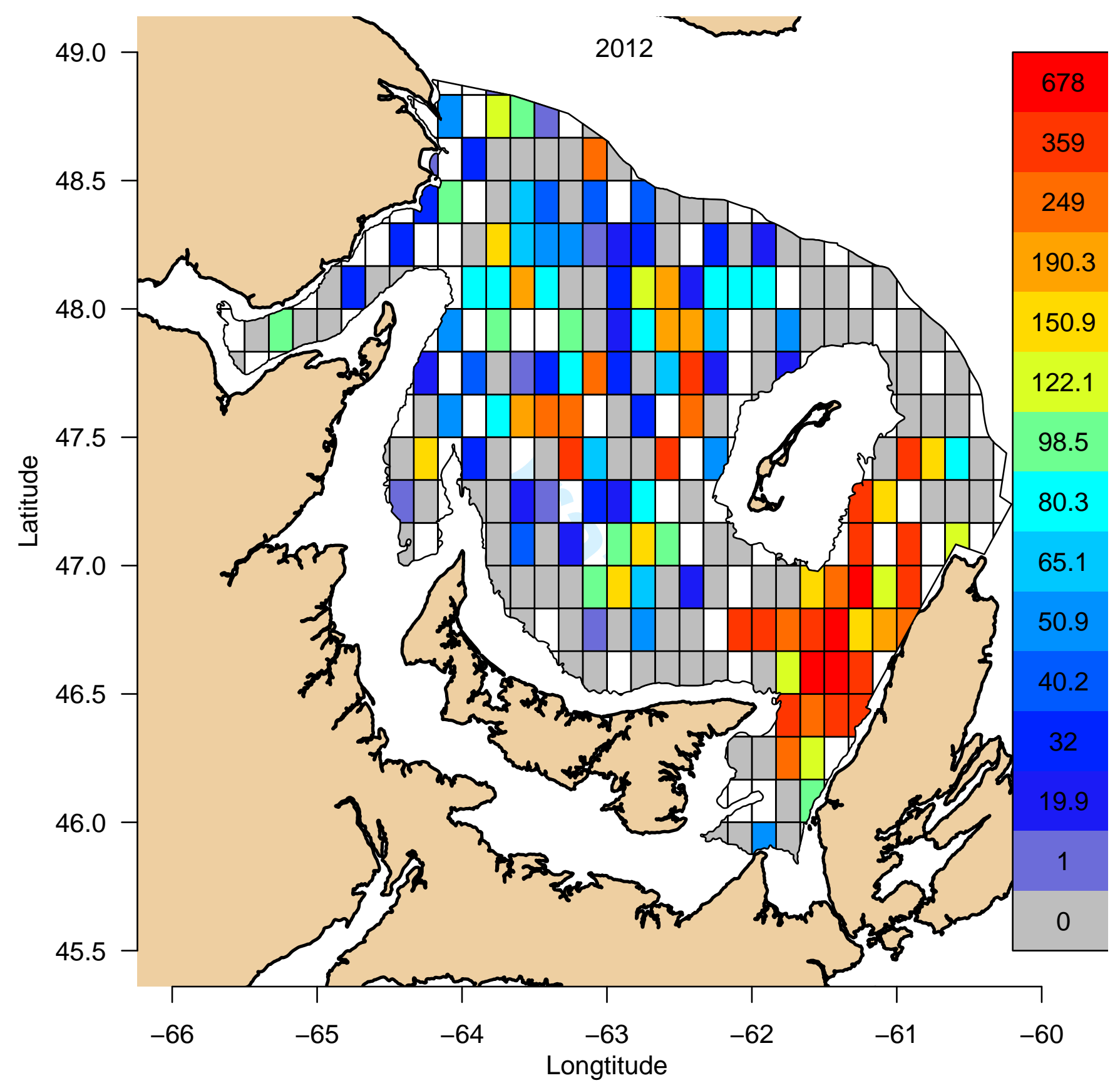

Figure SSEB.16. Snow crab survey exploitable biomass (shell conditions 3-5; tonnes per grid) results for 2012. Colors correspond to biomass levels, as indicated in the legend on the right-hand side. Darkest red grids indicate biomass $>98$ th percentile. 


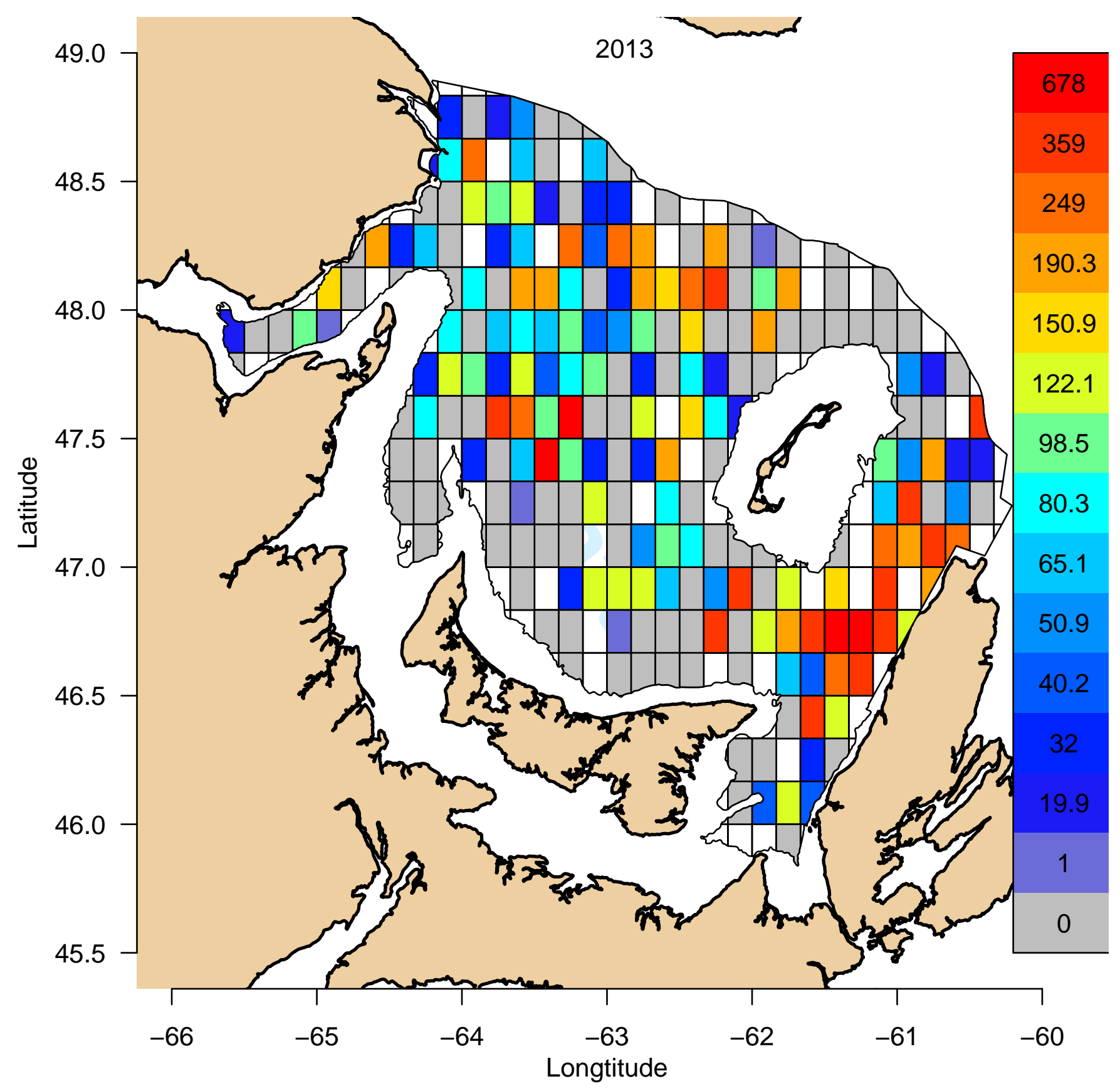

Figure SSEB.17. Snow crab survey exploitable biomass (shell conditions 3-5; tonnes per grid) results for 2013. Colors correspond to biomass levels, as indicated in the legend on the right-hand side. Darkest red grids indicate biomass $>98$ th percentile. 


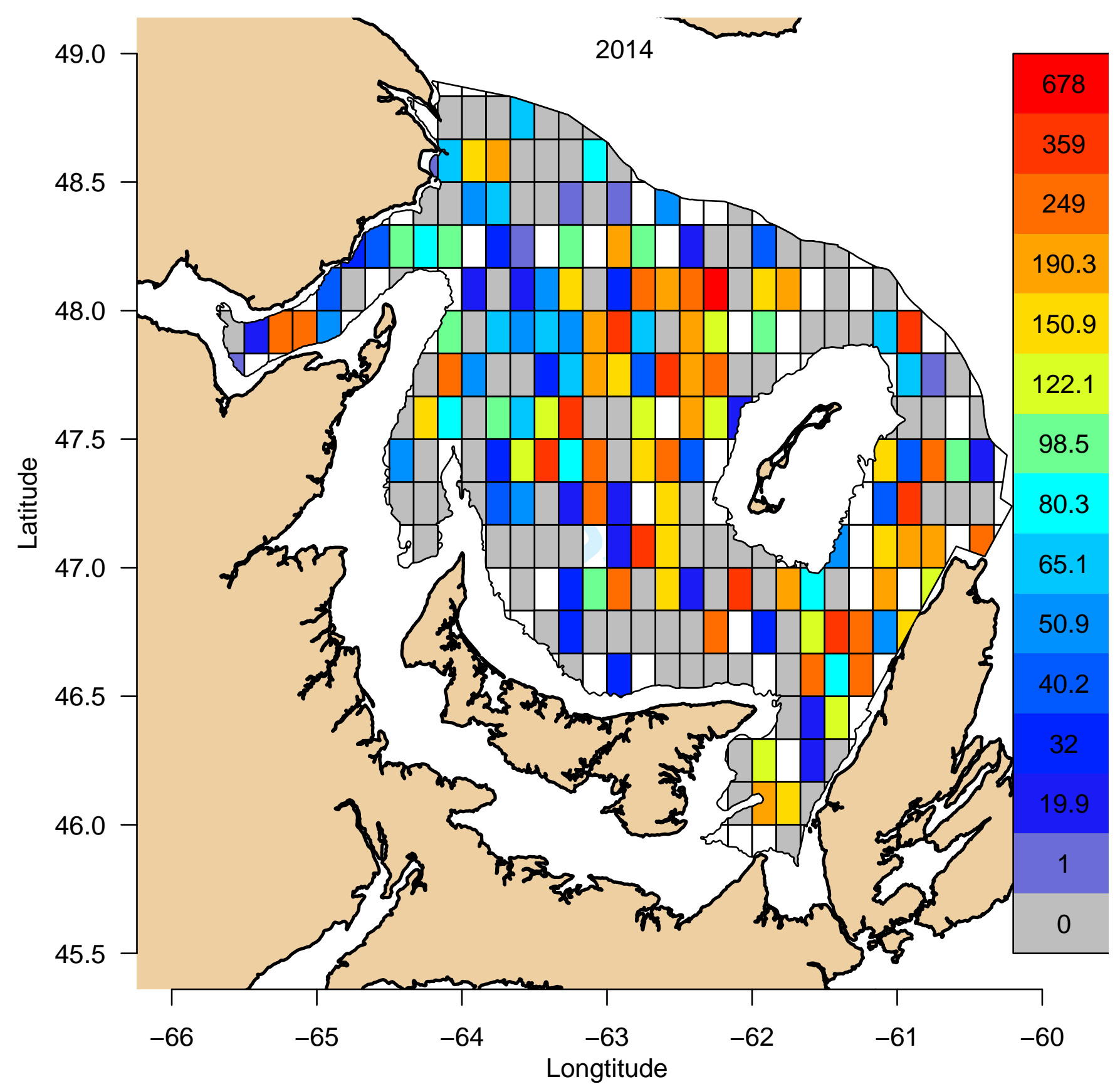

Figure SSEB.18. Snow crab survey exploitable biomass (shell conditions 3-5; tonnes per grid) results for 2014. Colors correspond to biomass levels, as indicated in the legend on the right-hand side. Darkest red grids indicate biomass $>98$ th percentile. 


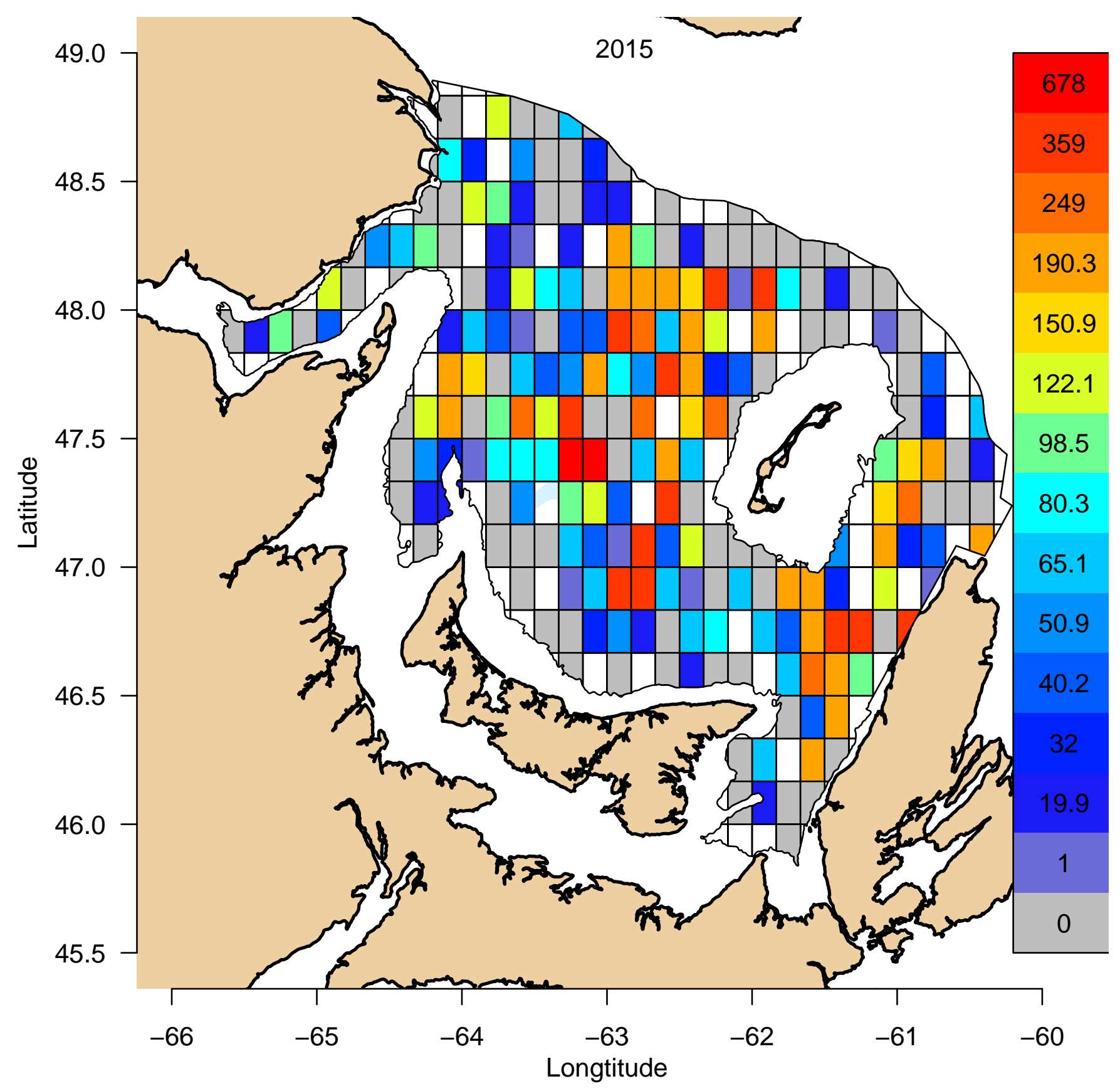

Figure SSEB.19. Snow crab survey exploitable biomass (shell conditions 3-5; tonnes per grid) results for 2015. Colors correspond to biomass levels, as indicated in the legend on the right-hand side. Darkest red grids indicate biomass $>98$ th percentile. 


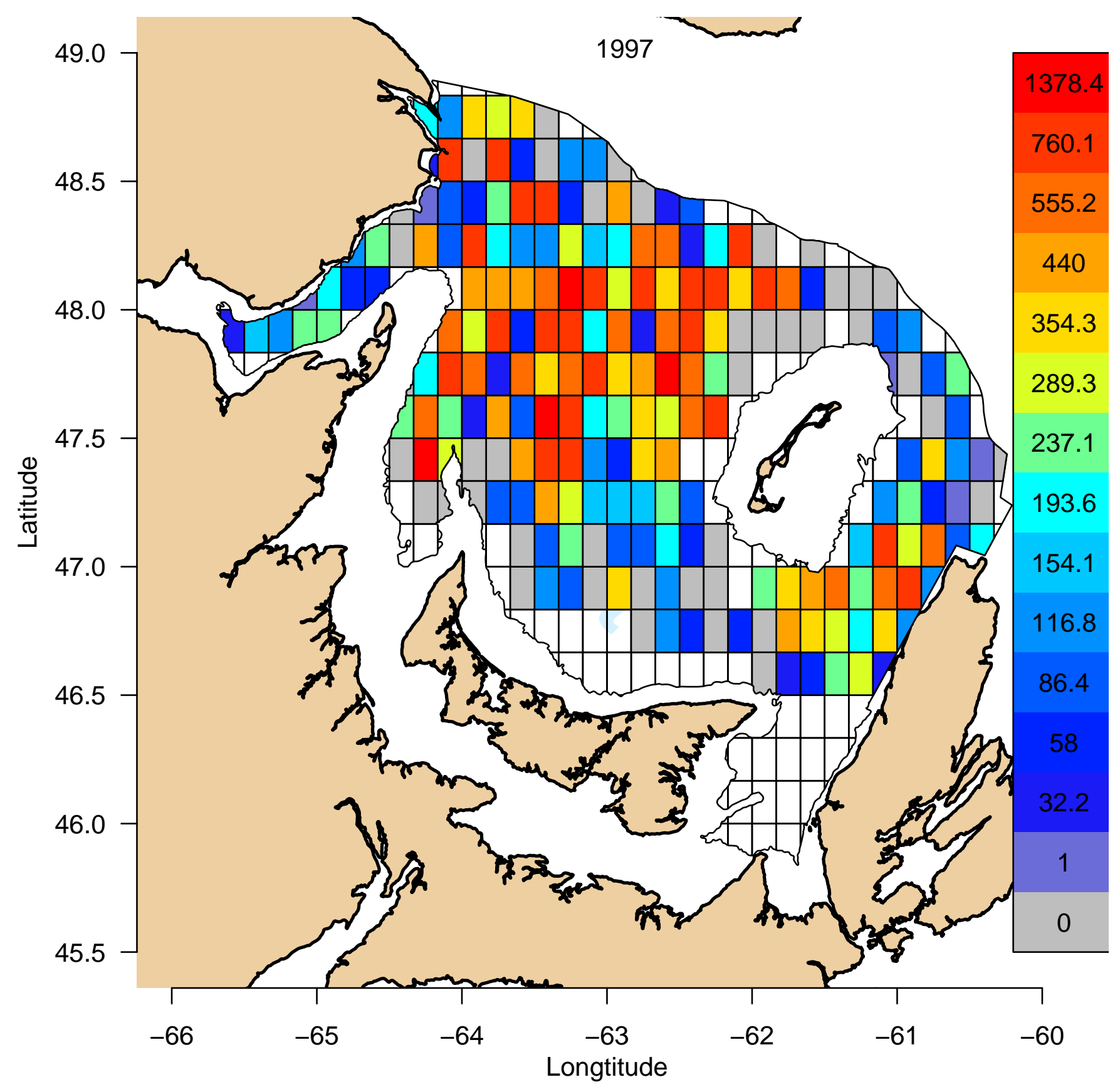

Figure SSTB.1. Snow crab survey total biomass (shell conditions 1-5; tonnes per grid) results for 1997. Colors correspond to biomass levels, as indicated in the legend on the right-hand side. Darkest red grids indicate biomass > 98th percentile. 


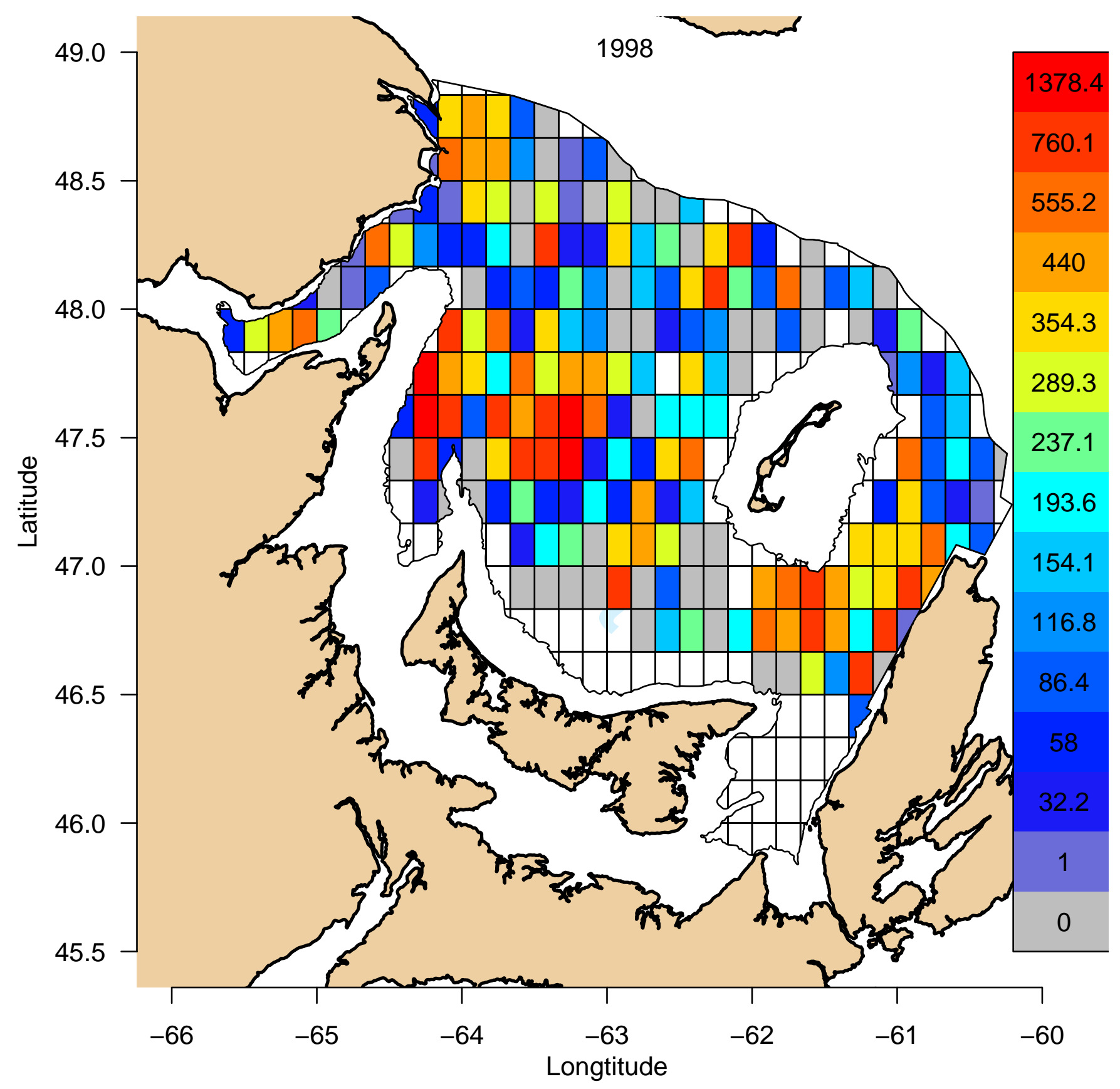

Figure SSTB.2. Snow crab survey total biomass (shell conditions 1-5; tonnes per grid) results for 1998. Colors correspond to biomass levels, as indicated in the legend on the right-hand side. Darkest red grids indicate biomass $>98$ th percentile. 


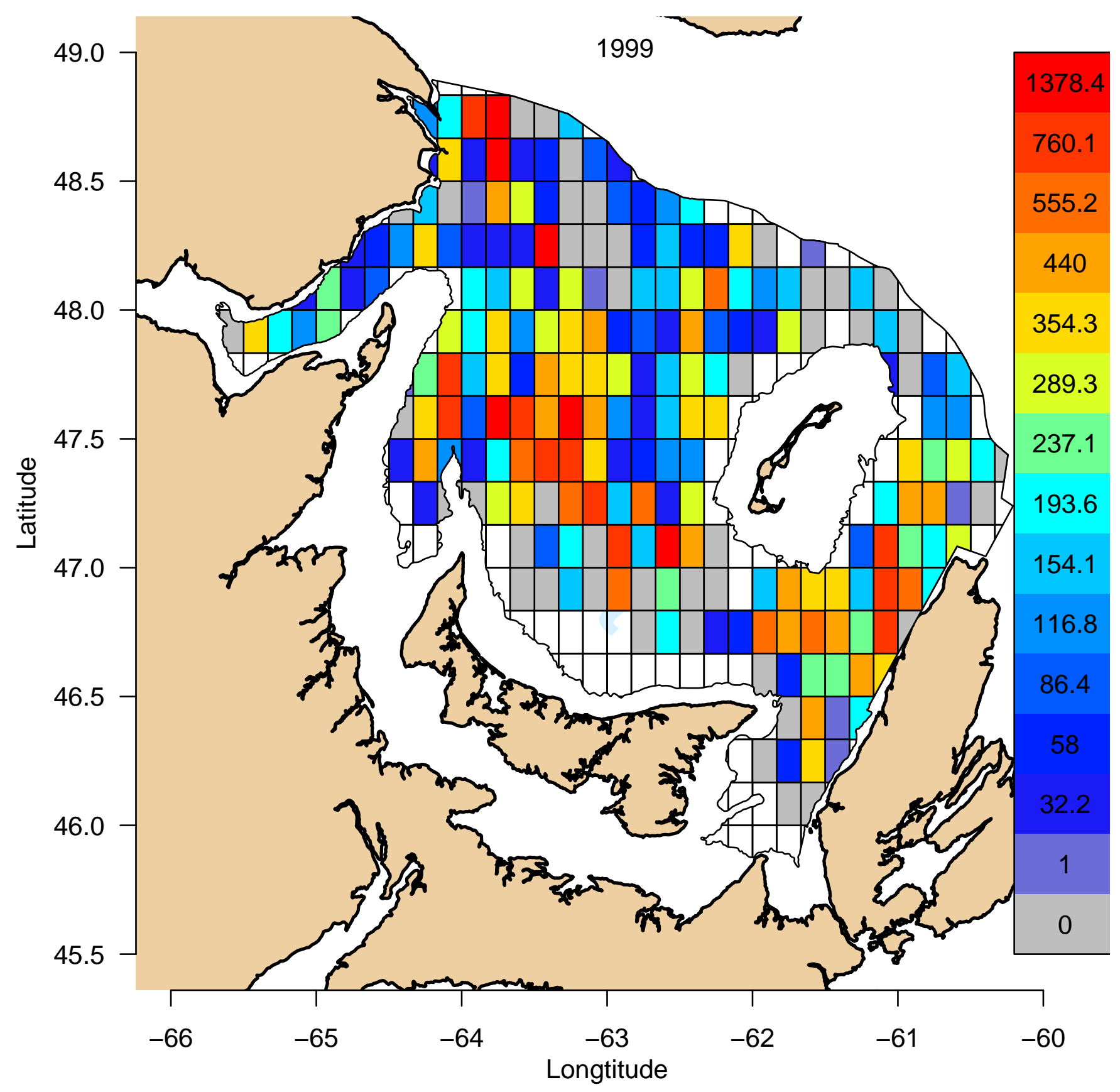

Figure SSTB.3. Snow crab survey total biomass (shell conditions 1-5; tonnes per grid) results for 1999. Colors correspond to biomass levels, as indicated in the legend on the right-hand side. Darkest red grids indicate biomass $>98$ th percentile. 


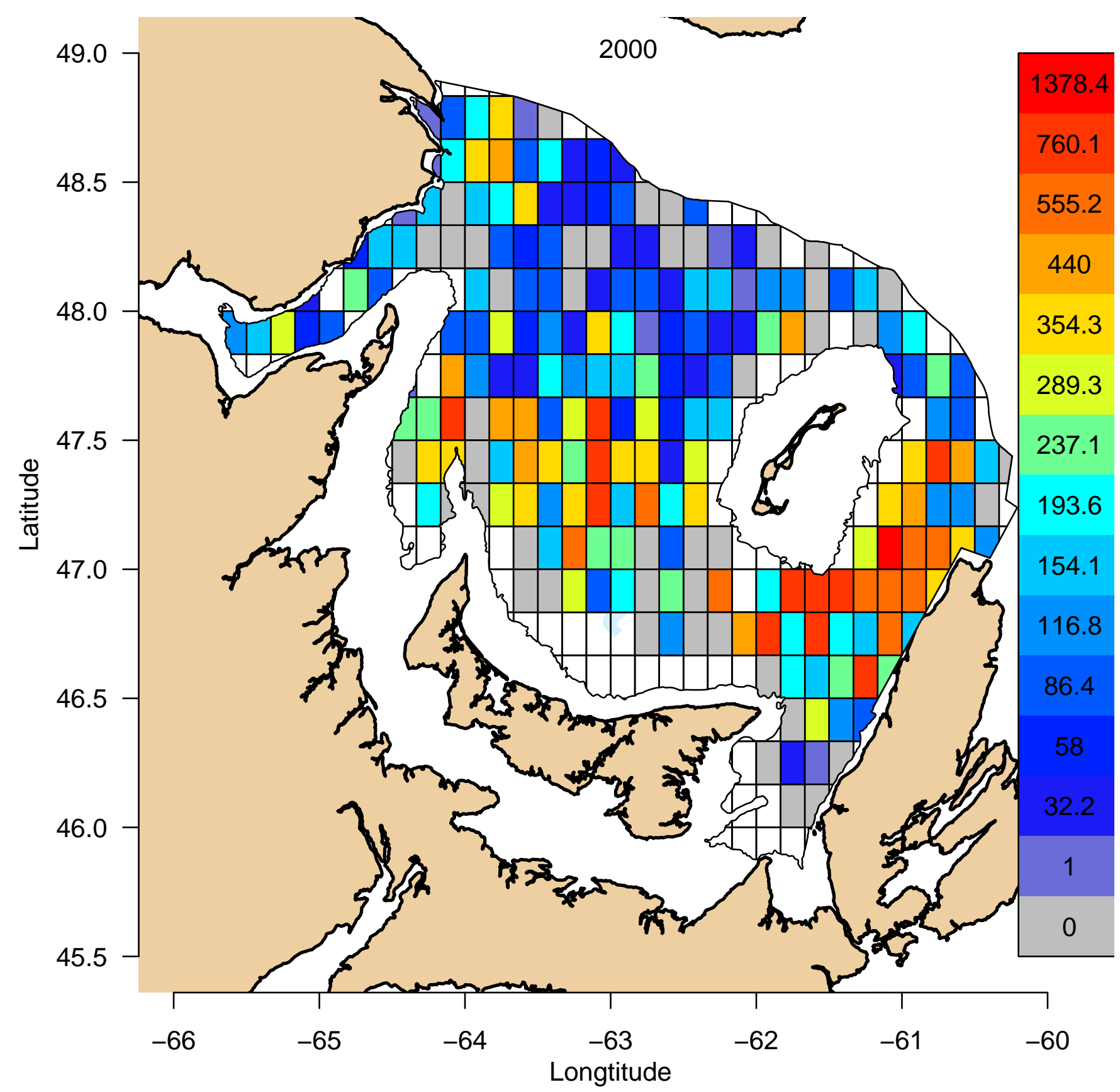

Figure SSTB.4. Snow crab survey total biomass (shell conditions 1-5; tonnes per grid) results for 2000. Colors correspond to biomass levels, as indicated in the legend on the right-hand side. Darkest red grids indicate biomass > 98th percentile. 


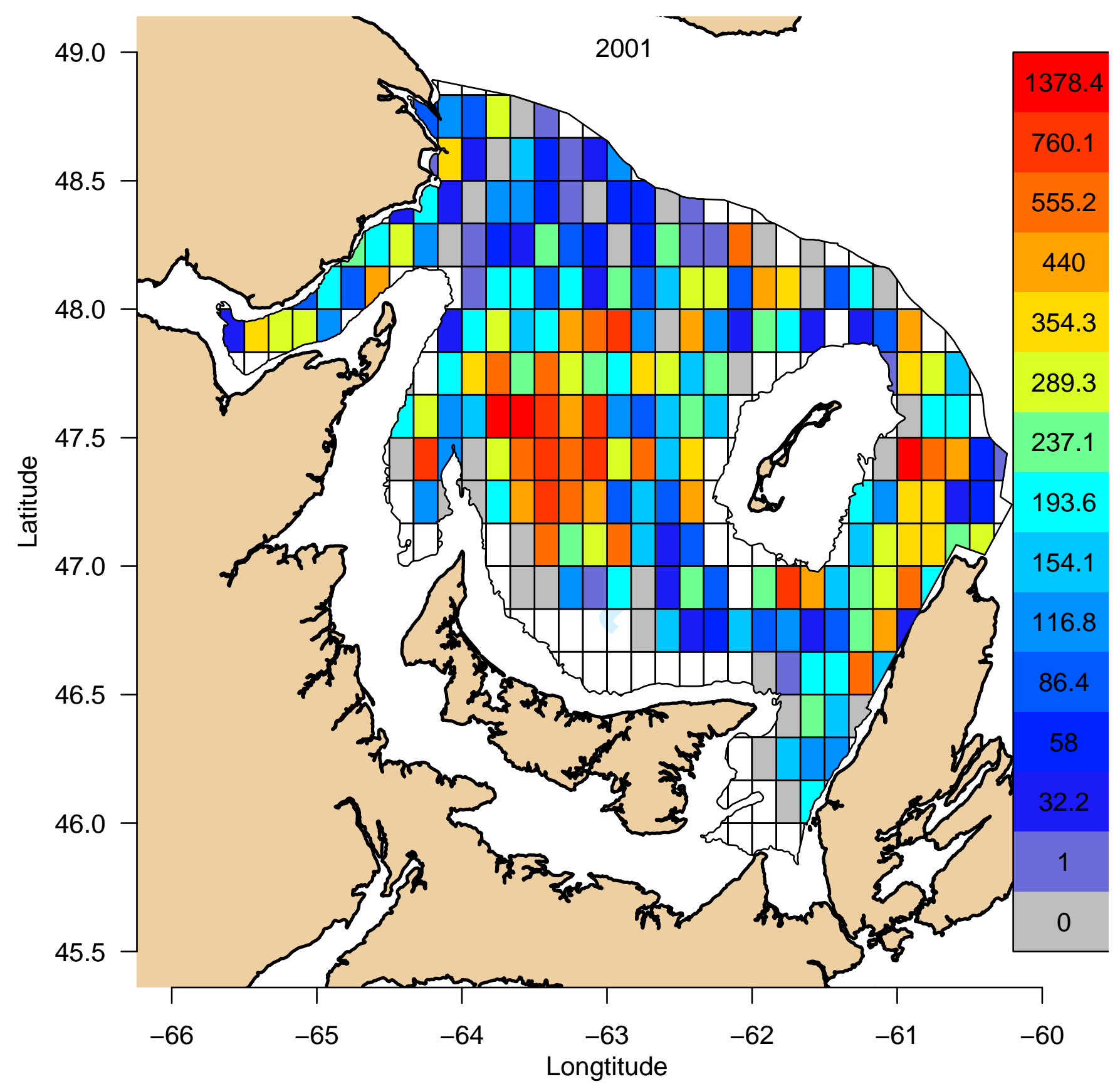

Figure SSTB.5. Snow crab survey total biomass (shell conditions 1-5; tonnes per grid) results for 2001. Colors correspond to biomass levels, as indicated in the legend on the right-hand side. Darkest red grids indicate biomass > 98th percentile. 


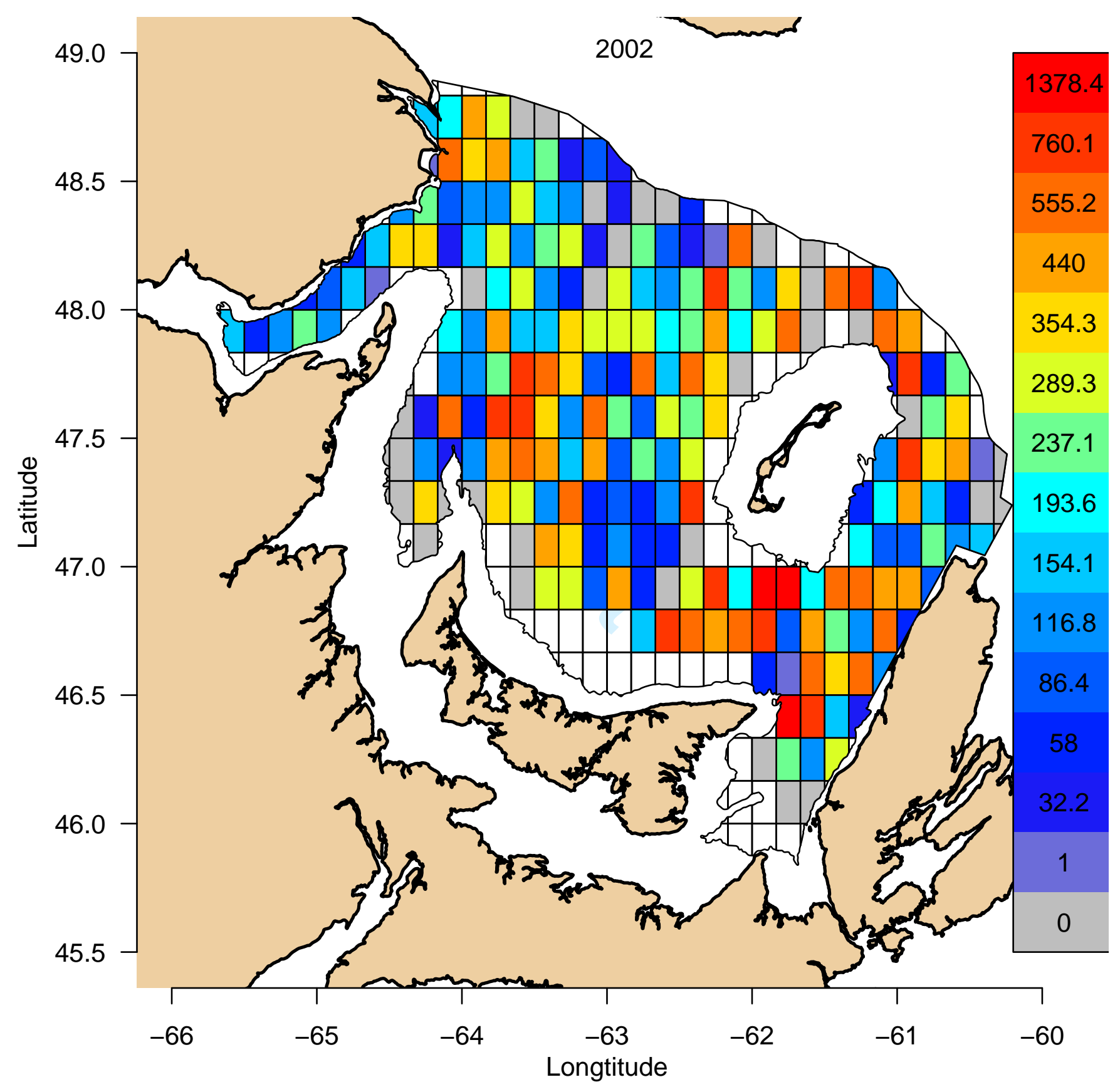

Figure SSTB.6. Snow crab survey total biomass (shell conditions 1-5; tonnes per grid) results for 2002. Colors correspond to biomass levels, as indicated in the legend on the right-hand side. Darkest red grids indicate biomass $>98$ th percentile. 


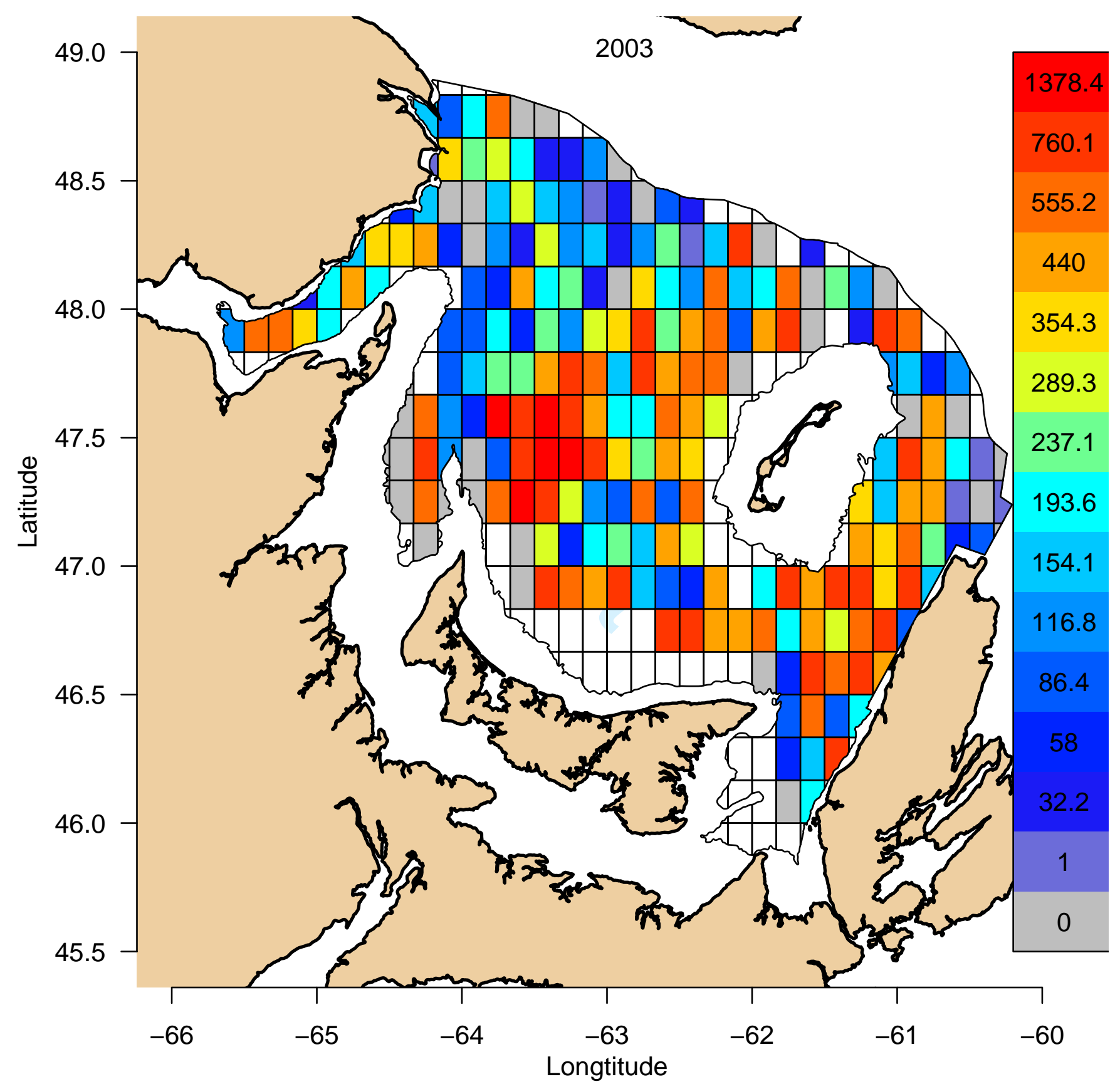

Figure SSTB.7. Snow crab survey total biomass (shell conditions 1-5; tonnes per grid) results for 2003. Colors correspond to biomass levels, as indicated in the legend on the right-hand side. Darkest red grids indicate biomass $>98$ th percentile. 


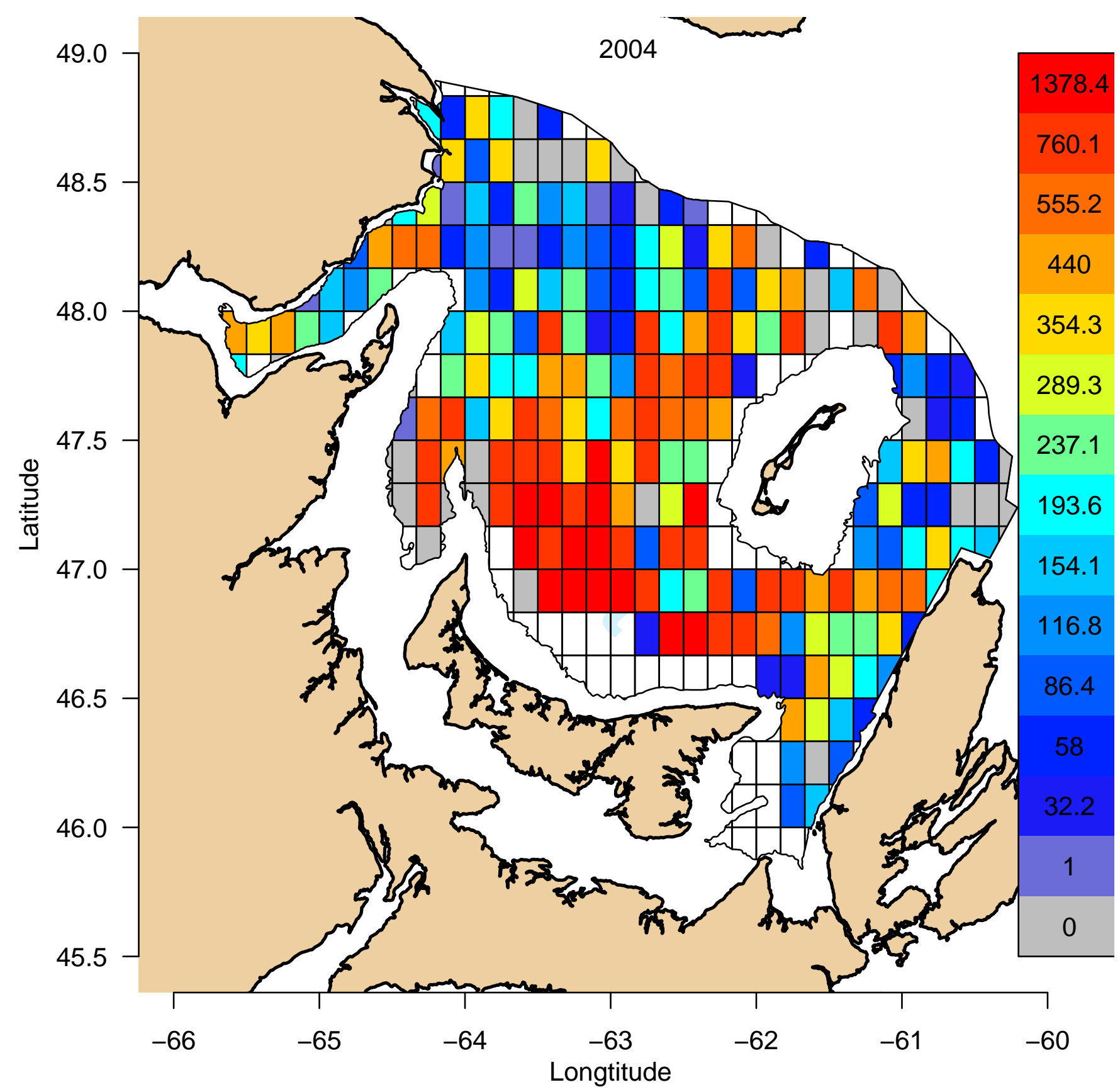

Figure SSTB.8. Snow crab survey total biomass (shell conditions 1-5; tonnes per grid) results for 2004. Colors correspond to biomass levels, as indicated in the legend on the right-hand side. Darkest red grids indicate biomass > 98th percentile. 


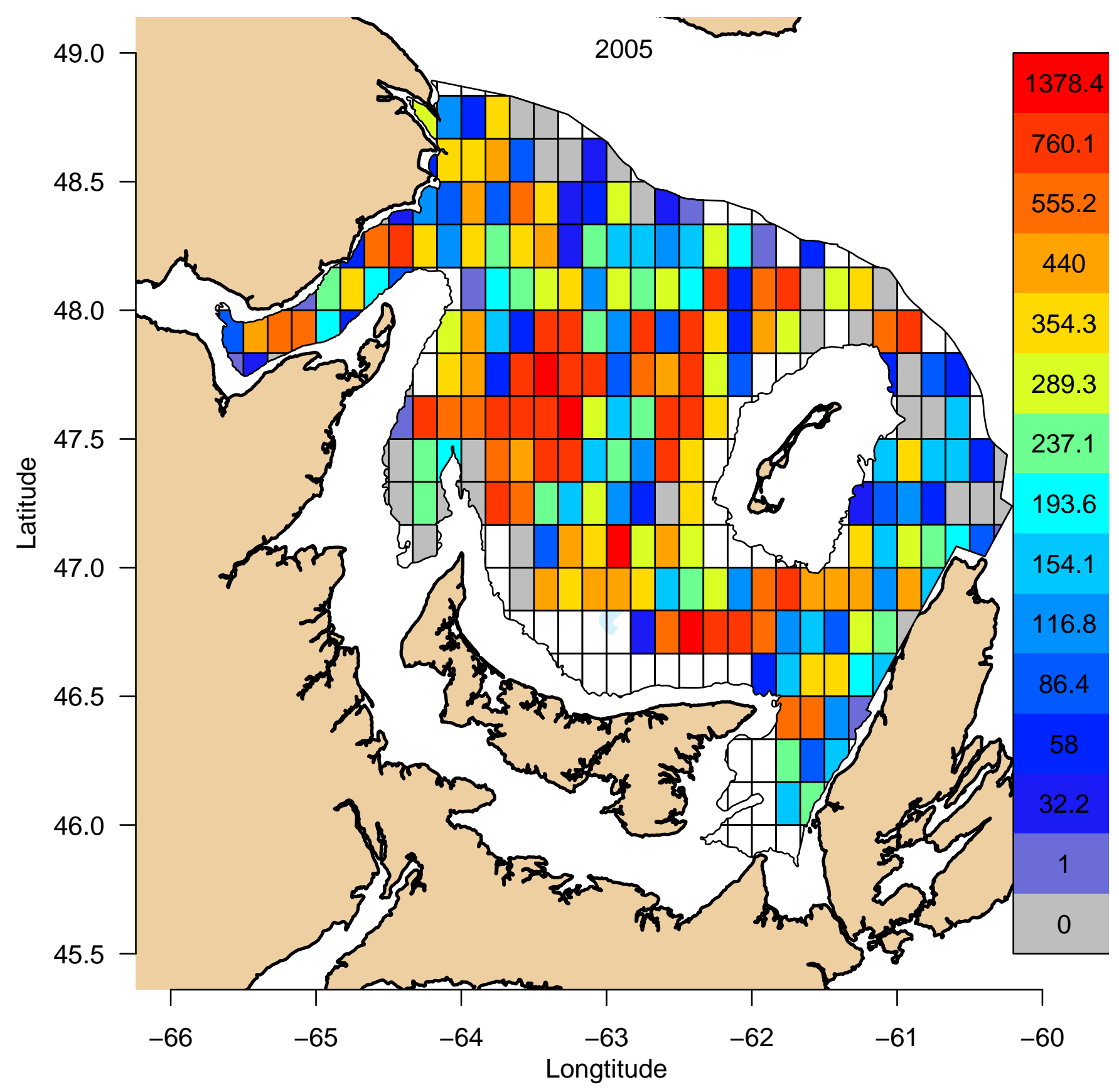

Figure SSTB.9. Snow crab survey total biomass (shell conditions 1-5; tonnes per grid) results for 2005. Colors correspond to biomass levels, as indicated in the legend on the right-hand side. Darkest red grids indicate biomass > 98th percentile. 


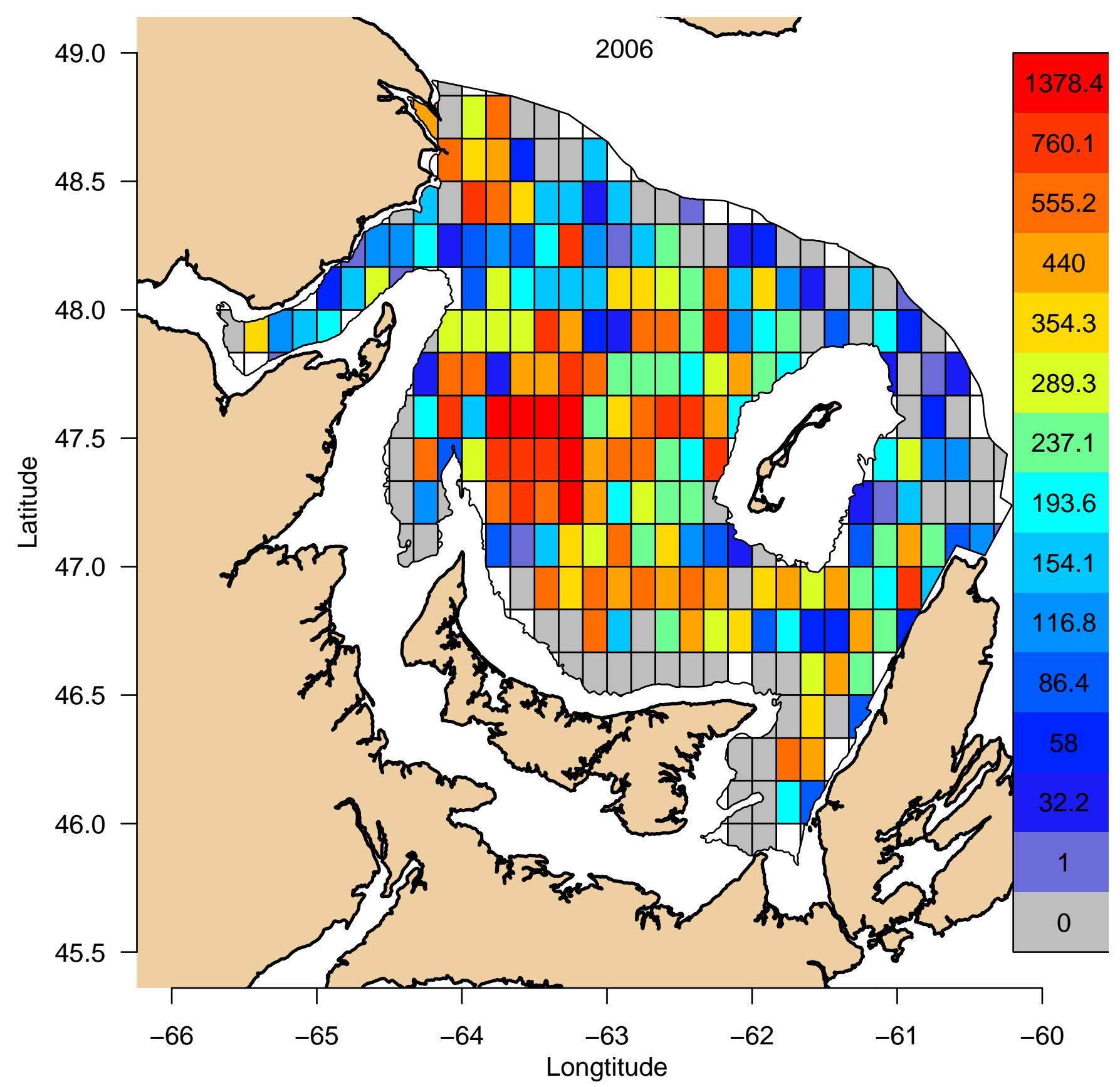

Figure SSTB.10. Snow crab survey total biomass (shell conditions 1-5; tonnes per grid) results for 2006. Colors correspond to biomass levels, as indicated in the legend on the right-hand side. Darkest red grids indicate biomass $>98$ th percentile. 


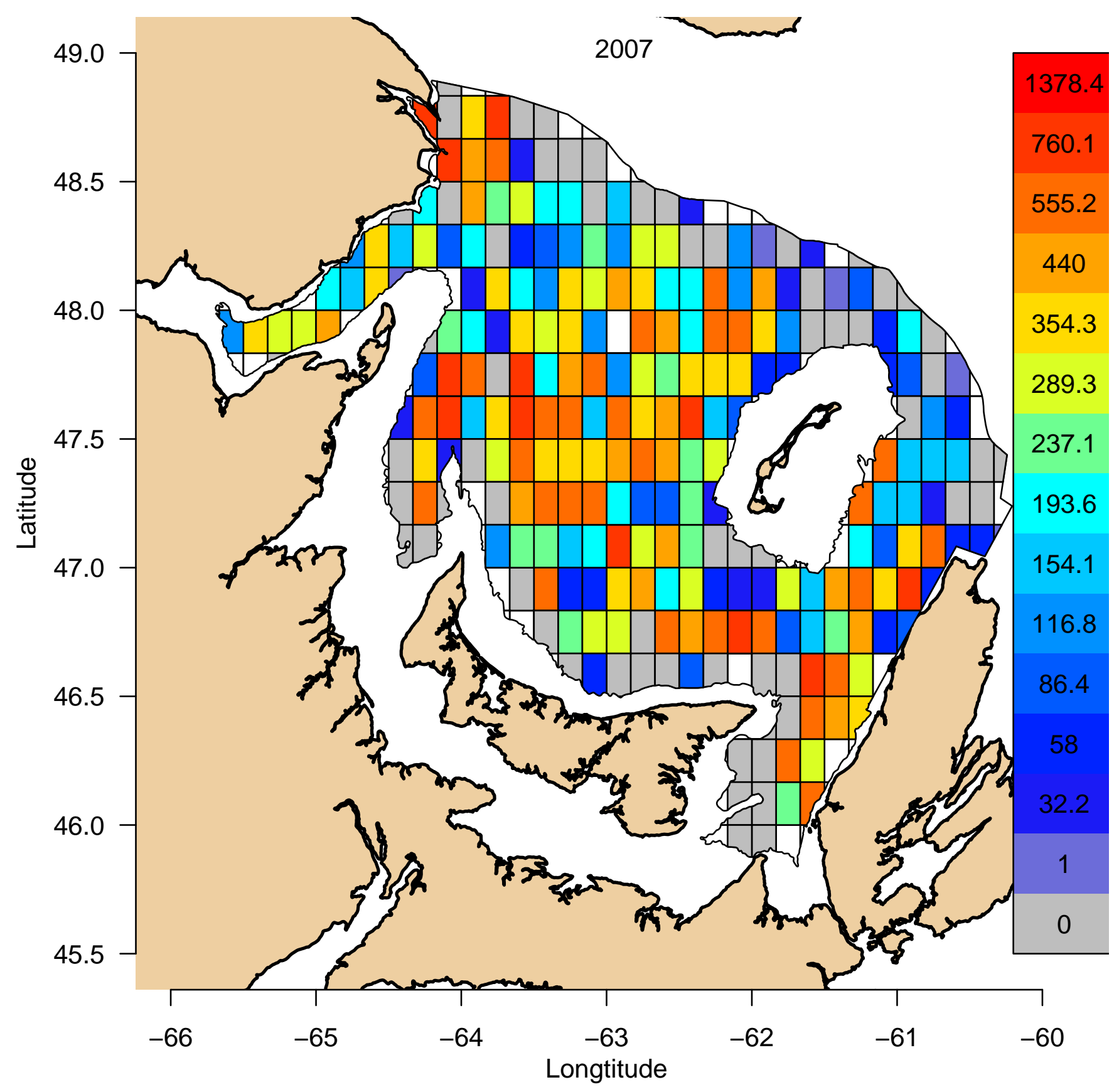

Figure SSTB.11. Snow crab survey total biomass (shell conditions 1-5; tonnes per grid) results for 2007. Colors correspond to biomass levels, as indicated in the legend on the right-hand side. Darkest red grids indicate biomass $>98$ th percentile. 


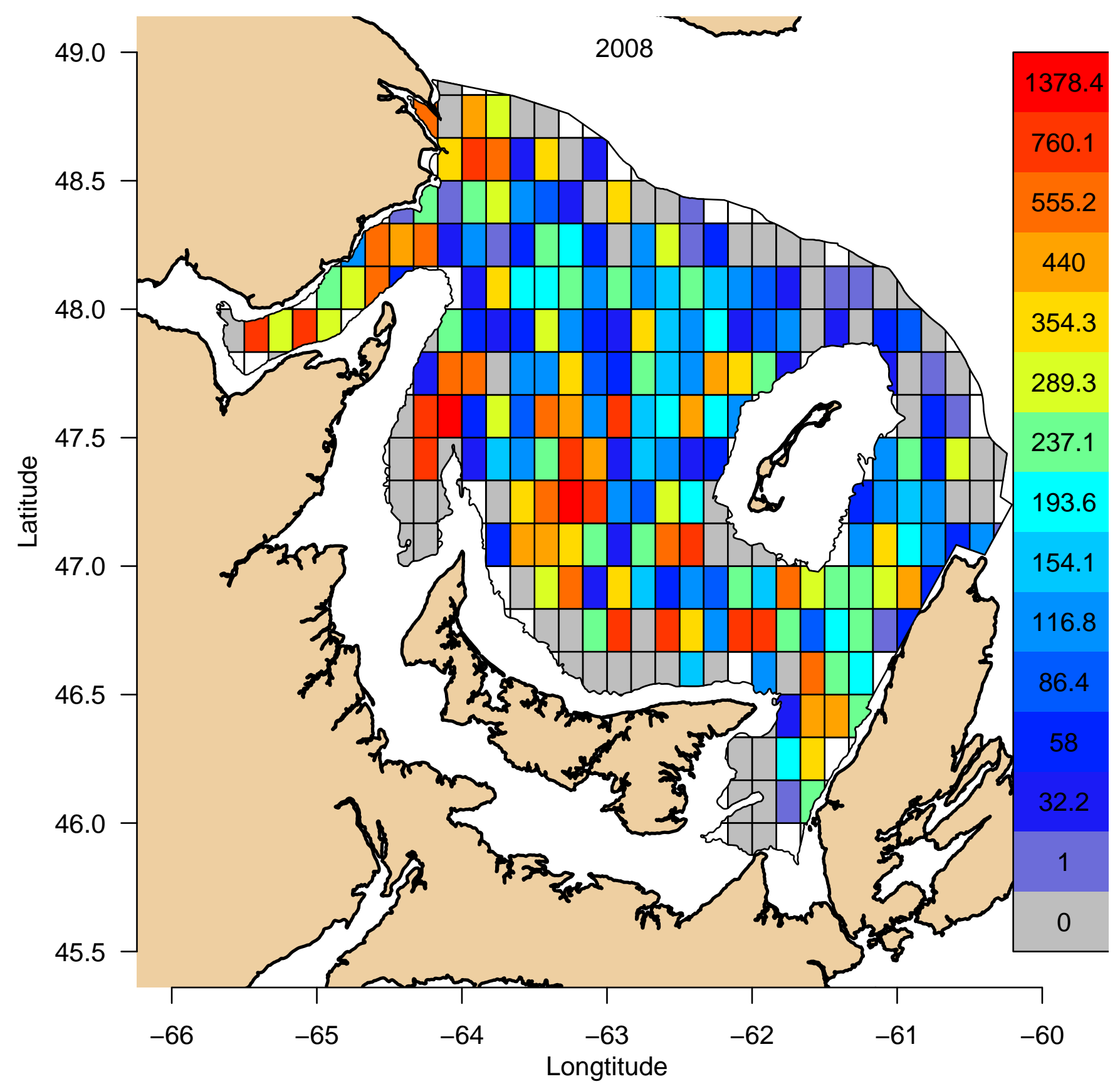

Figure SSTB.12. Snow crab survey total biomass (shell conditions 1-5; tonnes per grid) results for 2008. Colors correspond to biomass levels, as indicated in the legend on the right-hand side. Darkest red grids indicate biomass > 98th percentile. 


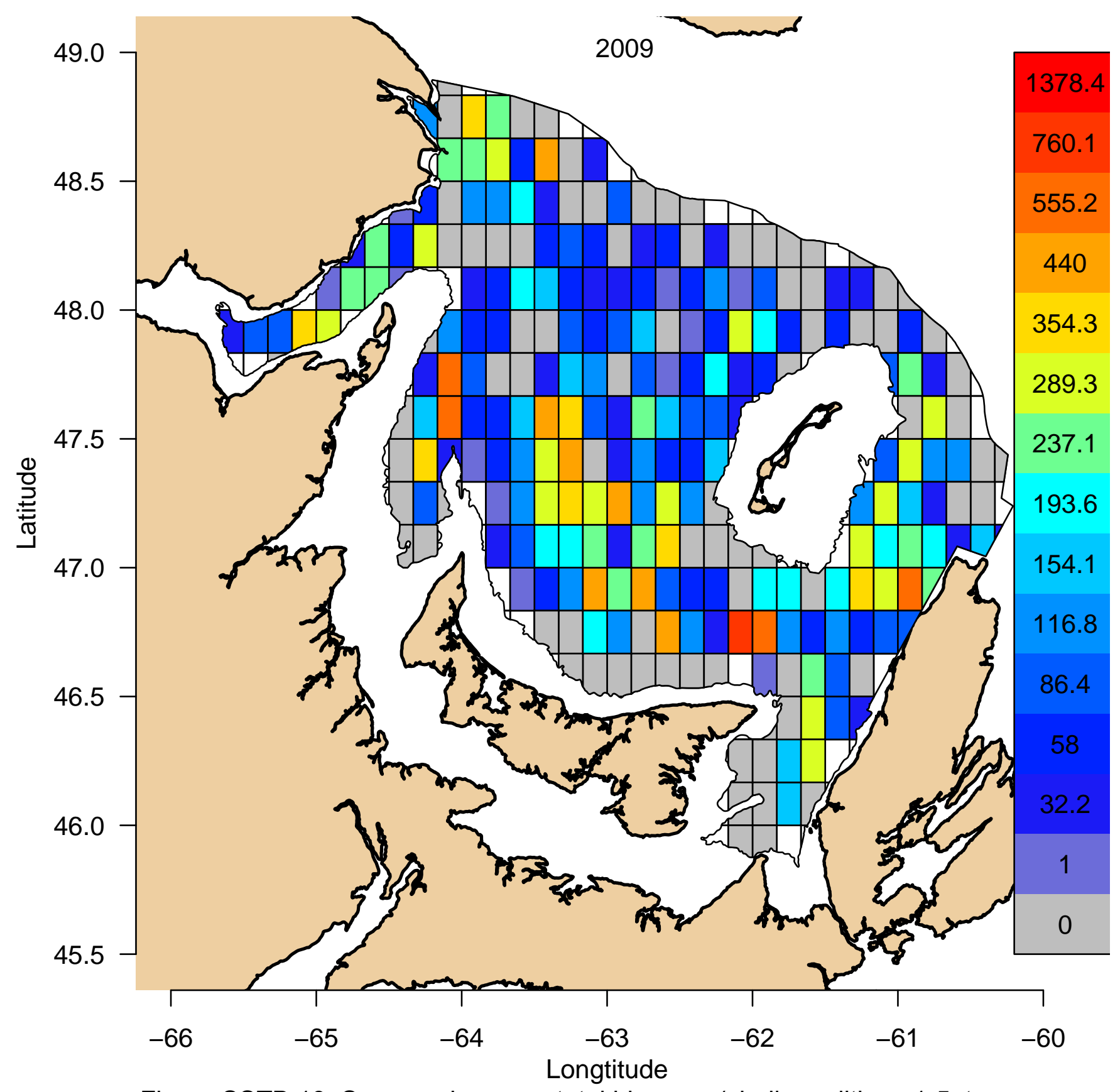

Figure SSTB.13. Snow crab survey total biomass (shell conditions 1-5; tonnes per grid) results for 2009. Colors correspond to biomass levels, as indicated in the legend on the right-hand side. Darkest red grids indicate biomass $>98$ th percentile. 


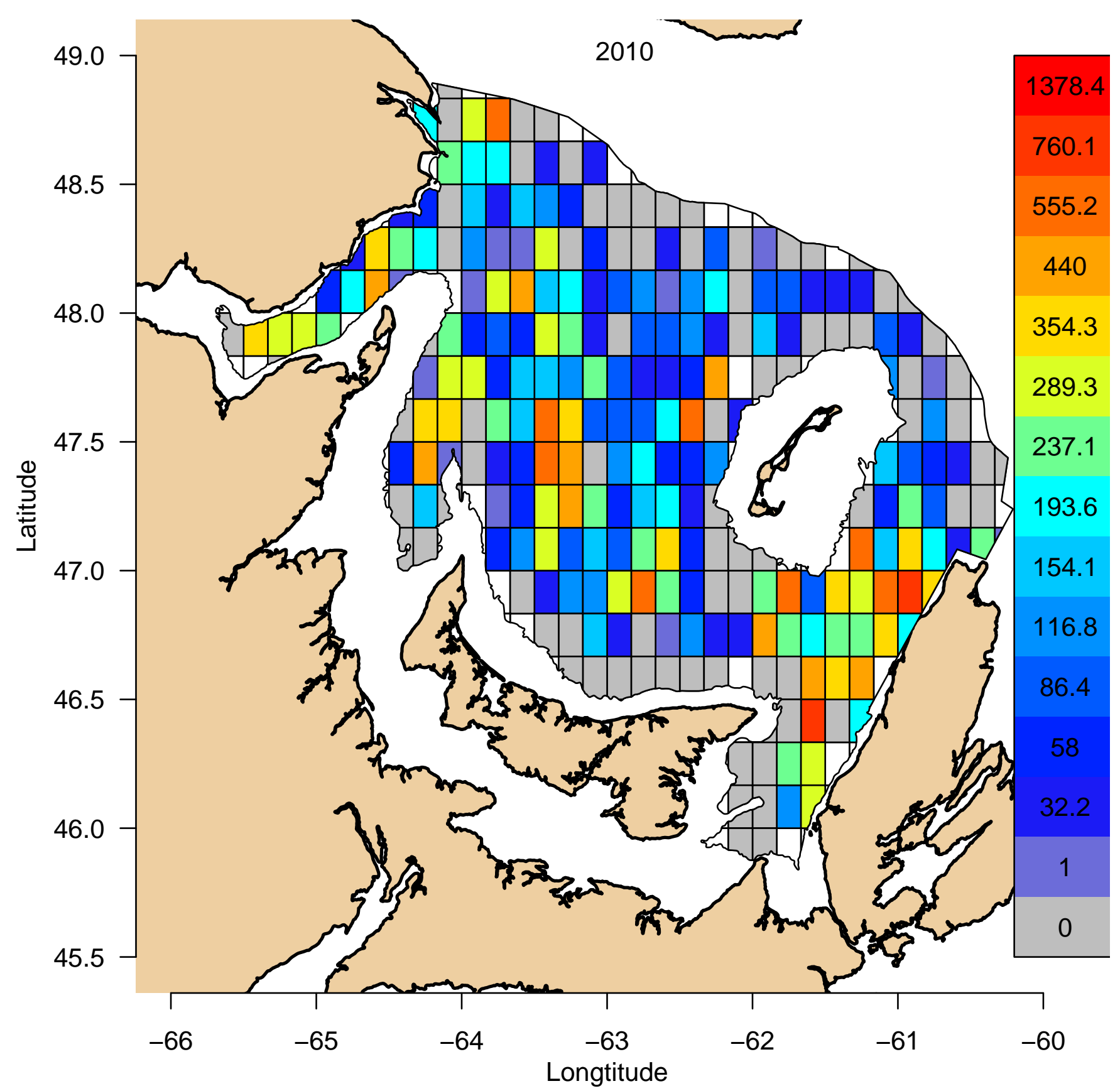

Figure SSTB.14. Snow crab survey total biomass (shell conditions 1-5; tonnes per grid) results for 2010. Colors correspond to biomass levels, as indicated in the legend on the right-hand side. Darkest red grids indicate biomass $>98$ th percentile. 


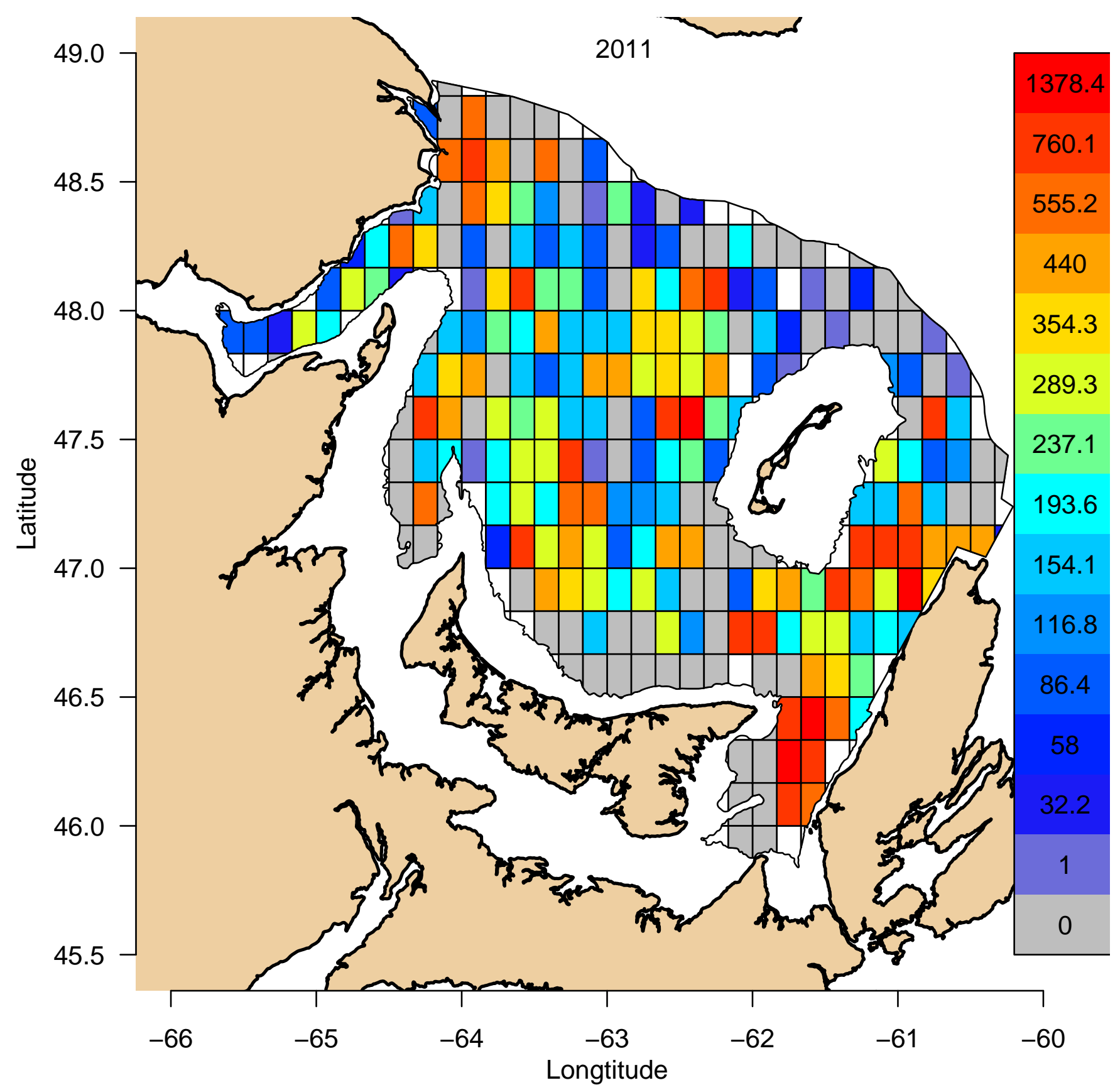

Figure SSTB.15. Snow crab survey total biomass (shell conditions 1-5; tonnes per grid) results for 2011. Colors correspond to biomass levels, as indicated in the legend on the right-hand side. Darkest red grids indicate biomass $>98$ th percentile. 


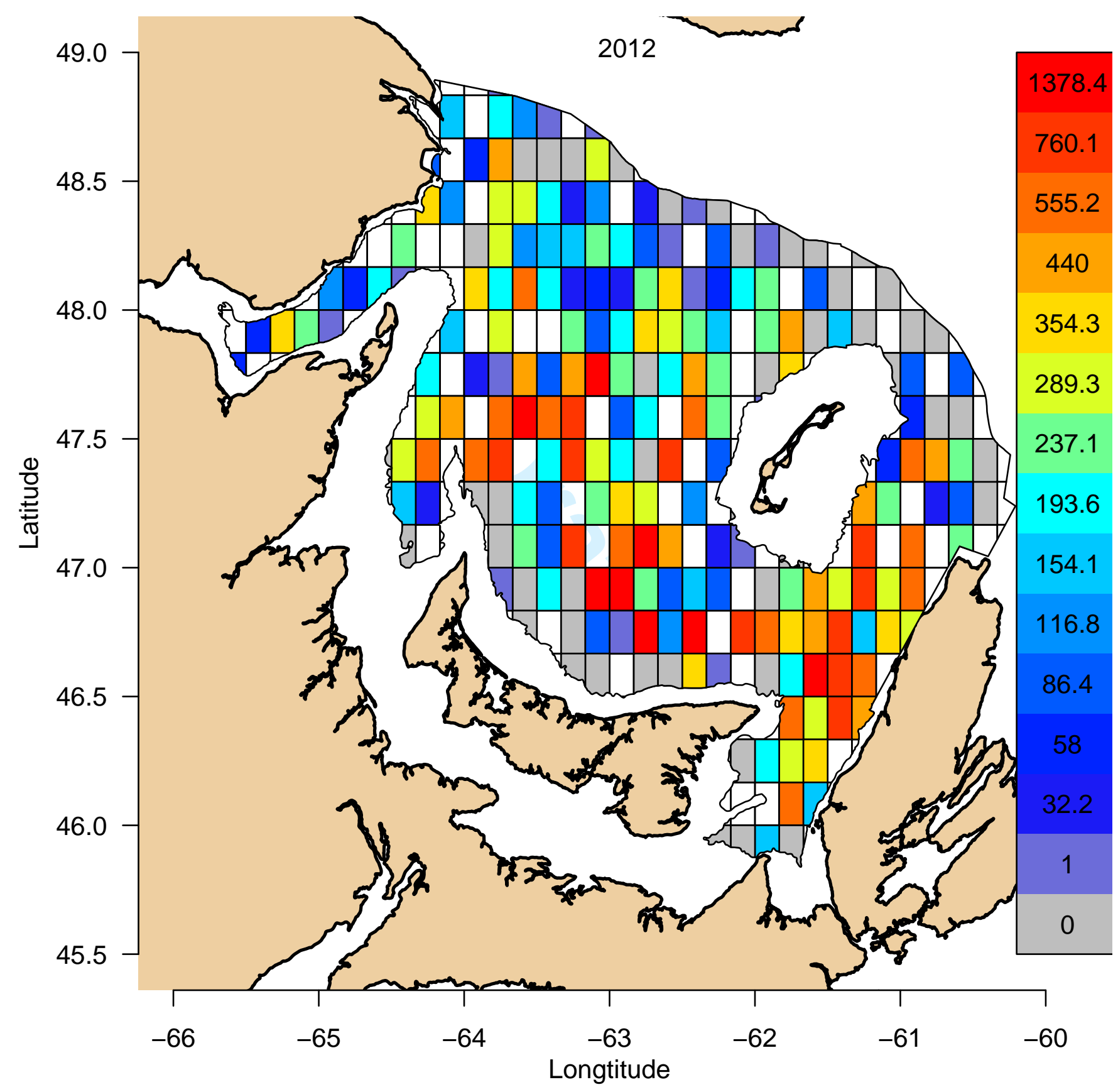

Figure SSTB.16. Snow crab survey total biomass (shell conditions 1-5; tonnes per grid) results for 2012. Colors correspond to biomass levels, as indicated in the legend on the right-hand side. Darkest red grids indicate biomass $>98$ th percentile. 


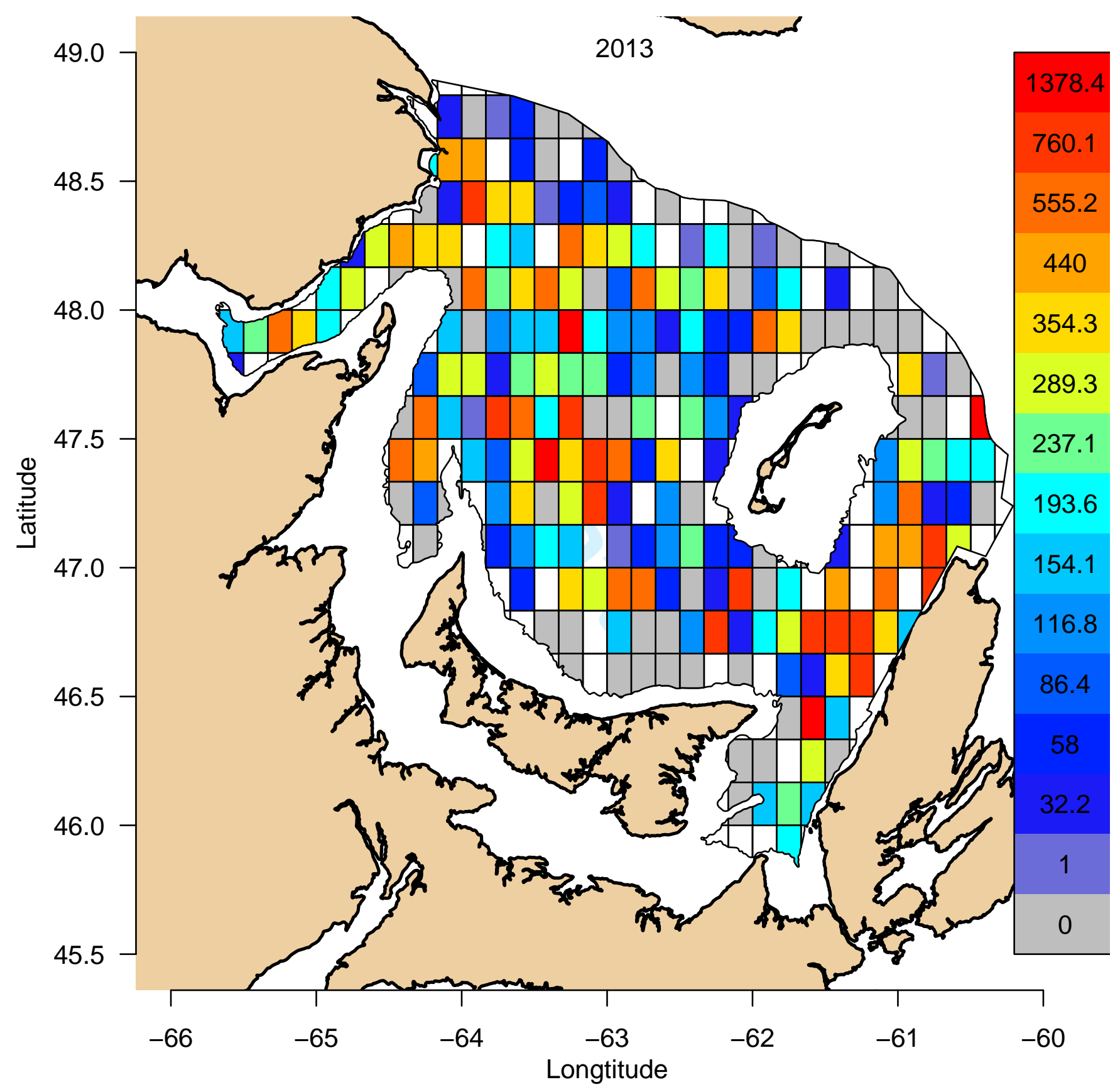

Figure SSTB.17. Snow crab survey total biomass (shell conditions 1-5; tonnes per grid) results for 2013. Colors correspond to biomass levels, as indicated in the legend on the right-hand side. Darkest red grids indicate biomass > 98th percentile. 


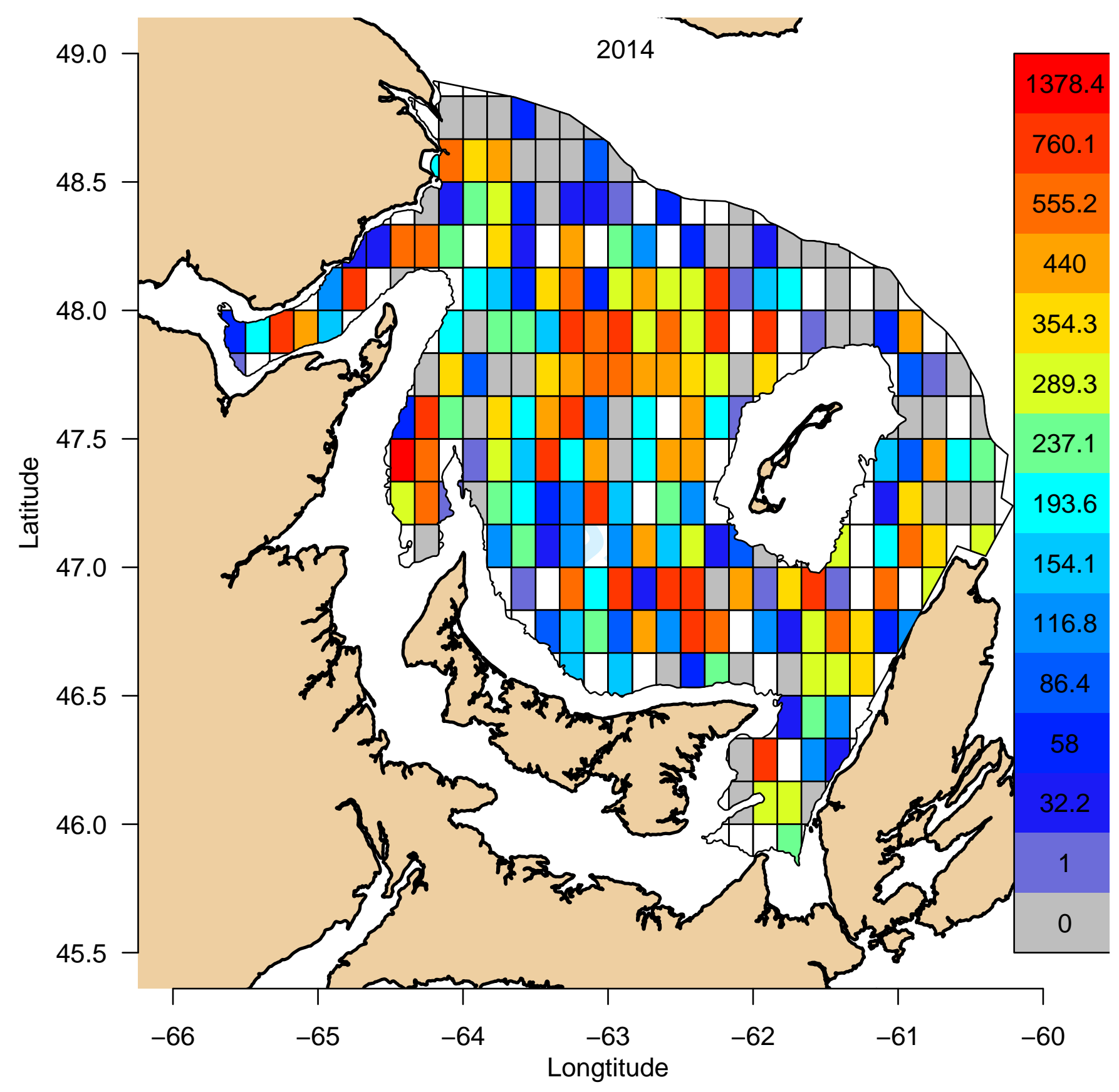

Figure SSTB.18. Snow crab survey total biomass (shell conditions 1-5; tonnes per grid) results for 2014. Colors correspond to biomass levels, as indicated in the legend on the right-hand side. Darkest red grids indicate biomass $>98$ th percentile. 


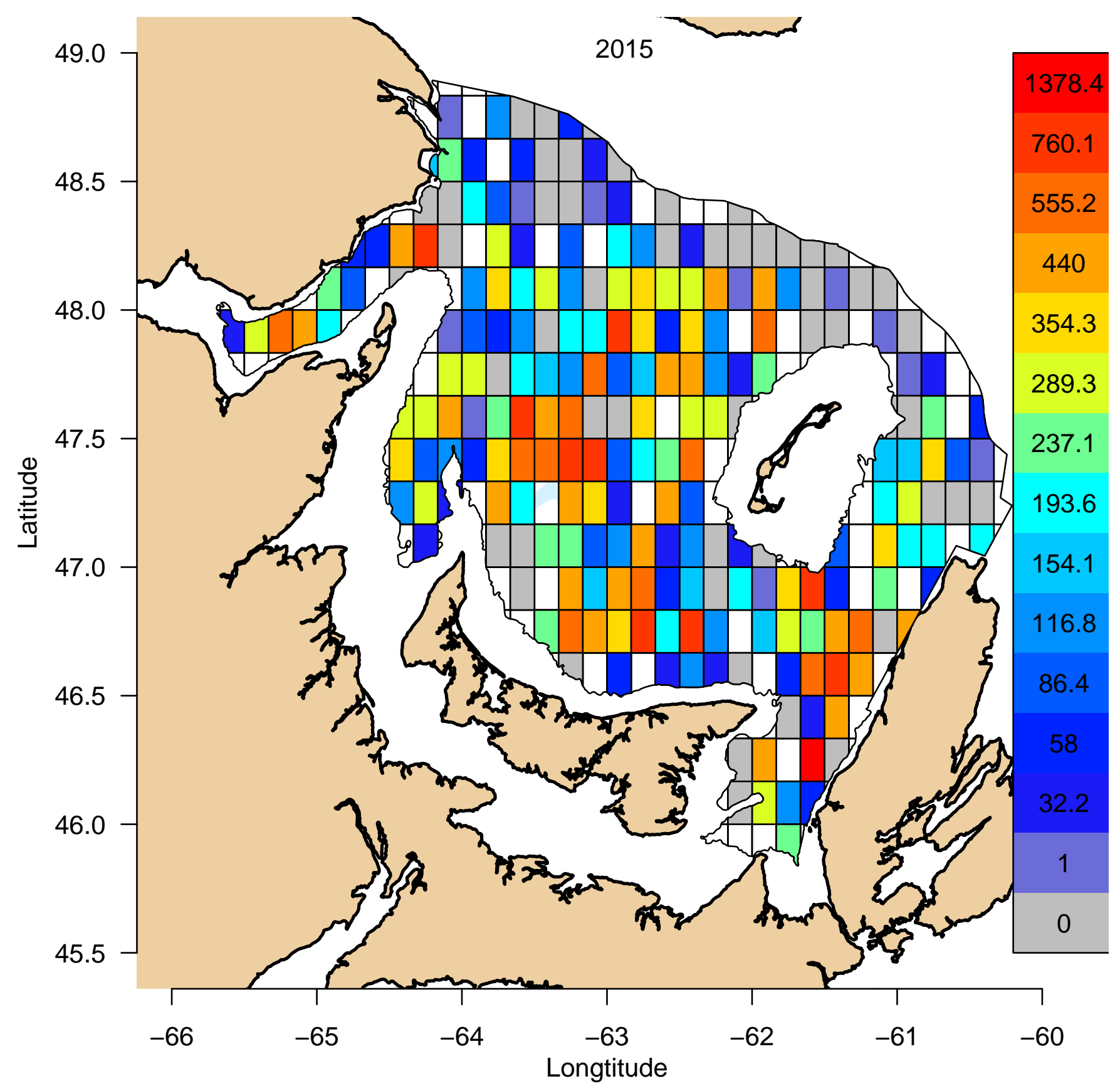

Figure SSTB.19. Snow crab survey total biomass (shell conditions 1-5; tonnes per grid) results for 2015. Colors correspond to biomass levels, as indicated in the legend on the right-hand side. Darkest red grids indicate biomass $>98$ th percentile. 